\title{
Catalytic [5 + 1]-Cycloadditions of Vinylcyclopropanes and Vinylidenes
}

Conner M. Farley, Kohei Sasakura, You-Yun Zhou, Vibha V. Kanale and Christopher Uyeda Department of Chemistry, Purdue University, West Lafayette, IN 47907, United States

Correspondence: cuyeda@purdue.edu

\section{Supporting Information}

1. General Information

2. Reaction Optimization Studies S4

3. Synthesis and Characterization of Vinylcyclopropane Substrates S7

4. Synthesis and Characterization of Vinylidene Substrates

5. Substrate Scope Studies and Product Characterization

6. $[5+1]$-Product Derivatization

S38

7. Mechanistic Studies

8. NMR Data for Vinylcyclopropanes and Vinylidenes

9. NMR Data for $[5+1]$-Products

10. IR Data for Vinylcyclopropanes and [5+1]-Products 


\section{General Information}

General considerations. Solvents were degassed and stored over activated $3 \AA$ molecular sieves prior to use. Deuterated solvents were purchased from Cambridge Isotope Laboratories, degassed, and stored over activated $3 \AA$ molecular sieves. Liquid reagents were degassed and stored over activated $3 \AA$ molecular sieves prior to use. Zn powder (325 mesh, 99.9\%) was purchased from Strem Chemicals, stored under inert atmosphere, and used without further purification. The (i-prNDI) $\mathrm{Ni}_{2}\left(\mathrm{C}_{6} \mathrm{H}_{6}\right)$ complex was prepared according to a previously reported procedure. ${ }^{1}$ The $( \pm)-t$-Bu-Quinox ligand was prepared according to a previously reported procedure. ${ }^{2}$ Unless otherwise noted, all 1,1-dichloroalkenes were prepared according to previously reported procedures.3,4 All other reagents and starting materials were purchased from commercial vendors and used without further purification unless otherwise noted.

Physical methods. ${ }^{1} \mathrm{H},{ }^{19} \mathrm{~F}$ and ${ }^{13} \mathrm{C}\left\{{ }^{1} \mathrm{H}\right\}$ NMR spectra were collected at room temperature on a Varian INOVA $300 \mathrm{MHz}$ or a Bruker AV-III-800 NMR spectrometer. ${ }^{1} \mathrm{H}$ and ${ }^{13} \mathrm{C}\left\{{ }^{1} \mathrm{H}\right\} \mathrm{NMR}$ spectra are reported in parts per million relative to tetramethylsilane, using the residual solvent resonances as an internal standard. High-resolution mass data were obtained using a Thermo Scientific LTQ Orbitrap XL mass spectrometer or a Thermo Electron Corporation MAT 95XP-Trap mass spectrometer. ATR-IR data were collected on a Thermo Scientific Nicolet Nexus spectrometer containing a $\mathrm{MCT}^{*}$ detector and $\mathrm{KBr}$ beam splitter with a range of $350-7400 \mathrm{~cm}^{-1}$. Optical rotation data for $\mathbf{2 9}$ and $\mathbf{3 0}$ were obtained at room temperature using a Rudolph Autopol III S2 Polarimeter.

X-Ray Crystallography. Data were collected, reflections were indexed and processed, and the files scaled and corrected for absorption using APEX3. ${ }^{5}$ The space groups were assigned and the structures were solved by direct methods using XPREP within the SHELXTL suite of programs $\mathrm{s}^{6,7}$ and refined by full matrix least squares against $\mathrm{F}^{2}$ with all reflections using Shelxl2018 ${ }^{8}$ using the graphical interface Shelxle. ${ }^{9}$ If not specified otherwise $\mathrm{H}$ atoms attached to carbon, boron and nitrogen atoms as well as hydroxyl hydrogens were positioned geometrically and constrained to ride on their parent atoms. C-H bond distances were constrained to $0.95 \AA$ for aromatic and alkene $\mathrm{C}-\mathrm{H}$ and $\mathrm{CH}_{2}$ and alkyne $\mathrm{C}-\mathrm{H}$ moieties, and to $1.00,0.99$ and $0.98 \AA$ A for aliphatic $\mathrm{C}-\mathrm{H}, \mathrm{CH}_{2}$ and $\mathrm{CH}_{3}$ moieties, respectively. Methyl $\mathrm{H}$ atoms were allowed to rotate but not to tip to best fit the experimental electron density. $\mathrm{U}_{\text {iso }}(\mathrm{H})$ values were set to a multiple of $\mathrm{U}_{\text {eq }}(\mathrm{C})$ with 1.5 for $\mathrm{CH}_{3}, \mathrm{NH}_{3}{ }^{+}$and $\mathrm{OH}$, and 1.2 for $\mathrm{C}-\mathrm{H}, \mathrm{CH}_{2}, \mathrm{~B}-\mathrm{H}, \mathrm{N}-\mathrm{H}$ and $\mathrm{NH}_{2}$ units, respectively. Additional data collection and refinement details, including description of disorder (where present) can be found in the Supporting Information. Complete crystallographic data, in CIF format, have been deposited with the Cambridge Crystallographic Data Centre. CCDC 1973505-1973507 contains the supplementary crystallographic data for this paper. These data can be obtained free of charge from The Cambridge Crystallographic Data Centre via www.ccdc.cam.ac.uk/data_request/cif.

\section{Bruker Quest with Mo radiation:}

Single crystals of $\mathbf{6}$ and $\mathbf{4 9}$ were coated with Fomblin oil and quickly transferred to the goniometer head of a Bruker Quest diffractometer with a fixed chi angle, a sealed tube fine focus X-ray tube, 
single crystal curved graphite incident beam monochromator, a Photon100 CMOS area detector and an Oxford Cryosystems low temperature device. Examination and data collection were performed with Mo K $\alpha$ radiation $(\lambda=0.71073 \AA$ ) at $150 \mathrm{~K}$.

\section{Bruker Quest with $\mathrm{Cu}$ radiation:}

Single crystals of $\mathbf{3 0}$ were coated with Fomblin oil and quickly transferred to the goniometer head of a Bruker Quest diffractometer with kappa geometry, an I- $\mu$-S microsource X-ray tube, laterally graded multilayer (Goebel) mirror single crystal for monochromatization, a Photon-II CMOS area detector and an Oxford Cryosystems low temperature device. Examination and data collection were performed with $\mathrm{Cu} \mathrm{K} \alpha$ radiation $(\lambda=1.54178 \AA$ ) $)$ at $150 \mathrm{~K}$. 


\section{Reaction Optimization Studies}

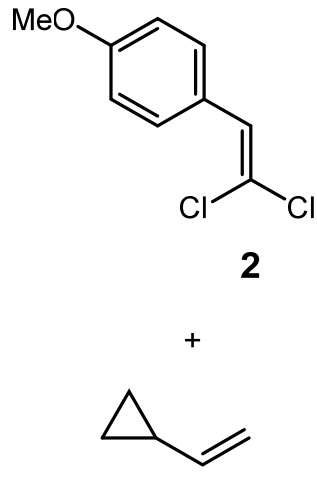

(3.0 equiv.)

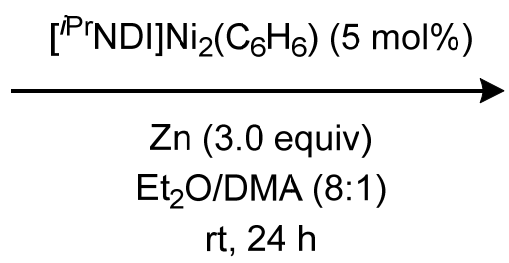

rt, $24 \mathrm{~h}$

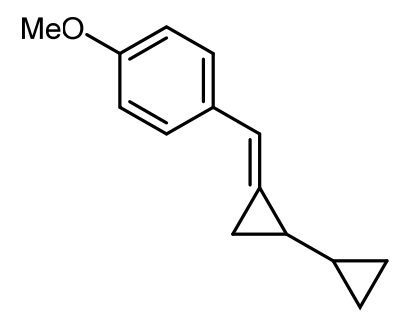

4

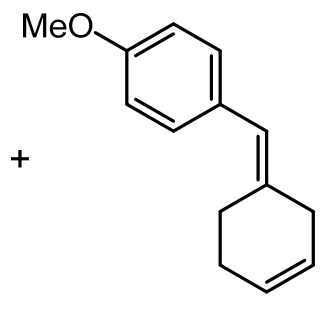

3

$E: Z=1: 2.7$

$87 \%$ Yield

$4: 3=1: 0.8$

Initial detection of the [5 + 1]-product. In an $\mathrm{N}_{2}$-filled glovebox, a 5-mL vial was charged with [ $\left.{ }^{\mathrm{iPrNDI}}\right] \mathrm{Ni}_{2}\left(\mathrm{C}_{6} \mathrm{H}_{6}\right)(3.6 \mathrm{mg}, 0.005 \mathrm{mmol}, 0.05$ equiv), Zn powder ( $19.6 \mathrm{mg}, 0.3 \mathrm{mmol}, 3.0$ equiv), and a magnetic stir bar. To this mixture was added a solution of 1-(2,2-dichlorovinyl)-4methoxybenzene (20.4 mg, $0.1 \mathrm{mmol}, 1.0$ equiv), vinylcyclopropane $(20.4 \mathrm{mg}, 0.3 \mathrm{mmol}, 3.0$ equiv), and 1,3,5-timethoxybenzene (16.8 $\mathrm{mg}, 0.1 \mathrm{mmol}$ ) dissolved in $\mathrm{Et}_{2} \mathrm{O}$ (0.8 mL) and DMA (0.1 $\mathrm{mL}$ ). The reaction was stirred at room temperature. After $16 \mathrm{~h}$, the crude reaction mixture was removed from the glovebox, opened to ambient atmosphere, and diluted with $\mathrm{Et}_{2} \mathrm{O}$. An aliquot was filtered through a glass fiber pad, concentrated, and analyzed by ${ }^{1} \mathrm{H}$ NMR.

General Procedure for ligand comparison study. In an $\mathrm{N}_{2}$-filled glovebox, a 5-mL vial was charged with $\mathrm{Co}(\mathrm{DME}) \mathrm{Br}_{2}(1.5 \mathrm{mg}, 0.005 \mathrm{mmol}, 0.05$ equiv), the ligand $(0.006 \mathrm{mmol}, 0.06$ equiv), Zn powder (19.6 mg, $0.3 \mathrm{mmol}, 3.0$ equiv), and a magnetic stir bar. To this mixture was added a solution of 1-(2,2-dichlorovinyl)-4-methoxybenzene (20.4 mg, $0.1 \mathrm{mmol}, 1.0$ equiv), (1cyclopropylvinyl)benzene ( $43.3 \mathrm{mg}, 0.3 \mathrm{mmol}, 3.0$ equiv), and 1,3,5-timethoxybenzene (16.8 $\mathrm{mg}$, $0.1 \mathrm{mmol})$ dissolved in DMA $(0.75 \mathrm{~mL})$. The reaction was stirred at room temperature. After $16 \mathrm{~h}$, the crude reaction mixture was removed from the glovebox, opened to ambient atmosphere, and diluted with $\mathrm{Et}_{2} \mathrm{O}$. An aliquot was filtered through a glass fiber pad, concentrated, and analyzed by ${ }^{1} \mathrm{H}$ NMR. 
<smiles>COc1ccc(C=C(Cl)Cl)cc1</smiles>

2<smiles>C=C(c1ccccc1)C1CC1</smiles>

5 (3.0 equiv)

\section{Co(DME)Br 2 (5 mol\%)}

Ligand (6 mol\%)

Zn (3.0 equiv)

DMA, rt<smiles>COc1ccc(C=C2CCC=C(c3ccccc3)C2)cc1</smiles>

\begin{tabular}{|c|c|c|c|c|}
\hline Entry & Ligand & Conversion of 2 & Yield 6 & E/Z Ratio of 6 \\
\hline 1 & L1 & $>99 \%$ & $94 \%$ & $>20: 1$ \\
\hline 2 & L2 & $>99 \%$ & $83 \%$ & $>20: 1$ \\
\hline 3 & L3 & $>99 \%$ & $15 \%$ & $>20: 1$ \\
\hline 4 & L4 & $11 \%$ & $0 \%$ & N/A \\
\hline 5 & L5 & $10 \%$ & $0 \%$ & N/A \\
\hline 6 & L6 & $22 \%$ & $0 \%$ & N/A \\
\hline 7 & L7 & $55 \%$ & $0 \%$ & $>20: 1$ \\
\hline 8 & L8 & $29 \%$ & $7 \%$ & \\
\hline
\end{tabular}<smiles>CC(C)CC1COC(c2ccc3ccccc3n2)=N1</smiles>

L1<smiles>BrCC1COC(C2=N[C@H](Cc3ccccc3)CO2)=N1</smiles>

L5<smiles>c1ccc2c(c1)C[C@H]1OC(c3ccc4ccccc4n3)=N[C@H]21</smiles>

L2<smiles>CC(=Nc1c(F)cccc1C(C)C)c1cccc(C(C)=Nc2c(F)cccc2C(C)C)n1</smiles>

L6<smiles>Cc1cccc(C2=NC(CC(C)C)CO2)n1</smiles>

L3<smiles>CCCc1cccc(C(C)C)c1/N=C/c1ccccn1</smiles>

L7<smiles>c1ccc2nc(-c3ccc4ccccc4n3)ccc2c1</smiles>

L4<smiles>c1ccc(-c2ccccn2)nc1</smiles>

L8 
General Procedure for standard condition comparison study. In an $\mathrm{N}_{2}$-filled glovebox, a 5-mL vial was charged with the metal source ( $0.005 \mathrm{mmol}, 0.05$ equiv), ( \pm )- $t$-Bu-Quinox (1.52 $\mathrm{mg}, 0.006 \mathrm{mmol}, 0.06$ equiv), the reductant ( $0.3 \mathrm{mmol}, 3.0$ equiv), and a magnetic stir bar. To this mixture was added a solution of the 1,1-dihaloalkene ( $0.1 \mathrm{mmol}, 1.0$ equiv), the vinylcyclopropane, and 1,3,5-timethoxybenzene (16.8 $\mathrm{mg}, 0.1 \mathrm{mmol}$ ) dissolved in DMA $(0.75 \mathrm{~mL})$. The reaction was stirred at room temperature. After $16 \mathrm{~h}$, the crude reaction mixture was removed from the glovebox, opened to ambient atmosphere, and diluted with $\mathrm{Et}_{2} \mathrm{O}$. An aliquot was filtered through a glass fiber pad, concentrated, and analyzed by ${ }^{1} \mathrm{H}$ NMR.

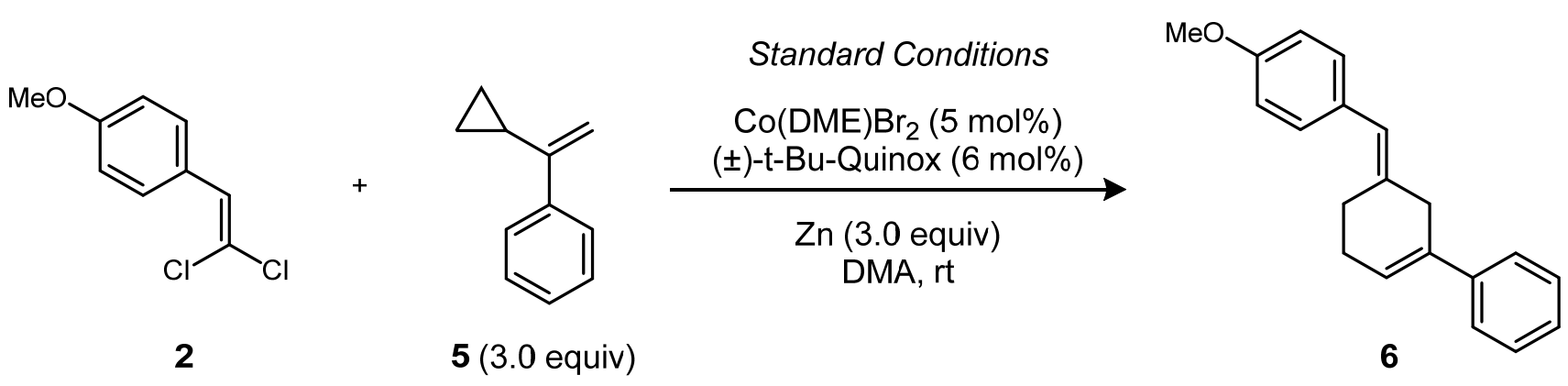

\begin{tabular}{|c|c|c|c|}
\hline Entry & Deviation from Standard Conditions & Yield 6 & $\mathrm{E} / \mathrm{Z}$ of 6 \\
\hline 1 & none & $97 \%$ & $>20: 1$ \\
\hline 2 & 3.0 equiv $\mathrm{Mn}$ instead of $\mathrm{Zn}$ & $93 \%$ & $>20: 1$ \\
\hline 3 & 3.0 equiv TDAE instead of $\mathrm{Zn}$ & $<1 \%$ & NA \\
\hline 4 & 7 instead of 2 & $15 \%$ & $>20: 1$ \\
\hline 5 & $\mathrm{Ni}(\mathrm{DME}) \mathrm{Br}_{2}$ or $\mathrm{Fe}(\mathrm{DME}) \mathrm{Br}_{2}$ instead of $\mathrm{Co}(\mathrm{DME}) \mathrm{Br}_{2}$ & $<1 \%$ & NA \\
\hline 6 & 1.5 equiv of 5 & $42 \%$ & $>20: 1$ \\
\hline 7 & 1.5 equiv of 6 at $60^{\circ} \mathrm{C}$ instead of rt & $73 \%$ & $>20: 1$ \\
\hline 8 & No $( \pm)-t$-Bu-Quinox & $<1 \%$ & NA \\
\hline 9 & No Zn & $<1 \%$ & NA \\
\hline 10 & 3.0 equiv $\mathrm{Cp}_{2} \mathrm{Co}$ instead of $\mathrm{Zn}$ & $<1 \%$ & NA \\
\hline 11 & 3.0 equiv $\mathrm{Cp}_{2} \mathrm{Co}$ and 5.0 equiv $\mathrm{ZnCl}_{2}$ instead of $\mathrm{Zn}$ & $63 \%$ & $>20: 1$ \\
\hline
\end{tabular}<smiles>COc1ccc(C=C(Br)Br)cc1</smiles> 


\section{Synthesis and Characterization of Vinylcyclopropane Substrates}

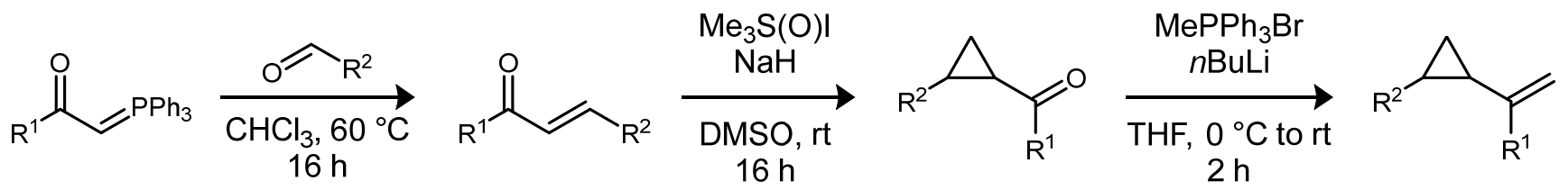

General procedure A: synthesis of $\alpha, \beta$-unsaturated ketones from aldehydes. A roundbottom flask was charged with a stir bar, the Wittig reagent (1.5 equiv), the aldehyde (1.0 equiv), and $\mathrm{CHCl}_{3}(0.5 \mathrm{M})$. The mixture was heated at reflux. After $16 \mathrm{~h}$, the crude reaction mixture was concentrated under reduced pressure. The residue was loaded directly onto a $\mathrm{SiO}_{2}$ column for purification.

General procedure B: synthesis of trans-cyclopropylketones from $\alpha, \beta$-unsaturated ketones. A flame-dried round bottom flask was charged with solid $\mathrm{NaH}$ (60\% in mineral oil, 1.2 equiv), trimethylsulfoxonium iodide (1.2 equiv), and a magnetic stir bar. The flask was placed under $\mathrm{N}_{2}$ atmosphere, and DMSO $(0.35 \mathrm{M})$ was added dropwise with stirring. After hydrogen evolution ceased, the reaction mixture was stirred for an additional $15 \mathrm{~min}$, during which time the solution became clear. The $\alpha, \beta$-unsaturated ketone (1.0 equiv) was added by syringe. The reaction was allowed to stir at room temperature. After $16 \mathrm{~h}$, the reaction was quenched with water, and the mixture was extracted $3 \mathrm{x}$ with $\mathrm{Et}_{2} \mathrm{O}$. The combined organic layers were dried over $\mathrm{MgSO}_{4}$, filtered, and concentrated under reduced pressure. The crude product was loaded directly onto a $\mathrm{SiO}_{2}$ column for purification.

General procedure C: synthesis of trans-vinylcyclopropanes from transcyclopropylketones. A flame-dried round-bottom flask was charged with a stir bar, $\mathrm{MePPh}_{3} \mathrm{Br}$ (1.5 equiv), and THF ( $\sim 0.2 \mathrm{M})$. The mixture was cooled to $0{ }^{\circ} \mathrm{C}$ under $\mathrm{N}_{2}$ atmosphere, followed by dropwise addition of $n \mathrm{BuLi}\left(2.5 \mathrm{M}\right.$ in hexanes, 1.5 equiv). The mixture was stirred at $0{ }^{\circ} \mathrm{C}$ for 30 min. A solution of the trans-cyclopropylketone in THF $(1 \mathrm{~mL})$ was added dropwise, and the reaction was then warmed to room temperature and stirred. After $2 \mathrm{~h}$, the reaction was quenched with a saturated aqueous solution of $\mathrm{NH}_{4} \mathrm{Cl}$ and extracted $3 \mathrm{x}$ with $\mathrm{Et}_{2} \mathrm{O}$. The combined organic layers were dried over $\mathrm{MgSO}_{4}$, filtered, and concentrated under reduced pressure. The crude product was loaded directly onto a $\mathrm{SiO}_{2}$ column for purification. 

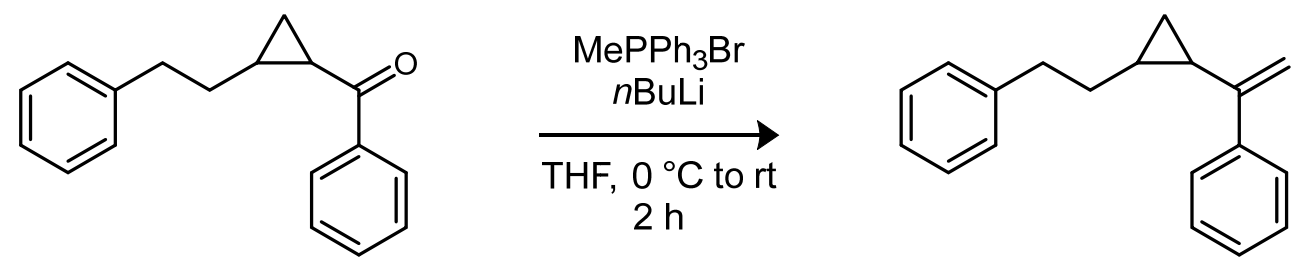

(1-(2-phenethylcyclopropyl)vinyl)benzene (S1). The reaction was conducted according to the general procedure $\mathrm{C}$ without modification using (2-phenethylcyclopropyl)(phenyl)methanone ${ }^{2}$ (1.06 g, $4.2 \mathrm{mmol}, 1.0$ equiv), $\mathrm{MePPh}_{3} \mathrm{Br}(2.25 \mathrm{~g}, 6.3 \mathrm{mmol}, 1.5$ equiv), and $n \mathrm{BuLi}$ ( $2.5 \mathrm{M}$ in hexanes, $2.52 \mathrm{~mL}, 6.3 \mathrm{mmol}, 1.5$ equiv) in THF $(25 \mathrm{~mL})$. The product was purified by column chromatography (100\% hexanes) to provide (1-(2-phenethylcyclopropyl)vinyl)benzene (949 mg, $91 \%$ yield) as a clear, colorless oil.

${ }^{1} \mathrm{H} \mathrm{NMR}\left(300 \mathrm{MHz}, \mathrm{CDCl}_{3}\right) \delta 7.54(\mathrm{~d}, J=6.8 \mathrm{~Hz}, 2 \mathrm{H}), 7.43-7.28(\mathrm{~m}, 4 \mathrm{H}), 7.24-7.12(\mathrm{~m}, 3 \mathrm{H})$, $5.23(\mathrm{~s}, 1 \mathrm{H}), 4.88(\mathrm{~s}, 1 \mathrm{H}), 2.77(\mathrm{t}, J=7.7 \mathrm{~Hz}, 2 \mathrm{H}), 2.01-1.74(\mathrm{~m}, 1 \mathrm{H}), 1.68-1.55(\mathrm{~m}, 1 \mathrm{H}), 1.50-$ $1.39(\mathrm{~m}, 1 \mathrm{H}), 1.08-0.91(\mathrm{~m}, 1 \mathrm{H}), 0.88-0.76(\mathrm{~m}, 1 \mathrm{H}), 0.73-0.59(\mathrm{~m}, 1 \mathrm{H})$.

${ }^{13} \mathrm{C}\left\{{ }^{1} \mathrm{H}\right\}$ NMR $\left(201 \mathrm{MHz}, \mathrm{CDCl}_{3}\right) \delta 149.3,142.3,141.9,128.5,128.3,128.2,127.4,126.2$, 125.7, 108.9, 36.3, 35.8, 23.4, 21.1, 14.0.

HRMS(ESI) (m/z): [M + H] ${ }^{+}$Calcd for $\mathrm{C}_{19} \mathrm{H}_{20}$ : 249.1638; found: 249.1643

TLC: $\mathrm{R}_{f}=0.72(100 \%$ hexanes $)$
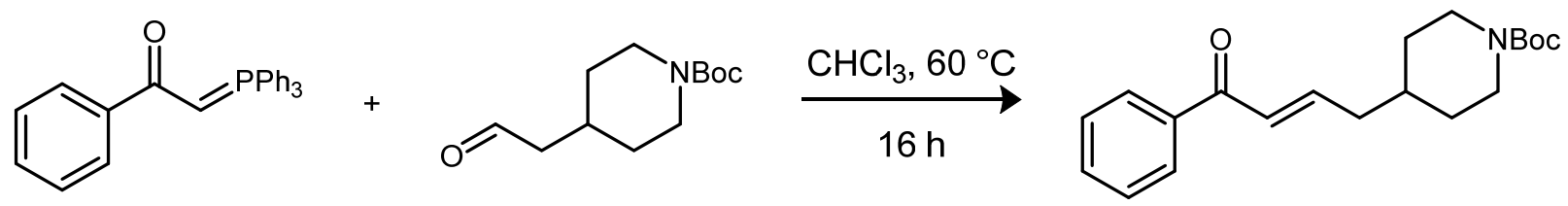

tert-butyl (E)-4-(4-oxo-4-phenylbut-2-en-1-yl)piperidine-1-carboxylate (S2). The reaction was conducted according to the general procedure A without modification using tert-butyl 4-(2oxoethyl)piperidine-1-carboxylate $\quad\left(\begin{array}{llllll}1.0 & \mathrm{~g} & 4.4 & \mathrm{mmol}, & 1.0 & \text { equiv }) \text { and }\end{array}\right.$ phenacyltriphenylphosphorane $\left(2.34 \mathrm{~g}, 6.2 \mathrm{mmol}, 1.5\right.$ equiv) in $\mathrm{CHCl}_{3}(10 \mathrm{~mL})$. The product was purified by column chromatography $\left(20 \% \mathrm{Et}_{2} \mathrm{O}\right.$ in hexanes) to provide tert-butyl (E)-4-(4-oxo-4phenylbut-2-en-1-yl)piperidine-1-carboxylate (1.35 g, 93\% yield) as an orange solid.

${ }^{1} \mathrm{H}$ NMR $\left(300 \mathrm{MHz}, \mathrm{CDCl}_{3}\right) \delta 7.93(\mathrm{~d}, J=7.3 \mathrm{~Hz}, 2 \mathrm{H}), 7.64-7.51(\mathrm{~m}, 1 \mathrm{H}), 7.48(\mathrm{t}, J=7.3 \mathrm{~Hz}$, $2 \mathrm{H}), 7.03(\mathrm{dt}, J=14.7,7.2 \mathrm{~Hz}, 1 \mathrm{H}), 6.90(\mathrm{~d}, J=15.4 \mathrm{~Hz}, 1 \mathrm{H}), 4.10(\mathrm{br} \mathrm{s}, 2 \mathrm{H}), 2.69(\mathrm{t}, J=12.7 \mathrm{~Hz}, 2 \mathrm{H})$, $2.28(\mathrm{t}, J=6.8 \mathrm{~Hz}, 2 \mathrm{H}), 1.70(\mathrm{~d}, J=12.9 \mathrm{~Hz}, 3 \mathrm{H}), 1.45(\mathrm{~s}, 9 \mathrm{H}), 1.33-0.98(\mathrm{~m}, 2 \mathrm{H})$.

${ }^{13} \mathrm{C}\left\{{ }^{1} \mathrm{H}\right\}$ NMR $\left(201 \mathrm{MHz}, \mathrm{CDCl}_{3}\right) \delta 190.5,154.8,147.3,137.8,132.7,128.6,128.5,127.4,79.4$, 41.0, 39.8, 35.8, 32.0, 28.5.

HRMS(ESI) (m/z): [M + Na] $]^{+}$Calcd for $\mathrm{C}_{20} \mathrm{H}_{27} \mathrm{NO}_{3}: 352.1883$; found: 352.1887

TLC: $\mathrm{R}_{f}=0.21\left(15 \% \mathrm{Et}_{2} \mathrm{O}\right.$ in hexanes) 

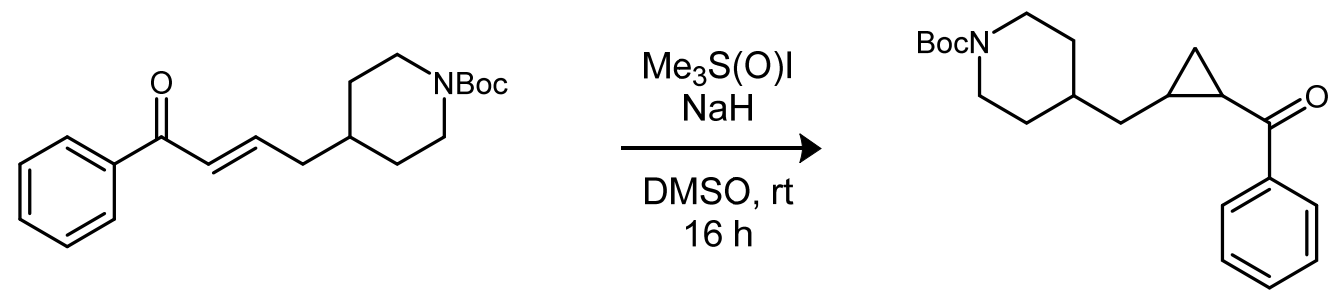

tert-butyl 4-((2-benzoylcyclopropyl)methyl)piperidine-1-carboxylate (S3). The reaction was conducted according to the general procedure B without modification using S2 (1.35 g, 4.4 mmol, 1.0 equiv), trimethylsulfoxonium iodide ( $1.08 \mathrm{~g}$, $4.9 \mathrm{mmol}, 1.2$ equiv), $\mathrm{NaH}$ ( $60 \%$ in mineral oil, $196 \mathrm{mg}, 1.2$ equiv). The product was purified by column chromatography (20\% $\mathrm{Et}_{2} \mathrm{O}$ in hexanes) to provide tert-butyl 4-((2-benzoylcyclopropyl)methyl)piperidine-1-carboxylate (741 $\mathrm{mg}, 53 \%$ yield) as a yellow oil.

${ }^{1} \mathrm{H} \mathrm{NMR}\left(300 \mathrm{MHz}, \mathrm{CDCl}_{3}\right) \delta 7.99(\mathrm{~d}, J=7.0 \mathrm{~Hz}, 2 \mathrm{H}), 7.63-7.53(\mathrm{~m}, 1 \mathrm{H}), 7.48(\mathrm{t}, J=7.3 \mathrm{~Hz}$, 2H), 4.08 (br s, 2H), $2.66(\mathrm{t}, J=12.9 \mathrm{~Hz}, 2 \mathrm{H}), 2.51-2.35(\mathrm{~m}, 1 \mathrm{H}), 1.80-1.66(\mathrm{~m}, 2 \mathrm{H}), 1.67-1.47$ (m, 4H), $1.45(\mathrm{~s}, 9 \mathrm{H}), 1.38-1.01(\mathrm{~m}, 3 \mathrm{H}), 1.00-0.79(\mathrm{~m}, 1 \mathrm{H})$.

${ }^{13} \mathrm{C}\left\{{ }^{1} \mathrm{H}\right\} \mathrm{NMR}\left(201 \mathrm{MHz}, \mathrm{CDCl}_{3}\right) \delta 199.9,154.8,137.9,132.7,128.5,128.0,79.2,40.4,36.5$, $32.1,28.5,25.6,25.1,24.5,19.2$.

HRMS(ESI) (m/z): [M + H] ${ }^{+}$Calcd for $\mathrm{C}_{21} \mathrm{H}_{29} \mathrm{NO}_{3}: 344.2220$; found: 344.2222

TLC: $\mathrm{R}_{f}=0.27\left(15 \% \mathrm{Et}_{2} \mathrm{O}\right.$ in hexanes)
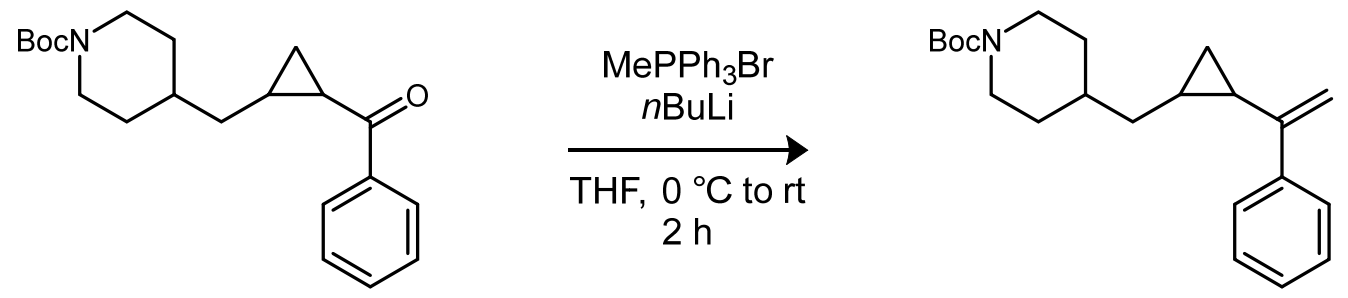

tert-butyl 4-((2-benzoylcyclopropyl)methyl)piperidine-1-carboxylate (S4). The reaction was conducted according to the general procedure $C$ without modification using $\mathbf{S} 3$ (741 $\mathrm{mg}, 2.16$ mmol, 1.0 equiv), $\mathrm{MePPh}_{3} \mathrm{Br}$ (1.14 g, $3.2 \mathrm{mmol}, 1.5$ equiv), and $n \mathrm{BuLi}$ (2.5 $\mathrm{M}$ in hexanes, $1.3 \mathrm{~mL}, 3.2$ $\mathrm{mmol}, 1.5$ equiv) in THF $(10 \mathrm{~mL})$. The product was purified by column chromatography $\left(10 \% \mathrm{Et}_{2} \mathrm{O}\right.$ in hexanes) to provide tert-butyl 4-((2-benzoylcyclopropyl)methyl)piperidine-1-carboxylate (448 $\mathrm{mg}, 61 \%$ yield) as a clear, colorless oil.

${ }^{1} \mathrm{H} \mathrm{NMR}\left(400 \mathrm{MHz}, \mathrm{CDCl}_{3}\right) \delta 7.51(\mathrm{~d}, J=7.0 \mathrm{~Hz}, 2 \mathrm{H}), 7.33(\mathrm{t}, J=7.2 \mathrm{~Hz}, 2 \mathrm{H}), 7.30-7.26(\mathrm{~m}$, $1 \mathrm{H}), 5.21(\mathrm{~s}, 1 \mathrm{H}), 4.87(\mathrm{~s}, 1 \mathrm{H}), 4.07(\mathrm{~s}, 2 \mathrm{H}), 2.67(\mathrm{~s}, 2 \mathrm{H}), 1.71(\mathrm{~d}, J=13.1 \mathrm{~Hz}, 2 \mathrm{H}), 1.62-1.49(\mathrm{~m}$, 2H), 1.45 (s, 9H), $1.41-1.34(\mathrm{~m}, 1 \mathrm{H}), 1.25-1.18(\mathrm{~m}, 1 \mathrm{H}), 1.18-1.05(\mathrm{~m}, 2 \mathrm{H}), 0.98-0.92(\mathrm{~m}, 1 \mathrm{H})$, $0.84-0.76(\mathrm{~m}, 1 \mathrm{H}), 0.69-0.56(\mathrm{~m}, 1 \mathrm{H})$.

${ }^{13} \mathrm{C}\left\{{ }^{1} \mathrm{H}\right\} \mathrm{NMR}\left(201 \mathrm{MHz}, \mathrm{CDCl}_{3}\right) \delta 154.9,149.4,141.8,128.2,127.4,126.1,108.8,79.2,41.2$, $36.7,32.2,28.5,23.3,18.9,14.3$.

HRMS(ESI) (m/z): [M + H] ${ }^{+}$Calcd for $\mathrm{C}_{22} \mathrm{H}_{31} \mathrm{NO}_{2}: 342.2428$; found: 342.2426

TLC: $\mathrm{R}_{f}=0.16\left(5 \% \mathrm{Et}_{2} \mathrm{O}\right.$ in hexanes) 
<smiles>Cc1ccc(CC/C=C/C(=O)c2ccccc2)o1</smiles>

(E)-5-(5-methylfuran-2-yl)-1-phenylpent-2-en-1-one (S5). The reaction was conducted according to the general procedure A without modification using 3-(5-methylfuran-2-yl)propanal (1.4 g, $10.1 \mathrm{mmol}, 1.0$ equiv) and phenacyltriphenylphosphorane ( $5.7 \mathrm{~g}, 15.2 \mathrm{mmol}, 1.5$ equiv) in $\mathrm{CHCl}_{3}(20 \mathrm{~mL})$. The product was purified by column chromatography $\left(20 \% \mathrm{Et}_{2} \mathrm{O}\right.$ in hexanes) to provide $(E)$-5-(5-methylfuran-2-yl)-1-phenylpent-2-en-1-one (2.06 g, 84\% yield) as an orange oil.

${ }^{1} \mathrm{H}$ NMR $\left(300 \mathrm{MHz}, \mathrm{CDCl}_{3}\right) \delta 7.91(\mathrm{~d}, J=8.2 \mathrm{~Hz}, 2 \mathrm{H}), 7.61-7.52(\mathrm{~m}, 1 \mathrm{H}), 7.47(\mathrm{t}, J=7.3 \mathrm{~Hz}$, $2 \mathrm{H}), 7.07(\mathrm{dt}, J=15.4,6.6 \mathrm{~Hz}, 1 \mathrm{H}), 6.90(\mathrm{~d}, J=15.5 \mathrm{~Hz}, 1 \mathrm{H}), 5.88(\mathrm{~d}, J=13.6 \mathrm{~Hz}, 2 \mathrm{H}), 2.82(\mathrm{t}, J=7.4$ $\mathrm{Hz}, 2 \mathrm{H}), 2.73-2.56(\mathrm{~m}, 2 \mathrm{H}), 2.26(\mathrm{~s}, 3 \mathrm{H})$.

${ }^{13} \mathrm{C}\left\{{ }^{1} \mathrm{H}\right\}$ NMR $\left(201 \mathrm{MHz}, \mathrm{CDCl}_{3}\right) \delta 190.9,152.6,150.6,148.2,137.9,132.7,128.6,128.5$, 126.6, 106.1, 106.0, 31.4, 26.8, 13.5 .

HRMS(APCI) (m/z): [M + H] $]^{+}$Calcd for $\mathrm{C}_{16} \mathrm{H}_{16} \mathrm{O}_{2}: 241.1223$; found: 241.1220

TLC: $\mathrm{R}_{f}=0.29\left(15 \% \mathrm{Et}_{2} \mathrm{O}\right.$ in hexanes)
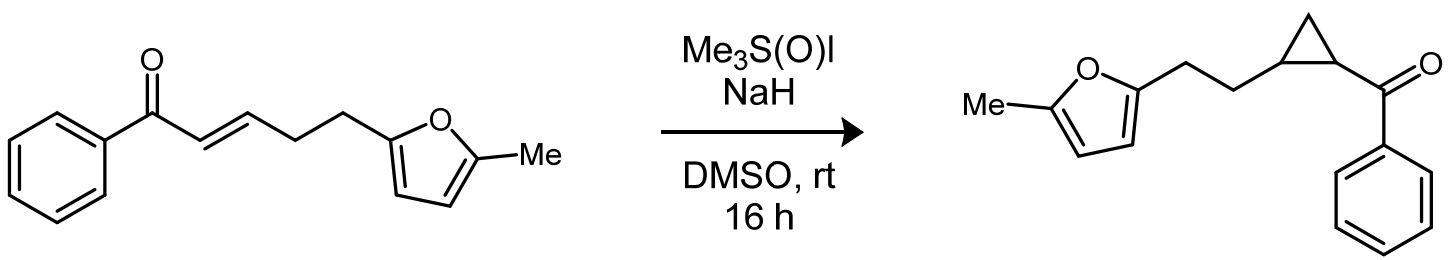

(2-(2-(5-methylfuran-2-yl)ethyl)cyclopropyl)(phenyl)methanone (S6). The reaction was conducted according to the general procedure B without modification using $\mathbf{S 5}(2.06 \mathrm{~g}, 8.1 \mathrm{mmol}$, 1.0 equiv), trimethylsulfoxonium iodide $(2.14 \mathrm{~g}, 9.71 \mathrm{mmol}, 1.2$ equiv), $\mathrm{NaH}$ ( $60 \%$ in mineral oil, $389 \mathrm{mg}, 1.2$ equiv). The product was purified by column chromatography (15\% $\mathrm{Et}_{2} \mathrm{O}$ in hexanes) to provide (2-(2-(5-methylfuran-2-yl)ethyl)cyclopropyl)(phenyl)methanone (1.02 g, 50\% yield) as a light yellow oil.

${ }^{1} \mathrm{H}$ NMR $\left(300 \mathrm{MHz}, \mathrm{CDCl}_{3}\right) \delta 7.96(\mathrm{~d}, J=7.0 \mathrm{~Hz}, 2 \mathrm{H}), 7.62-7.49(\mathrm{~m}, 1 \mathrm{H}), 7.46(\mathrm{t}, J=7.3 \mathrm{~Hz}$, $2 \mathrm{H}), 5.81(\mathrm{~d}, J=9.3 \mathrm{~Hz}, 2 \mathrm{H}), 2.71(\mathrm{t}, J=7.3 \mathrm{~Hz}, 2 \mathrm{H}), 2.48-2.40(\mathrm{~m}, 1 \mathrm{H}), 2.20(\mathrm{~s}, 3 \mathrm{H}), 1.90-1.70(\mathrm{~m}$, 2H), $1.68-1.58(\mathrm{~m}, 1 \mathrm{H}), 1.53-1.40(\mathrm{~m}, 1 \mathrm{H}), 1.02-0.85(\mathrm{~m}, 1 \mathrm{H})$.

${ }^{13} \mathrm{C}\left\{{ }^{1} \mathrm{H}\right\}$ NMR $\left(201 \mathrm{MHz}, \mathrm{CDCl}_{3}\right) \delta 199.9,153.4,150.3,138.0,132.6,128.4,128.0,105.9$, $105.8,32.2,27.8,26.4,25.0,18.7,13.5$.

HRMS(ESI) (m/z): [M + H] Calcd for $\mathrm{C}_{17} \mathrm{H}_{18} \mathrm{O}_{2}: 255.1380$; found: 255.1382

TLC: $\mathrm{R}_{f}=0.33\left(15 \% \mathrm{Et}_{2} \mathrm{O}\right.$ in hexanes) 

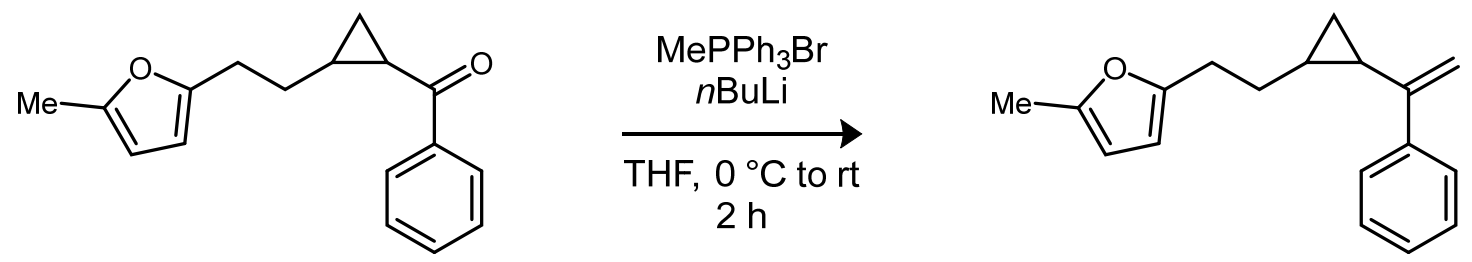

2-methyl-5-(2-(2-(1-phenylvinyl)cyclopropyl)ethyl)furan (S7). The reaction was conducted according to the general procedure $C$ without modification using $\mathbf{S 6}(1.02 \mathrm{~g}, 4.01 \mathrm{mmol}, 1.0$ equiv), $\mathrm{MePPh}_{3} \mathrm{Br}$ (2.15 g, $6.01 \mathrm{mmol}, 1.5$ equiv), and $n \mathrm{BuLi}$ (2.5 M in hexanes, $2.4 \mathrm{~mL}, 6.01 \mathrm{mmol}, 1.5$ equiv) in THF ( $20 \mathrm{~mL})$. The product was purified by column chromatography (100\% hexanes) to provide 2-methyl-5-(2-(2-(1-phenylvinyl)cyclopropyl)ethyl)furan (403 mg, 42\% yield) as a clear, colorless oil.

${ }^{1} \mathrm{H}$ NMR (400 MHz, CDCl 3$) \delta 7.54(\mathrm{~d}, J=6.8 \mathrm{~Hz}, 2 \mathrm{H}), 7.38-7.27(\mathrm{~m}, 3 \mathrm{H}), 5.92-5.80(\mathrm{~m}, 2 \mathrm{H})$, $5.23(\mathrm{~s}, 1 \mathrm{H}), 4.89(\mathrm{~s}, 1 \mathrm{H}), 2.72(\mathrm{t}, J=7.6 \mathrm{~Hz}, 2 \mathrm{H}), 2.25(\mathrm{~s}, 3 \mathrm{H}), 1.98-1.77(\mathrm{~m}, 1 \mathrm{H}), 1.72-1.50(\mathrm{~m}$, $1 \mathrm{H}), 1.50-1.34(\mathrm{~m}, 1 \mathrm{H}), 1.08-0.90(\mathrm{~m}, 1 \mathrm{H}), 0.88-0.73(\mathrm{~m}, 1 \mathrm{H}), 0.71-0.58(\mathrm{~m}, 1 \mathrm{H})$.

${ }^{13} \mathrm{C}\left\{{ }^{1} \mathrm{H}\right\}$ NMR $\left(201 \mathrm{MHz}, \mathrm{CDCl}_{3}\right) \delta 154.2,150.2,149.3,141.8,128.2,127.4,126.2,109.0$, 105.8, 105.4, 33.1, 28.0, 23.4, 21.0, 13.8, 13.5 .

HRMS(ESI) (m/z): [M + H] ${ }^{+}$Calcd for $\mathrm{C}_{18} \mathrm{H}_{20} \mathrm{O}: 253.1587$; found: 253.1585

TLC: $\mathrm{R}_{f}=0.47(100 \%$ hexanes $)$
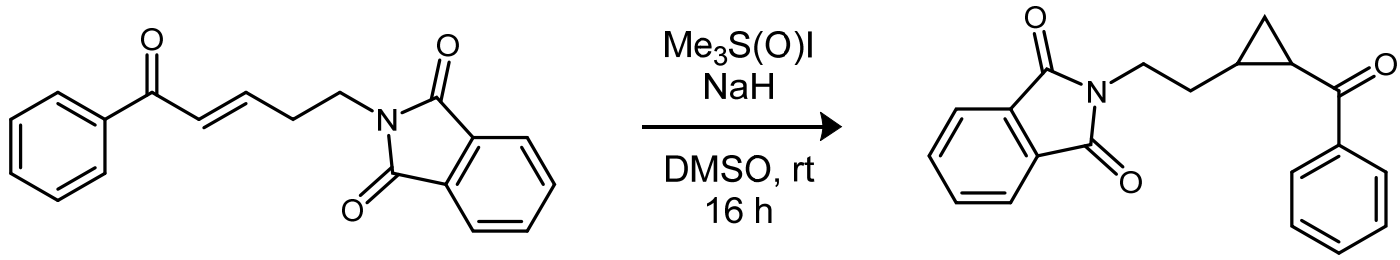

2-(2-(2-benzoylcyclopropyl)ethyl)isoindoline-1,3-dione (S8). The reaction was conducted according to the general procedure $B$ without modification using $(E)$-2-(5-oxo-5-phenylpent-3-en1-yl)isoindoline-1,3-dione ${ }^{2}$ (1.16 g, $3.8 \mathrm{mmol}, 1.0$ equiv), trimethylsulfoxonium iodide (1.0 g, 4.5 mmol, 1.2 equiv), $\mathrm{NaH}$ (60\% in mineral oil, $182 \mathrm{mg}, 1.2$ equiv). The product was purified by column chromatography $(30 \% \quad$ EtOAc in hexanes $)$ to provide 2-(2- $(2-$ benzoylcyclopropyl)ethyl)isoindoline-1,3-dione ( $424 \mathrm{mg}, 35 \%$ yield) as a white solid.

${ }^{1} \mathrm{H} \mathrm{NMR}\left(300 \mathrm{MHz}, \mathrm{CDCl}_{3}\right) \delta 7.91(\mathrm{~d}, J=6.9 \mathrm{~Hz}, 2 \mathrm{H}), 7.79-7.71(\mathrm{~m}, 2 \mathrm{H}), 7.69-7.61(\mathrm{~m}, 2 \mathrm{H})$, $7.51(\mathrm{t}, J=7.4 \mathrm{~Hz}, 1 \mathrm{H}), 7.39(\mathrm{t}, J=7.7 \mathrm{~Hz}, 2 \mathrm{H}), 3.81(\mathrm{t}, J=7.3 \mathrm{~Hz}, 2 \mathrm{H}), 2.56-2.37(\mathrm{~m}, 1 \mathrm{H}), 1.95-$ $1.72(\mathrm{~m}, 2 \mathrm{H}), 1.69-1.57(\mathrm{~m}, 1 \mathrm{H}), 1.53-1.34(\mathrm{~m}, 1 \mathrm{H}), 1.02-0.84(\mathrm{~m}, 1 \mathrm{H})$.

${ }^{13} \mathrm{C}\left\{{ }^{1} \mathrm{H}\right\}$ NMR $\left(201 \mathrm{MHz}, \mathrm{CDCl}_{3}\right) \delta 199.3,168.2,137.8,133.9,132.6,132.0,128.4,127.9$, 123.2, 37.5, 32.0, 24.5, 23.7, 18.2.

HRMS(ESI) (m/z): [M + H] ${ }^{+}$Calcd for $\mathrm{C}_{20} \mathrm{H}_{17} \mathrm{NO}_{3}: 320.1281$; found: 320.1284

TLC: $\mathrm{R}_{f}=0.21(30 \%$ EtOAc in hexanes) 

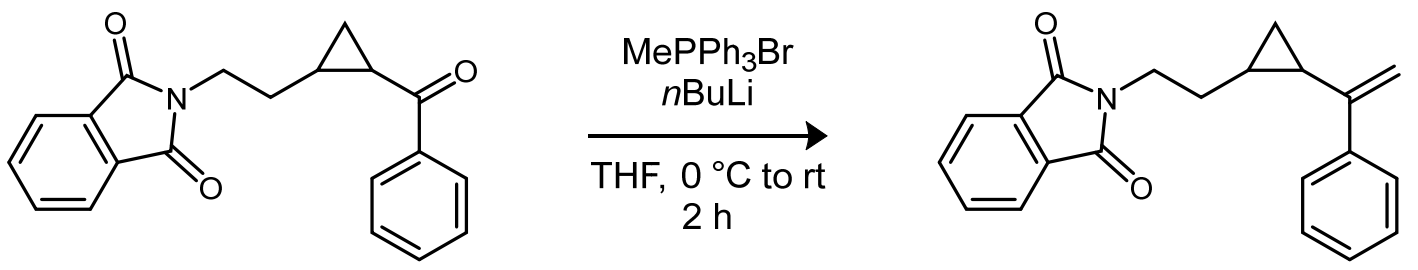

2-(2-(2-(1-phenylvinyl)cyclopropyl)ethyl)isoindoline-1,3-dione (S9). The reaction was conducted according to the general procedure $\mathrm{C}$ without modification using $\mathbf{S 8}$ (587 $\mathrm{mg}, 1.84$ mmol, 1.0 equiv), $\mathrm{MePPh}_{3} \mathrm{Br}$ (986 mg, $2.76 \mathrm{mmol}, 1.5$ equiv), and $n \mathrm{BuLi}(2.5 \mathrm{M}$ in hexanes, $1.1 \mathrm{~mL}$, $2.76 \mathrm{mmol}, 1.5$ equiv) in THF ( $20 \mathrm{~mL})$. The product was purified by column chromatography ( $15 \%$ EtOAc in hexanes) to provide 2-(2-(2-(1-phenylvinyl)cyclopropyl)ethyl)isoindoline-1,3-dione (84 $\mathrm{mg}, 14 \%$ yield) as a yellow oil.

${ }^{1} \mathrm{H} \mathrm{NMR}\left(300 \mathrm{MHz}, \mathrm{CDCl}_{3}\right) \delta 7.89-7.76(\mathrm{~m}, 2 \mathrm{H}), 7.76-7.62(\mathrm{~m}, 2 \mathrm{H}), 7.46(\mathrm{~d}, J=7.9 \mathrm{~Hz}, 2 \mathrm{H})$, $7.33-7.26(\mathrm{~m}, 3 \mathrm{H}), 5.19(\mathrm{~s}, 1 \mathrm{H}), 4.85(\mathrm{~s}, 1 \mathrm{H}), 3.83(\mathrm{t}, J=7.2 \mathrm{~Hz}, 2 \mathrm{H}), 2.08-1.87(\mathrm{~m}, 1 \mathrm{H}), 1.65-$ $1.56(\mathrm{~m}, 1 \mathrm{H}), 1.51-1.35(\mathrm{~m}, 1 \mathrm{H}), 1.09-0.87(\mathrm{~m}, 1 \mathrm{H}), 0.83-0.72(\mathrm{~m}, 1 \mathrm{H}), 0.72-0.59(\mathrm{~m}, 1 \mathrm{H})$. ${ }^{13} \mathrm{C}\left\{{ }^{1} \mathrm{H}\right\} \mathrm{NMR}\left(201 \mathrm{MHz}, \mathrm{CDCl}_{3}\right) \delta 168.4,148.7,141.5,133.9,132.2,128.1,127.4,126.1$, $123.2,109.3,37.8,32.8,23.0,18.3,13.5$.

HRMS(ESI) (m/z): [M + H] ${ }^{+}$Calcd for $\mathrm{C}_{21} \mathrm{H}_{19} \mathrm{NO}_{2}: 318.1489$; found: 318.1487

TLC: $\mathrm{R}_{f}=0.25\left(15 \% \mathrm{Et}_{2} \mathrm{O}\right.$ in hexanes)<smiles>O=CCC/C=C\CCCCCCCCCCCCCCCCCCCCCCC=CC(=O)c1ccccc1</smiles>

(2E,6Z)-1-phenyldodeca-2,6-dien-1-one (S10). The reaction was conducted according to the general procedure A without modification using cis-4-decenal $(1.0 \mathrm{~g}, 6.48 \mathrm{mmol}, 1.0$ equiv) and phenacyltriphenylphosphorane $\left(3.7 \mathrm{~g}, 9.72 \mathrm{mmol}, 1.5\right.$ equiv) in $\mathrm{CHCl}_{3}(20 \mathrm{~mL})$. The product was purified by column chromatography $\left(5 \% \mathrm{Et}_{2} \mathrm{O}\right.$ in hexanes) to provide $(2 E, 6 Z)-1$-phenyldodeca2,6-dien-1-one (1.50 g, 90\% yield) as yellow oil.

${ }^{1} \mathrm{H}$ NMR (300 MHz, $\left.\mathrm{CDCl}_{3}\right) \delta 7.92(\mathrm{~d}, J=7.0 \mathrm{~Hz}, 2 \mathrm{H}), 7.55(\mathrm{t}, J=7.3 \mathrm{~Hz}, 1 \mathrm{H}), 7.46(\mathrm{t}, J=7.3$ $\mathrm{Hz}, 2 \mathrm{H}), 7.06(\mathrm{dt}, J=15.5,6.5 \mathrm{~Hz}, 1 \mathrm{H}), 6.88(\mathrm{~d}, J=15.4 \mathrm{~Hz}, 1 \mathrm{H}), 5.54-5.26(\mathrm{~m}, 2 \mathrm{H}), 2.47-2.32(\mathrm{~m}$, $2 \mathrm{H}), 2.32-2.19(\mathrm{~m}, 2 \mathrm{H}), 2.03(\mathrm{q}, J=6.8 \mathrm{~Hz}, 2 \mathrm{H}), 1.42-1.19(\mathrm{~m}, 6 \mathrm{H}), 0.95-0.80(\mathrm{~m}, 3 \mathrm{H})$.

${ }^{13} \mathrm{C}\left\{{ }^{1} \mathrm{H}\right\} \mathrm{NMR}\left(201 \mathrm{MHz}, \mathrm{CDCl}_{3}\right) \delta 190.9,149.2,138.0,132.6,131.4,128.5,128.5,127.8$, $126.2,32.9,31.5,29.3,27.3,25.9,22.6,14.1$.

HRMS(ESI) (m/z): [M + H] Calcd for $\mathrm{C}_{18} \mathrm{H}_{24} \mathrm{O}: 256.1807$; found: 256.1814

TLC: $\mathrm{R}_{f}=0.39$ ( $5 \% \mathrm{Et}_{2} \mathrm{O}$ in hexanes) 

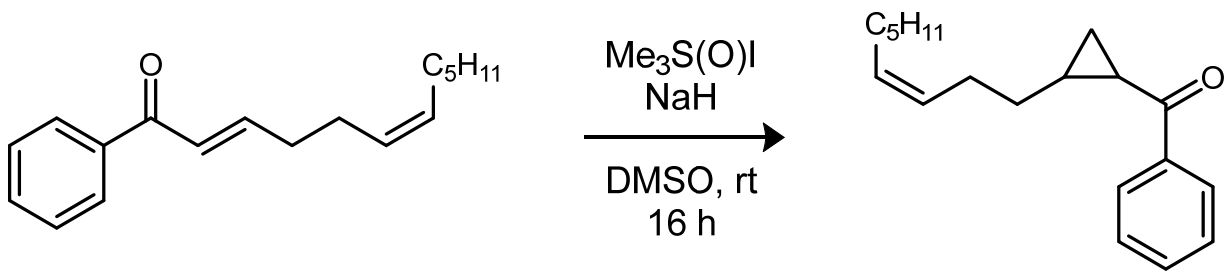

(Z)-(2-(non-3-en-1-yl)cyclopropyl)(phenyl)methanone (S11). The reaction was conducted according to the general procedure B without modification using $\mathbf{S 1 0}(1.5 \mathrm{~g}, 5.85 \mathrm{mmol}, 1.0$ equiv), trimethylsulfoxonium iodide ( $1.55 \mathrm{~g}, 7.0 \mathrm{mmol}, 1.2$ equiv), $\mathrm{NaH}$ (60\% in mineral oil, $182 \mathrm{mg}, 1.2$ equiv). The product was purified by column chromatography ( $5 \% \mathrm{Et}_{2} \mathrm{O}$ in hexanes) to provide $(Z)$ (2-(non-3-en-1-yl)cyclopropyl)(phenyl)methanone (778 mg, 49\% yield) as a yellow oil.

${ }^{1} \mathrm{H} \mathrm{NMR}\left(300 \mathrm{MHz}, \mathrm{CDCl}_{3}\right) \delta 8.01(\mathrm{~d}, J=7.0 \mathrm{~Hz}, 2 \mathrm{H}), 7.56(\mathrm{t}, J=7.3 \mathrm{~Hz}, 1 \mathrm{H}), 7.47(\mathrm{t}, J=7.3$ $\mathrm{Hz}, 2 \mathrm{H}), 5.45-5.31(\mathrm{~m}, 2 \mathrm{H}), 2.53-2.38(\mathrm{~m}, 1 \mathrm{H}), 2.24-2.08(\mathrm{~m}, 2 \mathrm{H}), 2.05-1.89(\mathrm{~m}, 2 \mathrm{H}), 1.70-$ $1.55(\mathrm{~m}, 1 \mathrm{H}), 1.55-1.38(\mathrm{~m}, 3 \mathrm{H}), 1.35-1.17(\mathrm{~m}, 6 \mathrm{H}), 0.99-0.90(\mathrm{~m}, 1 \mathrm{H}), 0.90-0.81(\mathrm{~m}, 3 \mathrm{H})$. ${ }^{13} \mathrm{C}\left\{{ }^{1} \mathrm{H}\right\}$ NMR $\left(201 \mathrm{MHz}, \mathrm{CDCl}_{3}\right) \delta 200.0,138.1,132.6,130.8,128.6,128.5,128.0,33.8,31.5$, 29.4, 27.2, 26.9, 26.9, 25.2, 22.6, 18.9, 14.1 .

HRMS(ESI) (m/z): [M + H]+ Calcd for $\mathrm{C}_{19} \mathrm{H}_{26} \mathrm{O}: 271.2056$; found: 271.2063

TLC: $\mathrm{R}_{f}=0.43\left(5 \% \mathrm{Et}_{2} \mathrm{O}\right.$ in hexanes)
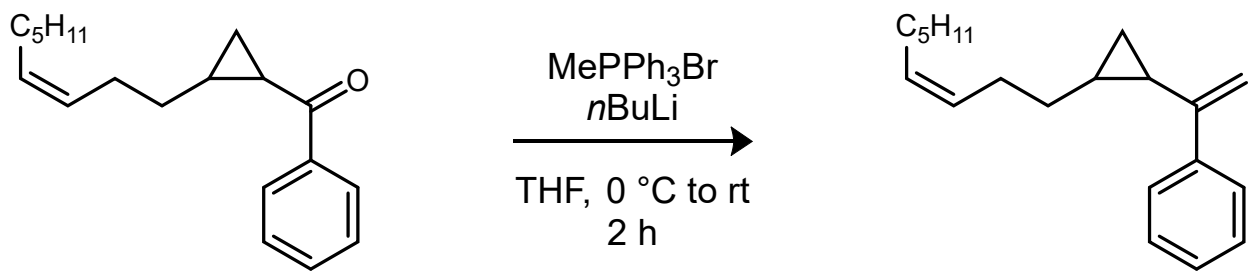

(Z)-(1-(2-(non-3-en-1-yl)cyclopropyl)vinyl)benzene (S12). The reaction was conducted according to the general procedure $\mathrm{C}$ without modification using $\mathbf{S 1 1}(778 \mathrm{mg}, 2.87 \mathrm{mmol}, 1.0$ equiv), $\mathrm{MePPh}_{3} \mathrm{Br}$ (1.54 g, $4.30 \mathrm{mmol}, 1.5$ equiv), and $n \mathrm{BuLi}(2.5 \mathrm{M}$ in hexanes, $1.7 \mathrm{~mL}, 4.30 \mathrm{mmol}$, 1.5 equiv) in THF ( $30 \mathrm{~mL}$ ). The product was purified by column chromatography ( $100 \%$ hexanes) to provide (Z)-(1-(2-(non-3-en-1-yl)cyclopropyl)vinyl)benzene (473 $\mathrm{mg}, 61 \%$ yield) as a clear, colorless oil.

${ }^{1} \mathrm{H} \mathrm{NMR}\left(300 \mathrm{MHz}, \mathrm{CDCl}_{3}\right) \delta 7.57(\mathrm{~d}, J=6.8 \mathrm{~Hz}, 2 \mathrm{H}), 7.39-7.27(\mathrm{~m}, 3 \mathrm{H}), 5.49-5.33(\mathrm{~m}, 2 \mathrm{H})$, $5.24(\mathrm{~s}, 1 \mathrm{H}), 4.89(\mathrm{~s}, 1 \mathrm{H}), 2.32-2.12(\mathrm{~m}, 2 \mathrm{H}), 2.12-1.95(\mathrm{~m}, 2 \mathrm{H}), 1.74-1.53(\mathrm{~m}, 1 \mathrm{H}), 1.50-1.24$ (m, 9H), $1.03-0.88(\mathrm{~m}, 3 \mathrm{H}), 0.86-0.76(\mathrm{~m}, 1 \mathrm{H}), 0.74-0.57(\mathrm{~m}, 1 \mathrm{H})$.

${ }^{13} \mathrm{C}\left\{{ }^{1} \mathrm{H}\right\}$ NMR $\left(201 \mathrm{MHz}, \mathrm{CDCl}_{3}\right) \delta 149.5,141.9,130.3,129.3,128.1,127.4,126.1,108.7,34.5$, 31.6, 29.5, 27.2, 27.1, 23.4, 22.6, 21.3, 14.1, 13.9.

HRMS(APCI) (m/z): [M + H] ${ }^{+}$Calcd for $\mathrm{C}_{20} \mathrm{H}_{28}: 269.2264$; found: 269.2262

TLC: $\mathrm{R}_{f}=0.84(100 \%$ hexanes $)$ 
<smiles>O=C(/C=C/CCOCc1ccccc1)c1ccccc1</smiles>
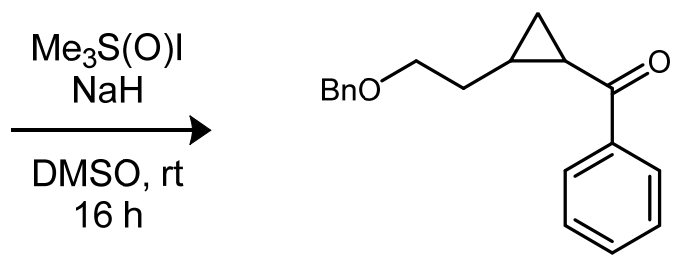

(2-(2-(benzyloxy)ethyl)cyclopropyl)(phenyl)methanone (S13). The reaction was conducted according to the general procedure B without modification using $(E)$-5-(benzyloxy)-1-phenylpent2-en-1-one ${ }^{2}$ ( $613 \mathrm{mg}, 2.30 \mathrm{mmol}, 1.0$ equiv), trimethylsulfoxonium iodide ( $608 \mathrm{~g}, 2.76 \mathrm{mmol}, 1.2$ equiv), $\mathrm{NaH}(60 \%$ in mineral oil, $110 \mathrm{mg}, 1.2$ equiv). The product was purified by column chromatography $\left(20 \% \quad \mathrm{Et}_{2} \mathrm{O} \quad\right.$ in hexanes $)$ to provide (2-(2(benzyloxy)ethyl)cyclopropyl)(phenyl)methanone (323 mg, $50 \%$ yield) as a yellow oil.

${ }^{1} \mathrm{H}$ NMR $\left(300 \mathrm{MHz}, \mathrm{CDCl}_{3}\right) \delta 7.99(\mathrm{~d}, J=6.9 \mathrm{~Hz}, 2 \mathrm{H}), 7.55(\mathrm{t}, J=7.4 \mathrm{~Hz}, 1 \mathrm{H}), 7.50-7.37(\mathrm{~m}$, $2 \mathrm{H}), 7.38-7.28(\mathrm{~m}, 5 \mathrm{H}), 4.50(\mathrm{~s}, 2 \mathrm{H}), 3.58(\mathrm{t}, J=6.2 \mathrm{~Hz}, 2 \mathrm{H}), 2.64-2.43(\mathrm{~m}, 1 \mathrm{H}), 1.96-1.77(\mathrm{~m}$, $1 \mathrm{H}), 1.77-1.60(\mathrm{~m}, 2 \mathrm{H}), 1.56-1.43(\mathrm{~m}, 1 \mathrm{H}), 1.04-0.85(\mathrm{~m}, 1 \mathrm{H})$.

${ }^{13} \mathrm{C}\left\{{ }^{1} \mathrm{H}\right\}$ NMR $\left(201 \mathrm{MHz}, \mathrm{CDCl}_{3}\right) \delta 200.0,138.4,138.0,132.6,128.5,128.4,128.0,127.6$, $127.5,73.1,69.8,33.7,25.0,24.2,18.2$.

HRMS(APCI) (m/z): [M + H] $]^{+}$Calcd for $\mathrm{C}_{19} \mathrm{H}_{20} \mathrm{O}_{2}: 281.1536$; found: 281.1538 TLC: $\mathrm{R}_{f}=0.22\left(20 \% \mathrm{Et}_{2} \mathrm{O}\right.$ in hexanes $)$
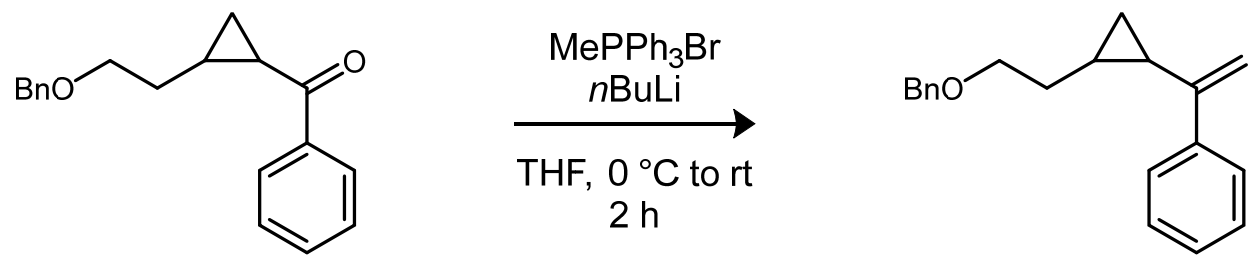

(1-(2-(2-(benzyloxy)ethyl)cyclopropyl)vinyl)benzene (S14). The reaction was conducted according to the general procedure $\mathrm{C}$ without modification using $\mathbf{S 1 3}(322 \mathrm{mg}$, $1.15 \mathrm{mmol}, 1.0$ equiv), $\mathrm{MePPh}_{3} \mathrm{Br}$ (616 mg, $1.73 \mathrm{mmol}, 1.5$ equiv), and $n \mathrm{BuLi}$ ( $2.5 \mathrm{M}$ in hexanes, $0.7 \mathrm{~mL}, 1.73 \mathrm{mmol}$, 1.5 equiv) in THF $(10 \mathrm{~mL})$. The product was purified by column chromatography $\left(5 \% \mathrm{Et}_{2} \mathrm{O}\right.$ in hexanes) to provide (1-(2-(2-(benzyloxy)ethyl)cyclopropyl)vinyl)benzene (209 $\mathrm{mg}, 65 \%$ yield) as a clear, colorless oil.

${ }^{1} \mathrm{H} \mathrm{NMR}\left(300 \mathrm{MHz}, \mathrm{CDCl}_{3}\right) \delta 7.56(\mathrm{~d}, J=6.5 \mathrm{~Hz}, 2 \mathrm{H}), 7.40-7.27(\mathrm{~m}, 8 \mathrm{H}), 5.26(\mathrm{~s}, 1 \mathrm{H}), 4.92$ (s, 1H), $4.55(\mathrm{~s}, 2 \mathrm{H}), 3.62(\mathrm{t}, J=6.6 \mathrm{~Hz}, 2 \mathrm{H}), 1.95-1.78(\mathrm{~m}, 1 \mathrm{H}), 1.74-1.60(\mathrm{~m}, 1 \mathrm{H}), 1.52-1.44(\mathrm{~m}$, $1 \mathrm{H}), 1.15-1.00(\mathrm{~m}, 1 \mathrm{H}), 0.91-0.77(\mathrm{~m}, 1 \mathrm{H}), 0.74-0.64(\mathrm{~m}, 1 \mathrm{H})$.

${ }^{13} \mathrm{C}\left\{{ }^{1} \mathrm{H}\right\}$ NMR $\left(201 \mathrm{MHz}, \mathrm{CDCl}_{3}\right) \delta 149.2,141.8,138.6,128.4,128.1,127.6,127.5,127.4$, 126.2, 109.1, 73.0, 70.2, 34.4, 23.1, 18.4, 13.6.

HRMS(ESI) (m/z): [M + H] Calcd for $\mathrm{C}_{20} \mathrm{H}_{22} \mathrm{O}: 279.1743$; found: 279.1742 TLC: $\mathrm{R}_{f}=0.67$ (5\% $\mathrm{Et}_{2} \mathrm{O}$ in hexanes) 
<smiles>C[C@@H]1CC1C(=O)c1ccccc1</smiles>

$>99 \%$ ee

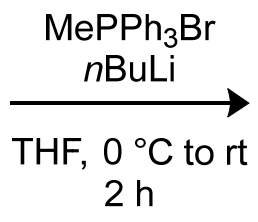

$>99 \%$ ee

(1-((1R,2R)-2-methylcyclopropyl)vinyl)benzene (29). The reaction was conducted according to the general procedure $\mathrm{C}$ without modification using $((1 R, 2 R)-2$ methylcyclopropyl)(phenyl)methanone ${ }^{10}\left(1.9 \mathrm{~g}, 11.9 \mathrm{mmol}, 1.0\right.$ equiv), $\mathrm{MePPh}_{3} \mathrm{Br}(6.40 \mathrm{~g}, 17.9$ mmol, 1.5 equiv), and $n$ BuLi ( $2.5 \mathrm{M}$ in hexanes, $7.16 \mathrm{~mL}, 17.9 \mathrm{mmol}, 1.5$ equiv) in THF (50 mL). The product was purified by column chromatography (100\% hexanes) to provide (1-((1R,2R)-2methylcyclopropyl)vinyl)benzene (786 mg, $42 \%$ yield) as a clear, colorless oil. $[\alpha]_{\mathrm{D}^{23}}=+71.8^{\circ}(\mathrm{c}$ $\left.0.204, \mathrm{CHCl}_{3}\right)$. Spectroscopic and mass spectrometry data were identical to those of the racemic product. ${ }^{11}$

HPLC: Chiralpak ${ }^{\circledR}$ OD-H column $(100 \%$ hexane, $1.0 \mathrm{~mL} / \mathrm{min}, \lambda=254 \mathrm{~nm}) \operatorname{tr}=6.54 \mathrm{~min}$ (minor), 7.22 min (major): 1: >99 er. 


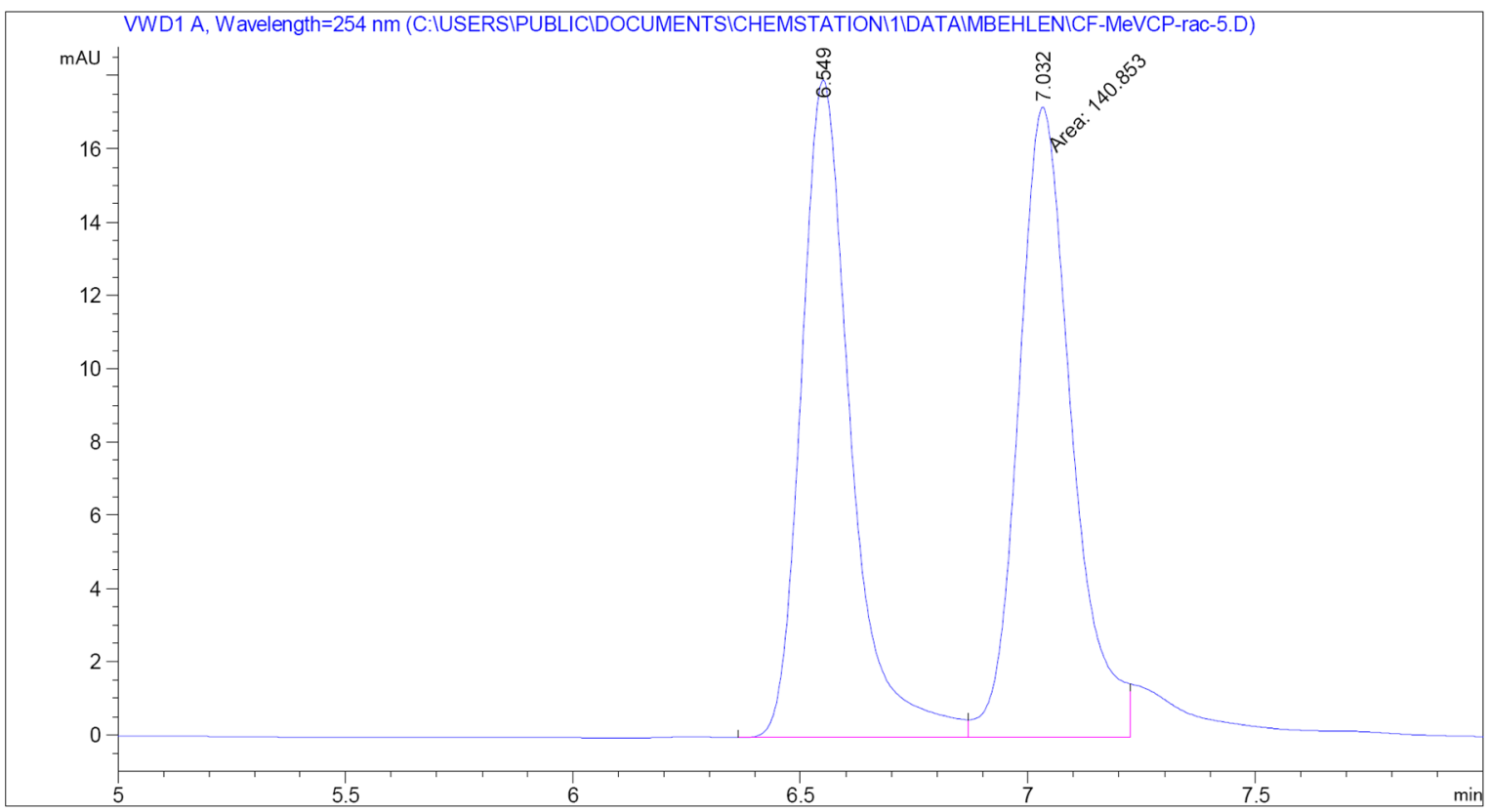

\begin{tabular}{|c|c|c|c|c|c|c|}
\hline $\begin{array}{c}\text { Peak } \\
\#\end{array}$ & $\begin{array}{c}\text { RetTime } \\
\text { [min] }\end{array}$ & Type & $\begin{array}{l}\text { Width } \\
\text { [min] }\end{array}$ & $\begin{array}{c}\text { Area } \\
{\left[\mathrm{mAU}^{*} \mathrm{~S}\right]}\end{array}$ & $\begin{array}{l}\text { Height } \\
{[\mathrm{mAU}]}\end{array}$ & $\begin{array}{c}\text { Area } \\
\frac{\circ}{8}\end{array}$ \\
\hline & 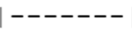 & & - & ---------- & -------- & -----1 \\
\hline 1 & 6.549 & BV & 0.1146 & 136.38600 & 17.95396 & 49.1945 \\
\hline 2 & 7.032 & $\mathrm{MF}$ & 0.1364 & 140.85257 & 17.21415 & 50.8055 \\
\hline
\end{tabular}

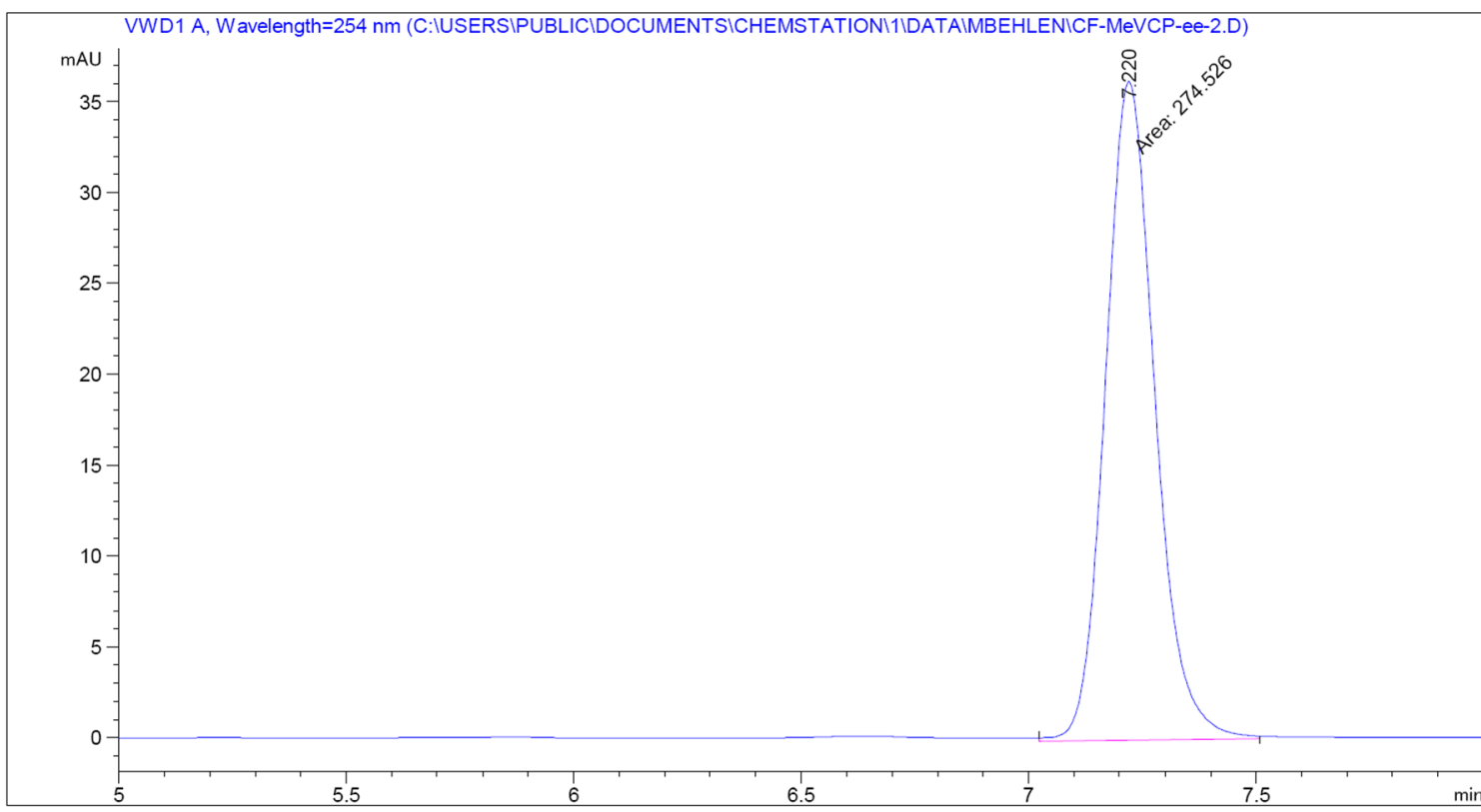

\begin{tabular}{|c|c|c|c|c|c|c|}
\hline $\begin{array}{c}\text { Peak } \\
\#\end{array}$ & $\begin{array}{c}\text { RetTime } \\
\text { [min] }\end{array}$ & Type & $\begin{array}{l}\text { Width } \\
\text { [min] }\end{array}$ & $\begin{array}{c}\text { Area } \\
{\left[\mathrm{mAU}^{\star} \mathrm{S}\right]}\end{array}$ & $\begin{array}{l}\text { Height } \\
\text { [mAU] }\end{array}$ & $\begin{array}{c}\text { Area } \\
\frac{\circ}{8}\end{array}$ \\
\hline-- & -1 & & & ------ & & $-------\mid$ \\
\hline 1 & 7.220 & MM & 0.1262 & 274.52582 & 2633 & 00.0000 \\
\hline
\end{tabular}

Figure S1. HPLC data for 29. (racemate, top; 29, bottom). 


\section{Synthesis and Characterization of Vinylidene Substrates}

General procedure for the synthesis of 1,1-dichlorolakenes from aldehydes. Under an $\mathrm{N}_{2}$ atmosphere, a round bottom flask equipped with a magnetic stir bar was charged with $\mathrm{Ph}_{3} \mathrm{P}$ (4.0 equiv) and $\mathrm{MeCN}$ ( $5 \mathrm{~mL} / \mathrm{mmol}$ ). With stirring, a solution of the aldehyde (1.0 equiv) and $\mathrm{CCl}_{4}$ (2.0 equiv) in MeCN ( $2 \mathrm{~mL} / \mathrm{mmol}$ ) was added dropwise over $1 \mathrm{~h}$. Following addition, the reaction mixture was stirred at room temperature for $2 \mathrm{~h}$. The reaction was then quenched by addition of $\mathrm{H}_{2} \mathrm{O}(100 \mathrm{~mL})$. The crude reaction mixture was extracted with $\mathrm{Et}_{2} \mathrm{O}$ (3 x $\left.50 \mathrm{~mL}\right)$. The combined organic phases were dried over $\mathrm{MgSO}_{4}$, filtered, and concentrated under reduced pressure. The crude residue was loaded directly onto a $\mathrm{SiO}_{2}$ column for purification.<smiles>COC(=O)CCCC=O</smiles>
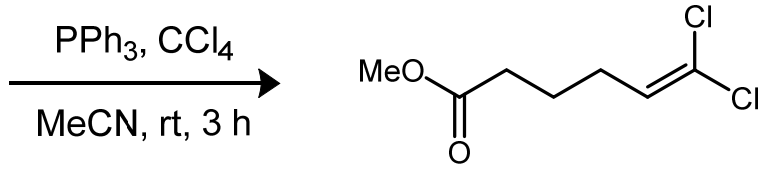

methyl 6,6-dichlorohex-5-enoate (S15). The reaction was conducted according to the general procedure without modification using methyl 5-oxopentanoate (1.8 g, $13.8 \mathrm{mmol}, 1.0$ equiv), triphenylphosphine (14.5 g, $55.3 \mathrm{mmol}, 4.0$ equiv), and $\mathrm{CCl}_{4}$ (4.26 g, $2.69 \mathrm{~mL}, 27.6 \mathrm{mmol}, 2.0$ equiv). The product was purified by column chromatography (10\% Et $2 \mathrm{O}$ in hexanes) to provide methyl 6,6-dichlorohex-5-enoate (1.19 g, 44\% yield) as a clear, colorless oil.

${ }^{1} \mathrm{H} \mathrm{NMR}\left(300 \mathrm{MHz} \mathrm{CDCl}_{3}\right) \delta 5.84(\mathrm{t}, J=7.5 \mathrm{~Hz}, 1 \mathrm{H}), 3.68(\mathrm{~s}, 3 \mathrm{H}), 2.34(\mathrm{t}, J=7.4 \mathrm{~Hz}, 2 \mathrm{H}), 2.22$ (q, $J=7.5 \mathrm{~Hz}, 2 \mathrm{H}), 1.85-1.65(\mathrm{~m}, 2 \mathrm{H})$.

${ }^{13} \mathrm{C}\left\{{ }^{1} \mathrm{H}\right\}$ NMR $(201 \mathrm{MHz}, \mathrm{CDCl} 3) \delta 173.4,128.6,120.9,51.6,33.1,28.8,23.3$.

HRMS(APCI) (m/z): [M + H] ${ }^{+}$Calcd for $\mathrm{C}_{7} \mathrm{H}_{10} \mathrm{Cl}_{2} \mathrm{O}_{2}$ : 197.0131; found: 197.0129

TLC: $\mathrm{R}_{f}=0.29\left(10 \% \mathrm{Et}_{2} \mathrm{O}\right.$ in hexanes)<smiles>CN([As])CCC=O</smiles>

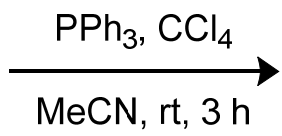<smiles>CN([AsH3])CCC=C(Cl)Cl</smiles>

N-(4,4-dichlorobut-3-en-1-yl)-N,4-dimethylbenzenesulfonamide (S16). The reaction was conducted according to the general procedure without modification using N,4-dimethyl-N-(3oxopropyl)benzenesulfonamide ${ }^{12}$ (1.89 g, $7.83 \mathrm{mmol}, 1.0$ equiv), triphenylphosphine (8.22 g, 31.3 mmol, 4.0 equiv), and $\mathrm{CCl}_{4}(2.41 \mathrm{~g}, 0.89 \mathrm{~mL}, 15.7 \mathrm{mmol}, 2.0$ equiv). The product was purified by column chromatography (30\% EtOAc in hexanes) to provide N-(4,4-dichlorobut-3-en-1-yl)-N,4dimethylbenzenesulfonamide ( $869 \mathrm{mg}, 36 \%$ yield) as a yellow oil.

${ }^{1} \mathrm{H}$ NMR (300 MHz, CDCl 3$) \delta 7.67(\mathrm{~d}, J=8.3 \mathrm{~Hz}, 2 \mathrm{H}), 7.32(\mathrm{~d}, J=7.9 \mathrm{~Hz}, 2 \mathrm{H}), 5.89$ (t, $J=7.2$ $\mathrm{Hz}, 1 \mathrm{H}), 3.08(\mathrm{t}, J=7.1 \mathrm{~Hz}, 2 \mathrm{H}), 2.74(\mathrm{~s}, 3 \mathrm{H}), 2.49-2.34(\mathrm{~m}, 5 \mathrm{H})$.

${ }^{13} \mathrm{C}\left\{{ }^{1} \mathrm{H}\right\} \mathrm{NMR}\left(201 \mathrm{MHz}, \mathrm{CDCl}_{3}\right) \delta 143.4,134.4,129.7,127.3,125.8,122.2,48.2,34.7,28.2$, 21.4.

HRMS(APCI) (m/z): [M + H] $]^{+}$Calcd for $\mathrm{C}_{12} \mathrm{H}_{15} \mathrm{Cl}_{2} \mathrm{NO}_{2} \mathrm{~S}$ : 308.0273; found: 308.0271

TLC: $\mathrm{R}_{f}=0.15\left(10 \% \mathrm{Et}_{2} \mathrm{O}\right.$ in hexanes $)$ 


\section{Substrate Scope Studies and Product Characterization}<smiles>ClC(Cl)=C[Tl]</smiles>

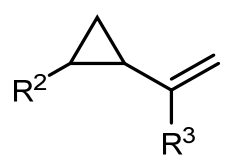

3.0 Equiv.
$\mathrm{Co}(\mathrm{DME}) \mathrm{Br}_{2}(5 \mathrm{~mol} \%)$

$( \pm)-t$-Bu-Quinox (6 mol\%)

$$
\text { Zn (3.0 equiv) }
$$

DMA, rt

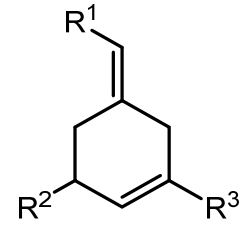

General Procedure for the [5 + 1]-Cycloaddition Reaction. In an N2-filled glovebox, a 5$\mathrm{mL}$ vial was charged with $\mathrm{Co}(\mathrm{DME}) \mathrm{Br}_{2}(1.54 \mathrm{mg}, 0.005 \mathrm{mmol}, 0.05$ equiv), $( \pm)-t$-Bu-Quinox (1.52 $\mathrm{mg}, 0.006 \mathrm{mmol}, 0.06$ equiv), $\mathrm{Zn}$ powder (19.6 mg, $0.3 \mathrm{mmol}, 3.0$ equiv), and a magnetic stir bar. To this mixture was added a solution of the 1,1-dichloroalkene (0.1 mmol, 1.0 equiv) and the vinylcyclopropane $(0.3 \mathrm{mmol}, 3.0$ equiv) dissolved in DMA $(0.75 \mathrm{~mL})$. The reaction was stirred at room temperature. After $16 \mathrm{~h}$, the crude reaction mixture was removed from the glovebox, opened to ambient atmosphere, and loaded directly onto $\mathrm{SiO}_{2}$ column for purification.<smiles>COc1ccc(C=C2CC=CCC2)cc1</smiles>

(E)-1-(cyclohex-3-en-1-ylidenemethyl)-4-methoxybenzene (3). The reaction was conducted according to the general procedure without modification using 1-(2,2-dichlorovinyl)-4methoxybenzene (20.4 mg, $0.1 \mathrm{mmol}, 1.0$ equiv) and vinylcyclopropane (20.4 mg, $0.3 \mathrm{mmol}, 3.0$ equiv). Isolated yields were determined following column chromatography $\left(\mathrm{SiO}_{2}, 15 \% \mathrm{CH}_{2} \mathrm{Cl}_{2}\right.$ in hexanes).

$14.1 \mathrm{mg}$ isolated (70\% yield), colorless oil, $E / Z=>20: 1$

${ }^{1} \mathrm{H}$ NMR $\left(300 \mathrm{MHz} \mathrm{CDCl}_{3}\right) \delta 7.16(\mathrm{~d}, J=8.7 \mathrm{~Hz}, 2 \mathrm{H}), 6.87(\mathrm{~d}, J=8.7 \mathrm{~Hz}, 2 \mathrm{H}), 6.33(\mathrm{~s}, 1 \mathrm{H}), 5.81$ - $5.65(\mathrm{~m}, 2 \mathrm{H}), 3.81(\mathrm{~s}, 3 \mathrm{H}), 2.89(\mathrm{~s}, 2 \mathrm{H}), 2.55(\mathrm{t}, J=6.4 \mathrm{~Hz}, 2 \mathrm{H}), 2.25-2.10(\mathrm{~m}, 2 \mathrm{H})$. ${ }^{13} \mathrm{C}\left\{{ }^{1} \mathrm{H}\right\} \operatorname{NMR}\left(201 \mathrm{MHz}, \mathrm{CDCl}_{3}\right) \delta 157.7,137.9,130.5,129.8,126.8,126.6,122.1,113.4,55.1$, 35.6, 27.1, 25.8.

HRMS(APCI) (m/z): [M + H]+ Calcd for $\mathrm{C}_{14} \mathrm{H}_{16} \mathrm{O}: 201.1274$; found: 201.1272

TLC: $\mathrm{R}_{f}=0.51\left(5 \% \mathrm{Et}_{2} \mathrm{O}\right.$ in hexanes) 


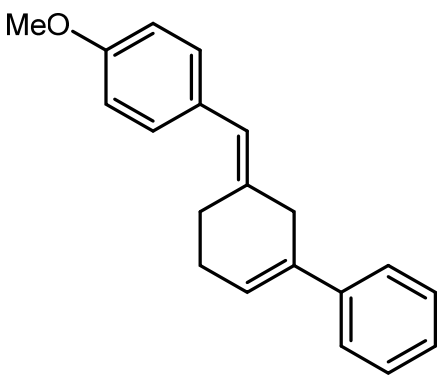

(E)-3-(4-methoxybenzylidene)-2,3,4,5-tetrahydro-1,1'-biphenyl (6). The reaction was conducted according to the general procedure without modification using 1-(2,2-dichlorovinyl)4-methoxybenzene ( $20.4 \mathrm{mg}, 0.1 \mathrm{mmol}, 1.0$ equiv) and (1-cyclopropylvinyl)benzene ( $43.3 \mathrm{mg}, 0.3$ mmol, 3.0 equiv). Isolated yields were determined following column chromatography $\left(\mathrm{SiO}_{2}, 15 \%\right.$ $\mathrm{CH}_{2} \mathrm{Cl}_{2}$ in hexanes). Single crystals of 6 suitable for X-ray diffraction analysis were obtained by evaporation of a saturated $\mathrm{Et}_{2} \mathrm{O}$ solution at room temperature.

$25.9 \mathrm{mg}$ isolated ( $94 \%$ yield), white solid, $E / Z=>20: 1$

${ }^{1} \mathrm{H}$ NMR $\left(300 \mathrm{MHz}, \mathrm{CDCl}_{3}\right) \delta 7.43(\mathrm{~d}, J=7.2 \mathrm{~Hz}, 2 \mathrm{H}), 7.34(\mathrm{t}, J=7.2 \mathrm{~Hz}, 2 \mathrm{H}), 7.25-7.22(\mathrm{~m}$, $1 \mathrm{H}), 7.19(\mathrm{~d}, J=8.8 \mathrm{~Hz}, 2 \mathrm{H}), 6.88(\mathrm{~d}, J=8.5 \mathrm{~Hz}, 2 \mathrm{H}), 6.43(\mathrm{~s}, 1 \mathrm{H}), 6.26-6.11(\mathrm{~m}, 1 \mathrm{H}), 3.82(\mathrm{~s}, 3 \mathrm{H})$, $3.29(\mathrm{~s}, 2 \mathrm{H}), 2.61(\mathrm{t}, J=6.4 \mathrm{~Hz}, 2 \mathrm{H}), 2.43-2.30(\mathrm{~m}, 2 \mathrm{H})$.

${ }^{13} \mathrm{C}\left\{{ }^{1} \mathrm{H}\right\}$ NMR $\left(201 \mathrm{MHz}, \mathrm{CDCl}_{3}\right) \delta 157.9,141.5,137.8,136.4,130.6,129.9,128.3,126.9$, 125.1, 124.2, 122.8, 113.6, 55.3, 37.9, 27.5, 25.5.

HRMS(ESI) (m/z): [M + H] ${ }^{+}$Calcd for $\mathrm{C}_{20} \mathrm{H}_{20} \mathrm{O}: 277.1587$; found: 277.1589

TLC: $\mathrm{R}_{f}=0.46\left(5 \% \mathrm{Et}_{2} \mathrm{O}\right.$ in hexanes)

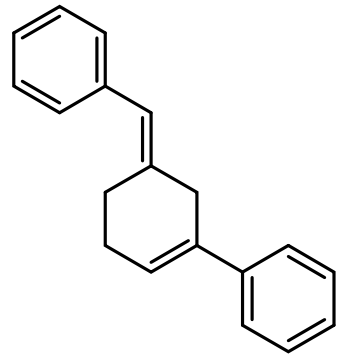

(E)-3-benzylidene-2,3,4,5-tetrahydro-1,1'-biphenyl (11). The reaction was conducted according to the general procedure without modification using 1-(2,2-dichlorovinyl)benzene (17.3 mg, $0.1 \mathrm{mmol}, 1.0$ equiv) and (1-cyclopropylvinyl)benzene ( $43.3 \mathrm{mg}, 0.3 \mathrm{mmol}, 3.0$ equiv). Isolated yields were determined following column chromatography $\left(\mathrm{SiO}_{2}, 15 \% \mathrm{CH}_{2} \mathrm{Cl}_{2}\right.$ in hexanes).

$21.5 \mathrm{mg}$ isolated ( $87 \%$ yield), white solid, $E / Z=>20: 1$

${ }^{1} \mathrm{H}$ NMR $\left(300 \mathrm{MHz}, \mathrm{CDCl}_{3}\right) \delta 7.44(\mathrm{~d}, J=8.0 \mathrm{~Hz}, 2 \mathrm{H}), 7.35(\mathrm{t}, J=7.3 \mathrm{~Hz}, 4 \mathrm{H}), 7.29-7.27(\mathrm{~m}$, $1 \mathrm{H}), 7.26-7.17(\mathrm{~m}, 3 \mathrm{H}), 6.50(\mathrm{~s}, 1 \mathrm{H}), 6.27-6.10(\mathrm{~m}, 1 \mathrm{H}), 3.39-3.25(\mathrm{~m}, 2 \mathrm{H}), 2.62(\mathrm{t}, J=6.4 \mathrm{~Hz}$, $2 \mathrm{H}), 2.47-2.27(\mathrm{~m}, 2 \mathrm{H})$.

${ }^{13} \mathrm{C}\left\{{ }^{1} \mathrm{H}\right\} \mathrm{NMR}\left(201 \mathrm{MHz}, \mathrm{CDCl}_{3}\right) \delta 141.5,139.0,138.0,136.3,128.9,128.3,128.1,126.9$, 126.1, 125.1, 124.2, 123.4, 38.0, 27.5, 25.5.

HRMS(APCI) (m/z): [M + H] ${ }^{+}$Calcd for $\mathrm{C}_{19} \mathrm{H}_{18}: 245.1325$; found: 245.1327

TLC: $\mathrm{R}_{f}=0.61\left(5 \% \mathrm{Et}_{2} \mathrm{O}\right.$ in hexanes) 


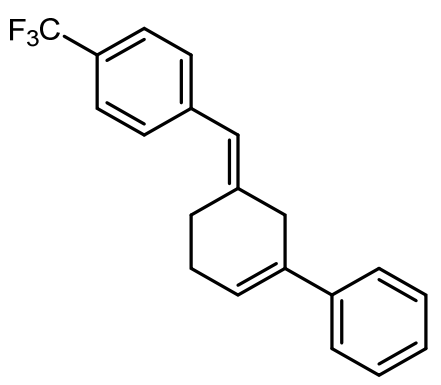

(E)-3-(4-(trifluoromethyl)benzylidene)-2,3,4,5-tetrahydro-1,1'-biphenyl (12). The reaction was conducted according to the general procedure without modification using 1-(2,2-

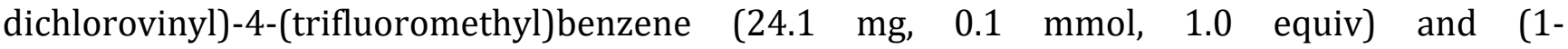
cyclopropylvinyl)benzene ( $43.3 \mathrm{mg}, 0.3 \mathrm{mmol}, 3.0$ equiv). Isolated yields were determined following column chromatography $\left(\mathrm{SiO}_{2}, 5 \% \mathrm{Et}_{2} \mathrm{O}\right.$ in hexanes).

$20.5 \mathrm{mg}$ isolated (65\% yield), white solid, $E / Z=>20: 1$

${ }^{1} \mathrm{H} \mathrm{NMR}\left(300 \mathrm{MHz}, \mathrm{CDCl}_{3}\right) \delta 7.59(\mathrm{~d}, J=8.1 \mathrm{~Hz}, 2 \mathrm{H}), 7.44(\mathrm{~d}, J=7.0 \mathrm{~Hz}, 2 \mathrm{H}), 7.40-7.32(\mathrm{~m}$, $4 \mathrm{H}), 7.32-7.27(\mathrm{~m}, 1 \mathrm{H}), 6.51(\mathrm{~s}, 1 \mathrm{H}), 6.27-6.14(\mathrm{~m}, 1 \mathrm{H}), 3.34(\mathrm{~s}, 2 \mathrm{H}), 2.61(\mathrm{t}, J=6.4 \mathrm{~Hz}, 2 \mathrm{H}), 2.47$ $-2.32(\mathrm{~m}, 2 \mathrm{H})$.

${ }^{13} \mathrm{C}\left\{{ }^{1} \mathrm{H}\right\}$ NMR $\left(201 \mathrm{MHz}, \mathrm{CDCl}_{3}\right) \delta 141.7,141.3,141.3,136.1,129.1,128.4,128.1\left(\mathrm{q},{ }^{2} \mathrm{JCF}_{\mathrm{CF}}=\right.$ $32.5 \mathrm{~Hz}), 127.0,125.1\left(\mathrm{q},{ }^{3} J_{\mathrm{CF}}=3.6 \mathrm{~Hz}\right), 125.1,124.1\left(\mathrm{q},{ }^{1} J_{\mathrm{CF}}=272.8 \mathrm{~Hz}\right), 124.0,122.3,38.0,27.4$, 25.6 .

${ }^{19} \mathrm{~F}$ NMR $\left(282 \mathrm{MHz}, \mathrm{CDCl}_{3}\right) \delta-63.86$.

HRMS(APCI) (m/z): [M + H] $]^{+}$Calcd for $\mathrm{C}_{20} \mathrm{H}_{17} \mathrm{~F}_{3}$ : 315.1355 ; found: 315.1348

TLC: $\mathrm{R}_{f}=0.51\left(5 \% \mathrm{Et}_{2} \mathrm{O}\right.$ in hexanes)

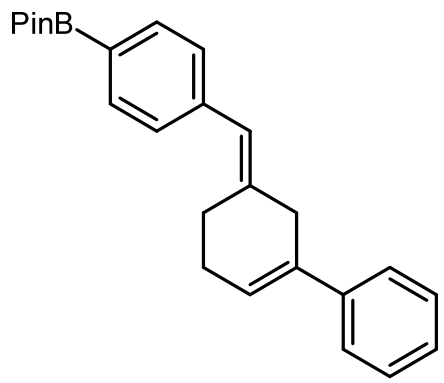

(E)-2-(4-((4,5-dihydro-[1,1'-biphenyl]-3(2H)-ylidene)methyl)phenyl)-4,4,5,5-tetramethyl1,3,2-dioxaborolane (13). The reaction was conducted according to the general procedure without modification using 2-(4-(2,2-dichlorovinyl)phenyl)-4,4,5,5-tetramethyl-1,3,2dioxaborolane (29.9 mg, $0.1 \mathrm{mmol}, 1.0$ equiv) and (1-cyclopropylvinyl)benzene ( $43.3 \mathrm{mg}, 0.3$ mmol, 3.0 equiv). Isolated yields were determined following column chromatography $\left(\mathrm{SiO}_{2}, 5 \%\right.$ $\mathrm{Et}_{2} \mathrm{O}$ in hexanes).

$22.3 \mathrm{mg}$ isolated (60\% yield), white solid, $E / Z=>20: 1$

${ }^{1} \mathrm{H}$ NMR $\left(300 \mathrm{MHz}, \mathrm{CDCl}_{3}\right) \delta 7.78(\mathrm{~d}, J=7.8 \mathrm{~Hz}, 2 \mathrm{H}), 7.43(\mathrm{~d}, J=7.6 \mathrm{~Hz}, 2 \mathrm{H}), 7.34(\mathrm{t}, J=7.5$ $\mathrm{Hz}, 2 \mathrm{H}), 7.29-7.26(\mathrm{~m}, 3 \mathrm{H}), 6.50(\mathrm{~s}, 1 \mathrm{H}), 6.19(\mathrm{~s}, 1 \mathrm{H}), 3.31(\mathrm{~s}, 2 \mathrm{H}), 2.62(\mathrm{t}, J=6.4 \mathrm{~Hz}, 2 \mathrm{H}), 2.43-$ $2.29(\mathrm{~m}, 2 \mathrm{H}), 1.35(\mathrm{~s}, 12 \mathrm{H})$. 
${ }^{13} \mathrm{C}\left\{{ }^{1} \mathrm{H}\right\}$ NMR $\left(201 \mathrm{MHz}, \mathrm{CDCl}_{3}\right) \delta 141.4,141.0,139.9,136.2,134.6,128.3,128.2,126.9$, $125.1,124.2,123.5,83.7,38.1,27.5,25.6,24.9$.

HRMS(APCI) (m/z): [M + H] ${ }^{+}$Calcd for $\mathrm{C}_{25} \mathrm{H}_{29} \mathrm{BO}_{2}: 372.2370$; found: 372.2370

TLC: $\mathrm{R}_{f}=0.46\left(5 \% \mathrm{Et}_{2} \mathrm{O}\right.$ in hexanes)

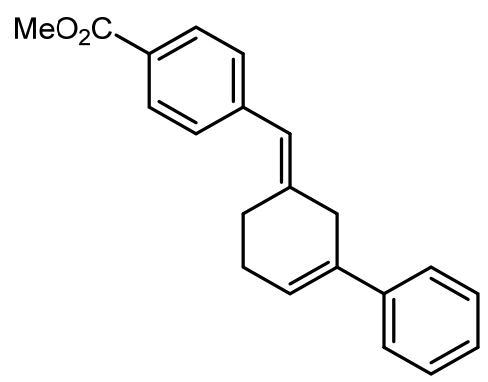

methyl (E)-4-((4,5-dihydro-[1,1'-biphenyl]-3(2H)-ylidene)methyl)benzoate (14). The reaction was conducted according to the general procedure without modification using methyl 4(2,2-dichlorovinyl)benzoate $(23.1 \mathrm{mg}, 0.1 \mathrm{mmol}, 1.0 \mathrm{equiv})$ and (1-cyclopropylvinyl)benzene $(43.3 \mathrm{mg}, \quad 0.3 \mathrm{mmol}, 3.0$ equiv). Isolated yields were determined following column chromatography $\left(\mathrm{SiO}_{2}, 15 \% \mathrm{Et}_{2} \mathrm{O}\right.$ in hexanes).

$21.2 \mathrm{mg}$ isolated (70\% yield), white solid, $E / Z=>20: 1$

${ }^{1} \mathrm{H} \mathrm{NMR}\left(300 \mathrm{MHz}, \mathrm{CDCl}_{3}\right) \delta 8.01(\mathrm{~d}, J=8.4 \mathrm{~Hz}, 2 \mathrm{H}), 7.43(\mathrm{~d}, J=7.0 \mathrm{~Hz}, 2 \mathrm{H}), 7.39-7.27(\mathrm{~m}$, $5 \mathrm{H}), 6.51(\mathrm{~s}, 1 \mathrm{H}), 6.27-6.12(\mathrm{~m}, 1 \mathrm{H}), 3.92(\mathrm{~s}, 3 \mathrm{H}), 3.33(\mathrm{~s}, 2 \mathrm{H}), 2.63(\mathrm{t}, J=6.4 \mathrm{~Hz}, 2 \mathrm{H}), 2.49-2.30$ $(\mathrm{m}, 2 \mathrm{H})$.

${ }^{13} \mathrm{C}\left\{{ }^{1} \mathrm{H}\right\}$ NMR $\left(201 \mathrm{MHz}, \mathrm{CDCl}_{3}\right) \delta 167.0,142.9,141.3,136.1,129.5,128.8,128.4,127.8$, 127.0, 125.1, 124.0, 122.8, 52.0, 38.1, 27.4, 25.7.

HRMS(APCI) (m/z): [M + H] $]^{+}$Calcd for $\mathrm{C}_{21} \mathrm{H}_{20} \mathrm{O}_{2}: 305.1536$; found: 305.1538

TLC: $\mathrm{R}_{f}=0.31\left(5 \% \mathrm{Et}_{2} \mathrm{O}\right.$ in hexanes)

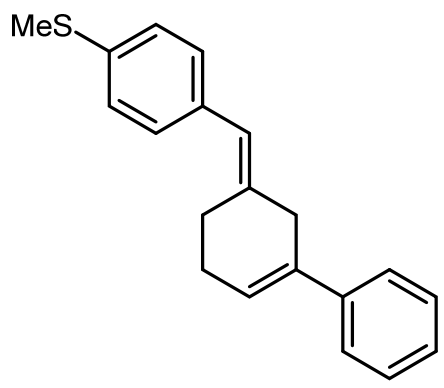

(E)-(4-((4,5-dihydro-[1,1'-biphenyl]-3(2H)-ylidene)methyl)phenyl)(methyl)sulfane (15). The reaction was conducted according to the general procedure without modification using (4(2,2-dichlorovinyl)phenyl)(methyl)sulfane $(21.9 \mathrm{mg}, \quad 0.1 \mathrm{mmol}, \quad 1.0$ equiv) and (1cyclopropylvinyl)benzene ( $43.3 \mathrm{mg}, 0.3 \mathrm{mmol}, 3.0$ equiv). Isolated yields were determined following column chromatography $\left(\mathrm{SiO}_{2}, 15 \% \mathrm{CH}_{2} \mathrm{Cl}_{2}\right.$ in hexanes).

$24.2 \mathrm{mg}$ isolated (83\% yield), white solid, $E / Z=>20: 1$ 
${ }^{1} \mathrm{H}$ NMR (300 MHz, $\left.\mathrm{CDCl}_{3}\right) \delta 7.43(\mathrm{~d}, J=8.1 \mathrm{~Hz}, 2 \mathrm{H}), 7.34(\mathrm{t}, J=7.5 \mathrm{~Hz}, 2 \mathrm{H}), 7.25(\mathrm{~s}, 1 \mathrm{H}), 7.24$ - $7.13(\mathrm{~m}, 4 \mathrm{H}), 6.44(\mathrm{~s}, 1 \mathrm{H}), 6.26-6.11(\mathrm{~m}, 1 \mathrm{H}), 3.30(\mathrm{~s}, 2 \mathrm{H}), 2.61(\mathrm{t}, J=6.5 \mathrm{~Hz}, 2 \mathrm{H}), 2.50(\mathrm{~s}, 3 \mathrm{H})$, $2.42-2.28(\mathrm{~m}, 2 \mathrm{H})$.

${ }^{13} \mathrm{C}\left\{{ }^{1} \mathrm{H}\right\} \mathrm{NMR}\left(201 \mathrm{MHz}, \mathrm{CDCl}_{3}\right) \delta 141.4,139.1,136.3,135.9,135.0,129.3,128.3,126.9$, $126.6,125.1,124.1,122.8,38.0,27.4,25.6,16.1$.

HRMS(ESI) (m/z): [M + H] $]^{+}$Calcd for $\mathrm{C}_{20} \mathrm{H}_{20} \mathrm{~S}: 293.1359$; found: 293.1355

TLC: $\mathrm{R}_{f}=0.45\left(5 \% \mathrm{Et}_{2} \mathrm{O}\right.$ in hexanes)<smiles>CCOC(=O)N1CCC(=C2CCC=C(c3ccccc3)C2)CC1</smiles>

ethyl 4-(4,5-dihydro-[1,1'-biphenyl]-3(2H)-ylidene)piperidine-1-carboxylate (16). The reaction was conducted according to the general procedure without modification using ethyl 4(dichloromethylene)piperidine-1-carboxylate $(23.8 \mathrm{mg}, 0.1 \mathrm{mmol}, 1.0 \mathrm{equiv})$ and (1cyclopropylvinyl)benzene (43.3 $\mathrm{mg}, 0.3 \mathrm{mmol}, 3.0$ equiv). Isolated yields were determined following column chromatography ( $\mathrm{SiO}_{2}, 20 \% \mathrm{Et}_{2} \mathrm{O}$ in hexanes).

$28.0 \mathrm{mg}$ isolated ( $90 \%$ yield), white solid

${ }^{1} \mathrm{H}$ NMR (300 MHz, CDCl $) \delta 7.41(\mathrm{~d}, J=7.1 \mathrm{~Hz}, 2 \mathrm{H}), 7.39-7.27(\mathrm{~m}, 2 \mathrm{H}), 7.28-7.18(\mathrm{~m}, 1 \mathrm{H})$, $6.25-6.08(\mathrm{~m}, 1 \mathrm{H}), 4.15(\mathrm{q}, J=7.1 \mathrm{~Hz}, 2 \mathrm{H}), 3.47(\mathrm{~s}, 4 \mathrm{H}), 3.20(\mathrm{~s}, 2 \mathrm{H}), 2.47-2.22(\mathrm{~m}, 8 \mathrm{H}), 1.27(\mathrm{t}, J$ $=7.1 \mathrm{~Hz}, 3 \mathrm{H})$.

${ }^{13} \mathrm{C}\left\{{ }^{1} \mathrm{H}\right\}$ NMR $\left(201 \mathrm{MHz}, \mathrm{CDCl}_{3}\right) \delta 155.7,142.0,136.3,128.3,128.0,126.8,126.0,125.1$, 124.7, 61.2, 44.8, 44.4, 31.6, 29.3, 28.9, 27.3, 26.0, 14.8 .

HRMS(ESI) (m/z): [M + H] ${ }^{+}$Calcd for $\mathrm{C}_{20} \mathrm{H}_{25} \mathrm{NO}_{2}$ : 312.1985 ; found: 312.1956

TLC: $\mathrm{R}_{f}=0.33\left(15 \% \mathrm{Et}_{2} \mathrm{O}\right.$ in hexanes)<smiles>C1=C(c2ccccc2)CCCC1=C1CCOCC1</smiles>

4-(4,5-dihydro-[1,1'-biphenyl]-3(2H)-ylidene)tetrahydro-2H-pyran (17). The reaction was conducted according to the general procedure without modification using 4-

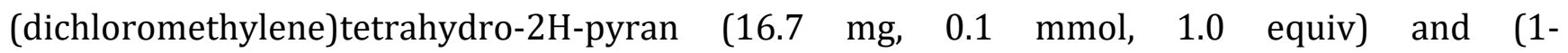


cyclopropylvinyl)benzene ( $43.3 \mathrm{mg}, 0.3 \mathrm{mmol}, 3.0$ equiv). Isolated yields were determined following column chromatography $\left(\mathrm{SiO}_{2}, 10 \% \mathrm{Et}_{2} \mathrm{O}\right.$ in hexanes).

$18.2 \mathrm{mg}$ isolated (76\% yield), white solid

${ }^{1} \mathrm{H}$ NMR $\left(300 \mathrm{MHz}, \mathrm{CDCl}_{3}\right) \delta 7.41(\mathrm{~d}, J=7.7 \mathrm{~Hz}, 2 \mathrm{H}), 7.33(\mathrm{t}, J=7.5 \mathrm{~Hz}, 2 \mathrm{H}), 7.23(\mathrm{~d}, J=7.3$ $\mathrm{Hz}, 1 \mathrm{H}), 6.30-6.06(\mathrm{~m}, 1 \mathrm{H}), 3.69(\mathrm{q}, J=5.4 \mathrm{~Hz}, 4 \mathrm{H}), 3.20(\mathrm{~s}, 2 \mathrm{H}), 2.46-2.34(\mathrm{~m}, 6 \mathrm{H}), 2.34-2.21$ (m, 2H).

${ }^{13} \mathrm{C}\left\{{ }^{1} \mathrm{H}\right\}$ NMR $\left(201 \mathrm{MHz}, \mathrm{CDCl}_{3}\right) \delta 141.9,136.3,128.2,126.9,126.7,125.7,125.0,124.6,69.1$, 68.8, 31.3, 30.8, 30.5, 27.3, 25.6.

HRMS(APCI) (m/z): [M + H] Calcd for $\mathrm{C}_{17} \mathrm{H}_{20} \mathrm{O}: 241.1587$; found: 241.1590

TLC: $\mathrm{R}_{f}=0.74\left(15 \% \mathrm{Et}_{2} \mathrm{O}\right.$ in hexanes)

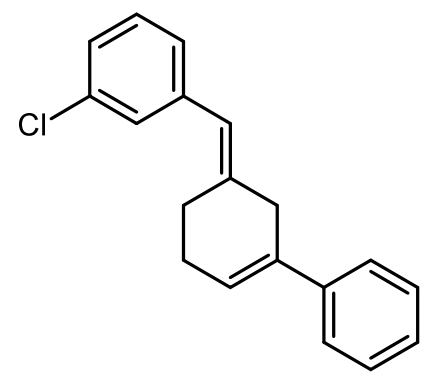

(E)-3-(3-chlorobenzylidene)-2,3,4,5-tetrahydro-1,1'-biphenyl (18). The reaction was conducted according to the general procedure without modification using 1-chloro-3-(2,2dichlorovinyl)benzene ( $20.7 \mathrm{mg}, 0.1 \mathrm{mmol}, 1.0$ equiv) and (1-cyclopropylvinyl)benzene ( $43.3 \mathrm{mg}$, $0.3 \mathrm{mmol}, 3.0$ equiv). Isolated yields were determined following column chromatography $\left(\mathrm{SiO}_{2}\right.$, $5 \% \mathrm{Et}_{2} \mathrm{O}$ in hexanes).

$25.1 \mathrm{mg}$ isolated (89\% yield), yellow oil, $E / Z=>20: 1$

${ }^{1} \mathrm{H}$ NMR $\left(300 \mathrm{MHz}, \mathrm{CDCl}_{3}\right) \delta 7.44(\mathrm{~d}, J=7.1 \mathrm{~Hz}, 2 \mathrm{H}), 7.35(\mathrm{t}, J=7.5 \mathrm{~Hz}, 2 \mathrm{H}), 7.31-7.26(\mathrm{~m}$, 2H), $7.25-7.17(\mathrm{~m}, 2 \mathrm{H}), 7.13(\mathrm{~d}, J=7.5 \mathrm{~Hz}, 1 \mathrm{H}), 6.43(\mathrm{~s}, 1 \mathrm{H}), 6.27-6.11(\mathrm{~m}, 1 \mathrm{H}), 3.31(\mathrm{~s}, 2 \mathrm{H}), 2.60$ $(\mathrm{t}, J=6.4 \mathrm{~Hz}, 2 \mathrm{H}), 2.47-2.31(\mathrm{~m}, 2 \mathrm{H})$.

${ }^{13} \mathrm{C}\left\{{ }^{1} \mathrm{H}\right\}$ NMR $\left(201 \mathrm{MHz}, \mathrm{CDCl}_{3}\right) \delta 141.3,140.5,139.8,136.1,134.0,129.4,128.8,128.4$, 127.0, 127.0, 126.2, 125.1, 124.1, 122.2, 37.9, 27.4, 25.6.

HRMS(APCI) (m/z): [M - H] $]^{+}$Calcd for $\mathrm{C}_{19} \mathrm{H}_{17} \mathrm{Cl}: 279.0935$; found: 279.0931

TLC: $\mathrm{R}_{f}=0.69$ (5\% $\mathrm{Et}_{2} \mathrm{O}$ in hexanes)

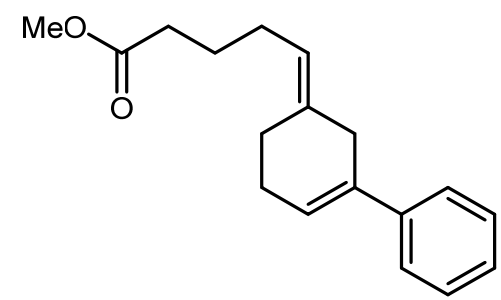

methyl (E)-5-(4,5-dihydro-[1,1'-biphenyl]-3(2H)-ylidene)pentanoate (19). The reaction was conducted according to the general procedure without modification using methyl 6,6-dichlorohex5 -enoate (19.7 mg, $0.1 \mathrm{mmol}, 1.0$ equiv) and (1-cyclopropylvinyl)benzene ( $43.3 \mathrm{mg}, 0.3 \mathrm{mmol}, 3.0$ 
equiv). Isolated yields were determined following column chromatography $\left(\mathrm{SiO}_{2}, 5 \% \mathrm{Et}_{2} \mathrm{O}\right.$ in hexanes).

$15.5 \mathrm{mg}$ isolated ( $57 \%$ yield), colorless oil, $E / Z=5: 1$

${ }^{1} \mathrm{H}$ NMR $\left(300 \mathrm{MHz}, \mathrm{CDCl}_{3}\right) \delta 7.39(\mathrm{~d}, J=8.3 \mathrm{~Hz}, 2 \mathrm{H}), 7.32(\mathrm{t}, J=7.4 \mathrm{~Hz}, 3 \mathrm{H}), 7.24(\mathrm{~d}, J=7.1$ $\mathrm{Hz}, 2 \mathrm{H}), 6.25-6.04(\mathrm{~m}, 1 \mathrm{H}), 5.32(\mathrm{t}, J=7.3 \mathrm{~Hz}, 1 \mathrm{H}), 3.66(\mathrm{~s}, 3 \mathrm{H}), 3.13(\mathrm{~s}, 2 \mathrm{H}), 2.44-2.23(\mathrm{~m}, 6 \mathrm{H})$, $2.13(\mathrm{q}, J=7.3 \mathrm{~Hz}, 2 \mathrm{H}), 1.73(\mathrm{q}, J=7.3 \mathrm{~Hz}, 2 \mathrm{H})$.

${ }^{13} \mathrm{C}\left\{{ }^{1} \mathrm{H}\right\}$ NMR $\left(201 \mathrm{MHz}, \mathrm{CDCl}_{3}\right) \delta 174.1,141.5,136.5,136.4,128.2,126.7,124.9,124.0$, $121.5,51.4,37.4,33.4,27.3,26.4,25.2,24.7$.

HRMS(ESI) (m/z): [M+ H] Calcd for $\mathrm{C}_{18} \mathrm{H}_{22} \mathrm{O}_{2}: 271.1693$; found: 271.1691

TLC: $\mathrm{R}_{f}=0.41\left(10 \% \mathrm{Et}_{2} \mathrm{O}\right.$ in hexanes)

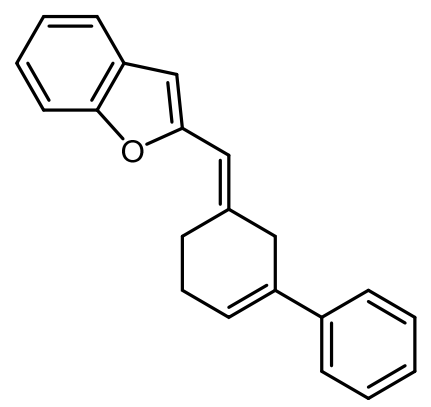

(E)-2-((4,5-dihydro-[1,1'-biphenyl]-3(2H)-ylidene)methyl)thiophene (20). The reaction was conducted according to the general procedure without modification using 2-(2,2dichlorovinyl)benzofuran ( $21.3 \mathrm{mg}, 0.1 \mathrm{mmol}, 1.0$ equiv) and (1-cyclopropylvinyl)benzene (43.3 $\mathrm{mg}, 0.3 \mathrm{mmol}, 3.0$ equiv). Isolated yields were determined following column chromatography $\left(\mathrm{SiO}_{2}, 5 \% \mathrm{Et}_{2} \mathrm{O}\right.$ in hexanes).

$16.7 \mathrm{mg}$ isolated (58\% yield), white solid, $E / Z=>20: 1$

${ }^{1} \mathrm{H}$ NMR $\left(300 \mathrm{MHz}, \mathrm{CDCl}_{3}\right) \delta 7.53(\mathrm{~d}, J=7.2 \mathrm{~Hz}, 1 \mathrm{H}), 7.45(\mathrm{t}, J=7.5 \mathrm{~Hz}, 3 \mathrm{H}), 7.36(\mathrm{t}, J=7.4$ $\mathrm{Hz}, 2 \mathrm{H}), 7.31-7.27(\mathrm{~m}, 1 \mathrm{H}), 7.26-7.16(\mathrm{~m}, 2 \mathrm{H}), 6.57(\mathrm{~s}, 1 \mathrm{H}), 6.34(\mathrm{~s}, 1 \mathrm{H}), 6.27-6.20(\mathrm{~m}, 1 \mathrm{H}), 3.36$ $(\mathrm{s}, 2 \mathrm{H}), 3.01(\mathrm{t}, J=6.5 \mathrm{~Hz}, 2 \mathrm{H}), 2.53-2.42(\mathrm{~m}, 2 \mathrm{H})$.

${ }^{13} \mathrm{C}\left\{{ }^{1} \mathrm{H}\right\}$ NMR $\left(201 \mathrm{MHz}, \mathrm{CDCl}_{3}\right) \delta 155.2,154.3,142.8,141.2,136.0,128.9,128.4,127.0$, 125.1, 124.4, 123.8, 122.7, 120.4, 112.4, 110.9, 104.6, 38.3, 27.3, 26.8.

HRMS(APCI) (m/z): [M + H] Calcd for $\mathrm{C}_{21} \mathrm{H}_{18} \mathrm{O}: 287.1430$; found: 287.1433

TLC: $\mathrm{R}_{f}=0.49\left(5 \% \mathrm{Et}_{2} \mathrm{O}\right.$ in hexanes) 


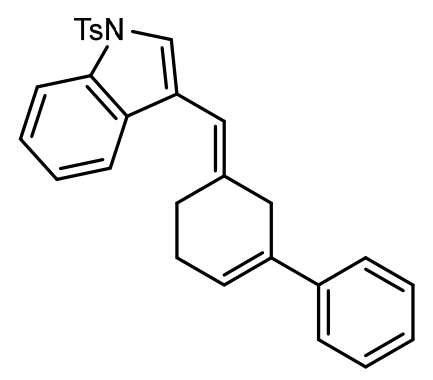

(E)-3-((4,5-dihydro-[1,1'-biphenyl]-3(2H)-ylidene)methyl)-1-tosyl-1H-indole (21). The reaction was conducted according to the general procedure without modification using 3-(2,2dichlorovinyl)-1-tosyl-1H-indole ( $36.6 \mathrm{mg}, 0.1 \mathrm{mmol}, 1.0 \mathrm{equiv}$ ) and (1-cyclopropylvinyl)benzene (43.3 $\mathrm{mg}, \quad 0.3 \mathrm{mmol}, \quad 3.0$ equiv). Isolated yields were determined following column chromatography $\left(\mathrm{SiO}_{2}, 30 \% \mathrm{CH}_{2} \mathrm{Cl}_{2}\right.$ in hexanes).

$35.2 \mathrm{mg}$ isolated ( $80 \%$ yield), white solid, $E / Z=>20: 1$

${ }^{1} \mathrm{H}$ NMR $\left(300 \mathrm{MHz}, \mathrm{CDCl}_{3}\right) \delta 7.99(\mathrm{~d}, J=8.2 \mathrm{~Hz}, 1 \mathrm{H}), 7.77(\mathrm{~d}, J=8.4 \mathrm{~Hz}, 2 \mathrm{H}), 7.55-7.46(\mathrm{~m}$, 2H), $7.47-7.40(\mathrm{~m}, 2 \mathrm{H}), 7.35(\mathrm{t}, J=7.5 \mathrm{~Hz}, 3 \mathrm{H}), 7.31-7.27(\mathrm{~m}, 1 \mathrm{H}), 7.25-7.18(\mathrm{~m}, 3 \mathrm{H}), 6.36(\mathrm{~s}$, $1 \mathrm{H}), 6.25-6.18(\mathrm{~m}, 1 \mathrm{H}), 3.38(\mathrm{~s}, 2 \mathrm{H}), 2.62(\mathrm{t}, J=6.5 \mathrm{~Hz}, 2 \mathrm{H}), 2.45-2.36(\mathrm{~m}, 2 \mathrm{H}), 2.34(\mathrm{~s}, 3 \mathrm{H})$.

${ }^{13} \mathrm{C}\left\{{ }^{1} \mathrm{H}\right\}$ NMR $\left(201 \mathrm{MHz}, \mathrm{CDCl}_{3}\right) \delta 144.8,141.6,141.3,136.2,135.3,134.8,131.2,129.8$, $128.4,127.0,126.8,125.1,124.8,124.2,123.2,123.1,119.8,119.5,113.6,112.1,37.8,27.2,26.6$, 21.6.

HRMS(APCI) (m/z): [M + H] ${ }^{+}$Calcd for $\mathrm{C}_{28} \mathrm{H}_{25} \mathrm{NO}_{2} \mathrm{~S}: 440.1674$; found: 440.1675

TLC: $\mathrm{R}_{f}=0.30\left(15 \% \mathrm{Et}_{2} \mathrm{O}\right.$ in hexanes)

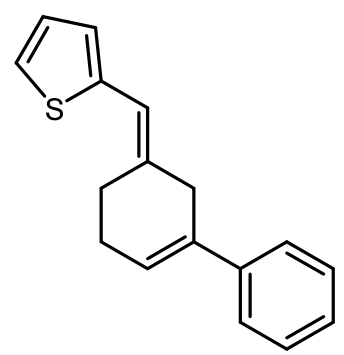

(E)-2-((4,5-dihydro-[1,1'-biphenyl]-3(2H)-ylidene)methyl)thiophene (22). The reaction was conducted according to the general procedure without modification using methyl 2-(2,2dichlorovinyl)thiophene $(17.9 \mathrm{mg}, 0.1 \mathrm{mmol}, 1.0$ equiv) and (1-cyclopropylvinyl)benzene (43.3 $\mathrm{mg}, 0.3 \mathrm{mmol}, 3.0$ equiv). Isolated yields were determined following column chromatography $\left(\mathrm{SiO}_{2}, 5 \% \mathrm{CH}_{2} \mathrm{Cl}_{2}\right.$ in hexanes).

$18.0 \mathrm{mg}$ isolated $(71 \%$ yield), yellow oil, $E / Z=>20: 1$

${ }^{1} \mathrm{H} \mathrm{NMR}\left(300 \mathrm{MHz}, \mathrm{CDCl}_{3}\right) \delta 7.42(\mathrm{~d}, J=8.1 \mathrm{~Hz}, 2 \mathrm{H}), 7.34(\mathrm{t}, J=7.4 \mathrm{~Hz}, 2 \mathrm{H}), 7.28(\mathrm{t}, J=1.5$ $\mathrm{Hz}, 1 \mathrm{H}), 7.21(\mathrm{~d}, J=5.1 \mathrm{~Hz}, 1 \mathrm{H}), 7.00(\mathrm{dd}, J=5.1,3.5 \mathrm{~Hz}, 1 \mathrm{H}), 6.94(\mathrm{~d}, J=3.4 \mathrm{~Hz}, 1 \mathrm{H}), 6.55(\mathrm{~s}, 1 \mathrm{H})$, $6.25-6.14(\mathrm{~m}, 1 \mathrm{H}), 3.30(\mathrm{~s}, 2 \mathrm{H}), 2.78(\mathrm{t}, J=6.5 \mathrm{~Hz}, 2 \mathrm{H}), 2.50-2.36(\mathrm{~m}, 2 \mathrm{H})$.

${ }^{13} \mathrm{C}\left\{{ }^{1} \mathrm{H}\right\}$ NMR $\left(201 \mathrm{MHz}, \mathrm{CDCl}_{3}\right) \delta 141.3,140.5,138.7,136.4,128.3,126.9,126.7,126.5$, $125.1,124.2,123.9,116.3,38.1,27.0,26.5$.

HRMS(APCI) (m/z): [M + H] Calcd for $\mathrm{C}_{17} \mathrm{H}_{16} \mathrm{~S}: 253.1046$; found: 253.1048

TLC: $\mathrm{R}_{f}=0.41\left(5 \% \mathrm{Et}_{2} \mathrm{O}\right.$ in hexanes) 


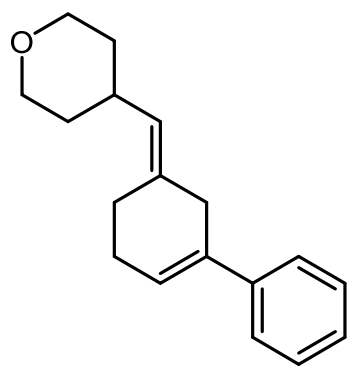

4-((4,5-dihydro-[1,1'-biphenyl]-3(2H)-ylidene)methyl)tetrahydro-2H-pyran (23). The reaction was conducted according to the general procedure without modification using 4-(2,2dichlorovinyl)tetrahydro-2H-pyran $(18.1 \mathrm{mg}, \quad 0.1 \mathrm{mmol}, \quad 1.0$ equiv $)$ and (1cyclopropylvinyl)benzene ( $43.3 \mathrm{mg}, 0.3 \mathrm{mmol}, 3.0$ equiv). Isolated yields were determined following column chromatography $\left(\mathrm{SiO}_{2}, 10 \% \mathrm{Et}_{2} \mathrm{O}\right.$ in hexanes).

$19.6 \mathrm{mg}$ isolated (77\% yield), white solid, $E / Z=12.5: 1$

${ }^{1} \mathrm{H}$ NMR $\left(300 \mathrm{MHz}, \mathrm{CDCl}_{3}\right) \delta 7.39(\mathrm{~d}, J=6.8 \mathrm{~Hz}, 2 \mathrm{H}), 7.32(\mathrm{t}, J=7.5 \mathrm{~Hz}, 2 \mathrm{H}), 7.22(\mathrm{~d}, J=7.1$ $\mathrm{Hz}, 1 \mathrm{H}), 6.14(\mathrm{~s}, 1 \mathrm{H}), 5.19(\mathrm{~d}, J=8.8 \mathrm{~Hz}, 1 \mathrm{H}), 3.96(\mathrm{~d}, J=11.5 \mathrm{~Hz}, 2 \mathrm{H}), 3.46(\mathrm{t}, J=9.7 \mathrm{~Hz}, 2 \mathrm{H}), 3.11$ (s, 2H), $2.65-2.43(\mathrm{~m}, 1 \mathrm{H}), 2.41-2.26(\mathrm{~m}, 4 \mathrm{H}), 1.57-1.38(\mathrm{~m}, 4 \mathrm{H})$.

${ }^{13} \mathrm{C}\left\{{ }^{1} \mathrm{H}\right\}$ NMR $\left(201 \mathrm{MHz}, \mathrm{CDCl}_{3}\right) \delta 141.5,136.7,133.2,128.3,127.5,126.8,125.0,124.0,67.8$, 67.3, 37.5, 33.4, 31.2, 27.7, 25.3.

HRMS(APCI) (m/z): [M + H] ${ }^{+}$Calcd for $\mathrm{C}_{18} \mathrm{H}_{22} \mathrm{O}: 255.1743$; found: 255.1740

TLC: $\mathrm{R}_{f}=0.68\left(15 \% \mathrm{Et}_{2} \mathrm{O}\right.$ in hexanes)

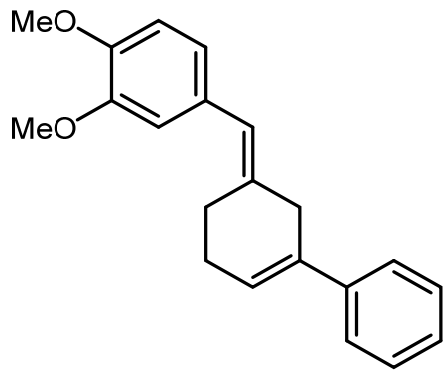

(E)-3-(3,4-dimethoxybenzylidene)-2,3,4,5-tetrahydro-1,1'-biphenyl (24). The reaction was conducted according to the general procedure without modification using 4-(2,2-dichlorovinyl)1,2-dimethoxybenzene ( $23.3 \mathrm{mg}, 0.1 \mathrm{mmol}, 1.0$ equiv) and (1-cyclopropylvinyl)benzene ( $43.3 \mathrm{mg}$, $0.3 \mathrm{mmol}, 3.0$ equiv). Isolated yields were determined following column chromatography $\left(\mathrm{SiO}_{2}\right.$, $5 \% \mathrm{Et}_{2} \mathrm{O}$ in hexanes).

$30.5 \mathrm{mg}$ isolated (99\% yield), white solid, $E / Z=>20: 1$

${ }^{1} \mathrm{H}$ NMR $\left(300 \mathrm{MHz}, \mathrm{CDCl}_{3}\right) \delta 7.43(\mathrm{~d}, J=7.0 \mathrm{~Hz}, 2 \mathrm{H}), 7.34(\mathrm{t}, J=7.4 \mathrm{~Hz}, 2 \mathrm{H}), 7.28(\mathrm{t}, J=1.4$ $\mathrm{Hz}, 1 \mathrm{H}), 6.88-6.80(\mathrm{~m}, 2 \mathrm{H}), 6.79(\mathrm{~s}, 1 \mathrm{H}), 6.44(\mathrm{~s}, 1 \mathrm{H}), 6.25-6.12(\mathrm{~m}, 1 \mathrm{H}), 3.89(\mathrm{~s}, 6 \mathrm{H}), 3.30(\mathrm{~s}, 2 \mathrm{H})$, $2.63(\mathrm{t}, J=6.4 \mathrm{~Hz}, 2 \mathrm{H}), 2.44-2.31(\mathrm{~m}, 2 \mathrm{H})$.

${ }^{13} \mathrm{C}\left\{{ }^{1} \mathrm{H}\right\}$ NMR $\left(201 \mathrm{MHz}, \mathrm{CDCl}_{3}\right) \delta 148.6,147.5,141.5,138.1,136.4,130.9,128.3,126.9$, $125.1,124.1,123.1,121.1,112.2,111.0,55.9,55.8,37.9,27.5,25.6$.

HRMS(APCI) (m/z): [M + H] ${ }^{+}$Calcd for $\mathrm{C}_{21} \mathrm{H}_{22} \mathrm{O}_{2}: 307.1693$; found: 307.1691 TLC: $\mathrm{R}_{f}=0.28\left(5 \% \mathrm{Et}_{2} \mathrm{O}\right.$ in hexanes) 


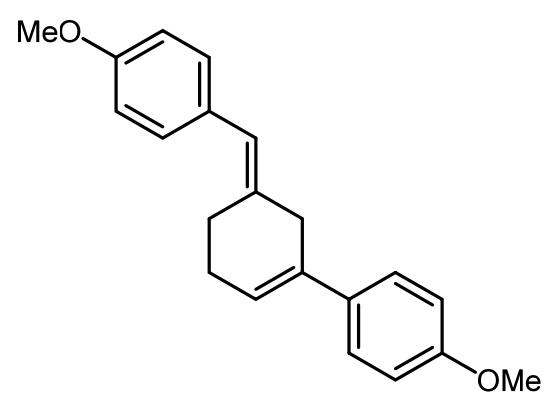

(E)-4'-methoxy-3-(4-methoxybenzylidene)-2,3,4,5-tetrahydro-1,1'-biphenyl (25). The reaction was conducted according to the general procedure without modification using 1-(2,2dichlorovinyl)-4-methoxybenzene ( $20.4 \mathrm{mg}, 0.1 \mathrm{mmol}, 1.0$ equiv) and 1-(1-cyclopropylvinyl)-4methoxybenzene ( $52.3 \mathrm{mg}, 0.3 \mathrm{mmol}, 3.0$ equiv). Isolated yields were determined following column chromatography ( $\mathrm{SiO}_{2}, 5 \% \mathrm{Et}_{2} \mathrm{O}$ in hexanes).

$23.2 \mathrm{mg}$ isolated (76\% yield), white solid, $E / Z=>20: 1$

$\left.{ }^{1} \mathrm{H} \mathrm{NMR} \mathrm{(300} \mathrm{MHz,} \mathrm{CDCl}_{3}\right) \delta 7.37(\mathrm{~d}, J=8.8 \mathrm{~Hz}, 2 \mathrm{H}), 7.19(\mathrm{~d}, J=8.5 \mathrm{~Hz}, 2 \mathrm{H}), 6.88(\mathrm{~d}, J=8.7$ $\mathrm{Hz}, 4 \mathrm{H}), 6.43$ (s, 1H), $6.19-5.96(\mathrm{~m}, 1 \mathrm{H}), 3.82(\mathrm{~s}, 6 \mathrm{H}), 3.26(\mathrm{~s}, 2 \mathrm{H}), 2.59$ (t, J = 6.3 Hz, 2H), $2.41-$ $2.30(\mathrm{~m}, 2 \mathrm{H})$.

${ }^{13} \mathrm{C}\left\{{ }^{1} \mathrm{H}\right\} \mathrm{NMR}\left(201 \mathrm{MHz}, \mathrm{CDCl}_{3}\right) \delta 158.7,157.9,137.9,135.7,134.2,130.6,129.9,126.1$, $122.7,122.5,113.7,113.6,55.3,55.3,38.0,27.4,25.5$.

HRMS(ESI) (m/z): [M + H] ${ }^{+}$Calcd for $\mathrm{C}_{21} \mathrm{H}_{22} \mathrm{O}_{2}$ : 307.1693; found: 307.1701

TLC: $\mathrm{R}_{f}=0.32\left(5 \% \mathrm{Et}_{2} \mathrm{O}\right.$ in hexanes)

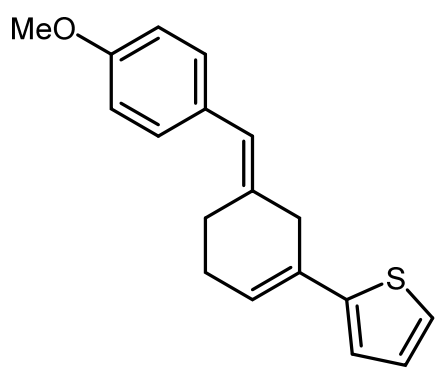

(E)-2-(5-(4-methoxybenzylidene)cyclohex-1-en-1-yl)thiophene (26). The reaction was conducted according to the general procedure without modification using 1-(2,2-dichlorovinyl)4-methoxybenzene (20.4 mg, $0.1 \mathrm{mmol}, 1.0$ equiv) and 2-(1-cyclopropylvinyl)thiophene ( $45.1 \mathrm{mg}$, $0.3 \mathrm{mmol}, 3.0$ equiv). Isolated yields were determined following column chromatography $\left(\mathrm{SiO}_{2}\right.$, $15 \% \mathrm{CH}_{2} \mathrm{Cl}_{2}$ in hexanes).

$24.3 \mathrm{mg}$ isolated ( $86 \%$ yield), white solid, $E / Z=>20: 1$

${ }^{1} \mathrm{H}$ NMR $\left(300 \mathrm{MHz}, \mathrm{CDCl}_{3}\right) \delta 7.21-7.15(\mathrm{~m}, 2 \mathrm{H}), 7.13(\mathrm{dd}, J=4.3,1.9 \mathrm{~Hz}, 1 \mathrm{H}), 7.02-6.95$ $(\mathrm{m}, 2 \mathrm{H}), 6.88(\mathrm{~d}, J=8.3 \mathrm{~Hz}, 2 \mathrm{H}), 6.44(\mathrm{~s}, 1 \mathrm{H}), 6.31-6.16(\mathrm{~m}, 1 \mathrm{H}), 3.82(\mathrm{~s}, 3 \mathrm{H}), 3.29(\mathrm{~s}, 2 \mathrm{H}), 2.59(\mathrm{t}$, $J=6.4 \mathrm{~Hz}, 2 \mathrm{H}), 2.41-2.28(\mathrm{~m}, 2 \mathrm{H})$.

${ }^{13} \mathrm{C}\left\{{ }^{1} \mathrm{H}\right\}$ NMR $\left(201 \mathrm{MHz}, \mathrm{CDCl}_{3}\right) \delta 158.0,145.9,137.0,131.0,130.4,129.9,127.2,123.4$, $123.2,123.0,121.4,113.6,55.3,37.9,27.2,25.5$.

HRMS(APCI) (m/z): [M + H] ${ }^{+}$Calcd for $\mathrm{C}_{18} \mathrm{H}_{18} \mathrm{OS}: 283.1151$; found: 283.1154

TLC: $\mathrm{R}_{f}=0.46\left(5 \% \mathrm{Et}_{2} \mathrm{O}\right.$ in hexanes) 


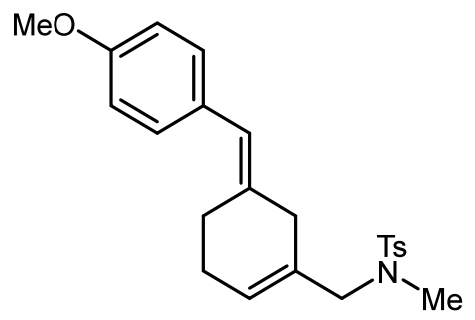

\section{(E)-N-((5-(4-methoxybenzylidene)cyclohex-1-en-1-yl)methyl)-N,4-}

dimethylbenzenesulfonamide (27). The reaction was conducted according to the general procedure without modification using 1-(2,2-dichlorovinyl)-4-methoxybenzene (20.4 mg, 0.1 mmol, 1.0 equiv) and $\mathrm{N}$-(2-cyclopropylallyl)- $\mathrm{N}$,4-dimethylbenzenesulfonamide $(79.6 \mathrm{mg}, 0.3$ mmol, 3.0 equiv). Isolated yields were determined following column chromatography $\left(\mathrm{SiO}_{2}, 15 \%\right.$ $\mathrm{CH}_{2} \mathrm{Cl}_{2}$ in hexanes).

$30.0 \mathrm{mg}$ isolated (75\% yield), yellow oil, $E / Z=>20: 1$

${ }^{1} \mathrm{H}$ NMR $\left(300 \mathrm{MHz}, \mathrm{CDCl}_{3}\right) \delta 7.69(\mathrm{~d}, J=8.1 \mathrm{~Hz}, 2 \mathrm{H}), 7.34(\mathrm{~d}, J=8.6 \mathrm{~Hz}, 2 \mathrm{H}), 7.15(\mathrm{~d}, J=8.9$ $\mathrm{Hz}, 2 \mathrm{H}), 6.86(\mathrm{~d}, J=8.7 \mathrm{~Hz}, 2 \mathrm{H}), 6.35(\mathrm{~s}, 1 \mathrm{H}), 5.63(\mathrm{~s}, 1 \mathrm{H}), 3.81(\mathrm{~s}, 3 \mathrm{H}), 3.50$ (s, 2H), $2.87(\mathrm{~s}, 2 \mathrm{H})$, $2.61(\mathrm{~s}, 3 \mathrm{H}), 2.50(\mathrm{t}, J=6.4 \mathrm{~Hz}, 2 \mathrm{H}), 2.44(\mathrm{~s}, 3 \mathrm{H}), 2.25-2.08(\mathrm{~m}, 2 \mathrm{H})$.

${ }^{13} \mathrm{C}\left\{{ }^{1} \mathrm{H}\right\}$ NMR $\left(201 \mathrm{MHz}, \mathrm{CDCl}_{3}\right) \delta 157.9,143.3,136.9,134.3,132.5,130.5,129.9,129.7$, $127.5,126.3,122.9,113.6,56.3,55.3,36.3,33.9,26.8,25.5,21.5$.

HRMS(ESI) (m/z): [M + H] ${ }^{+}$Calcd for $\mathrm{C}_{23} \mathrm{H}_{27} \mathrm{NO}_{3} \mathrm{~S}: 398.1784$; found: 398.1787

TLC: $\mathrm{R}_{f}=0.12\left(5 \% \mathrm{Et}_{2} \mathrm{O}\right.$ in hexanes)

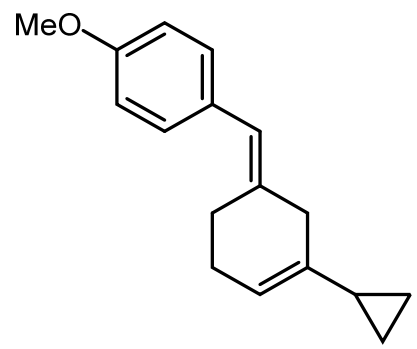

(E)-1-((3-cyclopropylcyclohex-3-en-1-ylidene)methyl)-4-methoxybenzene (28). The reaction was conducted according to the general procedure without modification using 1-(2,2dichlorovinyl)-4-methoxybenzene (20.4 $\mathrm{mg}, 0.1 \mathrm{mmol}, 1.0$ equiv) and ethene-1,1diyldicyclopropane $(32.5 \mathrm{mg}, 0.3 \mathrm{mmol}, 3.0$ equiv). Isolated yields were determined following column chromatography $\left(\mathrm{SiO}_{2}, 5 \% \mathrm{Et}_{2} \mathrm{O}\right.$ in hexanes).

$16.8 \mathrm{mg}$ isolated (70\% yield), colorless oil, $E / Z=>20: 1$

${ }^{1} \mathrm{H} \mathrm{NMR}\left(300 \mathrm{MHz}, \mathrm{CDCl}_{3}\right) \delta 7.15(\mathrm{~d}, J=8.8 \mathrm{~Hz}, 2 \mathrm{H}), 6.86(\mathrm{~d}, J=8.7 \mathrm{~Hz}, 2 \mathrm{H}), 6.32(\mathrm{~s}, 1 \mathrm{H}), 5.55$ - $5.44(\mathrm{~m}, 1 \mathrm{H}), 3.81(\mathrm{~s}, 3 \mathrm{H}), 2.74(\mathrm{~s}, 2 \mathrm{H}), 2.49(\mathrm{t}, J=6.5 \mathrm{~Hz}, 2 \mathrm{H}), 2.18-2.08(\mathrm{~m}, 2 \mathrm{H}), 1.43-1.27(\mathrm{~m}$, $1 \mathrm{H}), 0.70-0.53(\mathrm{~m}, 2 \mathrm{H}), 0.53-0.42(\mathrm{~m}, 2 \mathrm{H})$.

${ }^{13} \mathrm{C}\left\{{ }^{1} \mathrm{H}\right\}$ NMR $\left(201 \mathrm{MHz}, \mathrm{CDCl}_{3}\right) \delta 157.8,138.2,130.7,129.9,127.1,122.1,119.2,113.5,55.3$, 37.4, 26.9, 26.0, 16.9, 4.3.

HRMS(ESI) (m/z): [M + H]+ Calcd for $\mathrm{C}_{17} \mathrm{H}_{20} \mathrm{O}: 241.1587$; found: 241.1585

TLC: $\mathrm{R}_{f}=0.44\left(5 \% \mathrm{Et}_{2} \mathrm{O}\right.$ in hexanes) 


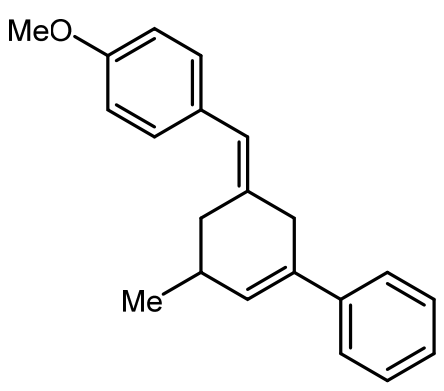

(E)-3-(4-methoxybenzylidene)-5-methyl-2,3,4,5-tetrahydro-1,1'-biphenyl (30). The reaction was conducted according to the general procedure without modification using 1-(2,2dichlorovinyl)-4-methoxybenzene $\quad(20.4 \mathrm{mg}, \quad 0.1 \mathrm{mmol}, \quad 1.0$ equiv) and (1-(2methylcyclopropyl)vinyl)benzene ( $47.5 \mathrm{mg}, 0.3 \mathrm{mmol}, 3.0$ equiv). Isolated yields were determined following column chromatography $\left(\mathrm{SiO}_{2}, 5 \% \mathrm{Et}_{2} \mathrm{O}\right.$ in hexanes).

$20.3 \mathrm{mg}$ isolated (70\% yield), white solid, $E / Z=12.4: 1$

$\left.{ }^{1} \mathrm{H} \mathrm{NMR} \mathrm{(300} \mathrm{MHz,} \mathrm{CDCl}_{3}\right) \delta 7.44(\mathrm{~d}, J=8.2 \mathrm{~Hz}, 2 \mathrm{H}), 7.35(\mathrm{t}, J=7.1 \mathrm{~Hz}, 2 \mathrm{H}), 7.20(\mathrm{~d}, J=8.2$ $\mathrm{Hz}, 3 \mathrm{H}), 6.89(\mathrm{~d}, J=8.7 \mathrm{~Hz}, 2 \mathrm{H}), 6.45(\mathrm{~s}, 1 \mathrm{H}), 6.03(\mathrm{~s}, 1 \mathrm{H}), 3.82(\mathrm{~s}, 3 \mathrm{H}), 3.37(\mathrm{~d}, J=19.2 \mathrm{~Hz}, 1 \mathrm{H}), 3.17$ (d, $J=18.7 \mathrm{~Hz}, 1 \mathrm{H}), 2.88(\mathrm{dd}, J=12.8,5.5 \mathrm{~Hz}, 1 \mathrm{H}), 2.50(\mathrm{~s}, 1 \mathrm{H}), 2.05(\mathrm{dd}, J=13.0,8.7 \mathrm{~Hz}, 1 \mathrm{H}), 1.09$ $(\mathrm{d}, J=7.0 \mathrm{~Hz}, 3 \mathrm{H})$.

${ }^{13} \mathrm{C}\left\{{ }^{1} \mathrm{H}\right\}$ NMR $\left(201 \mathrm{MHz}, \mathrm{CDCl}_{3}\right) \delta 157.8,141.3,137.0,135.3,133.6,130.4,129.9,128.2$, 126.9, 125.1, 123.2, 113.5, 55.2, 37.7, 33.8, 32.8, 21.7.

HRMS(APCI) (m/z): [M - H] ${ }^{+}$Calcd for $\mathrm{C}_{21} \mathrm{H}_{22} \mathrm{O}: 291.1743$; found: 291.1740

TLC: $\mathrm{R}_{f}=0.43\left(5 \% \mathrm{Et}_{2} \mathrm{O}\right.$ in hexanes)<smiles>COc1ccc(/C=C2\CC(c3ccccc3)=CC(CCc3ccccc3)C2)cc1</smiles>

(E)-3-(4-methoxybenzylidene)-5-phenethyl-2,3,4,5-tetrahydro-1,1'-biphenyl (31). The reaction was conducted according to the general procedure without modification using 1-(2,2dichlorovinyl)-4-methoxybenzene $\quad(20.4 \mathrm{mg}, \quad 0.1 \mathrm{mmol}, \quad 1.0$ equiv) and (1-(2phenethylcyclopropyl)vinyl)benzene $(74.5 \mathrm{mg}, 0.3 \mathrm{mmol}, 3.0$ equiv). Isolated yields were determined following column chromatography $\left(\mathrm{SiO}_{2}, 5 \% \mathrm{Et}_{2} \mathrm{O}\right.$ in hexanes).

$27.8 \mathrm{mg}$ isolated (70\% yield), colorless oil, $E / Z=10.0: 1$

${ }^{1} \mathrm{H}$ NMR $\left(300 \mathrm{MHz}, \mathrm{CDCl}_{3}\right) \delta 7.45(\mathrm{~d}, J=7.0 \mathrm{~Hz}, 2 \mathrm{H}), 7.36(\mathrm{t}, J=7.4 \mathrm{~Hz}, 2 \mathrm{H}), 7.32-7.25(\mathrm{~m}$, $1 \mathrm{H}), 7.25(\mathrm{~d}, J=4.0 \mathrm{~Hz}, 2 \mathrm{H}), 7.19(\mathrm{~d}, J=8.6 \mathrm{~Hz}, 3 \mathrm{H}), 7.12(\mathrm{~d}, J=6.7 \mathrm{~Hz}, 2 \mathrm{H}), 6.91(\mathrm{~d}, J=8.7 \mathrm{~Hz}, 2 \mathrm{H})$, $6.48(\mathrm{~s}, 1 \mathrm{H}), 6.17-6.05(\mathrm{~m}, 1 \mathrm{H}), 3.84(\mathrm{~s}, 3 \mathrm{H}), 3.37$ (d, $J=18.4 \mathrm{~Hz}, 1 \mathrm{H}), 3.23(\mathrm{~d}, J=18.5 \mathrm{~Hz}, 1 \mathrm{H}), 2.88$ (dd, $J=12.7,5.4 \mathrm{~Hz}, 1 \mathrm{H}), 2.64(\mathrm{t}, J=8.0 \mathrm{~Hz}, 2 \mathrm{H}), 2.53-2.36(\mathrm{~m}, 1 \mathrm{H}), 2.27(\mathrm{dd}, J=13.4,8.6 \mathrm{~Hz}, 1 \mathrm{H}$ ), $1.87-1.57(\mathrm{~m}, 2 \mathrm{H})$. 
${ }^{13} \mathrm{C}\left\{{ }^{1} \mathrm{H}\right\}$ NMR $\left(201 \mathrm{MHz}, \mathrm{CDCl}_{3}\right) \delta 157.9,142.3,141.3,136.7,136.0,133.7,133.6,130.5$, $129.9,128.3,128.2,126.9,125.6,125.1,123.6,113.6,55.2,38.0,37.7,37.2,33.2,31.2$.

HRMS(APCI) (m/z): [M - H] ${ }^{+}$Calcd for $\mathrm{C}_{28} \mathrm{H}_{28} \mathrm{O}: 381.2213$; found: 381.2210

TLC: $\mathrm{R}_{f}=0.39$ (5\% $\mathrm{Et}_{2} \mathrm{O}$ in hexanes)

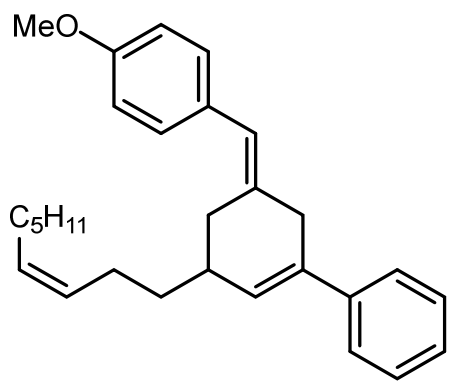

\section{3-((E)-4-methoxybenzylidene)-5-((Z)-non-3-en-1-yl)-2,3,4,5-tetrahydro-1,1'-biphenyl}

(32). The reaction was conducted according to the general procedure without modification using 1-(2,2-dichlorovinyl)-4-methoxybenzene $(20.4 \mathrm{mg}, 0.1 \mathrm{mmol}, 1.0$ equiv) and (Z)-(1-(2-(non-3-en1-yl)cyclopropyl)vinyl)benzene ( $80.5 \mathrm{mg}, 0.3 \mathrm{mmol}, 3.0$ equiv). Isolated yields were determined following column chromatography $\left(\mathrm{SiO}_{2}, 15 \% \mathrm{CH}_{2} \mathrm{Cl}_{2}\right.$ in hexanes).

$27.9 \mathrm{mg}$ isolated (70\% yield), colorless oil, $E / Z=14.6: 1$

${ }^{1} \mathrm{H} \mathrm{NMR}\left(300 \mathrm{MHz}, \mathrm{CDCl}_{3}\right) \delta 7.44(\mathrm{~d}, J=8.2 \mathrm{~Hz}, 2 \mathrm{H}), 7.34(\mathrm{t}, J=7.6 \mathrm{~Hz}, 2 \mathrm{H}), 7.28(\mathrm{t}, J=1.4$ $\mathrm{Hz}, 1 \mathrm{H}), 7.19$ (d, J = 8.8 Hz, 2H), $6.88(\mathrm{~d}, J=8.7 \mathrm{~Hz}, 2 \mathrm{H}), 6.45(\mathrm{~s}, 1 \mathrm{H}), 6.15-6.04(\mathrm{~m}, 1 \mathrm{H}), 5.44-5.22$ $(\mathrm{m}, 2 \mathrm{H}), 3.82(\mathrm{~s}, 3 \mathrm{H}), 3.36(\mathrm{~d}, J=18.4 \mathrm{~Hz}, 1 \mathrm{H}), 3.20(\mathrm{~d}, J=18.4 \mathrm{~Hz}, 1 \mathrm{H}), 2.84(\mathrm{dd}, J=12.8,5.5 \mathrm{~Hz}$, $1 \mathrm{H}), 2.42(\mathrm{~s}, 1 \mathrm{H}), 2.17(\mathrm{dd}, J=12.6,7.7 \mathrm{~Hz}, 1 \mathrm{H}), 2.07(\mathrm{q}, J=7.3 \mathrm{~Hz}, 2 \mathrm{H}), 1.96(\mathrm{q}, J=6.7 \mathrm{~Hz}, 2 \mathrm{H}), 1.53$ $-1.16(\mathrm{~m}, 8 \mathrm{H}), 0.92-0.84(\mathrm{~m}, 3 \mathrm{H})$.

${ }^{13} \mathrm{C}\left\{{ }^{1} \mathrm{H}\right\}$ NMR $\left(201 \mathrm{MHz}, \mathrm{CDCl}_{3}\right) \delta 157.9,141.5,136.9,135.8,130.6,130.4,129.9,129.3$, 129.0, 128.3, 126.9, 125.2, 123.5, 113.6, 55.2, 38.1, 37.3, 36.1, 31.5, 31.5, 29.4, 27.2, 24.6, 22.6, 14.1. HRMS(APCI) (m/z): [M - H] ${ }^{+}$Calcd for $\mathrm{C}_{29} \mathrm{H}_{36} \mathrm{O}: 399.2682$; found: 399.2686 TLC: $\mathrm{R}_{f}=0.44$ (5\% $\mathrm{Et}_{2} \mathrm{O}$ in hexanes)<smiles>COc1ccc(/C=C2\CC(c3ccccc3)=CC(CCOC(=O)c3ccccc3)C2)cc1</smiles>

(E)-5-(2-(benzyloxy)ethyl)-3-(4-methoxybenzylidene)-2,3,4,5-tetrahydro-1,1'-biphenyl (33). The reaction was conducted according to the general procedure without modification using 1-(2,2-dichlorovinyl)-4-methoxybenzene $(20.4 \mathrm{mg}, 0.1 \mathrm{mmol}, 1.0$ equiv) and (1-(2-(2(benzyloxy)ethyl)cyclopropyl)vinyl)benzene ( $83.5 \mathrm{mg}, 0.3 \mathrm{mmol}, 3.0$ equiv). Isolated yields were determined following column chromatography $\left(\mathrm{SiO}_{2}, 20 \% \mathrm{Et}_{2} \mathrm{O}\right.$ in hexanes). 
$25.1 \mathrm{mg}$ isolated (61\% yield), colorless oil, $E / Z=19.2: 1$

${ }^{1} \mathrm{H}$ NMR $\left(300 \mathrm{MHz}, \mathrm{CDCl}_{3}\right) \delta 7.42(\mathrm{~d}, J=8.1 \mathrm{~Hz}, 3 \mathrm{H}), 7.39-7.27(\mathrm{~m}, 7 \mathrm{H}), 7.18(\mathrm{~d}, J=8.6 \mathrm{~Hz}$, $2 \mathrm{H}), 6.84(\mathrm{~d}, J=8.7 \mathrm{~Hz}, 2 \mathrm{H}), 6.46(\mathrm{~s}, 1 \mathrm{H}), 6.13-6.03(\mathrm{~m}, 1 \mathrm{H}), 4.48(\mathrm{~s}, 2 \mathrm{H}), 3.80(\mathrm{~s}, 3 \mathrm{H}), 3.62-3.45$ (m, 2H), $3.35(\mathrm{~d}, J=18.3 \mathrm{~Hz}, 1 \mathrm{H}), 3.21(\mathrm{~d}, J=17.5 \mathrm{~Hz}, 1 \mathrm{H}), 2.86(\mathrm{dd}, J=12.8,5.5 \mathrm{~Hz}, 1 \mathrm{H}), 2.65(\mathrm{~s}$, $1 \mathrm{H}), 2.20(\mathrm{dd}, J=12.8,7.8 \mathrm{~Hz}, 1 \mathrm{H}), 1.89-1.72(\mathrm{~m}, 1 \mathrm{H}), 1.72-1.58(\mathrm{~m}, 1 \mathrm{H})$.

${ }^{13} \mathrm{C}\left\{{ }^{1} \mathrm{H}\right\}$ NMR $\left(201 \mathrm{MHz}, \mathrm{CDCl}_{3}\right) \delta 161.5,157.9,141.3,138.6,136.7,136.0,130.5,129.9$, $128.7,128.3,127.6,127.0,125.2,123.6,113.6,92.9,73.0,68.1,55.3,38.0,35.9,34.8,31.5$.

HRMS(ESI) (m/z): [M + Na] ${ }^{+}$Calcd for $\mathrm{C}_{29} \mathrm{H}_{30} \mathrm{O}_{2}: 433.2138$; found: 433.2142

TLC: $\mathrm{R}_{f}=0.37$ (5\% $\mathrm{Et}_{2} \mathrm{O}$ in hexanes)

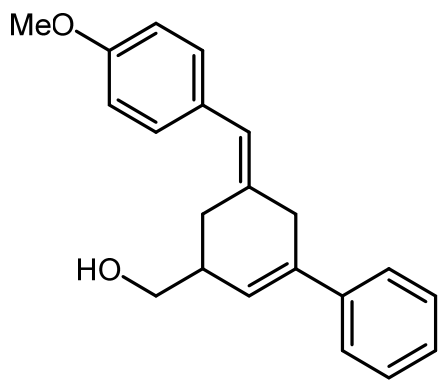

\section{(E)-(5-(4-methoxybenzylidene)-3,4,5,6-tetrahydro-[1,1'-biphenyl]-3-yl)methanol (34).}

The reaction was conducted according to the general procedure without modification using 1-(2,2dichlorovinyl)-4-methoxybenzene $\quad(20.4 \mathrm{mg}, \quad 0.1 \mathrm{mmol}, \quad 1.0$ equiv) and (2-(1phenylvinyl)cyclopropyl)methanol ${ }^{13}(52.3 \mathrm{mg}, 0.3 \mathrm{mmol}, 3.0$ equiv). Isolated yields were determined following column chromatography $\left(\mathrm{SiO}_{2}, 40 \% \mathrm{Et}_{2} \mathrm{O}\right.$ in hexanes).

$21.7 \mathrm{mg}$ isolated (68\% yield), light yellow solid, $E / Z=>20: 1$

${ }^{1} \mathrm{H} \mathrm{NMR}\left(300 \mathrm{MHz}, \mathrm{CDCl}_{3}\right) \delta 7.45(\mathrm{~d}, J=7.7 \mathrm{~Hz}, 2 \mathrm{H}), 7.35(\mathrm{t}, J=7.5 \mathrm{~Hz}, 2 \mathrm{H}), 7.29(\mathrm{~s}, 1 \mathrm{H}), 7.21$ $(\mathrm{d}, J=8.5 \mathrm{~Hz}, 2 \mathrm{H}), 6.89(\mathrm{~d}, J=8.4 \mathrm{~Hz}, 2 \mathrm{H}), 6.49(\mathrm{~s}, 1 \mathrm{H}), 6.09(\mathrm{~s}, 1 \mathrm{H}), 3.82(\mathrm{~s}, 3 \mathrm{H}), 3.61(\mathrm{t}, J=5.5 \mathrm{~Hz}$, $2 \mathrm{H}), 3.37(\mathrm{~d}, J=19.0 \mathrm{~Hz}, 1 \mathrm{H}), 3.25(\mathrm{~d}, J=18.7 \mathrm{~Hz}, 1 \mathrm{H}), 2.79(\mathrm{dd}, J=12.8,5.6 \mathrm{~Hz}, 1 \mathrm{H}), 2.63(\mathrm{~s}, 1 \mathrm{H})$, $2.43(\mathrm{dd}, J=12.9,7.4 \mathrm{~Hz}, 1 \mathrm{H}), 1.29-1.22(\mathrm{~m}, 1 \mathrm{H})$.

${ }^{13} \mathrm{C}\left\{{ }^{1} \mathrm{H}\right\} \mathrm{NMR}\left(201 \mathrm{MHz}, \mathrm{CDCl}_{3}\right) \delta 158.0,141.0,138.5,135.9,130.2,129.9,128.4,127.3$, 125.2, 124.5, 124.2, 113.7, 66.5, 55.3, 40.7, 38.1, 28.1.

HRMS(ESI) (m/z): [M + H] ${ }^{+}$Calcd for $\mathrm{C}_{21} \mathrm{H}_{22} \mathrm{O}_{2}: 307.1693$; found: 307.1690

TLC: $\mathrm{R}_{f}=0.21$ (30\% $\mathrm{Et}_{2} \mathrm{O}$ in hexanes) 


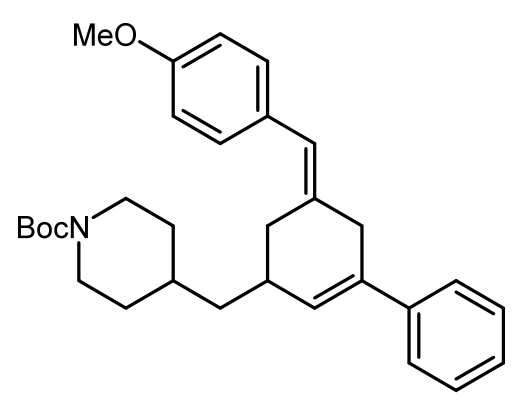

tert-butyl-4-((5-(4-methoxybenzylidene)-3,4,5,6-tetrahydro-[1,1'-biphenyl]-3-

yl)methyl)piperidine-1-carboxylate (35). The reaction was conducted according to the general procedure without modification using 1-(2,2-dichlorovinyl)-4-methoxybenzene (20.4 mg, 0.1 mmol, 1.0 equiv) and tert-butyl 4-((2-(1-phenylvinyl)cyclopropyl)methyl)piperidine-1carboxylate (102.5 mg, $0.3 \mathrm{mmol}, 3.0$ equiv). Isolated yields were determined following column chromatography $\left(\mathrm{SiO}_{2}, 20 \% \mathrm{Et}_{2} \mathrm{O}\right.$ in hexanes).

$39.6 \mathrm{mg}$ isolated ( $84 \%$ yield), yellow solid, $E / Z=8.6: 1$

${ }^{1} \mathrm{H}$ NMR (300 MHz, $\left.\mathrm{CDCl}_{3}\right) \delta 7.43(\mathrm{~d}, J=7.3 \mathrm{~Hz}, 2 \mathrm{H}), 7.34(\mathrm{t}, J=7.5 \mathrm{~Hz}, 3 \mathrm{H}), 7.17(\mathrm{~d}, J=8.5$ $\mathrm{Hz}, 2 \mathrm{H}), 6.87(\mathrm{~d}, J=8.6 \mathrm{~Hz}, 2 \mathrm{H}), 6.48(\mathrm{~s}, 1 \mathrm{H}), 6.05(\mathrm{~s}, 1 \mathrm{H}), 4.05(\mathrm{~s}, 2 \mathrm{H}), 3.82(\mathrm{~s}, 3 \mathrm{H}), 3.33(\mathrm{~d}, J=17.7$ $\mathrm{Hz}, 1 \mathrm{H}$ ), 3.23 (d, $J=18.7 \mathrm{~Hz}, 1 \mathrm{H}$ ), 2.73 (dd, $J=12.9,5.4 \mathrm{~Hz}, 1 \mathrm{H}$ ), 2.56 (s, 3H), 2.22 (dd, $J=12.7,7.3$ $\mathrm{Hz}, 1 \mathrm{H}), 1.73-1.47(\mathrm{~m}, 2 \mathrm{H}), 1.45(\mathrm{~s}, 10 \mathrm{H}), 1.40-1.16(\mathrm{~m}, 4 \mathrm{H}), 1.03(\mathrm{q}, J=11.6,9.7 \mathrm{~Hz}, 1 \mathrm{H})$.

${ }^{13} \mathrm{C}\left\{{ }^{1} \mathrm{H}\right\}$ NMR $\left(201 \mathrm{MHz}, \mathrm{CDCl}_{3}\right) \delta 158.0,154.9,141.3,136.7,136.0,130.5,129.9,128.8$, 128.3, 127.0, 125.2, 123.8, 113.6, 79.2, 55.3, 42.7, 38.1, 36.5, 34.4, 33.2, 31.5, 29.7, 28.5.

HRMS(APCI) (m/z): [M + H] $]^{+}$Calcd for $\mathrm{C}_{31} \mathrm{H}_{40} \mathrm{NO}_{3}: 474.3003$; found: 474.2988 TLC: $\mathrm{R}_{f}=0.13\left(15 \% \mathrm{Et}_{2} \mathrm{O}\right.$ in hexanes)

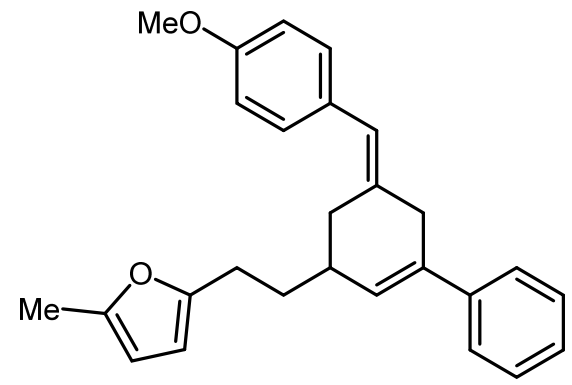

\section{(E)-2-(2-(5-(4-methoxybenzylidene)-3,4,5,6-tetrahydro-[1,1'-biphenyl]-3-yl)ethyl)-5-}

methylfuran (36). The reaction was conducted according to the general procedure without modification using 1-(2,2-dichlorovinyl)-4-methoxybenzene (20.4 mg, $0.1 \mathrm{mmol}, 1.0$ equiv) and 2methyl-5-(2-(2-(1-phenylvinyl)cyclopropyl)ethyl)furan $(75.7 \mathrm{mg}, 0.3 \mathrm{mmol}, 3.0$ equiv). Isolated yields were determined following column chromatography $\left(\mathrm{SiO}_{2}, 15 \% \mathrm{CH}_{2} \mathrm{Cl}_{2}\right.$ in hexanes).

$31.0 \mathrm{mg}$ isolated ( $81 \%$ yield), colorless oil, $E / Z=16.2: 1$

${ }^{1} \mathrm{H}$ NMR $\left(300 \mathrm{MHz}, \mathrm{CDCl}_{3}\right) \delta 7.44(\mathrm{~d}, J=8.1 \mathrm{~Hz}, 2 \mathrm{H}), 7.34(\mathrm{t}, J=7.3 \mathrm{~Hz}, 2 \mathrm{H}), 7.28(\mathrm{t}, J=1.4$ $\mathrm{Hz}, 1 \mathrm{H}), 7.18(\mathrm{~d}, J=8.8 \mathrm{~Hz}, 2 \mathrm{H}), 6.88(\mathrm{~d}, J=8.7 \mathrm{~Hz}, 2 \mathrm{H}), 6.46(\mathrm{~s}, 1 \mathrm{H}), 6.14-6.02(\mathrm{~m}, 1 \mathrm{H}), 5.82(\mathrm{~d}, J$ $=3.9 \mathrm{~Hz}, 2 \mathrm{H}), 3.83(\mathrm{~s}, 3 \mathrm{H}), 3.37(\mathrm{~d}, J=18.5 \mathrm{~Hz}, 1 \mathrm{H}), 3.20(\mathrm{~d}, J=18.7 \mathrm{~Hz}, 1 \mathrm{H}), 2.88(\mathrm{dd}, J=12.8,5.5$ 
$\mathrm{Hz}, 1 \mathrm{H}), 2.61(\mathrm{t}, J=7.8 \mathrm{~Hz}, 2 \mathrm{H}), 2.44(\mathrm{~s}, 1 \mathrm{H}), 2.25(\mathrm{~s}, 3 \mathrm{H}), 2.16(\mathrm{dd}, J=12.8,8.2 \mathrm{~Hz}, 1 \mathrm{H}), 1.88-1.74$ (m, 1H), $1.74-1.59(\mathrm{~m}, 1 \mathrm{H})$.

${ }^{13} \mathrm{C}\left\{{ }^{1} \mathrm{H}\right\}$ NMR $\left(201 \mathrm{MHz}, \mathrm{CDCl}_{3}\right) \delta 158.0,154.1,150.2,141.4,136.8,136.2,130.5,129.9$, 128.6, 128.3, 127.0, 125.2, 123.6, 113.6, 105.8, 105.5, 55.3, 38.0, 37.1, 34.4, 31.4, 25.5, 13.5 .

HRMS(APCI) (m/z): [M + H] ${ }^{+}$Calcd for $\mathrm{C}_{27} \mathrm{H}_{28} \mathrm{O}: 385.2162$; found: 385.2166

TLC: $\mathrm{R}_{f}=0.33\left(5 \% \mathrm{Et}_{2} \mathrm{O}\right.$ in hexanes)

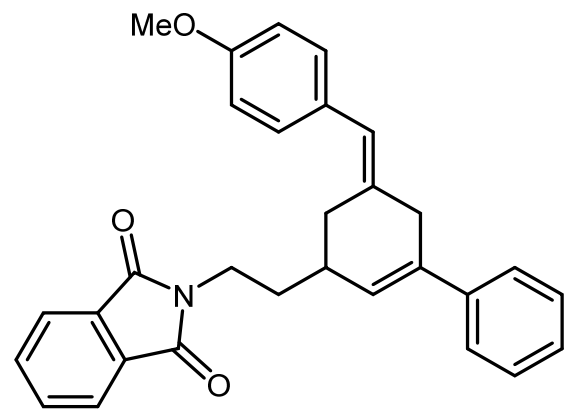

\section{(E)-2-(2-(5-(4-methoxybenzylidene)-3,4,5,6-tetrahydro-[1,1'-biphenyl]-3-}

yl)ethyl)isoindoline-1,3-dione (37). The reaction was conducted according to the general procedure without modification using 1-(2,2-dichlorovinyl)-4-methoxybenzene (20.4 mg, 0.1 mmol, 1.0 equiv ) and 2-(2-(2-(1-phenylvinyl)cyclopropyl)ethyl)isoindoline-1,3-dione $(95.2 \mathrm{mg}$, $0.3 \mathrm{mmol}, 3.0$ equiv). Isolated yields were determined following column chromatography $\left(\mathrm{SiO}_{2}\right.$, $10 \% \mathrm{Et}_{2} \mathrm{O}$ in hexanes).

$34.2 \mathrm{mg}$ isolated (76\% yield), yellow oil, $E / Z=12.1: 1$

${ }^{1} \mathrm{H}$ NMR $\left(300 \mathrm{MHz}, \mathrm{CDCl}_{3}\right) \delta 7.82(\mathrm{dd}, J=5.5,3.1 \mathrm{~Hz}, 2 \mathrm{H}), 7.69(\mathrm{dd}, J=5.4,3.1 \mathrm{~Hz}, 2 \mathrm{H}), 7.43$ (d, $J=6.8 \mathrm{~Hz}, 2 \mathrm{H}), 7.33(\mathrm{t}, J=7.4 \mathrm{~Hz}, 2 \mathrm{H}), 7.26-7.24(\mathrm{~m}, 1 \mathrm{H}), 7.19$ (d, $J=8.5 \mathrm{~Hz}, 2 \mathrm{H}), 6.89$ (d, $J=$ $8.6 \mathrm{~Hz}, 2 \mathrm{H}), 6.47(\mathrm{~s}, 1 \mathrm{H}), 6.16(\mathrm{~s}, 1 \mathrm{H}), 3.82(\mathrm{~s}, 3 \mathrm{H}), 3.78-3.64(\mathrm{~m}, 2 \mathrm{H}), 3.37$ (d, $J=19.9 \mathrm{~Hz}, 1 \mathrm{H})$, $3.20(\mathrm{~d}, J=18.6 \mathrm{~Hz}, 1 \mathrm{H}), 2.87(\mathrm{dd}, J=12.8,5.4 \mathrm{~Hz}, 1 \mathrm{H}), 2.45(\mathrm{~s}, 1 \mathrm{H}), 2.31(\mathrm{dd}, J=12.9,8.0 \mathrm{~Hz}, 1 \mathrm{H}$ ), $1.79(\mathrm{q}, J=7.1 \mathrm{~Hz}, 2 \mathrm{H})$.

${ }^{13} \mathrm{C}\left\{{ }^{1} \mathrm{H}\right\}$ NMR $\left(201 \mathrm{MHz}, \mathrm{CDCl}_{3}\right) \delta 168.4,158.0,141.1,136.7,136.1,133.9,132.1,130.3$, $130.0,128.3,127.4,127.1,125.2,124.1,123.2,113.7,55.3,37.9,35.8,35.3,34.5,31.2$.

HRMS(ESI) (m/z): [M + H] ${ }^{+}$Calcd for $\mathrm{C}_{30} \mathrm{H}_{27} \mathrm{NO}_{3}: 450.2063$; found: 450.2067 TLC: $\mathrm{R}_{f}=0.13\left(15 \% \mathrm{Et}_{2} \mathrm{O}\right.$ in hexanes) 


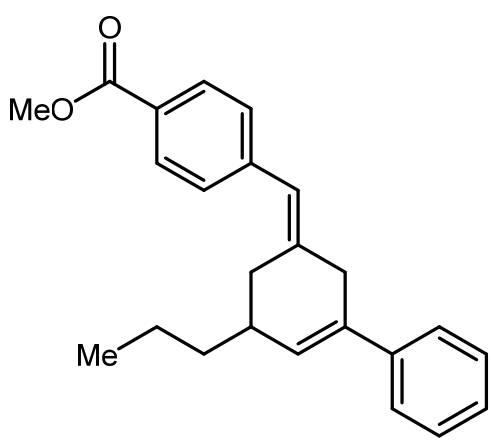

methyl (E)-4-((5-propyl-4,5-dihydro-[1,1'-biphenyl]-3(2H)-ylidene)methyl)benzoate (S17). The reaction was conducted according to the general procedure without modification using methyl 4-(2,2-dichlorovinyl)benzoate $(23.1 \mathrm{mg}, \quad 0.1 \mathrm{mmol}, \quad 1.0$ equiv) and (1-(2propylcyclopropyl)vinyl)benzene ${ }^{13}(55.9 \mathrm{mg}, 0.3 \mathrm{mmol}, 3.0$ equiv). Isolated yields were determined following column chromatography $\left(\mathrm{SiO}_{2}, 10 \% \mathrm{Et}_{2} \mathrm{O}\right.$ in hexanes).

$25.2 \mathrm{mg}$ isolated (73\% yield), colorless oil, $E / Z=8.2: 1$

$\left.{ }^{1} \mathrm{H} \mathrm{NMR} \mathrm{(300} \mathrm{MHz,} \mathrm{CDCl}_{3}\right) \delta 8.02(\mathrm{~d}, J=8.4 \mathrm{~Hz}, 2 \mathrm{H}), 7.44(\mathrm{~d}, J=8.2 \mathrm{~Hz}, 2 \mathrm{H}), 7.38-7.29(\mathrm{~m}$, 4H), $7.25-7.19(\mathrm{~m}, 1 \mathrm{H}), 6.53(\mathrm{~s}, 1 \mathrm{H}), 6.10(\mathrm{~s}, 1 \mathrm{H}), 3.93(\mathrm{~s}, 3 \mathrm{H}), 3.40(\mathrm{~d}, J=18.8 \mathrm{~Hz}, 1 \mathrm{H}), 3.23(\mathrm{~d}, J$ $=18.5 \mathrm{~Hz}, 1 \mathrm{H}), 2.84(\mathrm{dd}, J=12.9,5.4 \mathrm{~Hz}, 1 \mathrm{H}), 2.42(\mathrm{~s}, 1 \mathrm{H}), 2.16(\mathrm{dd}, J=13.2,8.2 \mathrm{~Hz}, 1 \mathrm{H}), 1.50-1.22$ (m, 4H), $1.01-0.77(\mathrm{~m}, 3 \mathrm{H})$.

${ }^{13} \mathrm{C}\left\{{ }^{1} \mathrm{H}\right\}$ NMR $\left(201 \mathrm{MHz}, \mathrm{CDCl}_{3}\right) \delta 167.0,142.9,141.2,140.7,135.2,129.4,129.2,128.7$, $128.3,127.0,125.1,123.2,52.0,38.3,38.2,37.6,31.8,20.0,14.1$.

HRMS(ESI) (m/z): [M + H] Calcd for $\mathrm{C}_{24} \mathrm{H}_{26} \mathrm{O}_{2}: 347.2006$; found: 347.2005

TLC: $\mathrm{R}_{f}=0.40\left(10 \% \mathrm{Et}_{2} \mathrm{O}\right.$ in hexanes)

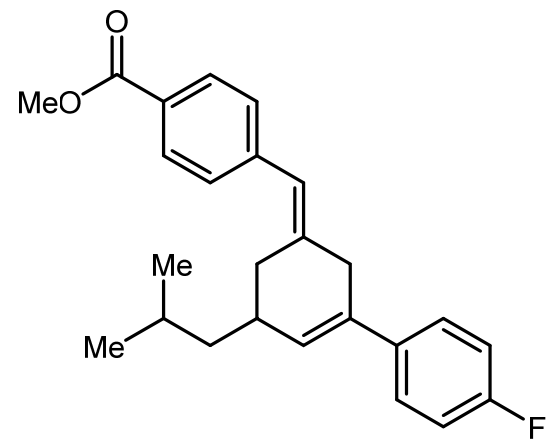

methyl

(E)-4-((4'-fluoro-5-isobutyl-4,5-dihydro-[1,1'-biphenyl]-3(2H)ylidene)methyl)benzoate (S18). The reaction was conducted according to the general procedure without modification using methyl 4-(2,2-dichlorovinyl)benzoate $(23.1 \mathrm{mg}, 0.1 \mathrm{mmol}$, 1.0 equiv) and 1-fluoro-4-(1-(2-isobutylcyclopropyl)vinyl)benzene ${ }^{2}$ (65.5 mg, $0.3 \mathrm{mmol}, 3.0$ equiv). Isolated yields were determined following column chromatography $\left(\mathrm{SiO}_{2}, 10 \% \mathrm{Et}_{2} \mathrm{O}\right.$ in hexanes).

$24.2 \mathrm{mg}$ isolated (64\% yield), colorless oil, $E / Z=8.1: 1$

${ }^{1} \mathrm{H}$ NMR $\left(300 \mathrm{MHz}, \mathrm{CDCl}_{3}\right) \delta 8.01(\mathrm{~d}, J=8.3 \mathrm{~Hz}, 2 \mathrm{H}), 7.46-7.34(\mathrm{~m}, 2 \mathrm{H}), 7.31(\mathrm{~d}, J=8.0 \mathrm{~Hz}$, 2H), $7.03(\mathrm{t}, J=8.8 \mathrm{~Hz}, 2 \mathrm{H}), 6.53(\mathrm{~s}, 1 \mathrm{H}), 6.08-5.95(\mathrm{~m}, 1 \mathrm{H}), 3.93(\mathrm{~s}, 3 \mathrm{H}), 3.34(\mathrm{~d}, J=20.3 \mathrm{~Hz}, 1 \mathrm{H})$, 
$3.20(\mathrm{~d}, J=17.3 \mathrm{~Hz}, 1 \mathrm{H}), 2.79(\mathrm{dd}, J=12.9,5.5 \mathrm{~Hz}, 1 \mathrm{H}), 2.48(\mathrm{~s}, 1 \mathrm{H}), 2.22-2.10(\mathrm{~m}, 1 \mathrm{H}), 1.67-1.52$ (m, 1H), $1.33-1.13(\mathrm{~m}, 2 \mathrm{H}), 0.86(\mathrm{dd}, J=6.6,4.2 \mathrm{~Hz}, 6 \mathrm{H})$.

${ }^{13} \mathrm{C}\left\{{ }^{1} \mathrm{H}\right\}$ NMR $\left(201 \mathrm{MHz}, \mathrm{CDCl}_{3}\right) \delta 167.0,162.0\left(\mathrm{~d},{ }^{1} \mathrm{JFF}_{\mathrm{CF}}=245.7 \mathrm{~Hz}\right), 142.8,140.2,137.3(\mathrm{~d}$, $\left.{ }^{4} J_{\mathrm{CF}}=3.1 \mathrm{~Hz}\right), 134.3,129.4,129.1,128.7,127.7,126.6\left(\mathrm{~d},{ }^{3} J_{\mathrm{CF}}=8.0 \mathrm{~Hz}\right), 123.4,115.0\left(\mathrm{~d},{ }^{2} J_{\mathrm{CF}}=21.3\right.$ $\mathrm{Hz}), 52.0,45.4,38.3,35.3,31.8,25.1,22.8,22.4$.

${ }^{19} \mathrm{~F}$ NMR $\left(282 \mathrm{MHz}, \mathrm{CDCl}_{3}\right) \delta-117.34$.

HRMS(ESI) (m/z): [M + H] ${ }^{+}$Calcd for $\mathrm{C}_{25} \mathrm{H}_{27} \mathrm{FO}_{2}: 379.2068$; found: 379.2070

TLC: $\mathrm{R}_{f}=0.30\left(10 \% \mathrm{Et}_{2} \mathrm{O}\right.$ in hexanes)

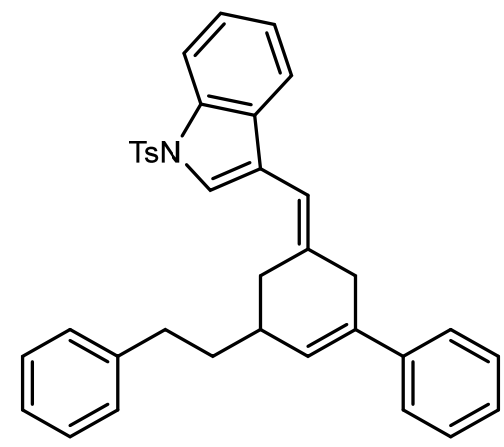

\section{(E)-3-((5-phenethyl-4,5-dihydro-[1,1'-biphenyl]-3(2H)-ylidene)methyl)-1-tosyl-1H-indole}

(S19). The reaction was conducted according to the general procedure without modification using 3-(2,2-dichlorovinyl)-1-tosyl-1H-indole (36.6 $\mathrm{mg}, \quad 0.1 \mathrm{mmol}, \quad 1.0$ equiv) and (1-(2phenethylcyclopropyl)vinyl)benzene $(74.5 \mathrm{mg}, 0.3 \mathrm{mmol}, 3.0$ equiv). Isolated yields were determined following column chromatography $\left(\mathrm{SiO}_{2}, 30 \% \mathrm{Et}_{2} \mathrm{O}\right.$ in hexanes).

$39.4 \mathrm{mg}$ isolated $(72 \%$ yield), light yellow solid, $E / Z=3.9: 1$

$\left.{ }^{1} \mathrm{H} \mathrm{NMR} \mathrm{(300} \mathrm{MHz,} \mathrm{CDCl} 3\right) \delta 8.05(\mathrm{~d}, J=8.3 \mathrm{~Hz}, 1 \mathrm{H}), 7.76(\mathrm{~d}, J=8.4 \mathrm{~Hz}, 2 \mathrm{H}), 7.51(\mathrm{~d}, J=7.8$ $\mathrm{Hz}, 2 \mathrm{H}), 7.45(\mathrm{~d}, J=7.0 \mathrm{~Hz}, 2 \mathrm{H}), 7.36(\mathrm{t}, J=7.3 \mathrm{~Hz}, 3 \mathrm{H}), 7.32-7.27(\mathrm{~m}, 2 \mathrm{H}), 7.18-7.07(\mathrm{~m}, 5 \mathrm{H})$, $6.92(\mathrm{~d}, J=5.8 \mathrm{~Hz}, 2 \mathrm{H}), 6.41(\mathrm{~s}, 1 \mathrm{H}), 6.18-6.08(\mathrm{~m}, 1 \mathrm{H}), 3.48-3.27(\mathrm{~m}, 2 \mathrm{H}), 2.85-2.73(\mathrm{~m}, 1 \mathrm{H})$, $2.56-2.36(\mathrm{~m}, 4 \mathrm{H}), 2.24(\mathrm{~s}, 3 \mathrm{H}), 1.80-1.57(\mathrm{~m}, 2 \mathrm{H})$.

${ }^{13} \mathrm{C}\left\{{ }^{1} \mathrm{H}\right\}$ NMR $\left(201 \mathrm{MHz}, \mathrm{CDCl}_{3}\right) \delta 144.8,142.0,141.1,140.5,135.7,135.1,134.8,131.2$, $129.8,128.6,128.3,128.2,128.2,127.0,126.6,125.6,125.1,124.8,123.2,123.0,119.8,119.5$, 113.6, 113.1, 37.9, 37.7, 37.2, 33.6, 32.1, 21.4.

HRMS(APCI) (m/z): [M + H] ${ }^{+}$Calcd for $\mathrm{C}_{36} \mathrm{H}_{33} \mathrm{NO}_{2} \mathrm{~S}: 544.2305$; found: 544.2302 TLC: $\mathrm{R}_{f}=0.28\left(15 \% \mathrm{Et}_{2} \mathrm{O}\right.$ in hexanes) 
<smiles>COc1ccc(/C=C2\CC(c3ccccc3)=C[C@H](C)C2)cc1</smiles>

(R,E)-3-(4-methoxybenzylidene)-5-methyl-2,3,4,5-tetrahydro-1,1'-biphenyl (30). The reaction was conducted according to the general procedure without modification using 1-(2,2dichlorovinyl)-4-methoxybenzene $(20.4 \mathrm{mg}, \quad 0.1 \mathrm{mmol}, 1.0 \mathrm{equiv})$ and (1-((1R,2R)-2methylcyclopropyl)vinyl)benzene ( $47.5 \mathrm{mg}, 0.3 \mathrm{mmol}$, 3.0 equiv). Isolated yields were determined following column chromatography $\left(\mathrm{SiO}_{2}, 5 \% \mathrm{Et}_{2} \mathrm{O}\right.$ in hexanes). Spectroscopic and mass spectrometry data were identical to those of the racemic product. Single crystals of $\mathbf{3 0}$ suitable for $\mathrm{X}$-ray diffraction analysis were obtained by cooling a saturated $\mathrm{Et}_{2} \mathrm{O}$ solution to $-20^{\circ} \mathrm{C}$.

$20.2 \mathrm{mg}$ isolated $(70 \%$ yield), white solid, $E / Z=12.3: 1$

$[\alpha]_{\mathrm{D}}{ }^{23}=-181^{\circ}\left(\mathrm{c} 0.184, \mathrm{CHCl}_{3}\right)$.

HPLC: Chiralpak ${ }^{\circledR}$ OJ-H column (hexane/IPA $=98: 2,1.0 \mathrm{~mL} / \mathrm{min}, \lambda=254 \mathrm{~nm}$ ) tr $=15.64 \mathrm{~min}$ (major), 16.61 min (minor): >99:1 er.

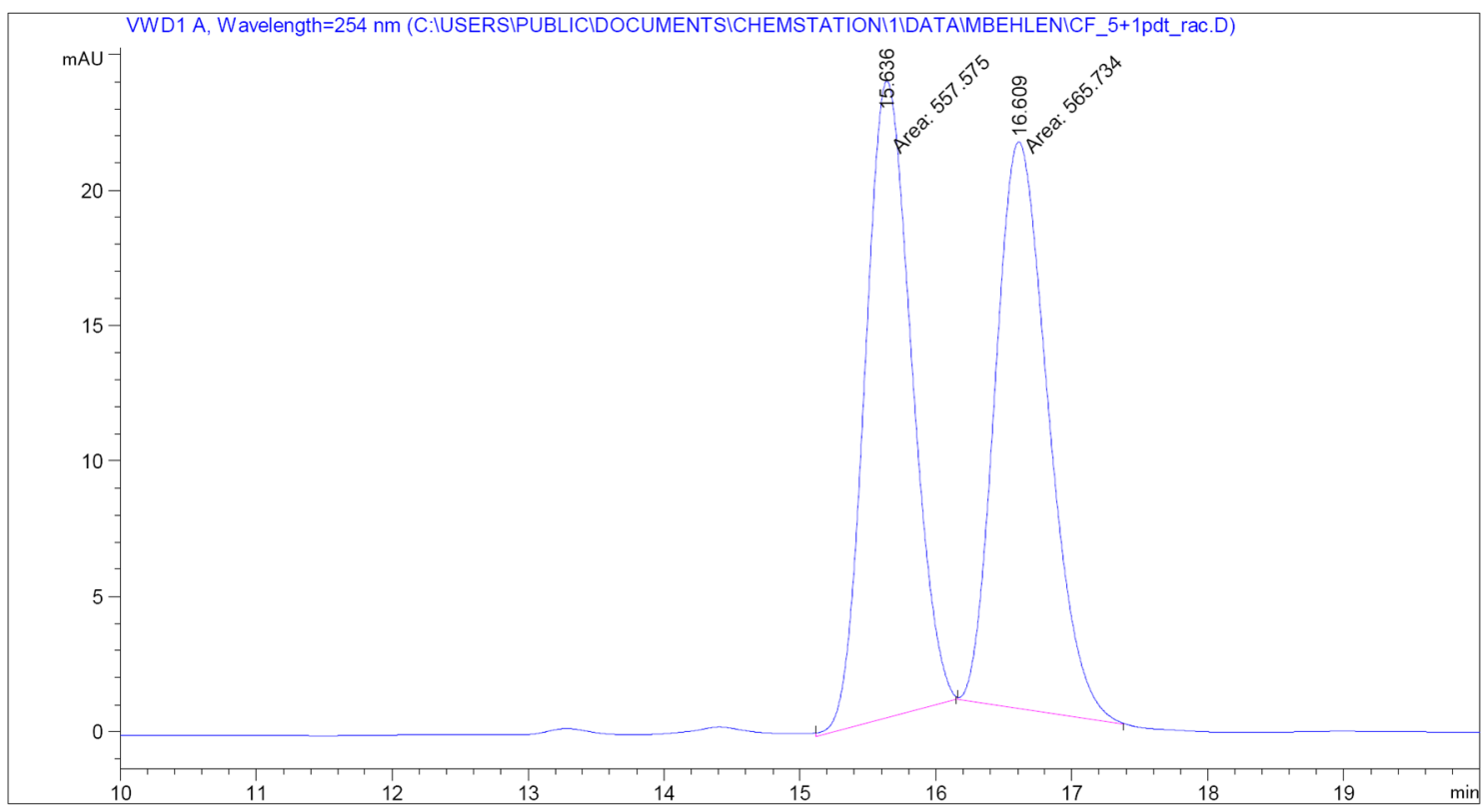

\begin{tabular}{|c|c|c|c|c|c|c|}
\hline $\begin{array}{c}\text { Peak } \\
\#\end{array}$ & $\begin{array}{c}\text { RetTime } \\
\text { [min] }\end{array}$ & Type & $\begin{array}{l}\text { Width } \\
\text { [min] }\end{array}$ & $\begin{array}{c}\text { Area } \\
{\left[\mathrm{mAU}^{\star} \mathrm{s}\right]}\end{array}$ & $\begin{array}{l}\text { Height } \\
{[\mathrm{mAU}]}\end{array}$ & $\begin{array}{c}\text { Area } \\
\quad \frac{\circ}{8}\end{array}$ \\
\hline & & & ------ & ---------- & ---- & ----- \\
\hline 1 & 15.636 & $\mathrm{MM} \mathrm{T}$ & 0.4793 & 557.57458 & 23.52308 & 49.6368 \\
\hline 2 & 16.609 & $\mathrm{MM} \mathrm{T}$ & 0.4504 & 565.73358 & 20.93578 & 50.3632 \\
\hline
\end{tabular}



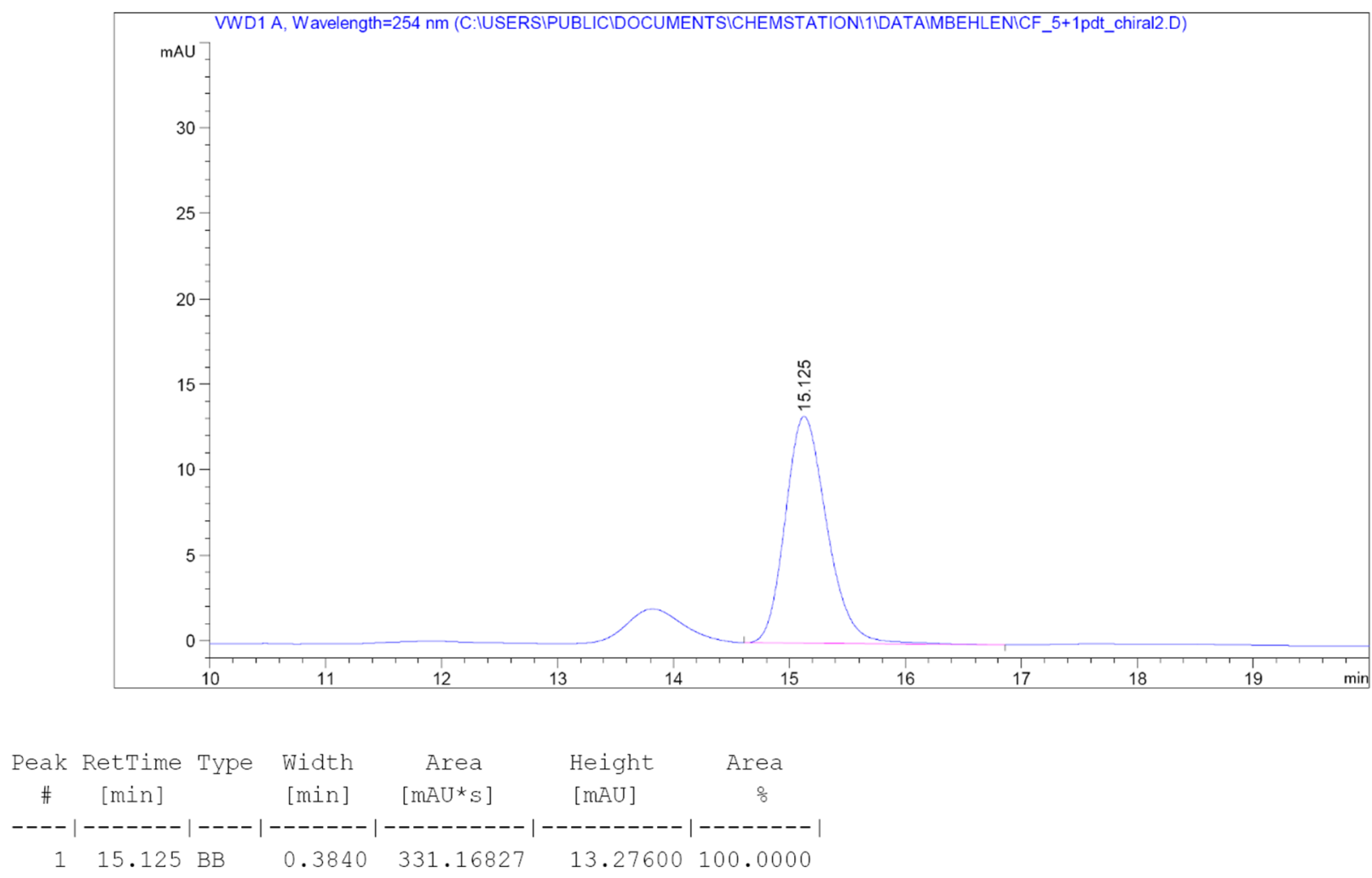

Figure S2. HPLC data for 30. (racemate, top; $(R)-\mathbf{3 0}$, bottom).

\section{1,1-Dichloroalkenes:}<smiles>ClC(Cl)=Cc1ccccn1</smiles><smiles>C=C(Cl)Cl</smiles><smiles>ClC(Cl)=CC=CCCC=Cc1ccccc1</smiles>

Vinylcyclopropanes:<smiles>C=CC1CC1(F)F</smiles><smiles></smiles><smiles>C=CC1CC1c1ccccc1</smiles><smiles>C=C(O[Na])C1CC1</smiles><smiles>Oc1ccccc1</smiles>

Figure S3. Substrates that were found to be ineffective in the $[5+1]$-cycloaddition (no detectable product using the general procedure). 


\section{6. $[5+1]$-Product Derivatization}

General procedure for the dehydrogenation reaction. A flame-dried round bottom flask was charged with the [5 + 1]-product (1.0 equiv), $\mathrm{Pd} / \mathrm{C}(10 \mathrm{wt} \%$ on activated carbon, $10 \mathrm{wt} \%$ relative to the [5+1]-product), $1.0 \mathrm{~mL}$ ethylene glycol, and a magnetic stir bar. The reaction was refluxed at $200{ }^{\circ} \mathrm{C}$ and stirred. After $8 \mathrm{hr}$, the reaction was cooled to ambient temperature and extracted with $\mathrm{CH}_{2} \mathrm{Cl}_{2}(3 \times 5 \mathrm{~mL})$. The combined organic phases were dried over $\mathrm{MgSO}_{4}$, concentrated under reduced pressure, and loaded directly onto a $\mathrm{SiO}_{2}$ column for purification. ${ }^{14}$
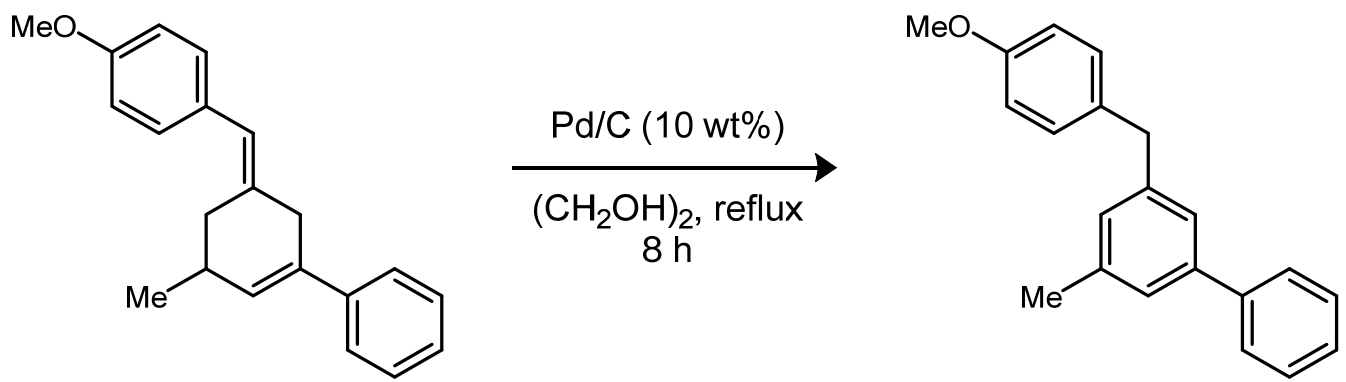

3-(4-methoxybenzyl)-5-methyl-1,1'-biphenyl (38). The reaction was conducted according to the general procedure without modification using 30 (19.7 mg, $0.068 \mathrm{mmol}, 1.0$ equiv) and $\mathrm{Pd} / \mathrm{C}$ $(10 \mathrm{wt} \%, 1.9 \mathrm{mg})$. Isolated yields were determined following column chromatography $\left(5 \% \mathrm{Et}_{2} \mathrm{O}\right.$ in hexanes).

$11.4 \mathrm{mg}$ isolated (77\% yield), colorless oil

${ }^{1} \mathrm{H}$ NMR $\left(300 \mathrm{MHz}, \mathrm{CDCl}_{3}\right) \delta 7.56(\mathrm{~d}, J=7.0 \mathrm{~Hz}, 2 \mathrm{H}), 7.41(\mathrm{t}, J=7.5 \mathrm{~Hz}, 2 \mathrm{H}), 7.33(\mathrm{~d}, J=7.2$ $\mathrm{Hz}, 1 \mathrm{H}), 7.23$ (d, $J=7.8 \mathrm{~Hz}, 2 \mathrm{H}), 7.15(\mathrm{~d}, J=8.6 \mathrm{~Hz}, 2 \mathrm{H}), 6.99$ (s, 1H), $6.84(\mathrm{~d}, J=8.6 \mathrm{~Hz}, 2 \mathrm{H}), 3.95$ (s, $2 \mathrm{H}), 3.79(\mathrm{~s}, 3 \mathrm{H}), 2.38(\mathrm{~s}, 3 \mathrm{H})$.

${ }^{13} \mathrm{C}\left\{{ }^{1} \mathrm{H}\right\} \mathrm{NMR}\left(201 \mathrm{MHz}, \mathrm{CDCl}_{3}\right) \delta 157.9,141.9,141.3,141.3,138.4,133.2,129.8,128.6$, $128.5,127.1,127.0,125.7,124.8,113.8,55.2,41.0,21.4$.

HRMS(ESI) (m/z): [M + H]+ Calcd for $\mathrm{C}_{21} \mathrm{H}_{20} \mathrm{O}: 289.1587$; found: 289.1583

TLC: $\mathrm{R}_{f}=0.42\left(5 \% \mathrm{Et}_{2} \mathrm{O}\right.$ in hexanes)
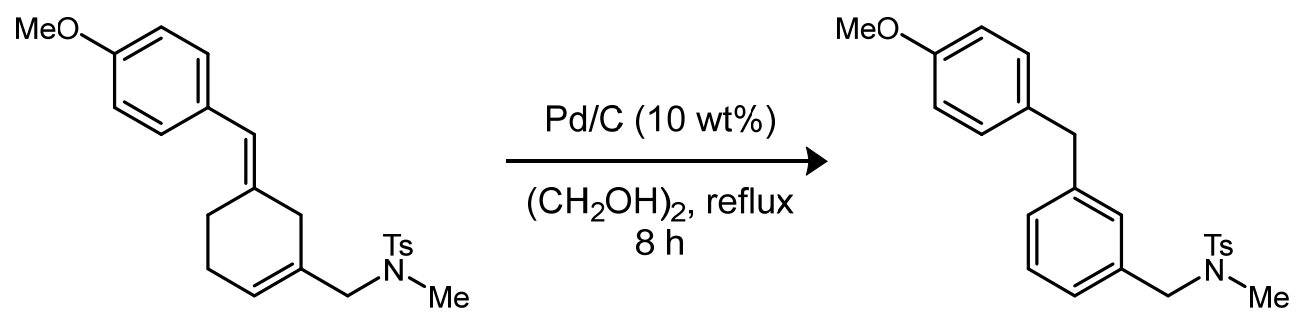

N-(3-(4-methoxybenzyl)benzyl)-N,4-dimethylbenzenesulfonamide (39). The reaction was conducted according to the general procedure without modification using 27 (29.6 mg, 0.074 mmol, 1.0 equiv.) and $\mathrm{Pd} / \mathrm{C}(10 \mathrm{wt} \%, 2.9 \mathrm{mg})$. Isolated yields were determined following column chromatography (20\% $\mathrm{Et}_{2} \mathrm{O}$ in hexanes).

$22.7 \mathrm{mg}$ isolated (77\% yield), yellow solid 
${ }^{1} \mathrm{H}$ NMR $\left(300 \mathrm{MHz}, \mathrm{CDCl}_{3}\right) \delta 7.72(\mathrm{~d}, J=8.3 \mathrm{~Hz}, 2 \mathrm{H}), 7.35(\mathrm{~d}, J=8.6 \mathrm{~Hz}, 2 \mathrm{H}), 7.23(\mathrm{~d}, J=8.1$ $\mathrm{Hz}, 1 \mathrm{H}), 7.19-7.00(\mathrm{~m}, 5 \mathrm{H}), 6.82(\mathrm{~d}, J=8.7 \mathrm{~Hz}, 2 \mathrm{H}), 4.08(\mathrm{~s}, 2 \mathrm{H}), 3.89(\mathrm{~s}, 2 \mathrm{H}), 3.78(\mathrm{~s}, 3 \mathrm{H}), 2.56$ (s, $3 \mathrm{H}), 2.45(\mathrm{~s}, 3 \mathrm{H})$.

${ }^{13} \mathrm{C}\left\{{ }^{1} \mathrm{H}\right\}$ NMR $\left(201 \mathrm{MHz}, \mathrm{CDCl}_{3}\right) \delta 158.0,143.4,142.1,135.8,134.3,133.0,129.8,129.7$, 128.8, 128.8, 128.4, 127.6, 126.1, 113.9, 55.3, 54.1, 40.9, 34.4, 21.6.

HRMS(ESI) (m/z): [M + H] ${ }^{+}$Calcd for $\mathrm{C}_{23} \mathrm{H}_{25} \mathrm{NO}_{3} \mathrm{~S}: 396.1628$; found: 396.1630

TLC: $\mathrm{R}_{f}=0.15\left(5 \% \mathrm{Et}_{2} \mathrm{O}\right.$ in hexanes)
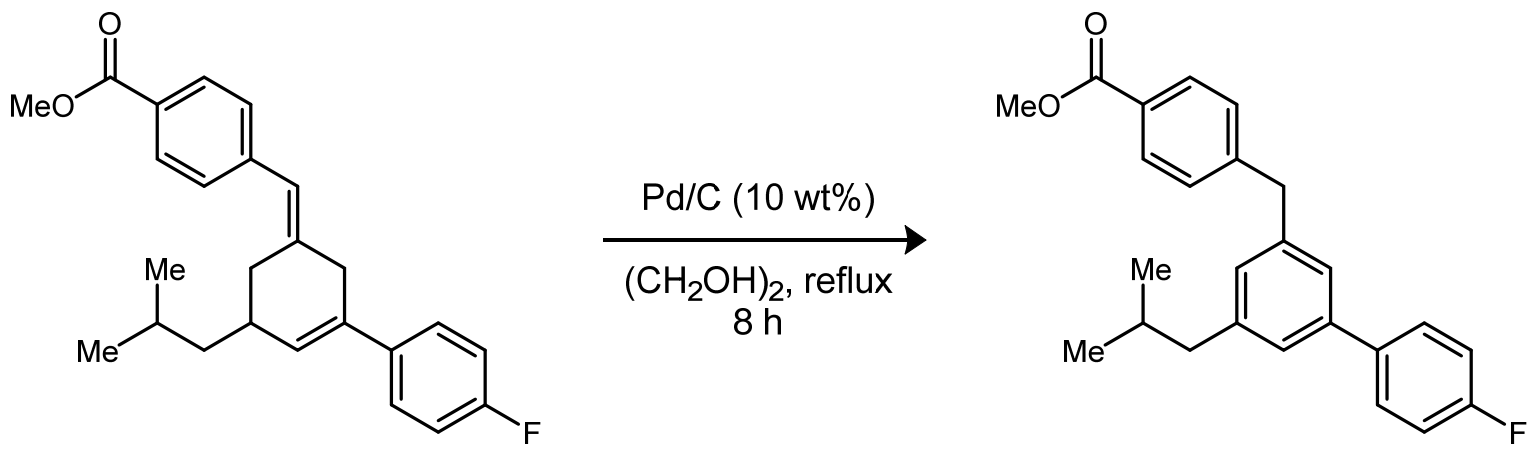

methyl 4-((4'-fluoro-5-isobutyl-[1,1'-biphenyl]-3-yl)methyl)benzoate (40). The reaction was conducted according to the general procedure without modification using $\mathbf{S 1 8}$ (24.6 $\mathrm{mg}, 0.065$ mmol, 1.0 equiv) and $\mathrm{Pd} / \mathrm{C}(10 \mathrm{wt} \%, 2.5 \mathrm{mg}$ ). Isolated yields were determined following column chromatography $\left(10 \% \mathrm{Et}_{2} \mathrm{O}\right.$ in hexanes).

$12.2 \mathrm{mg}$ isolated ( $50 \%$ yield), colorless oil

${ }^{1} \mathrm{H}$ NMR $\left(300 \mathrm{MHz}, \mathrm{CDCl}_{3}\right) \delta 7.97(\mathrm{~d}, J=8.2 \mathrm{~Hz}, 2 \mathrm{H}), 7.57-7.44(\mathrm{~m}, 2 \mathrm{H}), 7.29(\mathrm{~d}, J=8.7 \mathrm{~Hz}$, $2 \mathrm{H}), 7.17(\mathrm{~d}, J=5.7 \mathrm{~Hz}, 2 \mathrm{H}), 7.09(\mathrm{t}, J=8.7 \mathrm{~Hz}, 2 \mathrm{H}), 6.94(\mathrm{~s}, 1 \mathrm{H}), 4.06(\mathrm{~s}, 2 \mathrm{H}), 3.90(\mathrm{~s}, 3 \mathrm{H}), 2.49(\mathrm{~d}, J$ $=7.1 \mathrm{~Hz}, 2 \mathrm{H}), 1.96-1.79(\mathrm{~m}, 1 \mathrm{H}), 0.91(\mathrm{~d}, J=6.6 \mathrm{~Hz}, 6 \mathrm{H})$.

${ }^{13} \mathrm{C}\left\{{ }^{1} \mathrm{H}\right\}$ NMR $\left(201 \mathrm{MHz}, \mathrm{CDCl}_{3}\right) \delta 167.0,162.3\left(\mathrm{~d},{ }^{1} \mathrm{~J}_{\mathrm{CF}}=246.6 \mathrm{~Hz}\right), 146.4,142.6,140.3,137.3$ $\left(\mathrm{d},{ }^{4} J_{\mathrm{CF}}=3.0 \mathrm{~Hz}\right), 129.8,128.8,128.7,128.6\left(\mathrm{~d},{ }^{3} J_{\mathrm{CF}}=8.3 \mathrm{~Hz}\right), 128.0,125.9,125.0,115.4\left(\mathrm{~d},{ }^{2} J_{\mathrm{CF}}=\right.$ $21.4 \mathrm{~Hz}), 52.0,45.3,41.9,30.2,22.3$.

${ }^{19} \mathrm{~F}$ NMR $\left(282 \mathrm{MHz}, \mathrm{CDCl}_{3}\right) \delta-117.47$.

HRMS(ESI) (m/z): [M + H] ${ }^{+}$Calcd for $\mathrm{C}_{25} \mathrm{H}_{25} \mathrm{FO}_{2}: 377.1911$; found: 377.1909

TLC: $\mathrm{R}_{f}=0.47$ (10\% $\mathrm{Et}_{2} \mathrm{O}$ in hexanes) 
<smiles>CCCC1C=C(c2ccccc2)C/C(=C/c2ccc(C(=O)OC)cc2)C1</smiles><smiles>CCCc1cc(Cc2ccc(C(=O)OC)cc2)cc(-c2ccccc2)c1</smiles>

methyl 4-((5-propyl-[1,1'-biphenyl]-3-yl)methyl)benzoate (41). The reaction was conducted according to the general procedure without modification using $\mathbf{S 1 7}$ (25.3 $\mathrm{mg}, 0.073 \mathrm{mmol}, 1.0$ equiv) and $\mathrm{Pd} / \mathrm{C}(10 \mathrm{wt} \%, 2.5 \mathrm{mg})$. Isolated yields were determined following column chromatography (10\% $\mathrm{Et}_{2} \mathrm{O}$ in hexanes).

$13.3 \mathrm{mg}$ isolated (53\% yield), colorless oil

${ }^{1} \mathrm{H}$ NMR (300 MHz, CDCl 3$) \delta 7.97(\mathrm{~d}, J=8.3 \mathrm{~Hz}, 2 \mathrm{H}), 7.55(\mathrm{~d}, J=7.0 \mathrm{~Hz}, 2 \mathrm{H}), 7.41(\mathrm{t}, J=7.3$ $\mathrm{Hz}, 2 \mathrm{H}), 7.36-7.27(\mathrm{~m}, 4 \mathrm{H}), 7.21(\mathrm{~s}, 1 \mathrm{H}), 6.98(\mathrm{~s}, 1 \mathrm{H}), 4.07(\mathrm{~s}, 2 \mathrm{H}), 3.90(\mathrm{~d}, J=8.1 \mathrm{~Hz}, 3 \mathrm{H}), 2.61(\mathrm{t}$, $J=8.1 \mathrm{~Hz}, 2 \mathrm{H}), 1.73-1.57(\mathrm{~m}, 2 \mathrm{H}), 0.95(\mathrm{t}, J=7.3 \mathrm{~Hz}, 3 \mathrm{H})$.

${ }^{13} \mathrm{C}\left\{{ }^{1} \mathrm{H}\right\} \mathrm{NMR}\left(201 \mathrm{MHz}, \mathrm{CDCl}_{3}\right) \delta 167.0,146.5,143.5,141.4,141.2,140.0,129.8,128.9$, 128.6, 128.0, 127.1, 125.5, 125.2, 51.9, 41.9, 38.0, 24.5, 13.8 .

HRMS(ESI) (m/z): [M + H] Calcd for $\mathrm{C}_{22} \mathrm{H}_{24} \mathrm{O}_{2}: 345.1849$; found: 245.1847

TLC: $\mathrm{R}_{f}=0.53\left(10 \% \mathrm{Et}_{2} \mathrm{O}\right.$ in hexanes $)$<smiles>[3H]N(C)CCC=C(Cl)Cl</smiles><smiles>C=C(c1ccccc1)C1CC1C</smiles>

3.0 Equiv.
$\mathrm{Co}(\mathrm{DME}) \mathrm{Br}_{2}(5 \mathrm{~mol} \%)$

${ }^{t}$ Bu-Quinox (6 mol\%)

Zn (3.0 Equiv.)

DMA, $25^{\circ} \mathrm{C}$

$48 \mathrm{~h}$<smiles>CC1C=C(c2ccccc2)CC(=CCCN(C)C)C1</smiles>

(E)-N,4-dimethyl- $N$-(3-(5-methyl-4,5-dihydro-[1,1'-biphenyl]-3(2H)-

ylidene)propyl)benzenesulfonamide (S20). In an $\mathrm{N}_{2}$-filled glovebox, a 5-mL vial was charged with Co(DME)Br2 (1.54 mg, $0.005 \mathrm{mmol}, 0.05$ equiv), ( \pm )- $t$-Bu-Quinox (1.52 mg, $0.006 \mathrm{mmol}, 0.06$ equiv), $\mathrm{Zn}$ powder (19.6 mg, $0.3 \mathrm{mmol}, 3.0$ equiv), and a magnetic stir bar. To this mixture was added a solution of $\mathbf{S 1 6}(30.8 \mathrm{mg}, \quad 0.1 \mathrm{mmol}, 1.0$ equiv) and (1-(2methylcyclopropyl)vinyl)benzene ( $43.3 \mathrm{mg}, 0.3 \mathrm{mmol}, 3.0$ equiv) in DMA $(0.75 \mathrm{~mL})$. The reaction was stirred at room temperature. After $48 \mathrm{~h}$, the crude reaction mixture was removed from the glovebox, opened to ambient atmosphere, and loaded directly onto a $\mathrm{SiO}_{2}$ column for purification (30\% $\mathrm{Et}_{2} \mathrm{O}$ in hexanes). Compound $\mathbf{S 2 0}$ is highly unstable and was used immediately in the dehydrogenation reaction.

$20.9 \mathrm{mg}$ isolated (53\% yield), yellow oil 

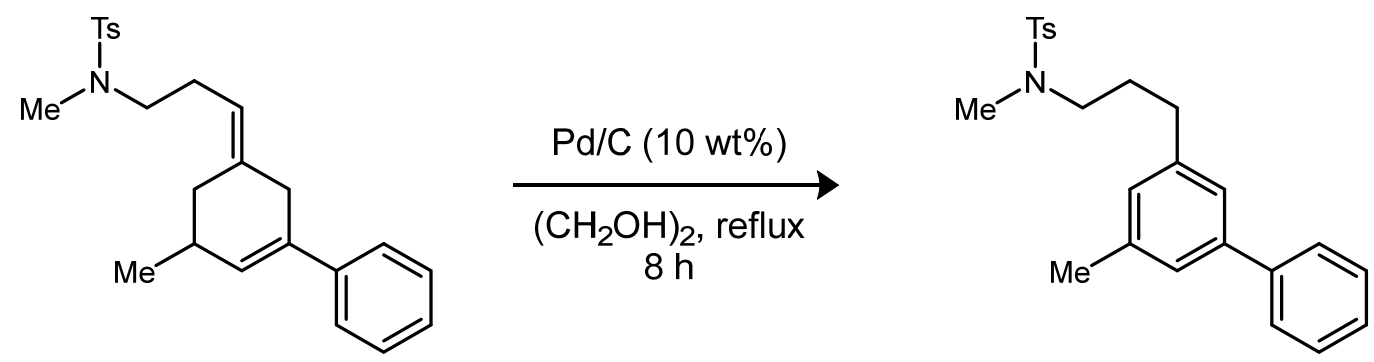

$\boldsymbol{N}$,4-dimethyl- $\boldsymbol{N}$-(3-(5-methyl-[1,1'-biphenyl]-3-yl)propyl)benzenesulfonamide (42). The reaction was conducted according to the general procedure without modification using S20 (20.9 $\mathrm{mg}, 0.053 \mathrm{mmol}, 1.0$ equiv.) and $\mathrm{Pd} / \mathrm{C}(10 \mathrm{wt} \%, 2.1 \mathrm{mg})$. Isolated yields were determined following column chromatography (30\% $\mathrm{Et}_{2} \mathrm{O}$ in hexanes).

$7.3 \mathrm{mg}$ isolated (35\% yield), colorless oil

${ }^{1} \mathrm{H}$ NMR $\left(300 \mathrm{MHz}, \mathrm{CDCl}_{3}\right) \delta 7.66(\mathrm{~d}, J=8.2 \mathrm{~Hz}, 2 \mathrm{H}), 7.58(\mathrm{~d}, J=7.0 \mathrm{~Hz}, 2 \mathrm{H}), 7.49-7.37(\mathrm{~m}$, $2 \mathrm{H}), 7.35(\mathrm{~d}, J=7.0 \mathrm{~Hz}, 1 \mathrm{H}), 7.29(\mathrm{~d}, J=7.0 \mathrm{~Hz}, 2 \mathrm{H}), 7.22(\mathrm{~d}, J=13.5 \mathrm{~Hz}, 2 \mathrm{H}), 7.00(\mathrm{~s}, 1 \mathrm{H}), 3.06(\mathrm{t}, J$ $=7.1 \mathrm{~Hz}, 2 \mathrm{H}), 2.73(\mathrm{~s}, 3 \mathrm{H}), 2.69(\mathrm{t}, J=7.4 \mathrm{~Hz}, 2 \mathrm{H}), 2.41(\mathrm{~s}, 3 \mathrm{H}), 2.40(\mathrm{~s}, 3 \mathrm{H}), 1.98-1.78(\mathrm{~m}, 2 \mathrm{H})$.

${ }^{13} \mathrm{C}\left\{{ }^{1} \mathrm{H}\right\}$ NMR $\left(201 \mathrm{MHz}, \mathrm{CDCl}_{3}\right) \delta 143.2,141.7,141.3,141.3,138.4,134.5,129.6,128.6$, 128.1, 127.4, 127.1, 127.1, 125.7, 124.4, 49.7, 34.7, 32.7, 29.3, 21.4, 21.4.

HRMS(APCI) (m/z): [M + H] ${ }^{+}$Calcd for $\mathrm{C}_{24} \mathrm{H}_{27} \mathrm{NO}_{2} \mathrm{~S}: 394.1835$; found: 394.1833

TLC: $\mathrm{R}_{f}=0.21\left(15 \% \mathrm{Et}_{2} \mathrm{O}\right.$ in hexanes)
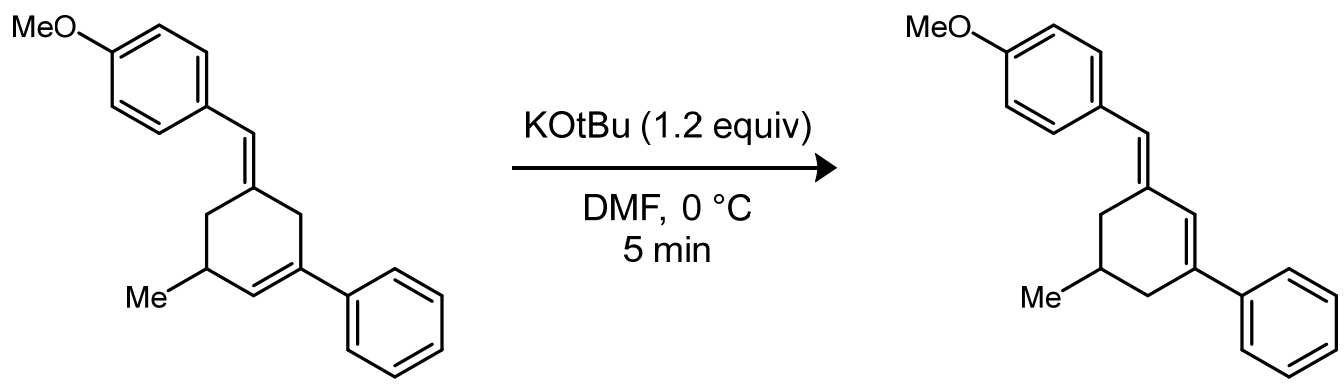

(E)-5-(4-methoxybenzylidene)-3-methyl-2,3,4,5-tetrahydro-1,1'-biphenyl (43). A solution of 30 (24.3 mg, $0.083 \mathrm{mmol}, 1.0$ equiv.) in DMF ( $1 \mathrm{~mL}$ ) was cooled to $0{ }^{\circ} \mathrm{C}$ using an ice bath and stirred. To this solution was added KOtBu (10.8 mg, $0.097 \mathrm{mmol}, 1.2$ equiv) in one portion. The reaction was stirred at $0{ }^{\circ} \mathrm{C}$. After $5 \mathrm{~min}$, the reaction was quenched with $\mathrm{H}_{2} \mathrm{O}(1 \mathrm{~mL})$ and extracted with $\mathrm{Et}_{2} \mathrm{O}(3 \times 5 \mathrm{~mL})$. The combined organic phases were dried over $\mathrm{MgSO}_{4}$, concentrated under reduced pressure, and loaded directly onto a $\mathrm{SiO}_{2}$ column for purification (5\% $\mathrm{Et}_{2} \mathrm{O}$ in hexanes).

$18.8 \mathrm{mg}$ isolated (78\% yield), white solid

${ }^{1} \mathrm{H}$ NMR $\left(300 \mathrm{MHz}, \mathrm{CDCl}_{3}\right) \delta 7.50(\mathrm{~d}, J=7.2 \mathrm{~Hz}, 2 \mathrm{H}), 7.35(\mathrm{t}, J=7.6 \mathrm{~Hz}, 3 \mathrm{H}), 7.30(\mathrm{~d}, J=8.5$ $\mathrm{Hz}, 2 \mathrm{H}), 6.90(\mathrm{~d}, J=8.3 \mathrm{~Hz}, 2 \mathrm{H}), 6.66(\mathrm{~d}, J=2.0 \mathrm{~Hz}, 1 \mathrm{H}), 6.41(\mathrm{~s}, 1 \mathrm{H}), 3.83(\mathrm{~s}, 3 \mathrm{H}), 2.89(\mathrm{dd}, J=14.8$, $3.5 \mathrm{~Hz}, 1 \mathrm{H}$ ), 2.63 (dd, $J=16.8,4.4 \mathrm{~Hz}, 1 \mathrm{H}), 2.36-2.10(\mathrm{~m}, 2 \mathrm{H}), 1.93(\mathrm{~s}, 1 \mathrm{H}), 1.11$ (d, $J=6.5 \mathrm{~Hz}, 3 \mathrm{H}$ ).

${ }^{13} \mathrm{C}\left\{{ }^{1} \mathrm{H}\right\}$ NMR $\left(201 \mathrm{MHz}, \mathrm{CDCl}_{3}\right) \delta 158.1,141.5,137.4,136.7,130.7,130.3,128.7,128.4$, 127.3, 127.0, 125.0, 113.7, 55.3, 36.0, 34.9, 29.4, 21.8.

HRMS(ESI) (m/z): [M + H] ${ }^{+}$Calcd for $\mathrm{C}_{21} \mathrm{H}_{22} \mathrm{O}$ : 291.1743; found: 291.1741 
TLC: $\mathrm{R}_{f}=0.45\left(5 \% \mathrm{Et}_{2} \mathrm{O}\right.$ in hexanes $)$

General procedure for the Riley oxidation reaction. A flame-dried microwave vial was charged with the [5 + 1]-product (1.0 equiv), $\mathrm{SeO}_{2}$ (3.0 equiv), $1.0 \mathrm{~mL}$ 1,4-dioxane, and a magnetic stir bar. The reaction was heated to $100{ }^{\circ} \mathrm{C}$ and stirred. After $30 \mathrm{~min}$, the reaction was cooled to ambient temperature and loaded directly onto a $\mathrm{SiO}_{2}$ column for purification.
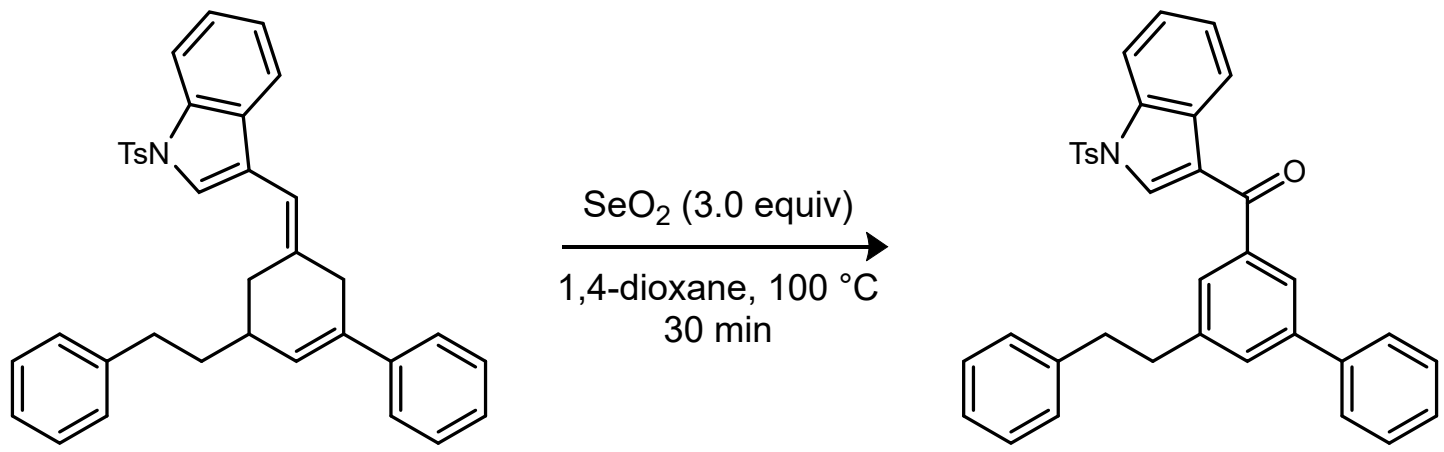

(5-phenethyl-[1,1'-biphenyl]-3-yl)(1-tosyl-1H-indol-3-yl)methanone (44). The reaction was conducted according to the general procedure without modification using $\mathbf{S 1 9}$ (39.4 $\mathrm{mg}, 0.072$ mmol, 1.0 equiv) and $\mathrm{SeO}_{2}(24.1 \mathrm{mg}, 0.22 \mathrm{mmol}, 3.0$ equiv). Isolated yields were determined following column chromatography ( $30 \% \mathrm{Et}_{2} \mathrm{O}$ in hexanes).

$20.7 \mathrm{mg}$ isolated ( $52 \%$ yield), yellow solid

${ }^{1} \mathrm{H}$ NMR $\left(300 \mathrm{MHz}, \mathrm{CDCl}_{3}\right) \delta 8.30(\mathrm{~d}, J=8.1 \mathrm{~Hz}, 1 \mathrm{H}), 8.13-7.95(\mathrm{~m}, 2 \mathrm{H}), 7.87(\mathrm{~s}, 1 \mathrm{H}), 7.80$ (d, $J=8.3 \mathrm{~Hz}, 2 \mathrm{H}), 7.68(\mathrm{~s}, 1 \mathrm{H}), 7.65-7.54(\mathrm{~m}, 3 \mathrm{H}), 7.52-7.38(\mathrm{~m}, 5 \mathrm{H}), 7.38-7.28(\mathrm{~m}, 4 \mathrm{H}), 7.23-$ $7.14(\mathrm{~m}, 3 \mathrm{H}), 3.19-2.94(\mathrm{~m}, 4 \mathrm{H}), 2.36(\mathrm{~s}, 3 \mathrm{H})$.

${ }^{13} \mathrm{C}\left\{{ }^{1} \mathrm{H}\right\}$ NMR $\left(201 \mathrm{MHz}, \mathrm{CDCl}_{3}\right) \delta 190.9,145.8,142.8,141.6,141.1,140.2,139.9,135.0$, $134.5,133.5,131.3,130.2,128.8,128.4,128.4,127.8,127.7,127.2,127.0,126.1,125.9,125.6$, 124.8, 122.8, 120.6, 113.17, 37.7, 30.3, 21.6.

HRMS(ESI) (m/z): [M + H] ${ }^{+}$Calcd for $\mathrm{C}_{36} \mathrm{H}_{29} \mathrm{NO}_{3} \mathrm{~S}: 556.1941$; found: 556.1937

TLC: $\mathrm{R}_{f}=0.24\left(30 \% \mathrm{Et}_{2} \mathrm{O}\right.$ in hexanes)
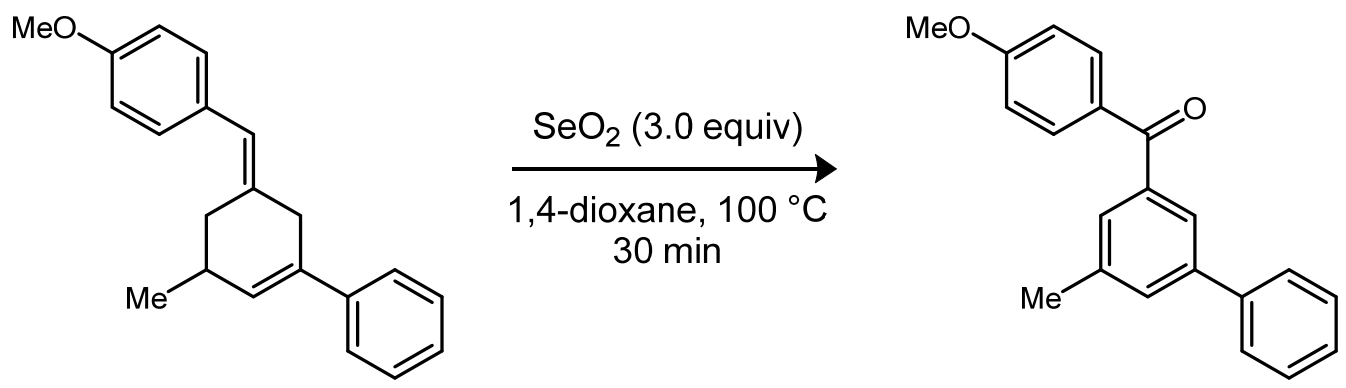

(4-methoxyphenyl)(5-methyl-[1,1'-biphenyl]-3-yl)methanone (45). The reaction was conducted according to the general procedure without modification using $\mathbf{3 0}$ (24.3 $\mathrm{mg}, 0.083$ mmol, 1.0 equiv.) and $\mathrm{SeO}_{2}$ (28.0 mg, $0.25 \mathrm{mmol}, 3.0$ equiv.). Isolated yields were determined following column chromatography (10\% EtOAc in hexanes). 
$11.2 \mathrm{mg}$ isolated (55\% yield), colorless oil

${ }^{1} \mathrm{H}$ NMR $\left(300 \mathrm{MHz}, \mathrm{CDCl}_{3}\right) \delta 7.87(\mathrm{~d}, J=8.8 \mathrm{~Hz}, 2 \mathrm{H}), 7.75(\mathrm{~s}, 1 \mathrm{H}), 7.60(\mathrm{~d}, J=8.1 \mathrm{~Hz}, 3 \mathrm{H}), 7.56$ $(\mathrm{s}, 1 \mathrm{H}), 7.45(\mathrm{t}, J=7.2 \mathrm{~Hz}, 2 \mathrm{H}), 7.37(\mathrm{~d}, J=5.8 \mathrm{~Hz}, 1 \mathrm{H}), 6.98(\mathrm{~d}, J=8.9 \mathrm{~Hz}, 2 \mathrm{H}), 3.90(\mathrm{~s}, 3 \mathrm{H}), 2.49(\mathrm{~s}$, $3 \mathrm{H})$.

${ }^{13} \mathrm{C}\left\{{ }^{1} \mathrm{H}\right\}$ NMR $\left(201 \mathrm{MHz}, \mathrm{CDCl}_{3}\right) \delta 195.7,163.3,141.1,140.4,138.9,138.6,132.6,131.4$, $130.3,129.1,128.8,127.6,127.2,125.7,113.6,55.5,21.5$.

HRMS(ESI) (m/z): [M + H] Calcd for $\mathrm{C}_{21} \mathrm{H}_{18} \mathrm{O}_{2}: 303.1380$; found: 303.1382

TLC: $\mathrm{R}_{f}=0.22\left(15 \% \mathrm{Et}_{2} \mathrm{O}\right.$ in hexanes)

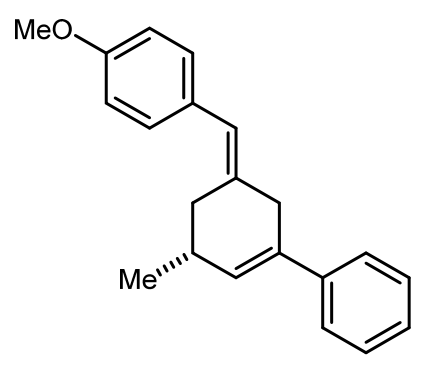

$30>99 \%$ ee
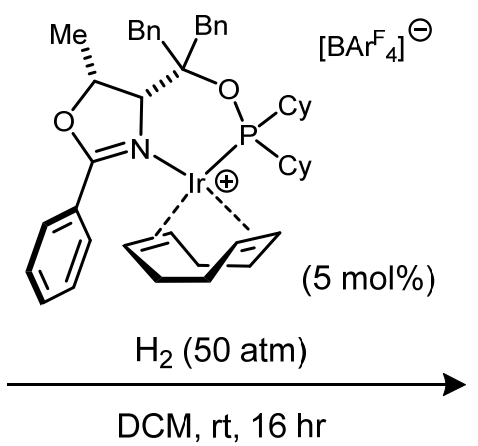

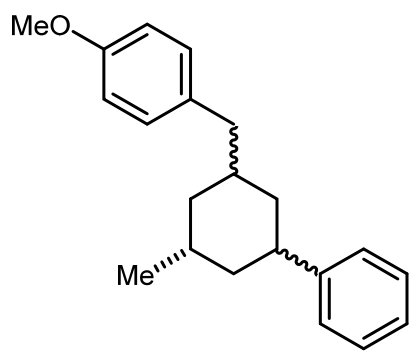

Attempted diastereoselective hydrogenation reaction. In an $\mathrm{N}_{2}$-filled glovebox, a 2dram vial was equipped with a magnetic stir bar, $30(20.3 \mathrm{mg}, 0.07 \mathrm{mmol}, 1.0$ equiv), $(R, R)$ [COD]Ir[Cy2 PThrePHOX] (6.0 mg, $0.0035 \mathrm{mmol}, 0.05$ equiv), and $\mathrm{CH}_{2} \mathrm{Cl}_{2}(1.0 \mathrm{~mL})$. The vial was capped and removed from the glovebox. A Parr bomb reactor vessel was placed under argon. The vial was uncapped and quickly placed into the Parr apparatus while under argon. The Parr apparatus was purged and backfilled with hydrogen (3x), then pressurized to 730 psi. After stirring for $16 \mathrm{~h}$, the crude reaction mixture was filtered through a glass fiber pad. The filtrate was concentrated under reduced pressure, and the crude residue was analyzed using by ${ }^{1} \mathrm{H}$ NMR spectroscopy. The hydrogenated product was obtained as a mixture of all four possible diastereomers. 


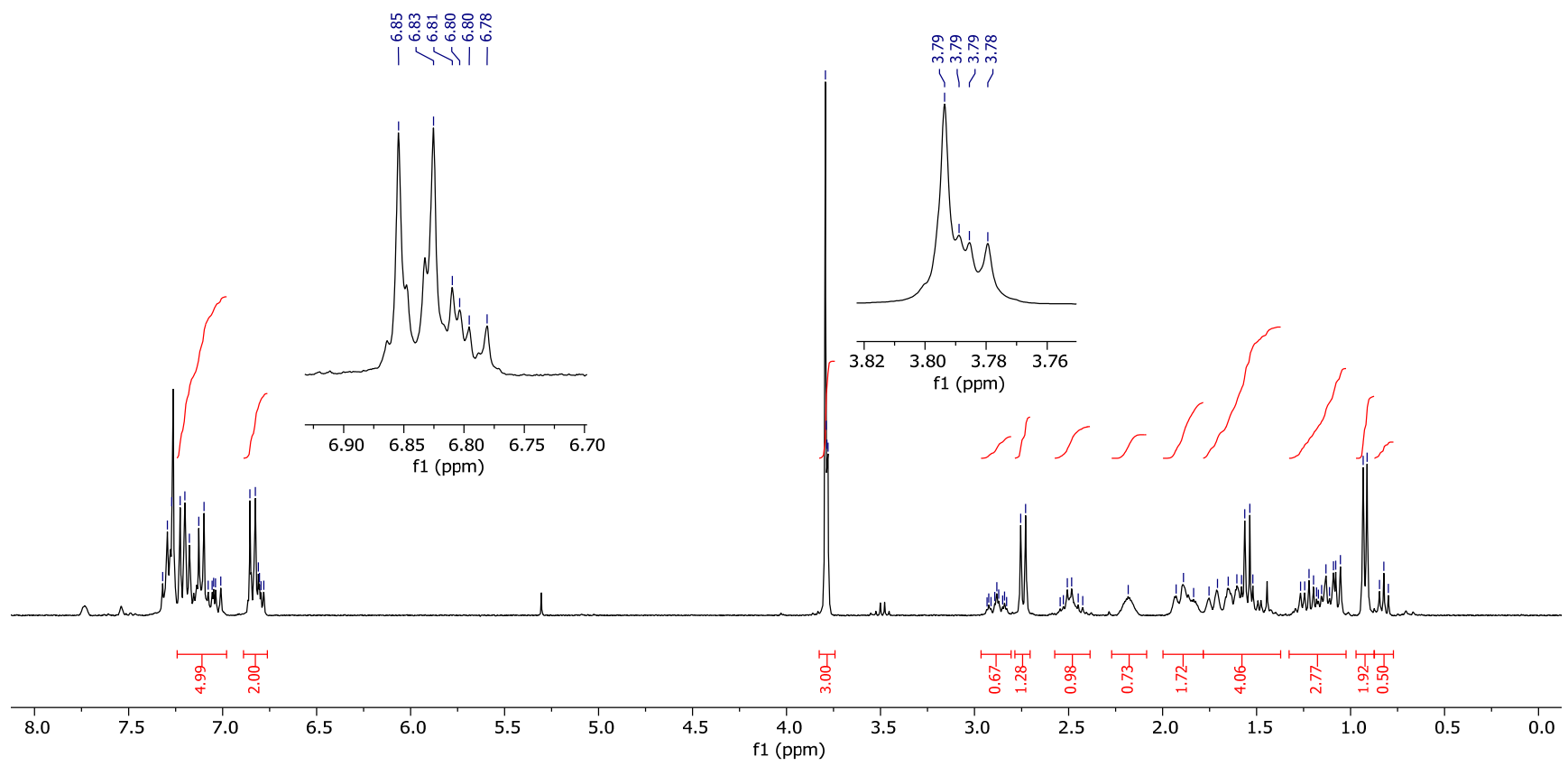

Figure S4. Crude ${ }^{1} \mathrm{H}$ NMR spectrum for the hydrogenation of $\mathbf{3 0}$ using a chiral Ir catalyst to provide a diastereomeric mixture of products $\left(300 \mathrm{MHz}, \mathrm{CDCl}_{3}, 295 \mathrm{~K}\right)$. 


\section{Mechanistic Studies}
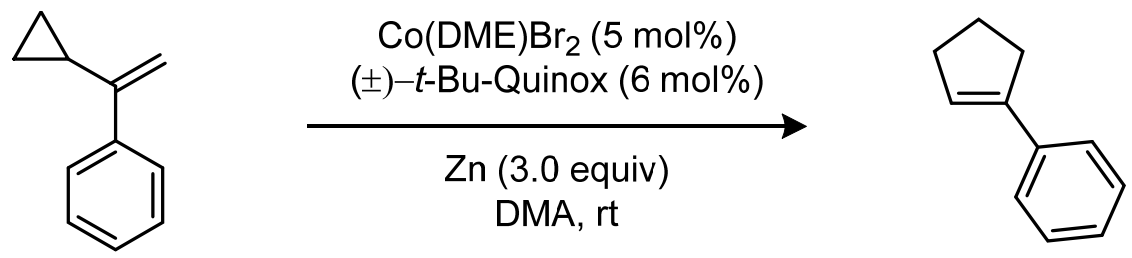

No Reaction

Vinylcyclopropane rearrangement experiment. In an $\mathrm{N}_{2}$-filled glovebox, a 5-mL vial was charged with Co(DME)Br2 (1.5 mg, $0.005 \mathrm{mmol}, 0.05$ equiv), ( \pm )-t-Bu-Quinox (1.5 mg, $0.006 \mathrm{mmol}$, 0.06 equiv), $\mathrm{Zn}$ powder (19.6 mg, $0.3 \mathrm{mmol}, 3.0$ equiv), and a magnetic stir bar. To this mixture was added a solution of (1-cyclopropylvinyl)benzene (43.3 mg, $0.3 \mathrm{mmol}, 3.0$ equiv) and 1,3,5timethoxybenzene $(16.8 \mathrm{mg}, 0.1 \mathrm{mmol})$ in DMA $(0.75 \mathrm{~mL})$. The reaction was stirred at room temperature. After $16 \mathrm{~h}$, the crude reaction mixture was removed from the glovebox, opened to ambient atmosphere, and diluted with $\mathrm{Et}_{2} \mathrm{O}$. An aliquot was filtered through a glass fiber pad and analyzed by ${ }^{1} \mathrm{H}$ NMR. The conversion of (1-cyclopropylvinyl)benzene was determined by integration against 1,3,5-timethoxybenzene ( $<1 \%$ conversion). 1-phenylcyclopentene was not detected in the mixture.

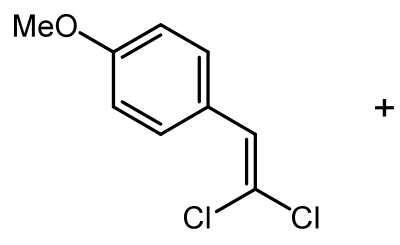

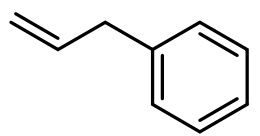

3.0 equiv.

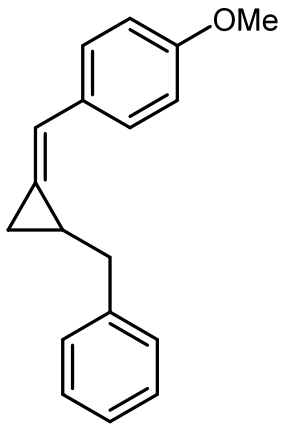

(Z)-1-((2-benzylcyclopropylidene)methyl)-4-methoxybenzene (47). In an $\mathrm{N}_{2}$-filled glovebox, a 5-mL vial was charged with $\mathrm{Co}(\mathrm{DME}) \mathrm{Br}_{2}(1.5 \mathrm{mg}, 0.005 \mathrm{mmol}, 0.05$ equiv), $( \pm)$ - $t$-Bu-Quinox (1.5 $\mathrm{mg}, 0.006 \mathrm{mmol}, 0.06$ equiv), Zn powder (19.6 mg, $0.3 \mathrm{mmol}, 3.0$ equiv), and a magnetic stir bar. To this mixture was added a solution of 1-(2,2-dichlorovinyl)-4-methoxybenzene (20.4 mg, 0.1 mmol, 1.0 equiv) and allylbenzene ( $35.4 \mathrm{mg}, 0.3 \mathrm{mmol}, 3.0$ equiv) in DMA $(0.75 \mathrm{~mL})$. The reaction was stirred at room temperature. After $16 \mathrm{~h}$, the crude reaction mixture was removed from the glovebox, opened to ambient atmosphere, and loaded directly onto a $\mathrm{SiO}_{2}$ column for purification (5\% $\mathrm{Et}_{2} \mathrm{O}$ in hexanes).

$16.0 \mathrm{mg}$ isolated (64\% yield), colorless oil, $E / Z=>20: 1$

${ }^{1} \mathrm{H}$ NMR $\left(300 \mathrm{MHz}, \mathrm{CDCl}_{3}\right) \delta 7.39(\mathrm{~d}, J=8.6 \mathrm{~Hz}, 2 \mathrm{H}), 7.35-7.28(\mathrm{~m}, 3 \mathrm{H}), 7.27-7.19(\mathrm{~m}, 2 \mathrm{H})$, $6.87(\mathrm{~d}, J=8.7 \mathrm{~Hz}, 2 \mathrm{H}), 6.68(\mathrm{~s}, 1 \mathrm{H}), 3.83(\mathrm{~s}, 3 \mathrm{H}), 3.25(\mathrm{dd}, J=14.6,4.5 \mathrm{~Hz}, 1 \mathrm{H}), 2.45$ (dd, $J=14.6$, $8.9 \mathrm{~Hz}, 1 \mathrm{H}), 2.19-1.93(\mathrm{~m}, 1 \mathrm{H}), 1.37(\mathrm{t}, J=8.7 \mathrm{~Hz}, 1 \mathrm{H}), 1.05-0.86(\mathrm{~m}, 1 \mathrm{H})$. 
${ }^{13} \mathrm{C}\left\{{ }^{1} \mathrm{H}\right\}$ NMR $\left(201 \mathrm{MHz}, \mathrm{CDCl}_{3}\right) \delta 158.5,140.5,130.7,128.6,128.2,127.8,126.4,126.1$, $118.8,113.9,55.2,37.7,17.6,7.8$.

HRMS(APCI) (m/z): [M - H] ${ }^{+}$Calcd for $\mathrm{C}_{18} \mathrm{H}_{18} \mathrm{O}: 249.1247$; found: 249.1127

TLC: $\mathrm{R}_{f}=0.47$ (5\% $\mathrm{Et}_{2} \mathrm{O}$ in hexanes)
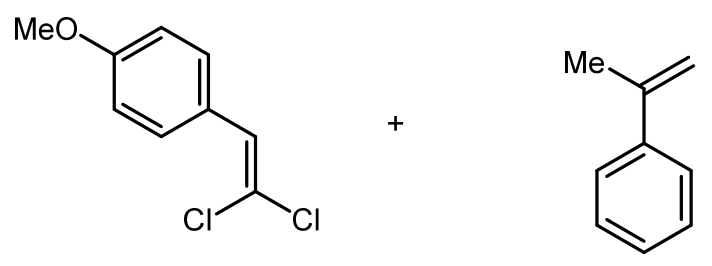

3.0 Equiv.

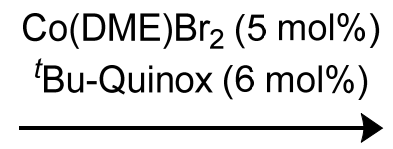

Zn (3.0 Equiv.)

DMA, $25^{\circ} \mathrm{C}$

$20 \mathrm{~h}$

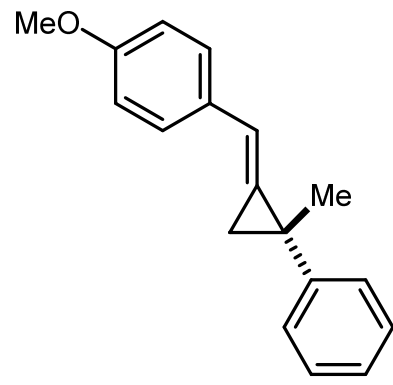

Not Detected

In an $\mathrm{N}_{2}$-filled glovebox, a 5-mL vial was charged with $\mathrm{Co}(\mathrm{DME}) \mathrm{Br}_{2}(1.5 \mathrm{mg}, 0.005 \mathrm{mmol}$, 0.05 equiv), ( \pm )- $t$-Bu-Quinox ( $1.5 \mathrm{mg}, 0.006 \mathrm{mmol}, 0.06$ equiv), $Z \mathrm{n}$ powder ( $19.6 \mathrm{mg}, 0.3 \mathrm{mmol}, 3.0$ equiv), and a magnetic stir bar. To this mixture was added a solution of 1-(2,2-dichlorovinyl)-4methoxybenzene ( $20.4 \mathrm{mg}, 0.1 \mathrm{mmol}, 1.0$ equiv), $\alpha$-methylstyrene ( $35.5 \mathrm{mg}, 0.3 \mathrm{mmol}, 3.0$ equiv), and 1,3,5-timethoxybenzene $(16.8 \mathrm{mg}, 0.1 \mathrm{mmol})$ in DMA $(0.75 \mathrm{~mL})$. The reaction was stirred at room temperature. After $16 \mathrm{~h}$, the crude reaction mixture was removed from the glovebox, opened to ambient atmosphere, and diluted with $\mathrm{Et}_{2} \mathrm{O}$. An aliquot was filtered through a glass fiber pad and analyzed by ${ }^{1} \mathrm{H}$ NMR. The conversion of 1-(2,2-dichlorovinyl)-4-methoxybenzene was determined by integration against 1,3,5-timethoxybenzene ( $>99 \%$ conversion). The product shown was not detected in the mixture.
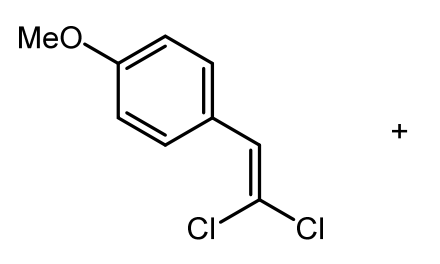

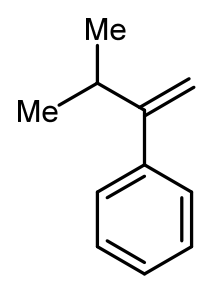

3.0 Equiv.

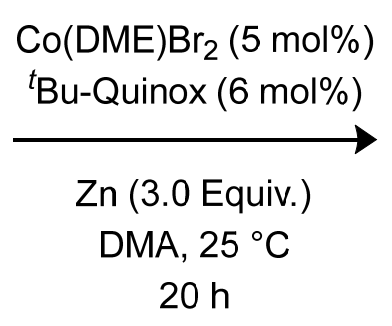

$20 \mathrm{~h}$

Not Detected

In an $\mathrm{N}_{2}$-filled glovebox, a 5-mL vial was charged with Co(DME)Br2 $(1.5 \mathrm{mg}, 0.005 \mathrm{mmol}$, 0.05 equiv), ( \pm )- $t$-Bu-Quinox ( $1.5 \mathrm{mg}, 0.006 \mathrm{mmol}, 0.06$ equiv), $\mathrm{Zn}$ powder ( $19.6 \mathrm{mg}, 0.3 \mathrm{mmol}, 3.0$ equiv), and a magnetic stir bar. To this mixture was added a solution of 1-(2,2-dichlorovinyl)-4methoxybenzene (20.4 mg, $0.1 \mathrm{mmol}, 1.0$ equiv), (3-methylbut-1-en-2-yl)benzene ( $43.9 \mathrm{mg}, 0.3$ mmol, 3.0 equiv), and 1,3,5-timethoxybenzene $(16.8 \mathrm{mg}, 0.1 \mathrm{mmol})$ in DMA (0.75 mL). The reaction was stirred at room temperature. After $16 \mathrm{~h}$, the crude reaction mixture was removed from the glovebox, opened to ambient atmosphere, and diluted with $\mathrm{Et}_{2} \mathrm{O}$. An aliquot was filtered through a glass fiber pad and analyzed by ${ }^{1} \mathrm{H}$ NMR. The conversion of 1-(2,2-dichlorovinyl)-4- 
methoxybenzene was determined by integration against 1,3,5-timethoxybenzene (>99\% conversion). The product shown was not detected in the mixture.
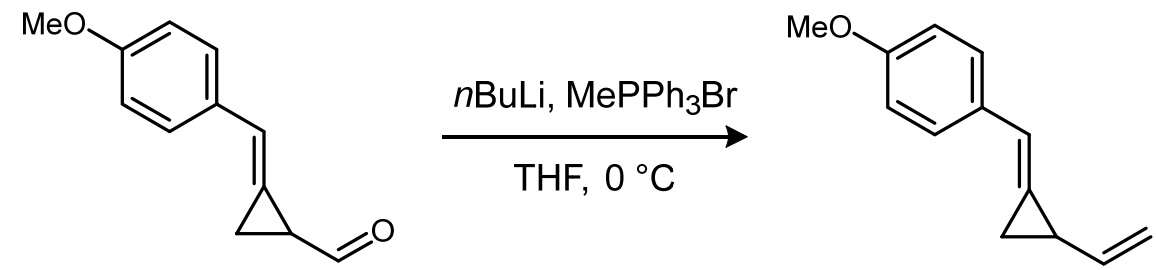

(E)-1-methoxy-4-((2-vinylcyclopropylidene)methyl)benzene (S21). A flame-dried roundbottom flask was charged with a stir bar, $\mathrm{MePPh}_{3} \mathrm{Br}$ (3.82 $\mathrm{g}, 10.6 \mathrm{mmol}, 1.5$ equiv), and THF (30 $\mathrm{mL}$ ). The mixture was cooled to $0{ }^{\circ} \mathrm{C}$ under $\mathrm{N}_{2}$ atmosphere, followed by dropwise addition of $n \mathrm{BuLi}$ (2.5 $\mathrm{M}$ in hexanes, $4.27 \mathrm{~mL}, 10.6 \mathrm{mmol}, 1.5$ equiv). The mixture was stirred at $0{ }^{\circ} \mathrm{C}$ for $30 \mathrm{~min}$. A solution of $(E)$-2-(4-methoxybenzylidene)cyclopropane-1-carbaldehyde ${ }^{15}(1.33 \mathrm{~g}, 7.1 \mathrm{mmol}, 1.0$ equiv) in THF ( $5 \mathrm{~mL}$ ) was added dropwise, and the reaction was then warmed to room temperature and stirred. After $2 \mathrm{hr}$, the reaction was quenched with a saturated aqueous solution of $\mathrm{NH}_{4} \mathrm{Cl}$ and extracted with $\mathrm{Et}_{2} \mathrm{O}(3 \times 20 \mathrm{~mL})$. The combined organic layers were dried over $\mathrm{MgSO}_{4}$, filtered, and concentrated under reduced pressure. The crude product was loaded directly onto a $\mathrm{SiO}_{2}$ column for purification (5\% $\mathrm{Et}_{2} \mathrm{O}$ in hexanes), providing (E)-1-methoxy-4-((2vinylcyclopropylidene)methyl)benzene as a white solid ( $846 \mathrm{mg}, 64 \%$ yield).

${ }^{1} \mathrm{H} \mathrm{NMR}\left(400 \mathrm{MHz}, \mathrm{CDCl}_{3}\right) \delta 7.47(\mathrm{~d}, J=8.9 \mathrm{~Hz}, 2 \mathrm{H}), 6.88(\mathrm{~d}, J=8.8 \mathrm{~Hz}, 2 \mathrm{H}), 6.76(\mathrm{~s}, 1 \mathrm{H}), 5.59$ $-5.39(\mathrm{~m}, 1 \mathrm{H}), 5.18(\mathrm{~d}, J=17.0 \mathrm{~Hz}, 1 \mathrm{H}), 4.96(\mathrm{~d}, J=11.8 \mathrm{~Hz}, 1 \mathrm{H}), 3.82(\mathrm{~s}, 3 \mathrm{H}), 2.27-2.08(\mathrm{~m}, 1 \mathrm{H})$, $1.84(\mathrm{t}, J=8.8 \mathrm{~Hz}, 1 \mathrm{H}), 1.40-1.28(\mathrm{~m}, 1 \mathrm{H})$.

${ }^{13} \mathrm{C}\left\{{ }^{1} \mathrm{H}\right\} \operatorname{NMR}\left(101 \mathrm{MHz}, \mathrm{CDCl}_{3}\right) \delta 158.7,139.4,130.5,127.8,125.4,118.5,113.8,113.1,55.2$, $17.4,12.8$.

HRMS(APCI) (m/z): [M + H] ${ }^{+}$Calcd for $\mathrm{C}_{13} \mathrm{H}_{14} \mathrm{O}: 187.1117$; found: 187.1122

TLC: $\mathrm{R}_{f}=0.49$ (5\% $\mathrm{Et}_{2} \mathrm{O}$ in hexanes)
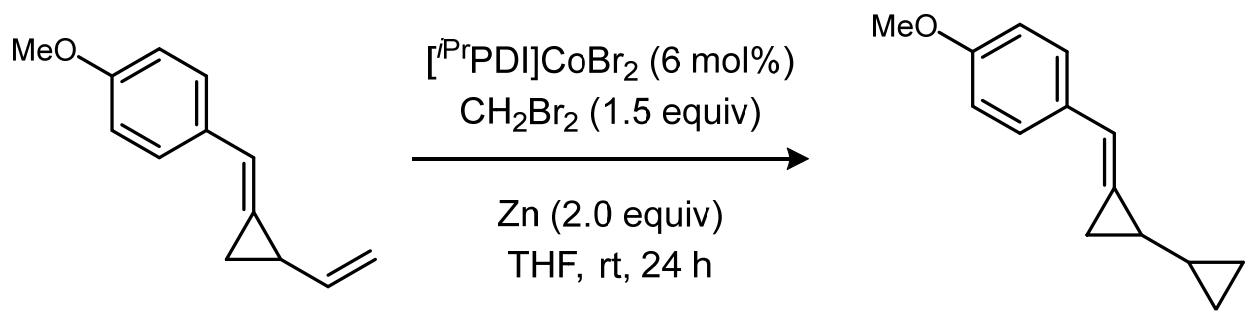

(E)-2-(4-methoxybenzylidene)-1,1'-bi(cyclopropane) (4). In an $\mathrm{N}_{2}$-filled glovebox, a $20 \mathrm{~mL}$ scintillation vial was charged with [iPrPDI] $\mathrm{CoBr}_{2}$ ( $44.8 \mathrm{mg}, 0.06 \mathrm{mmol}, 0.06$ equiv), Zn powder (140 $\mathrm{mg}, 2.14 \mathrm{mmol}, 6.0$ equiv), and a magnetic stir bar. To this mixture was added a solution of $\mathbf{S 2 1}$ (200 mg, 1.07 mmol, 1.0 equiv) and $\mathrm{CH}_{2} \mathrm{Br}_{2}$ (280 mg, $1.61 \mathrm{mmol}$, 1.5 equiv) in THF ( $8 \mathrm{~mL}$ ). The reaction was stirred at room temperature. After $16 \mathrm{~h}$, the crude reaction mixture was removed from the glovebox, opened to ambient atmosphere, concentrated under reduced pressure, and 
loaded directly onto a $\mathrm{SiO}_{2}$ column for purification (5\% $\mathrm{Et}_{2} \mathrm{O}$ in hexanes), providing $(E)$-2-(4methoxybenzylidene)-1,1'-bi(cyclopropane) as a yellow oil (190.5 mg, $89 \%$ yield). ${ }^{16}$

${ }^{1} \mathrm{H} \mathrm{NMR}\left(300 \mathrm{MHz}, \mathrm{CDCl}_{3}\right) \delta 7.46(\mathrm{~d}, J=8.8 \mathrm{~Hz}, 2 \mathrm{H}), 6.87(\mathrm{~d}, J=8.8 \mathrm{~Hz}, 2 \mathrm{H}), 6.62(\mathrm{q}, J=2.2$ $\mathrm{Hz}, 1 \mathrm{H}), 3.82$ (s, 3H), $1.75-1.66(\mathrm{~m}, 1 \mathrm{H}), 1.48(\mathrm{td}, J=8.7,2.3 \mathrm{~Hz}, 1 \mathrm{H}), 1.15-0.99(\mathrm{~m}, 2 \mathrm{H}), 0.52-$ $0.38(\mathrm{~m}, 1 \mathrm{H}), 0.31-0.04(\mathrm{~m}, 3 \mathrm{H})$.

${ }^{13} \mathrm{C}\left\{{ }^{1} \mathrm{H}\right\} \mathrm{NMR}\left(201 \mathrm{MHz}, \mathrm{CDCl}_{3}\right) \delta 158.5,131.0,127.6,124.8,117.5,113.8,55.2,14.7,11.6$, 9.6, 3.9, 0.9.

HRMS(APCI) (m/z): [M + H] Calcd for $\mathrm{C}_{14} \mathrm{H}_{16} \mathrm{O}: 201.1274$; found: 201.1277

TLC: $\mathrm{R}_{f}=0.57\left(5 \% \mathrm{Et}_{2} \mathrm{O}\right.$ in hexanes)
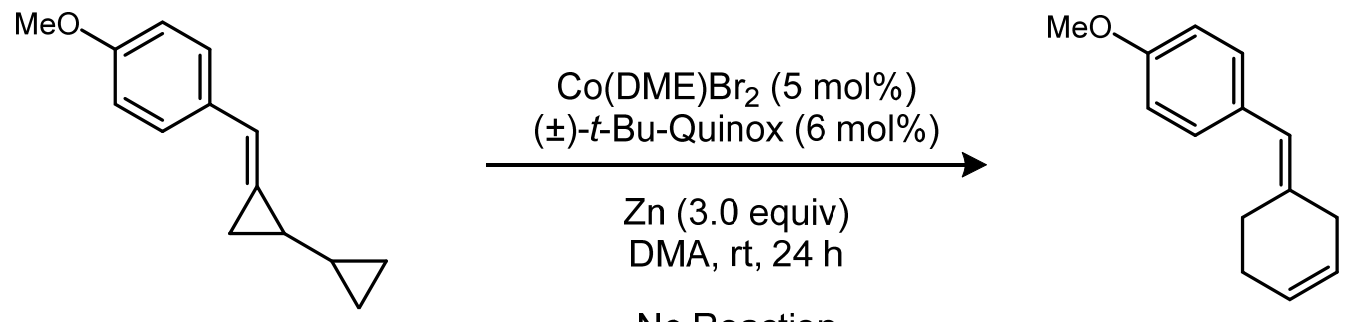

No Reaction

In an $\mathrm{N}_{2}$-filled glovebox, a 5-mL vial was charged with $\mathrm{Co}(\mathrm{DME}) \mathrm{Br}_{2}(1.5 \mathrm{mg}, 0.005 \mathrm{mmol}$, 0.05 equiv), ( \pm )- $t$-Bu-Quinox ( $1.5 \mathrm{mg}, 0.006 \mathrm{mmol}, 0.06$ equiv), $\mathrm{Zn}$ powder ( $19.6 \mathrm{mg}, 0.3 \mathrm{mmol}, 3.0$ equiv), and a magnetic stir bar. To this mixture was added a solution of $4(20.0 \mathrm{mg}, 0.1 \mathrm{mmol}, 1.0$ equiv) and 1,3,5-timethoxybenzene (16.8 $\mathrm{mg}, 0.1 \mathrm{mmol})$ in DMA $(0.75 \mathrm{~mL})$. The reaction was stirred at room temperature. After $16 \mathrm{~h}$, the crude reaction mixture was removed from the glovebox, opened to ambient atmosphere, and diluted with $\mathrm{Et}_{2} \mathrm{O}$. An aliquot was filtered through a glass fiber pad and analyzed by ${ }^{1} \mathrm{H}$ NMR. The conversion of $\mathbf{4}$ was determined by integration against 1,3,5-timethoxybenzene ( $<1 \%$ conversion). Compound 3 was not detected in the mixture.
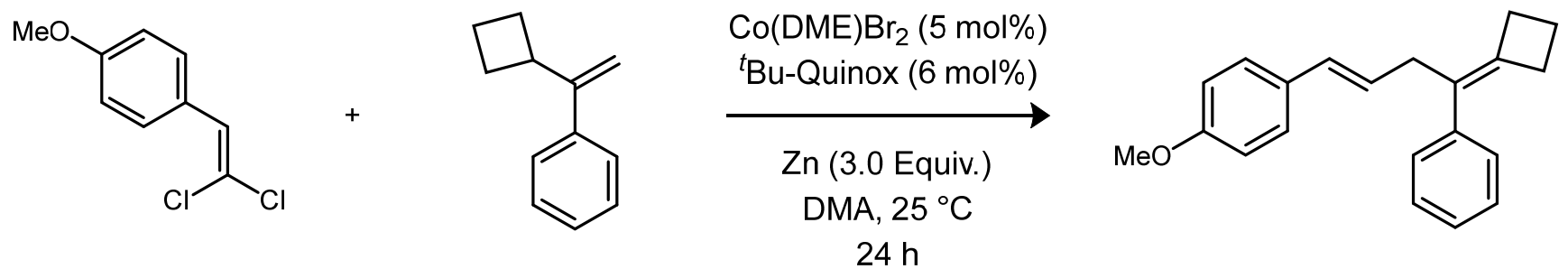

3.0 Equiv.

(E)-1-(4-cyclobutylidene-4-phenylbut-1-en-1-yl)-4-methoxybenzene (49). In an $\mathrm{N}_{2}$-filled glovebox, a 5-mL vial was charged with $\mathrm{Co}(\mathrm{DME}) \mathrm{Br}_{2}(1.5 \mathrm{mg}, 0.005 \mathrm{mmol}, 0.05$ equiv), ( \pm )- $t$ - $\mathrm{Bu}$ Quinox (1.5 mg, $0.006 \mathrm{mmol}, 0.06$ equiv), $\mathrm{Zn}$ powder (19.6 mg, $0.3 \mathrm{mmol}, 3.0$ equiv), and a magnetic stir bar. To this mixture was added a solution of 1-(2,2-dichlorovinyl)-4methoxybenzene (20.4 mg, $0.1 \mathrm{mmol}, 1.0$ equiv) and (1-cyclobutylvinyl)benzene ${ }^{17}$ (47.5 mg, 0.3 mmol, 3.0 equiv) in DMA $(0.75 \mathrm{~mL})$. The reaction was stirred at room temperature. After $16 \mathrm{~h}$, the crude reaction mixture was removed from the glovebox, opened to ambient atmosphere, and loaded directly onto a $\mathrm{SiO}_{2}$ column for purification (5\% $\mathrm{Et}_{2} \mathrm{O}$ in hexanes). Single crystals of $\mathbf{4 9}$ 
suitable for X-ray diffraction analysis were obtained by cooling the purified, neat material to -20 ${ }^{\circ} \mathrm{C}$.

$14.5 \mathrm{mg}$ isolated ( $50 \%$ yield), colorless oil, $E / Z=>20: 1$

${ }^{1} \mathrm{H}$ NMR $\left(300 \mathrm{MHz}, \mathrm{CDCl}_{3}\right) \delta 7.37-7.34(\mathrm{~m}, 1 \mathrm{H}), 7.33-7.28(\mathrm{~m}, 2 \mathrm{H}), 7.25(\mathrm{~d}, J=8.7 \mathrm{~Hz}, 2 \mathrm{H})$, $7.22-7.12(\mathrm{~m}, 2 \mathrm{H}), 6.82(\mathrm{~d}, J=8.6 \mathrm{~Hz}, 2 \mathrm{H}), 6.38(\mathrm{~d}, J=17.6 \mathrm{~Hz}, 1 \mathrm{H}), 6.17-6.02(\mathrm{~m}, 1 \mathrm{H}), 3.80(\mathrm{~s}$, $3 \mathrm{H}), 3.24(\mathrm{~d}, J=5.6 \mathrm{~Hz}, 2 \mathrm{H}), 3.00-2.85(\mathrm{~m}, 4 \mathrm{H}), 2.12-1.96(\mathrm{~m}, 2 \mathrm{H})$.

${ }^{13} \mathrm{C}\left\{{ }^{1} \mathrm{H}\right\}$ NMR $\left(201 \mathrm{MHz}, \mathrm{CDCl}_{3}\right) \delta 158.6,140.0,139.9,130.6,129.4,128.1,127.9,127.0$, $126.3,125.8,113.7,55.2,34.5,32.2,30.8,17.0$.

HRMS(APCI) (m/z): [M + H] Calcd for $\mathrm{C}_{21} \mathrm{H}_{22} \mathrm{O}: 291.1743$; found: 291.1749

TLC: $\mathrm{R}_{f}=0.54\left(5 \% \mathrm{Et}_{2} \mathrm{O}\right.$ in hexanes)

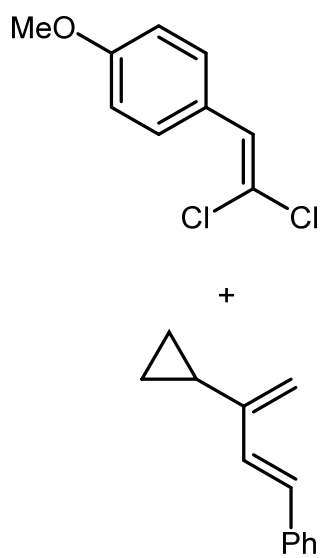

3.0 Equiv.
$\mathrm{Co}(\mathrm{DME}) \mathrm{Br}_{2}(5 \mathrm{~mol} \%)$

${ }^{t}$ Bu-Quinox (6 mol\%)

Zn (3.0 Equiv.)

DMA, $25^{\circ} \mathrm{C}$

$24 \mathrm{~h}$

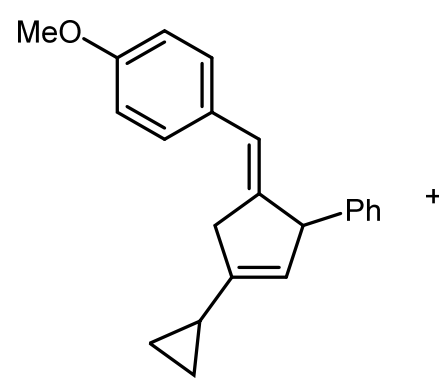

$52 \%$ Yield

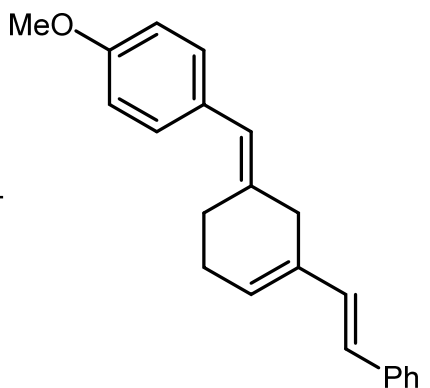

$6 \%$ Yield

[4+1] cycloaddition competition experiment. In an $\mathrm{N}_{2}$-filled glovebox, a 5-mL vial was charged with Co(DME)Br2 (1.5 mg, $0.005 \mathrm{mmol}, 0.05$ equiv), ( \pm )- $t$-Bu-Quinox (1.5 mg, $0.006 \mathrm{mmol}, 0.06$ equiv), $\mathrm{Zn}$ powder (19.6 mg, $0.3 \mathrm{mmol}, 3.0$ equiv), and a magnetic stir bar. To this mixture was added a solution of 1-(2,2-dichlorovinyl)-4-methoxybenzene (20.4 mg, $0.1 \mathrm{mmol}, 1.0$ equiv) and (E)-(3-cyclopropylbuta-1,3-dien-1-yl)benzene $(51.1 \mathrm{mg}, 0.3 \mathrm{mmol}, 3.0$ equiv) in DMA (0.75 mL). The reaction was stirred at room temperature. After $16 \mathrm{~h}$, the crude reaction mixture was removed from the glovebox, opened to ambient atmosphere, and loaded directly onto a $\mathrm{SiO}_{2}$ column for purification (5\% $\mathrm{Et}_{2} \mathrm{O}$ in hexanes). The [4+1] and [5+1] products were inseparable.

$17.5 \mathrm{mg}$ isolated (58\% yield), white solid

${ }^{1} \mathrm{H}$ NMR $\left(300 \mathrm{MHz}, \mathrm{CDCl}_{3}\right) \delta 7.23(\mathrm{~d}, \mathrm{~J}=7.2 \mathrm{~Hz}, 2 \mathrm{H}), 7.16(\mathrm{t}, \mathrm{J}=7.1,3 \mathrm{H}), 7.04(\mathrm{~d}, \mathrm{~J}=8.7 \mathrm{~Hz}$, $2 \mathrm{H}), 6.69(\mathrm{~d}, \mathrm{~J}=8.8 \mathrm{~Hz}, 2 \mathrm{H}), 5.53(\mathrm{~s}, 1 \mathrm{H}), 4.64(\mathrm{~s}, 1 \mathrm{H}), 3.73(\mathrm{~s}, 3 \mathrm{H}), 3.39(\mathrm{~d}, \mathrm{~J}=20.3 \mathrm{~Hz}, 1 \mathrm{H}), 3.08(\mathrm{~d}$, $\mathrm{J}=20.3 \mathrm{~Hz}, 1 \mathrm{H}), 1.54-1.46(\mathrm{~m}, 1 \mathrm{H}), 0.70-0.62(\mathrm{~m}, 2 \mathrm{H}), 0.57-0.48(\mathrm{~m}, 2 \mathrm{H})$.

${ }^{13} \mathrm{C}\left\{{ }^{1} \mathrm{H}\right\} \mathrm{NMR}\left(201 \mathrm{MHz}, \mathrm{CDCl}_{3}\right) \delta 157.83,144.29,142.85,142.22,130.03,129.48,128.52$, 127.29, 127.11, 125.83, 124.11, 113.33, 77.09, 76.93, 76.78, 55.08, 53.81, 41.98, 11.87, 5.52, 5.29. LRMS(ESI) (m/z): [M - H] Calcd for $\mathrm{C}_{22} \mathrm{H}_{22} \mathrm{O}: 302.4$; found: 301.2 TLC: $\mathrm{R}_{f}=0.53\left(5 \% \mathrm{Et}_{2} \mathrm{O}\right.$ in hexanes) 

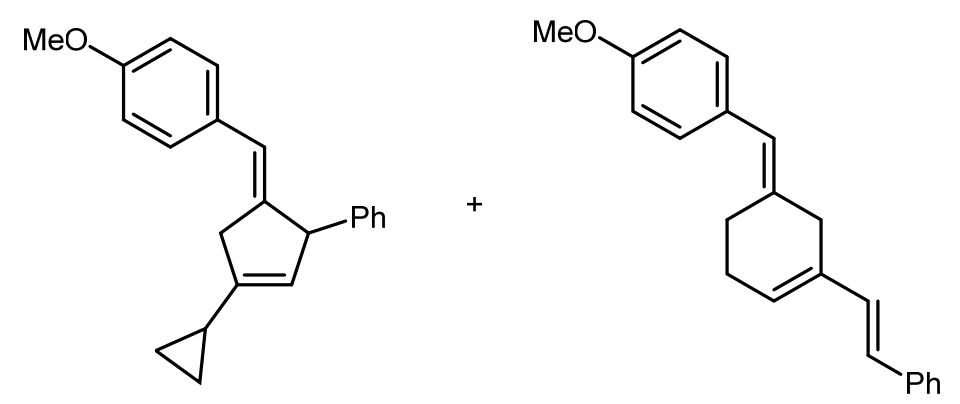

$9.7: 1$

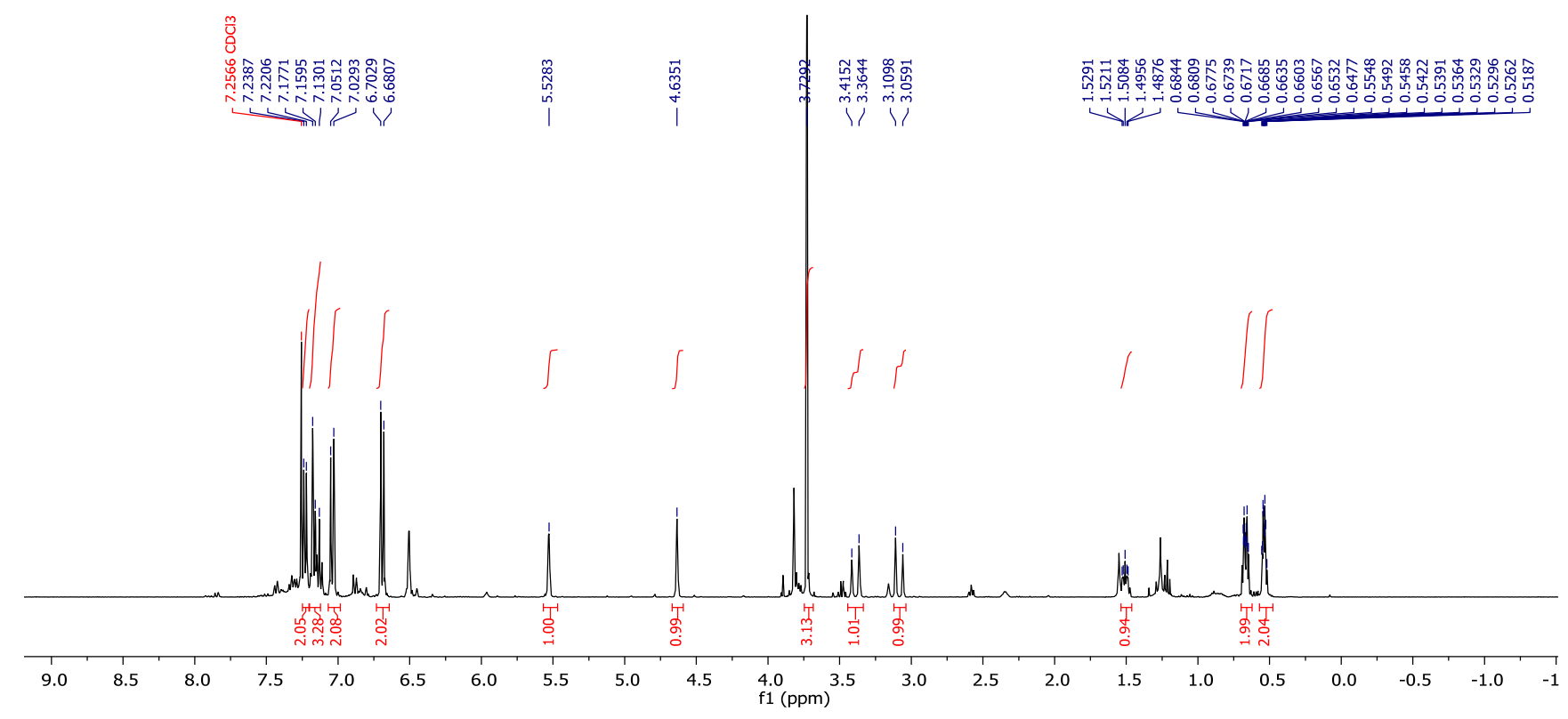

Figure S5. ${ }^{1} \mathrm{H}$ NMR of competition experiment (400 MHz, $\left.\mathrm{CDCl}_{3}, 295 \mathrm{~K}\right)$.

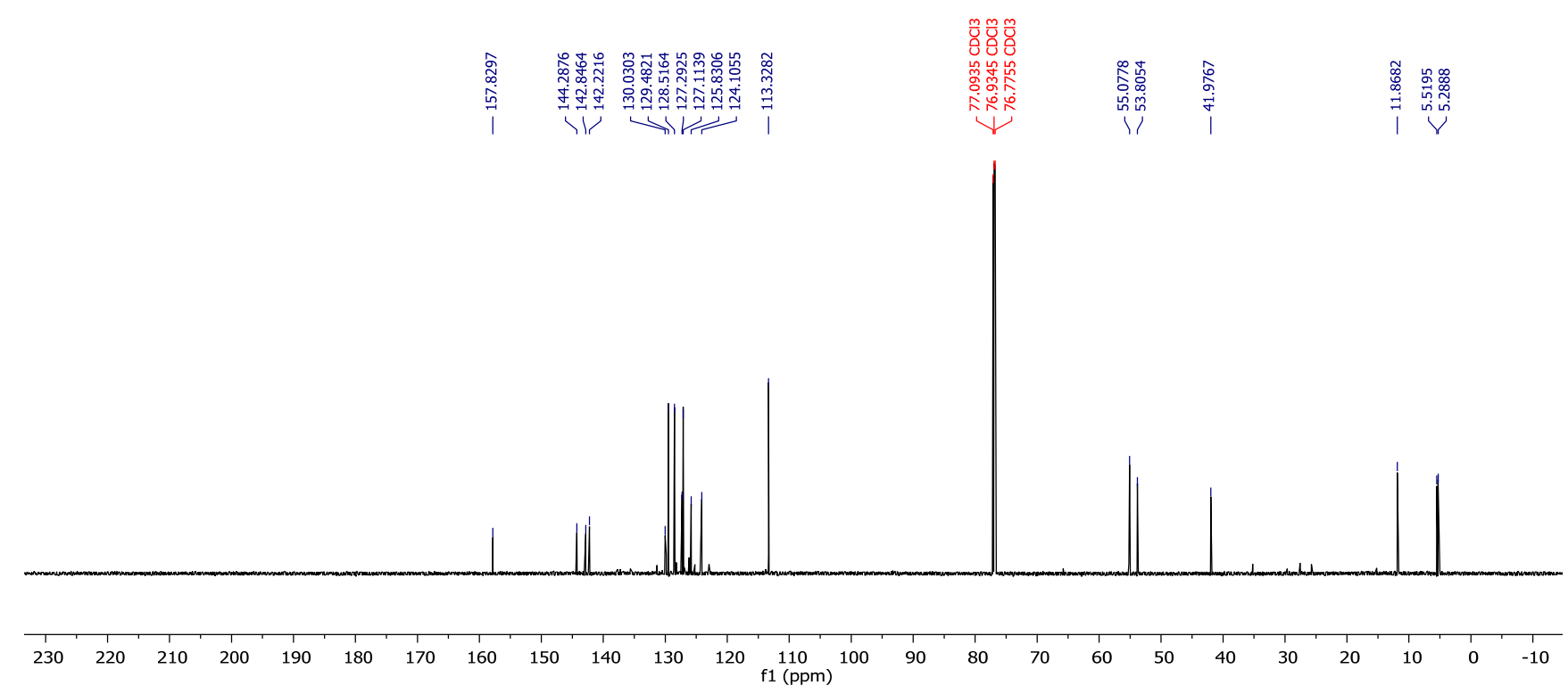

Figure S6. ${ }^{13} \mathrm{C}$ NMR of competition experiment $\left(201 \mathrm{MHz}, \mathrm{CDCl}_{3}, 295 \mathrm{~K}\right)$. 


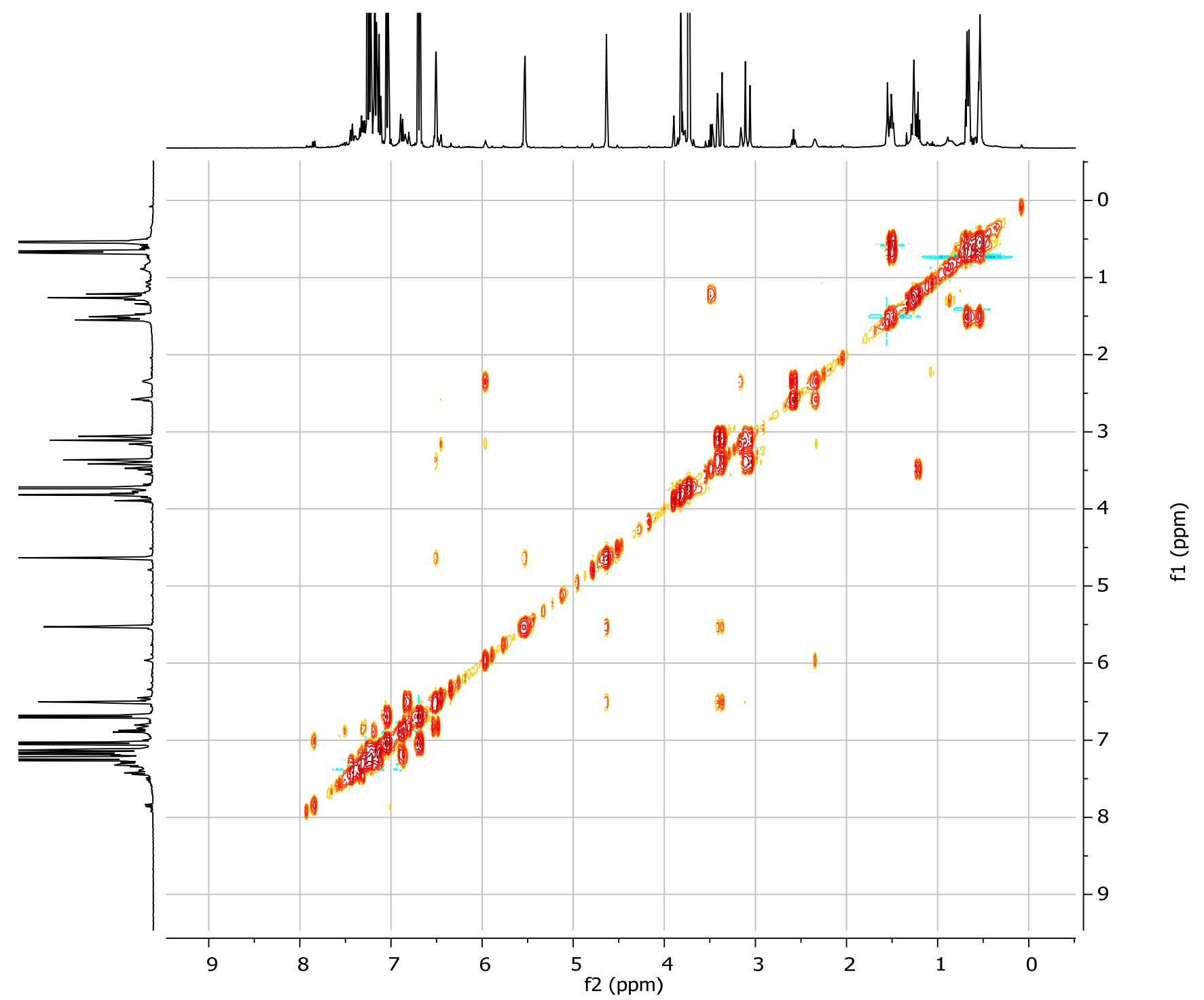

Figure S7. COSY of competition experiment $\left(400 \mathrm{MHz}, \mathrm{CDCl}_{3}, 295 \mathrm{~K}\right)$. 


\section{NMR Data for Vinylcyclopropanes and Vinylidenes}<smiles>C=C(c1ccccc1)C1CC1CCc1ccccc1</smiles>
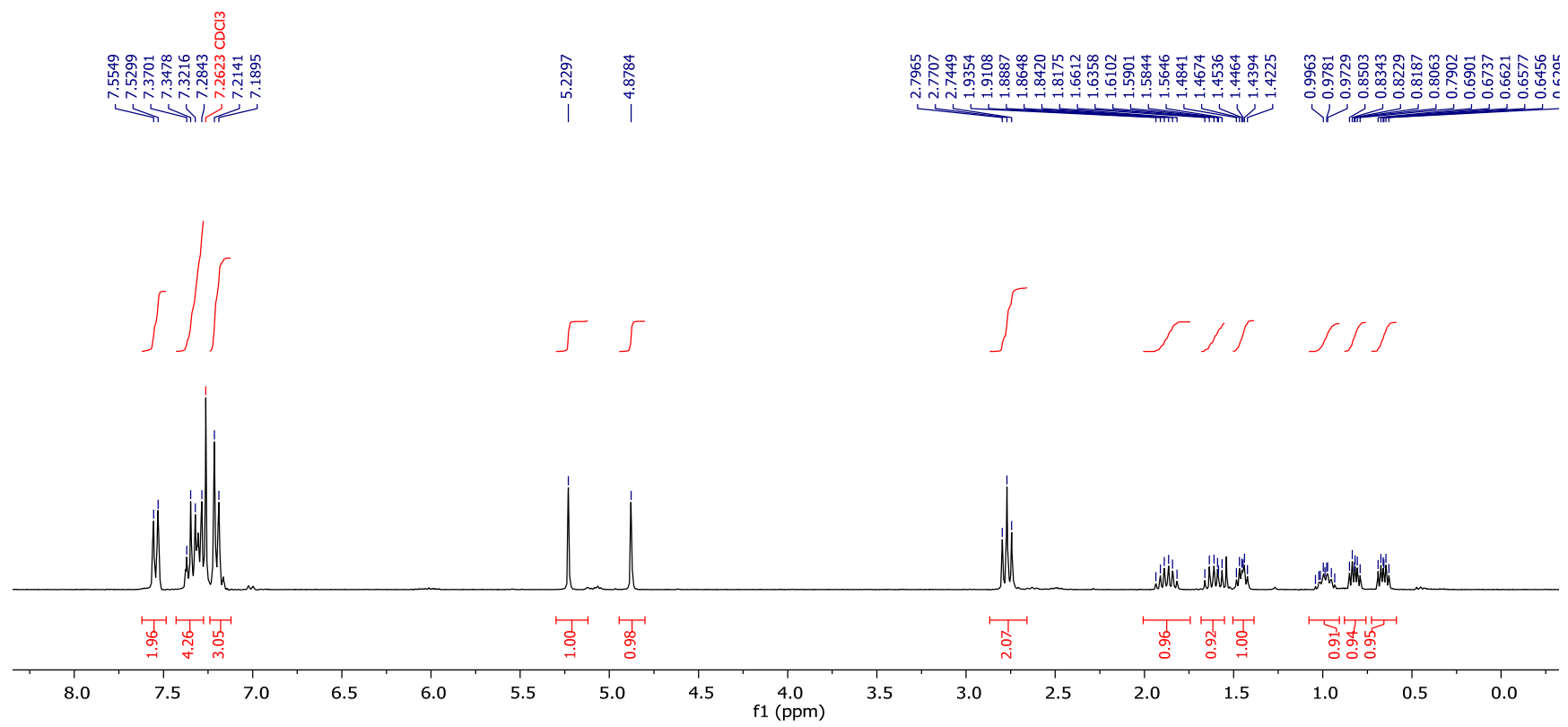

Figure S8. ${ }^{1} \mathrm{H}$ NMR of $\mathbf{S 1}\left(300 \mathrm{MHz}, \mathrm{CDCl}_{3}, 295 \mathrm{~K}\right)$.
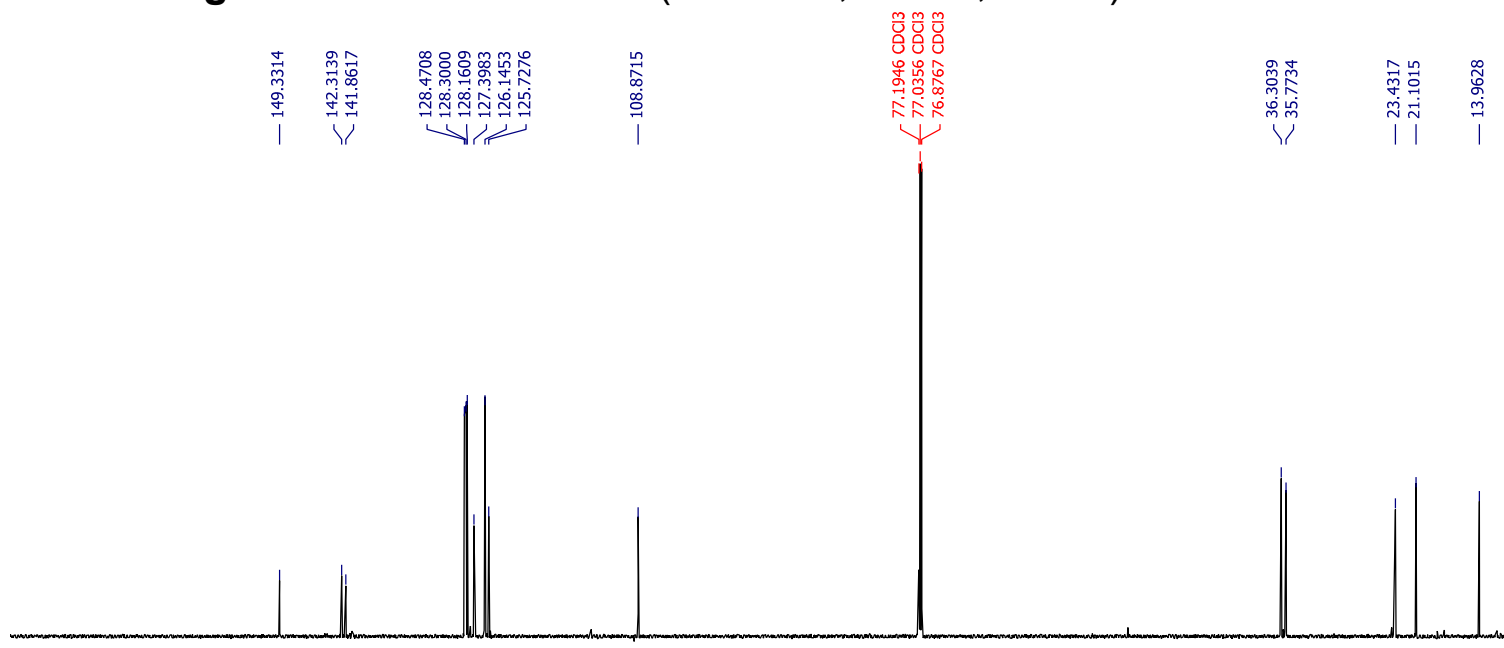

Figure S9. ${ }^{13} \mathrm{C}$ NMR of $\mathbf{S} 1\left(201 \mathrm{MHz}, \mathrm{CDCl} \mathbf{f l}_{3}, 295 \mathrm{~K}\right)$. 
<smiles>CC(C)(C)OC(=O)N1CCC(C/C=C/C(=O)c2ccccc2)CC1</smiles>

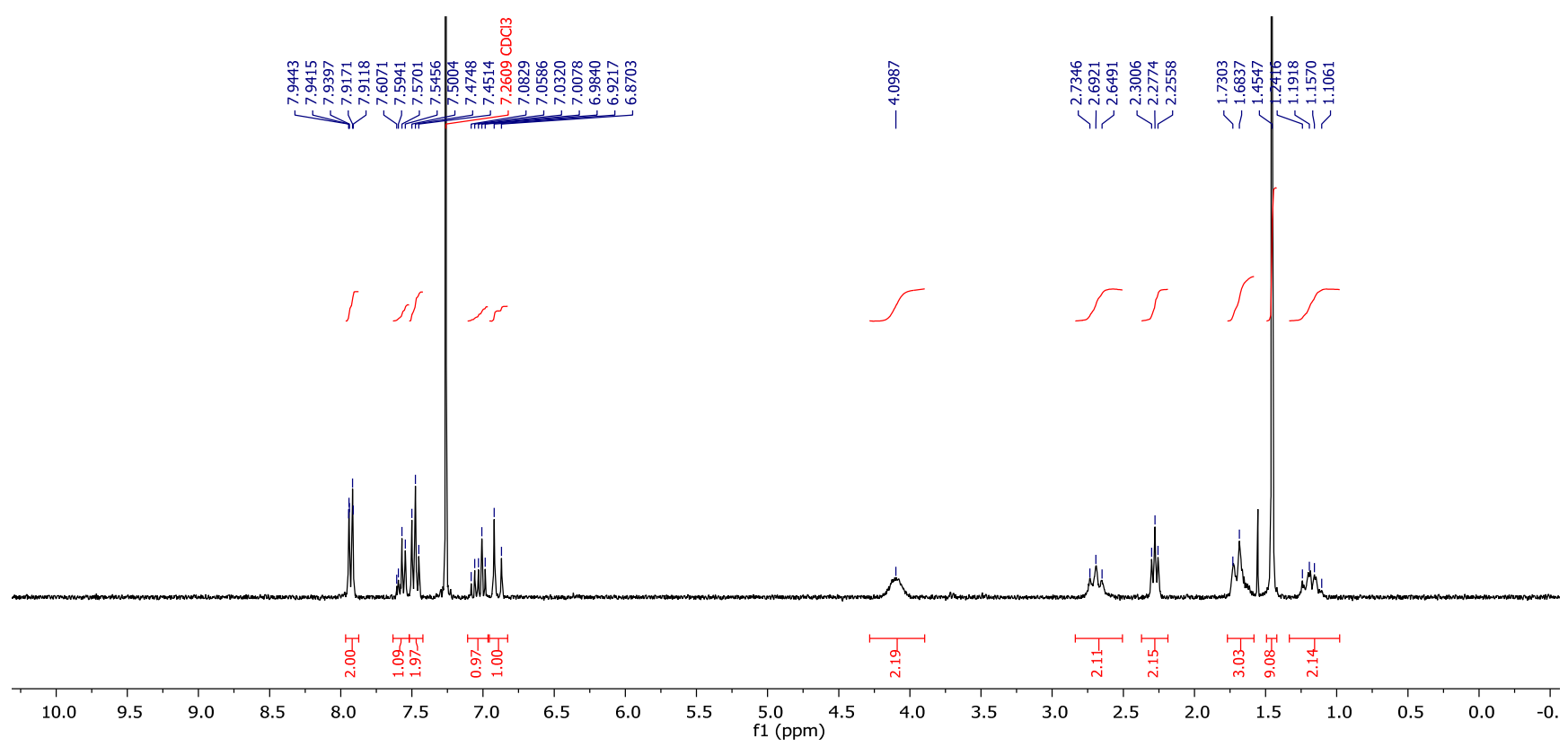

Figure S10. $\quad{ }^{1} \mathrm{H}$ NMR of $\mathbf{S 2}\left(300 \mathrm{MHz}, \mathrm{CDCl}_{3}, 295 \mathrm{~K}\right)$.
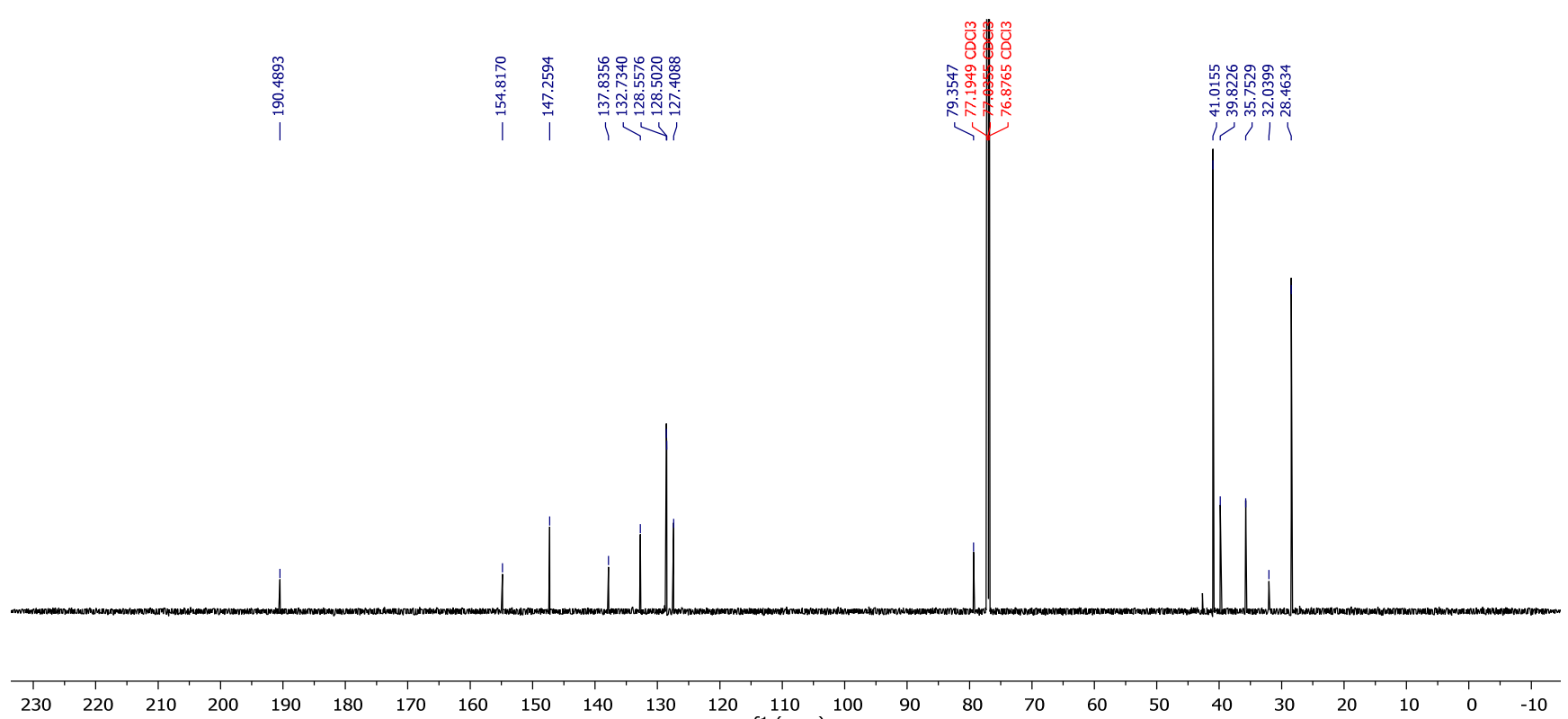

Figure S11. $\quad{ }^{13} \mathrm{C}$ NMR of $\mathbf{S} 2\left(201 \mathrm{MHz}, \mathrm{CDCl}_{3}, 295 \mathrm{~K}\right)$. 

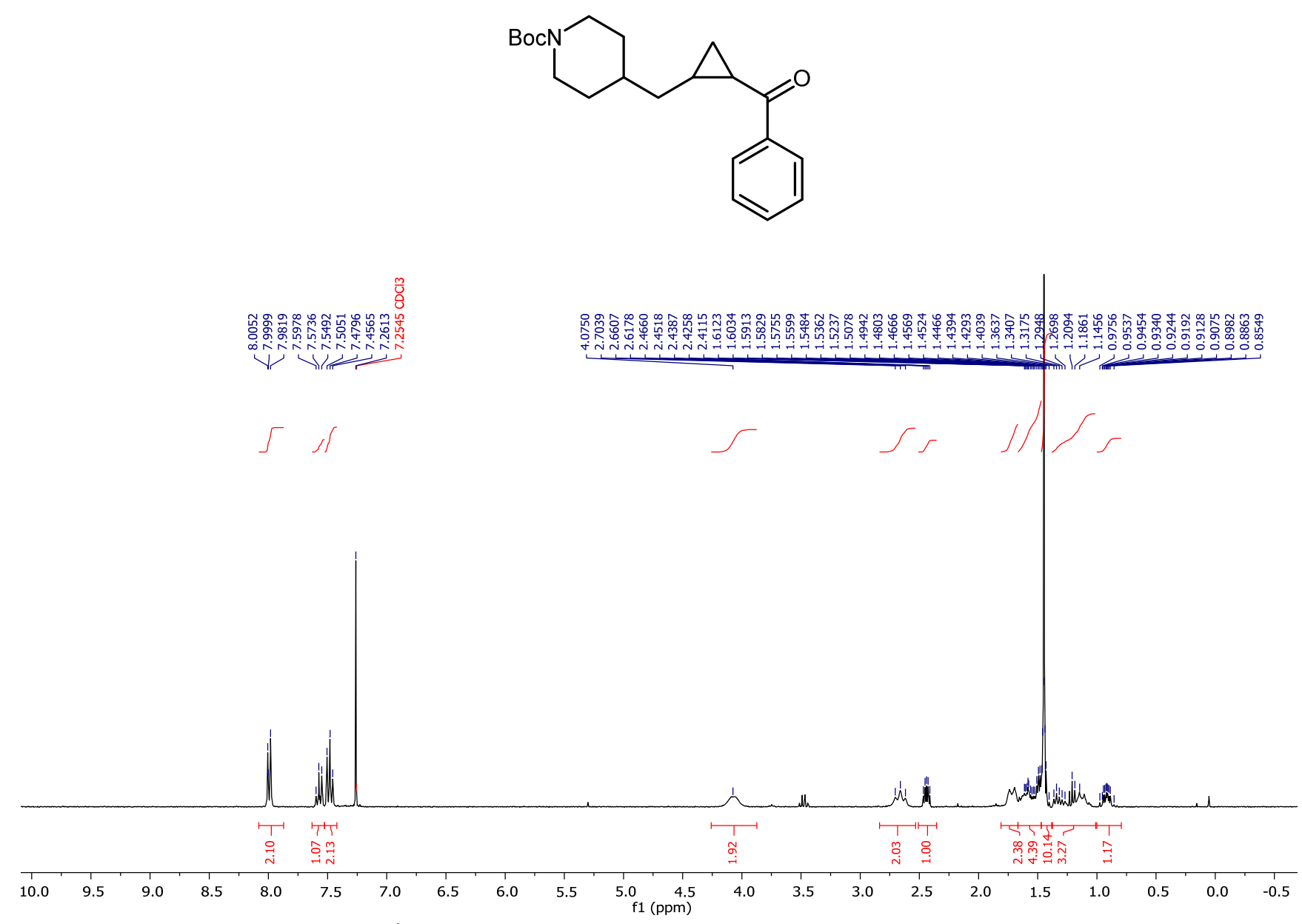

Figure S12. $\quad{ }^{1} \mathrm{H}$ NMR of $\mathbf{S 3}\left(300 \mathrm{MHz}, \mathrm{CDCl}_{3}, 295 \mathrm{~K}\right)$.
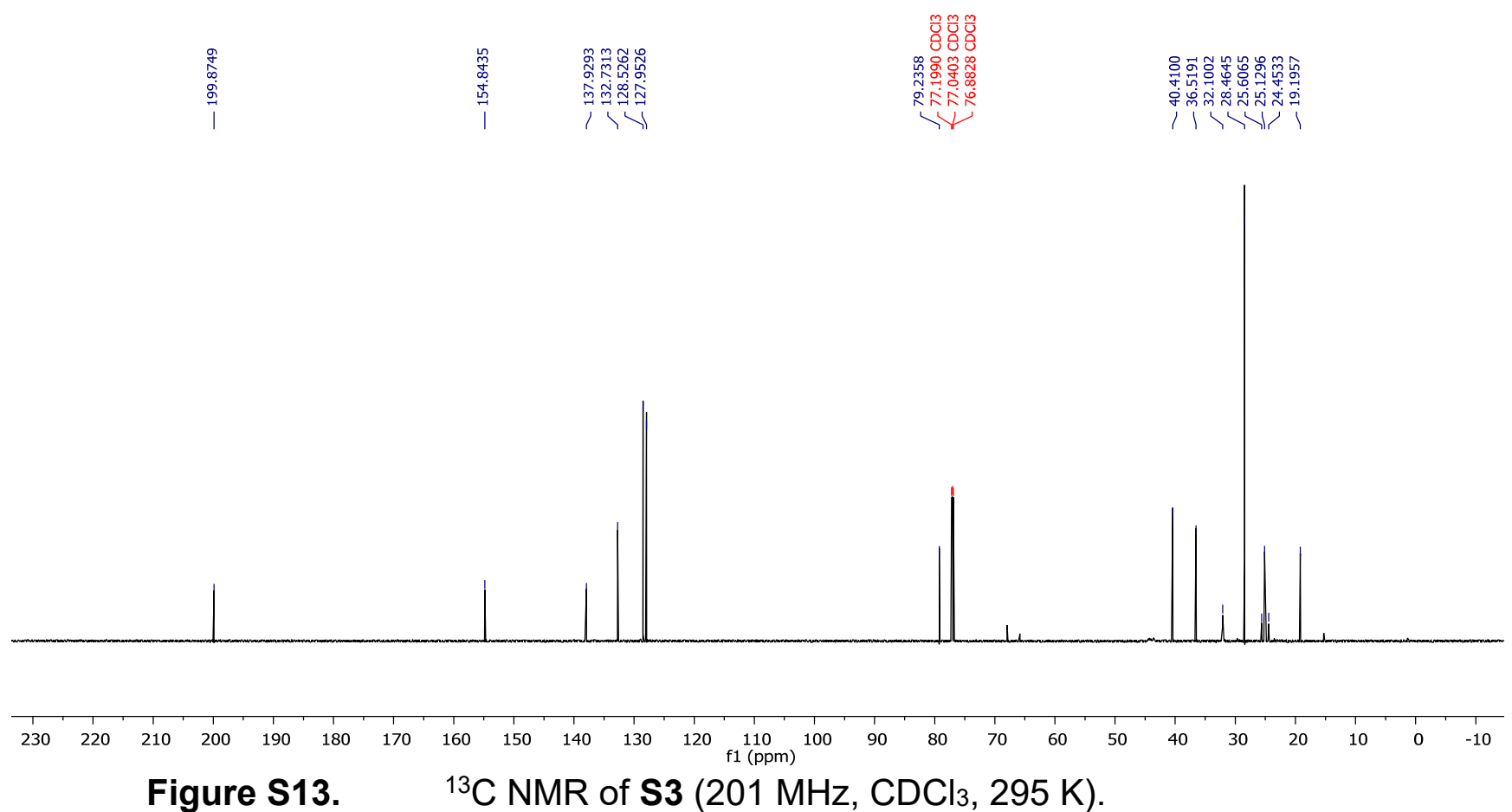

Figure S13. $\quad{ }^{13} \mathrm{C}$ NMR of $\mathbf{S 3}\left(201 \mathrm{MHz}, \mathrm{CDCl}_{3}, 295 \mathrm{~K}\right)$. 
<smiles>C=C(c1ccccc1)C1CC1CC1CCN(C(=O)OC(C)(C)C)CC1</smiles>

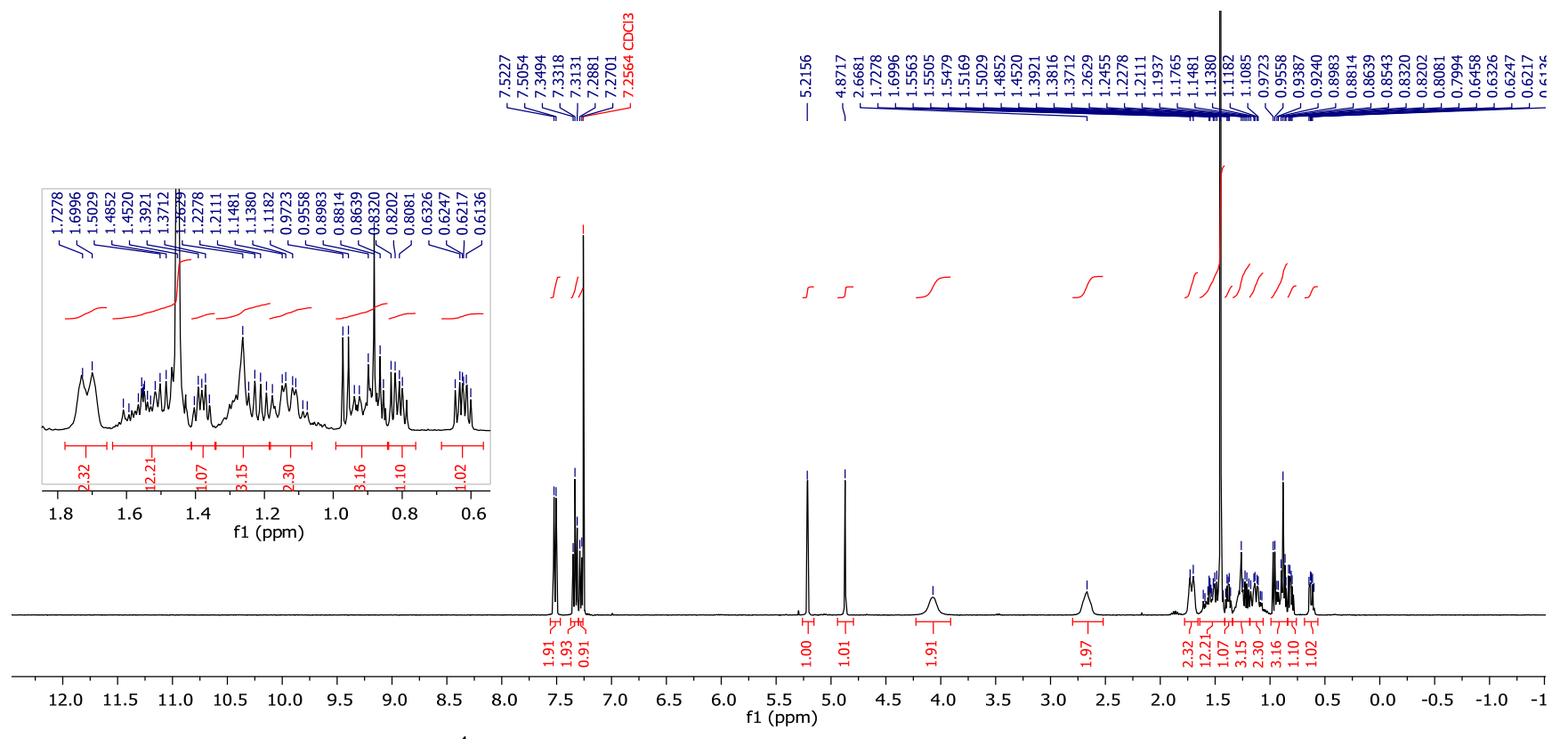

Figure S14. $\quad{ }^{1} \mathrm{H}$ NMR of $\mathbf{S 4}\left(300 \mathrm{MHz}, \mathrm{CDCl}_{3}, 295 \mathrm{~K}\right)$.
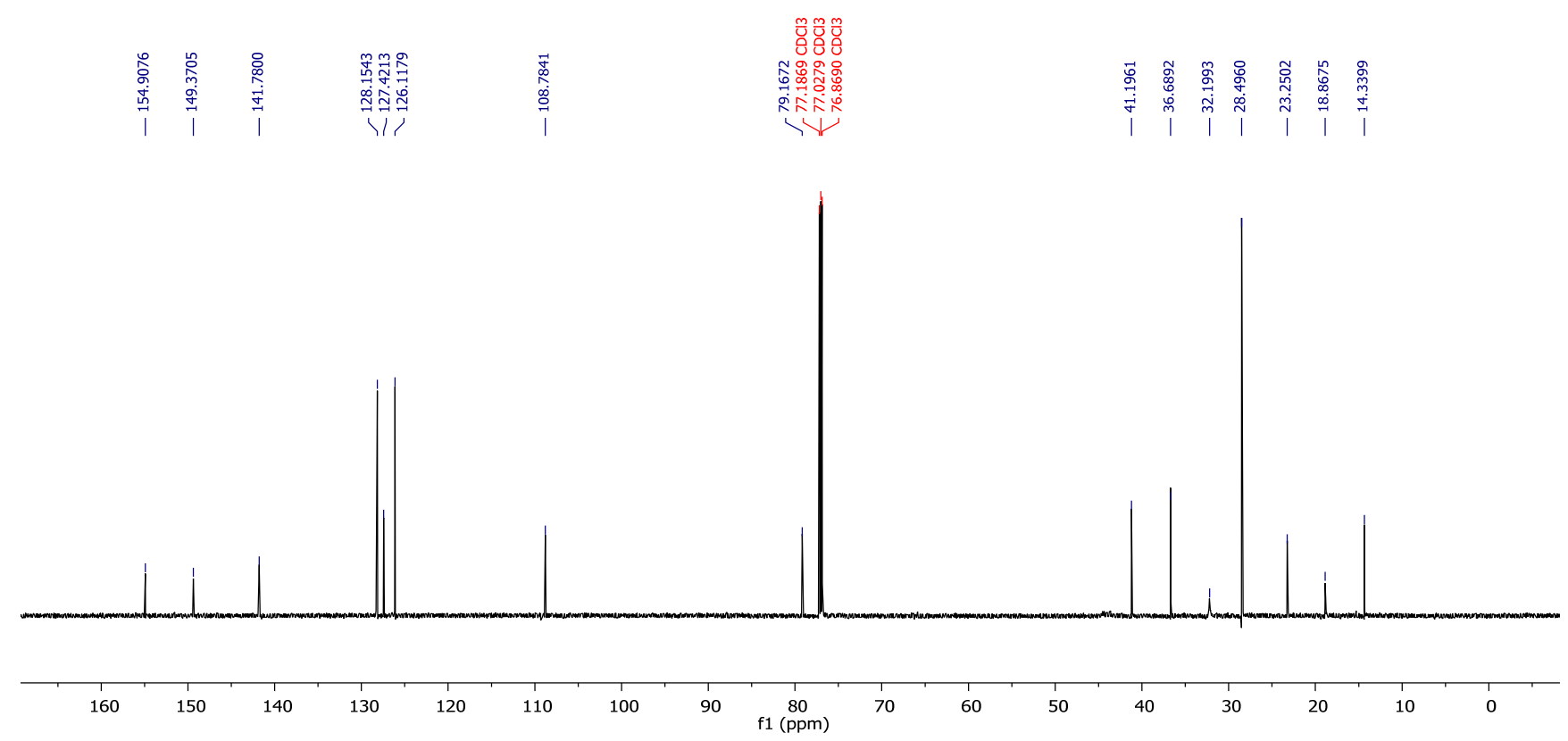

Figure S15. $\quad{ }^{13} \mathrm{C}$ NMR of $\mathbf{S 4}\left(201 \mathrm{MHz}, \mathrm{CDCl}_{3}, 295 \mathrm{~K}\right)$. 
<smiles>Cc1ccc(CC/C=C/C(=O)c2ccccc2)o1</smiles>
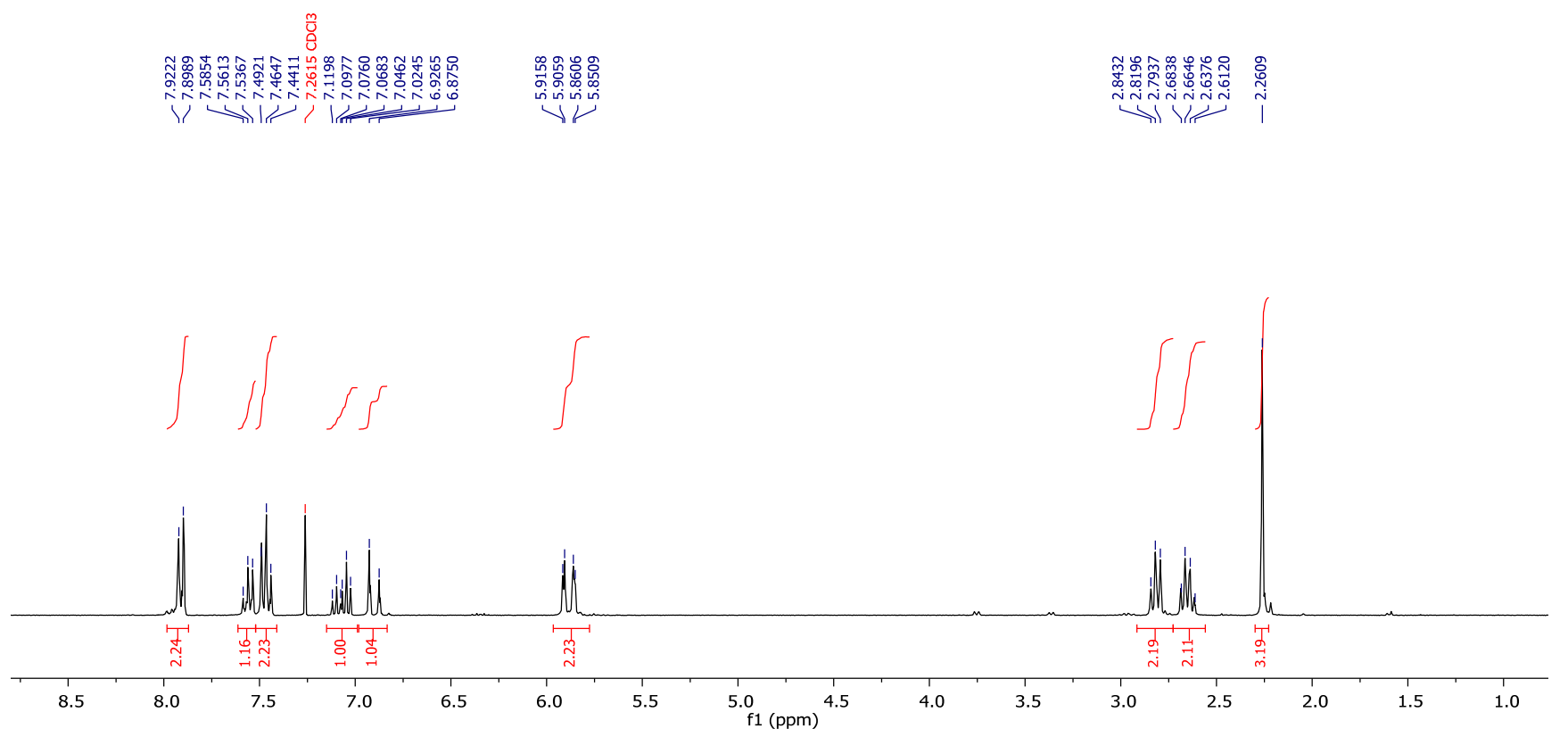

Figure S16. $\quad{ }^{1} \mathrm{H}$ NMR of $\mathbf{S 5}\left(300 \mathrm{MHz}, \mathrm{CDCl}_{3}, 295 \mathrm{~K}\right)$.
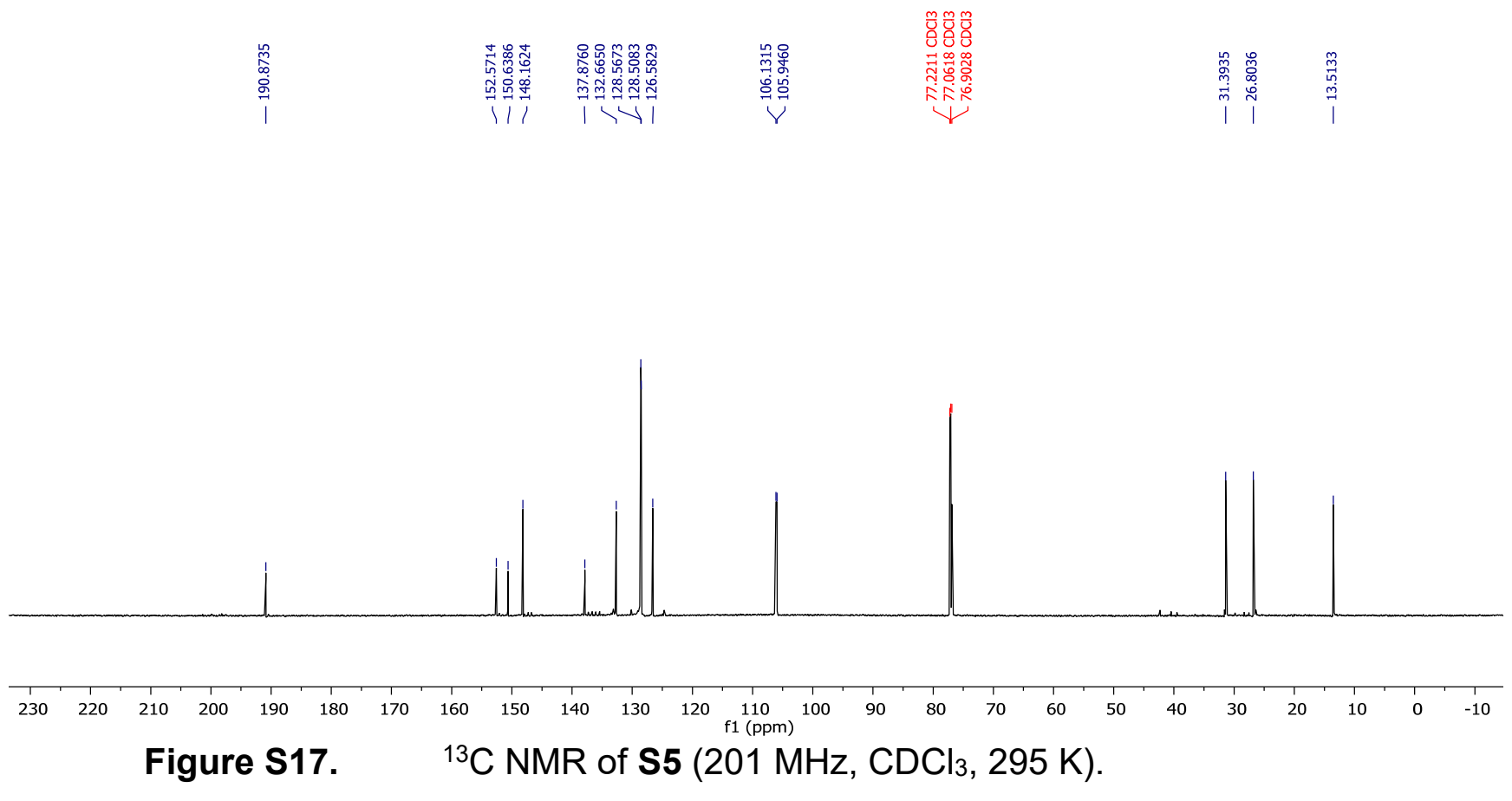

Figure S17. $\quad{ }^{13} \mathrm{C}$ NMR of $\mathbf{S} 5\left(201 \mathrm{MHz}, \mathrm{CDCl}_{3}, 295 \mathrm{~K}\right)$. 
<smiles>Cc1ccc(CCC2CC2C(=O)c2ccccc2)o1</smiles>

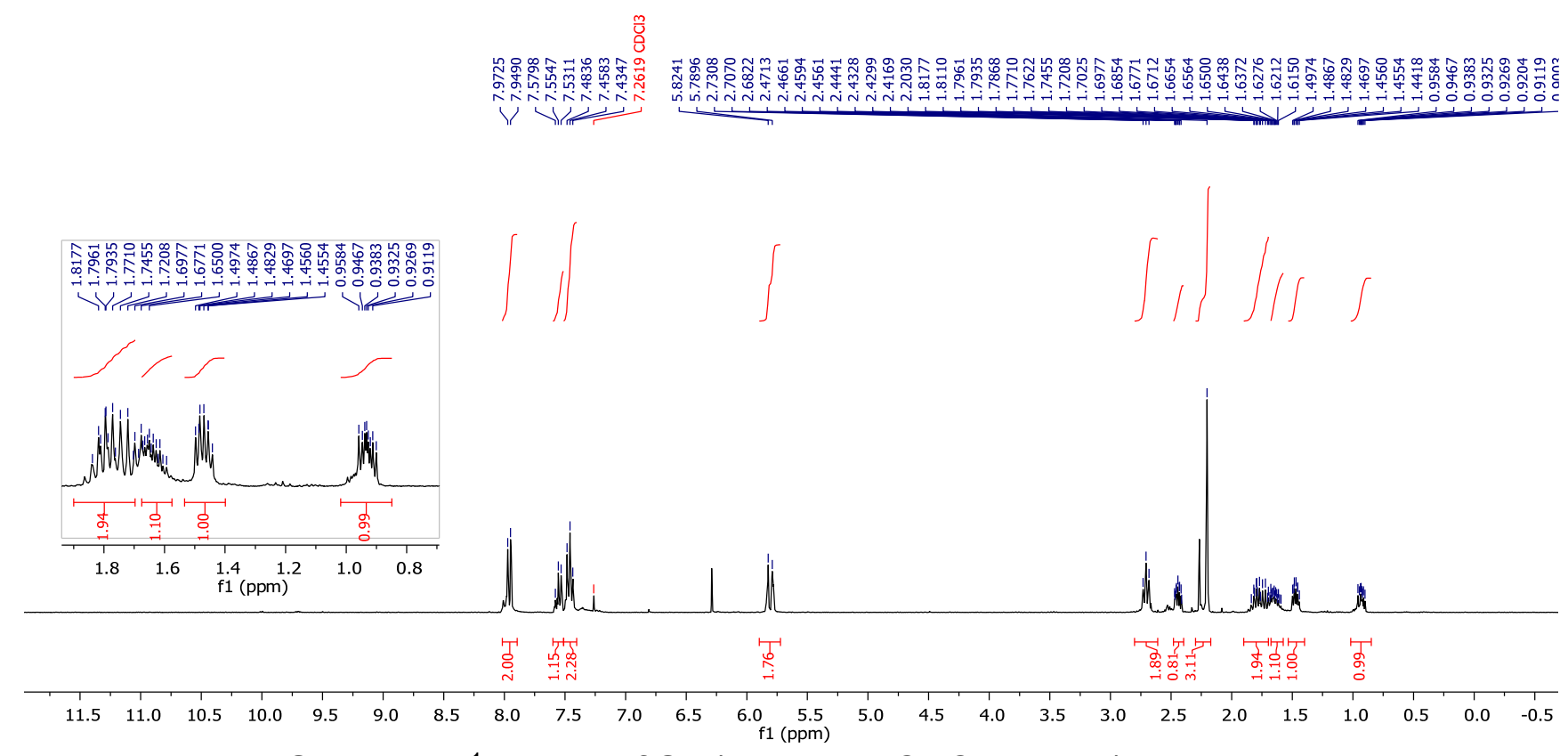

Figure S18. $\quad{ }^{1} \mathrm{H}$ NMR of $\mathbf{S 6}\left(300 \mathrm{MHz}, \mathrm{CDCl}_{3}, 295 \mathrm{~K}\right)$.

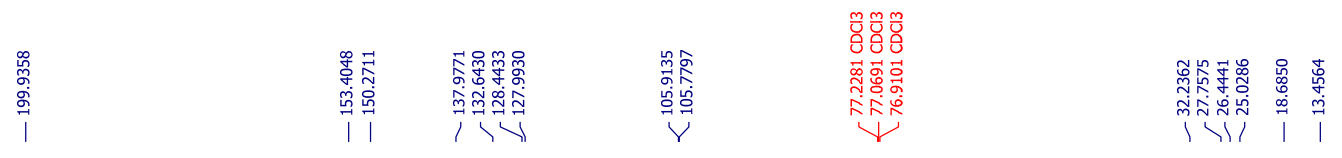

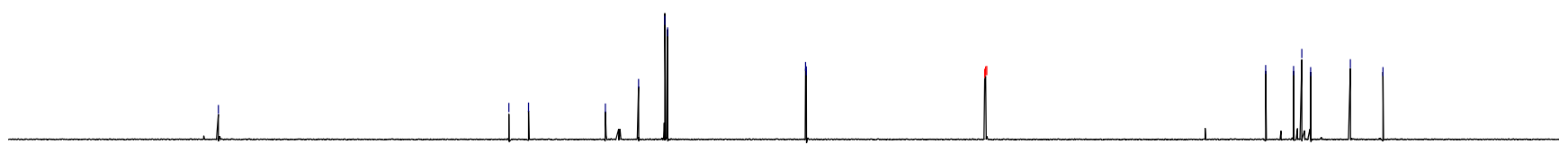

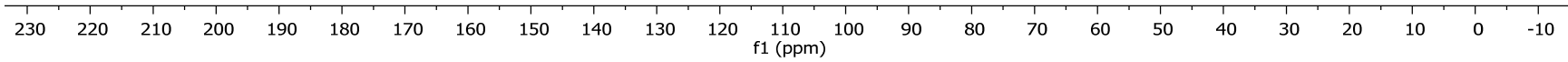

Figure S19. $\quad{ }^{13} \mathrm{C}$ NMR of $\mathbf{S 6}\left(201 \mathrm{MHz}, \mathrm{CDCl}_{3}, 295 \mathrm{~K}\right)$. 
<smiles>C=C(c1ccccc1)C1CC1CCc1ccc(C)o1</smiles>

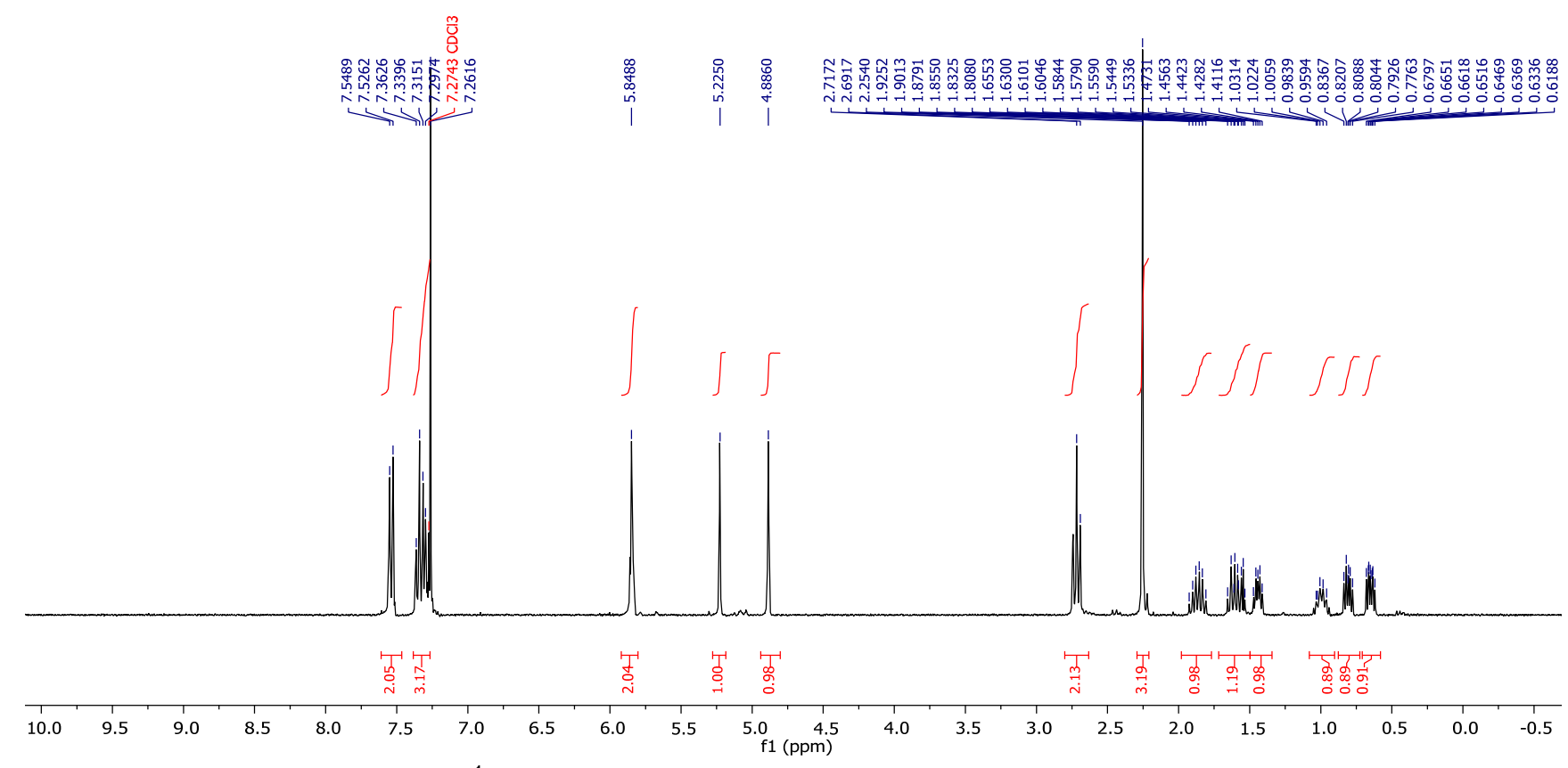

Figure S20. $\quad{ }^{1} \mathrm{H}$ NMR of $\mathbf{S 7}\left(300 \mathrm{MHz}, \mathrm{CDCl}_{3}, 295 \mathrm{~K}\right)$.
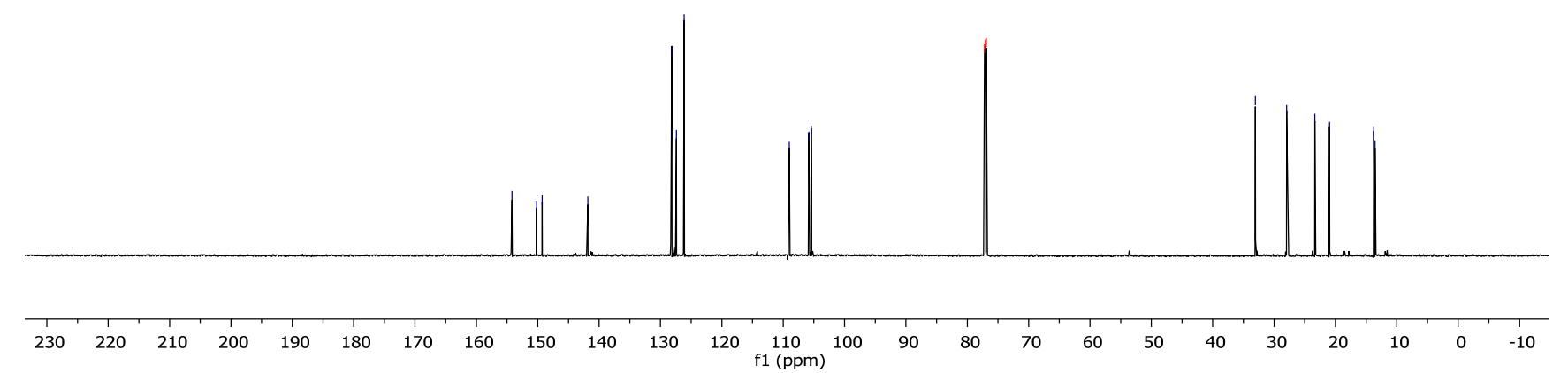

Figure S21. $\quad{ }^{13} \mathrm{C} \mathrm{NMR} \mathrm{of} \mathbf{S} 7\left(201 \mathrm{MHz}, \mathrm{CDCl}_{3}, 295 \mathrm{~K}\right)$. 
<smiles>O=c1c(=O)c2ccccc2c1=O</smiles>
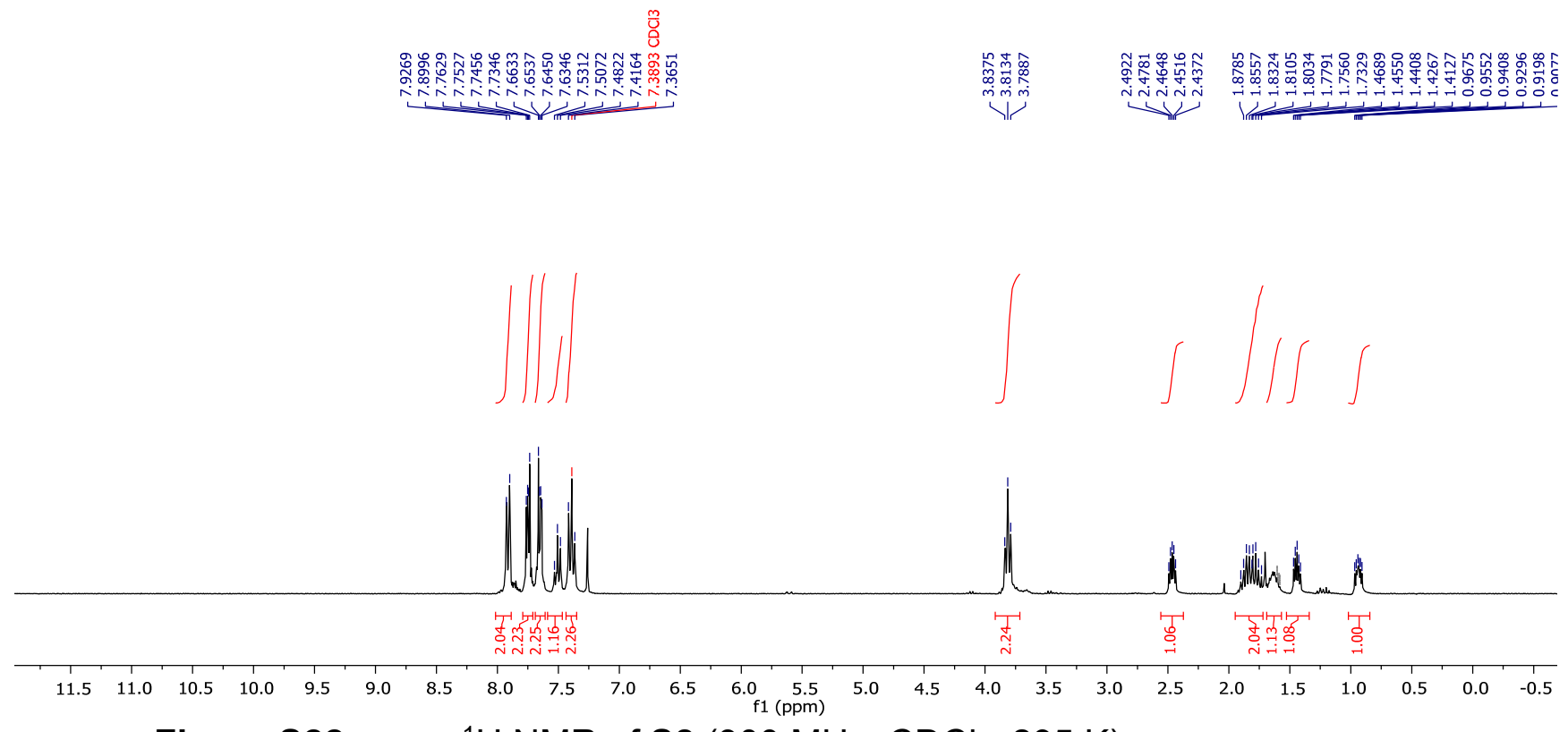

Figure S22. $\quad{ }^{1} \mathrm{H}$ NMR of $\mathbf{S 8}\left(300 \mathrm{MHz}, \mathrm{CDCl}_{3}, 295 \mathrm{~K}\right)$.

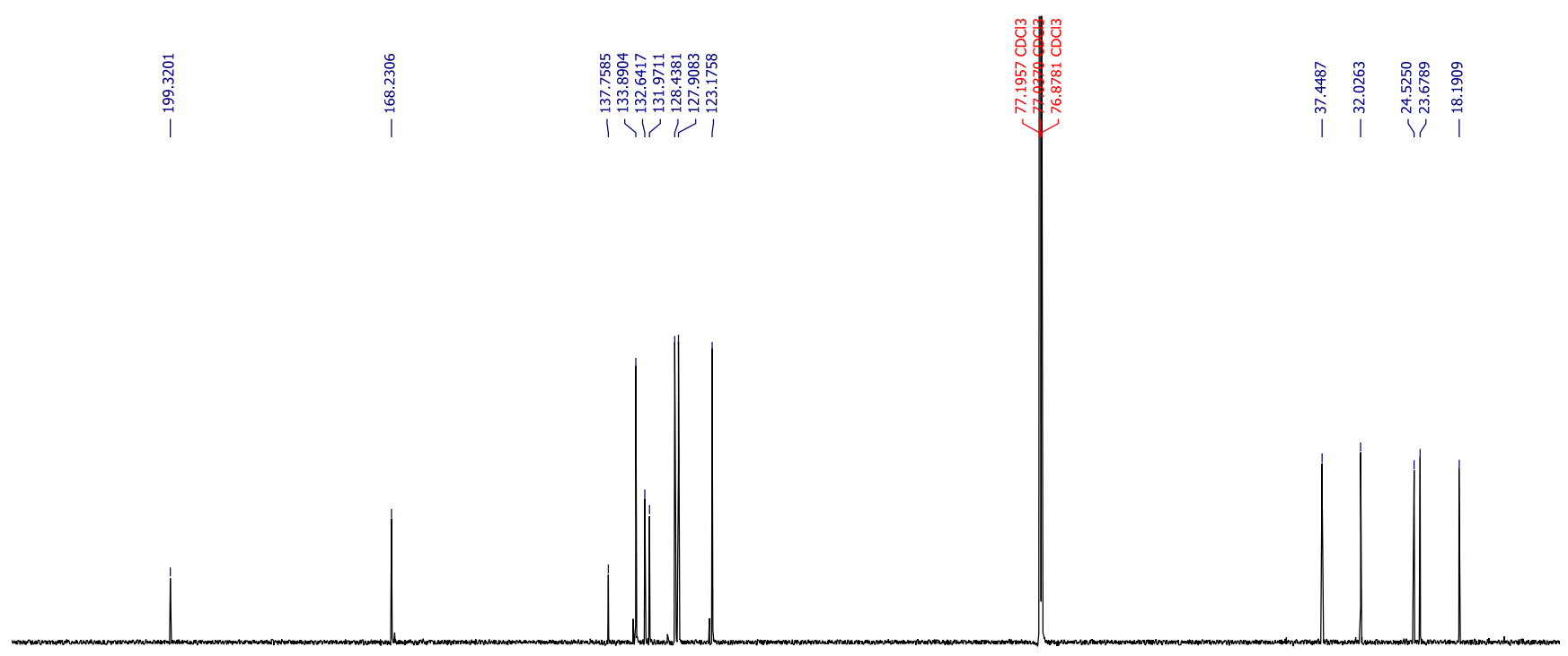

220

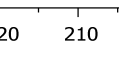

$200 \quad 190 \quad 180$
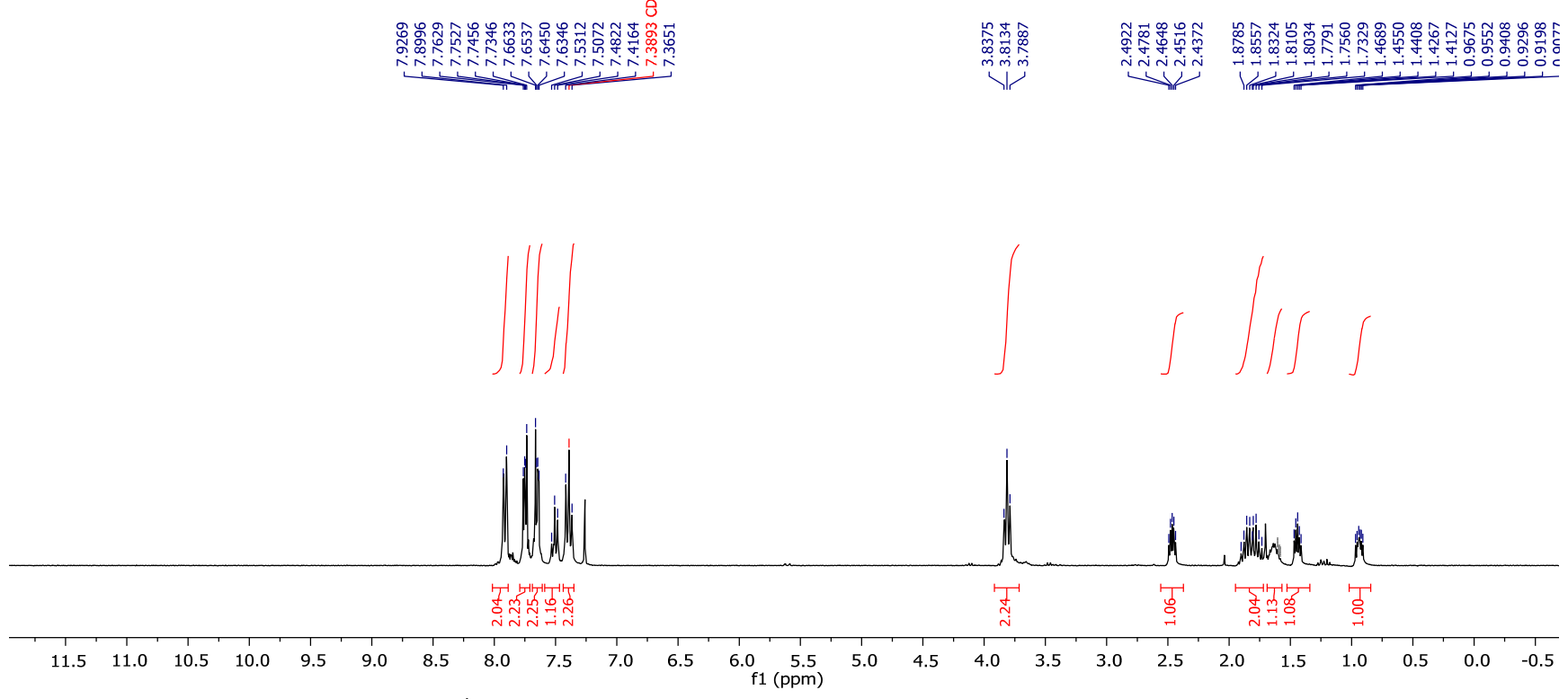
<smiles>C=C(c1ccccc1)C1CC1CCN1C(=O)c2ccccc2C1=O</smiles>

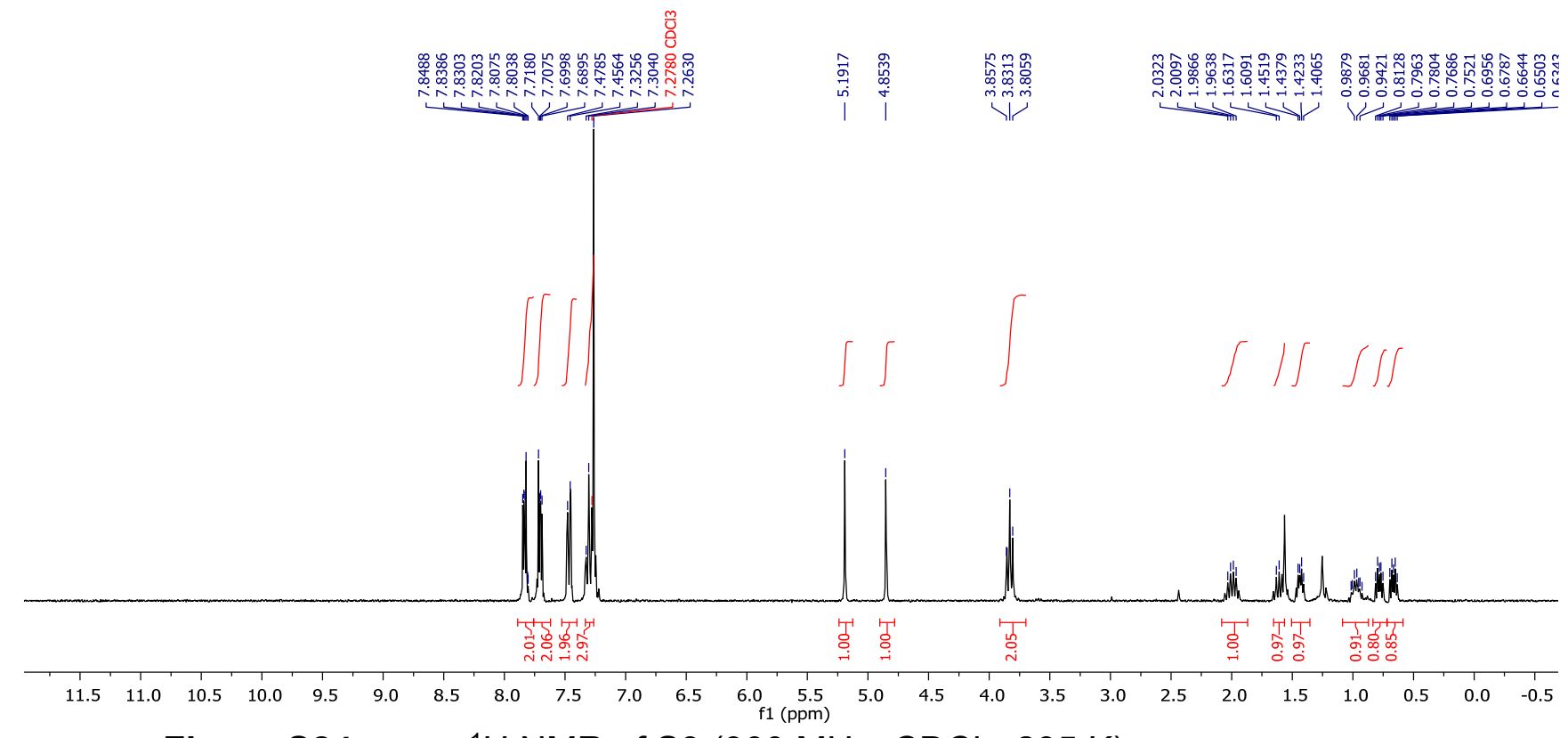

Figure S24. $\quad{ }^{1} \mathrm{H}$ NMR of $\mathbf{S 9}\left(300 \mathrm{MHz}, \mathrm{CDCl}_{3}, 295 \mathrm{~K}\right)$.

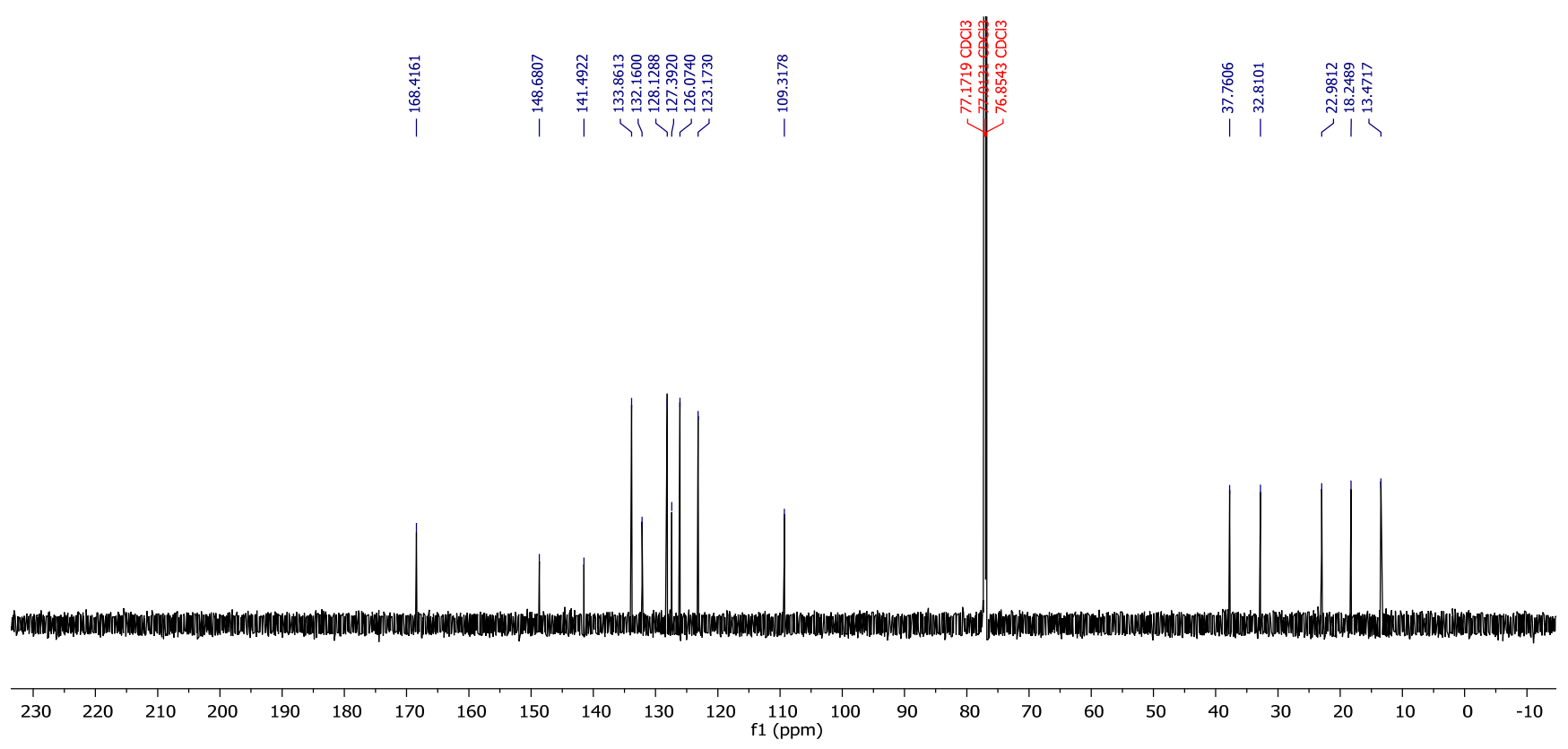

Figure S25. $\quad{ }^{13} \mathrm{C}$ NMR of $\mathbf{S 9}\left(201 \mathrm{MHz}, \mathrm{CDCl}_{3}, 295 \mathrm{~K}\right)$. 
<smiles>CCCCCCCC=CC(=O)c1ccccc1</smiles>
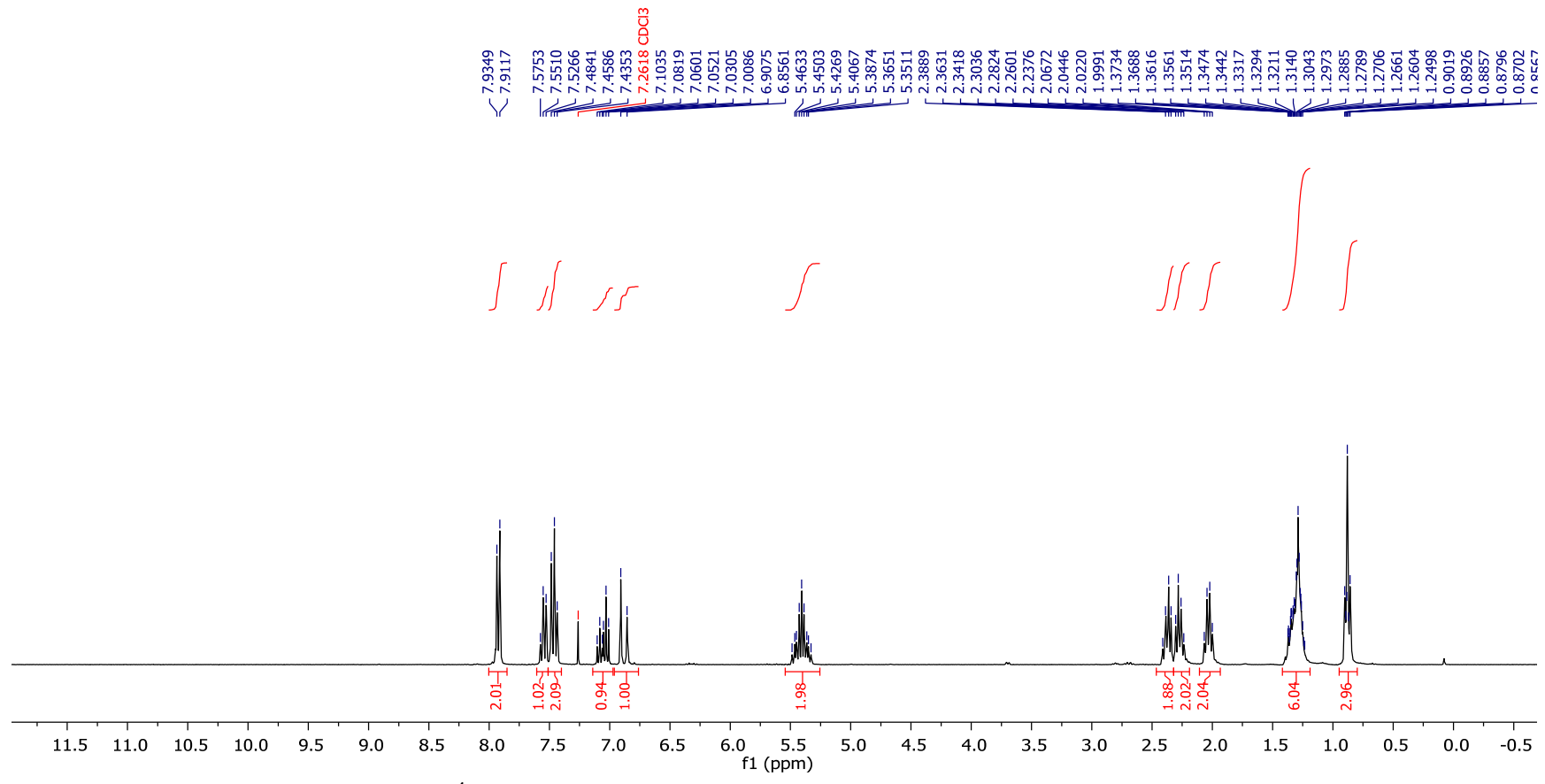

Figure S26. $\quad{ }^{1} \mathrm{H} \mathrm{NMR}$ of $\mathbf{S 1 0}\left(300 \mathrm{MHz}, \mathrm{CDCl}_{3}, 295 \mathrm{~K}\right)$.
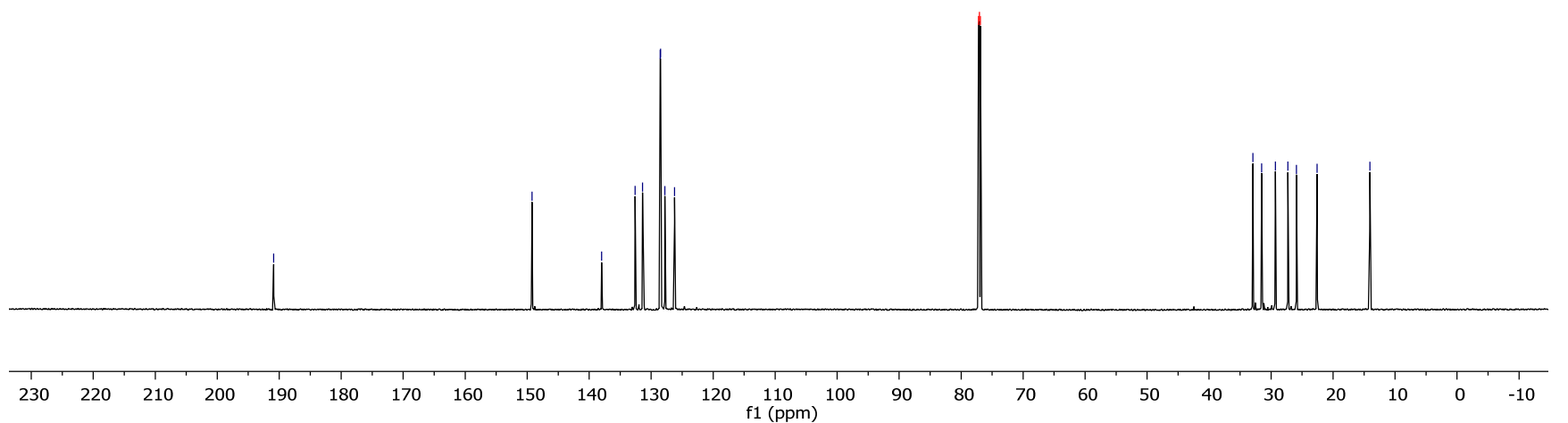

Figure S27. $\quad{ }^{13} \mathrm{C}$ NMR of $\mathbf{S} 10\left(201 \mathrm{MHz}, \mathrm{CDCl}_{3}, 295 \mathrm{~K}\right)$. 

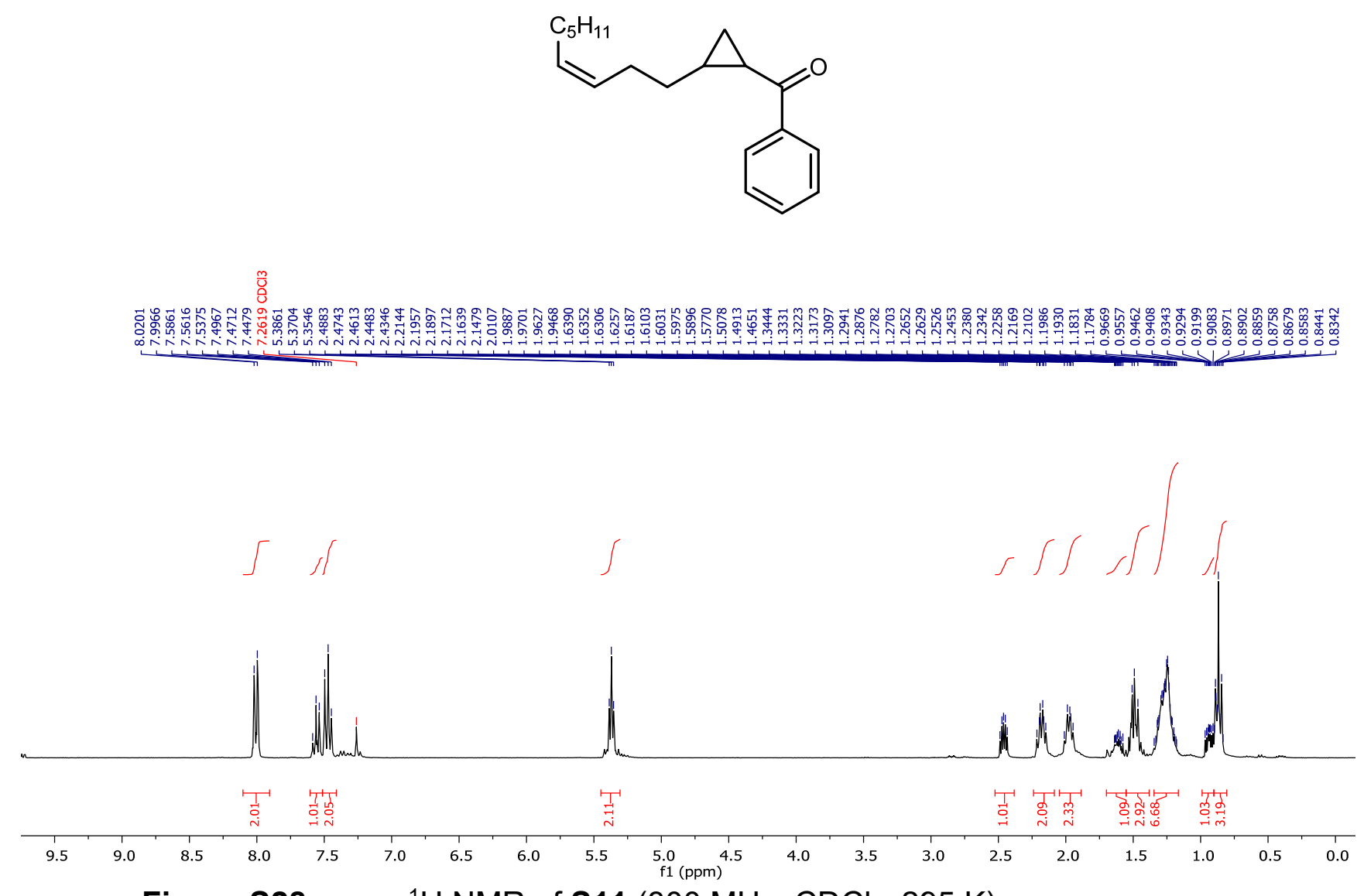

Figure S28. $\quad{ }^{1} \mathrm{H}$ NMR of $\mathbf{S} 11\left(300 \mathrm{MHz}, \mathrm{CDCl}_{3}, 295 \mathrm{~K}\right)$.
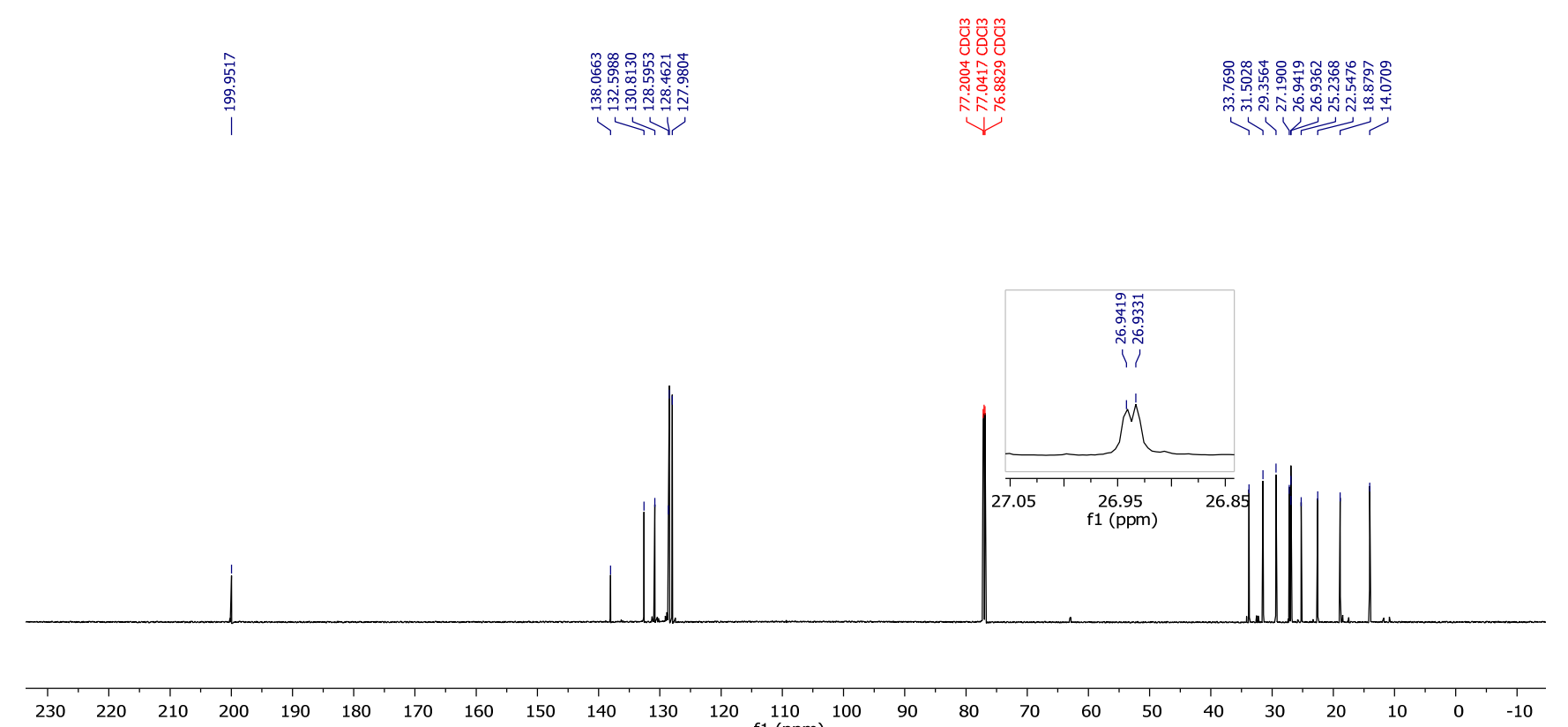

Figure S29. $\quad{ }^{13} \mathrm{C} \mathrm{NMR}$ of $\mathbf{S 1 1}\left(201 \mathrm{MHz}, \mathrm{CDCl}_{3}, 295 \mathrm{~K}\right)$. 

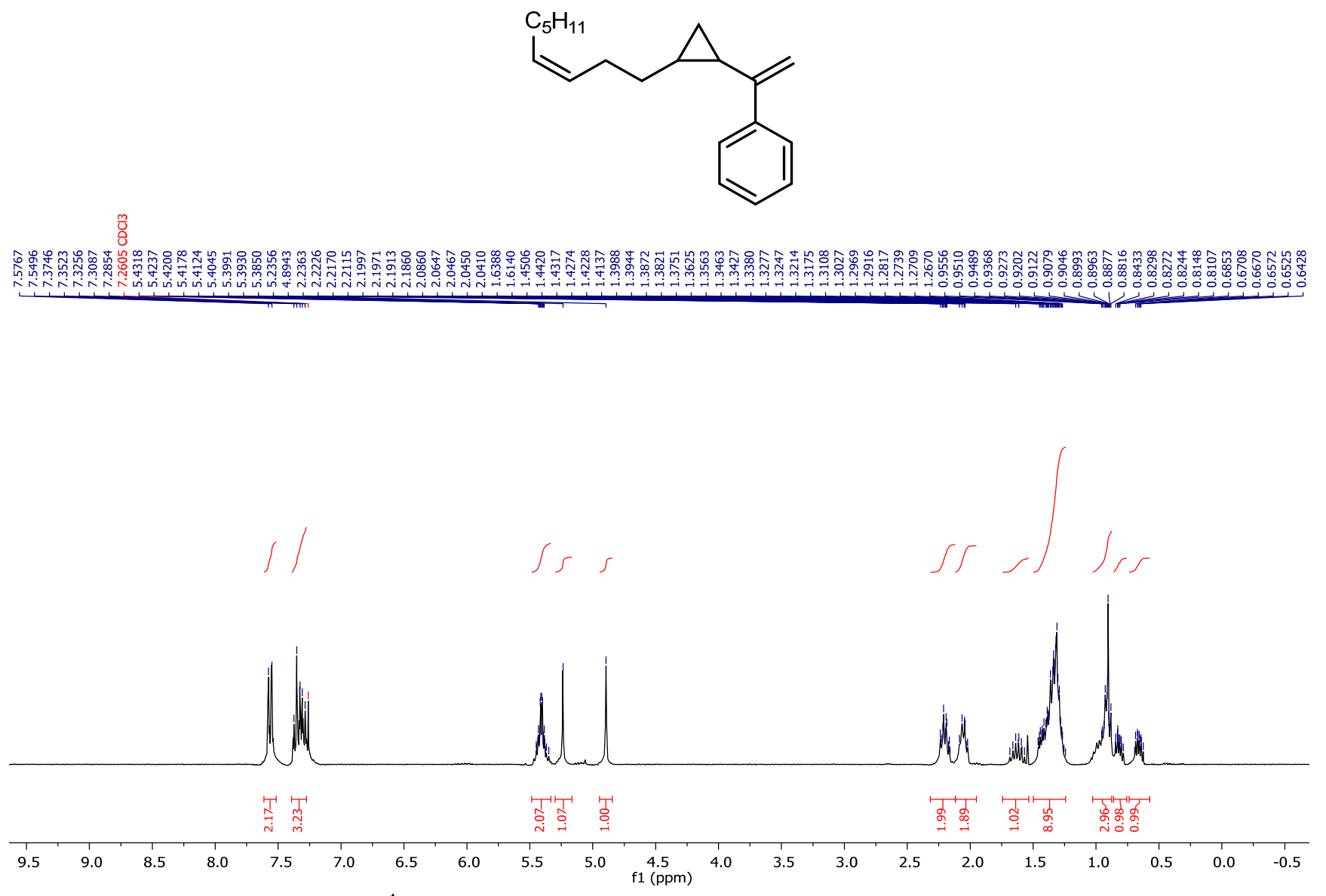

Figure S30. $\quad{ }^{1} \mathrm{H} \mathrm{NMR}$ of $\mathbf{S} 12\left(300 \mathrm{MHz}, \mathrm{CDCl}_{3}, 295 \mathrm{~K}\right)$.
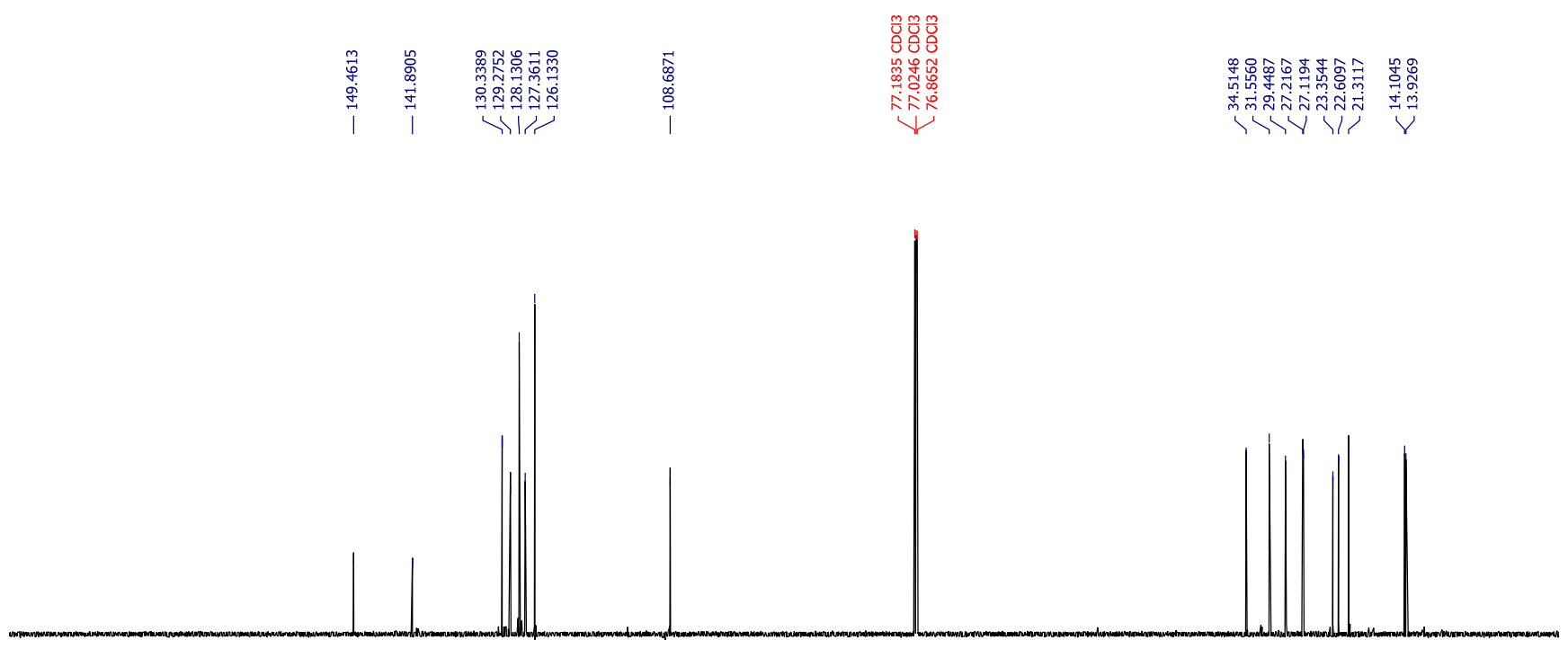

Figure S31. $\quad{ }^{13} \mathrm{C}$ NMR of $\mathbf{S 1 2}\left(201 \mathrm{MHz}, \mathrm{CDCl}_{3}, 295 \mathrm{~K}\right)$. 
<smiles>O=C(c1ccccc1)C1CC1CCO</smiles>

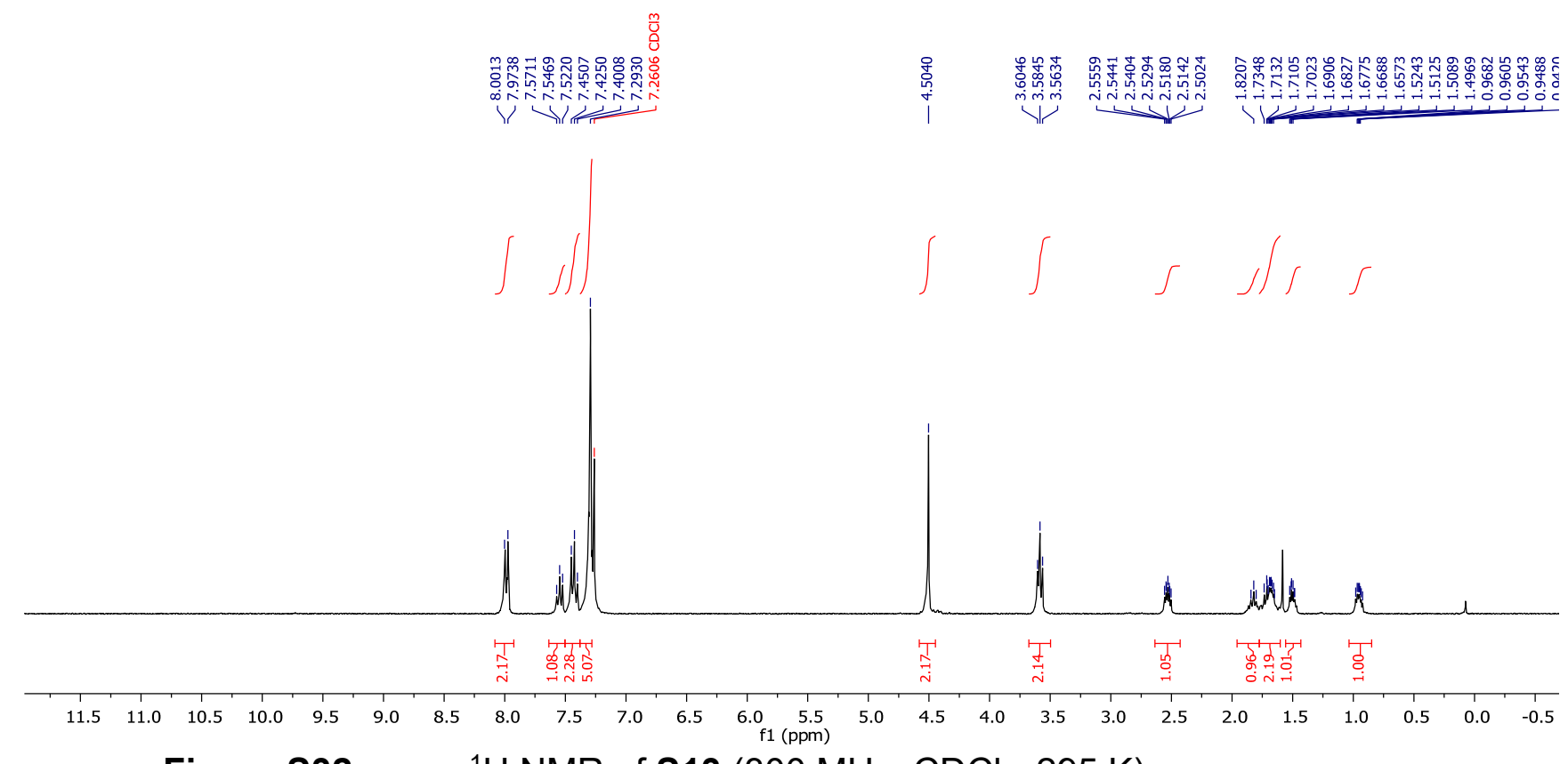

Figure S32. $\quad{ }^{1} \mathrm{H} \mathrm{NMR}$ of $\mathbf{S} 13\left(300 \mathrm{MHz}, \mathrm{CDCl}_{3}, 295 \mathrm{~K}\right)$.

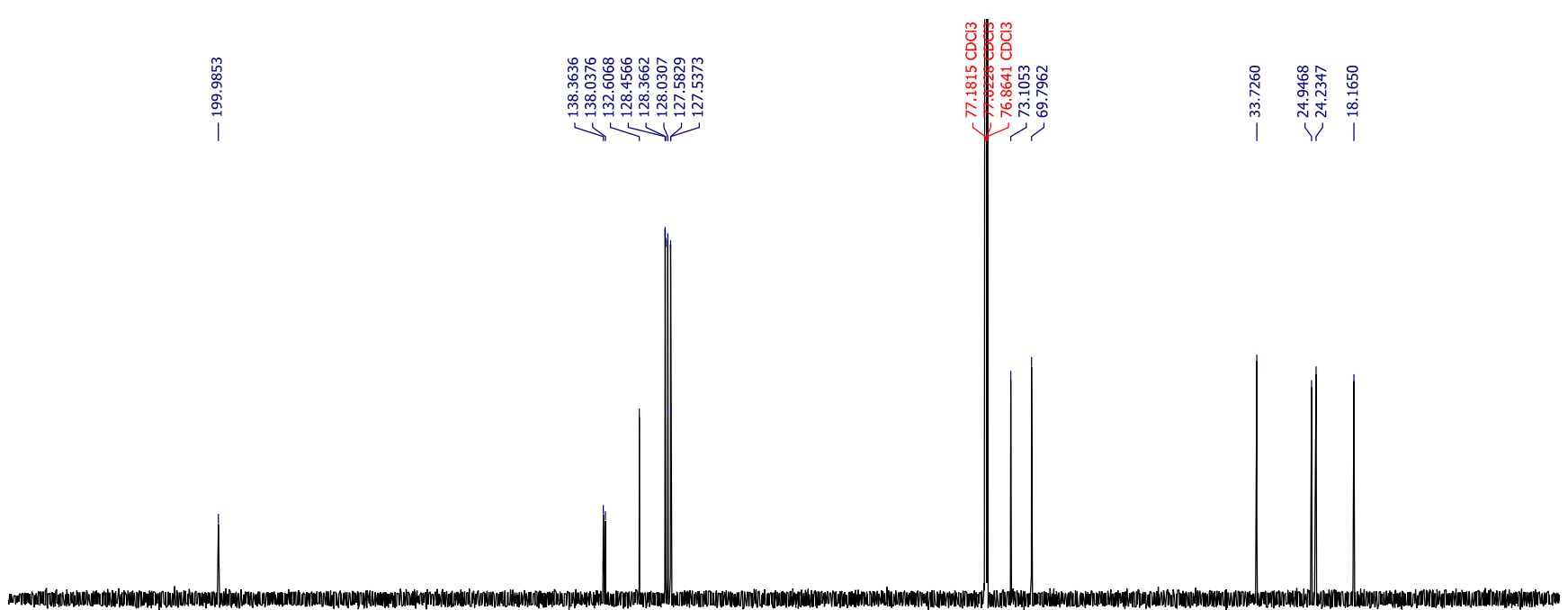

Figure S33. $\quad{ }^{13} \mathrm{C} N M R$ of $\mathbf{S} 13\left(201 \mathrm{MHz}, \mathrm{CDCl}_{3}, 295 \mathrm{~K}\right)$. 

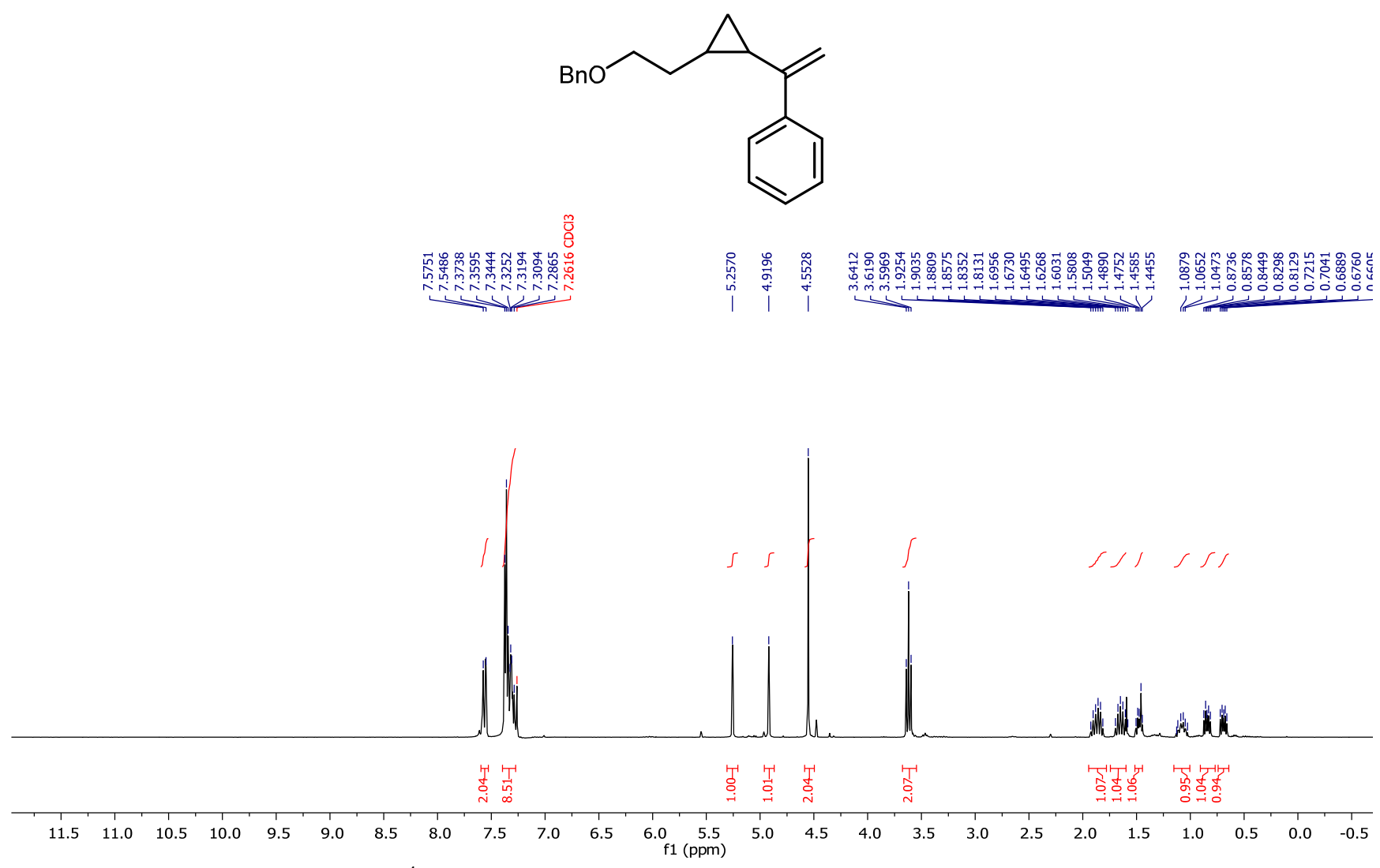

Figure S34. $\quad{ }^{1} \mathrm{H} \mathrm{NMR}$ of $\mathbf{S} 14\left(300 \mathrm{MHz}, \mathrm{CDCl}_{3}, 295 \mathrm{~K}\right)$.

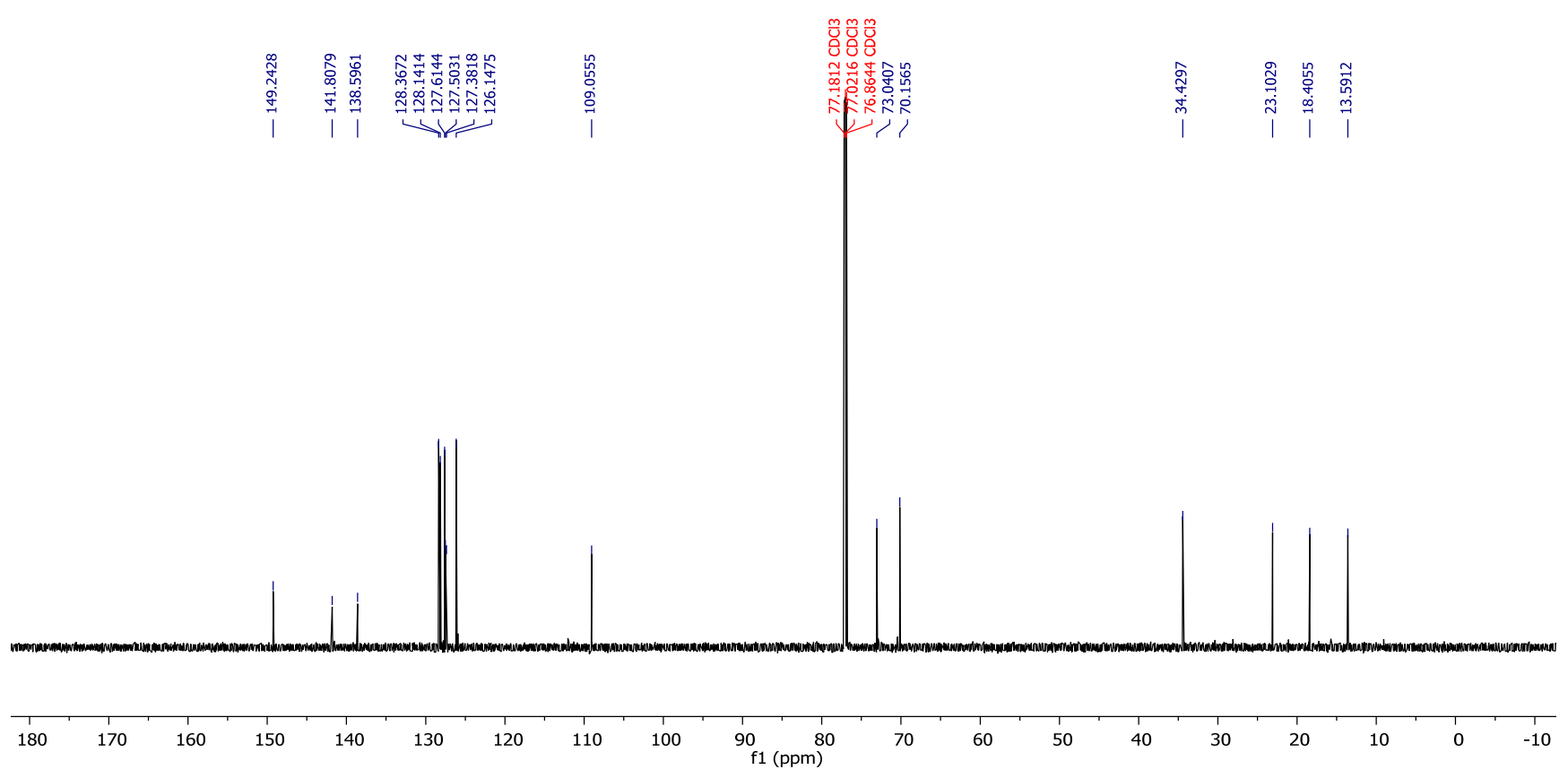

Figure S35. $\quad{ }^{13} \mathrm{C}$ NMR of $\mathbf{S 1 4}\left(201 \mathrm{MHz}, \mathrm{CDCl}_{3}, 295 \mathrm{~K}\right)$. 
<smiles>COC(=O)CCCC=C(Cl)Cl</smiles>

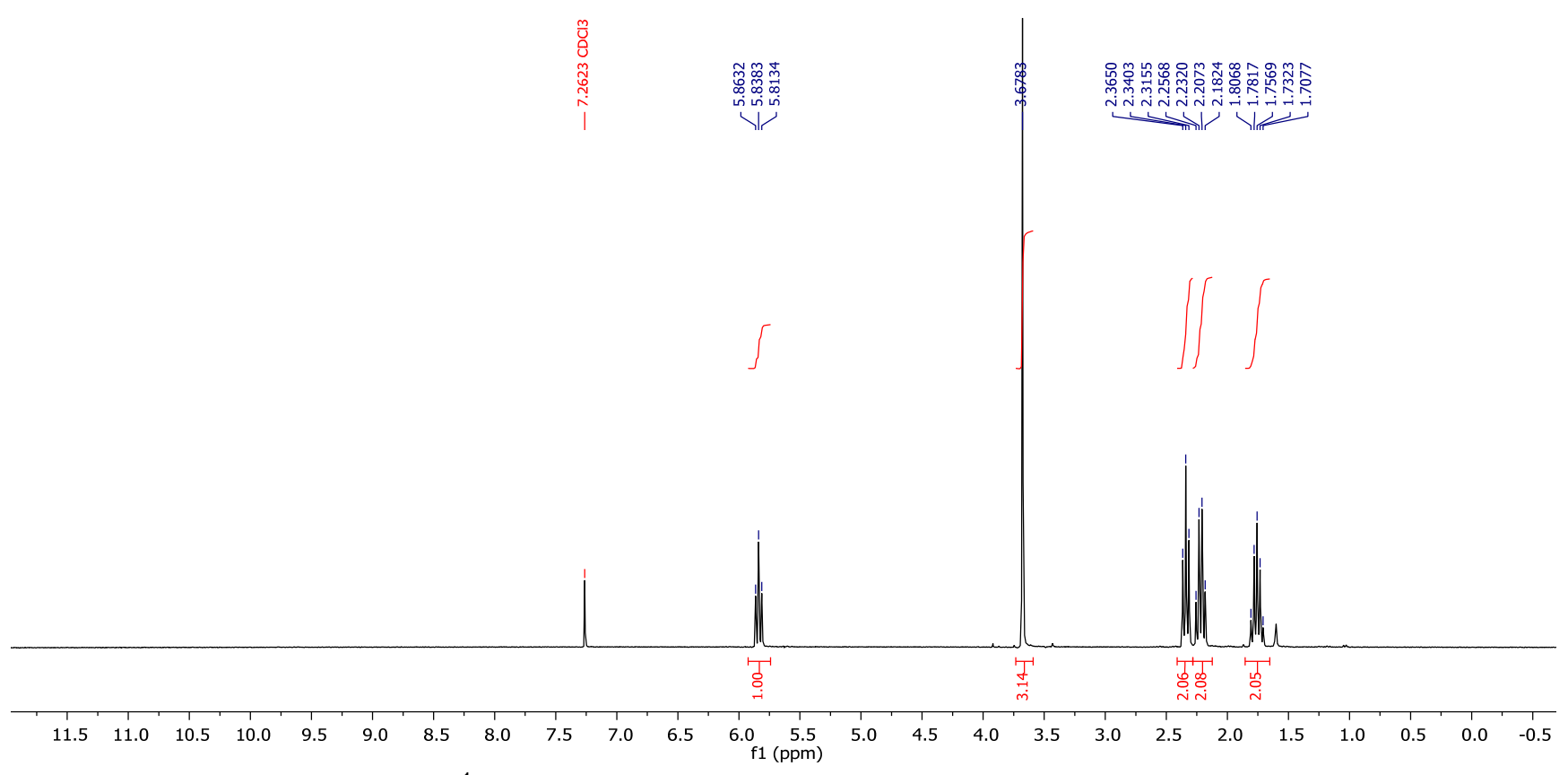

Figure S36. $\quad{ }^{1} \mathrm{H} \mathrm{NMR}$ of $\mathbf{S} 15\left(300 \mathrm{MHz}, \mathrm{CDCl}_{3}, 295 \mathrm{~K}\right)$.
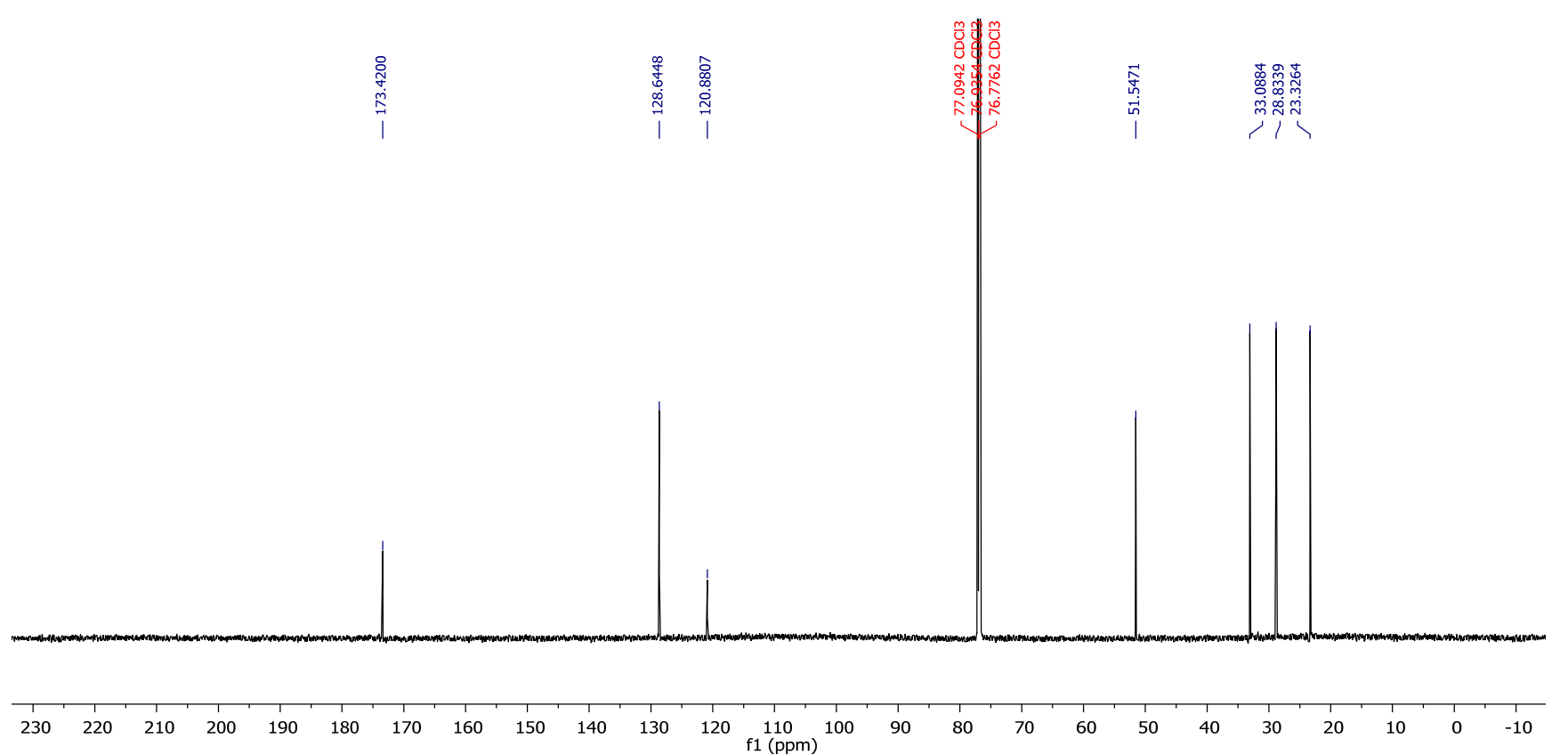

Figure S37. $\quad{ }^{13} \mathrm{C}$ NMR of $\mathbf{S} 15\left(201 \mathrm{MHz}, \mathrm{CDCl}_{3}, 295 \mathrm{~K}\right)$. 
<smiles>CN([13CH3])CCC=C(Cl)Cl</smiles>

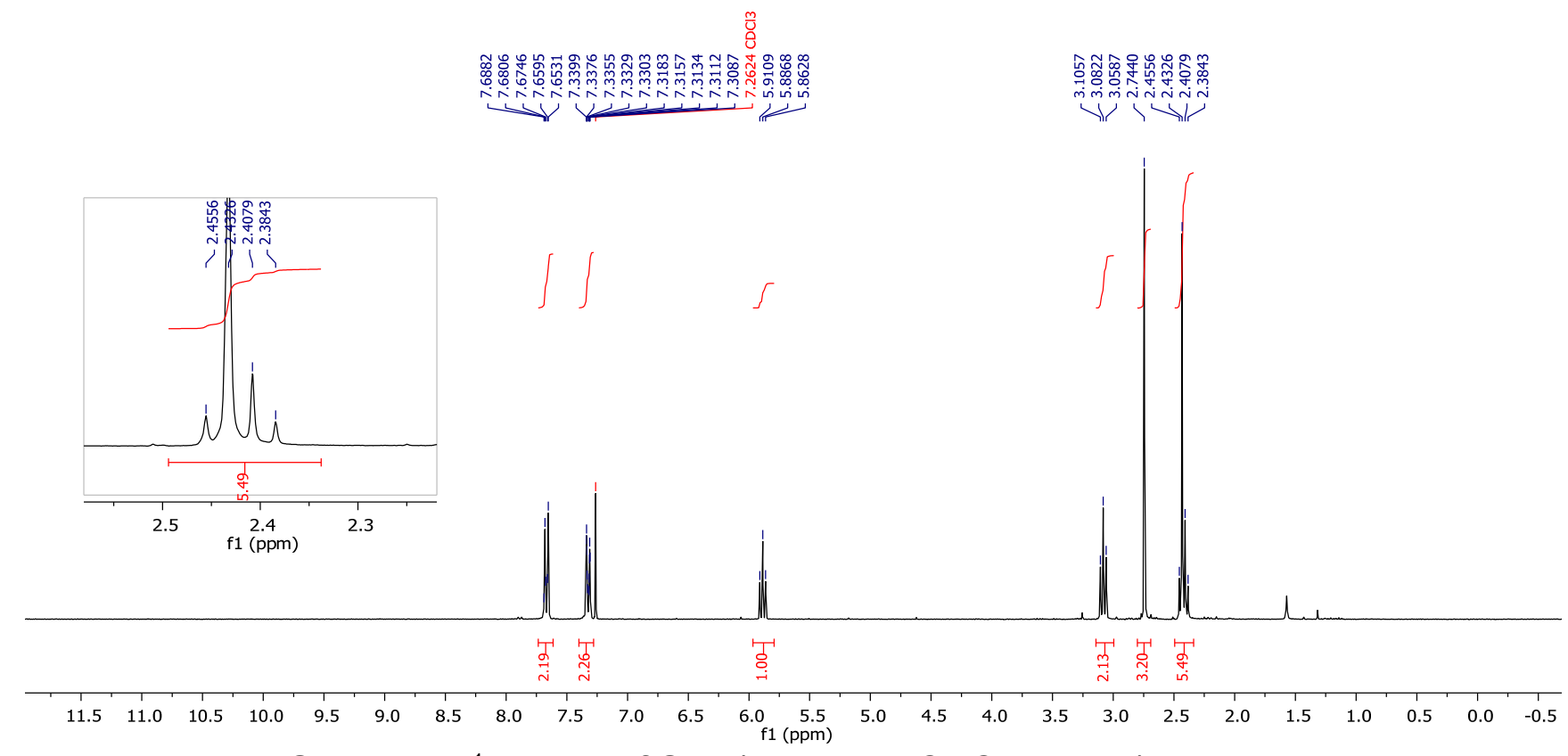

Figure S38. $\quad{ }^{1} \mathrm{H}$ NMR of $\mathbf{S 1 6}\left(300 \mathrm{MHz}, \mathrm{CDCl}_{3}, 295 \mathrm{~K}\right)$.

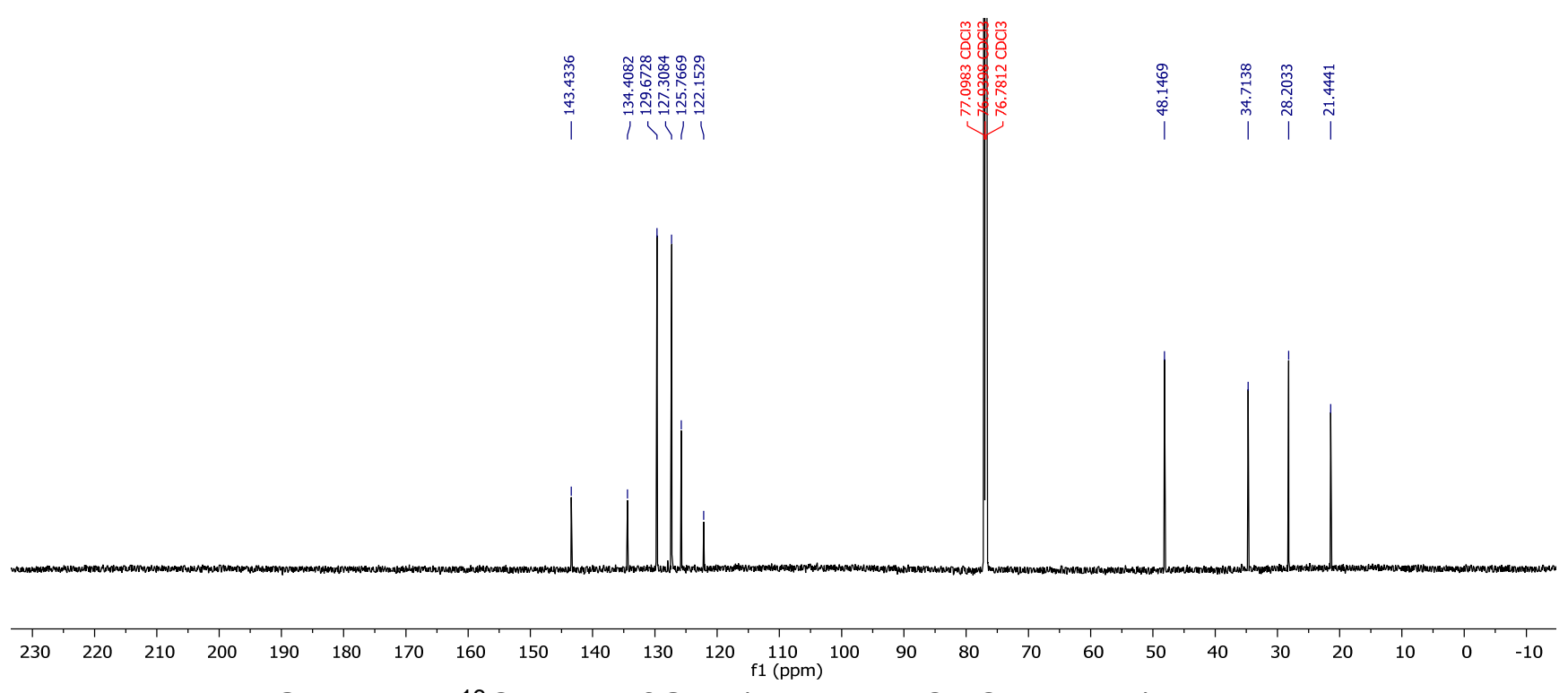

Figure S39. $\quad{ }^{13} \mathrm{C}$ NMR of $\mathbf{S 1 6}\left(201 \mathrm{MHz}, \mathrm{CDCl}_{3}, 295 \mathrm{~K}\right)$. 


\section{NMR Data for [5+1]-Products}
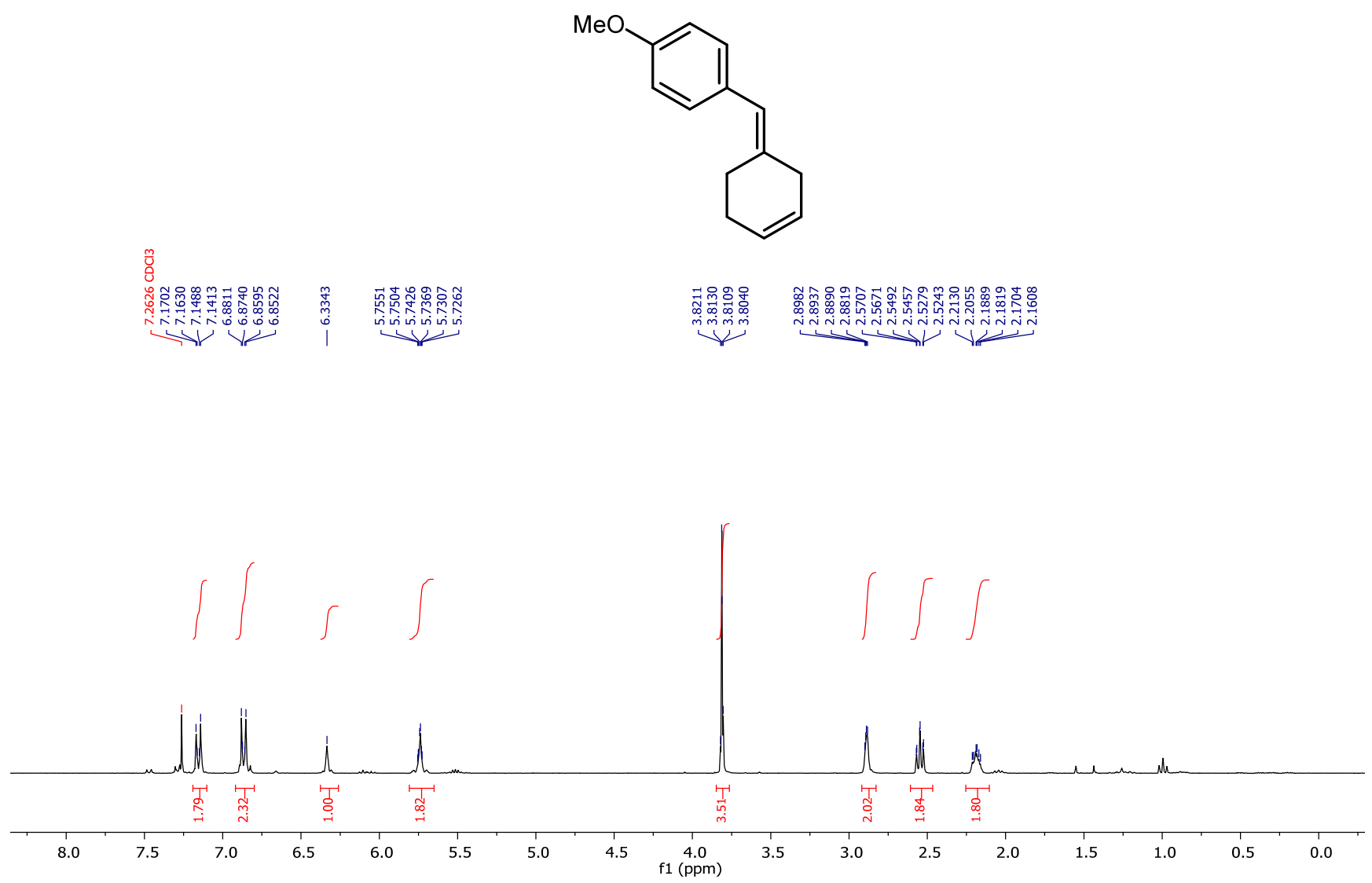

Figure S40. $\quad{ }^{1} \mathrm{H}$ NMR of $3\left(300 \mathrm{MHz}, \mathrm{CDCl}_{3}, 295 \mathrm{~K}\right)$.

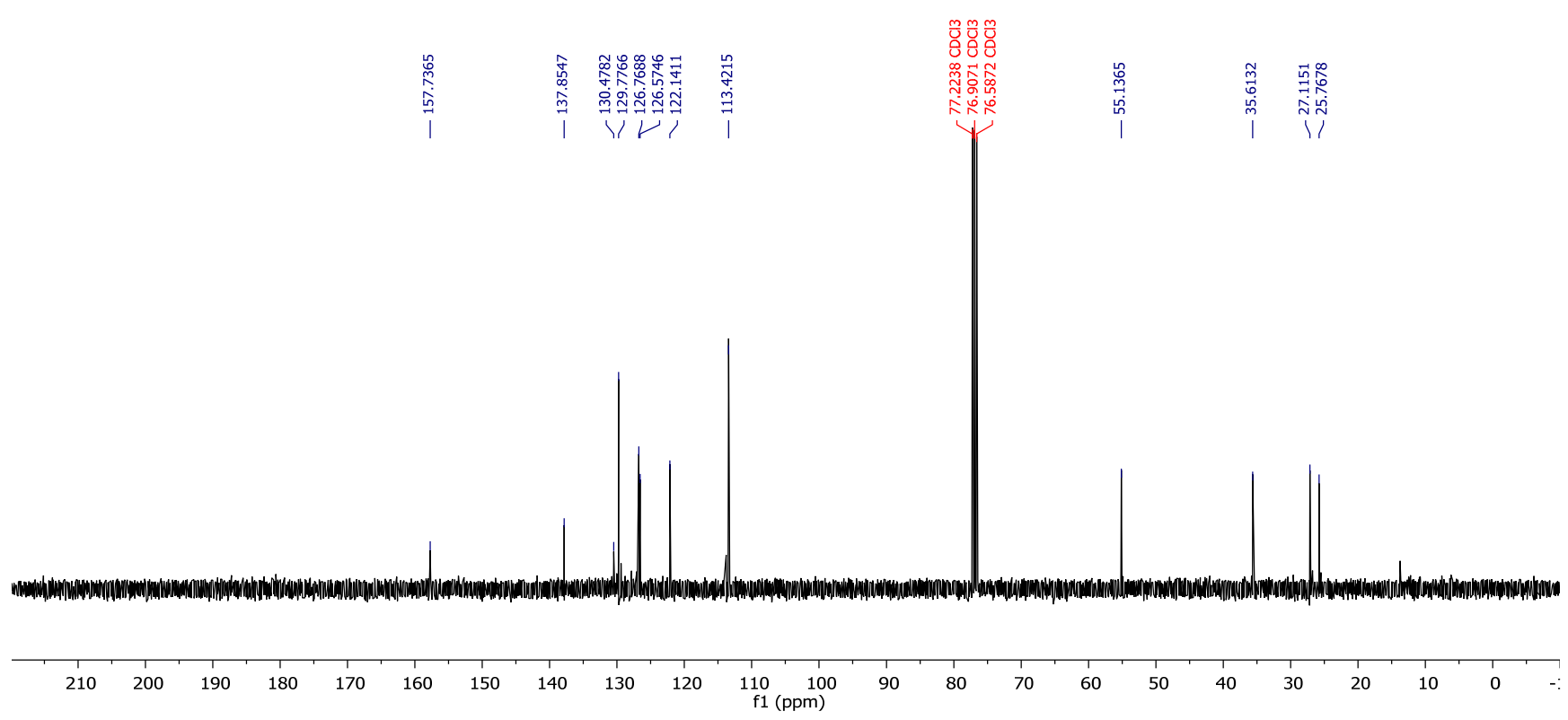

Figure S41. $\quad{ }^{13} \mathrm{C}$ NMR of $3\left(201 \mathrm{MHz}, \mathrm{CDCl}_{3}, 295 \mathrm{~K}\right)$. 


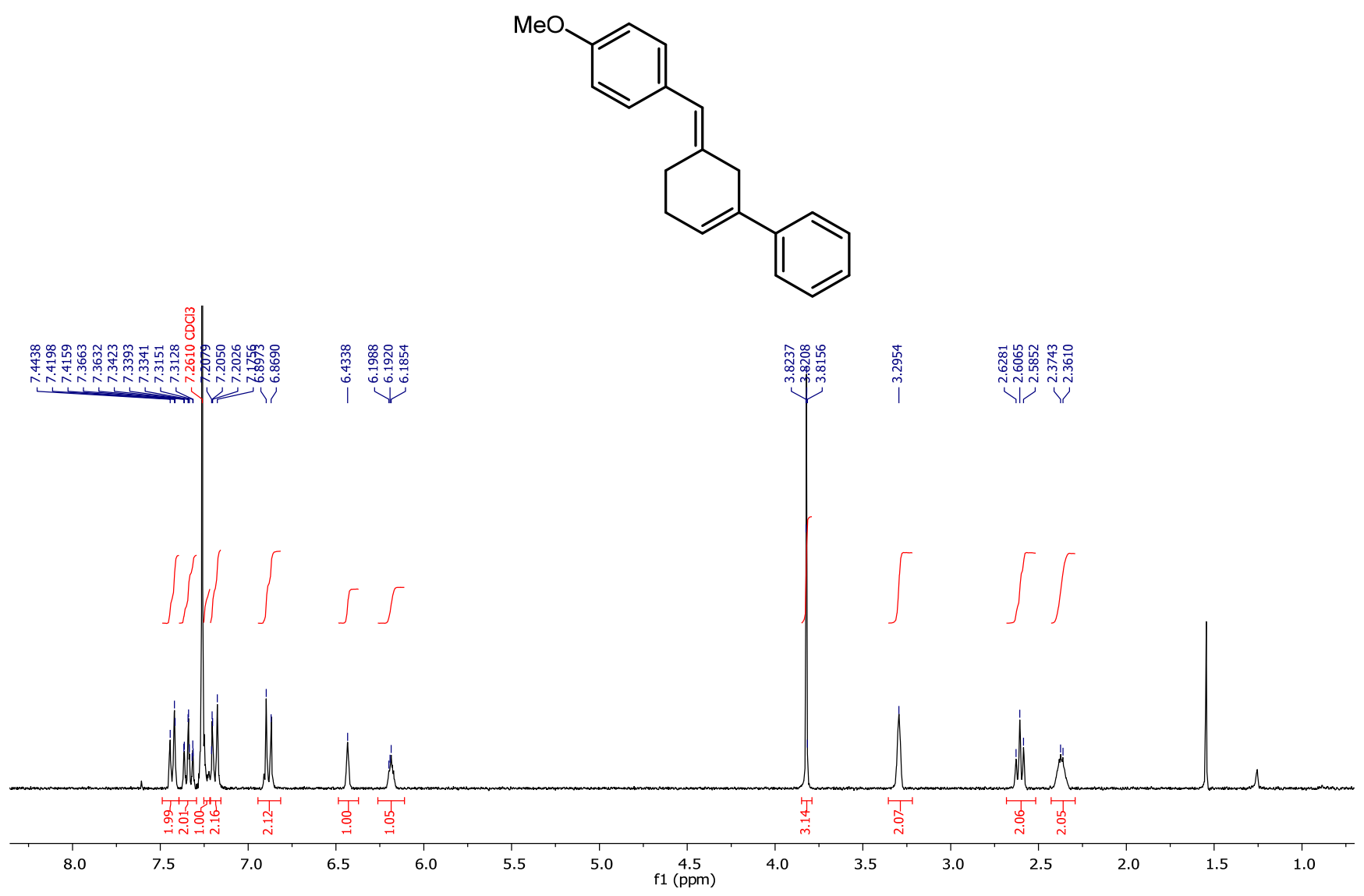

Figure S42. $\quad{ }^{1} \mathrm{H}$ NMR of $6\left(300 \mathrm{MHz}, \mathrm{CDCl}_{3}, 295 \mathrm{~K}\right)$.

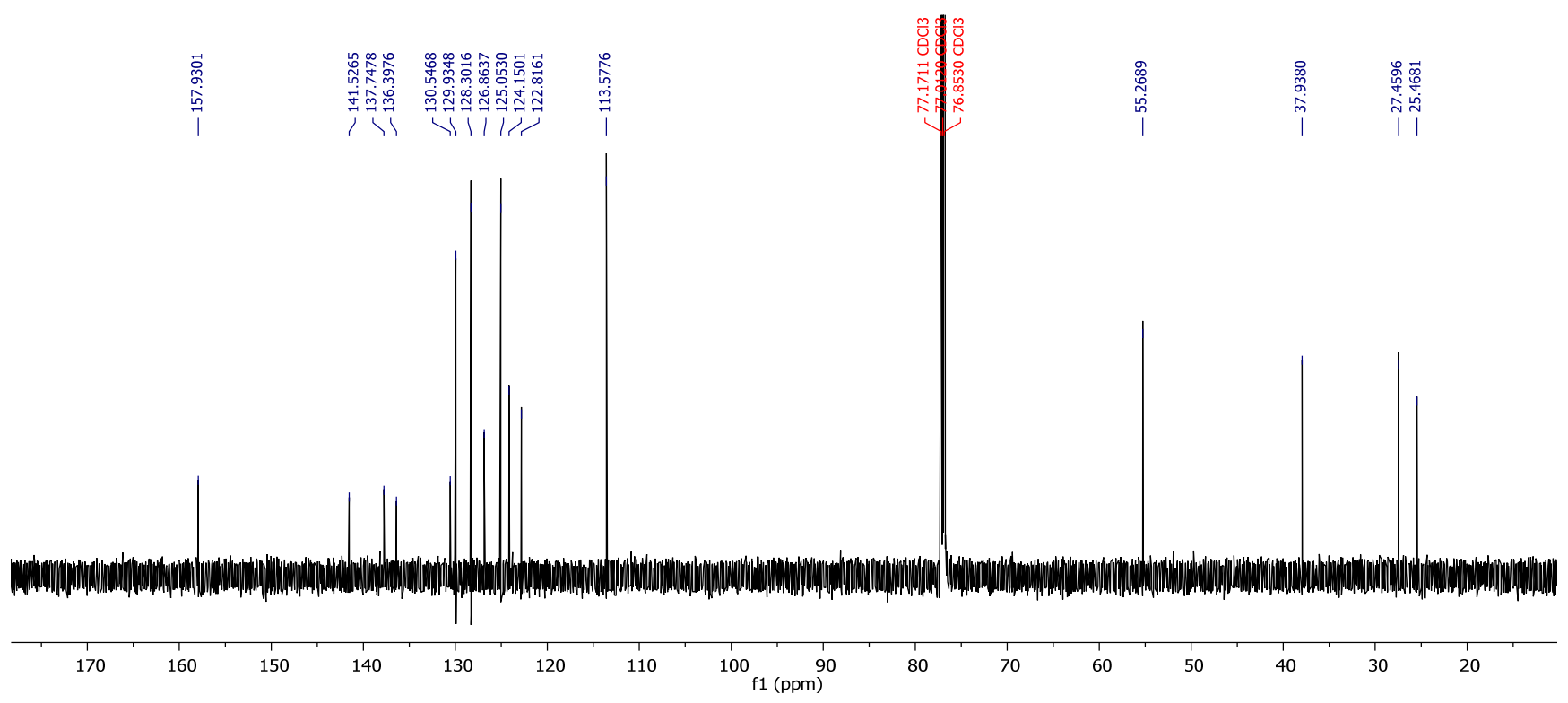

Figure S43. $\quad{ }^{13} \mathrm{C} \mathrm{NMR}$ of $6\left(201 \mathrm{MHz}, \mathrm{CDCl}_{3}, 295 \mathrm{~K}\right)$. 


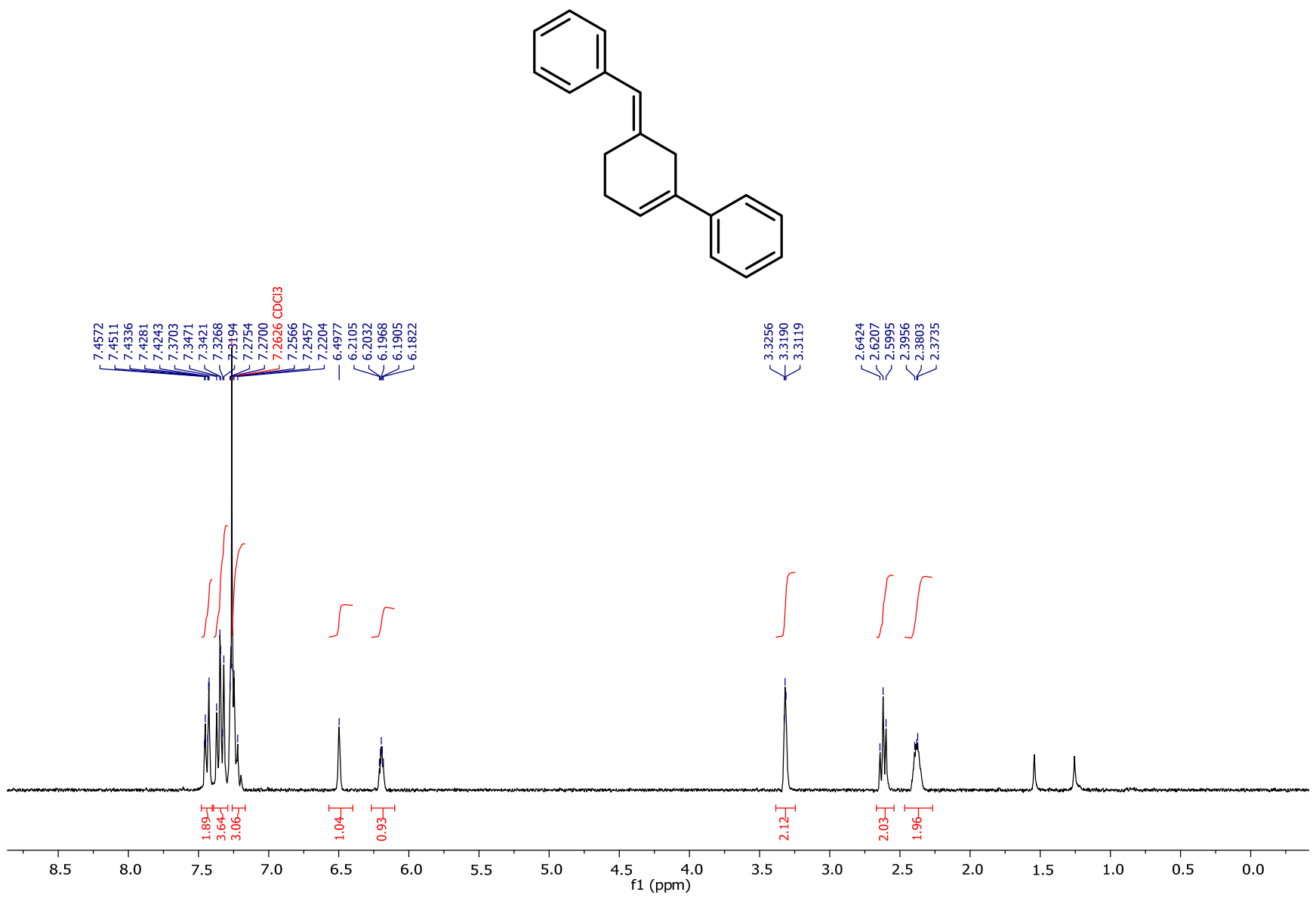

Figure S44. $\quad{ }^{1} \mathrm{H}$ NMR of $11\left(300 \mathrm{MHz}, \mathrm{CDCl}_{3}, 295 \mathrm{~K}\right)$.
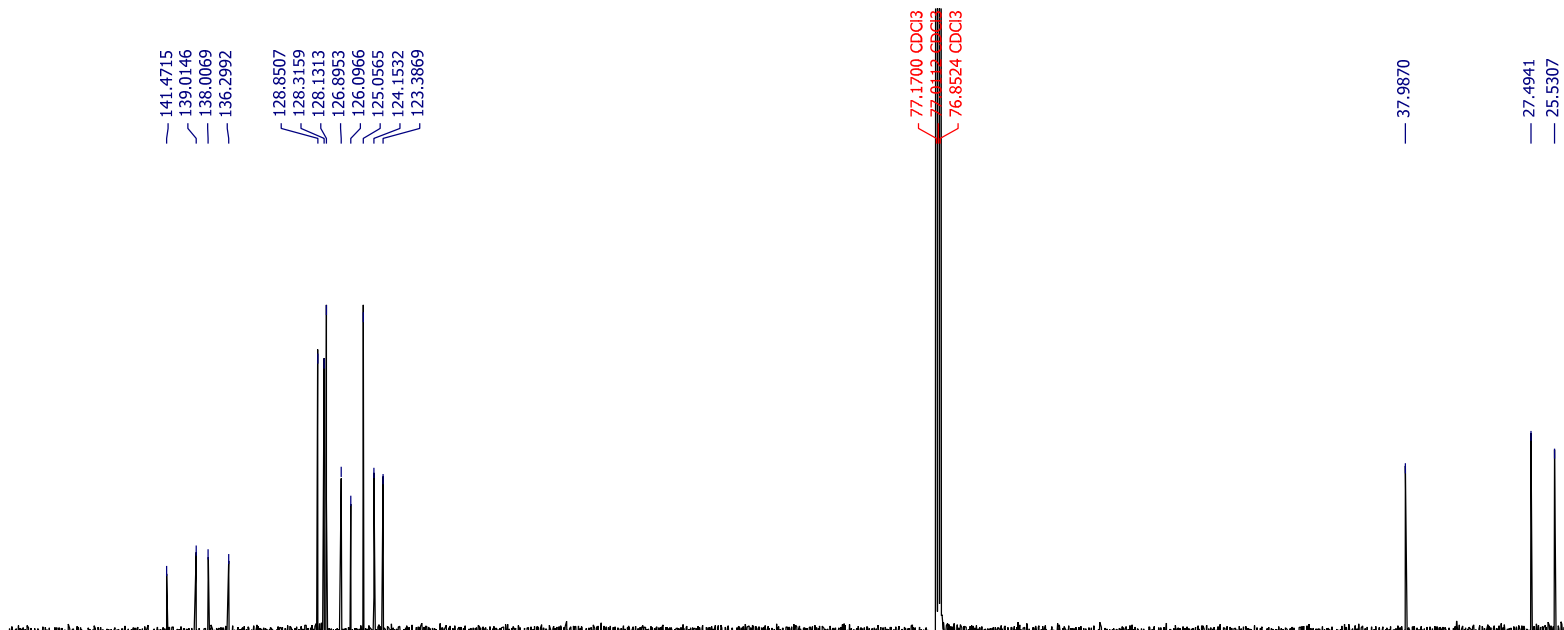

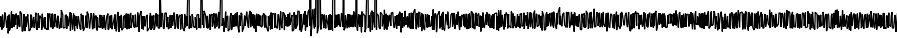

$\begin{array}{llllllllllllllllllllllllllllllllll}150 & 145 & 140 & 135 & 130 & 125 & 120 & 115 & 110 & 105 & 100 & 95 & 90 & 85 & 80 & 75 & 70 & 65 & 60 & 55 & 50 & 45 & 40 & 35 & 30 & 25 & 20 & 15\end{array}$ Figure S45. $\quad{ }^{13} \mathrm{C}$ NMR of $11\left(201 \mathrm{MHz}, \mathrm{CDCl}_{3}, 295 \mathrm{~K}\right)$. 

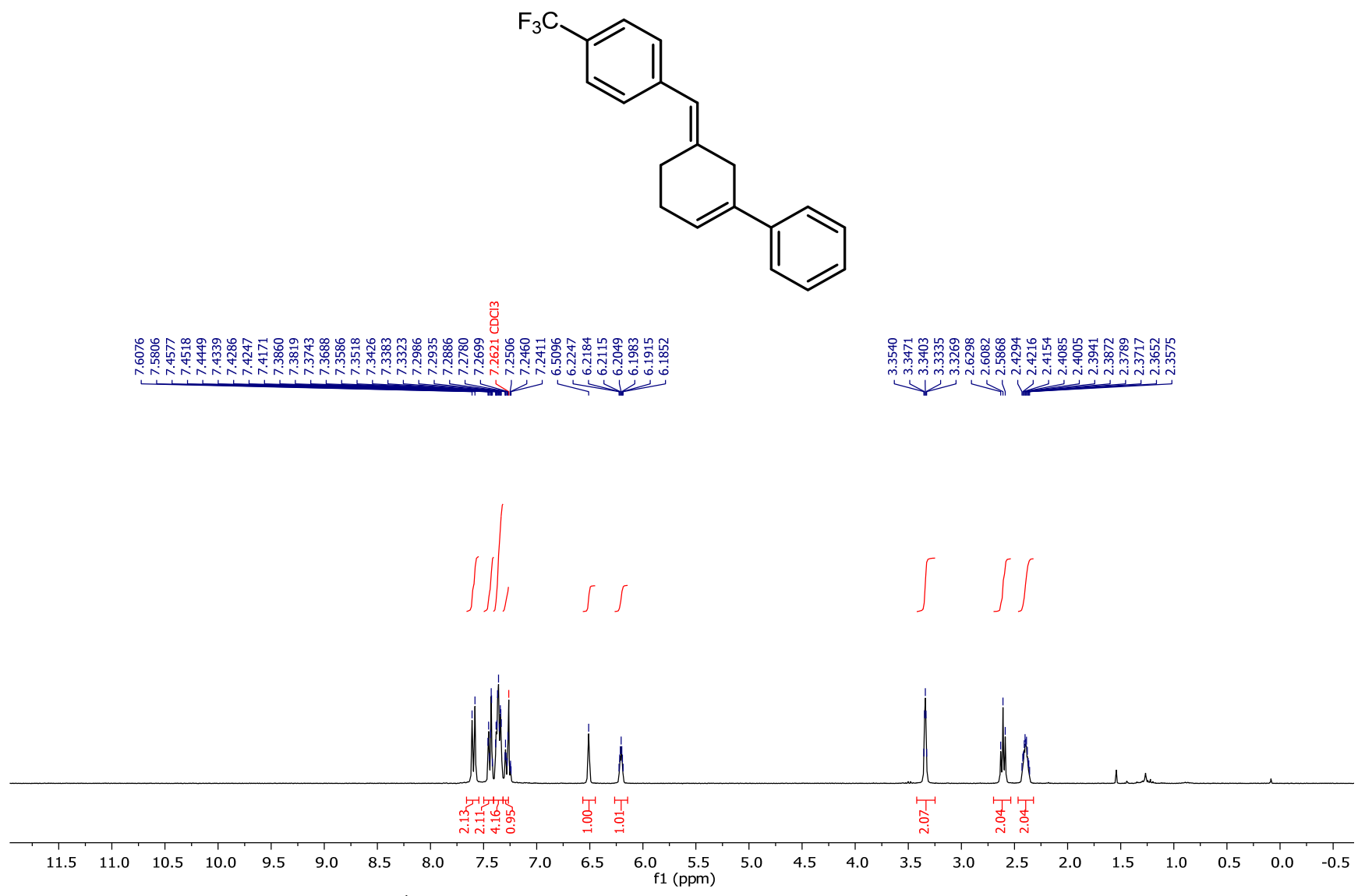

Figure S46. $\quad{ }^{1} \mathrm{H}$ NMR of $12\left(300 \mathrm{MHz}, \mathrm{CDCl}_{3}, 295 \mathrm{~K}\right)$.

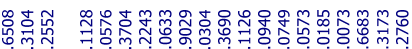

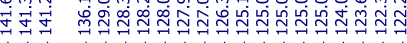

$>$
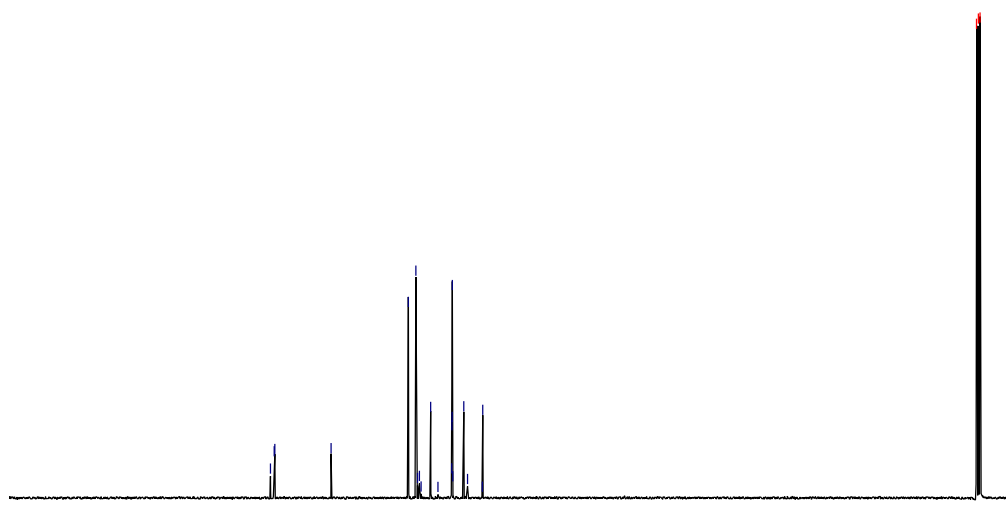

Figure S47.

${ }^{13} \mathrm{C}$ NMR of $12\left(201 \mathrm{MHz}, \mathrm{CDCl}_{3}, 295 \mathrm{~K}\right)$. 


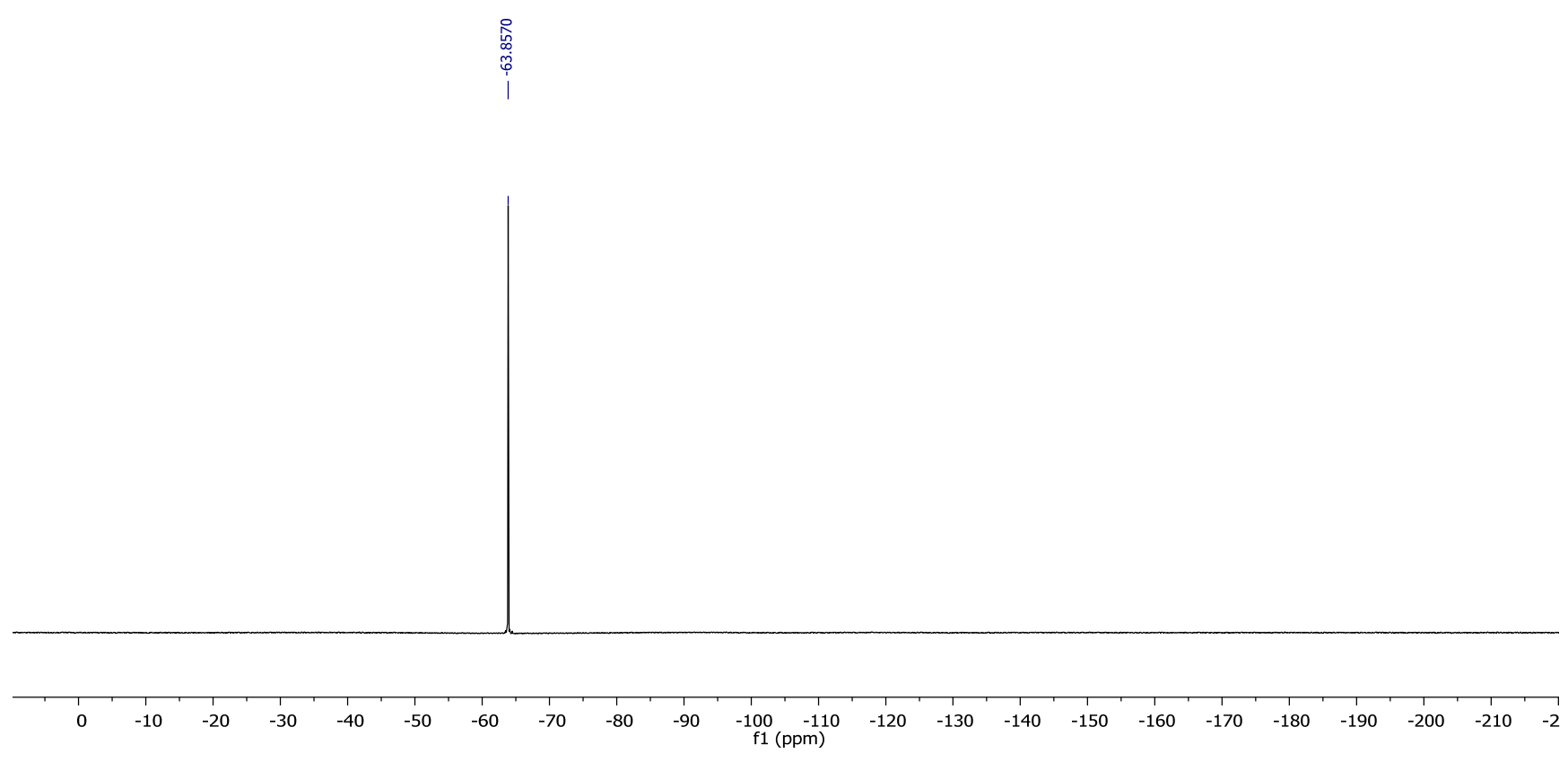

Figure S48. $\quad{ }^{19} \mathrm{~F} \mathrm{NMR} \mathrm{of} 12\left(282 \mathrm{MHz}, \mathrm{CDCl}_{3}, 295 \mathrm{~K}\right)$. 


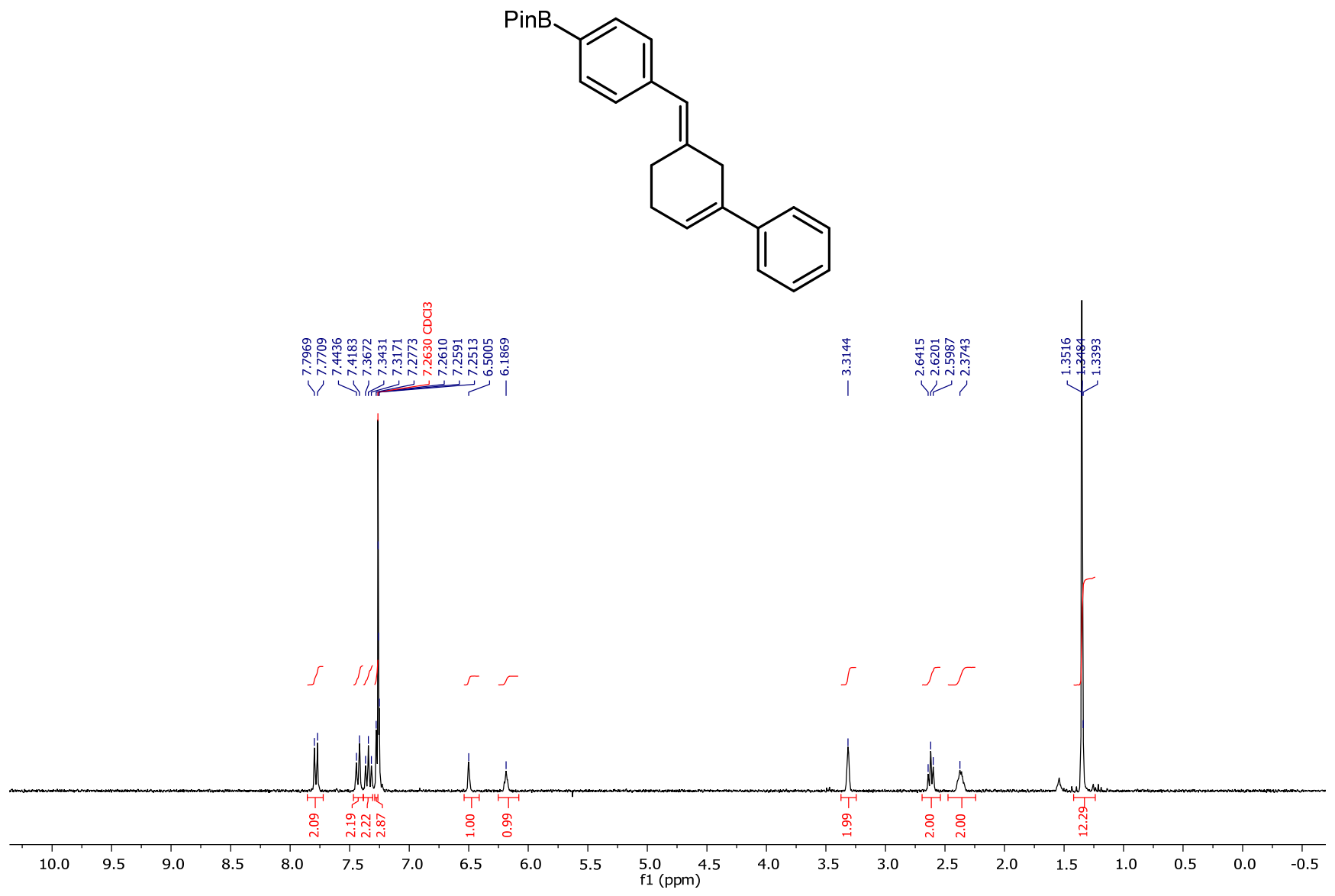

Figure S49. $\quad{ }^{1} \mathrm{H} \mathrm{NMR}$ of $13\left(300 \mathrm{MHz}, \mathrm{CDCl}_{3}, 295 \mathrm{~K}\right)$.

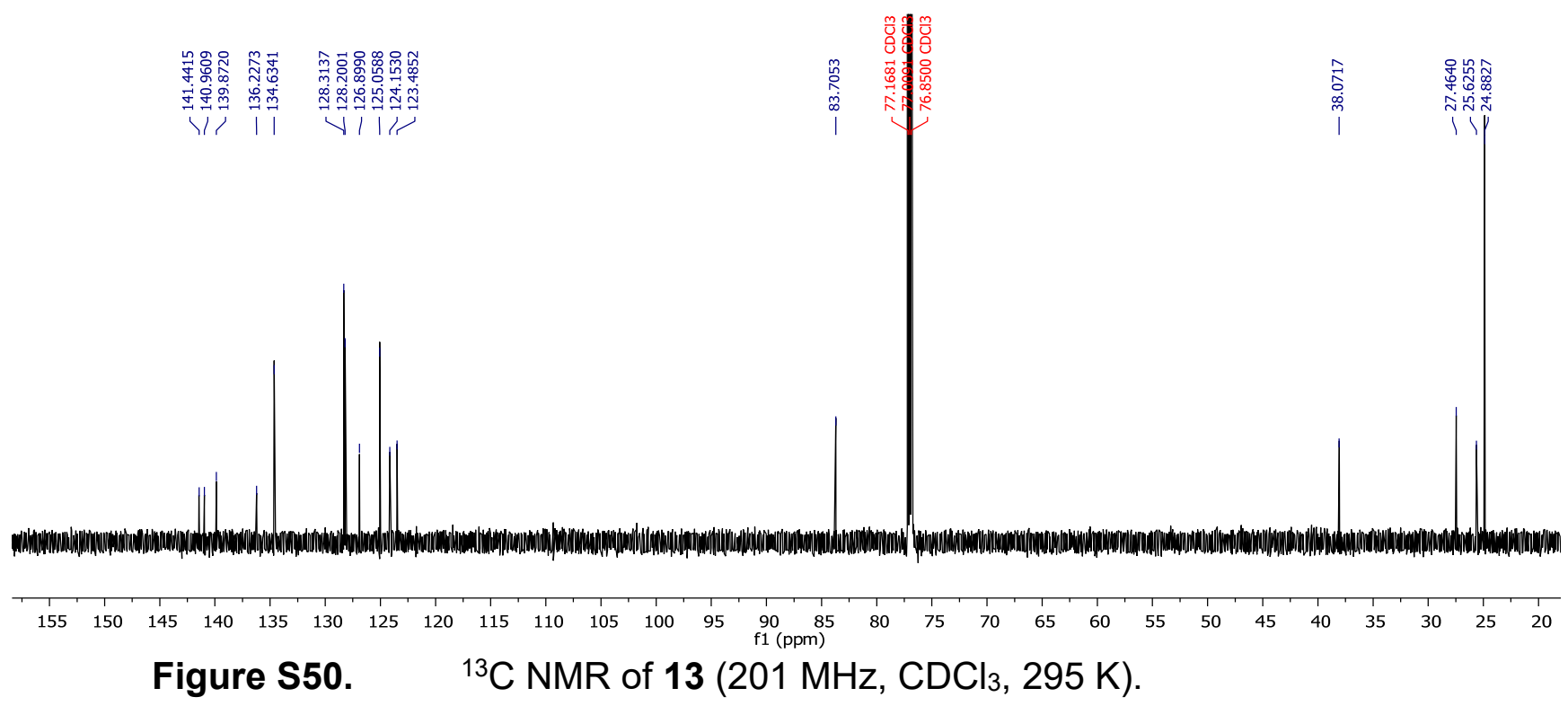




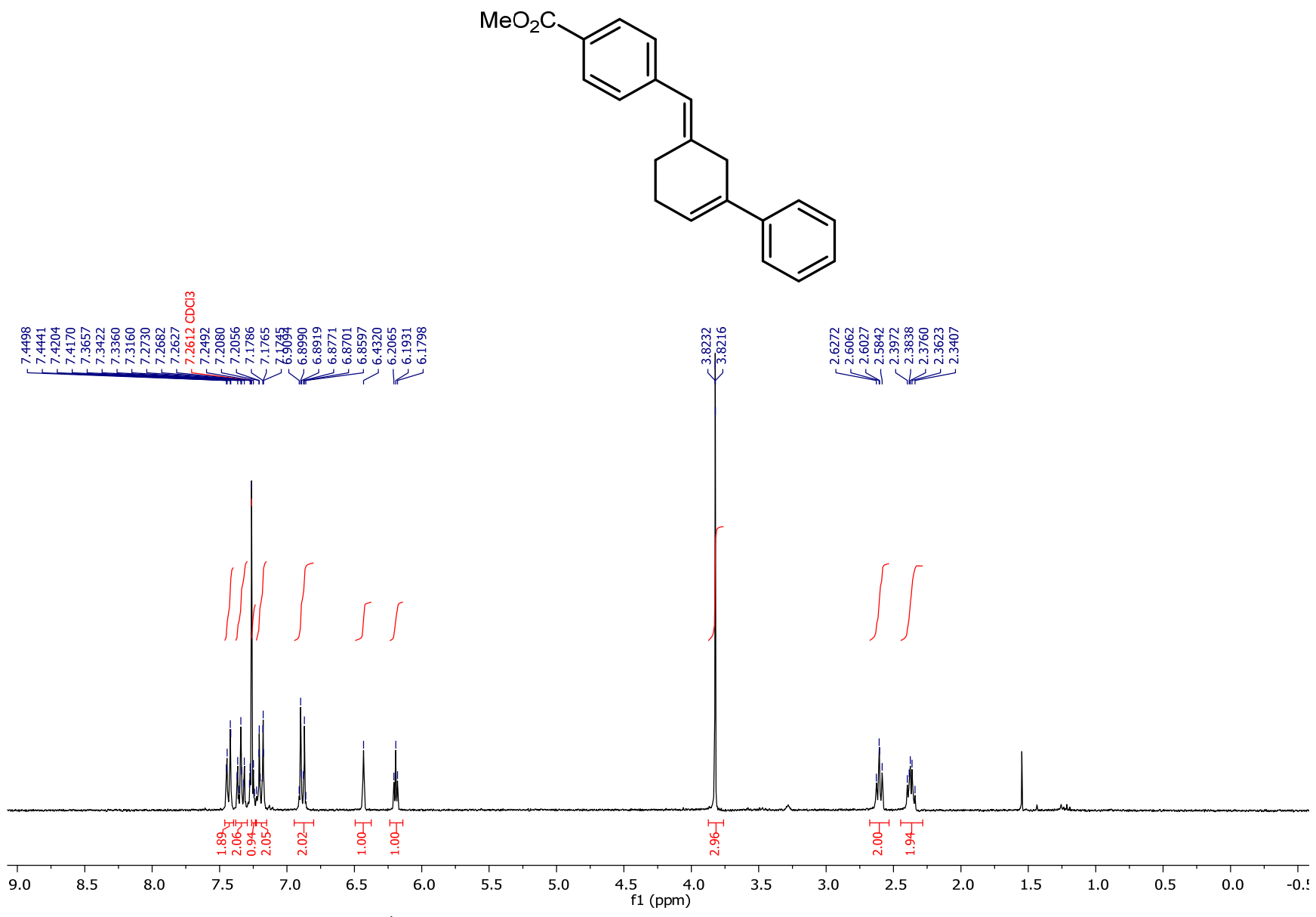

Figure S51. $\quad{ }^{1} \mathrm{H}$ NMR of $\mathbf{1 4}\left(300 \mathrm{MHz}, \mathrm{CDCl}_{3}, 295 \mathrm{~K}\right)$.

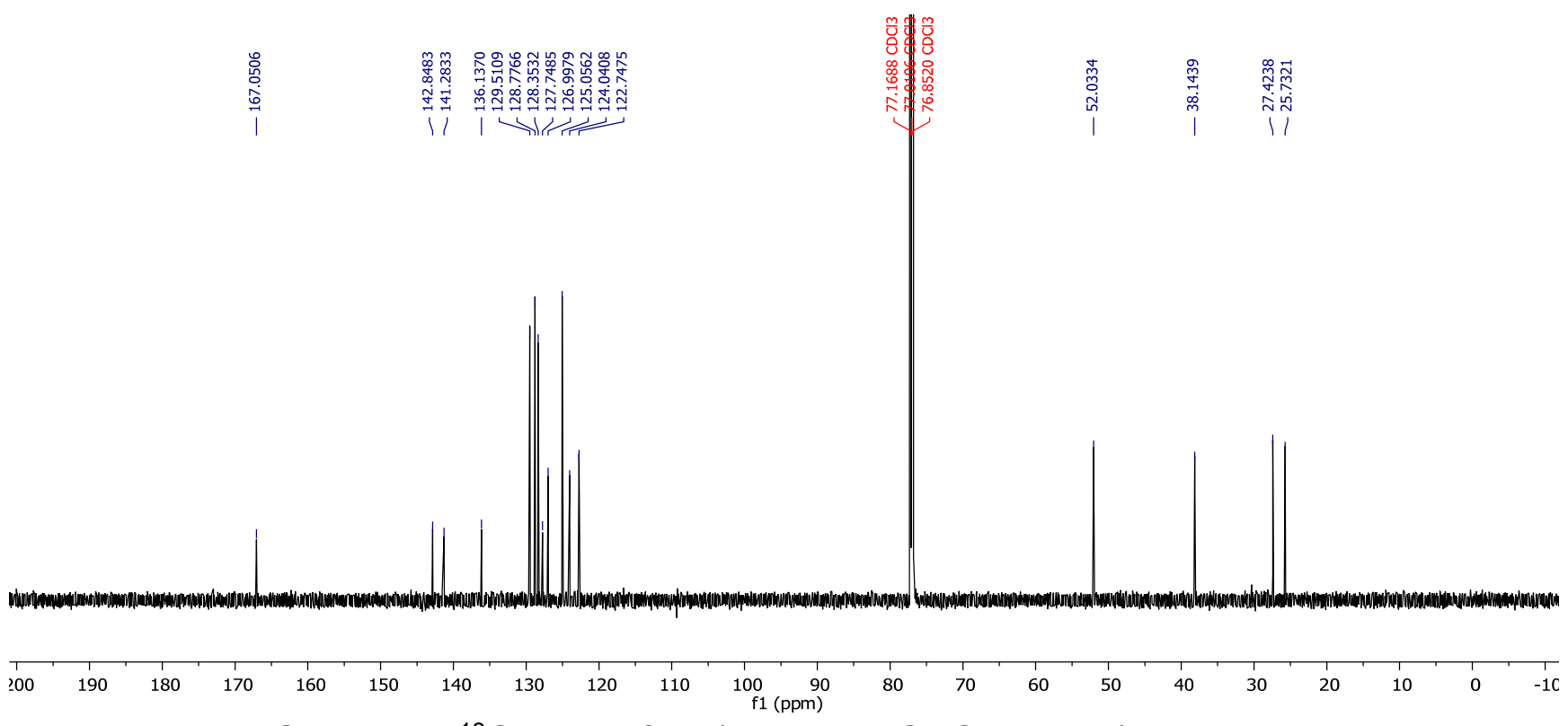

Figure S52. $\quad{ }^{13} \mathrm{C} \mathrm{NMR} \mathrm{of} 14\left(201 \mathrm{MHz}, \mathrm{CDCl}_{3}, 295 \mathrm{~K}\right)$. 


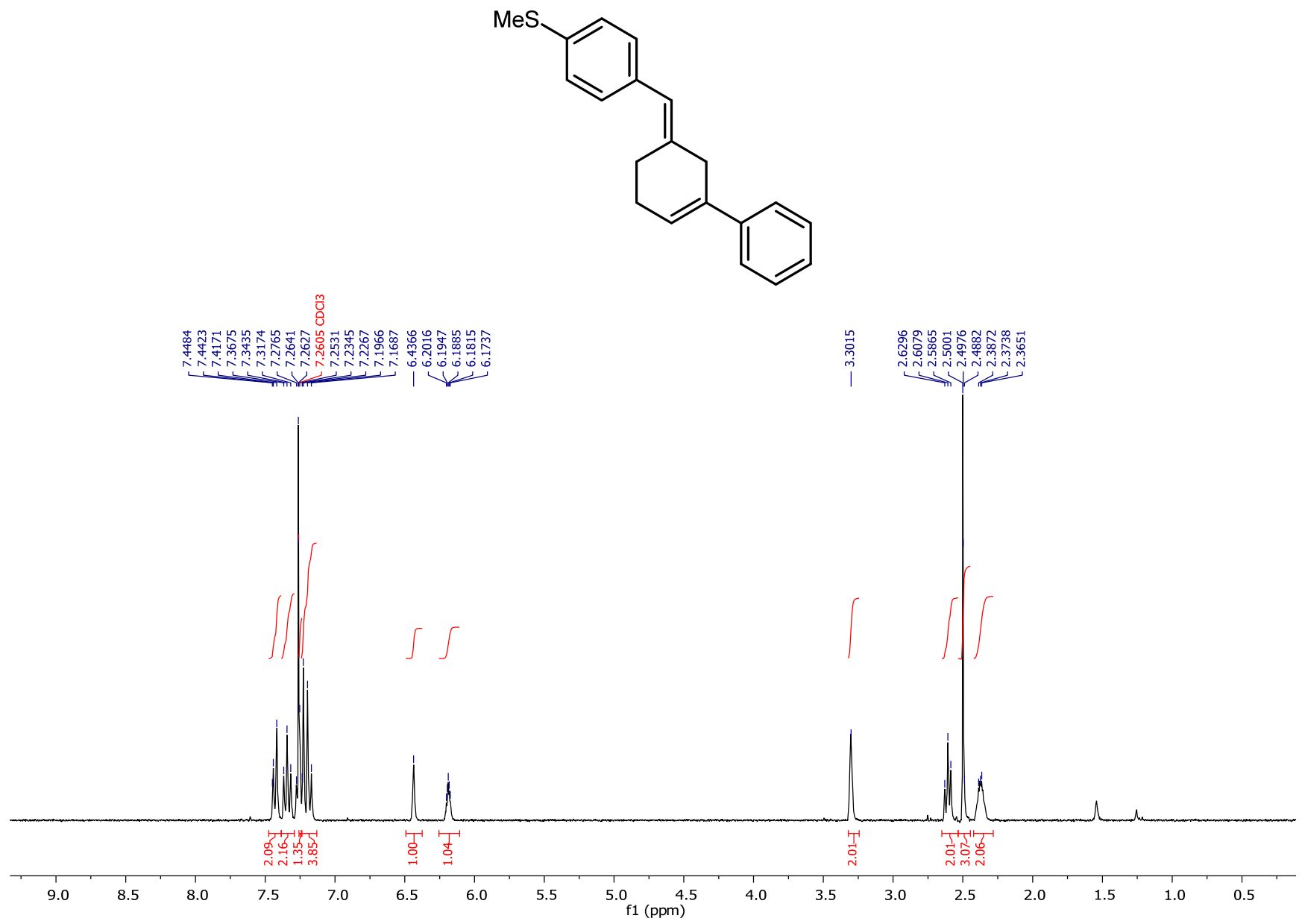

Figure S53. $\quad{ }^{1} \mathrm{H}$ NMR of $15\left(300 \mathrm{MHz}, \mathrm{CDCl}_{3}, 295 \mathrm{~K}\right)$.

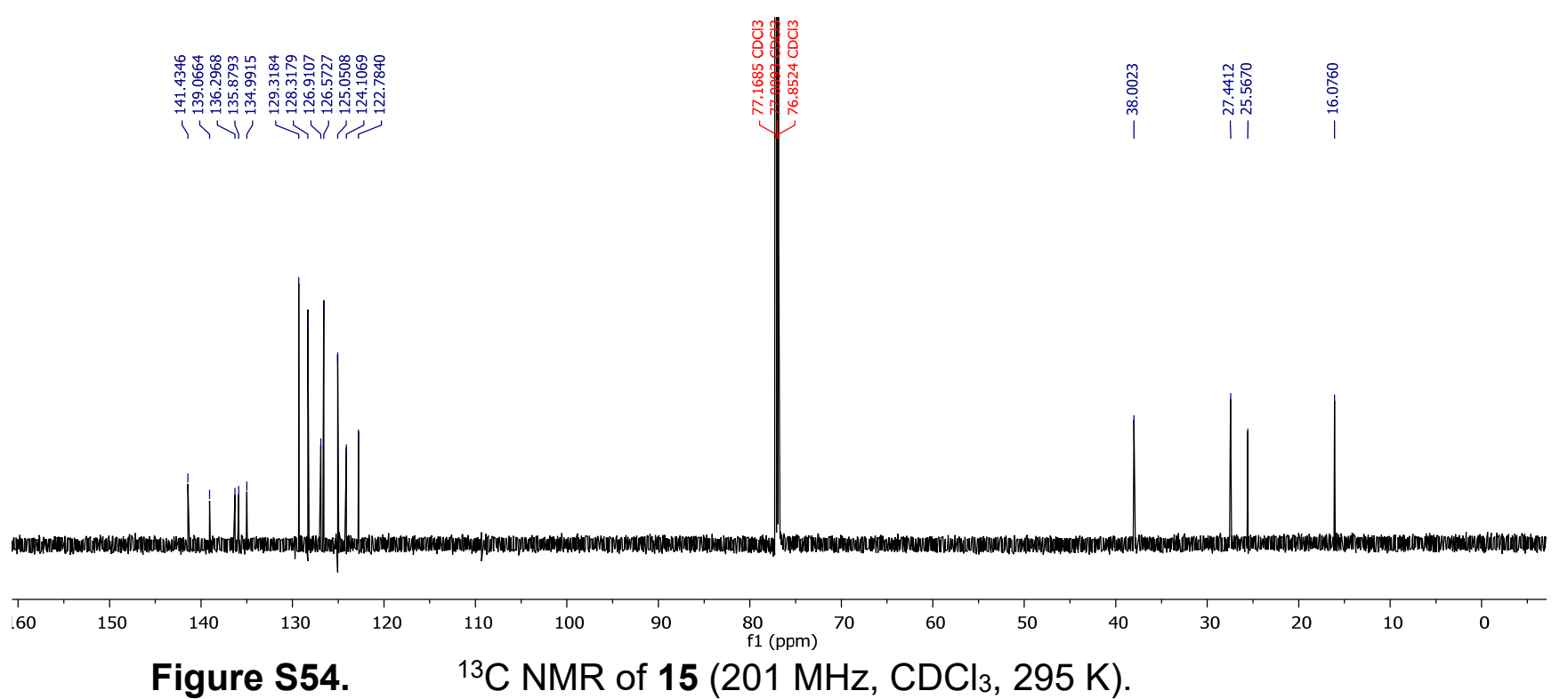

Figure S54. $\quad{ }^{13} \mathrm{C}$ NMR of $15\left(201 \mathrm{MHz}, \mathrm{CDCl}_{3}, 295 \mathrm{~K}\right)$. 


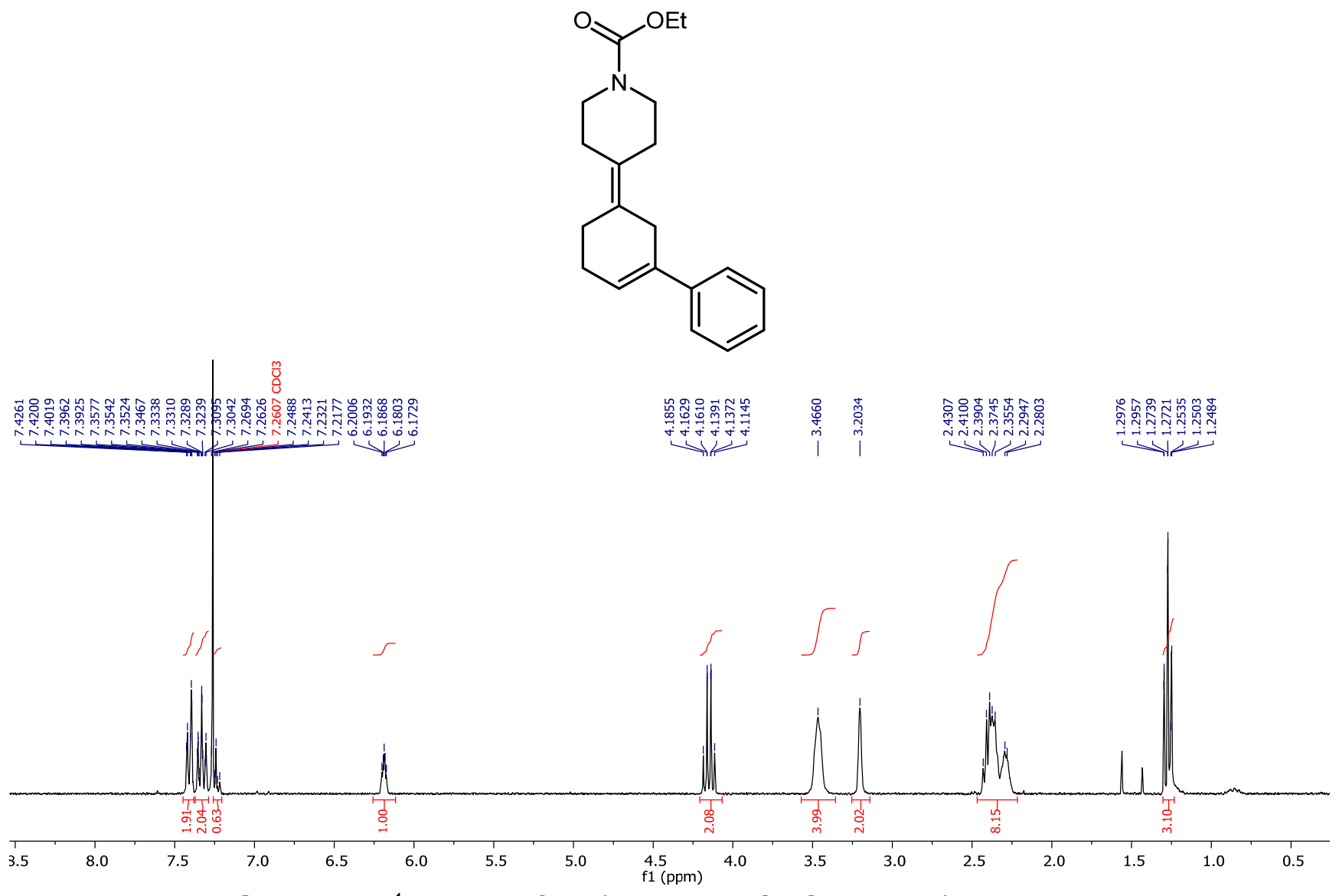

Figure S55. $\quad{ }^{1} \mathrm{H}$ NMR of $\mathbf{1 6}\left(300 \mathrm{MHz}, \mathrm{CDCl}_{3}, 295 \mathrm{~K}\right)$.

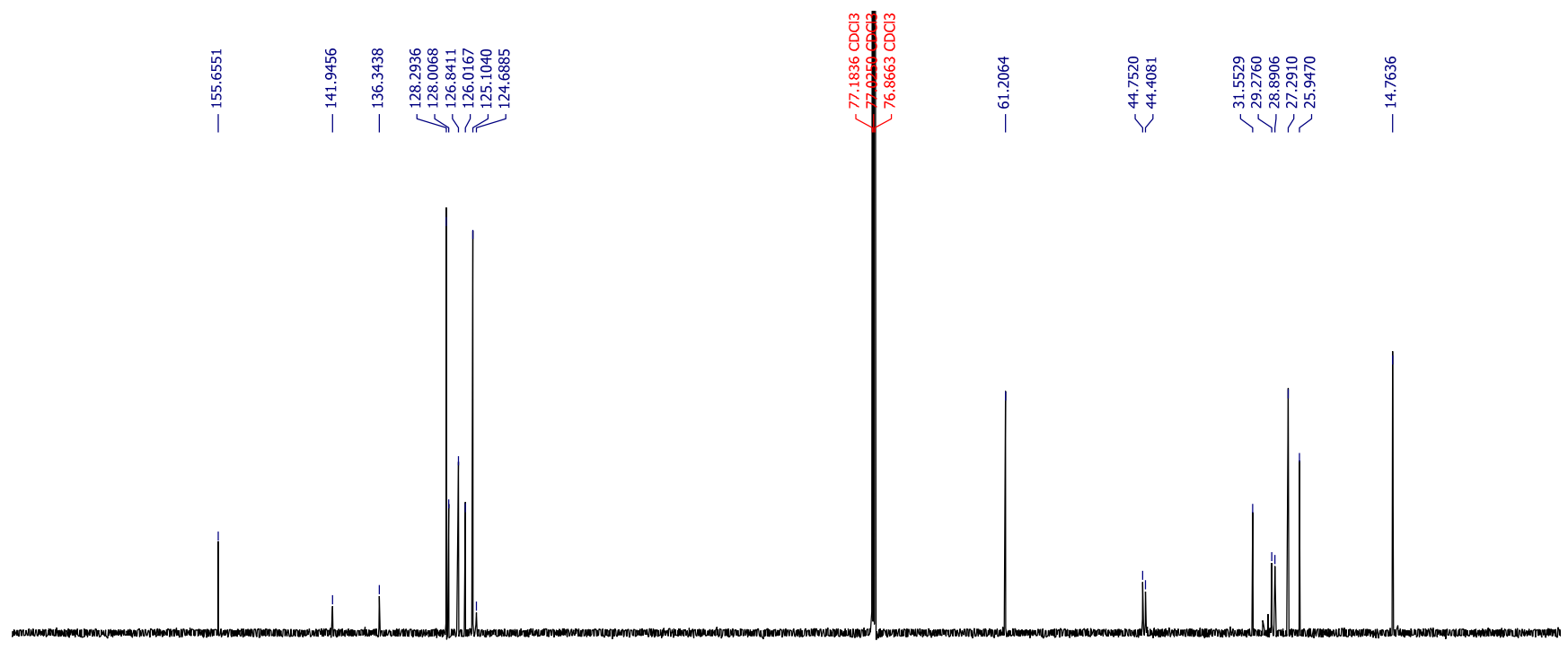

Figure S56. $\quad{ }^{13} \mathrm{C}$ NMR of $16\left(201 \mathrm{MHz}, \mathrm{CDCl}_{3}, 295 \mathrm{~K}\right)$. 
<smiles>C1=C(c2ccccc2)CC(=C2CCOCC2)CC1</smiles>

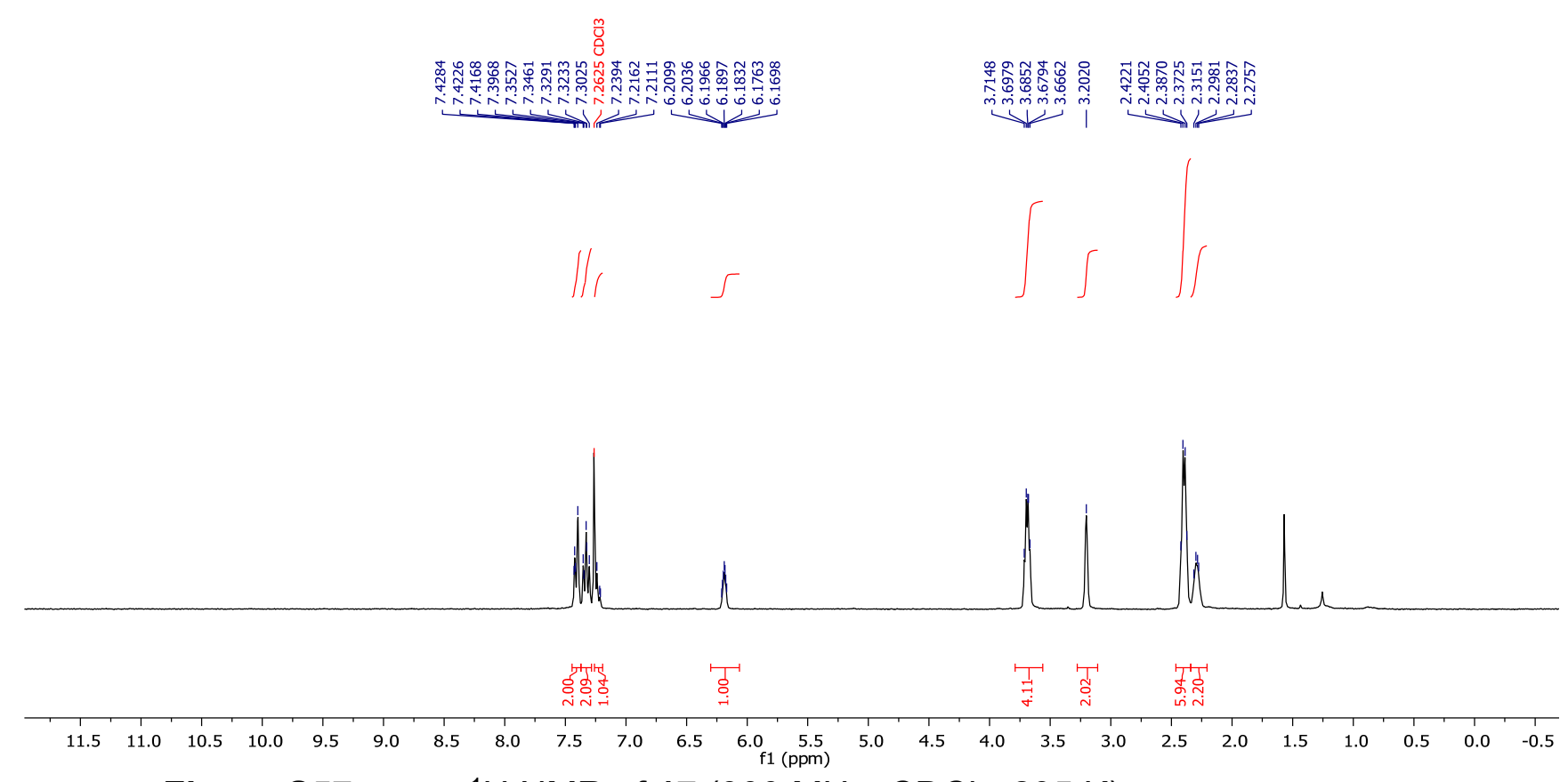

Figure S57. $\quad{ }^{1} \mathrm{H}$ NMR of $\mathbf{1 7}\left(300 \mathrm{MHz}, \mathrm{CDCl}_{3}, 295 \mathrm{~K}\right)$.

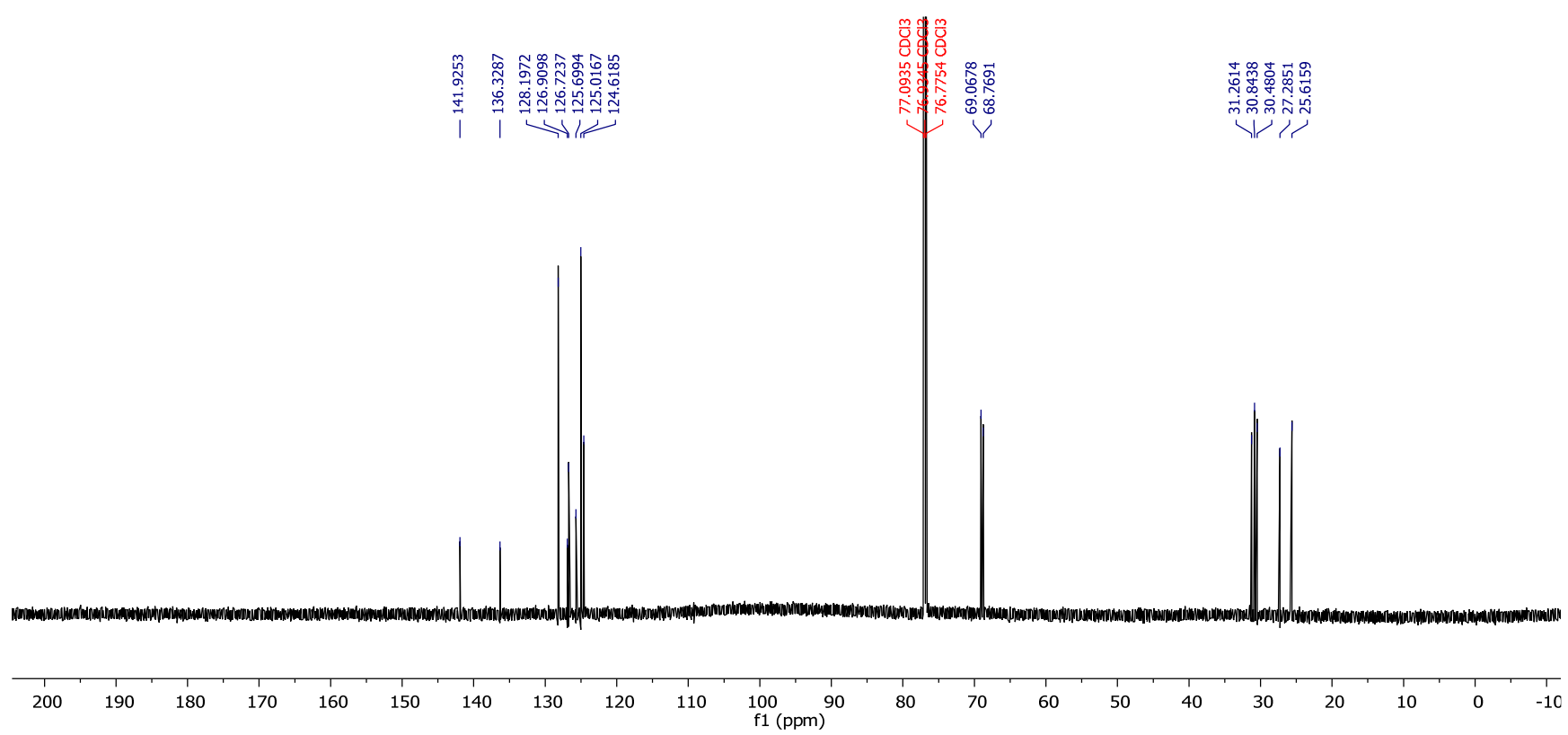

Figure S58. $\quad{ }^{13} \mathrm{C}$ NMR of $17\left(201 \mathrm{MHz}, \mathrm{CDCl}_{3}, 295 \mathrm{~K}\right)$. 


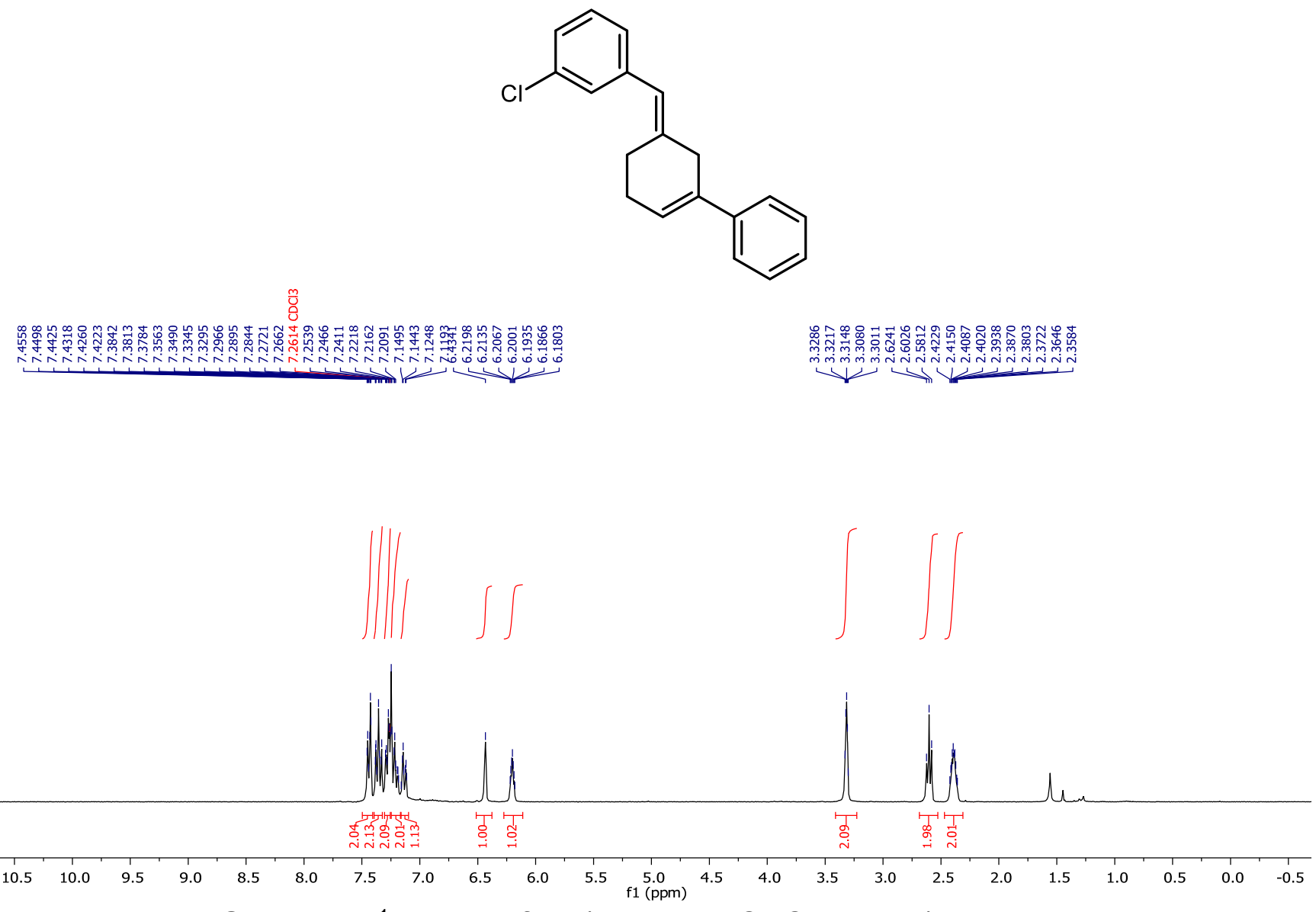

Figure S59. $\quad{ }^{1} \mathrm{H}$ NMR of $18\left(300 \mathrm{MHz}, \mathrm{CDCl}_{3}, 295 \mathrm{~K}\right)$.
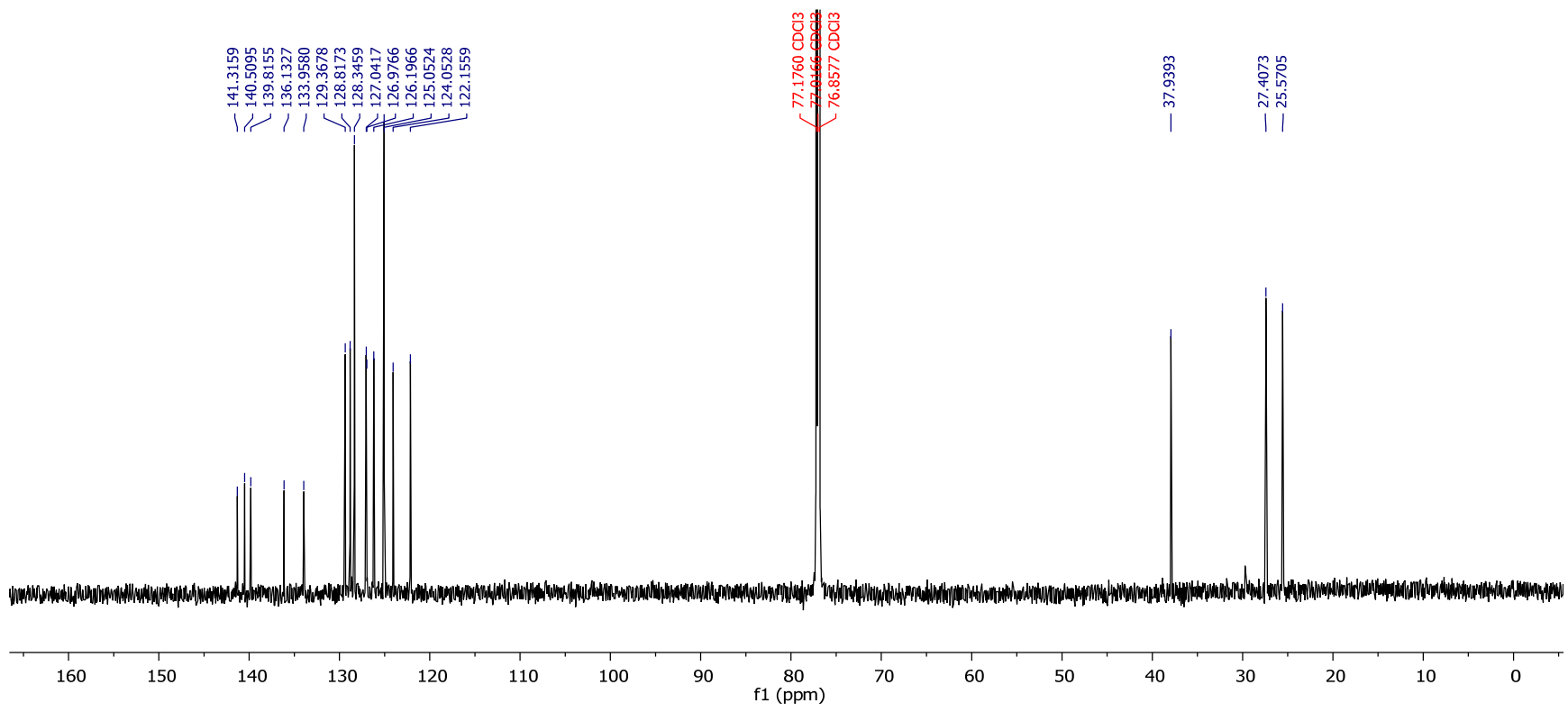

Figure S60. $\quad{ }^{13} \mathrm{C}$ NMR of $18\left(201 \mathrm{MHz}, \mathrm{CDCl}_{3}, 295 \mathrm{~K}\right)$. 


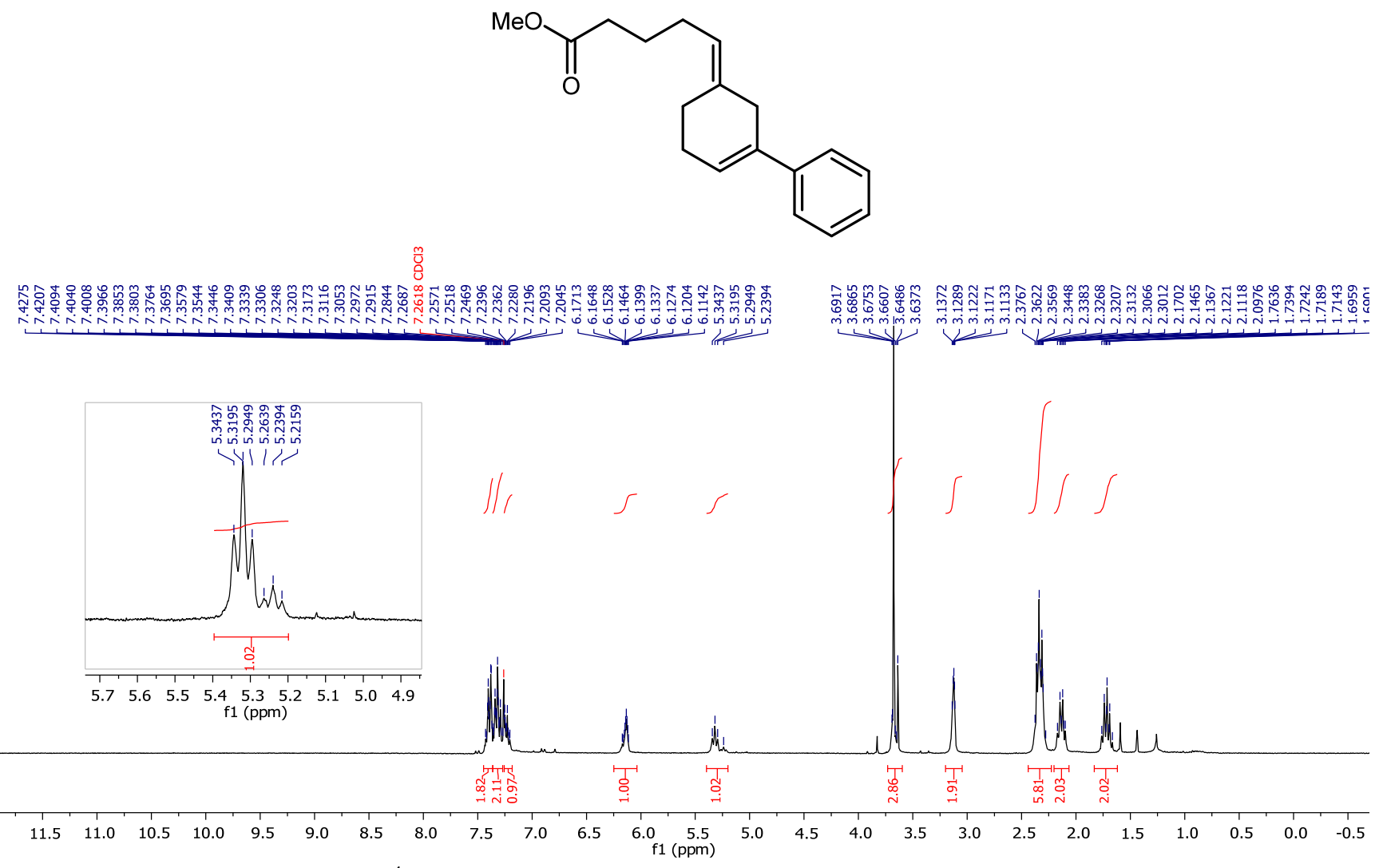

Figure S61. $\quad{ }^{1} \mathrm{H}$ NMR of $19\left(300 \mathrm{MHz}, \mathrm{CDCl}_{3}, 295 \mathrm{~K}\right)$.

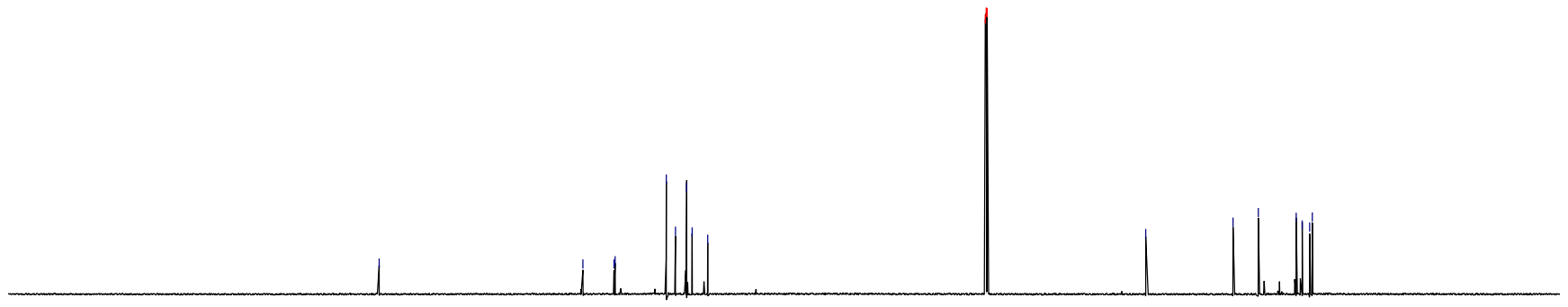

$\begin{array}{rlllllllllllllllllllllllllll}230 & 220 & 210 & 200 & 190 & 180 & 170 & 160 & 150 & 140 & 130 & 120 & 110 & 100 & 90 & 80 & 70 & 60 & 50 & 40 & 30 & 20 & 10 & 0 & -10\end{array}$ Figure S62. $\quad{ }^{13} \mathrm{C}$ NMR of $19\left(201 \mathrm{MHz}, \mathrm{CDCl}_{3}, 295 \mathrm{~K}\right)$. 


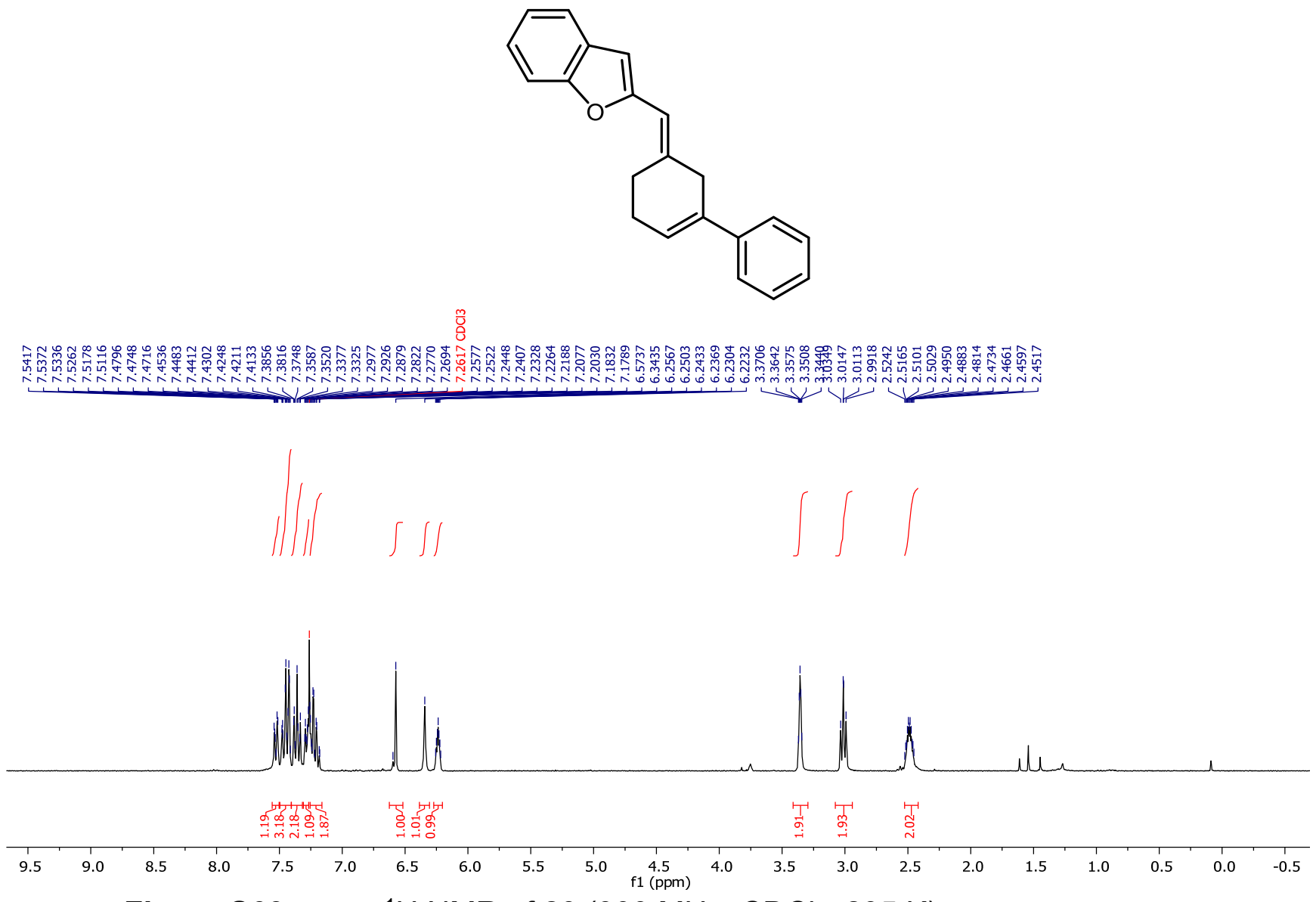

Figure S63. $\quad{ }^{1} \mathrm{H}$ NMR of $20\left(300 \mathrm{MHz}, \mathrm{CDCl}_{3}, 295 \mathrm{~K}\right)$.
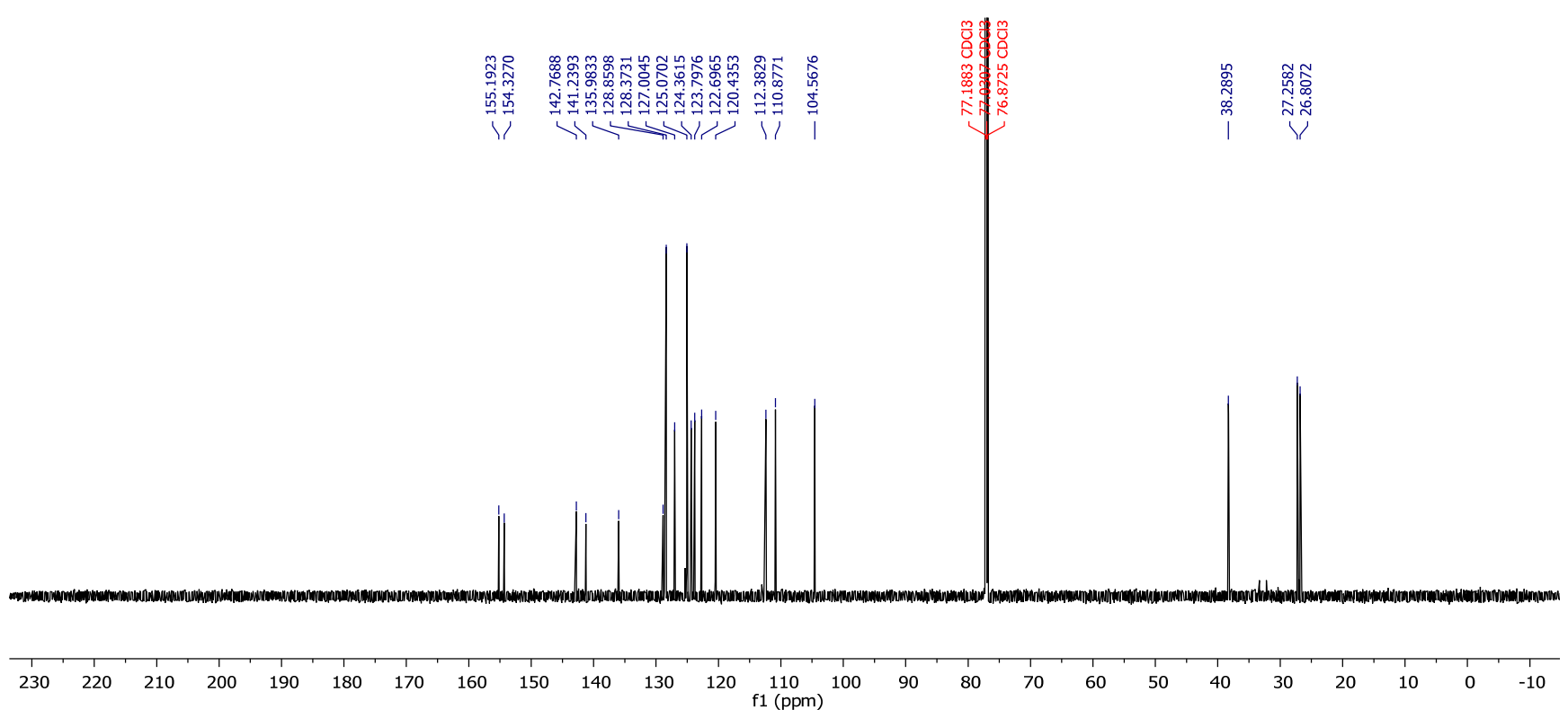

Figure S64. $\quad{ }^{13} \mathrm{C}$ NMR of $20\left(201 \mathrm{MHz}, \mathrm{CDCl}_{3}, 295 \mathrm{~K}\right)$. 


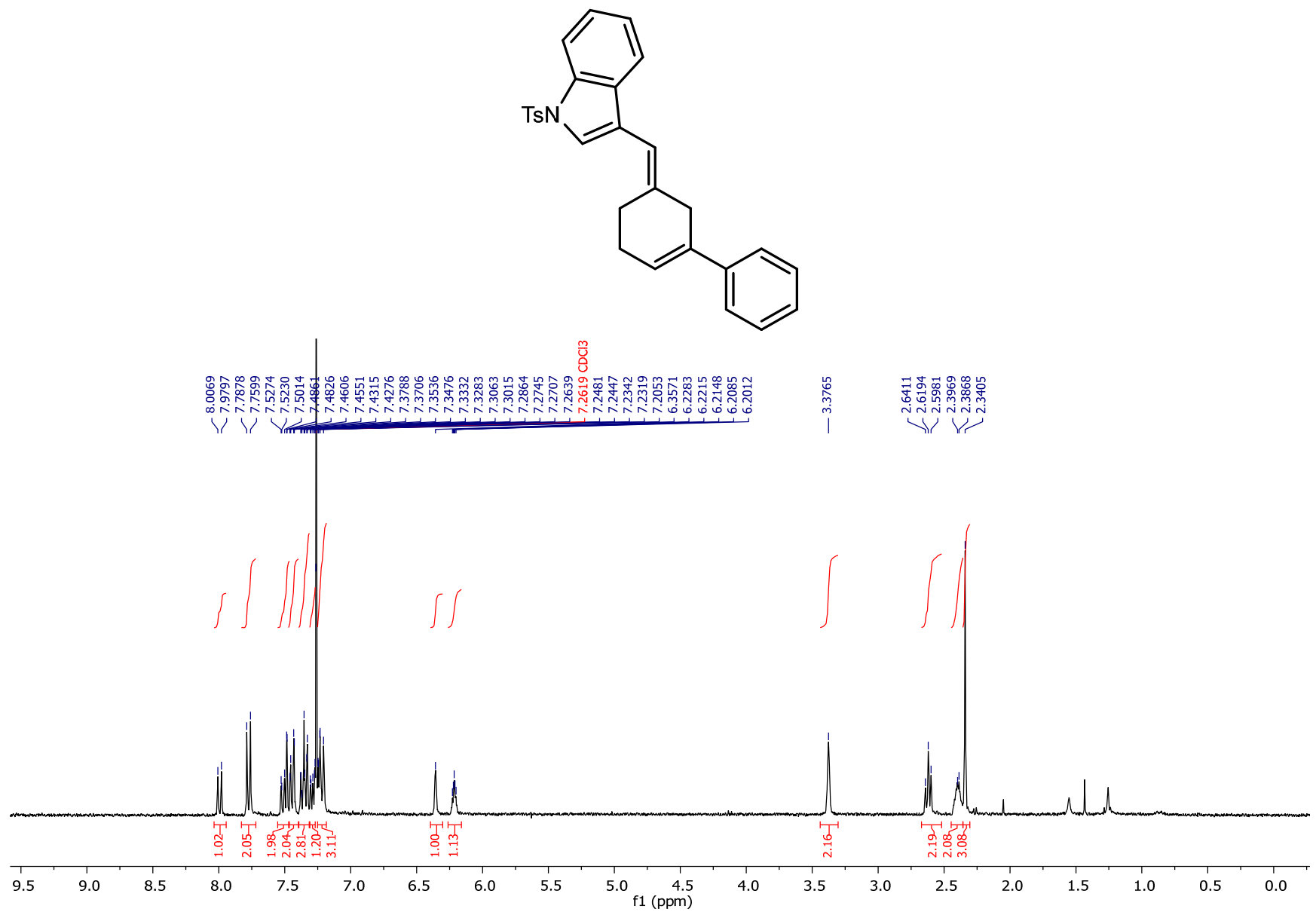

Figure S65. $\quad{ }^{1} \mathrm{H}$ NMR of $21\left(300 \mathrm{MHz}, \mathrm{CDCl}_{3}, 295 \mathrm{~K}\right)$.
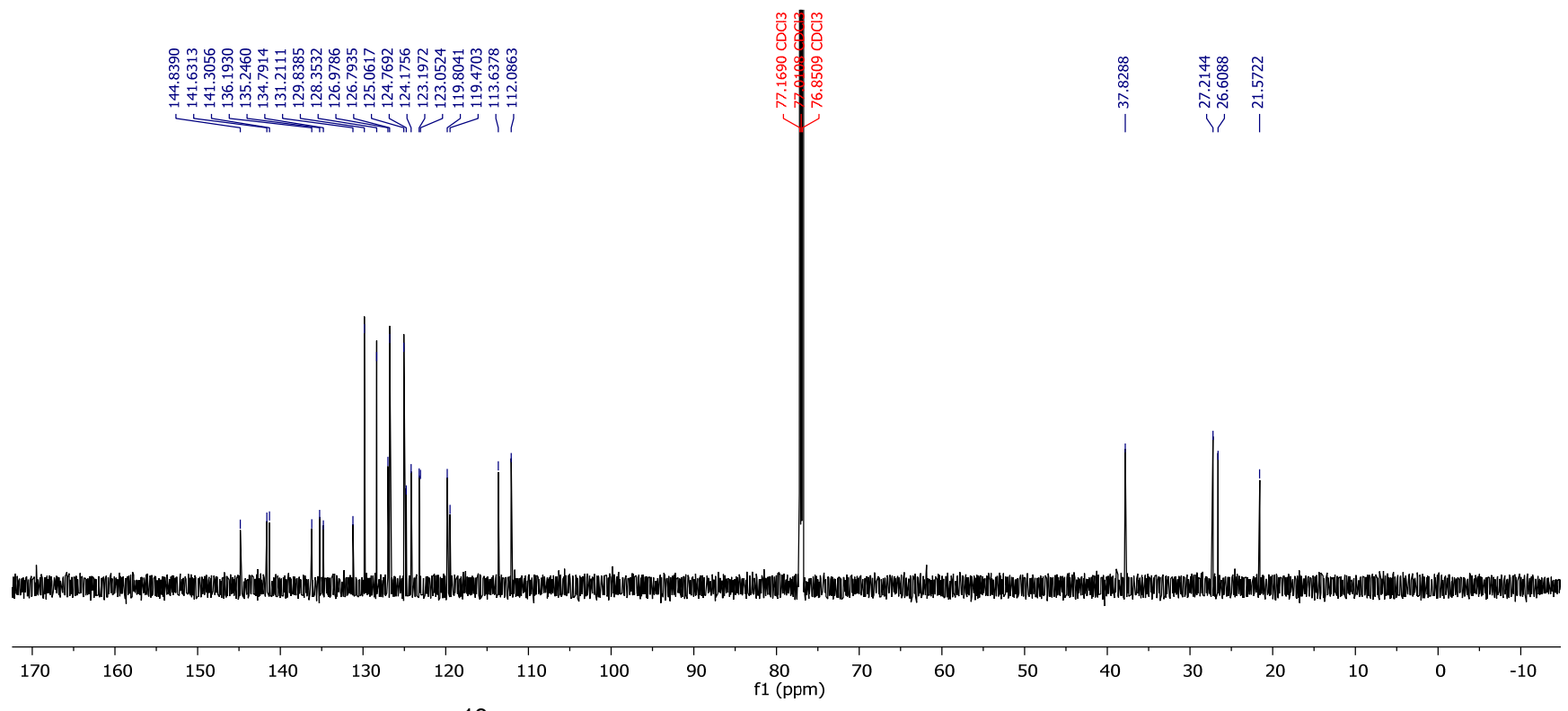

Figure S66. $\quad{ }^{13} \mathrm{C} \mathrm{NMR} \mathrm{of} 21\left(201 \mathrm{MHz}, \mathrm{CDCl}_{3}, 295 \mathrm{~K}\right)$. 
<smiles>C1=C(c2ccccc2)CCC=C1c1cccs1</smiles>
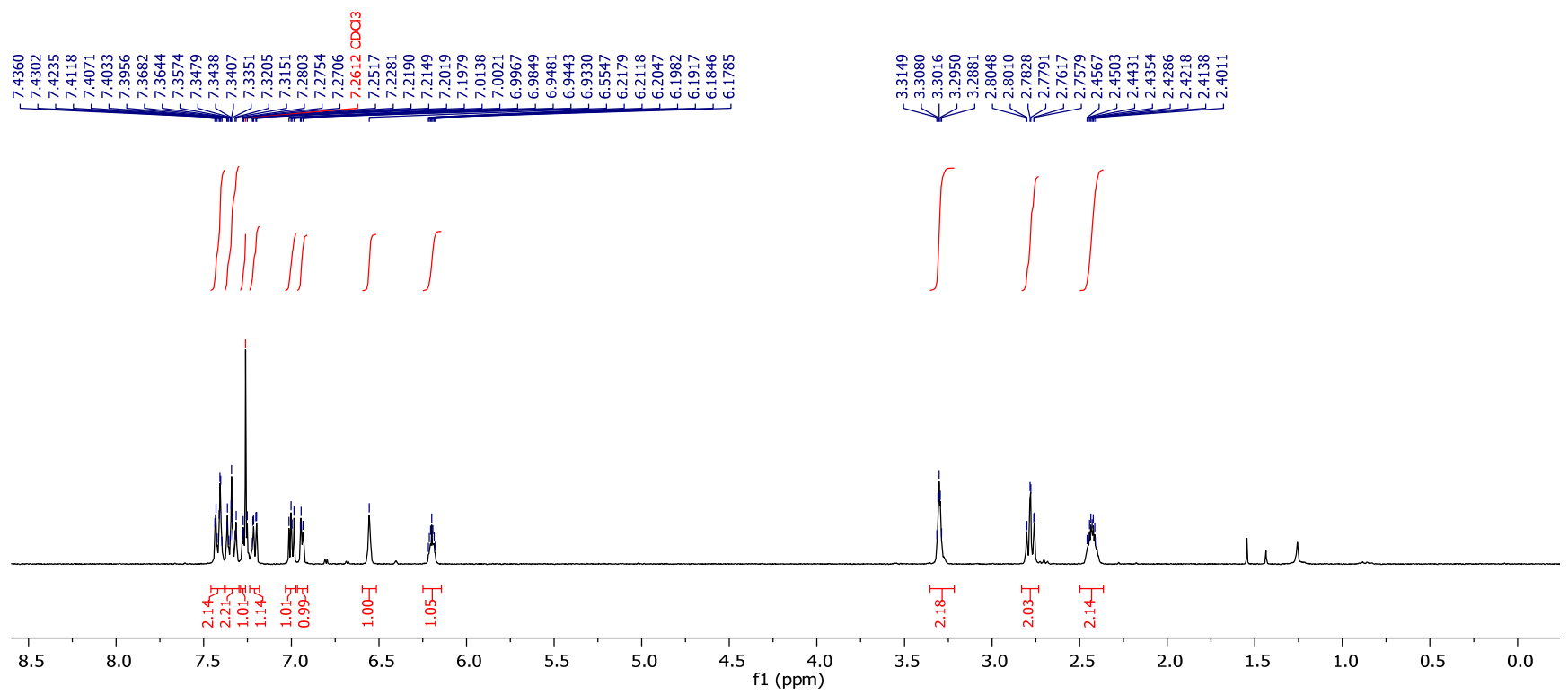

Figure S67. $\quad{ }^{1} \mathrm{H}$ NMR of $22\left(300 \mathrm{MHz}, \mathrm{CDCl}_{3}, 295 \mathrm{~K}\right)$.

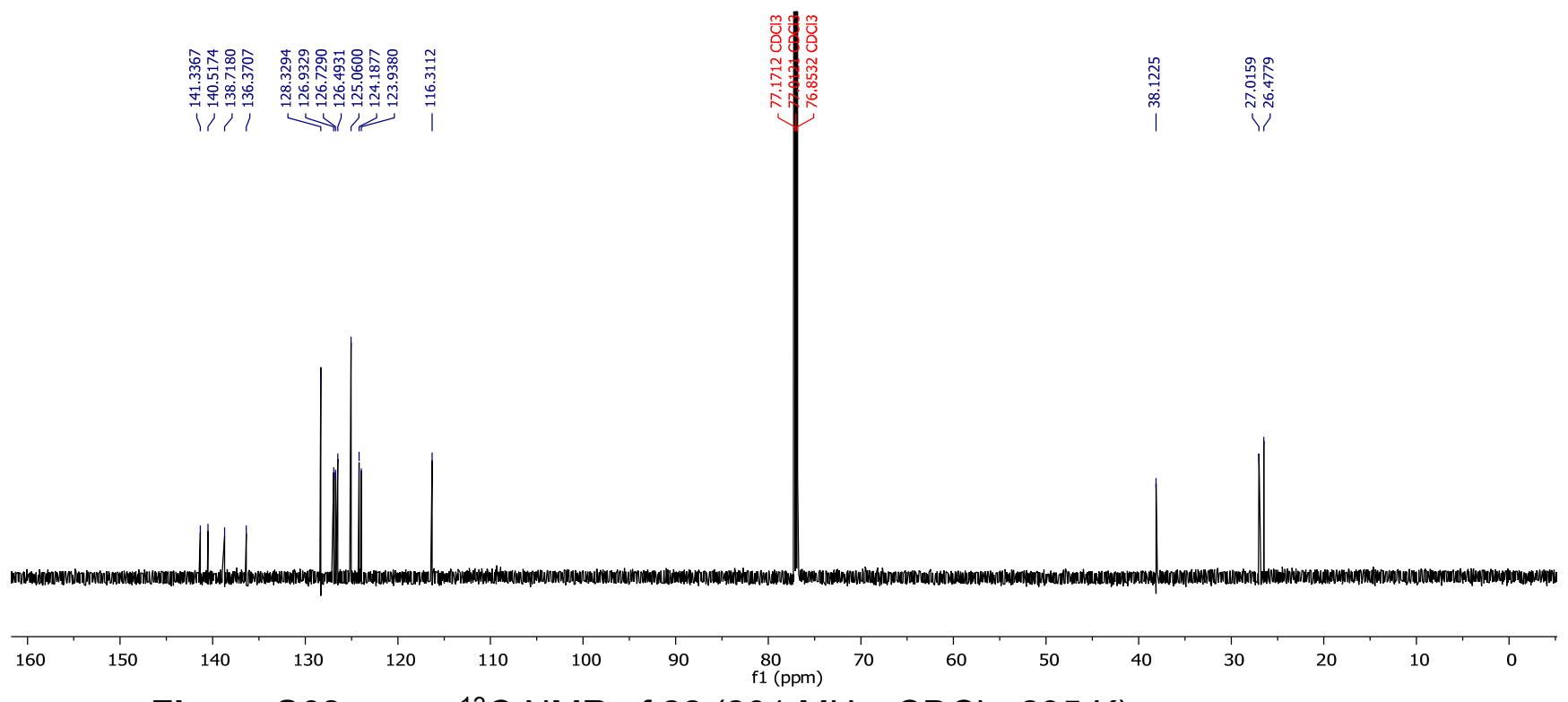

Figure S68. $\quad{ }^{13} \mathrm{C}$ NMR of $22\left(201 \mathrm{MHz}, \mathrm{CDCl}_{3}, 295 \mathrm{~K}\right)$. 
Supporting Information

583<smiles>C1=C(c2ccccc2)C/C(=C\C2CCOCC2)CC1</smiles>

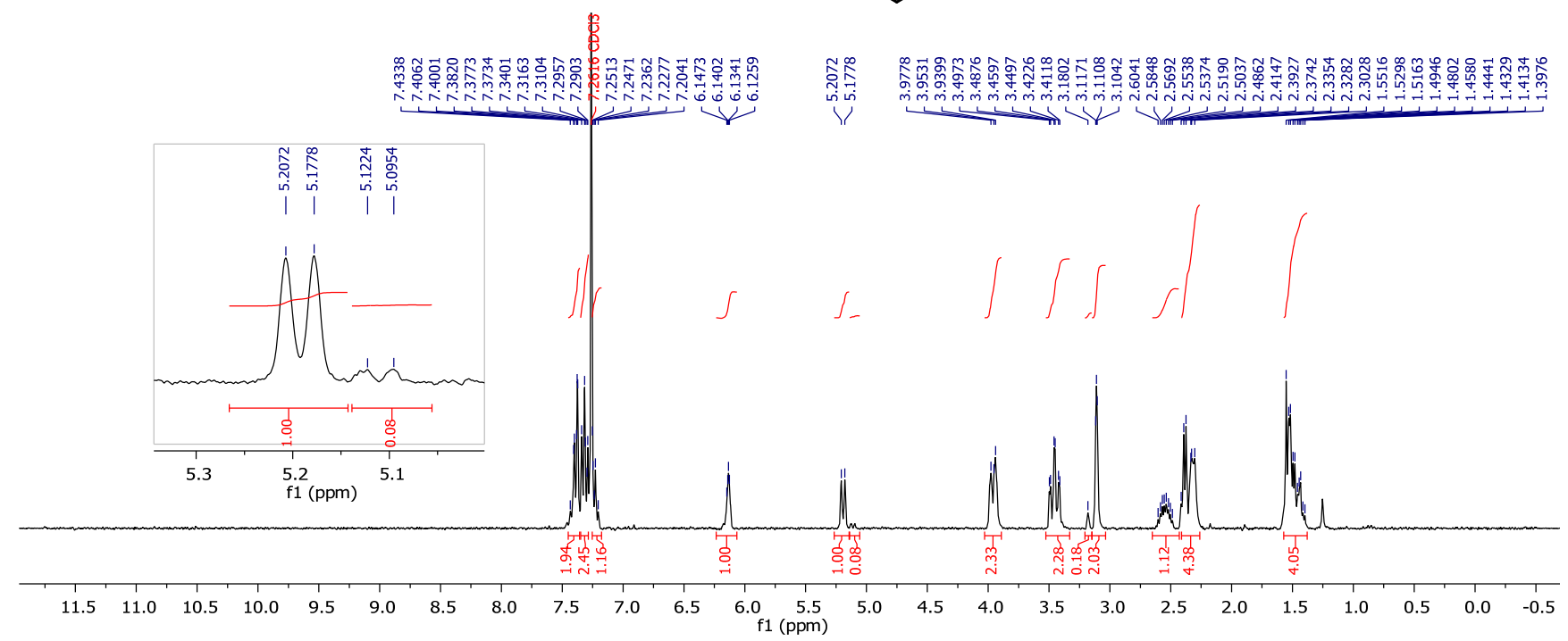

Figure S69. $\quad{ }^{1} \mathrm{H}$ NMR of $23\left(300 \mathrm{MHz}, \mathrm{CDCl}_{3}, 295 \mathrm{~K}\right)$.
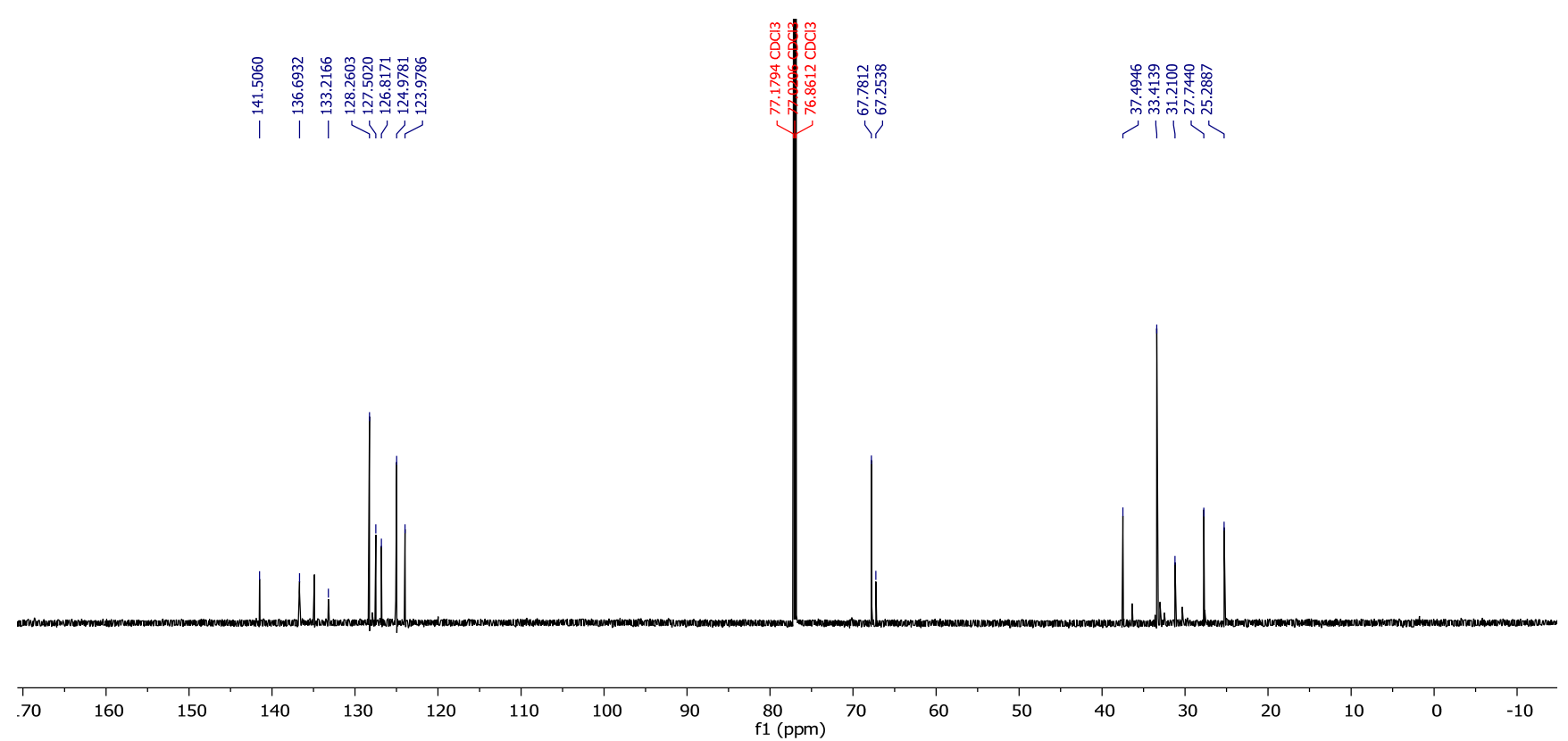

Figure S70.

${ }^{13} \mathrm{C}$ NMR of $23\left(201 \mathrm{MHz}, \mathrm{CDCl}_{3}, 295 \mathrm{~K}\right)$. 
<smiles>COc1ccc(C=C2CCC=C(c3ccccc3)C2)cc1OC</smiles>
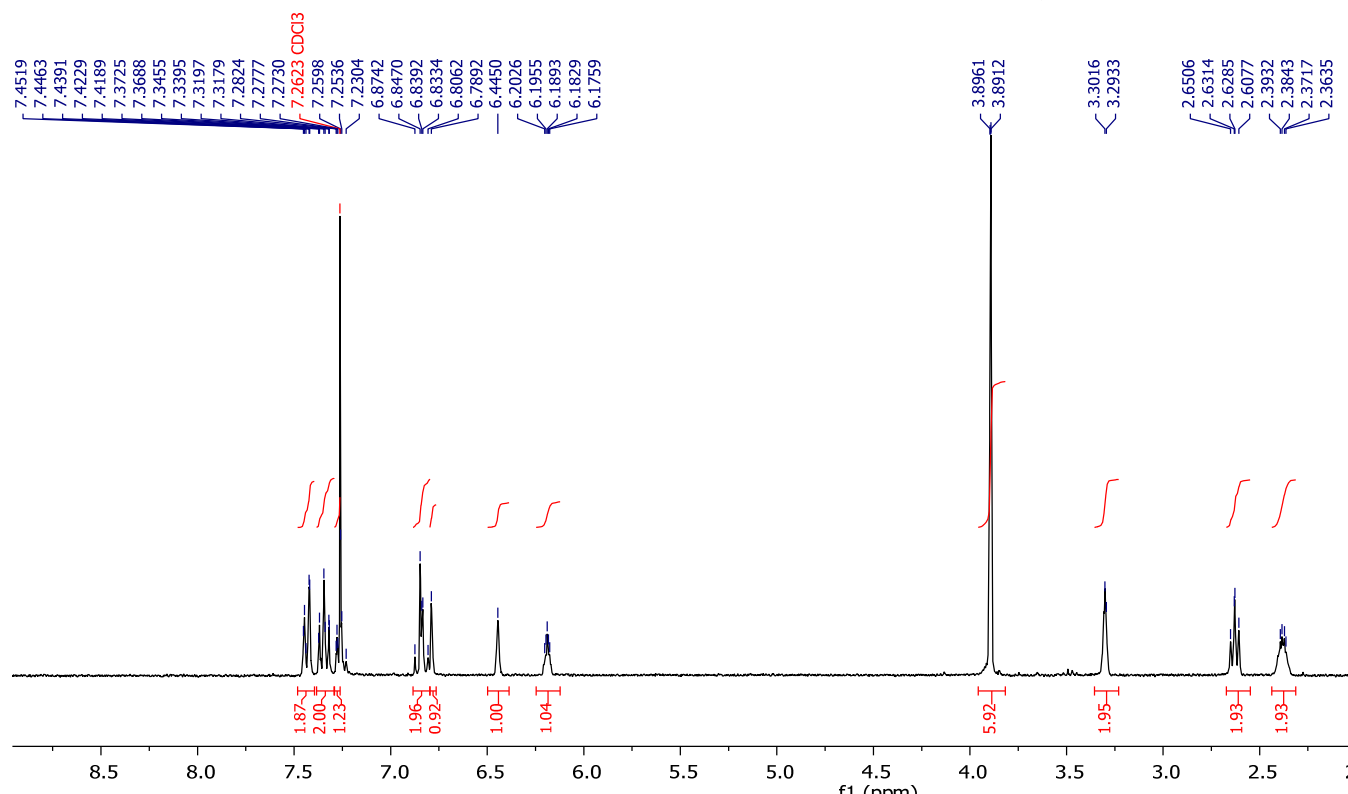

Figure S71. $\quad{ }^{1} \mathrm{H}$ NMR of $24\left(300 \mathrm{MHz}, \mathrm{CDCl}_{3}, 295 \mathrm{~K}\right)$.
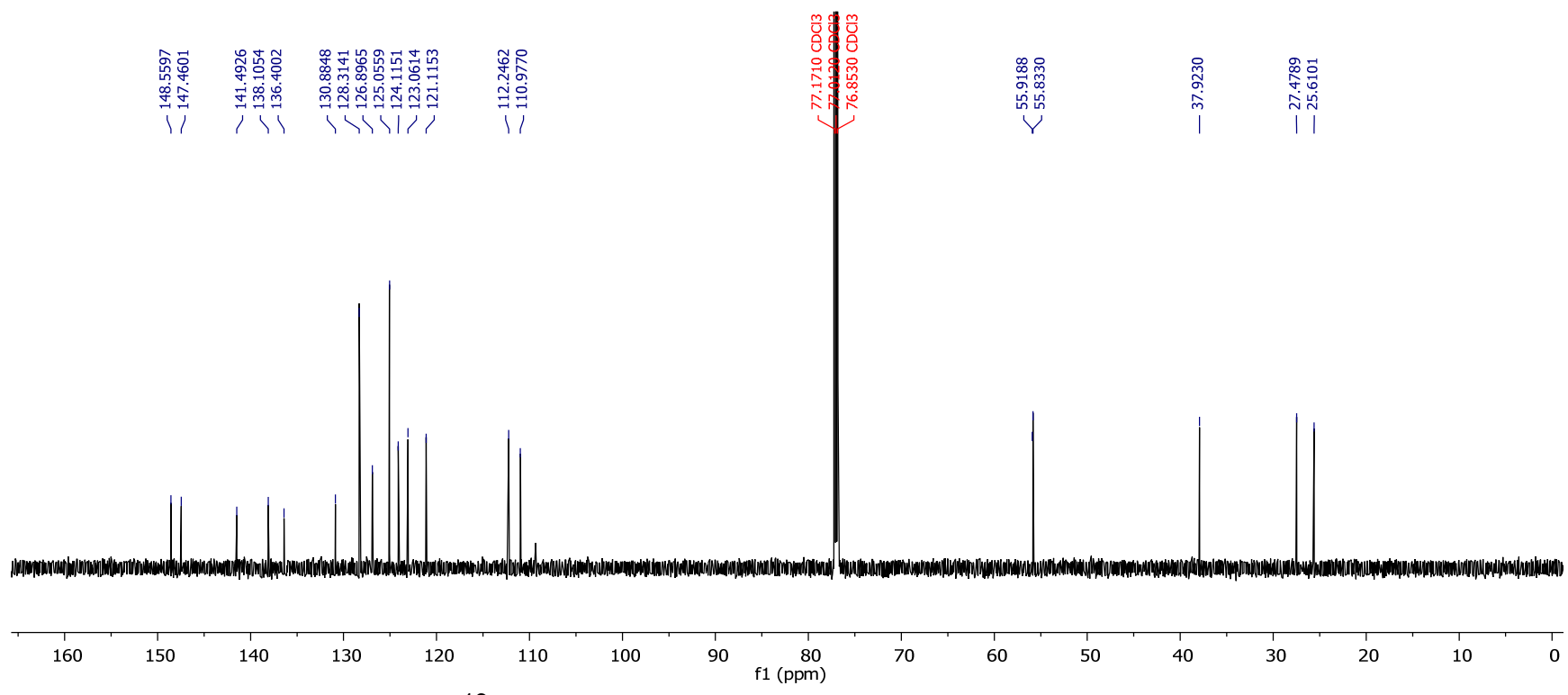

Figure S72. $\quad{ }^{13} \mathrm{C}$ NMR of $24\left(201 \mathrm{MHz}, \mathrm{CDCl}_{3}, 295 \mathrm{~K}\right)$. 


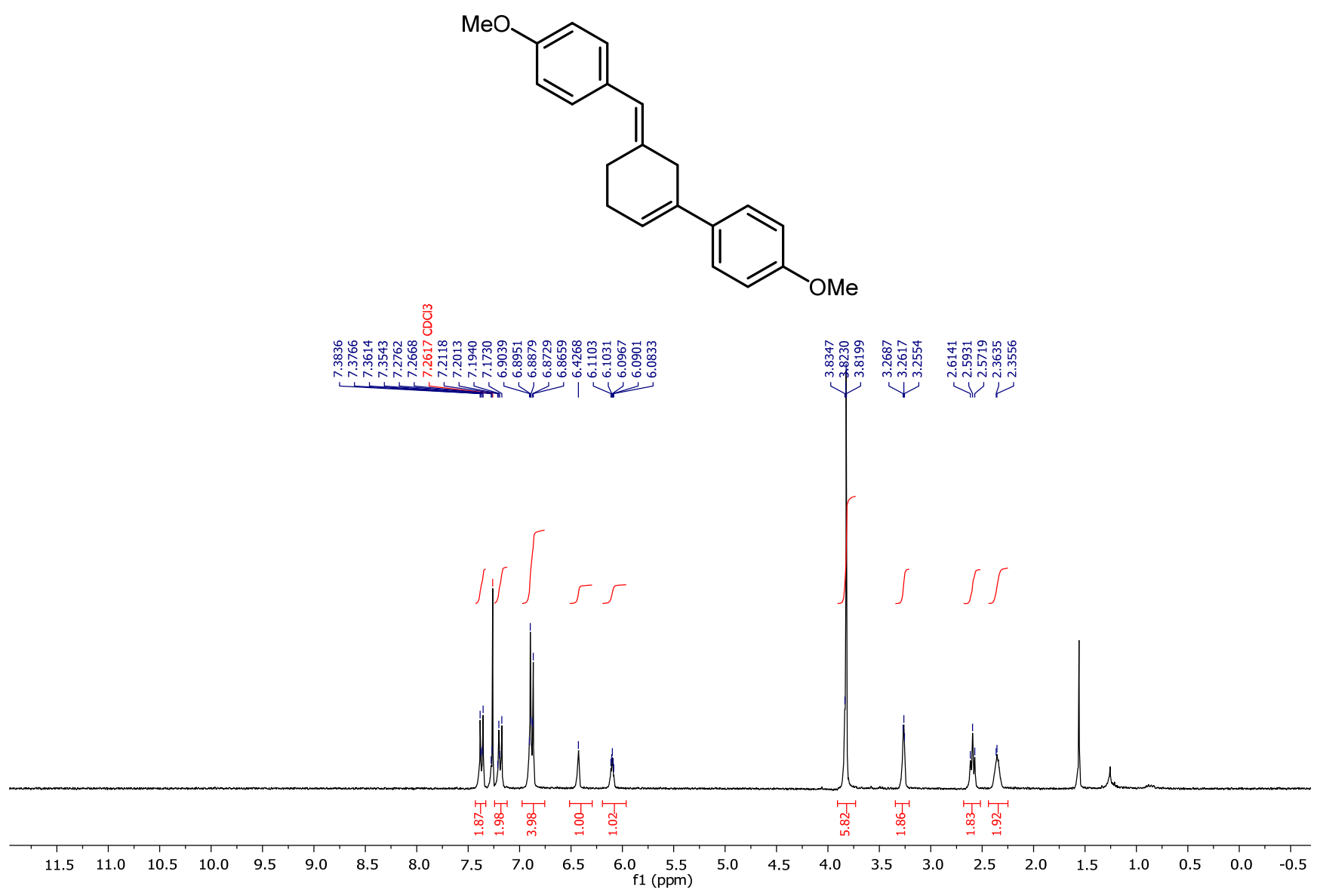

Figure S73. ${ }^{1} \mathrm{H}$ NMR of $25\left(300 \mathrm{MHz}, \mathrm{CDCl}_{3}, 295 \mathrm{~K}\right)$.

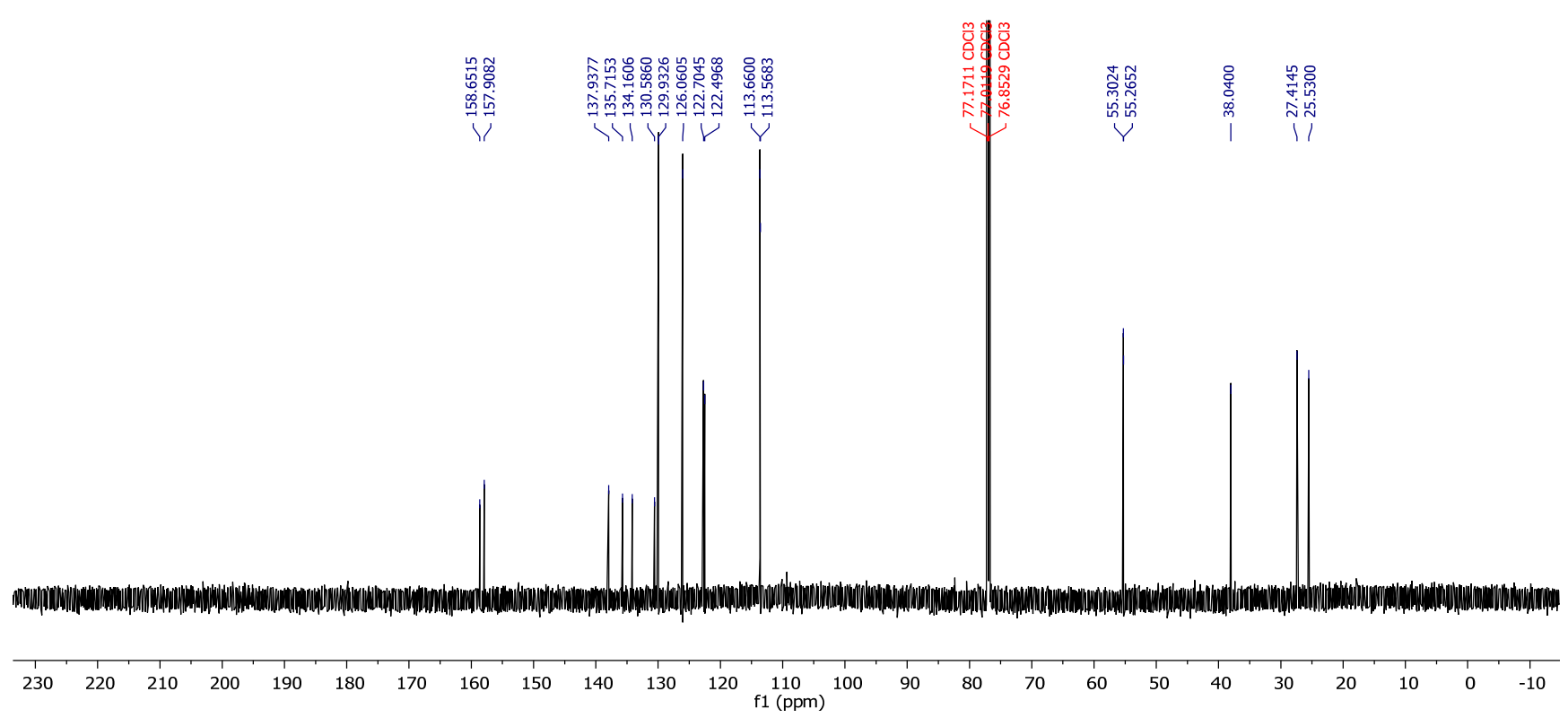

Figure S74. $\quad{ }^{13} \mathrm{C}$ NMR of $25\left(201 \mathrm{MHz}, \mathrm{CDCl}_{3}, 295 \mathrm{~K}\right)$. 


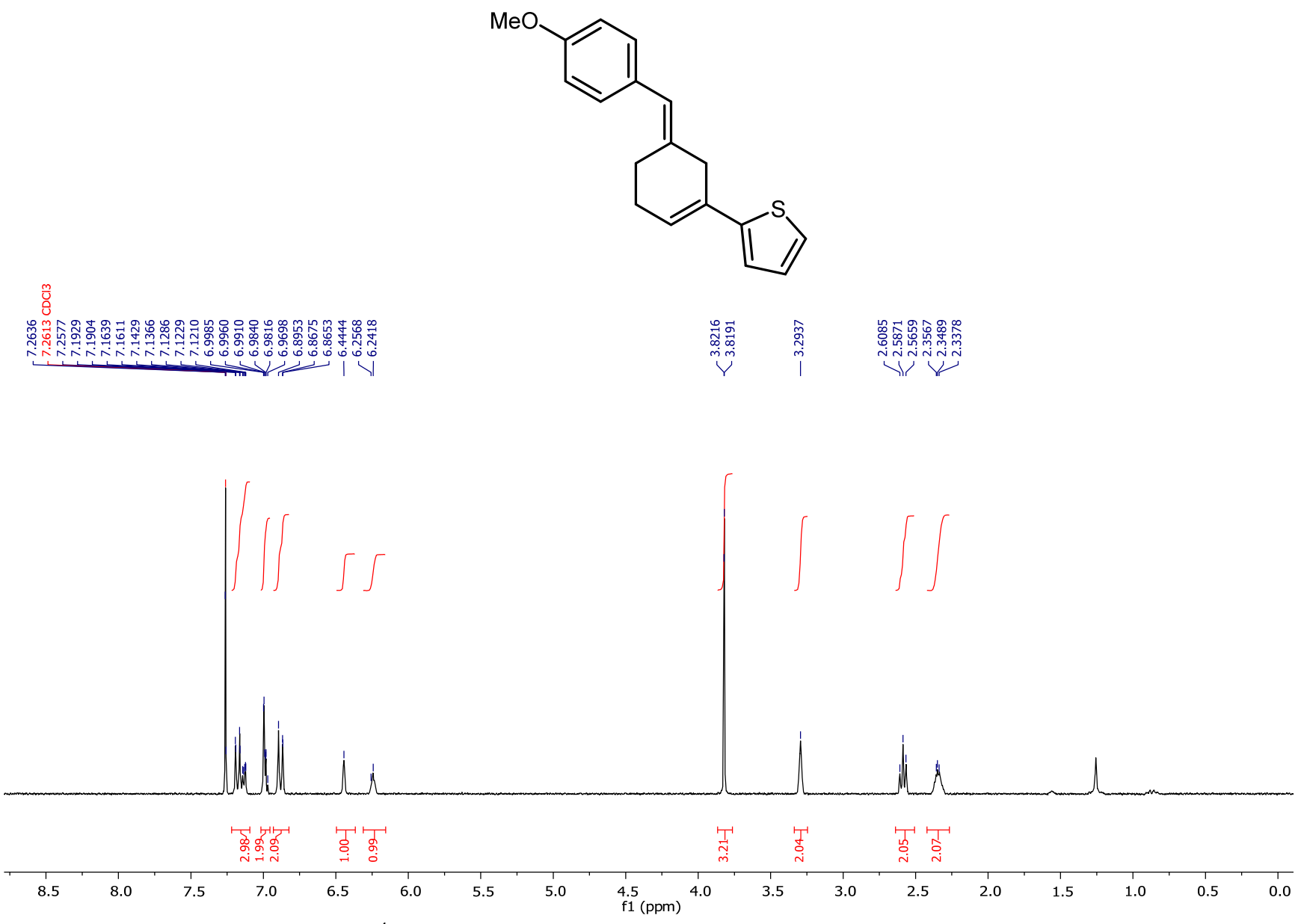

Figure S75. $\quad{ }^{1} \mathrm{H}$ NMR of $26\left(300 \mathrm{MHz}, \mathrm{CDCl}_{3}, 295 \mathrm{~K}\right)$.

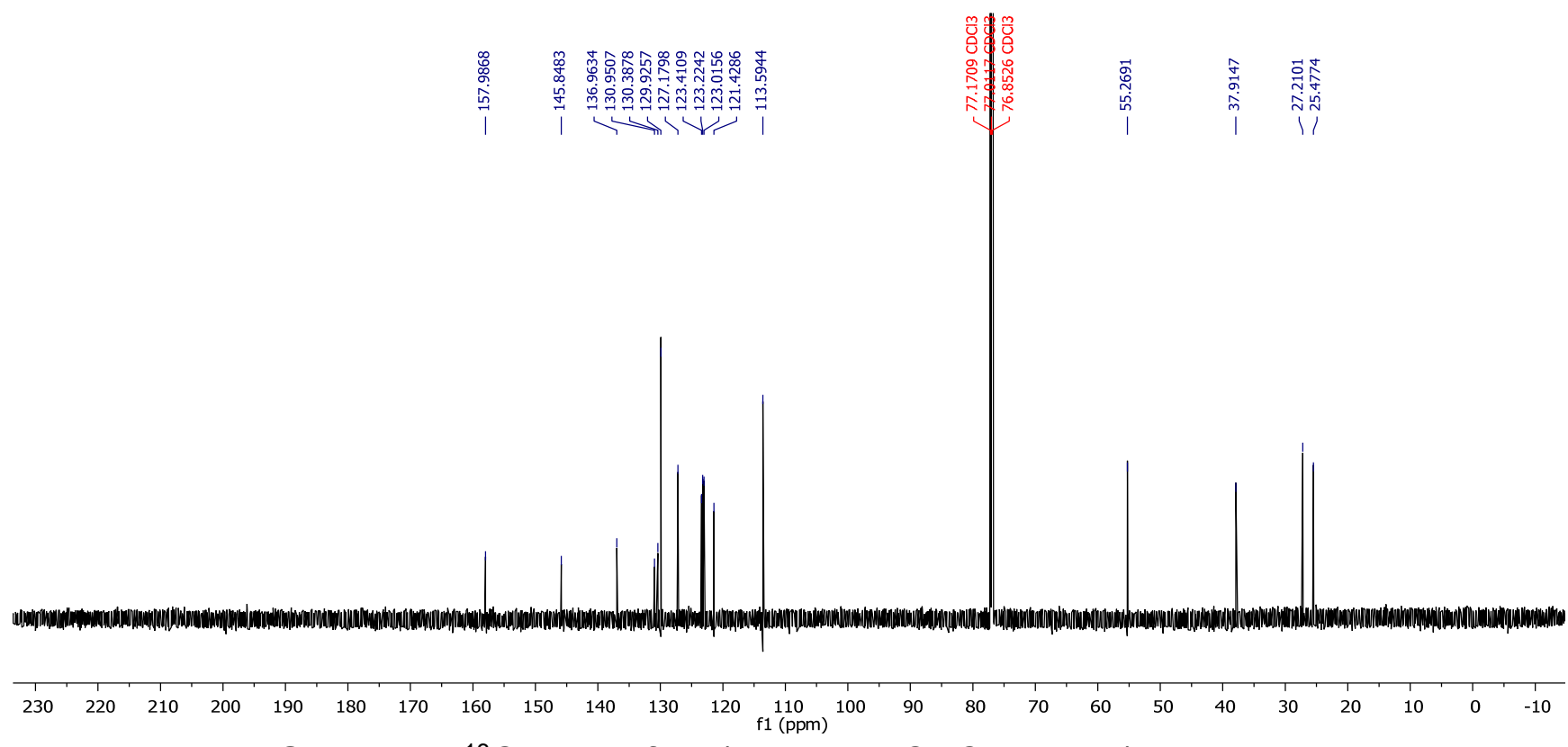

Figure S76. $\quad{ }^{13} \mathrm{C} \mathrm{NMR} \mathrm{of} 26\left(201 \mathrm{MHz}, \mathrm{CDCl}_{3}, 295 \mathrm{~K}\right)$. 

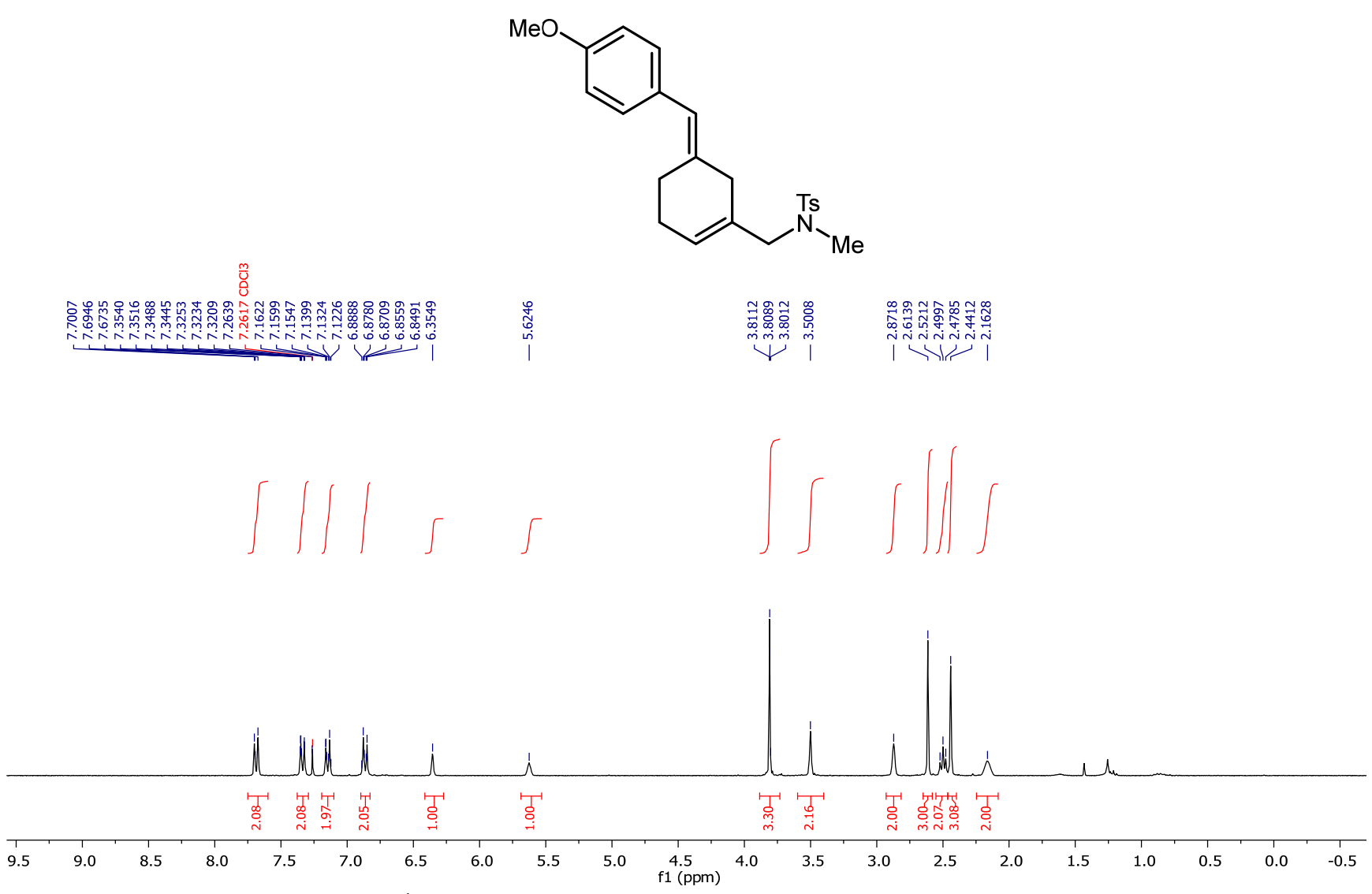

Figure S77. $\quad{ }^{1} \mathrm{H}$ NMR of $27\left(300 \mathrm{MHz}, \mathrm{CDCl}_{3}, 295 \mathrm{~K}\right)$.
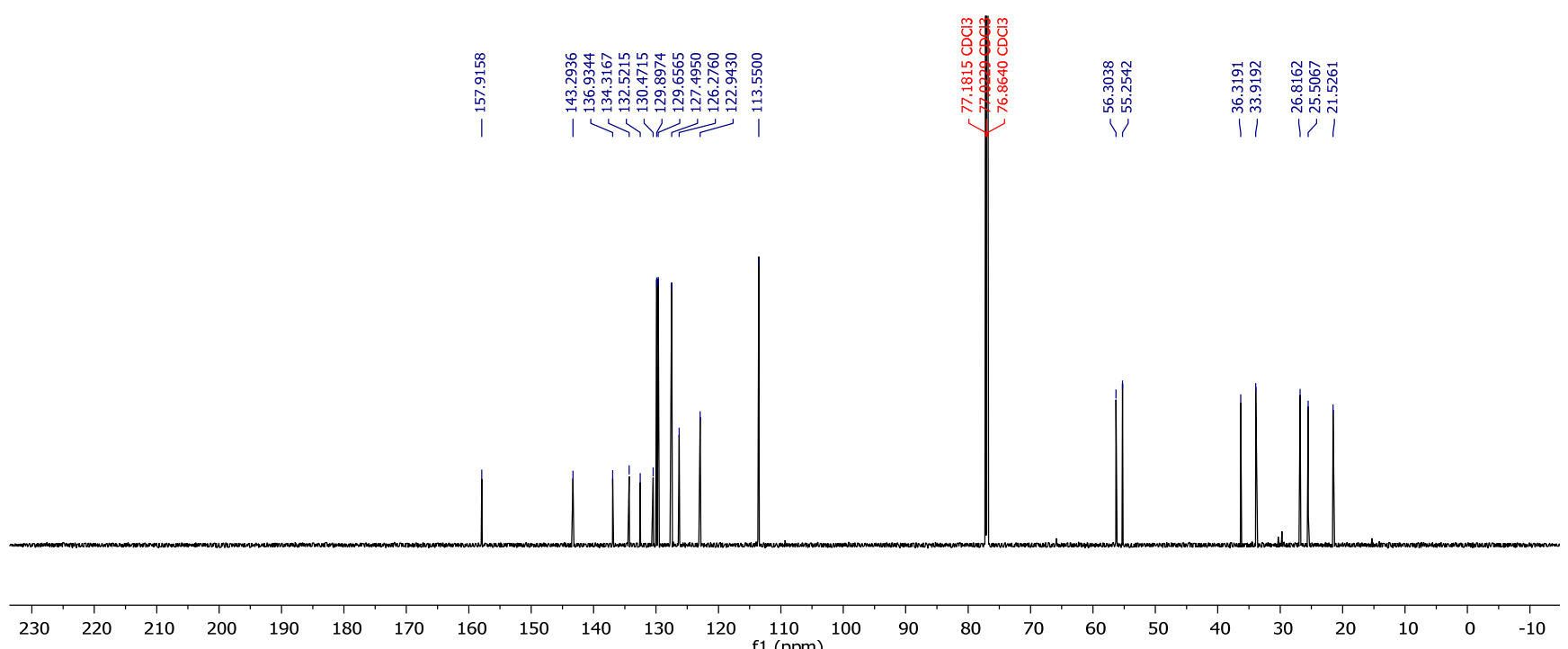

Figure S78. $\quad{ }^{13} \mathrm{C}$ NMR of $27\left(201 \mathrm{MHz}, \mathrm{CDCl}_{3}, 295 \mathrm{~K}\right)$. 


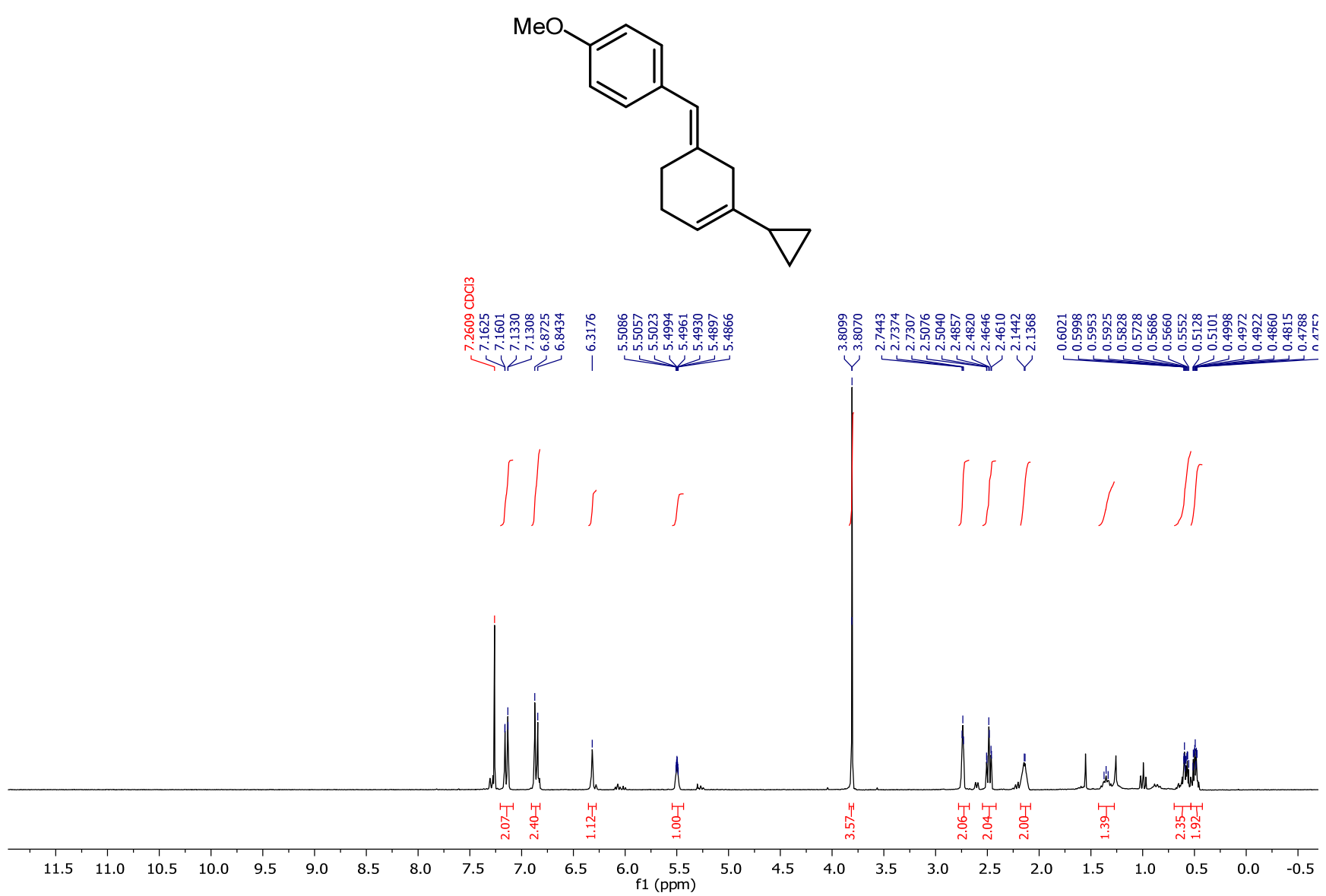

Figure S79. $\quad{ }^{1} \mathrm{H}$ NMR of $28\left(300 \mathrm{MHz}, \mathrm{CDCl}_{3}, 295 \mathrm{~K}\right)$.
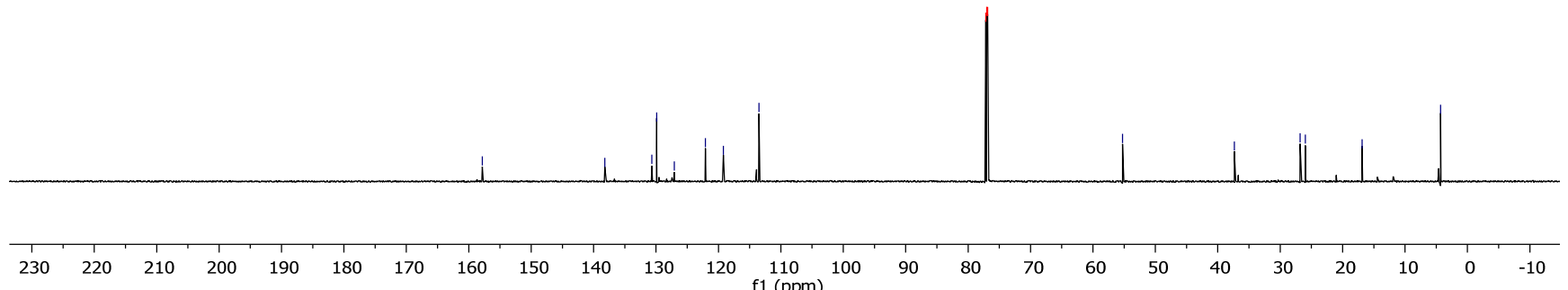
Figure S80. $\quad{ }^{13} \mathrm{C}$ NMR of $28\left(201 \mathrm{MHz}, \mathrm{CDCl}_{3}, 295 \mathrm{~K}\right)$. 


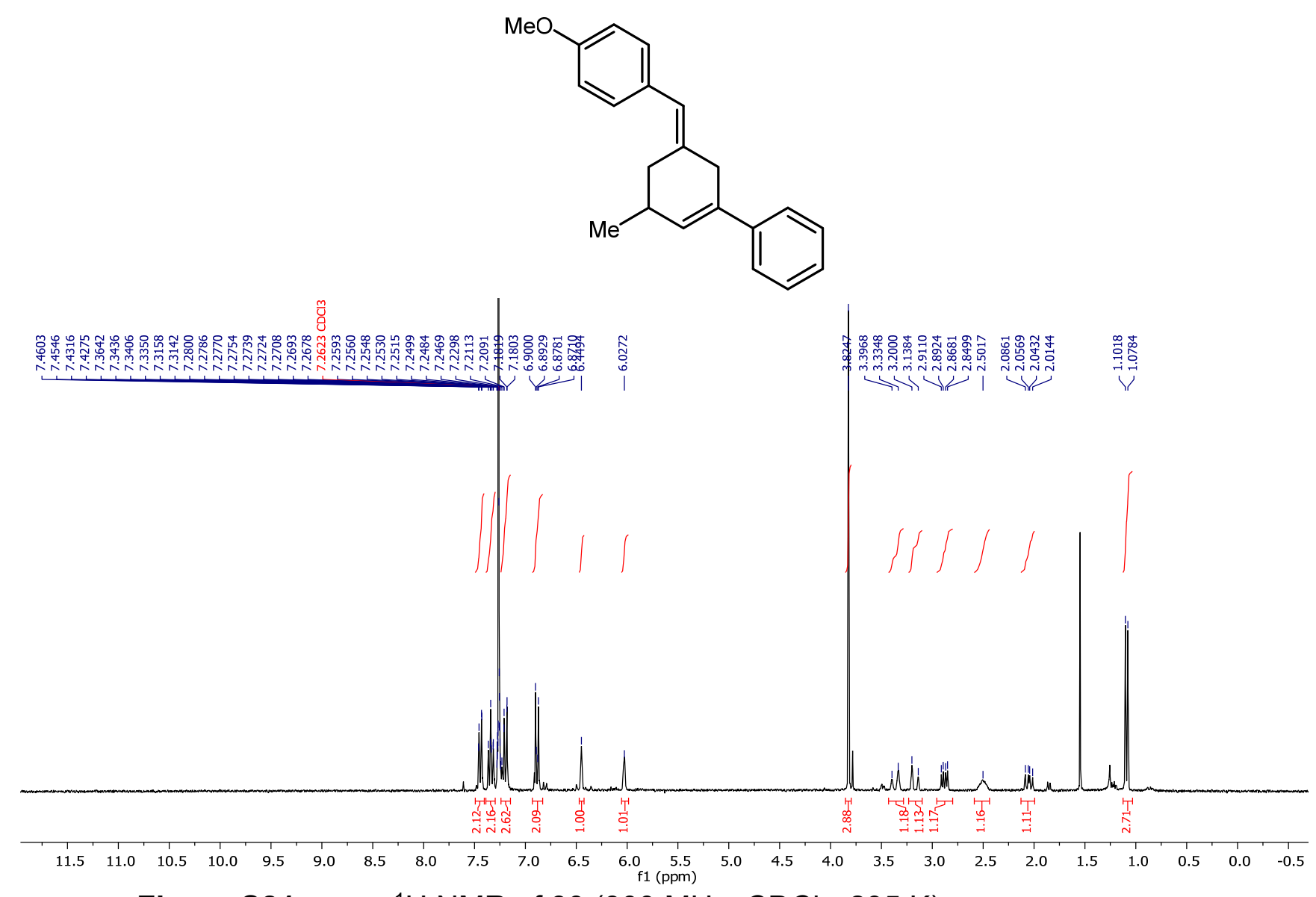

Figure S81. $\quad{ }^{1} \mathrm{H}$ NMR of $\mathbf{3 0}\left(300 \mathrm{MHz}, \mathrm{CDCl}_{3}, 295 \mathrm{~K}\right)$.
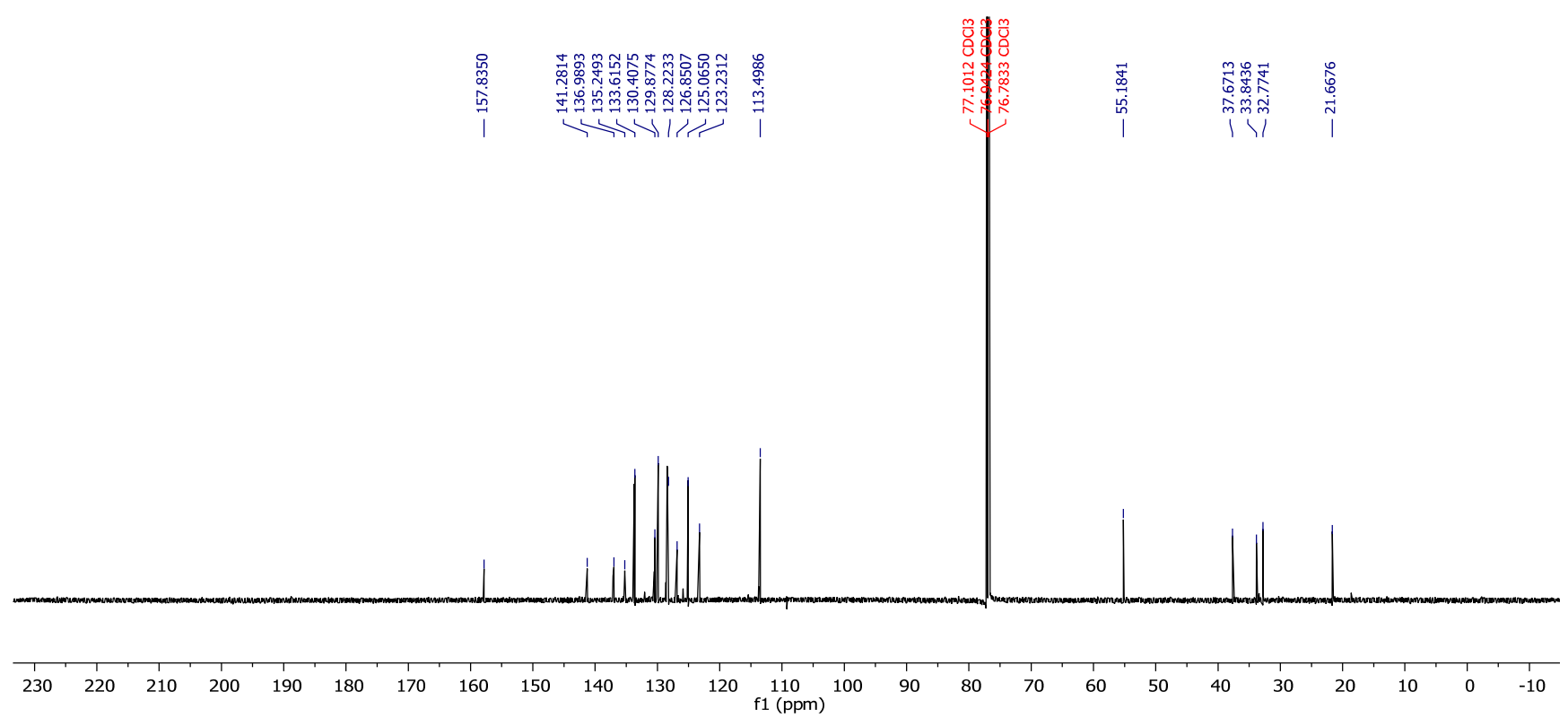

Figure S82. $\quad{ }^{13} \mathrm{C}$ NMR of $\mathbf{3 0}\left(201 \mathrm{MHz}, \mathrm{CDCl}_{3}, 295 \mathrm{~K}\right)$. 


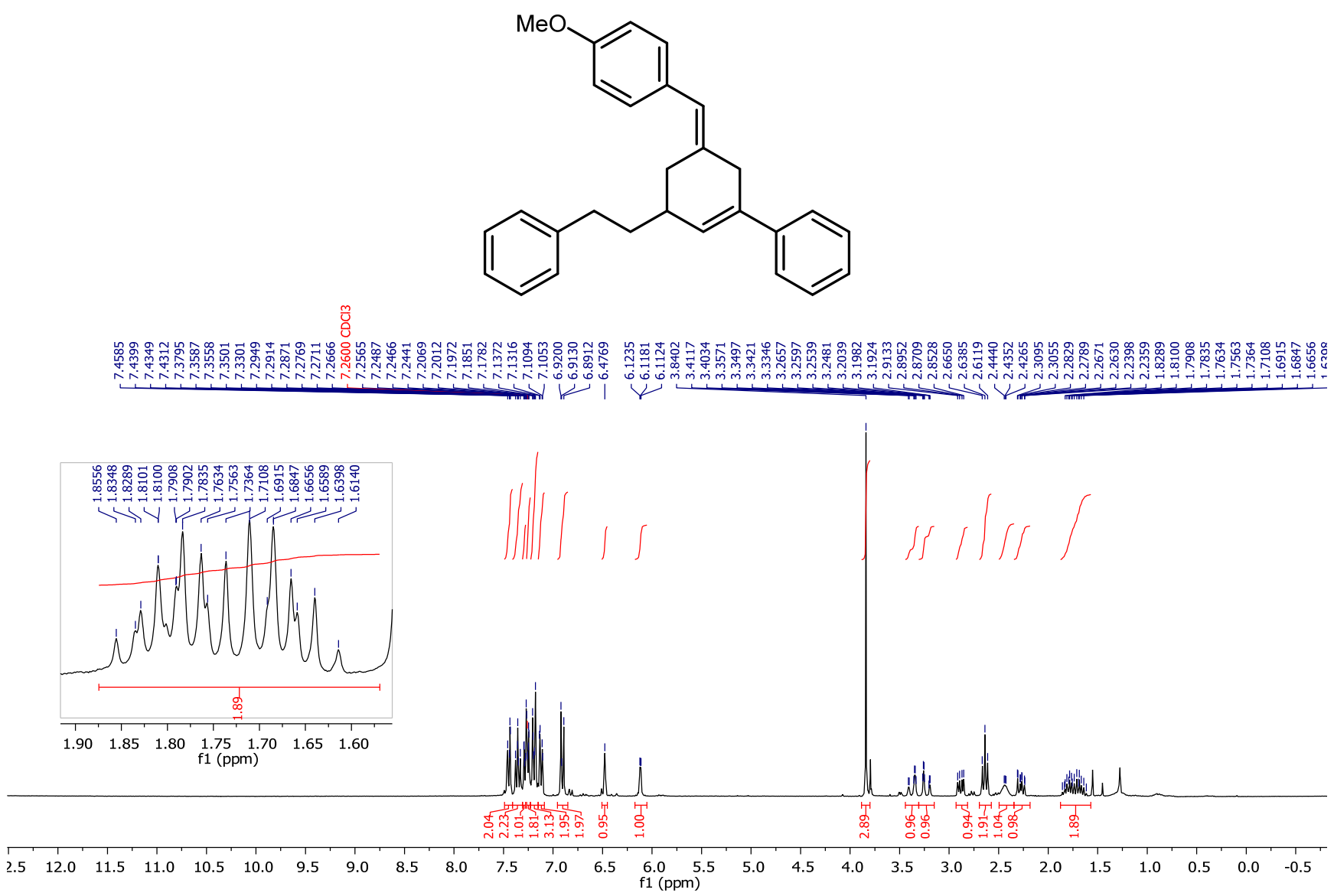

Figure S83. $\quad{ }^{1} \mathrm{H}$ NMR of $31\left(300 \mathrm{MHz}, \mathrm{CDCl}_{3}, 295 \mathrm{~K}\right)$.

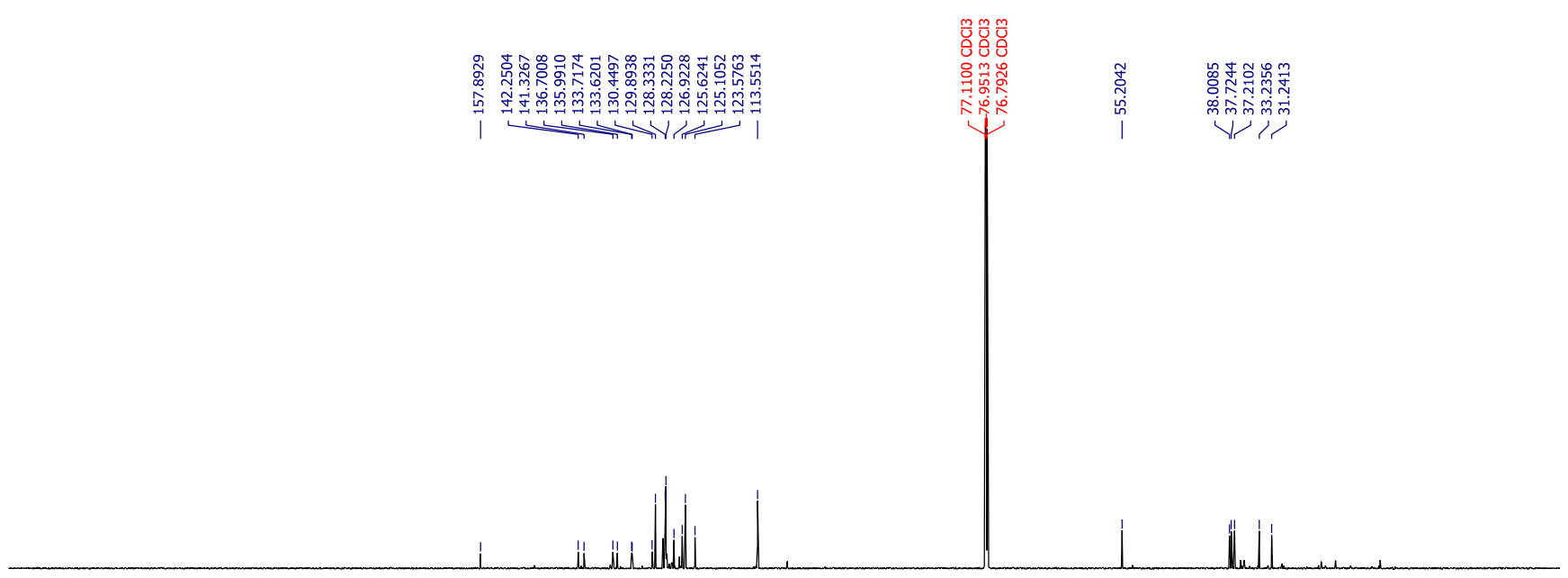

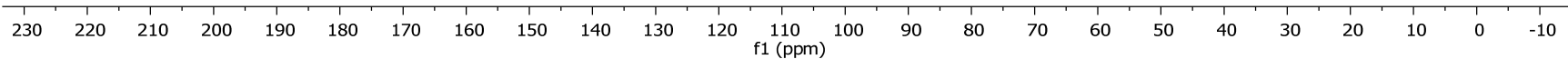

Figure S84. $\quad{ }^{13} \mathrm{C}$ NMR of $31\left(201 \mathrm{MHz}, \mathrm{CDCl}_{3}, 295 \mathrm{~K}\right)$. 


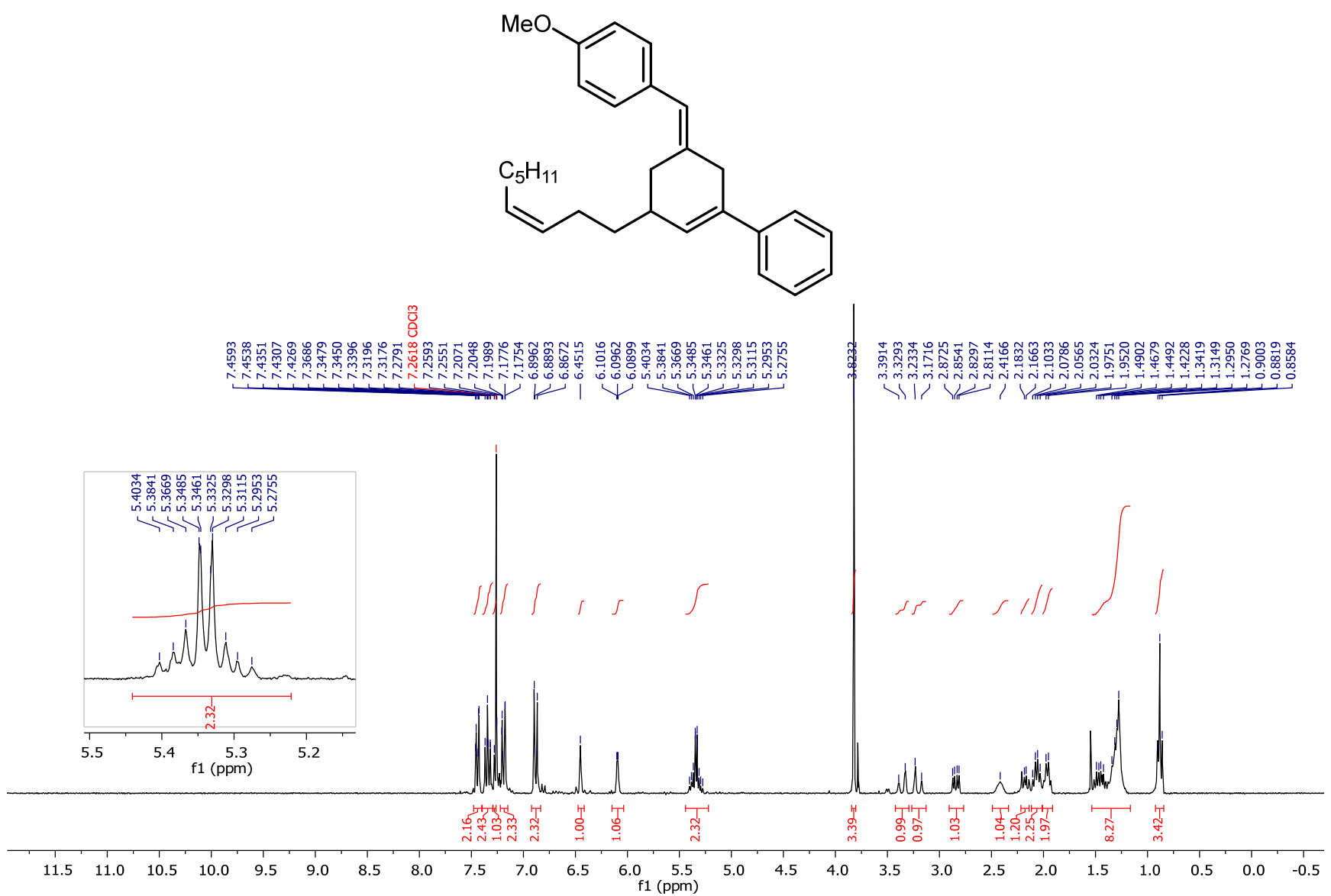

Figure S85. $\quad{ }^{1} \mathrm{H}$ NMR of $32\left(300 \mathrm{MHz}, \mathrm{CDCl}_{3}, 295 \mathrm{~K}\right)$.

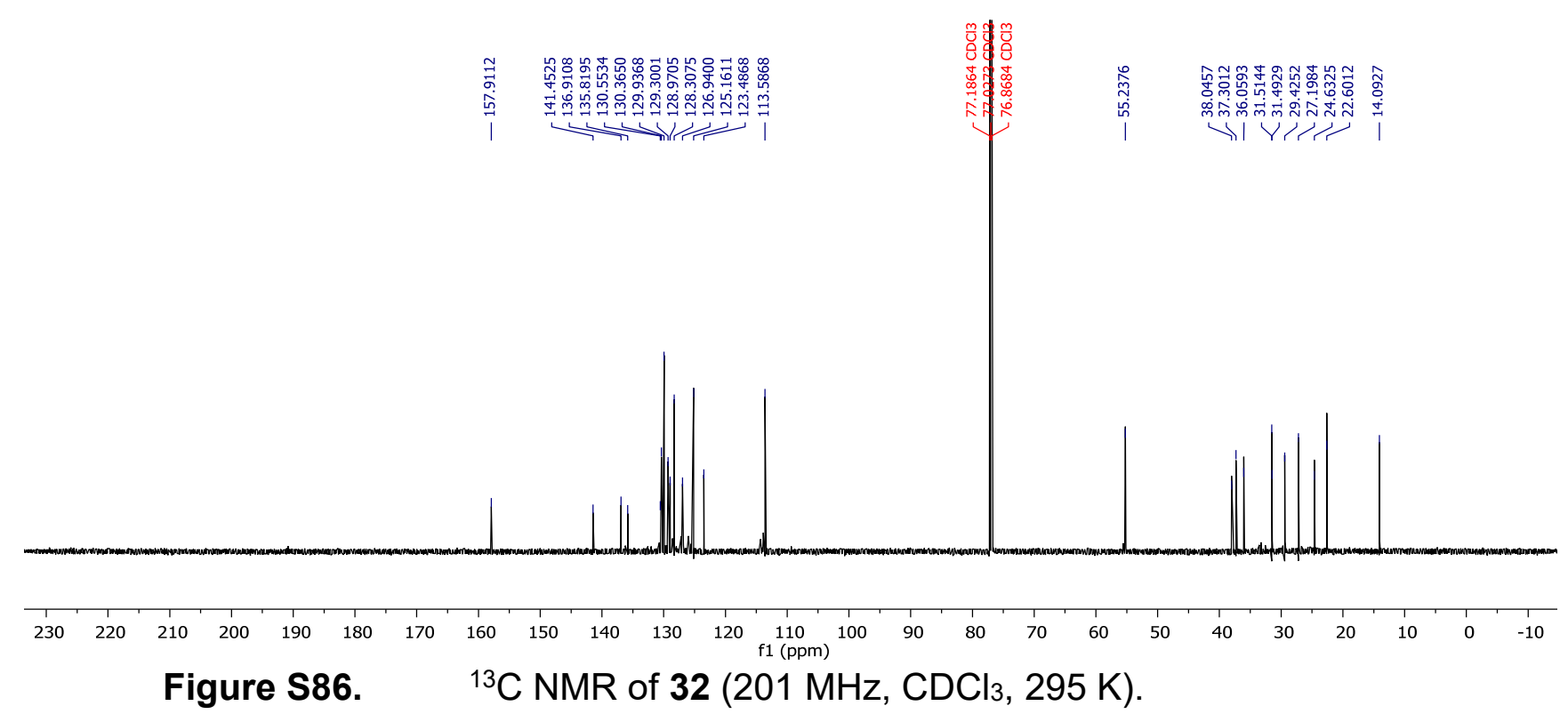

Figure S86. $\quad{ }^{13} \mathrm{C} \mathrm{NMR}$ of $32\left(201 \mathrm{MHz}, \mathrm{CDCl}_{3}, 295 \mathrm{~K}\right)$. 
<smiles>COc1ccc(/C=C2/CC(c3ccccc3)=CC(CCOCc3ccccc3)C2)cc1</smiles>
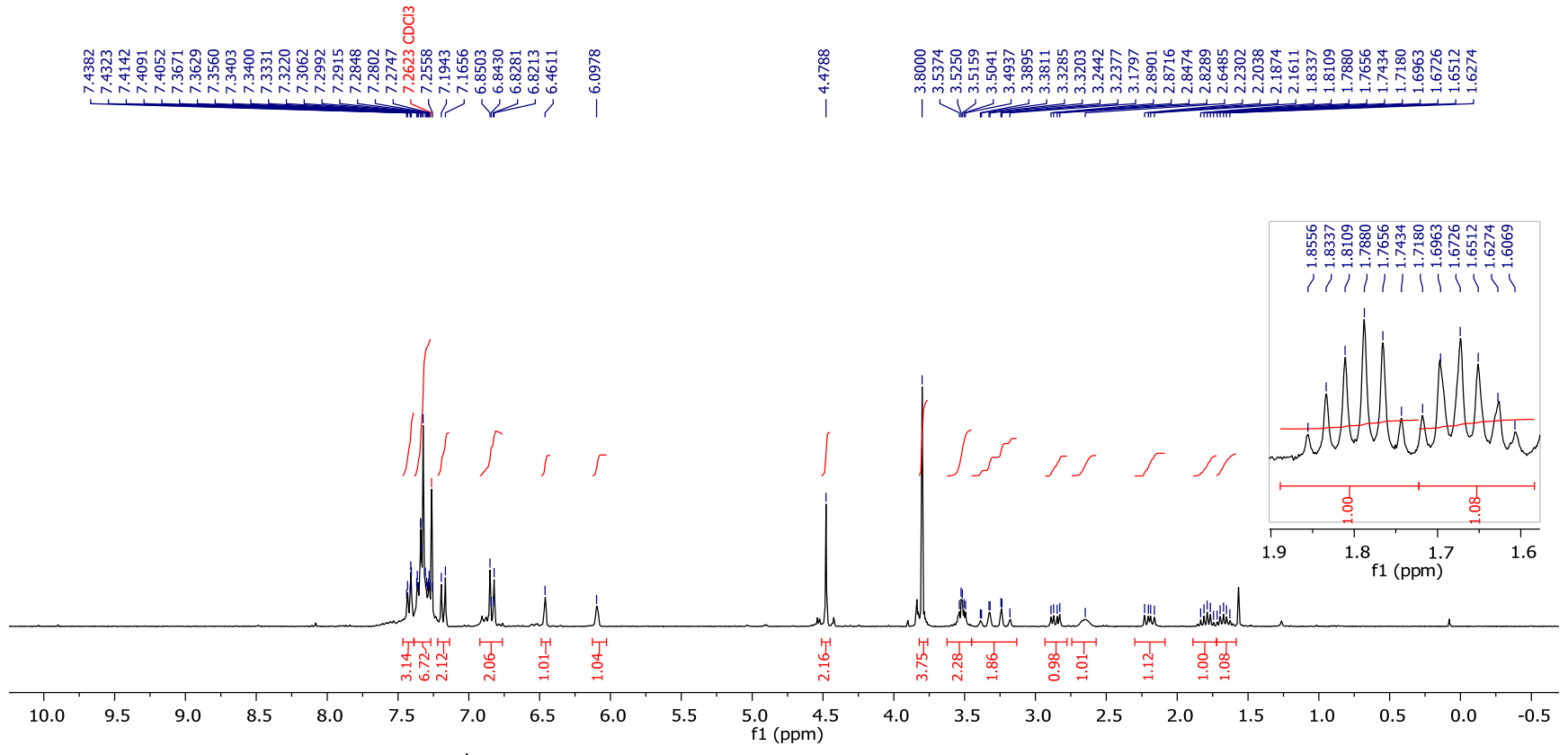

Figure S87. $\quad{ }^{1} \mathrm{H}$ NMR of $\mathbf{3 3}\left(300 \mathrm{MHz}, \mathrm{CDCl}_{3}, 295 \mathrm{~K}\right)$.

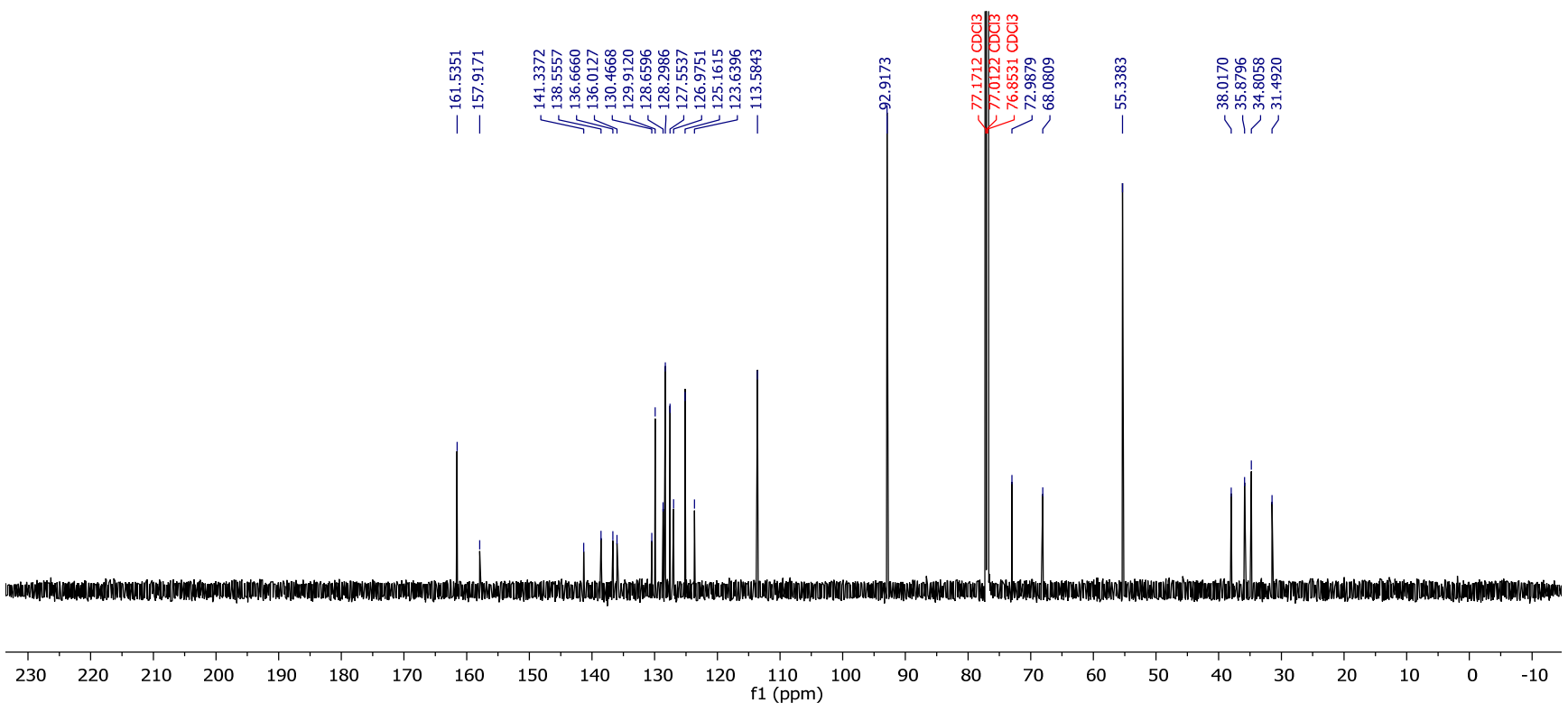

Figure S88. $\quad{ }^{13} \mathrm{C}$ NMR of $33\left(201 \mathrm{MHz}, \mathrm{CDCl}_{3}, 295 \mathrm{~K}\right)$. 


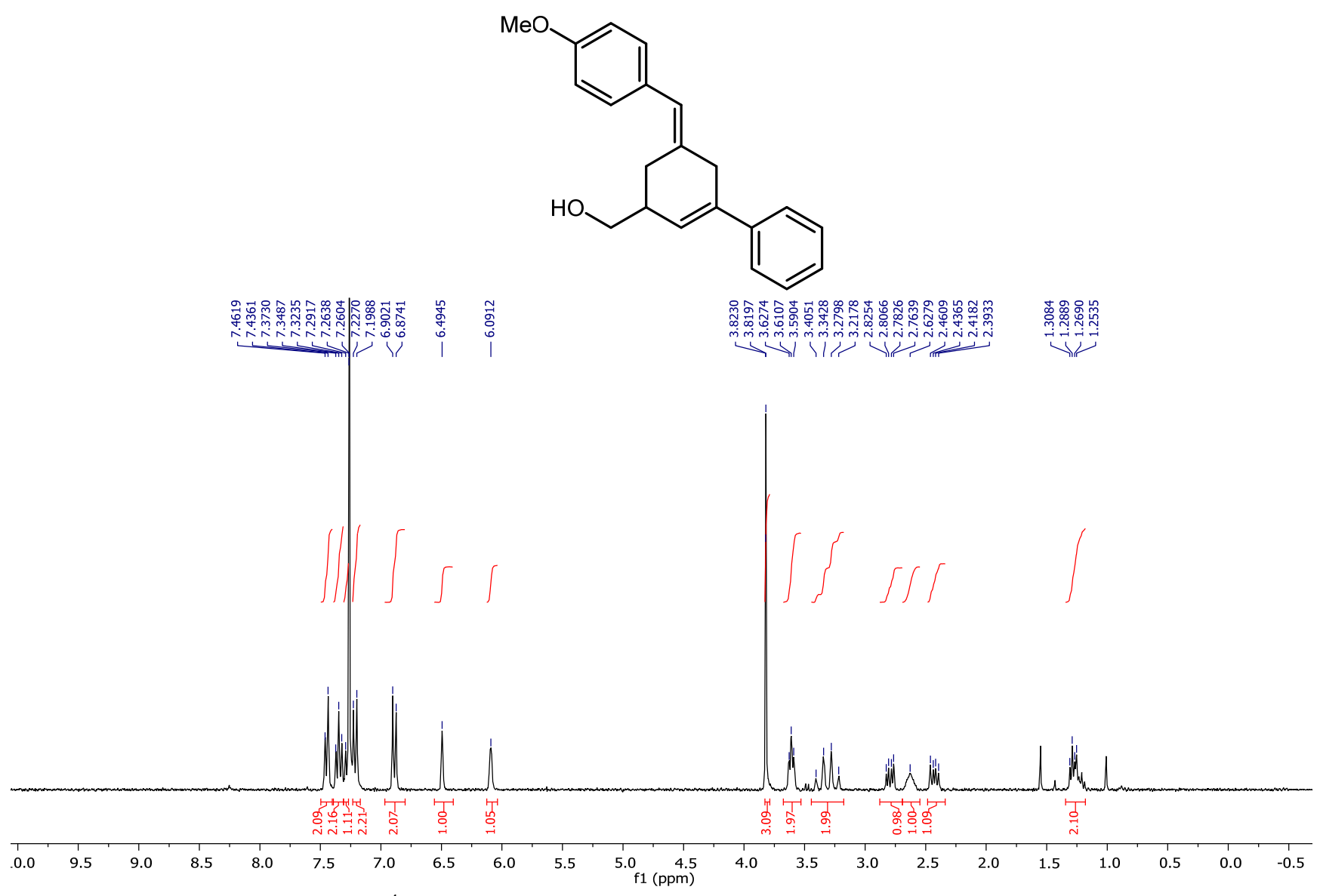

Figure S89. $\quad{ }^{1} \mathrm{H}$ NMR of $\mathbf{3 4}\left(300 \mathrm{MHz}, \mathrm{CDCl}_{3}, 295 \mathrm{~K}\right)$.

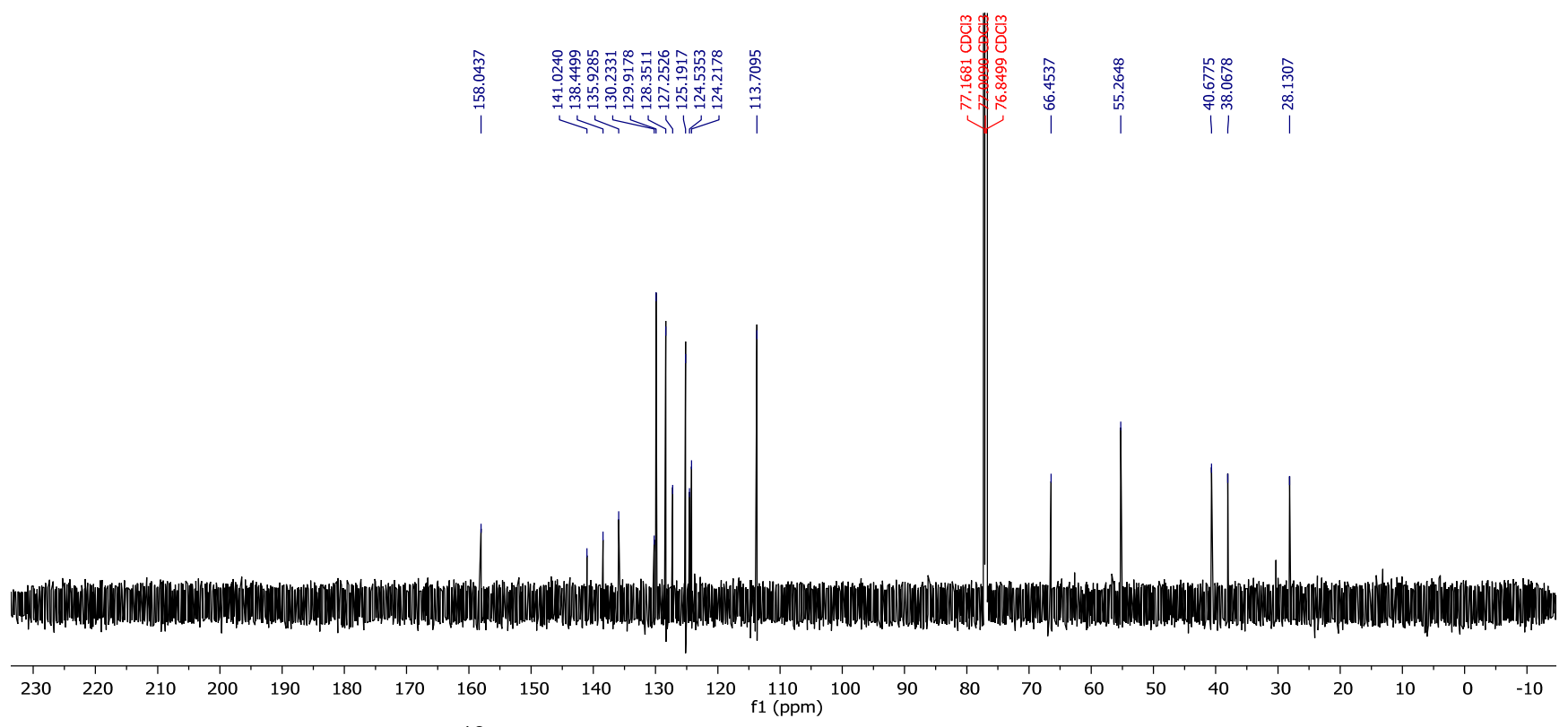

Figure S90. $\quad{ }^{13} \mathrm{C}$ NMR of $34\left(201 \mathrm{MHz}, \mathrm{CDCl}_{3}, 295 \mathrm{~K}\right)$. 


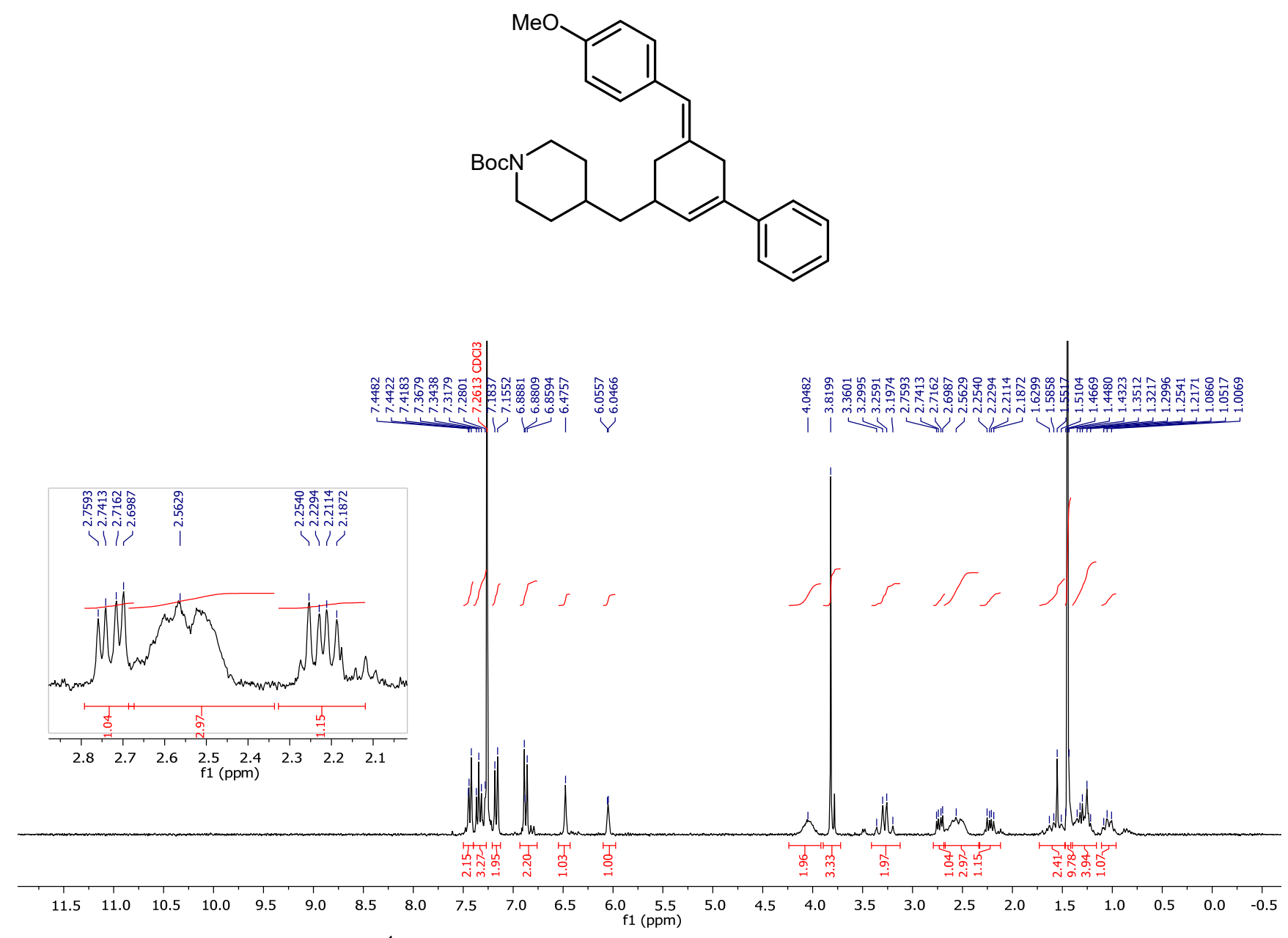

Figure S91. $\quad{ }^{1} \mathrm{H} \mathrm{NMR}$ of $\mathbf{3 5}\left(300 \mathrm{MHz}, \mathrm{CDCl}_{3}, 295 \mathrm{~K}\right)$.
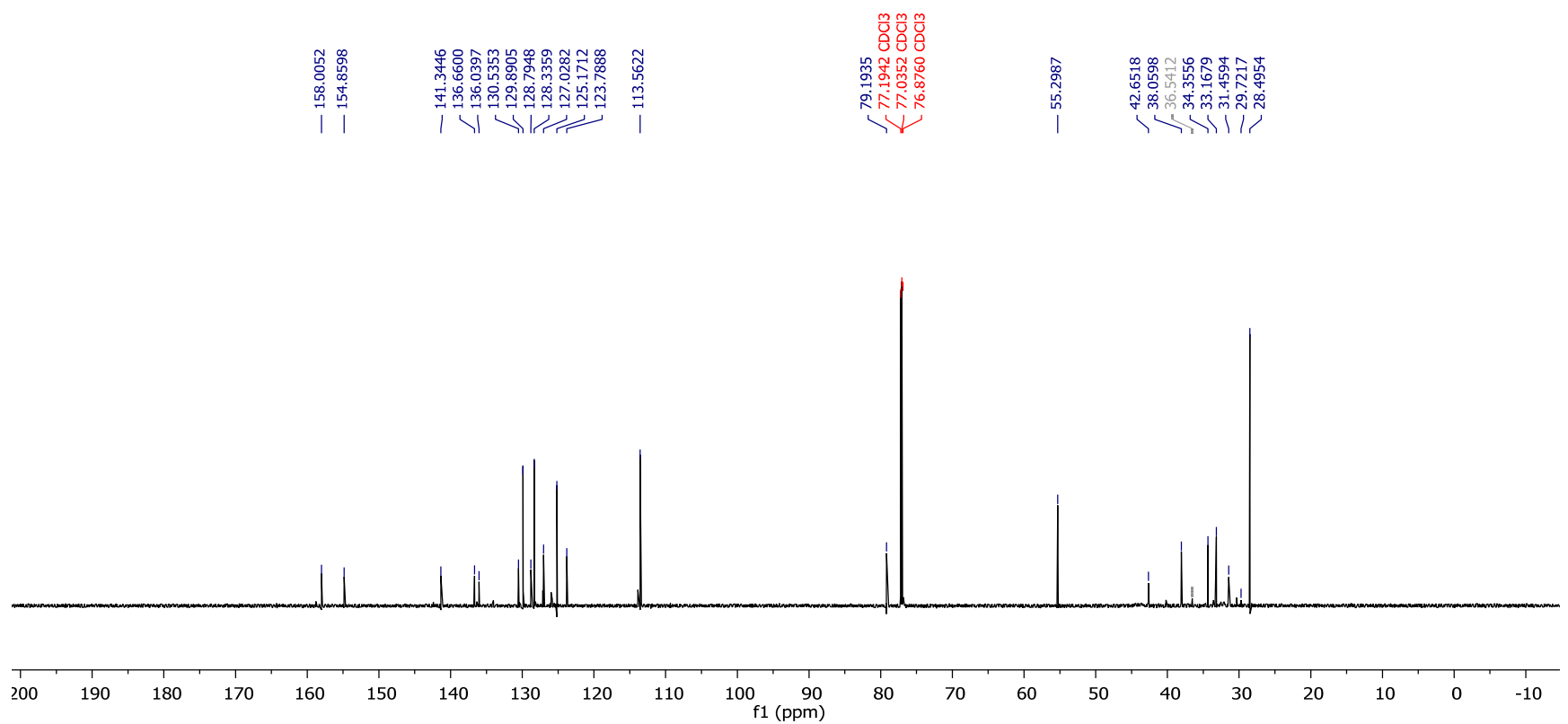

Figure S92. $\quad{ }^{13} \mathrm{C} \mathrm{NMR} \mathrm{of} 35\left(201 \mathrm{MHz}, \mathrm{CDCl}_{3}, 295 \mathrm{~K}\right)$. 
<smiles>COc1ccc(/C=C2/CC(c3ccccc3)=CC(CCc3ccc(C)o3)C2)cc1</smiles>

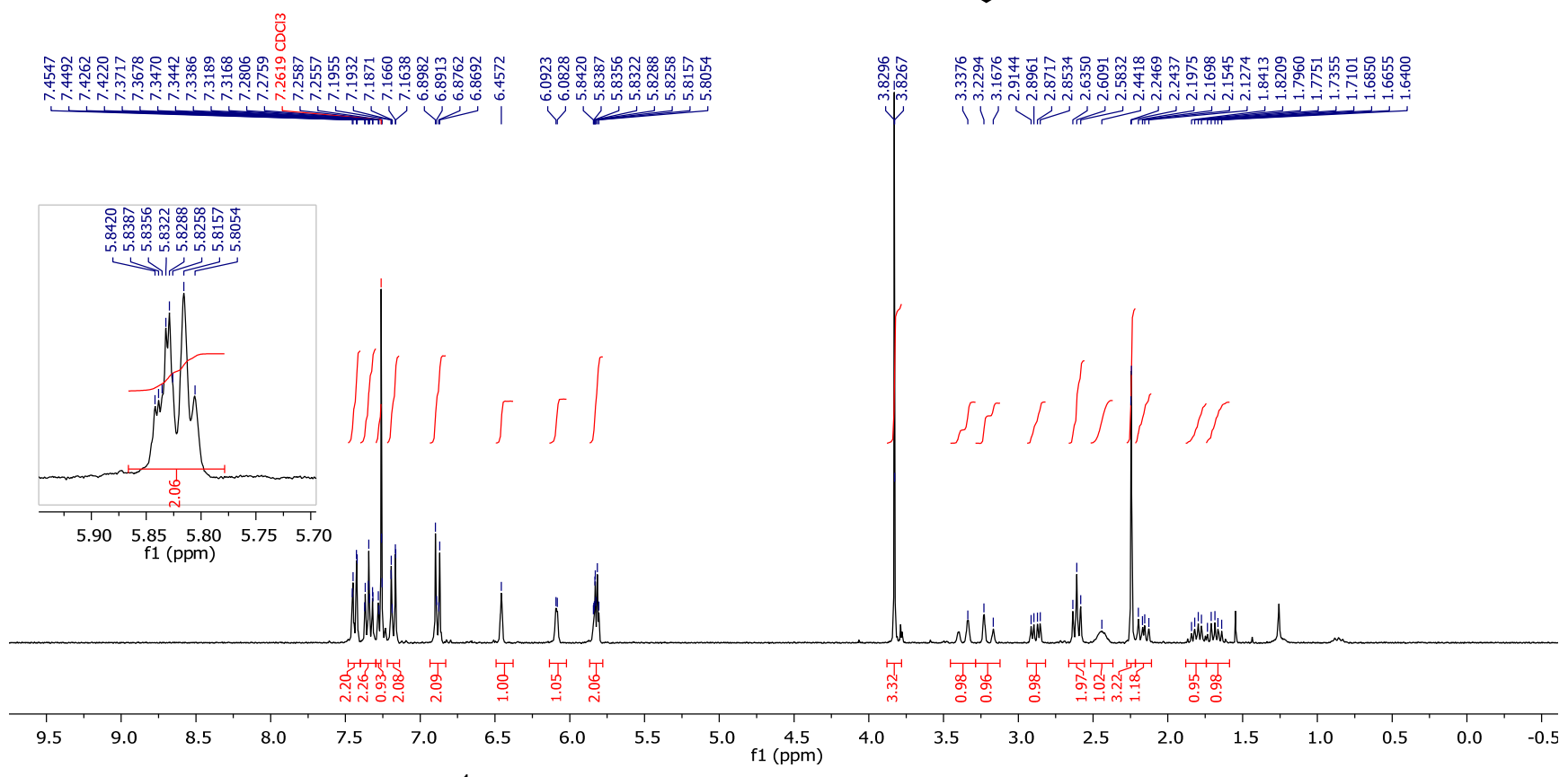

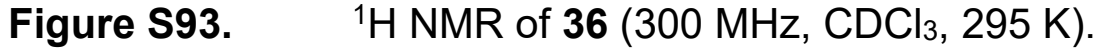

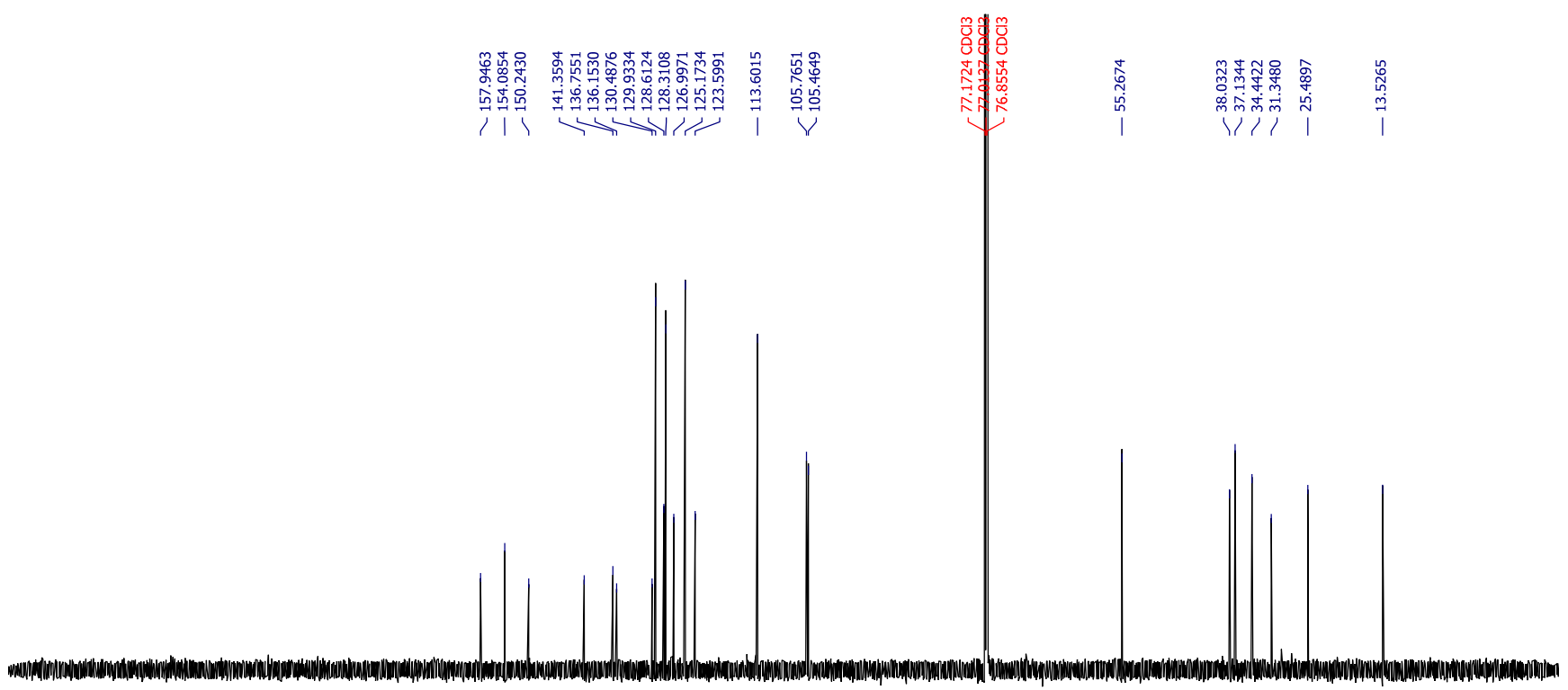

Figure S94. $\quad{ }^{13} \mathrm{C}$ NMR of $\mathbf{3 6}\left(201 \mathrm{MHz}, \mathrm{CDCl}_{3}, 295 \mathrm{~K}\right)$. 


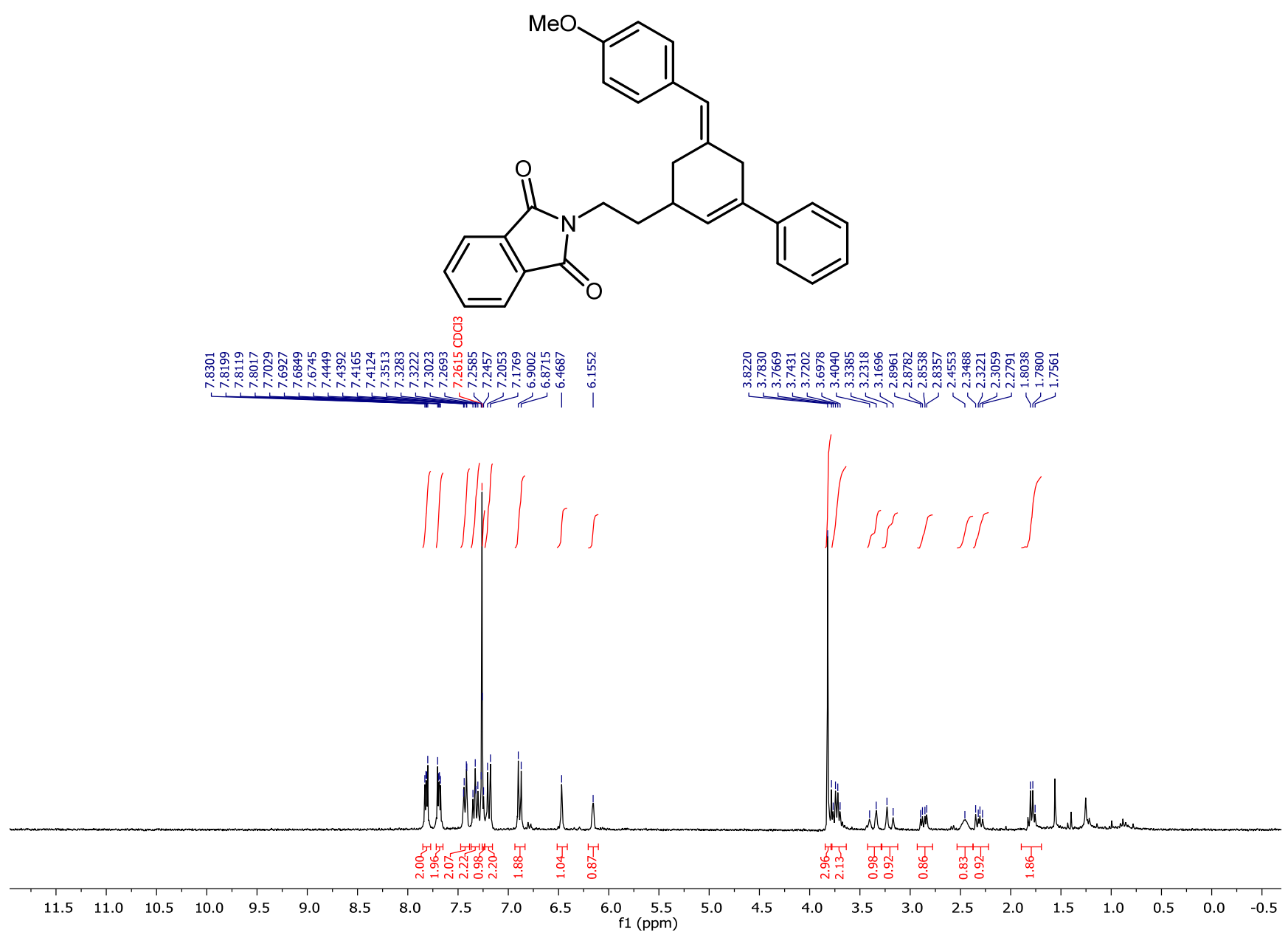

Figure S95. $\quad{ }^{1} \mathrm{H}$ NMR of $37\left(300 \mathrm{MHz}, \mathrm{CDCl}_{3}, 295 \mathrm{~K}\right)$.

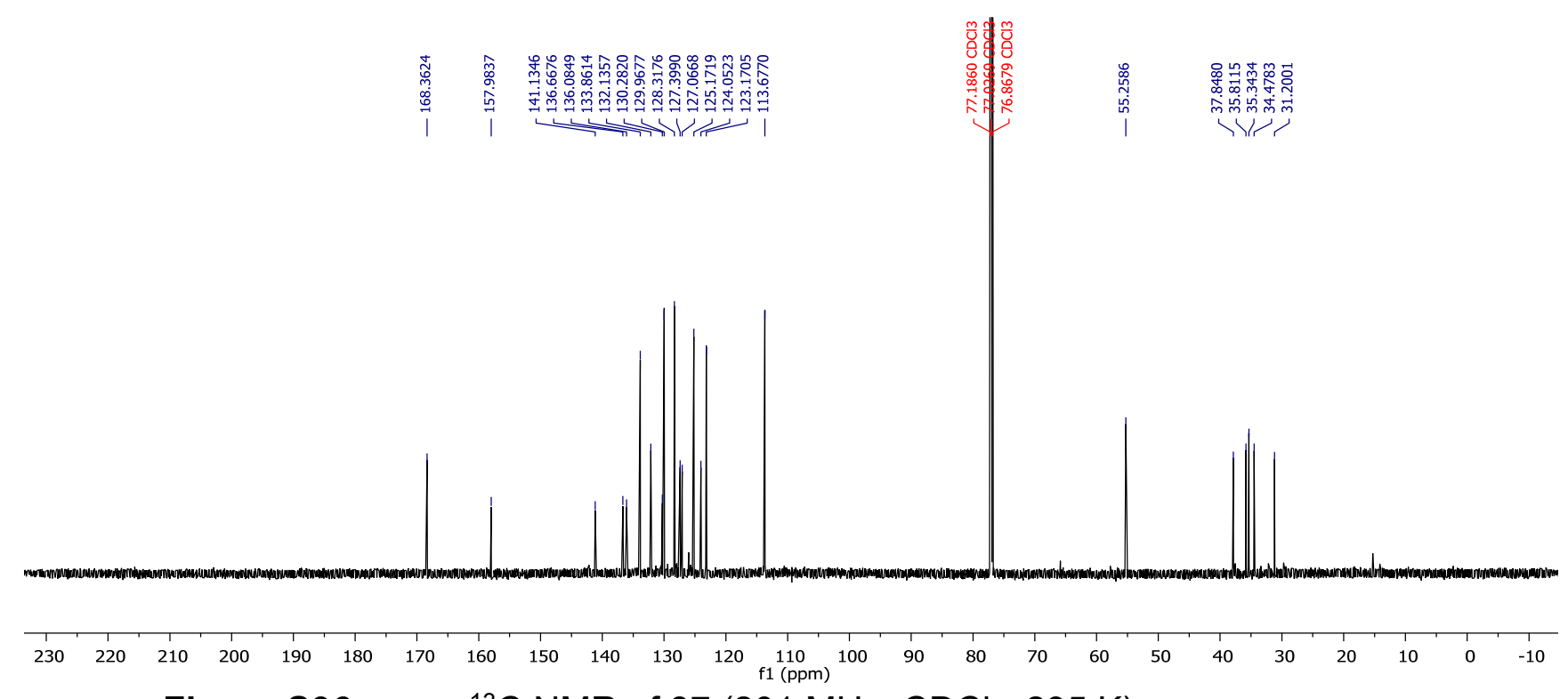

Figure S96. $\quad{ }^{13} \mathrm{C} \mathrm{NMR}$ of $37\left(201 \mathrm{MHz}, \mathrm{CDCl}_{3}, 295 \mathrm{~K}\right)$. 

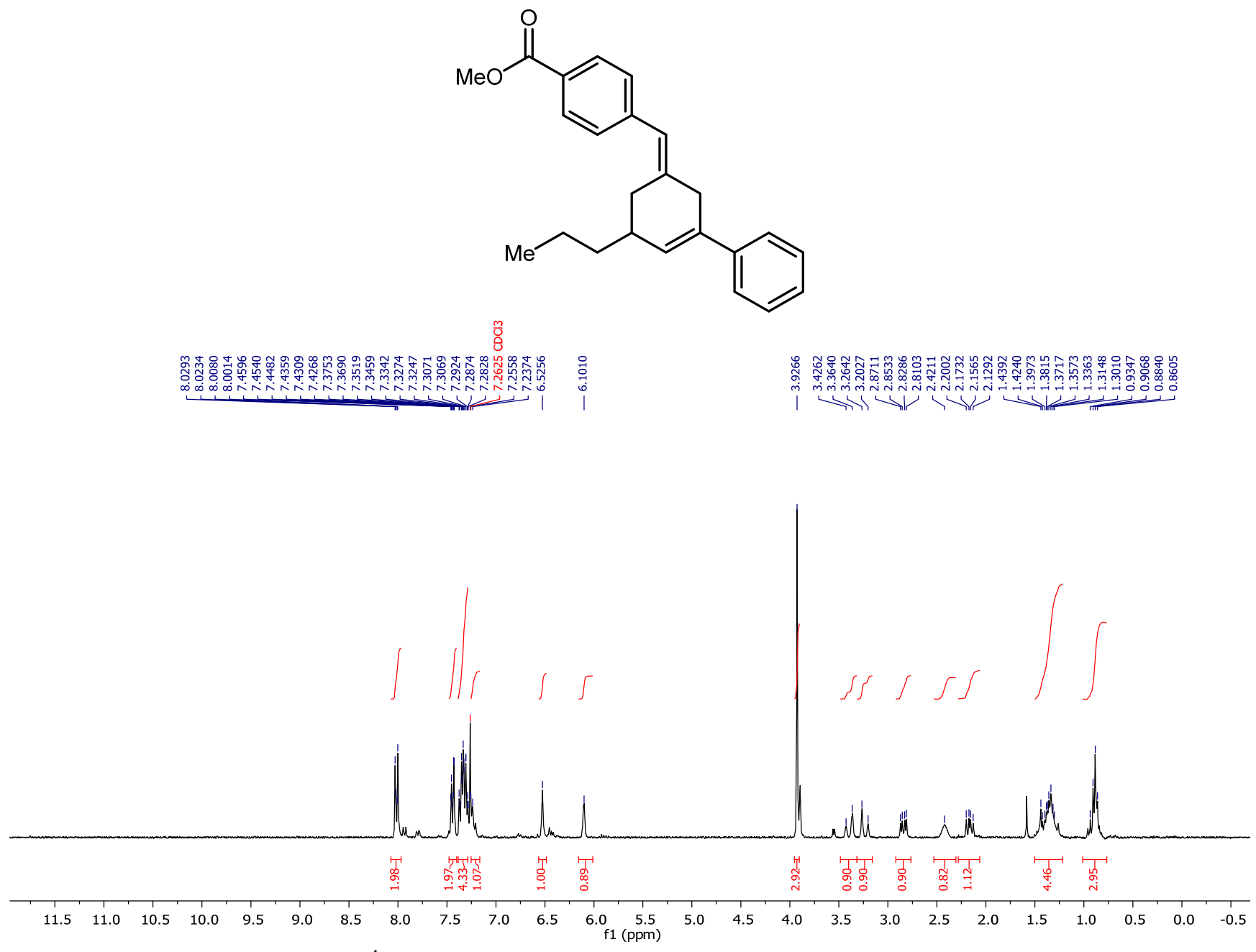

Figure S97. $\quad{ }^{1} \mathrm{H}$ NMR of $\mathbf{S 1 7}\left(300 \mathrm{MHz}, \mathrm{CDCl}_{3}, 295 \mathrm{~K}\right)$.
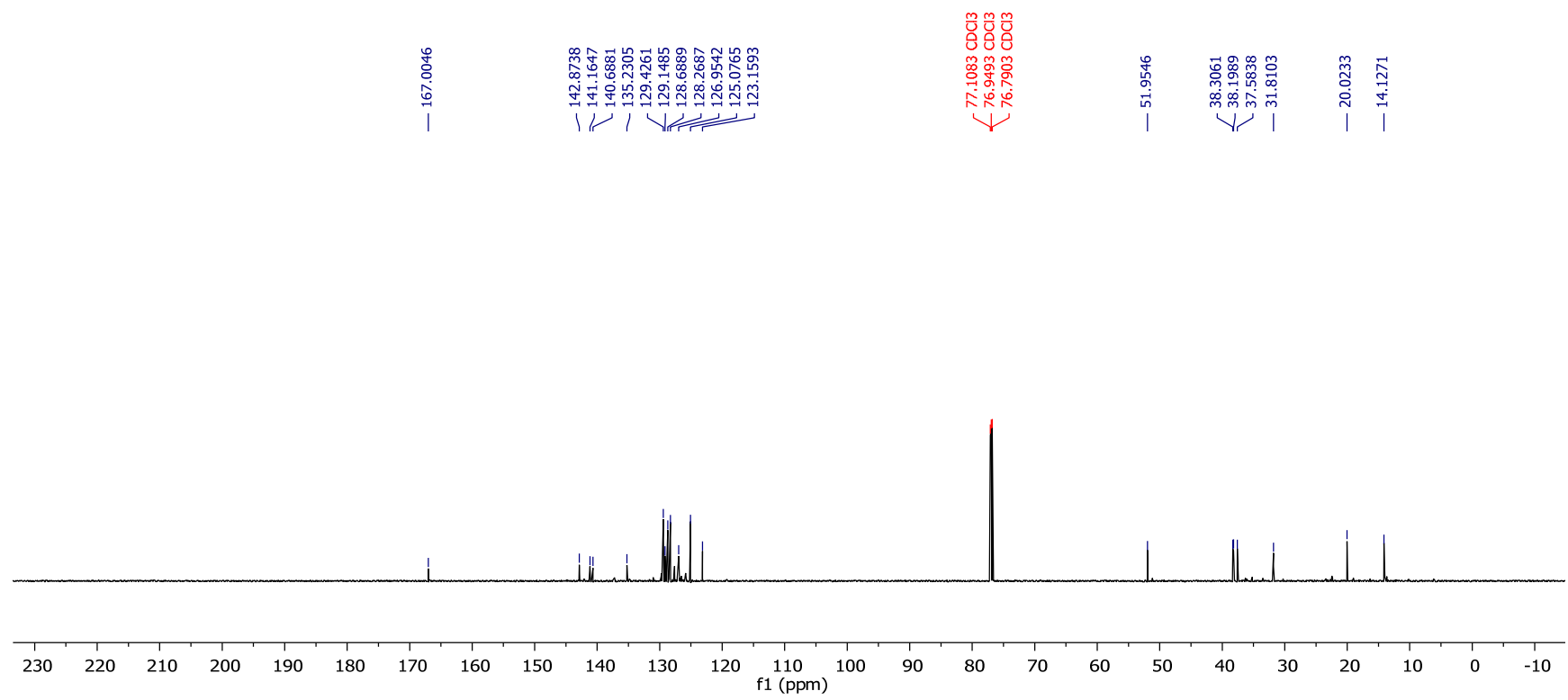

Figure S98. $\quad{ }^{13} \mathrm{C}$ NMR of $\mathbf{S} 17\left(201 \mathrm{MHz}, \mathrm{CDCl}_{3}, 295 \mathrm{~K}\right)$. 

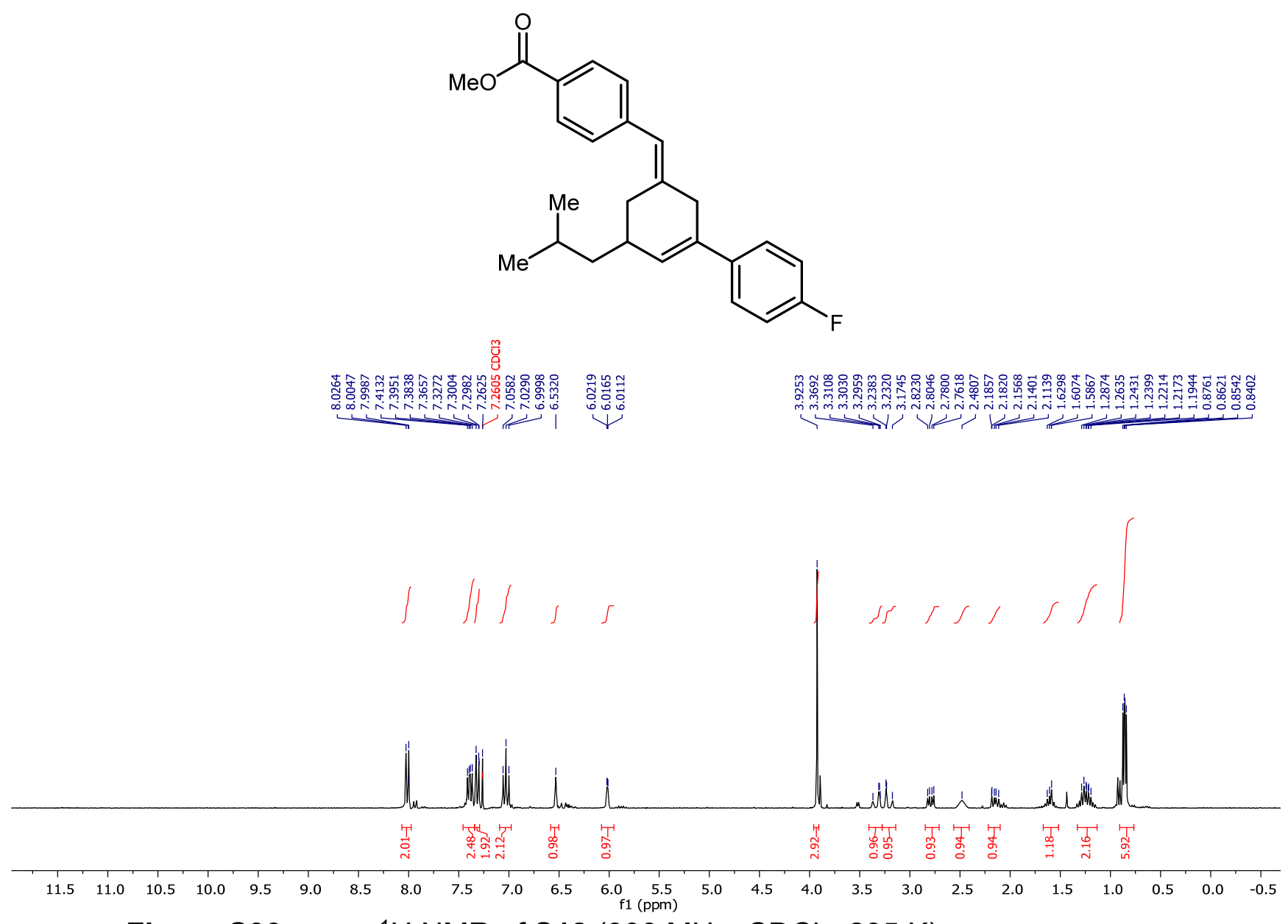

Figure S99. $\quad{ }^{1} \mathrm{H}$ NMR of $\mathbf{S} 18\left(300 \mathrm{MHz}, \mathrm{CDCl}_{3}, 295 \mathrm{~K}\right)$.

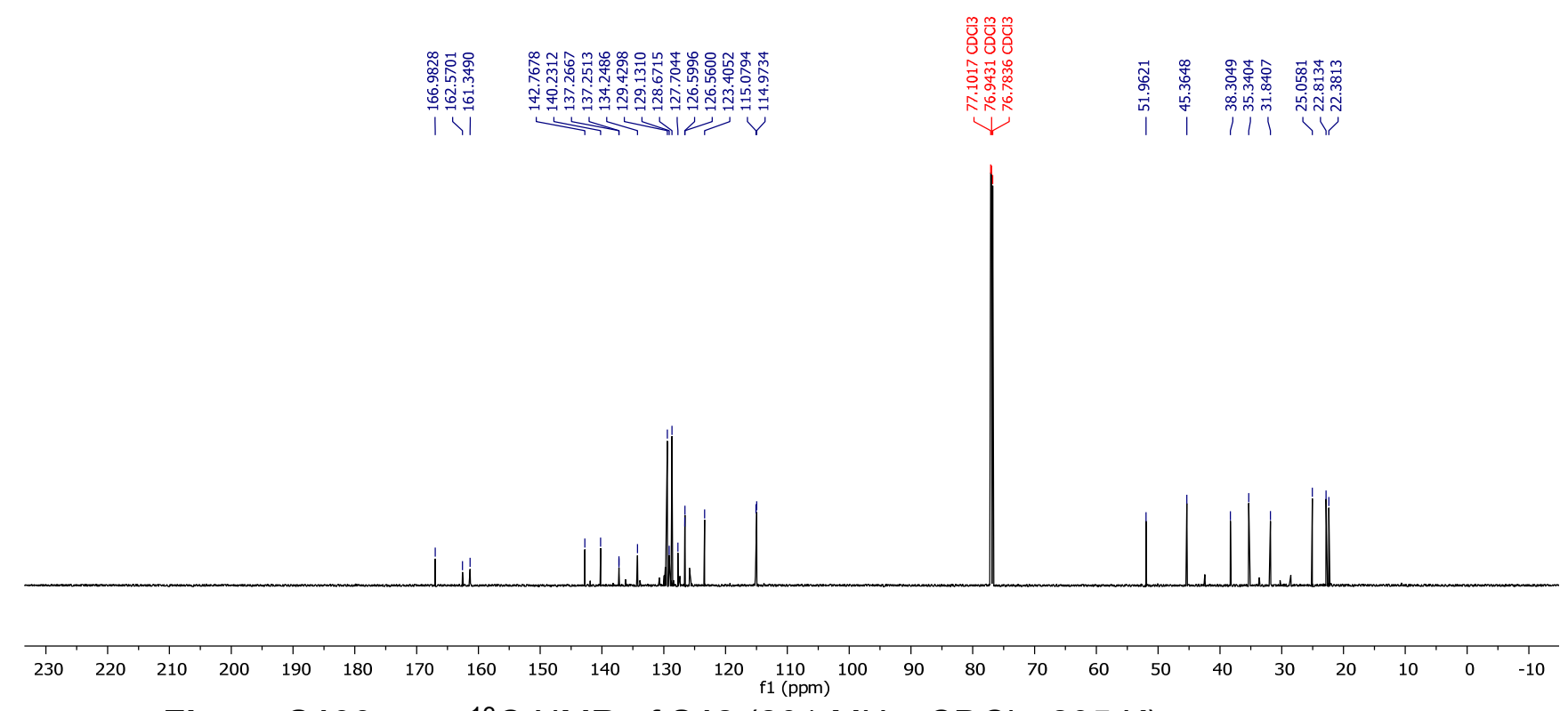

Figure S100. $\quad{ }^{13} \mathrm{C}$ NMR of $\mathbf{S} 18\left(201 \mathrm{MHz}, \mathrm{CDCl}_{3}, 295 \mathrm{~K}\right)$. 


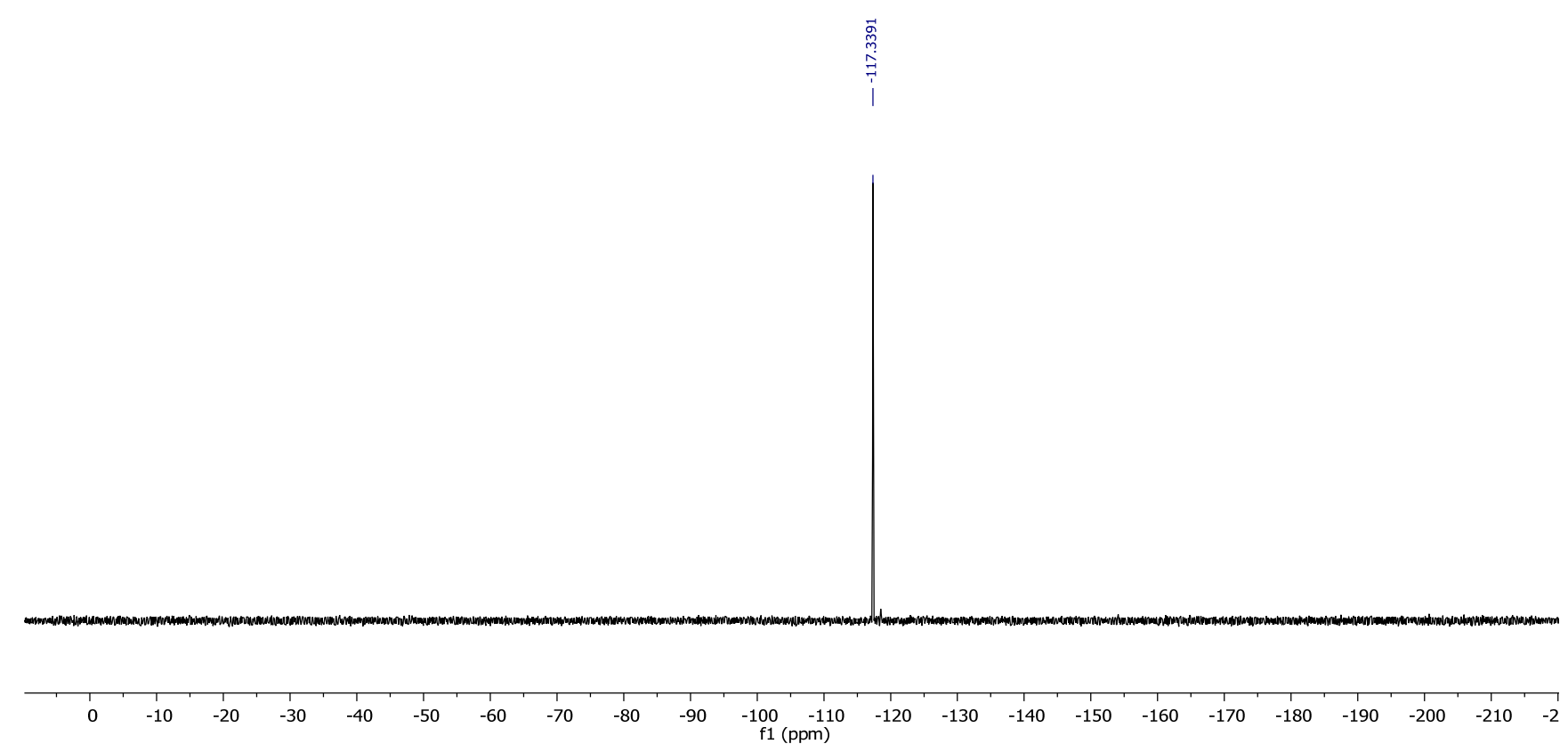

Figure S101. $\quad{ }^{19} \mathrm{~F} \mathrm{NMR} \mathrm{of} \mathbf{S} 18\left(282 \mathrm{MHz}, \mathrm{CDCl}_{3}, 295 \mathrm{~K}\right)$. 
<smiles>C(=C1CC(c2ccccc2)=CC(CCc2ccccc2)C1)c1c[nH]c2ccccc12</smiles>
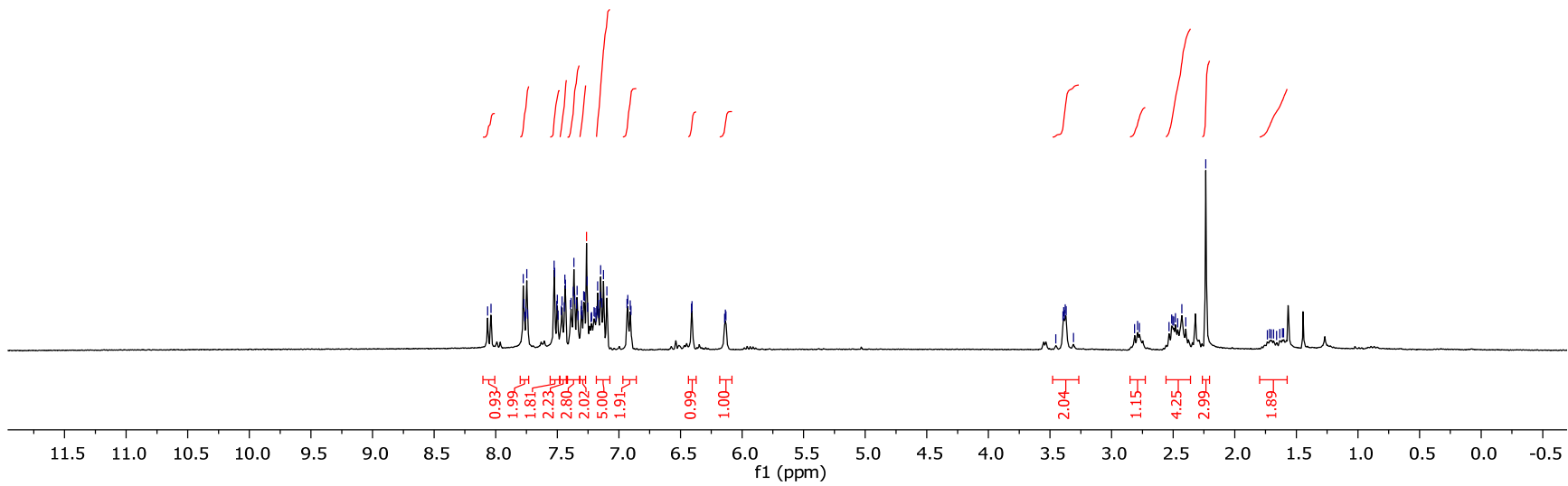

Figure S102. $\quad{ }^{1} \mathrm{H}$ NMR of $\mathbf{S} 19\left(300 \mathrm{MHz}, \mathrm{CDCl}_{3}, 295 \mathrm{~K}\right)$.

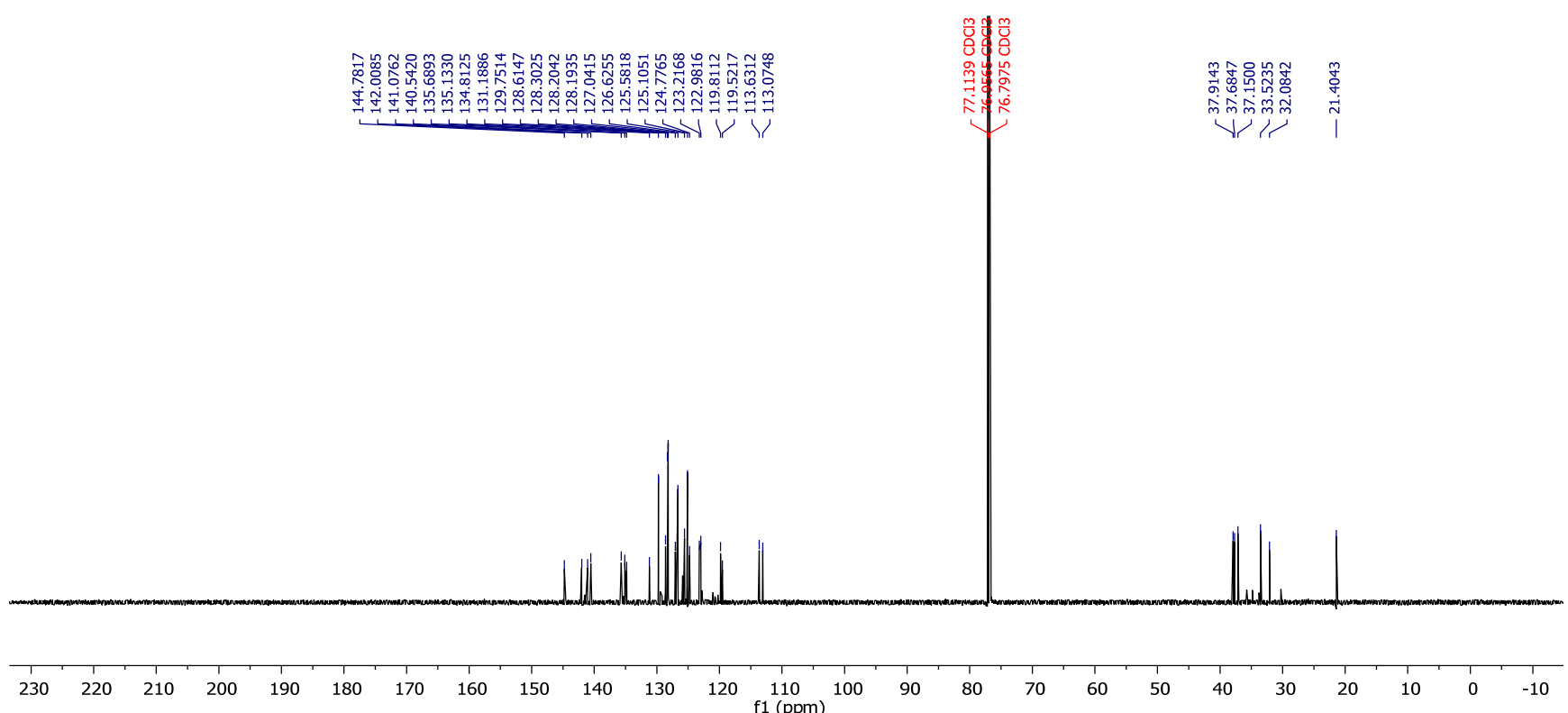

Figure S103. $\quad{ }^{13} \mathrm{C}$ NMR of $\mathbf{S} 19\left(201 \mathrm{MHz}, \mathrm{CDCl}_{3}, 295 \mathrm{~K}\right)$. 
<smiles>COc1ccc(Cc2cc(C)cc(-c3ccccc3)c2)cc1</smiles>

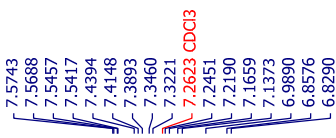

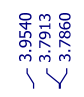
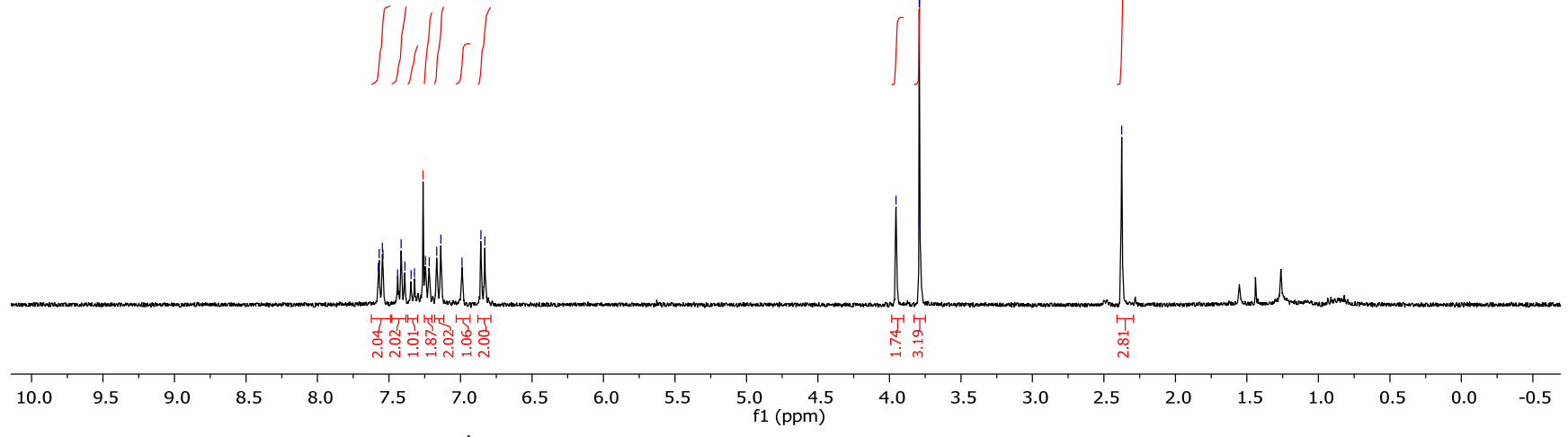

Figure S104. $\quad{ }^{1} \mathrm{H}$ NMR of $\mathbf{3 8}\left(300 \mathrm{MHz}, \mathrm{CDCl}_{3}, 295 \mathrm{~K}\right)$.

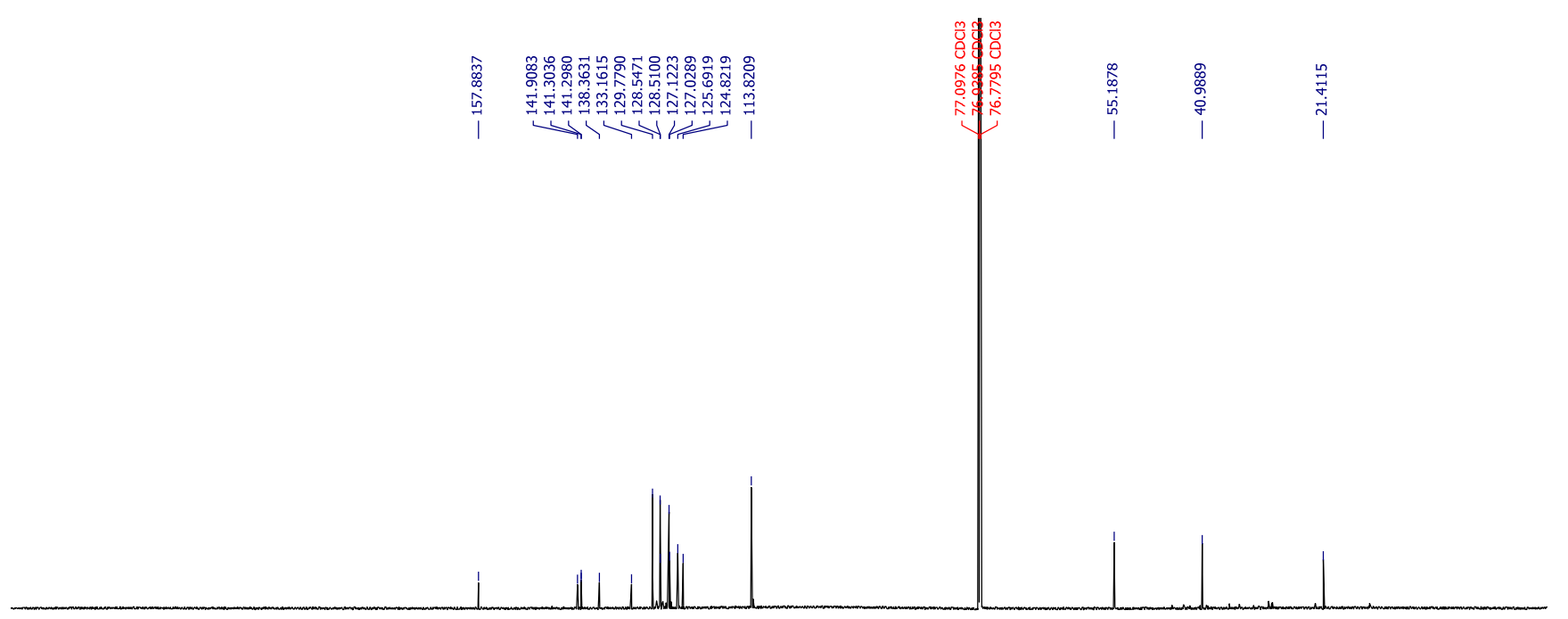

Figure S105. $\quad{ }^{13} \mathrm{C}$ NMR of $38\left(201 \mathrm{MHz}, \mathrm{CDCl}_{3}, 295 \mathrm{~K}\right)$. 

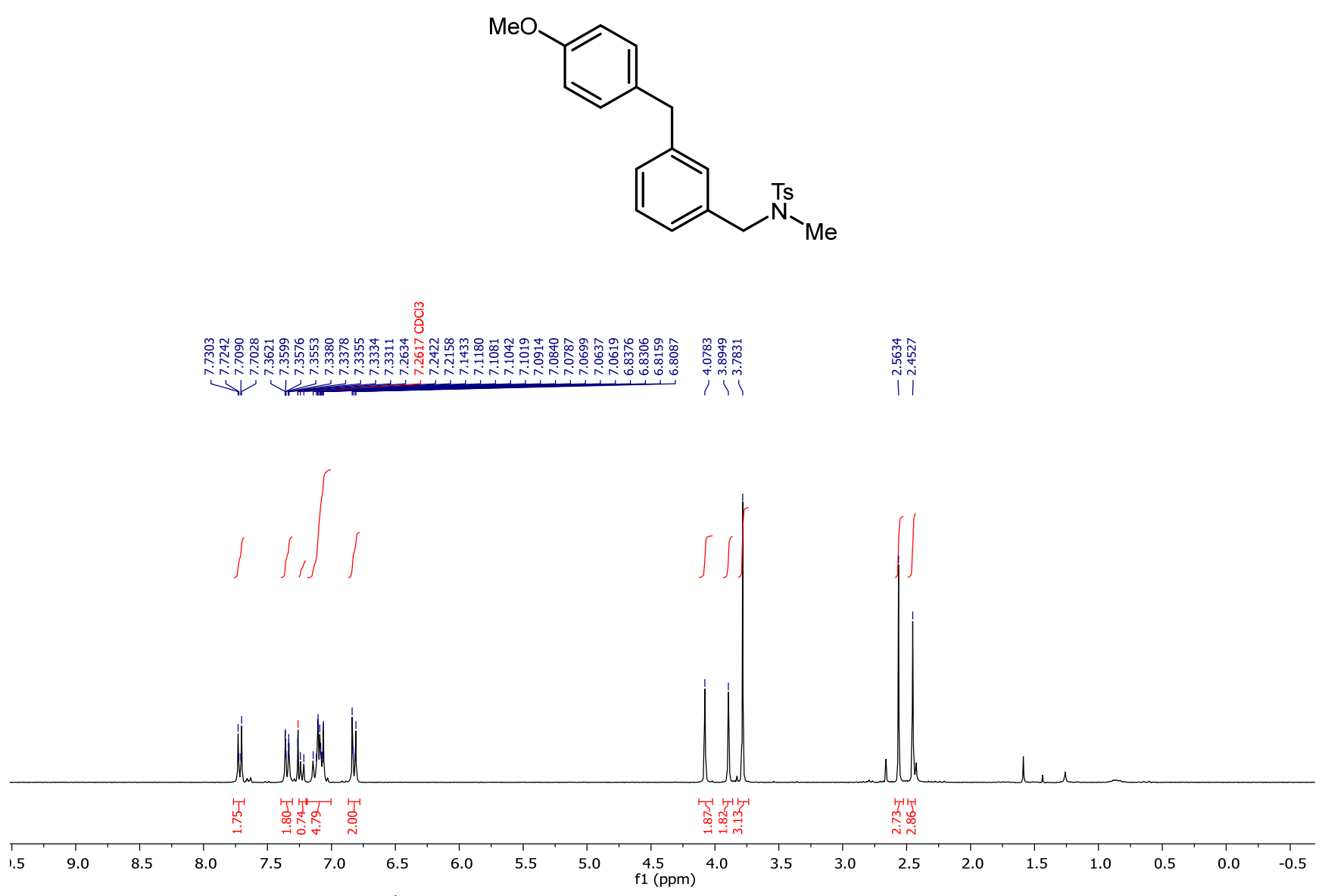

Figure S106. ${ }^{1} \mathrm{H} \mathrm{NMR}$ of $39\left(300 \mathrm{MHz}, \mathrm{CDCl}_{3}, 295 \mathrm{~K}\right)$.
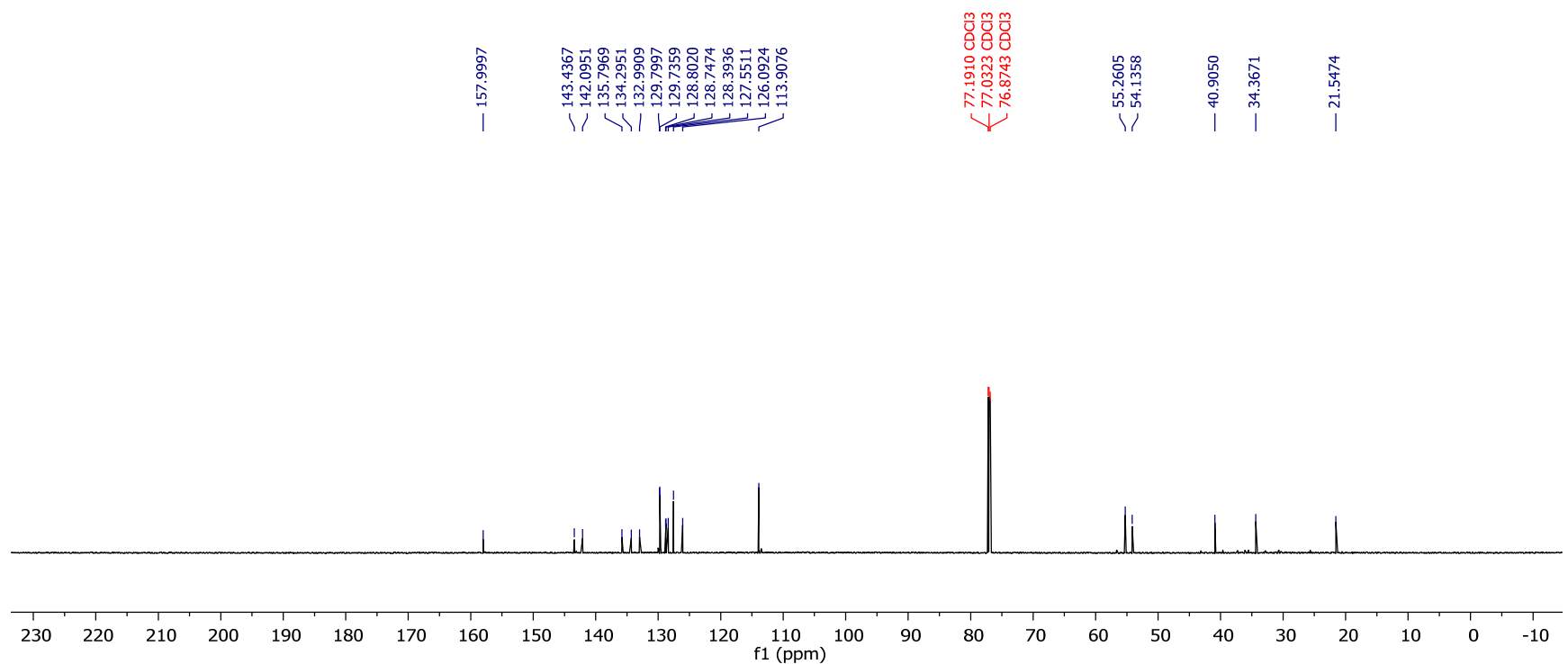

Figure S107. $\quad{ }^{13} \mathrm{C}$ NMR of $39\left(201 \mathrm{MHz}, \mathrm{CDCl}_{3}, 295 \mathrm{~K}\right)$. 

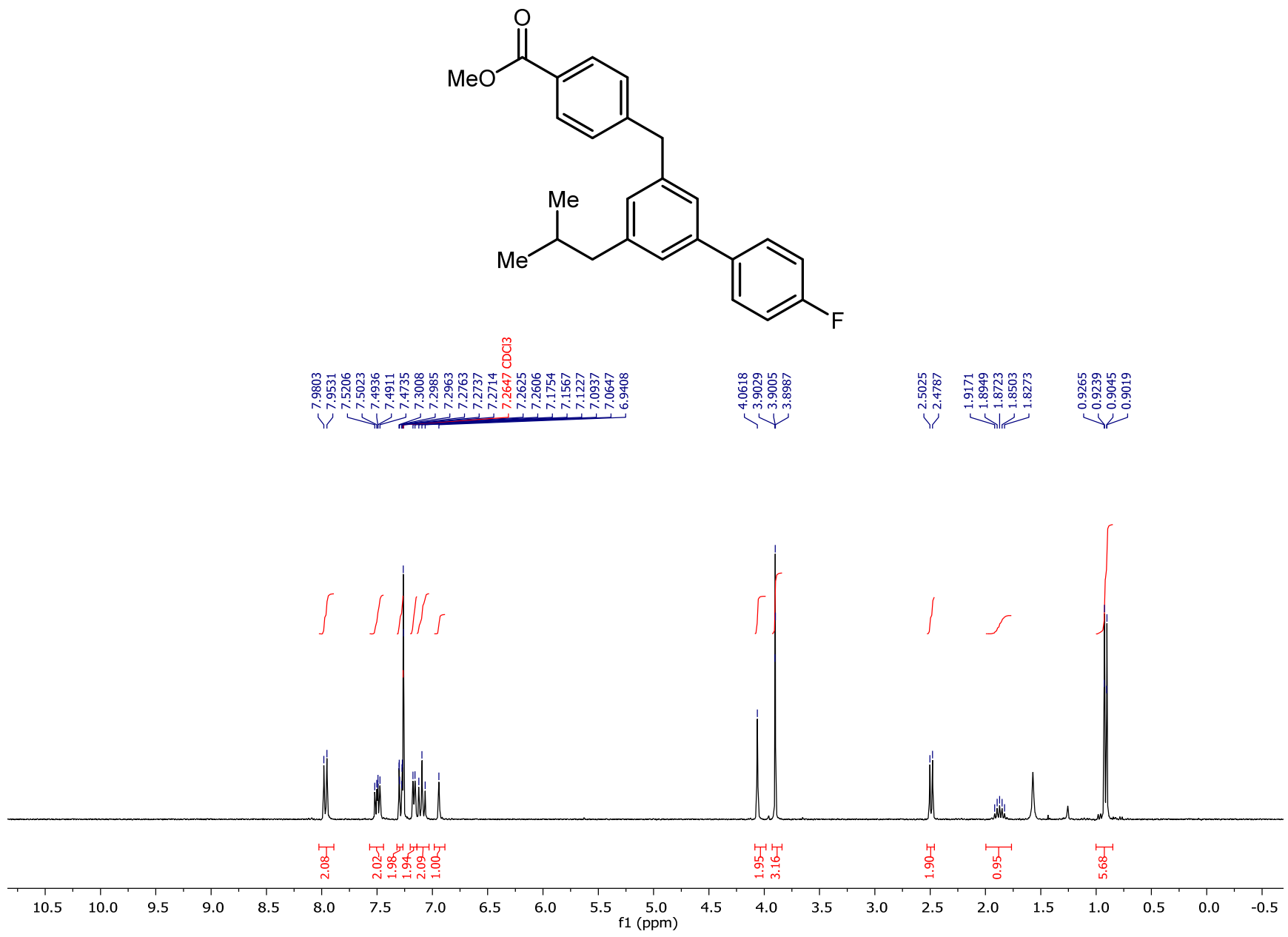

Figure S108. $\quad{ }^{1} \mathrm{H} \mathrm{NMR}$ of $\mathbf{4 0}\left(300 \mathrm{MHz}, \mathrm{CDCl}_{3}, 295 \mathrm{~K}\right)$.

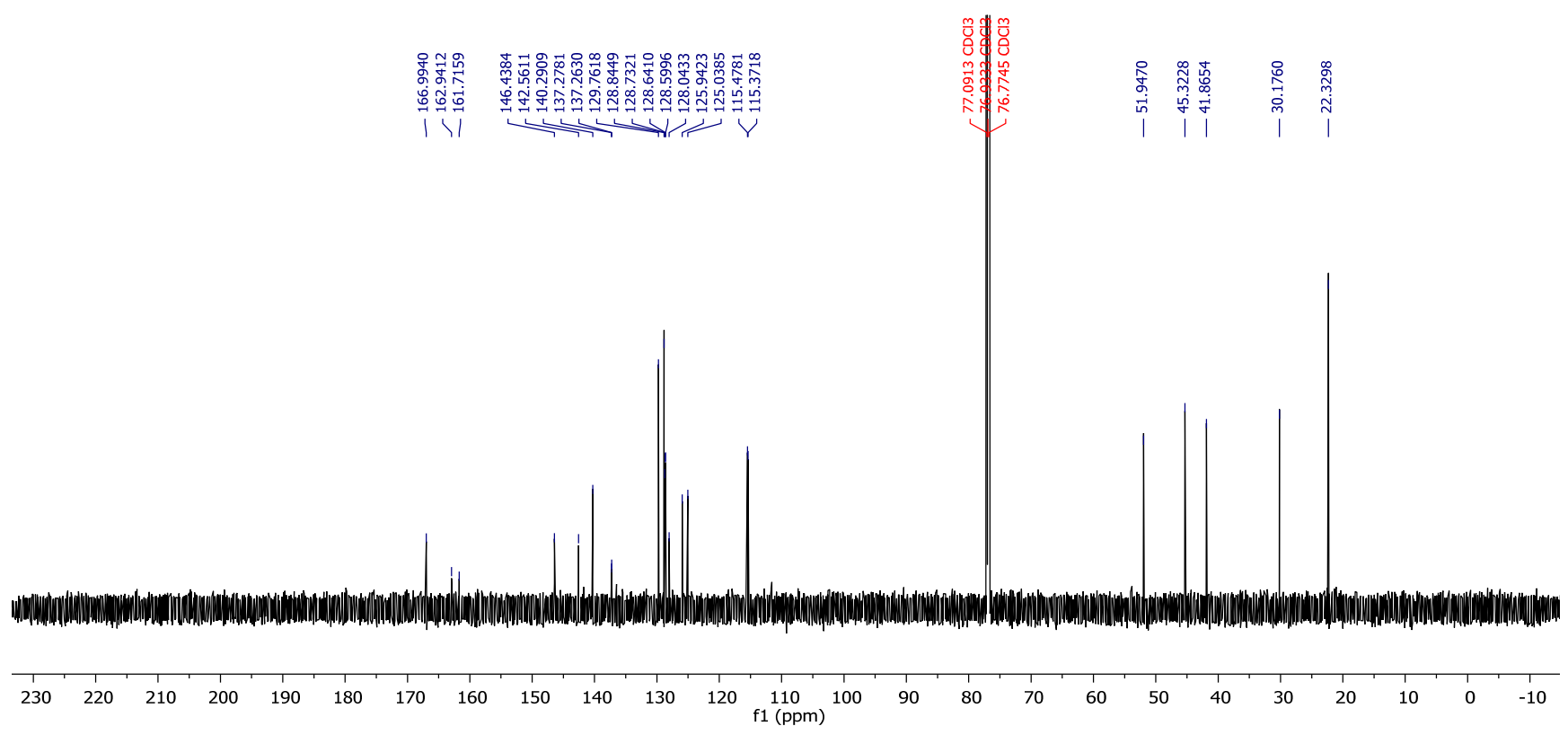

Figure S109. $\quad{ }^{13} \mathrm{C} \mathrm{NMR} \mathrm{of} \mathbf{4 0}\left(201 \mathrm{MHz}, \mathrm{CDCl}_{3}, 295 \mathrm{~K}\right)$. 


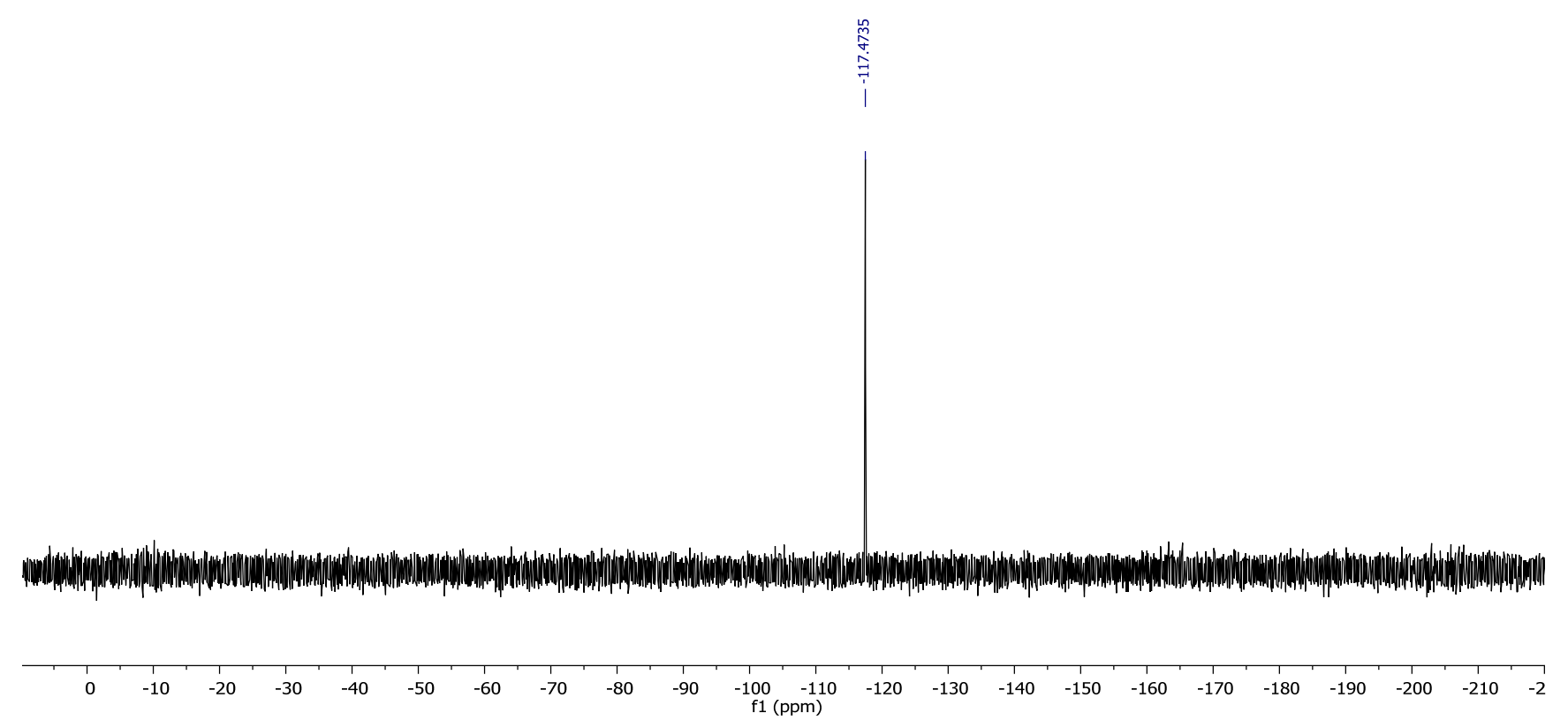

Figure S110. ${ }^{19} \mathrm{~F} \mathrm{NMR}$ of $39\left(282 \mathrm{MHz}, \mathrm{CDCl}_{3}, 295 \mathrm{~K}\right)$. 

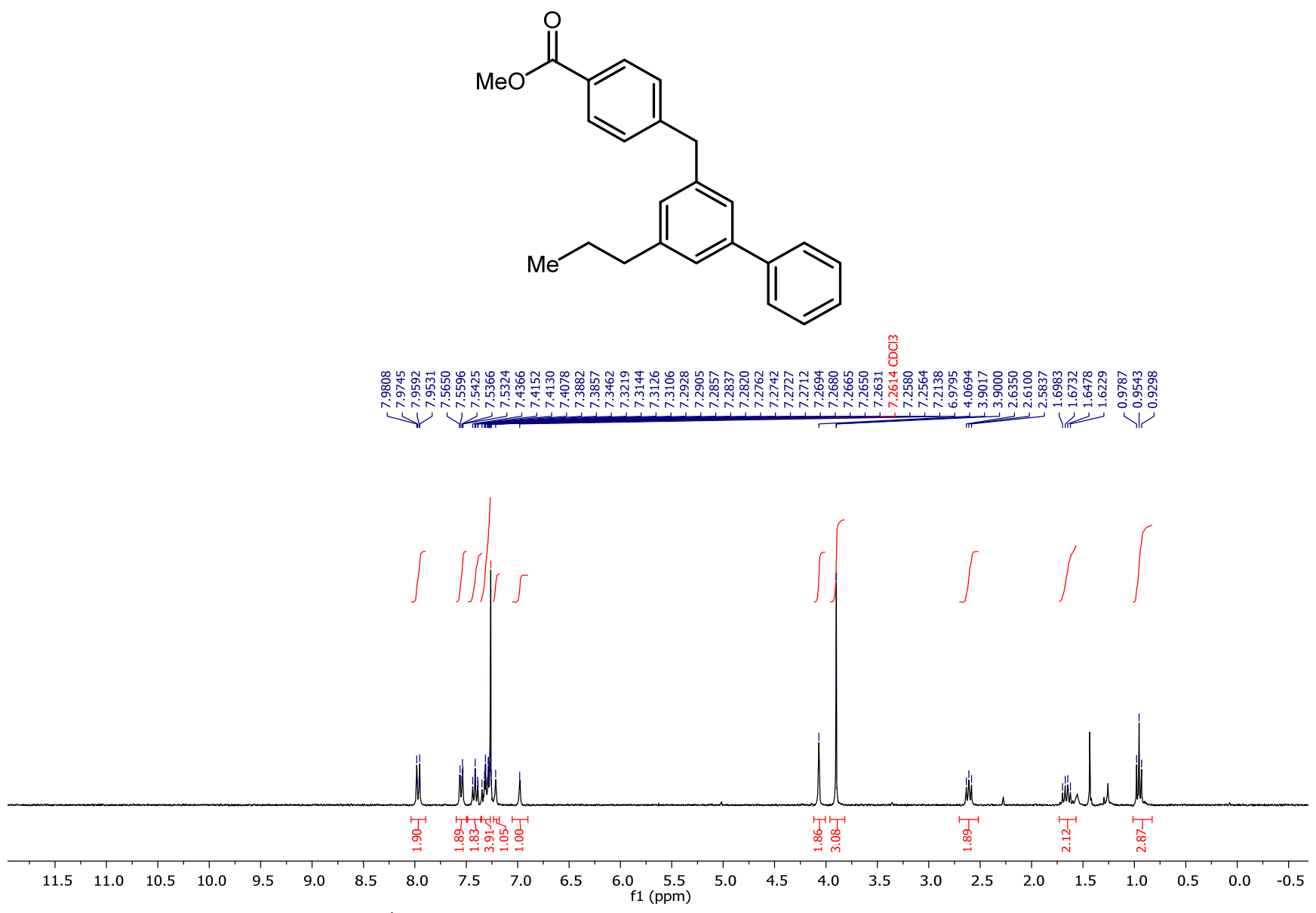

Figure S111. ${ }^{1} \mathrm{H}$ NMR of $41\left(300 \mathrm{MHz}, \mathrm{CDCl}_{3}, 295 \mathrm{~K}\right)$.

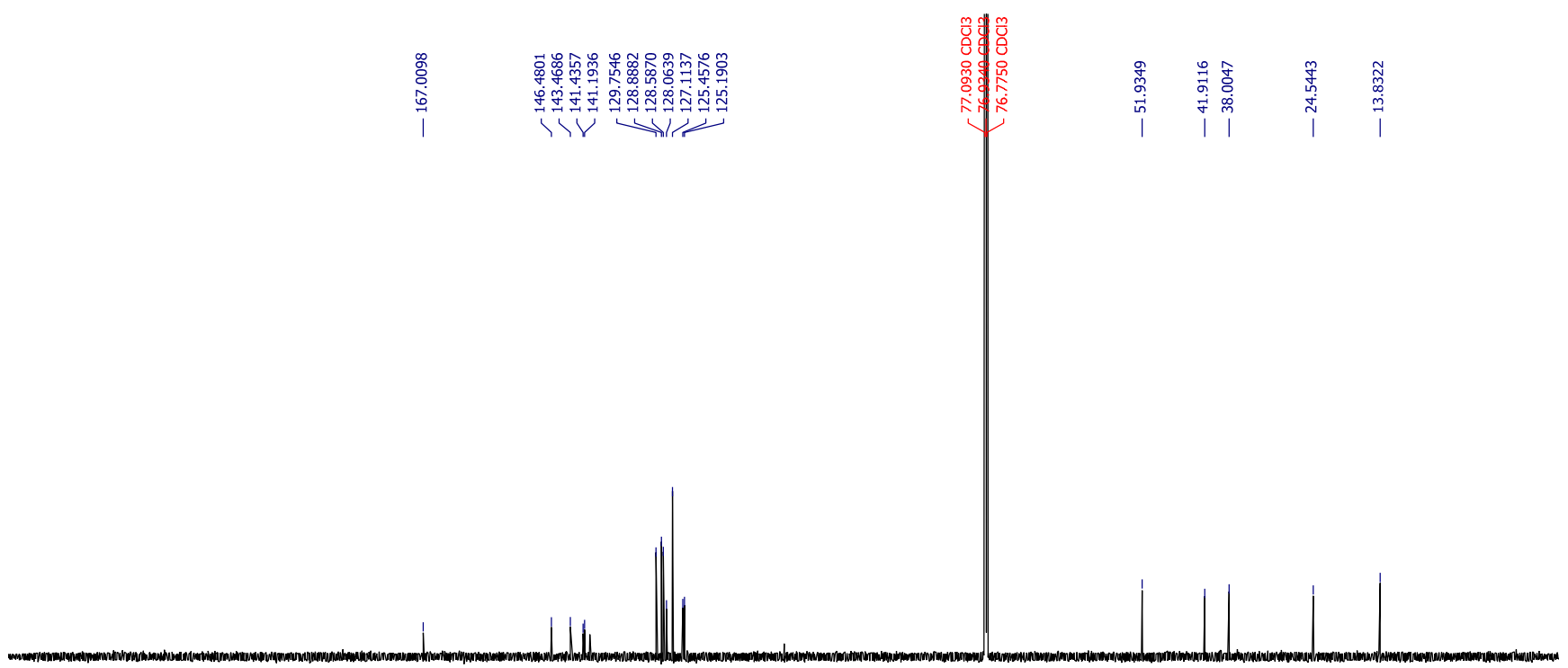

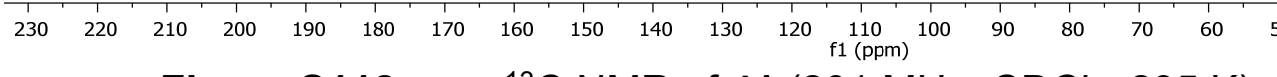

Figure S112. $\quad{ }^{13} \mathrm{C}$ NMR of $41\left(201 \mathrm{MHz}, \mathrm{CDCl}_{3}, 295 \mathrm{~K}\right)$. 
<smiles>Cc1cc(CCCN(C)S)cc(-c2ccccc2)c1</smiles>
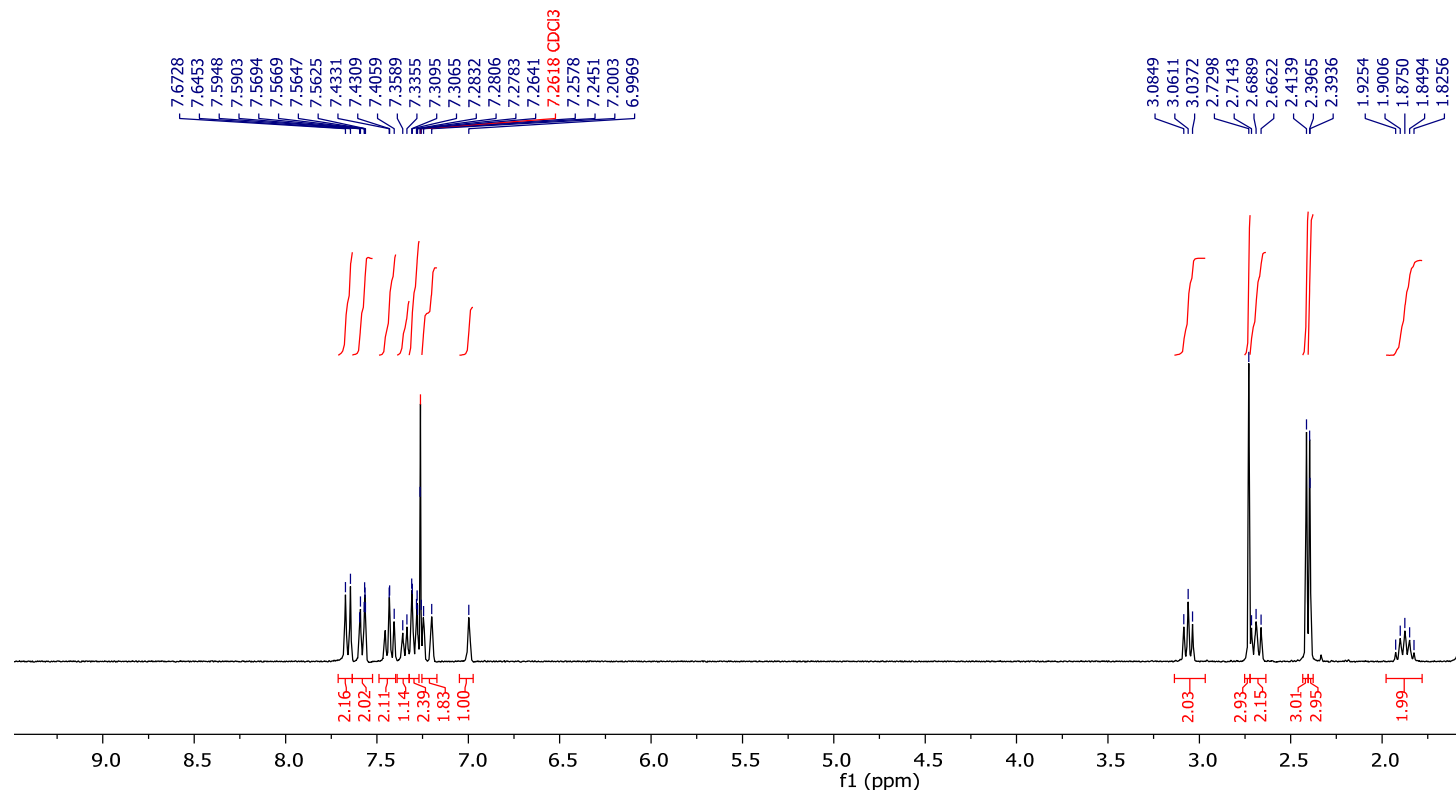

Figure S113. ${ }^{1} \mathrm{H}$ NMR of $42\left(300 \mathrm{MHz}, \mathrm{CDCl}_{3}, 295 \mathrm{~K}\right)$.

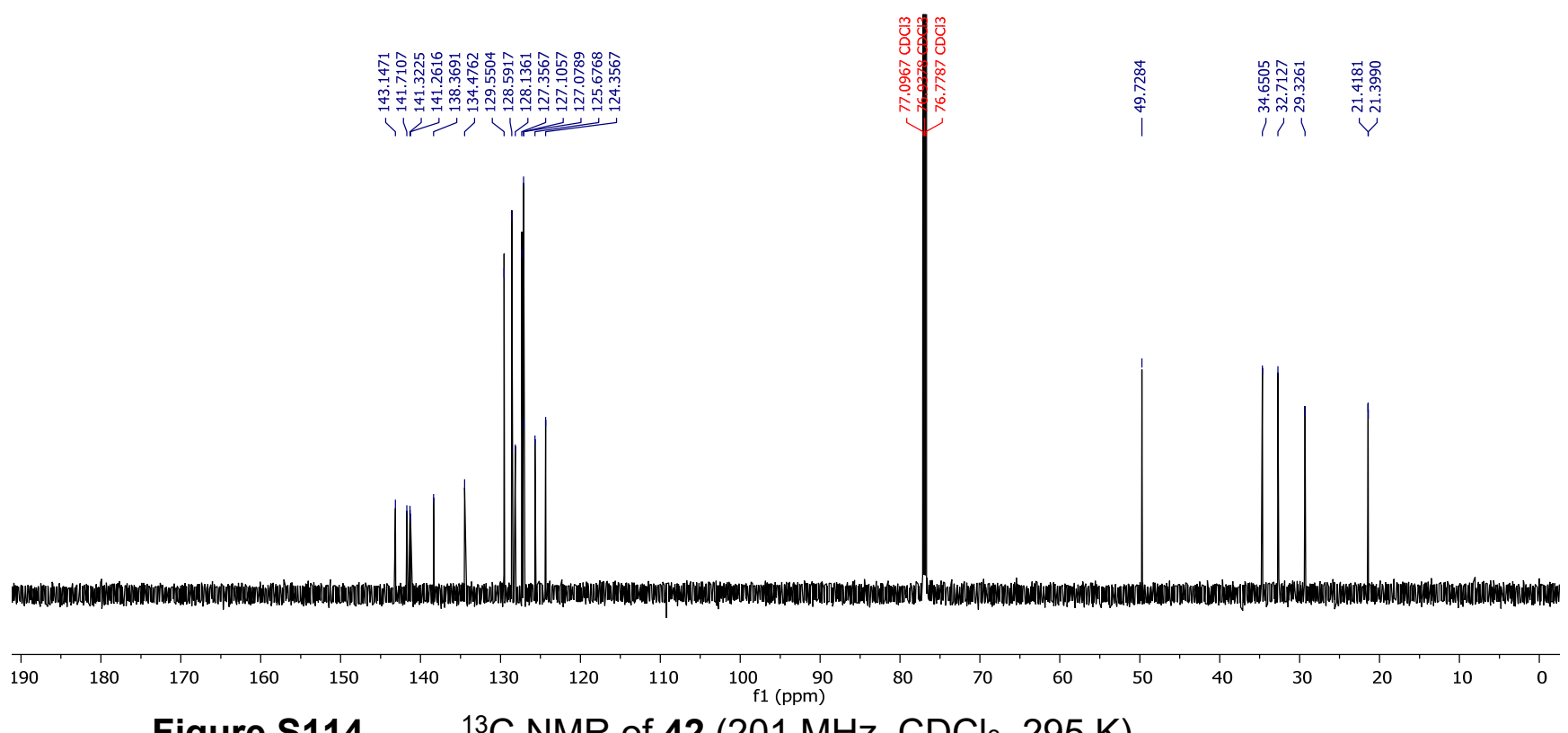

Figure S114. $\quad{ }^{13} \mathrm{C} \mathrm{NMR}$ of $42\left(201 \mathrm{MHz}, \mathrm{CDCl}_{3}, 295 \mathrm{~K}\right)$. 
<smiles>COc1ccc(/C=C2/C=C(c3ccccc3)CC(C)C2)cc1</smiles>

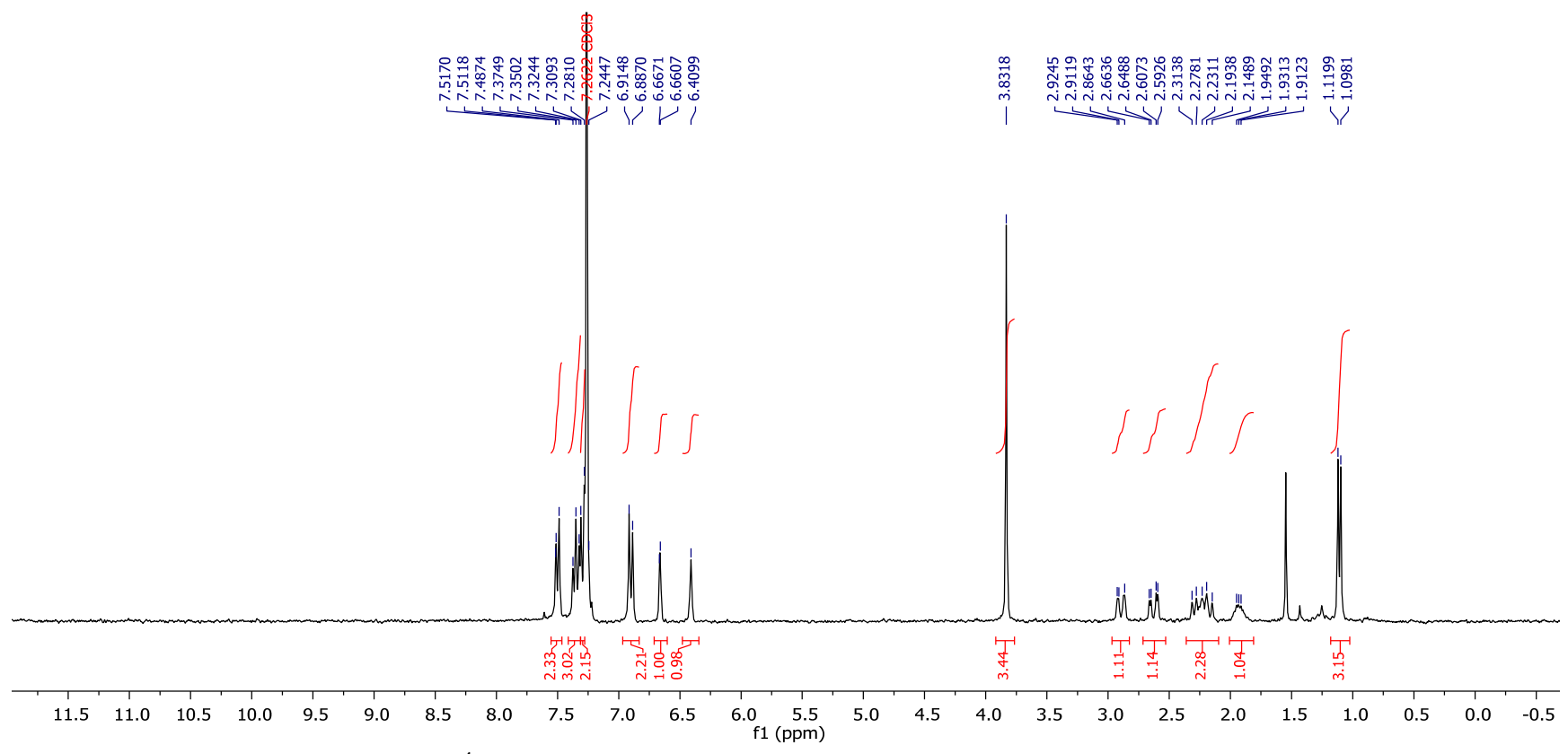

Figure S115. $\quad{ }^{1} \mathrm{H}$ NMR of $\mathbf{4 3}\left(300 \mathrm{MHz}, \mathrm{CDCl}_{3}, 295 \mathrm{~K}\right)$.

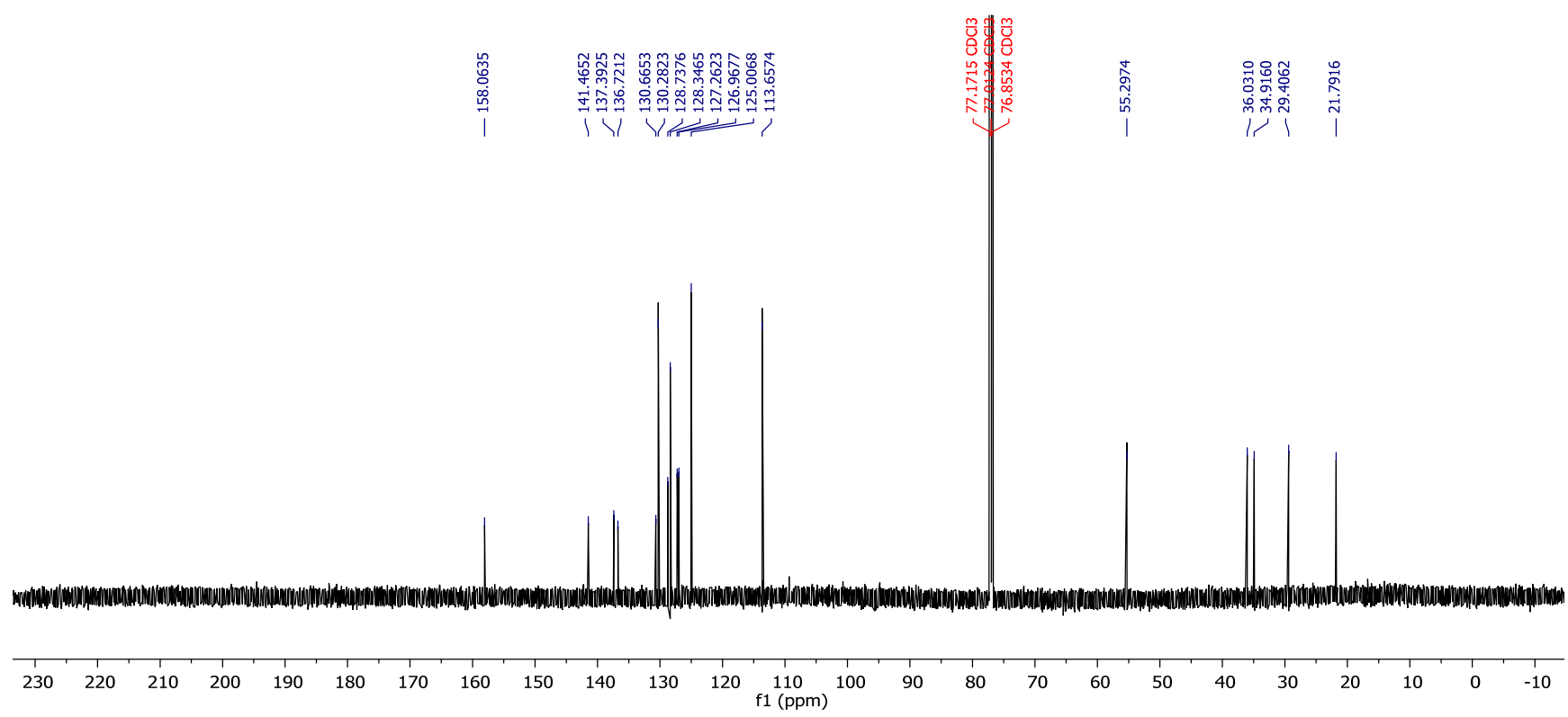

Figure S116. $\quad{ }^{13} \mathrm{C}$ NMR of $43\left(201 \mathrm{MHz}, \mathrm{CDCl}_{3}, 295 \mathrm{~K}\right)$. 


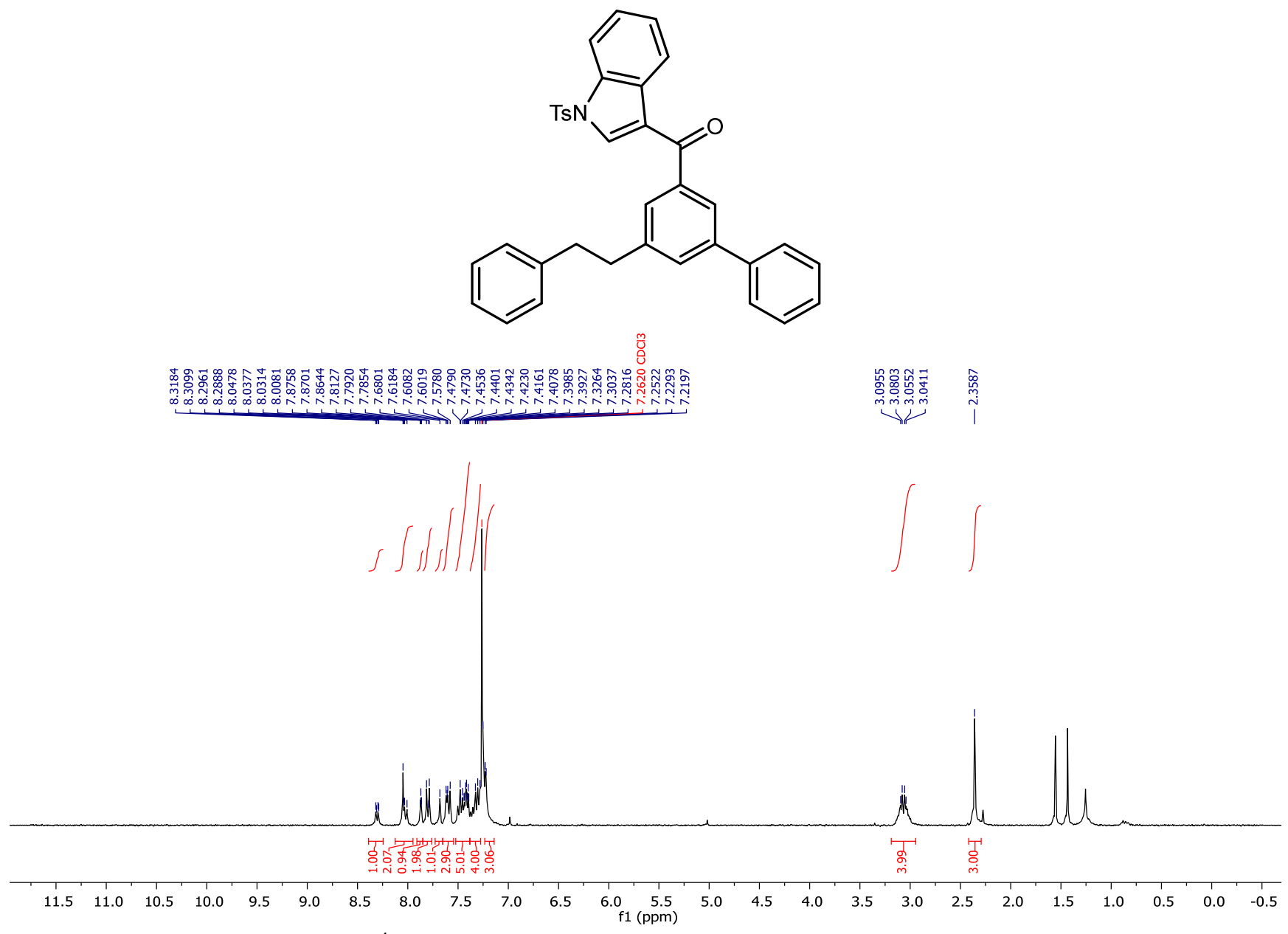

Figure S117. $\quad{ }^{1} \mathrm{H}$ NMR of $\mathbf{4 4}\left(300 \mathrm{MHz}, \mathrm{CDCl}_{3}, 295 \mathrm{~K}\right)$.

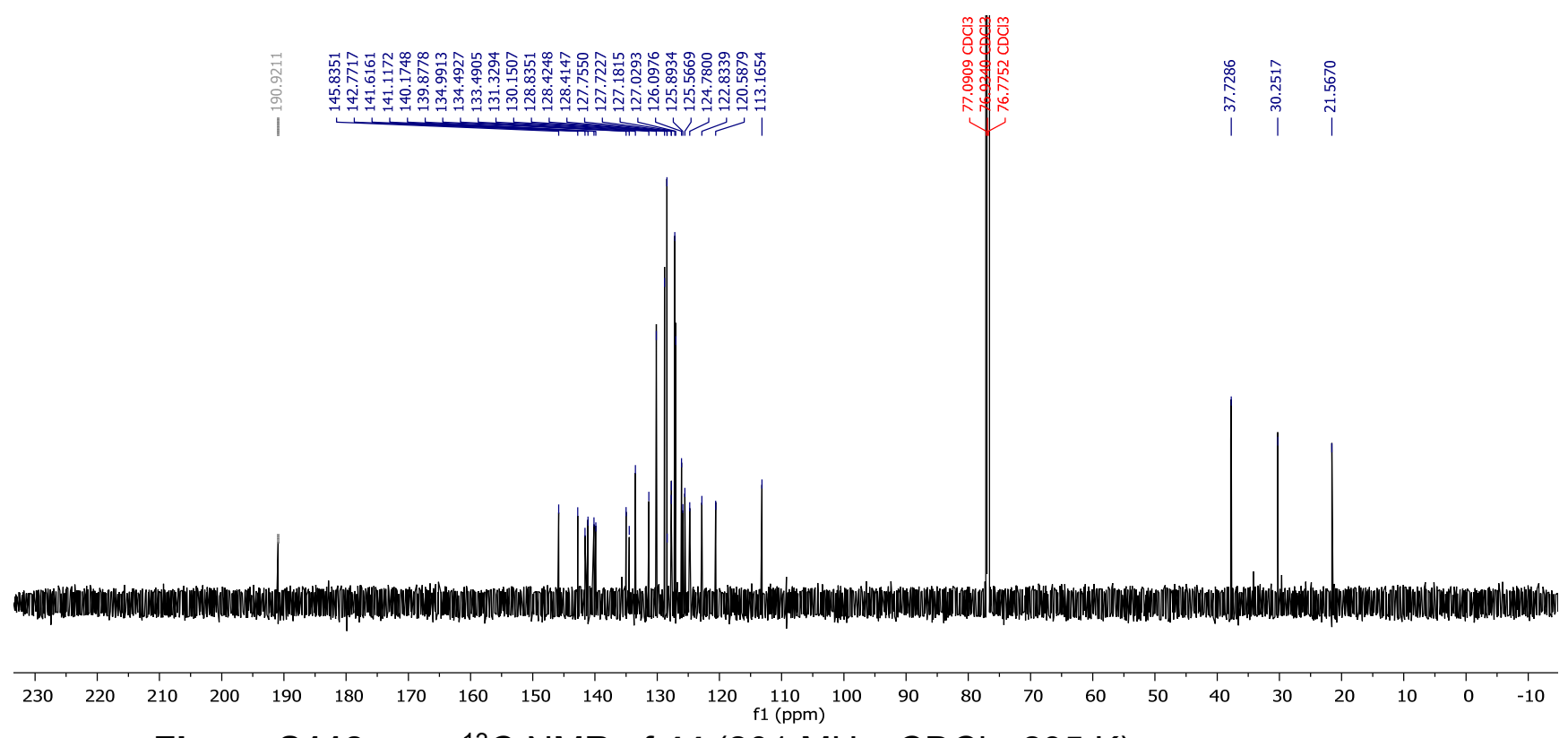

Figure S118. $\quad{ }^{13} \mathrm{C} \mathrm{NMR}$ of $44\left(201 \mathrm{MHz}, \mathrm{CDCl}_{3}, 295 \mathrm{~K}\right)$. 


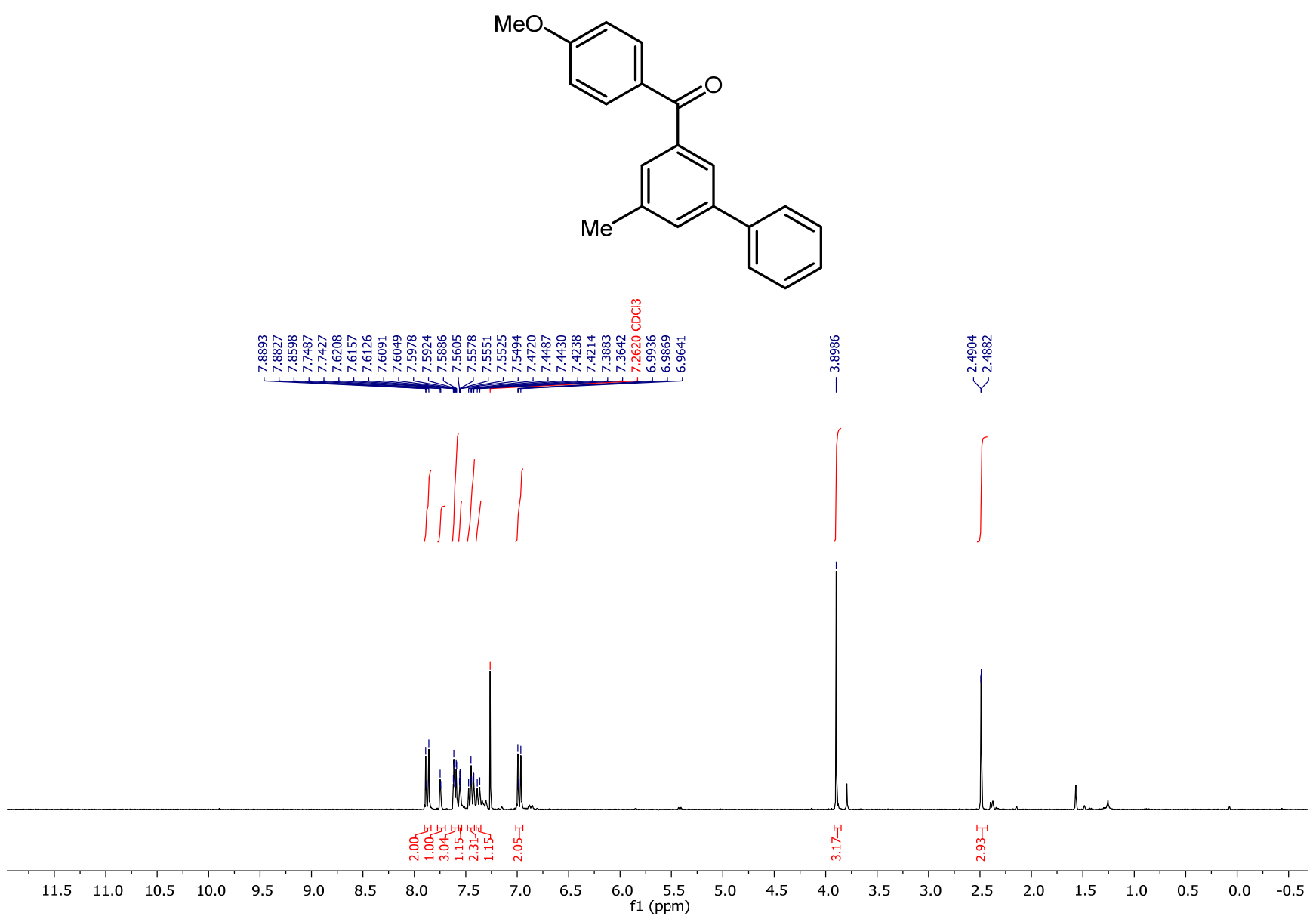

Figure S119. ${ }^{1} \mathrm{H}$ NMR of $45\left(300 \mathrm{MHz}, \mathrm{CDCl}_{3}, 295 \mathrm{~K}\right)$.

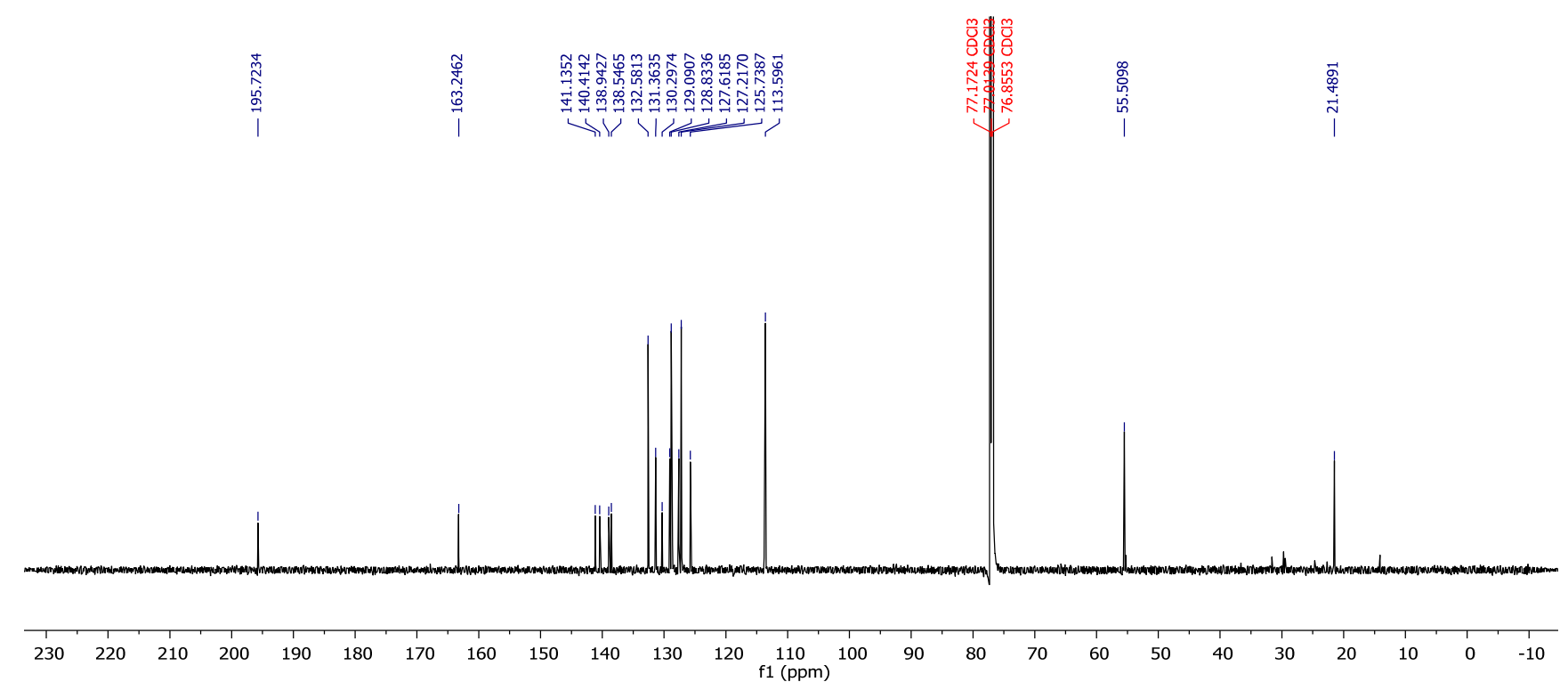

Figure S120. $\quad{ }^{13} \mathrm{C} \mathrm{NMR} \mathrm{of} 45\left(201 \mathrm{MHz}, \mathrm{CDCl}_{3}, 295 \mathrm{~K}\right)$. 
<smiles>COc1ccc(C=C2CC2Cc2ccccc2)cc1</smiles>
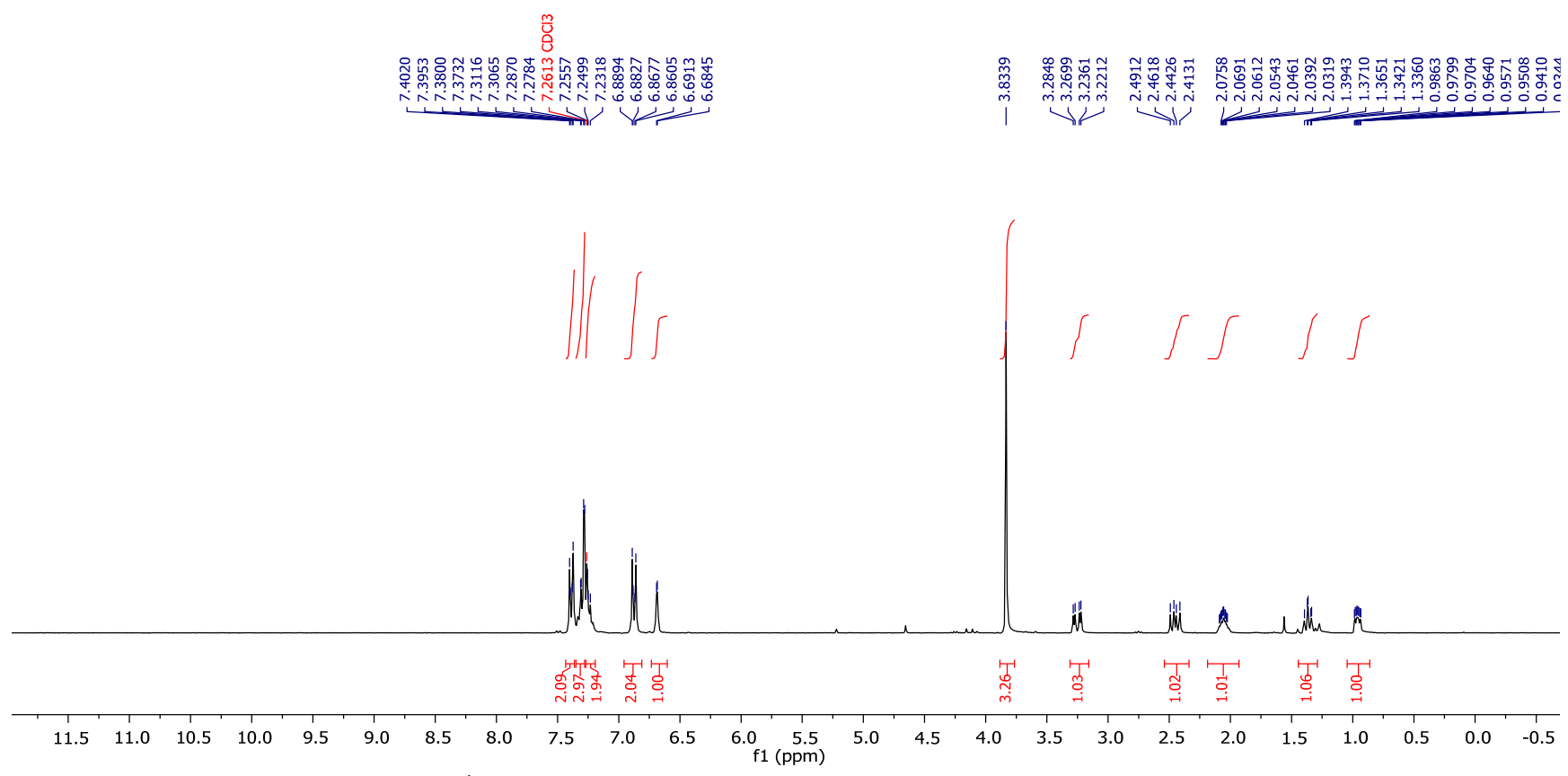

Figure S121. ${ }^{1} \mathrm{H}$ NMR of $47\left(300 \mathrm{MHz}, \mathrm{CDCl}_{3}, 295 \mathrm{~K}\right)$.

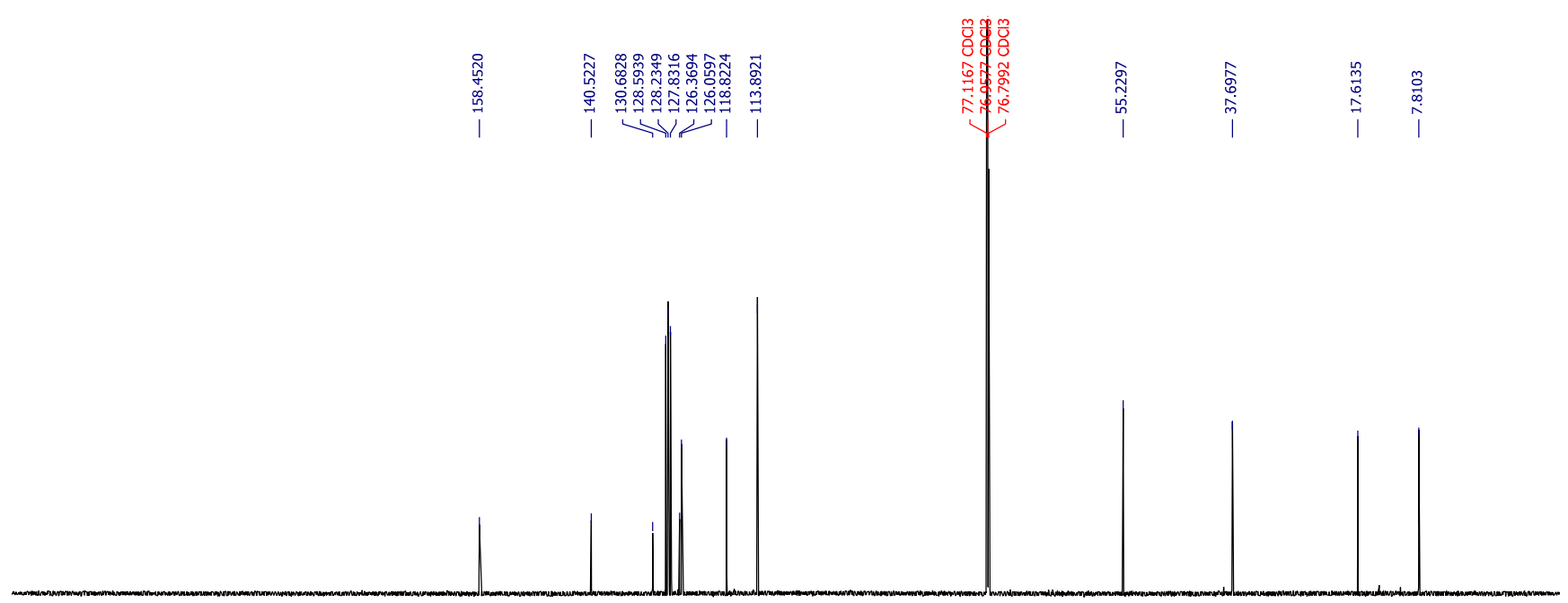

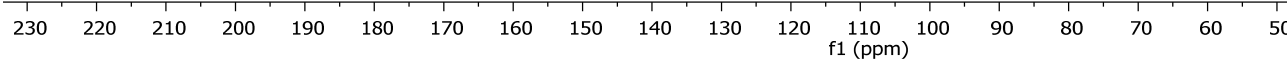

Figure S122. $\quad{ }^{13} \mathrm{C}$ NMR of $47\left(201 \mathrm{MHz}, \mathrm{CDCl}_{3}, 295 \mathrm{~K}\right)$. 

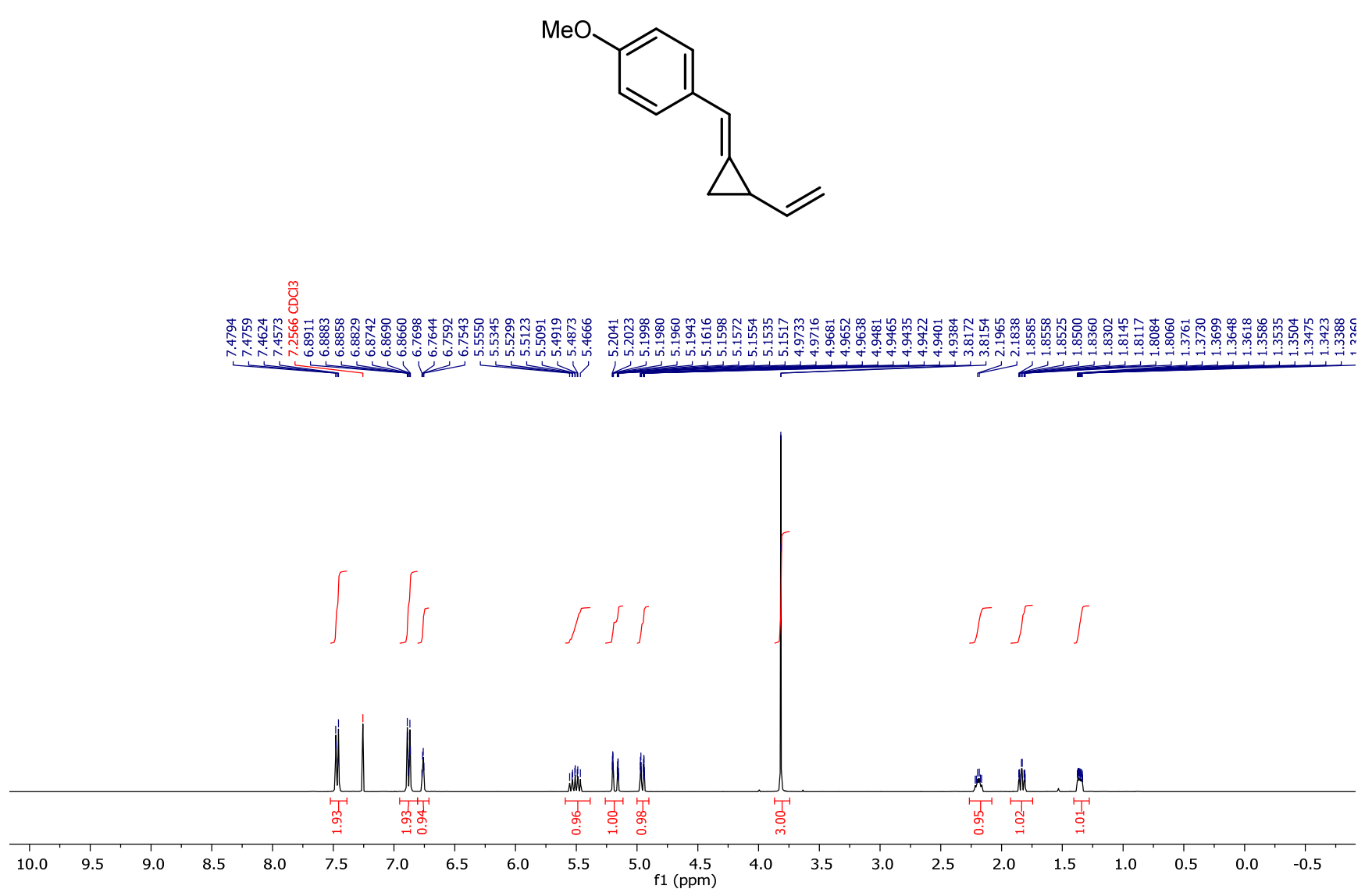

Figure S123. $\quad{ }^{1} \mathrm{H} \mathrm{NMR}$ of $\mathbf{S} 21\left(400 \mathrm{MHz}, \mathrm{CDCl}_{3}, 295 \mathrm{~K}\right)$.
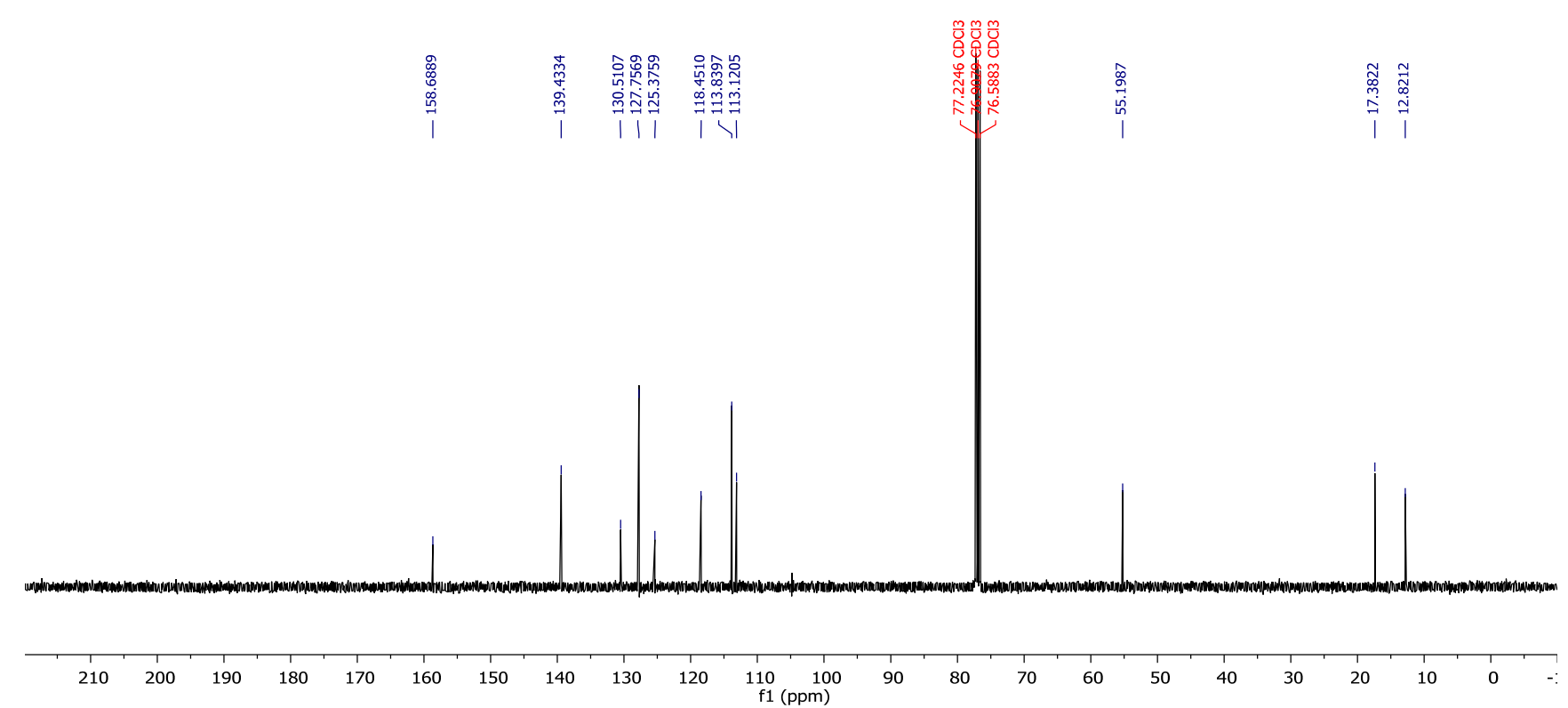

Figure S124. $\quad{ }^{13} \mathrm{C}$ NMR of $\mathbf{S 2 1}\left(101 \mathrm{MHz}, \mathrm{CDCl}_{3}, 295 \mathrm{~K}\right)$. 

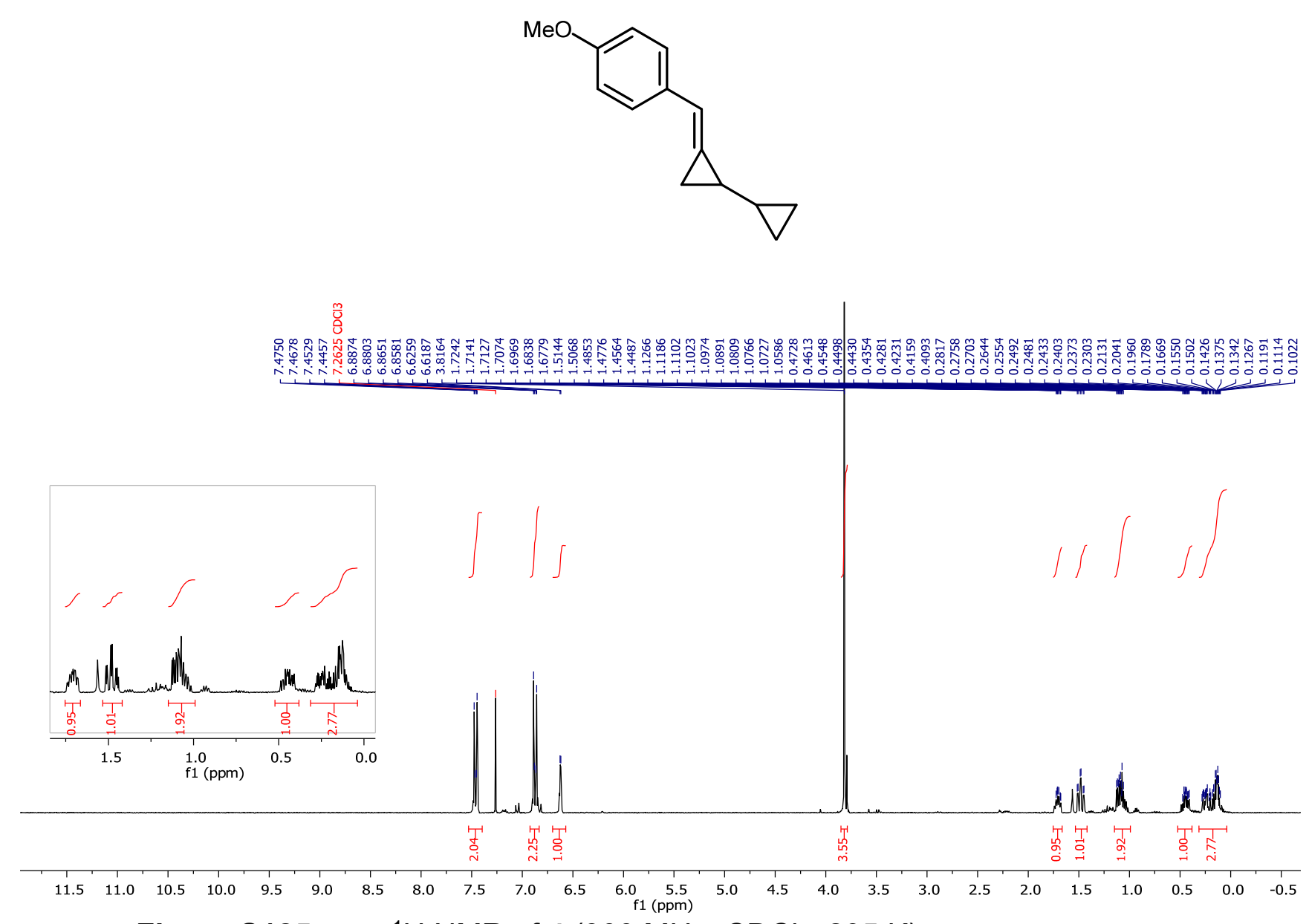

Figure S125. $\quad{ }^{1} \mathrm{H}$ NMR of $4\left(300 \mathrm{MHz}, \mathrm{CDCl}_{3}, 295 \mathrm{~K}\right)$.

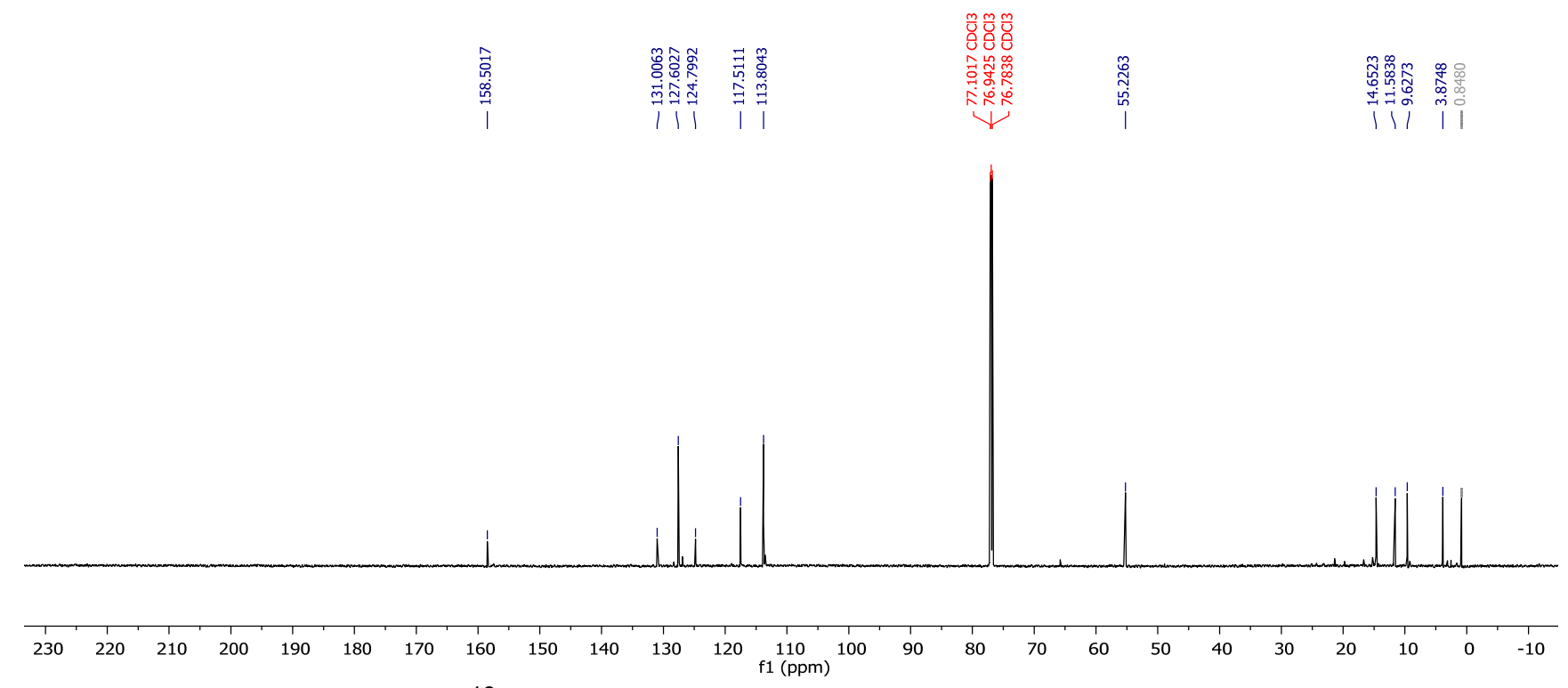

Figure S126. $\quad{ }^{13} \mathrm{C}$ NMR of $4\left(201 \mathrm{MHz}, \mathrm{CDCl}_{3}, 295 \mathrm{~K}\right)$. 
<smiles>COc1ccc(/C=C/CC(=C2CCC2)c2ccccc2)cc1</smiles>
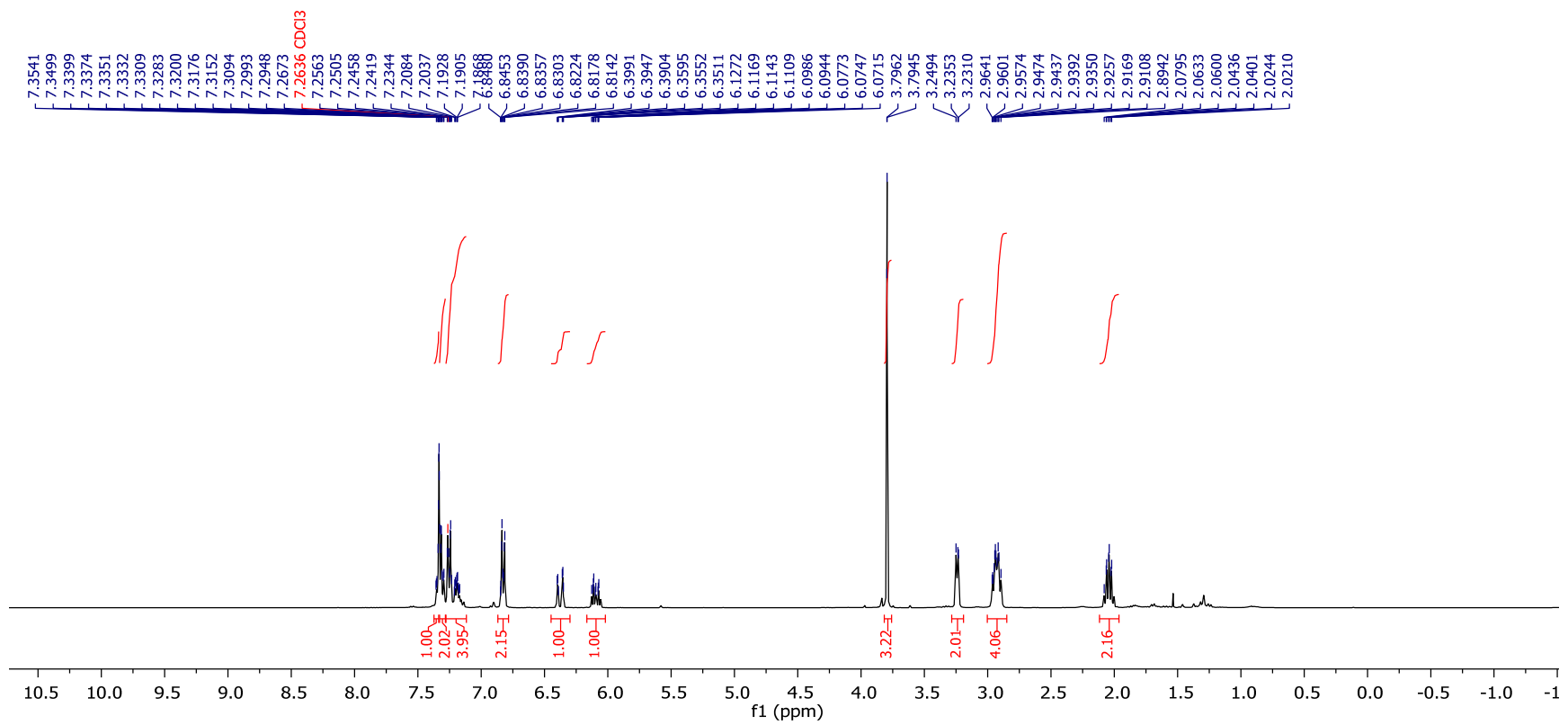

Figure S127. ${ }^{1} \mathrm{H}$ NMR of $49\left(300 \mathrm{MHz}, \mathrm{CDCl}_{3}, 295 \mathrm{~K}\right)$.
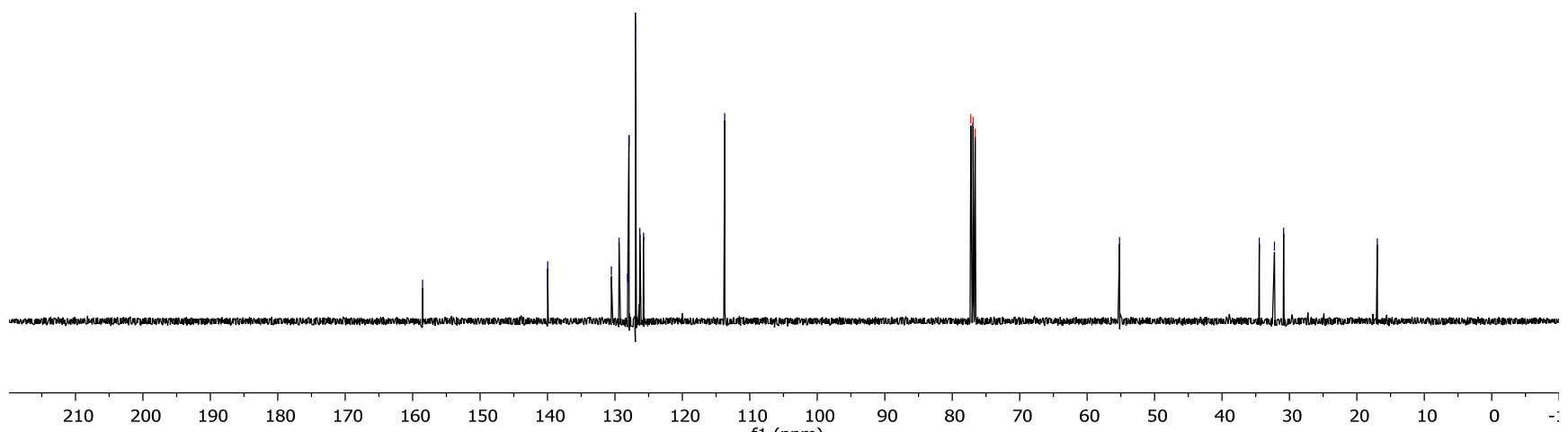

Figure S128. $\quad{ }^{13} \mathrm{C}$ NMR of $49\left(201 \mathrm{MHz}, \mathrm{CDCl}_{3}, 295 \mathrm{~K}\right)$. 


\section{IR Data for Vinylcyclopropanes and [5+1]-Products}<smiles>C=C(c1ccccc1)C1CC1CCc1ccccc1</smiles>

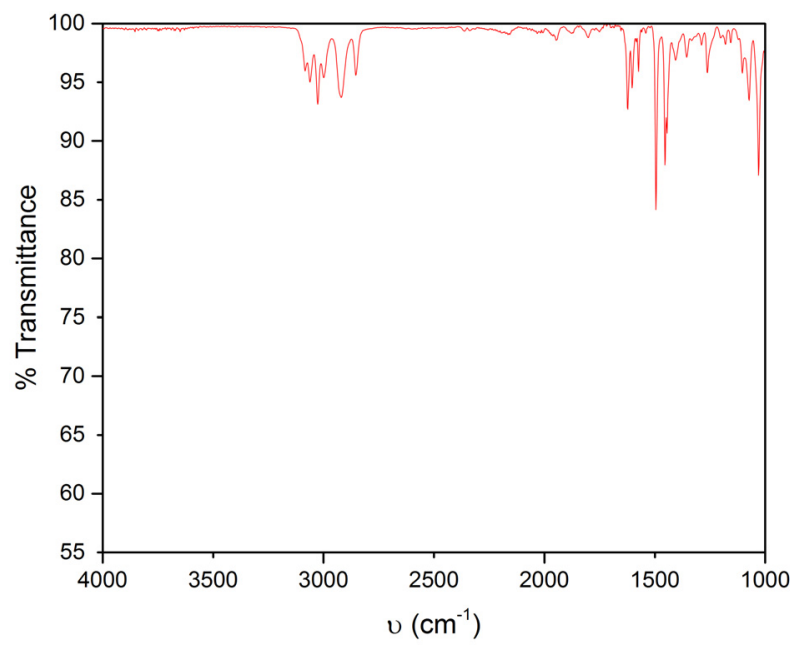

Figure S129. FT-IR of S1.<smiles>CC(C)(C)OC(=O)N1CCC(C/C=C/C(=O)c2ccccc2)CC1</smiles>

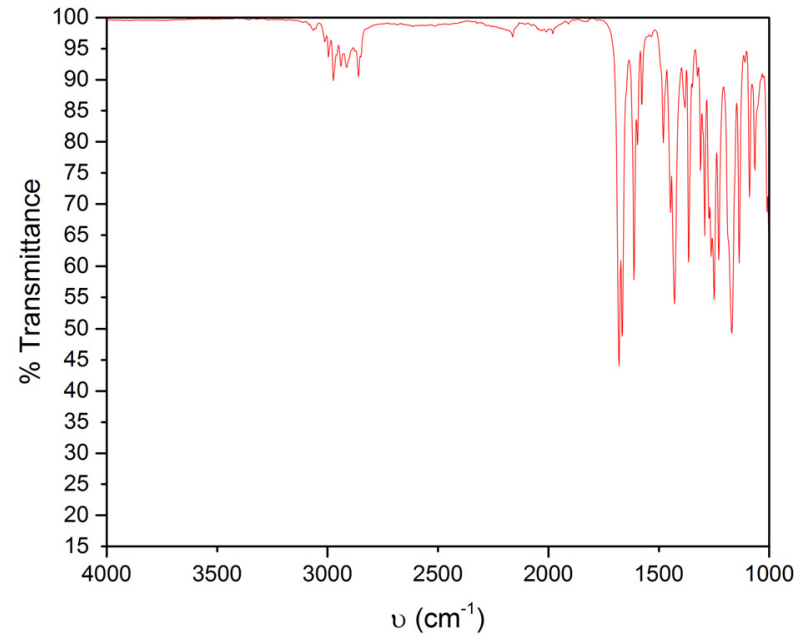

Figure S130. FT-IR of S2. 

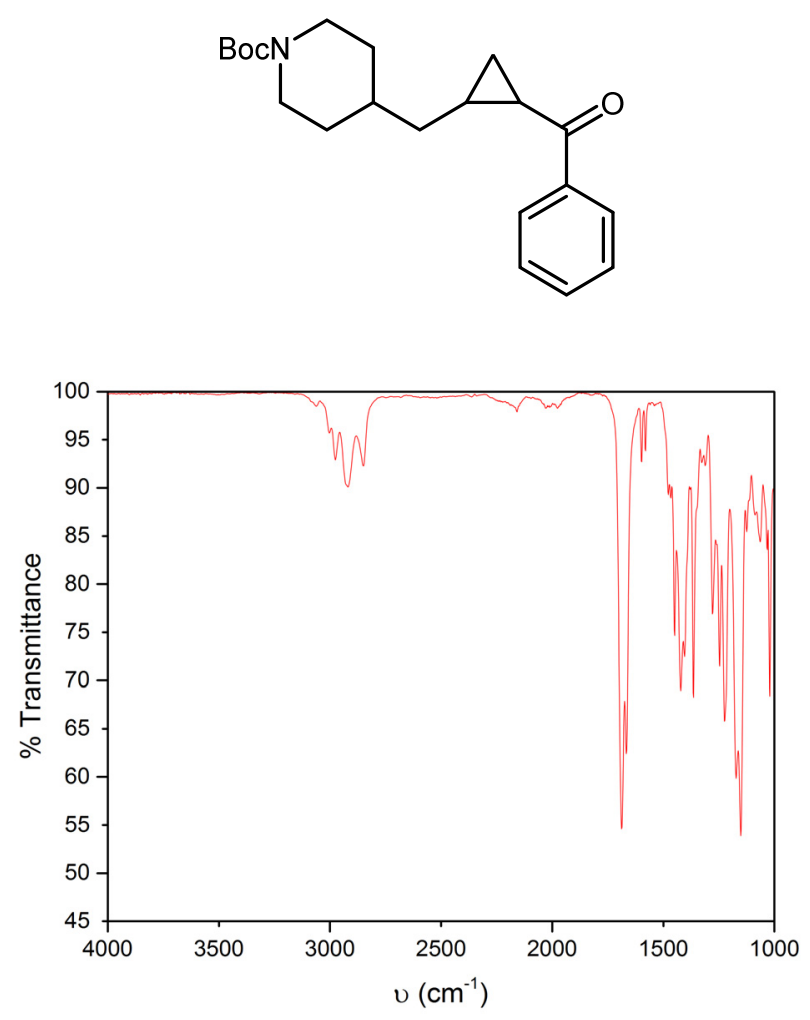

Figure S131. FT-IR of S3.
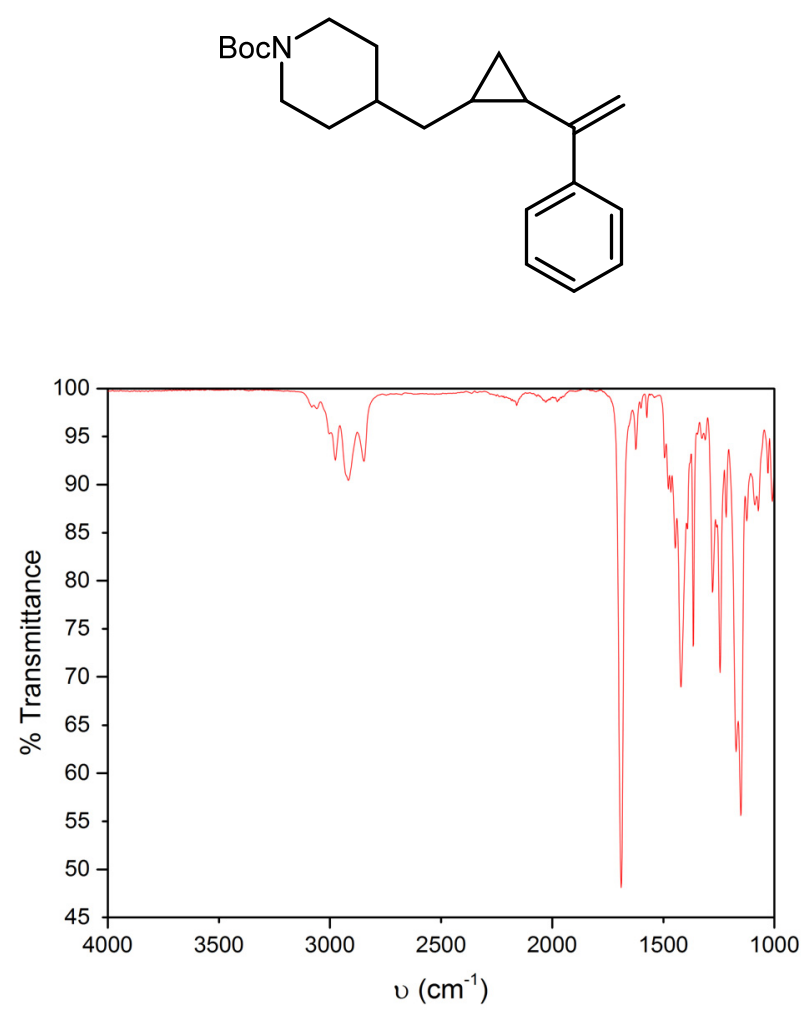

Figure S132. FT-IR of S4. 
<smiles>Cc1ccc(CC/C=C/C(=O)c2ccccc2)o1</smiles>

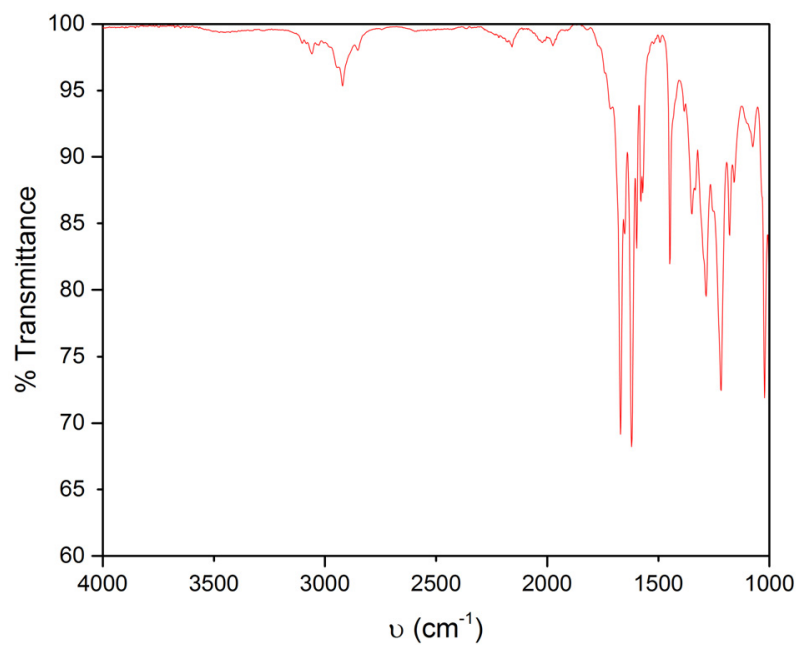

Figure S133. FT-IR of S5.
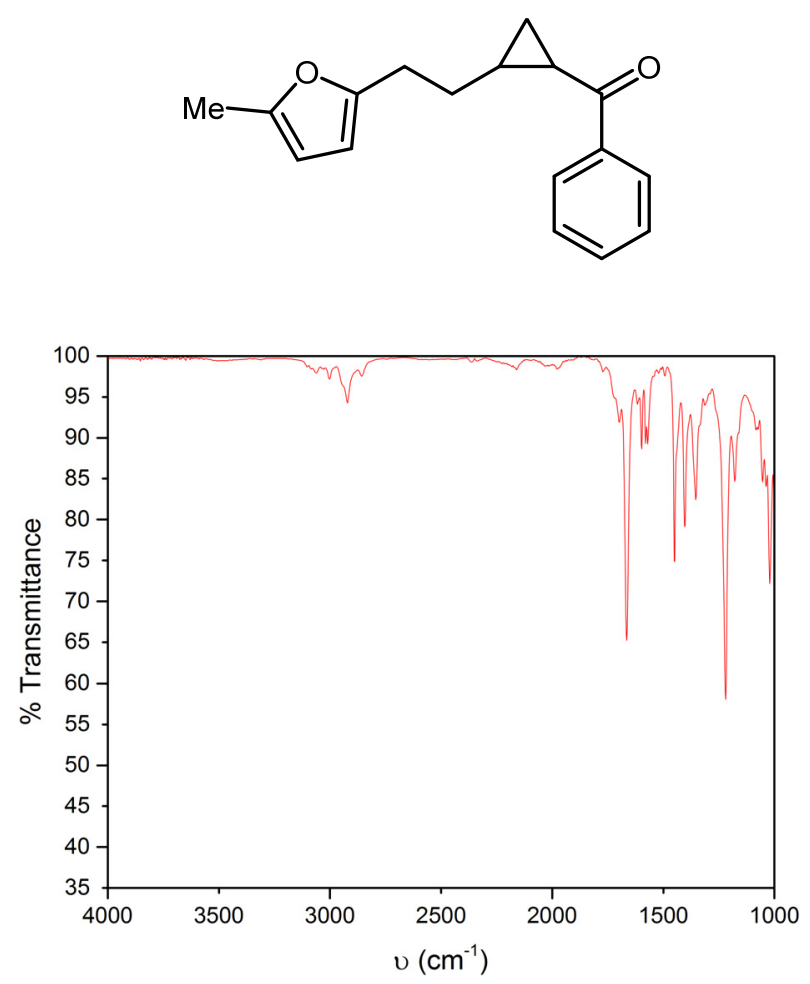

Figure S134. FT-IR of S6. 

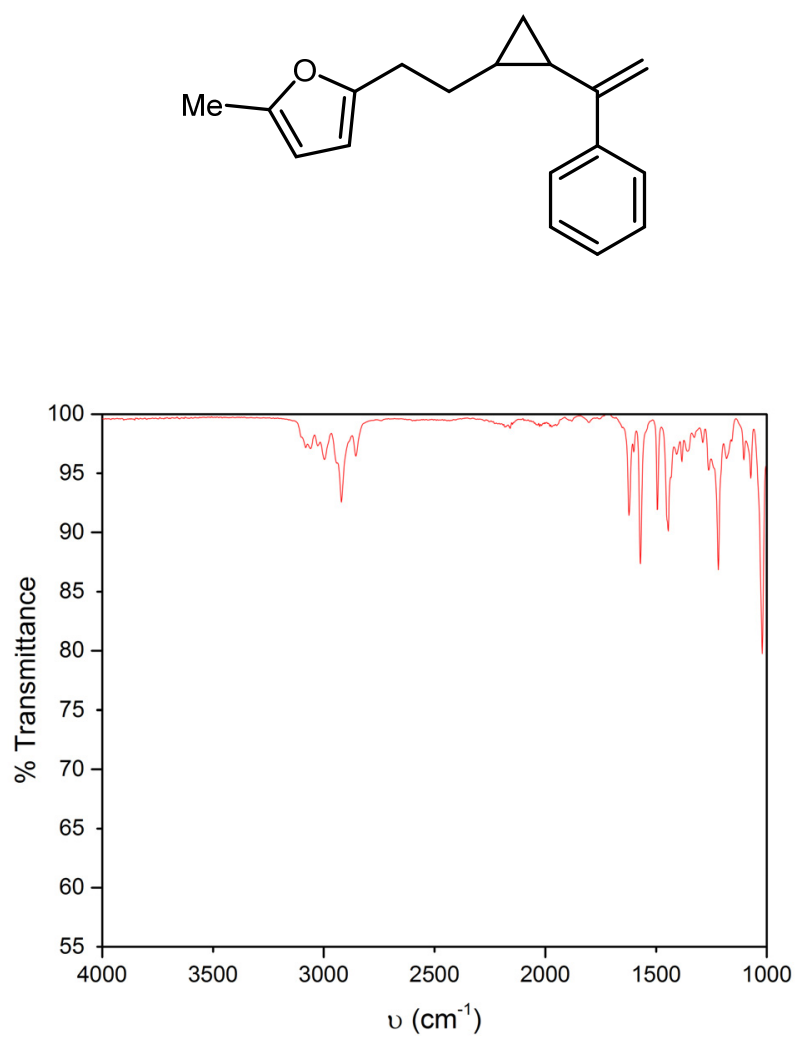

Figure S135. FT-IR of S7.<smiles>O=C(c1ccccc1)C1CC1CCN1C(=O)c2ccccc2C1=O</smiles>

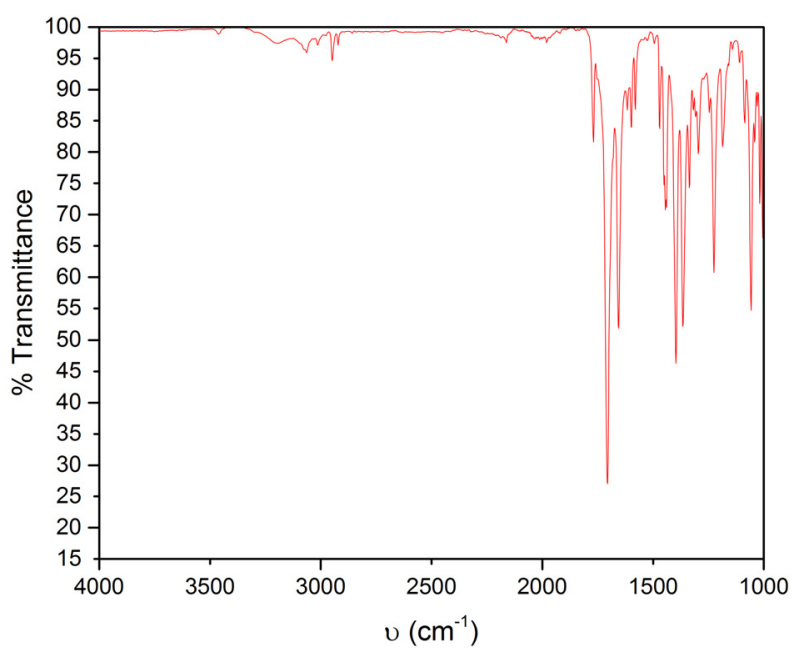

Figure S136. FT-IR of S8. 

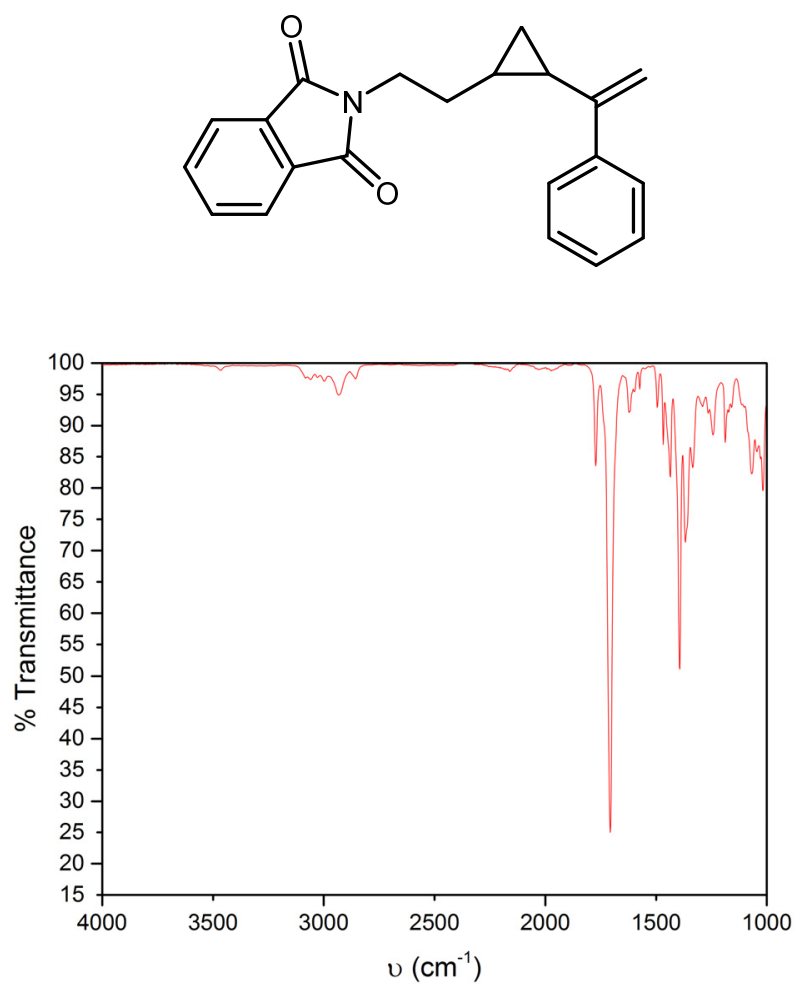

Figure S137. FT-IR of S9.<smiles>CCCCCCCC=CC(=O)c1ccccc1</smiles>

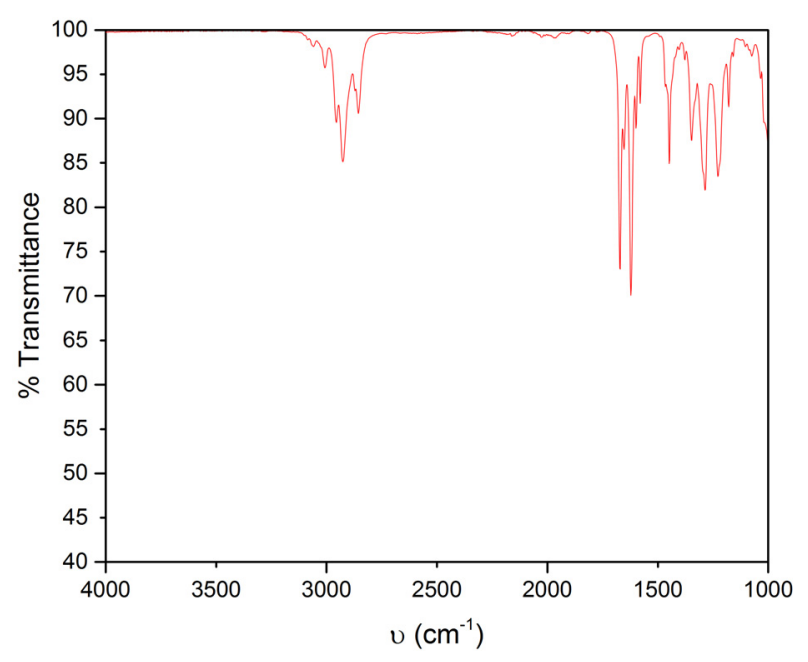

Figure S138. FT-IR of S10. 

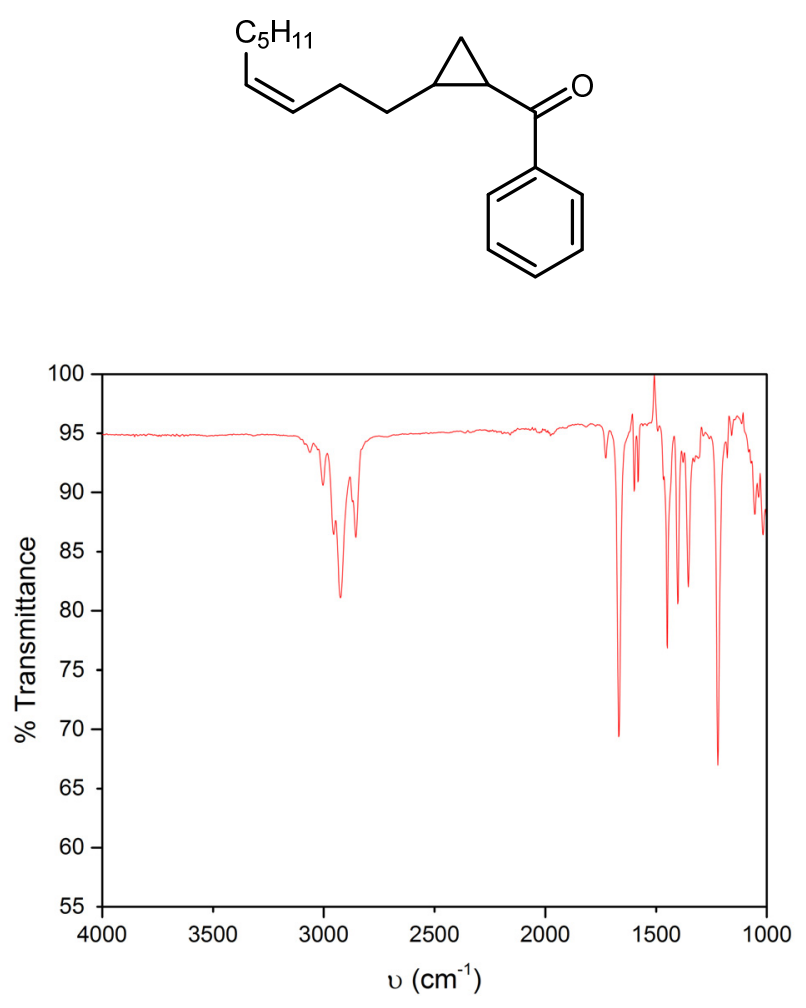

Figure S139. FT-IR of S11.
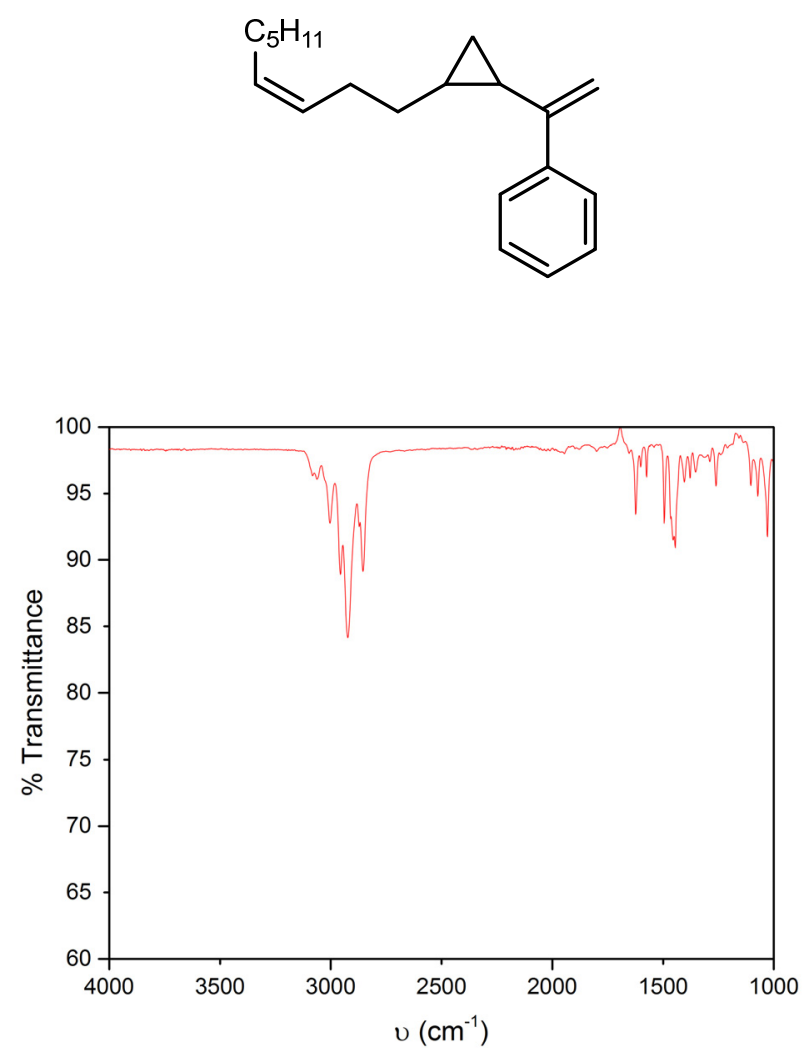

Figure S140. FT-IR of S12. 

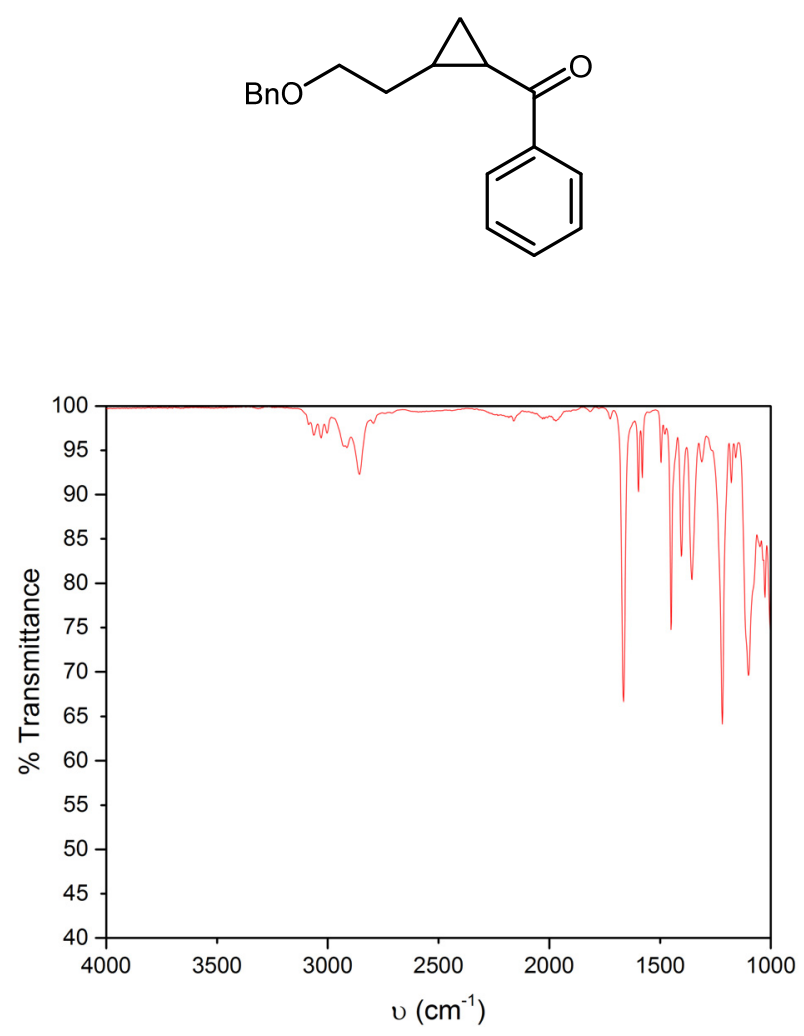

Figure S141. FT-IR of S13.
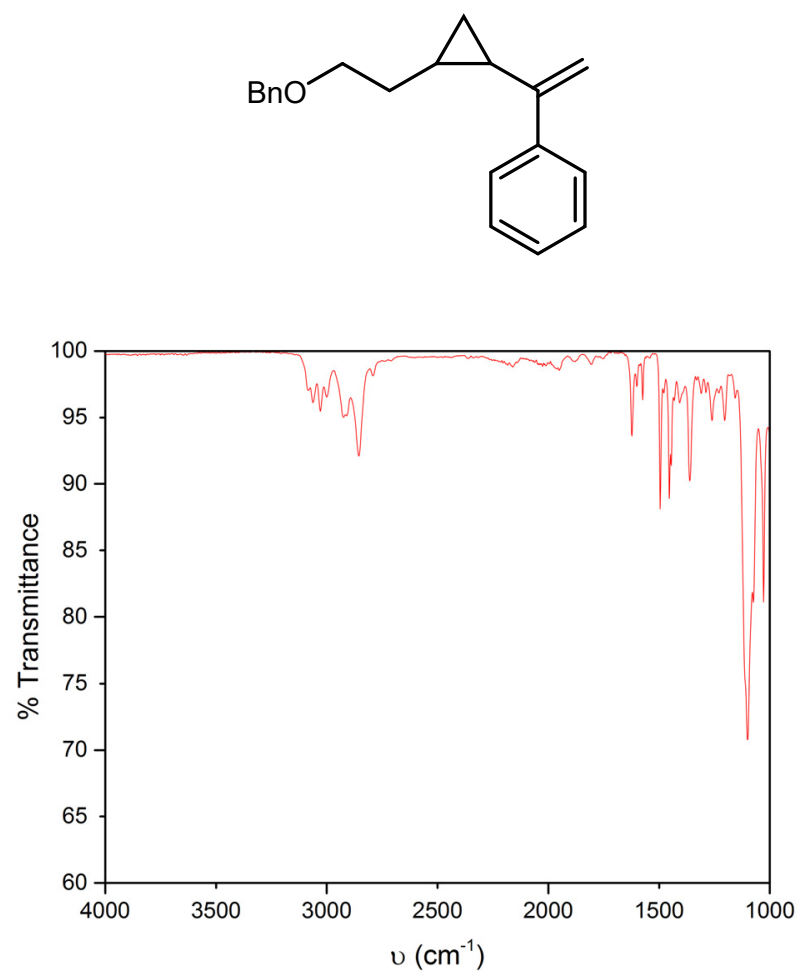

Figure S142. FT-IR of S14. 
<smiles>COC(=O)CCCC=C(Cl)Cl</smiles>

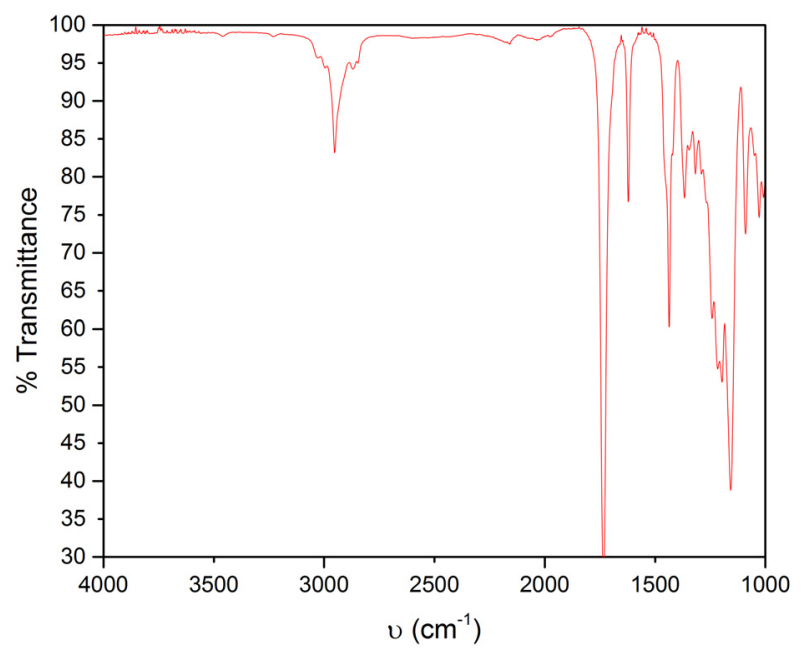

Figure S143. FT-IR of S15.<smiles>CN([125I])CCC=C(Cl)Cl</smiles>

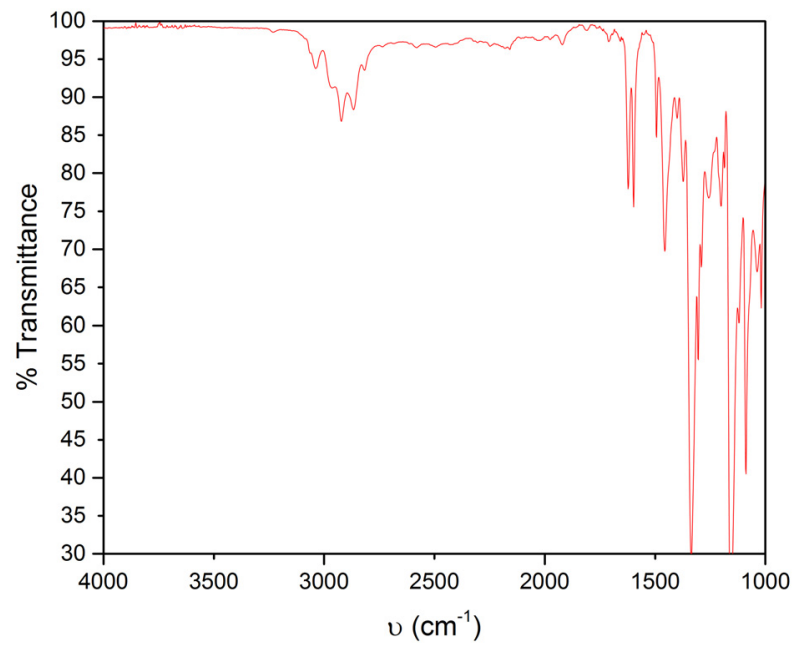

Figure S144. FT-IR of S16. 
<smiles>COc1ccc(C=C2CC=CCC2)cc1</smiles>

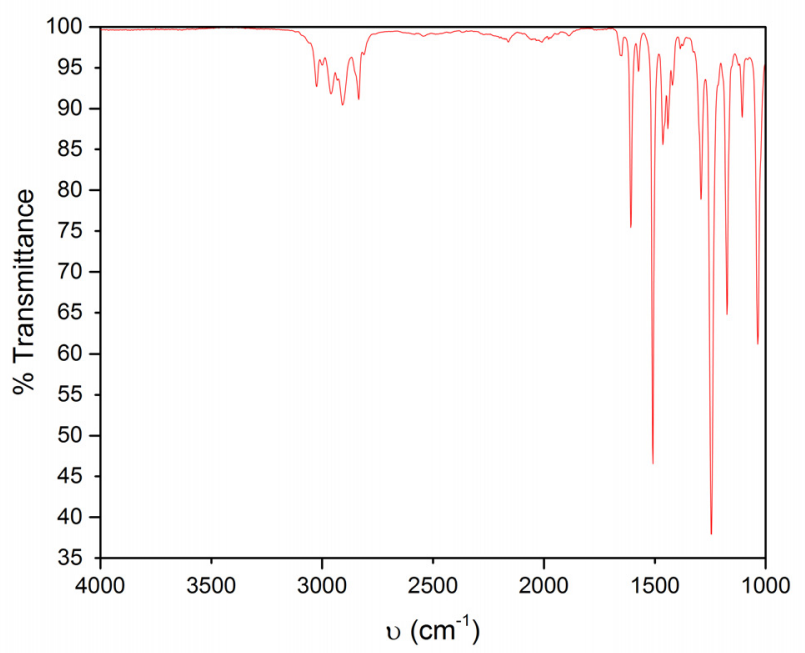

Figure S145. FT-IR of 3.

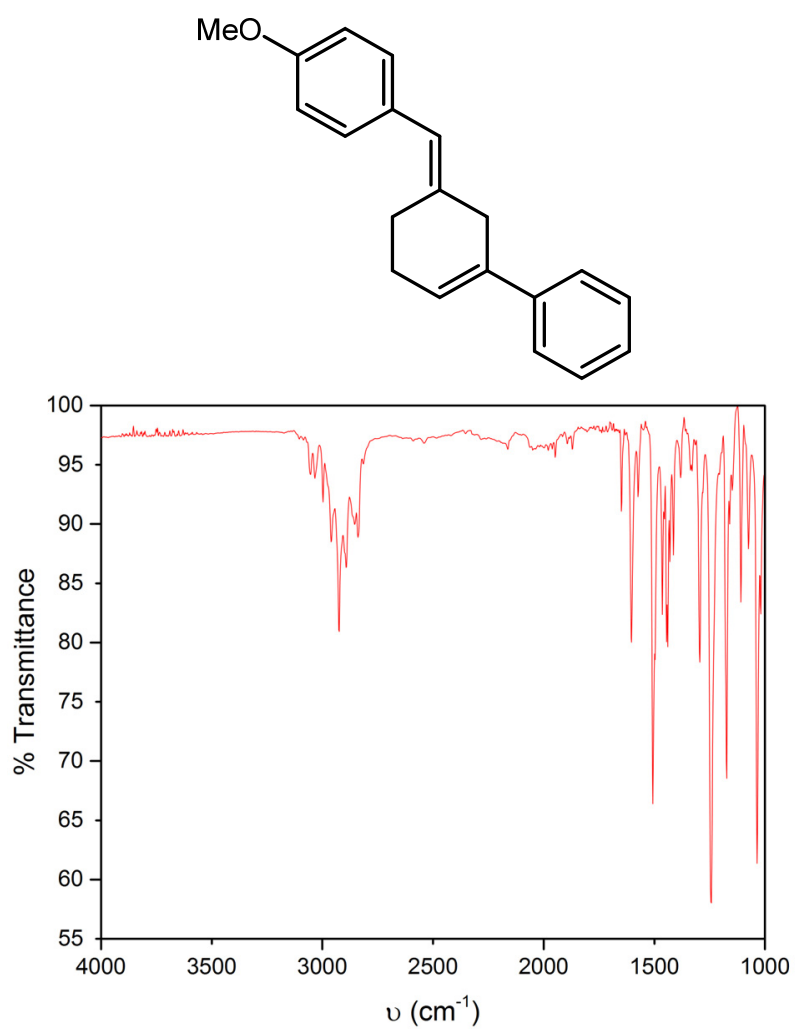

Figure S146. FT-IR of 6. 


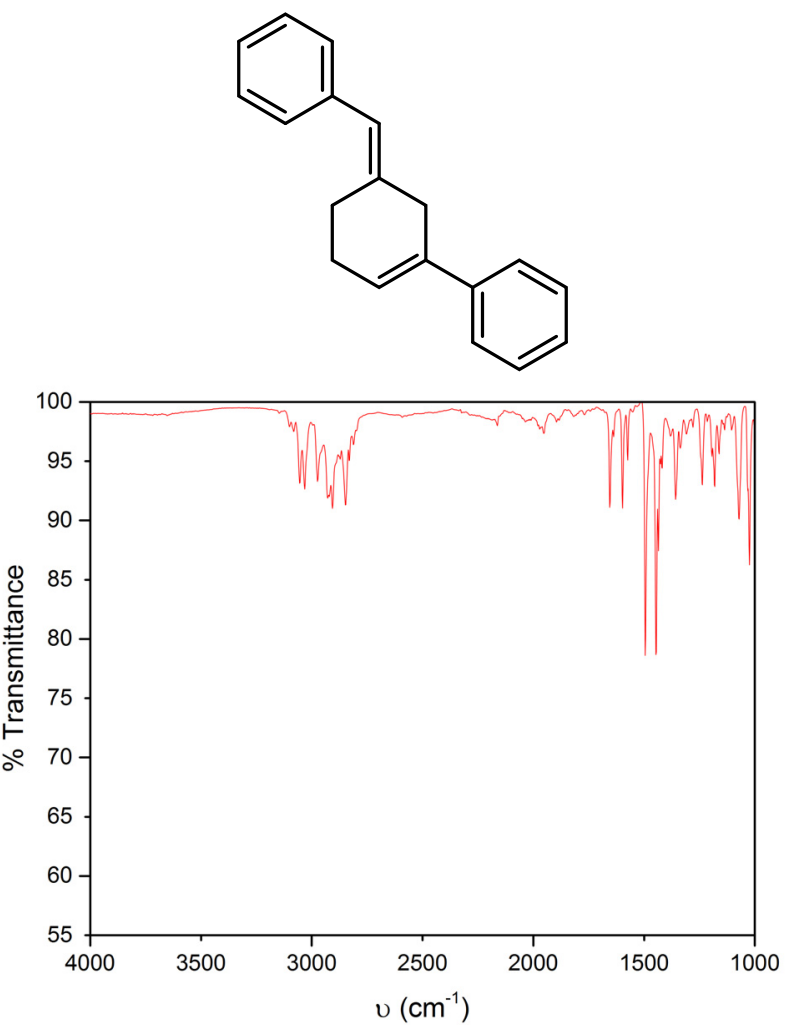

Figure S147. FT-IR of 11.
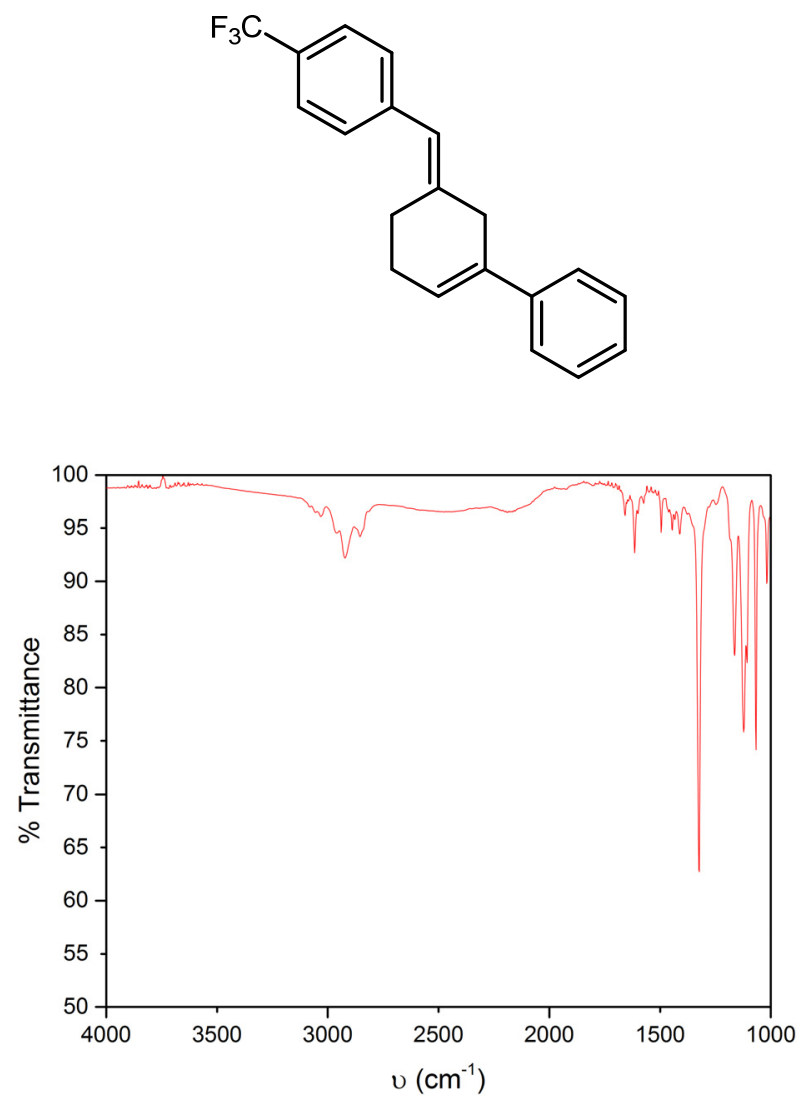

Figure S148. FT-IR of 12. 


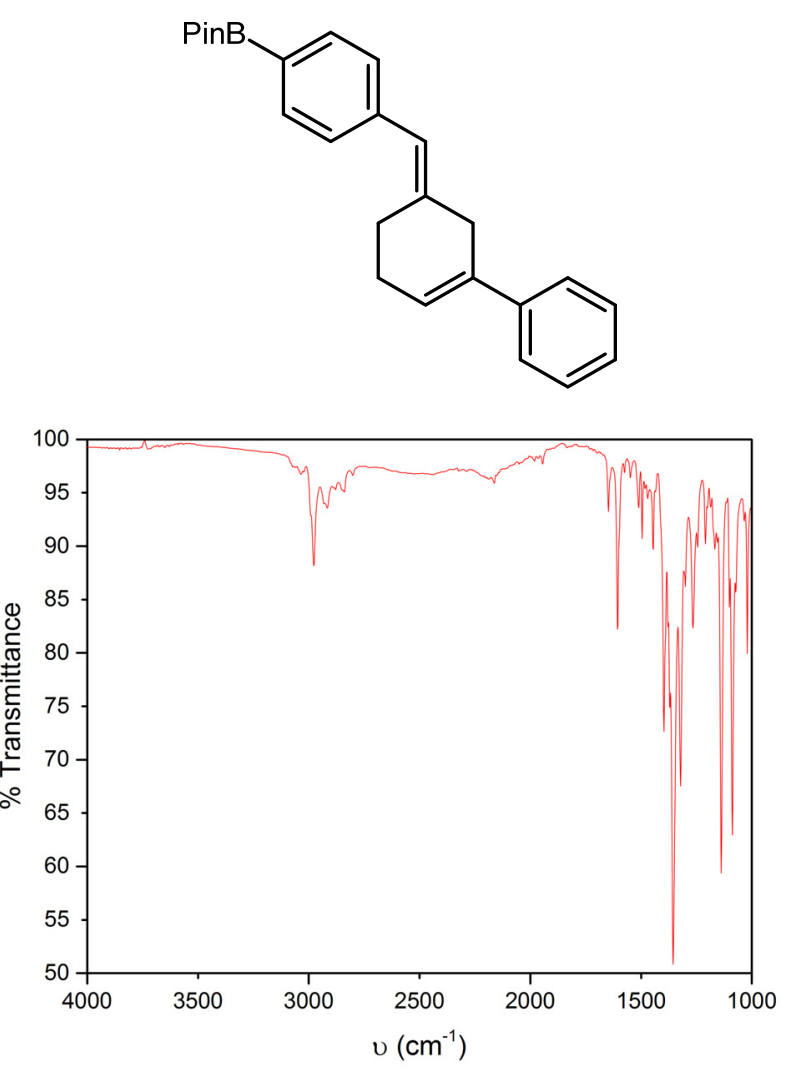

Figure S149. FT-IR of 13.
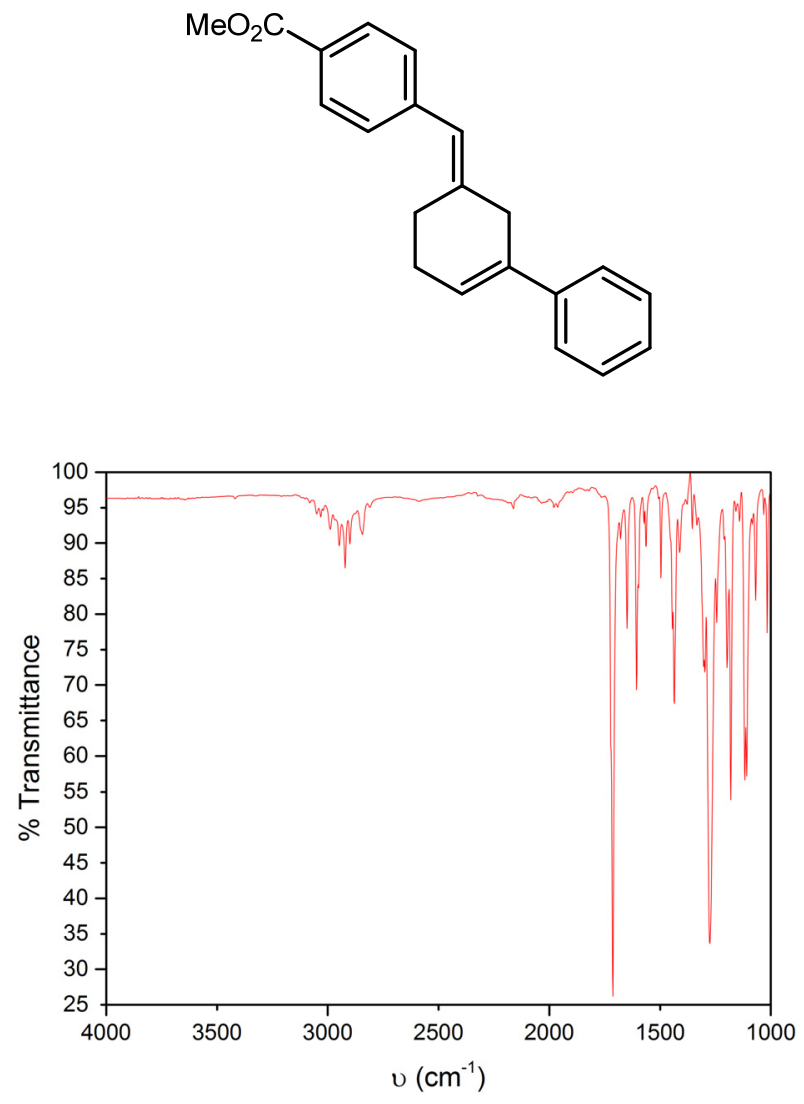

Figure S150. FT-IR of 14. 


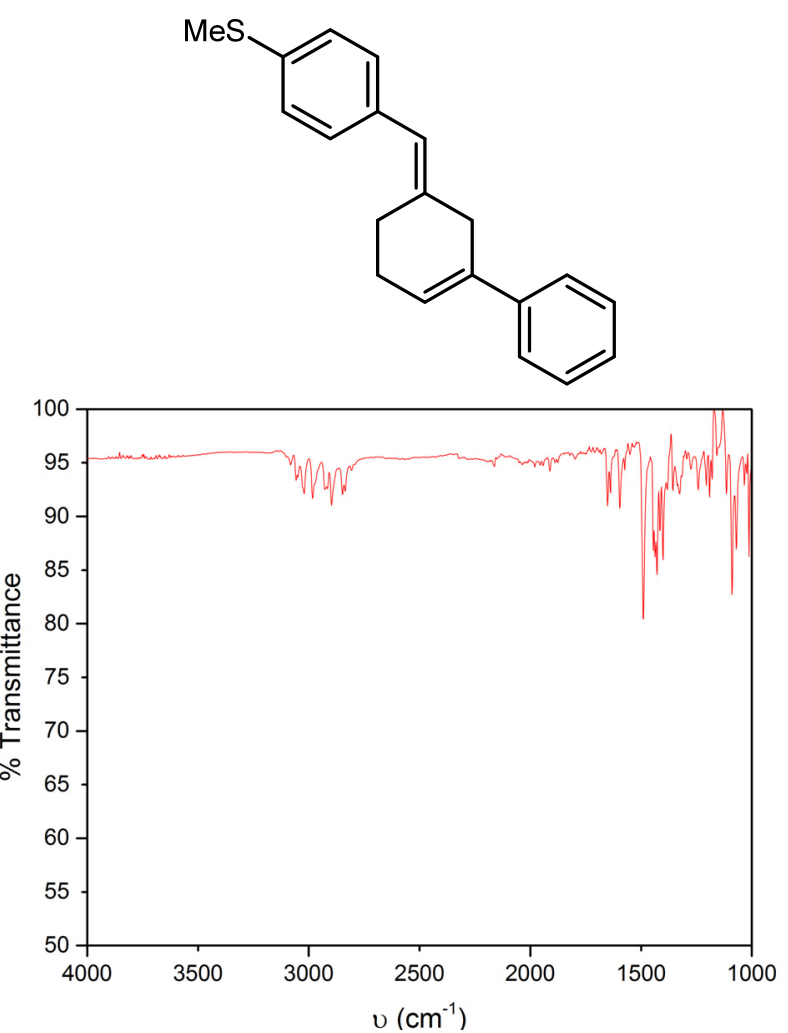

Figure S151. FT-IR of 15.
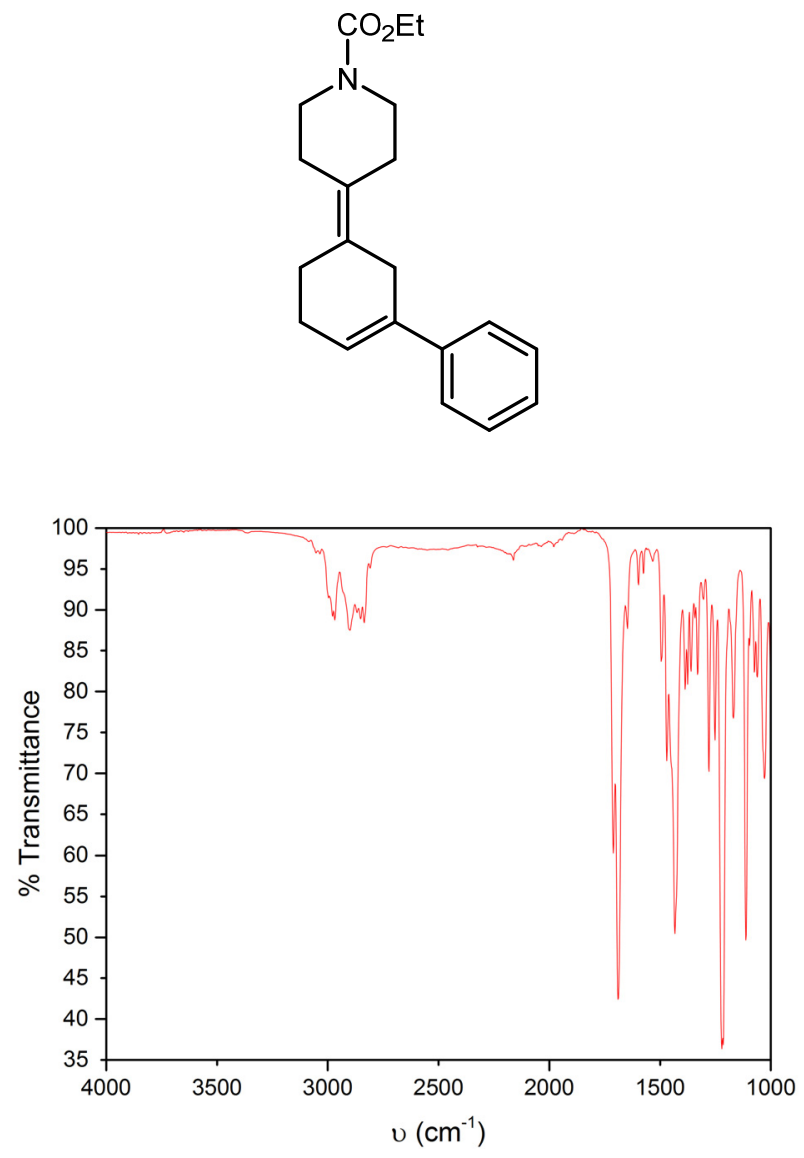

Figure S152. FT-IR of 16. 


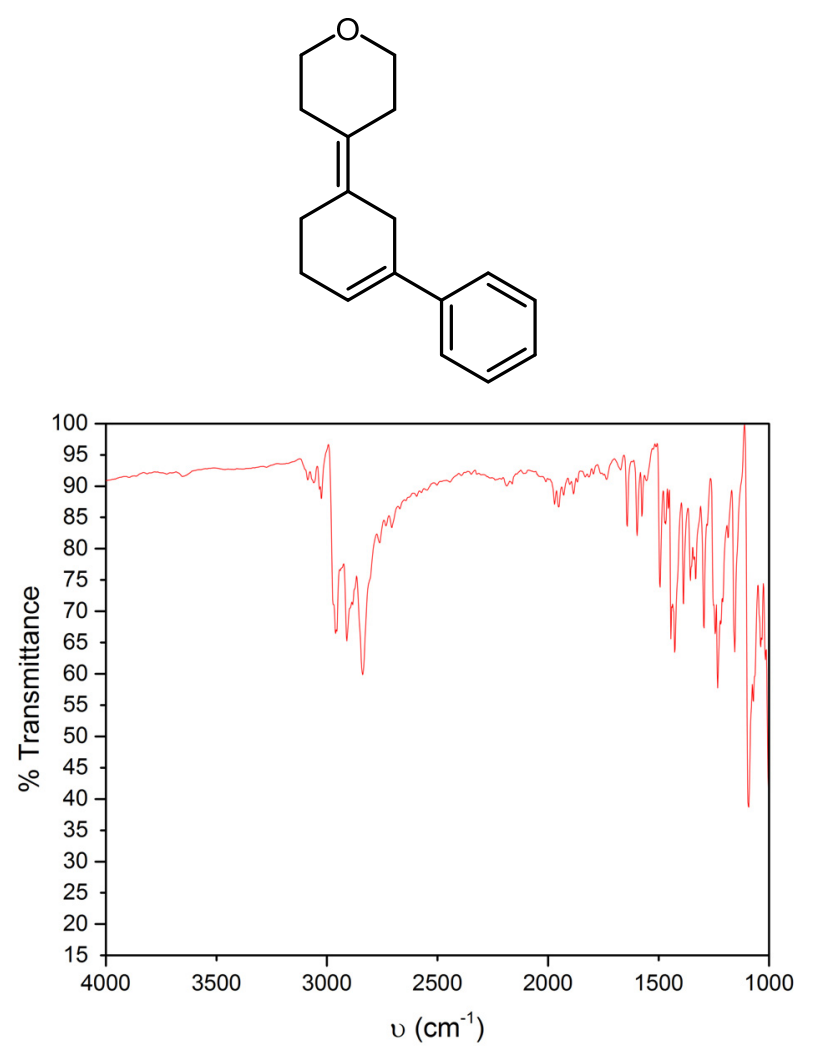

Figure S153. FT-IR of 17.

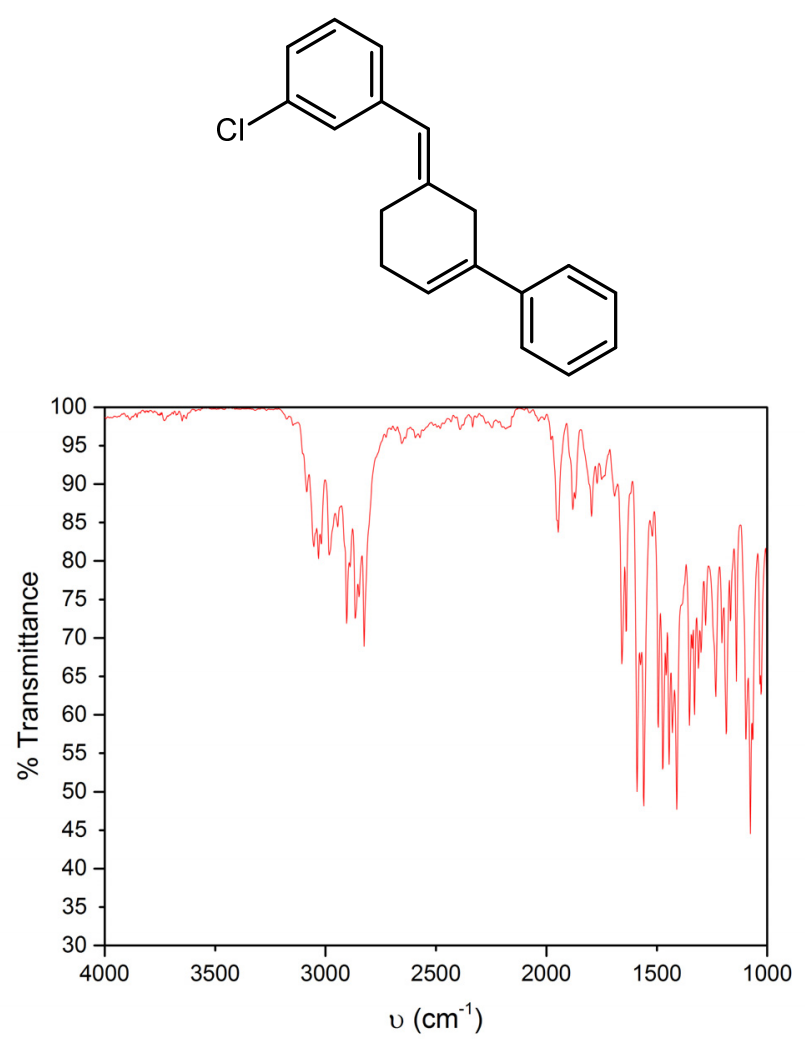

Figure S154. FT-IR of 18. 


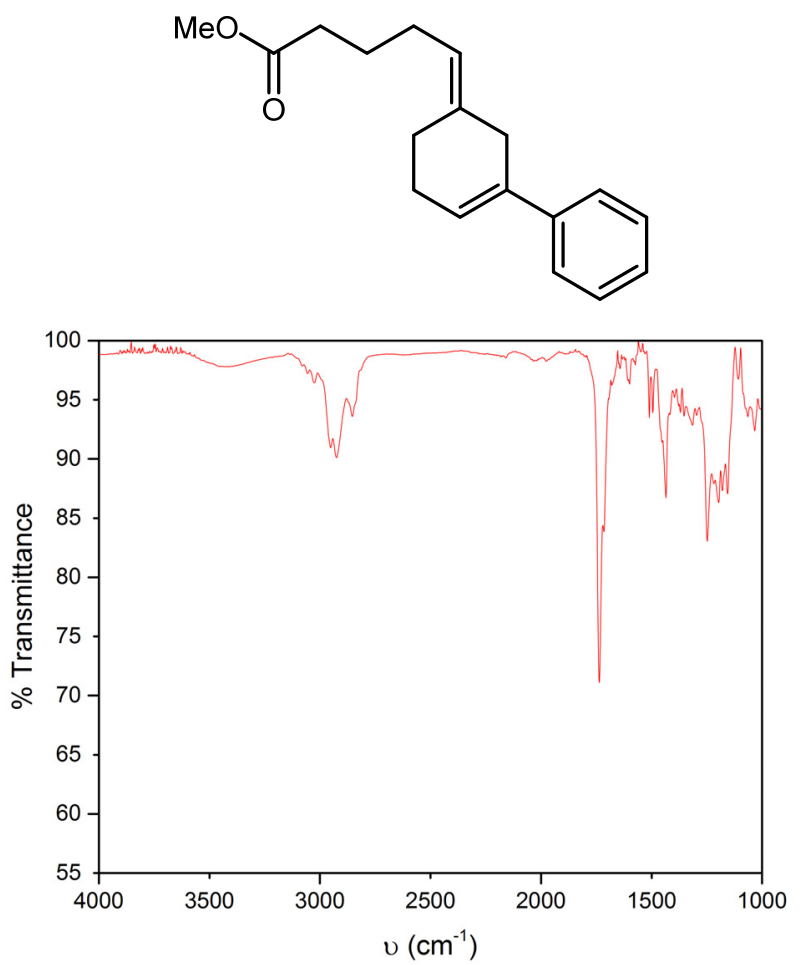

Figure S155. FT-IR of 19.
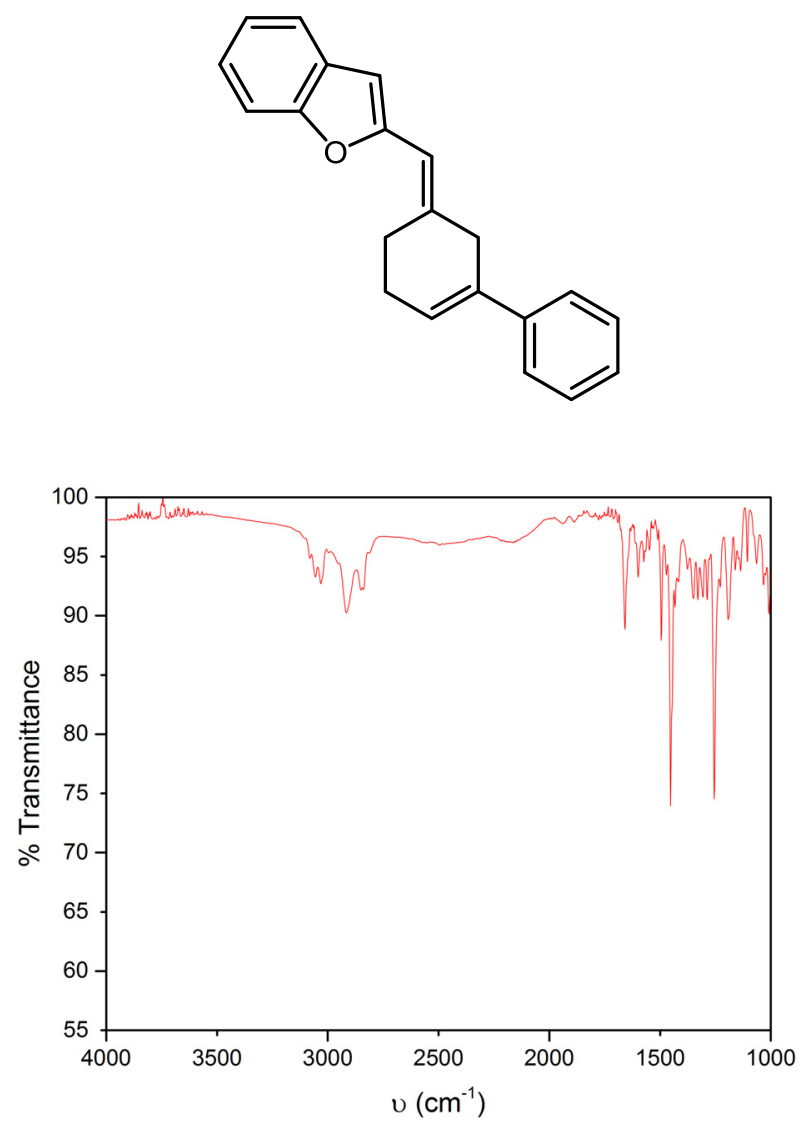

Figure S156. FT-IR of 20. 

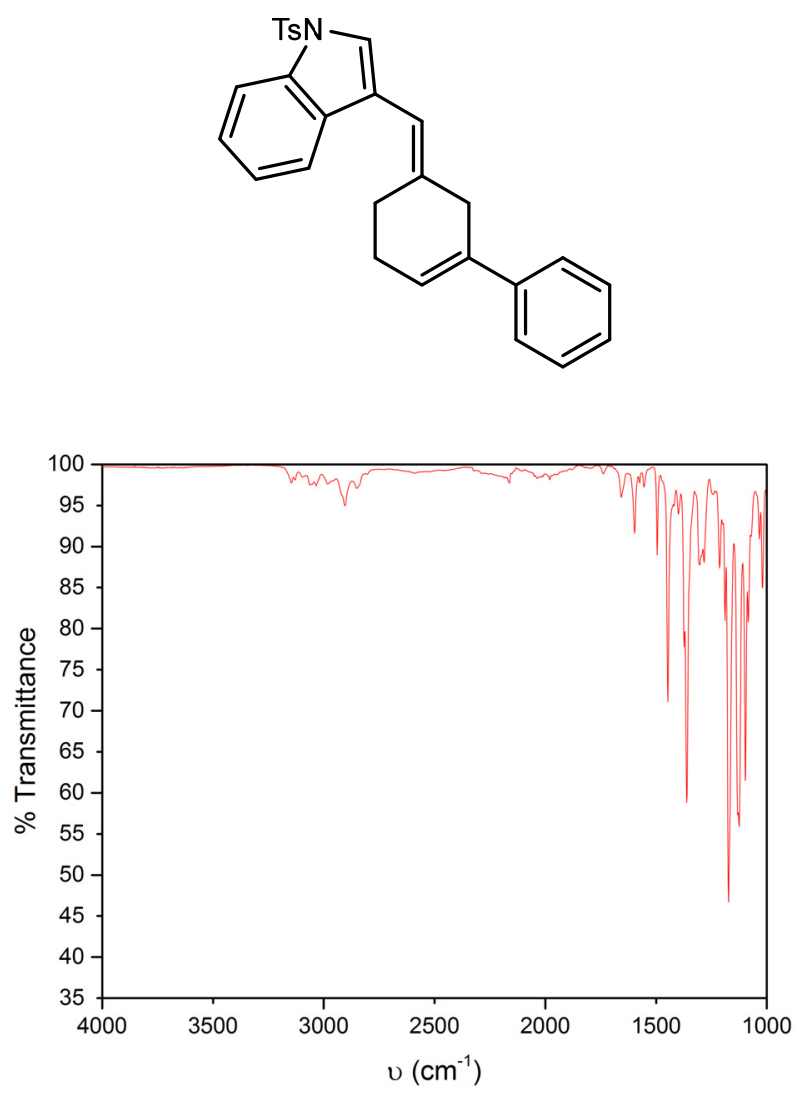

Figure S157. FT-IR of 21.
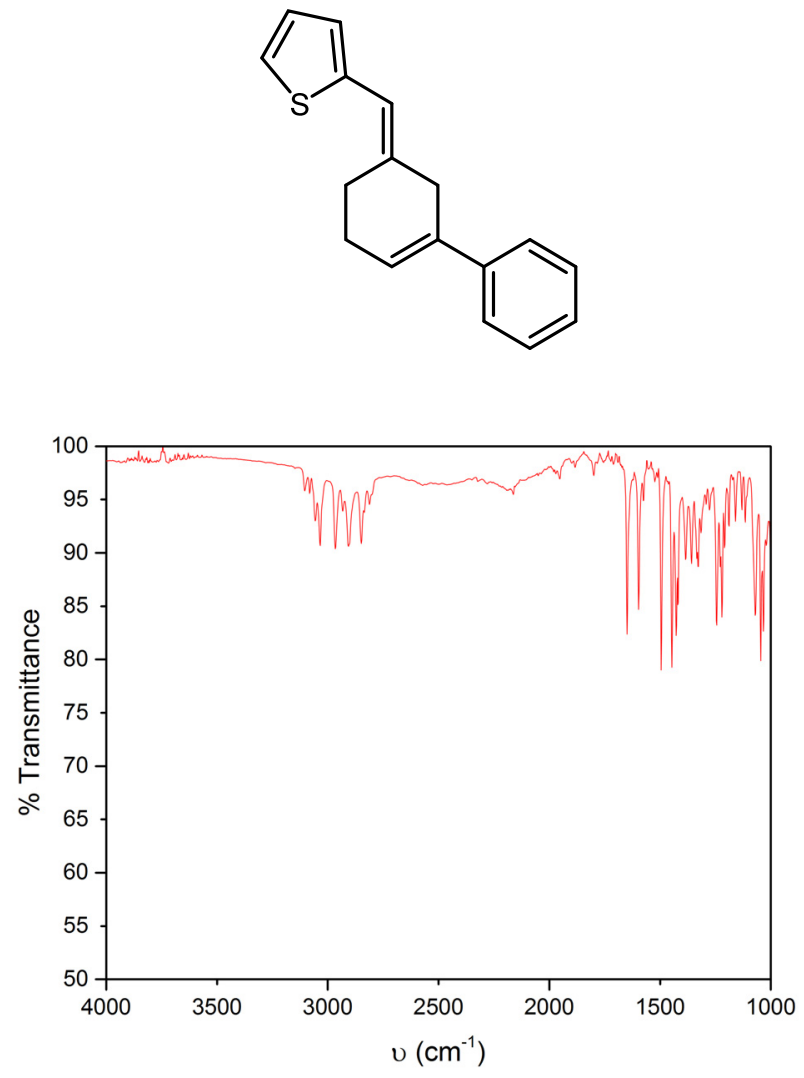

Figure S158. FT-IR of 22. 


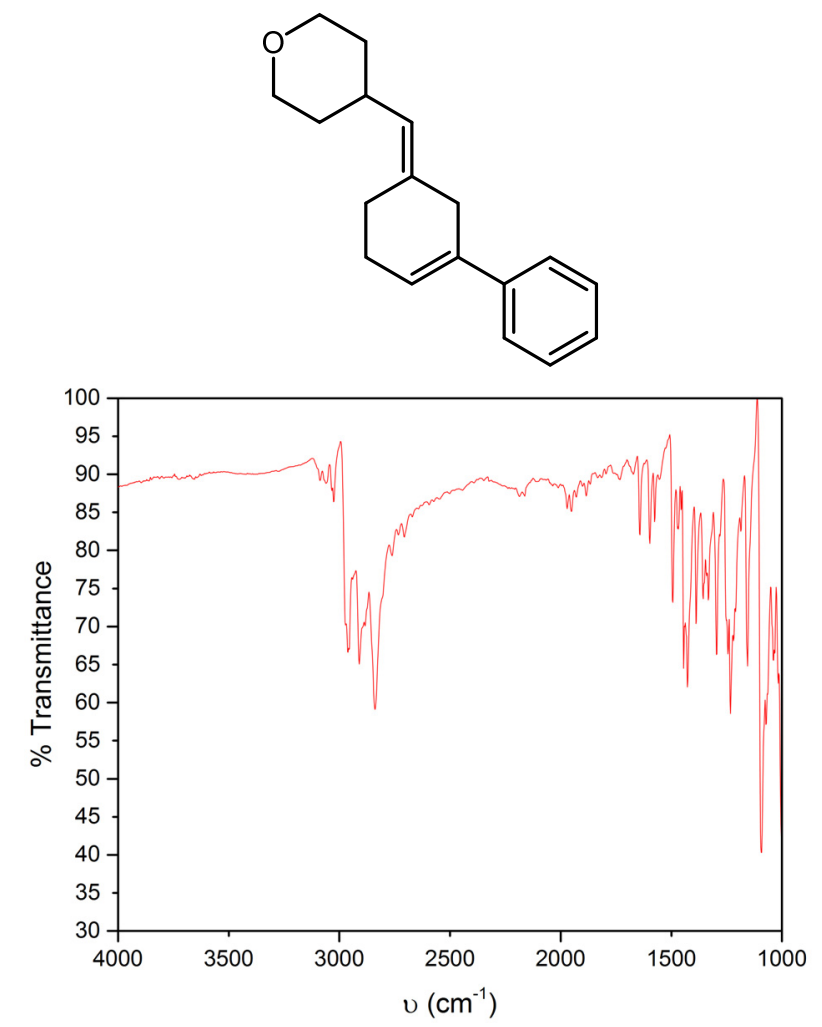

Figure S159. $\quad$ FT-IR of 23.

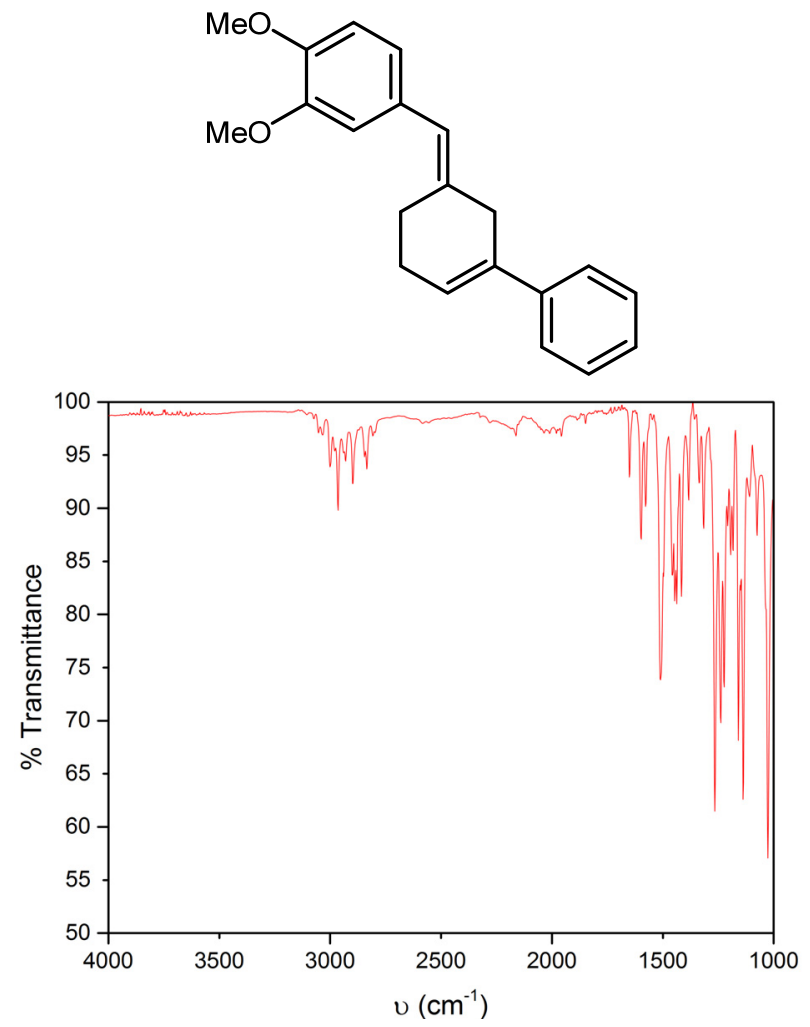

Figure S160. FT-IR of 24. 

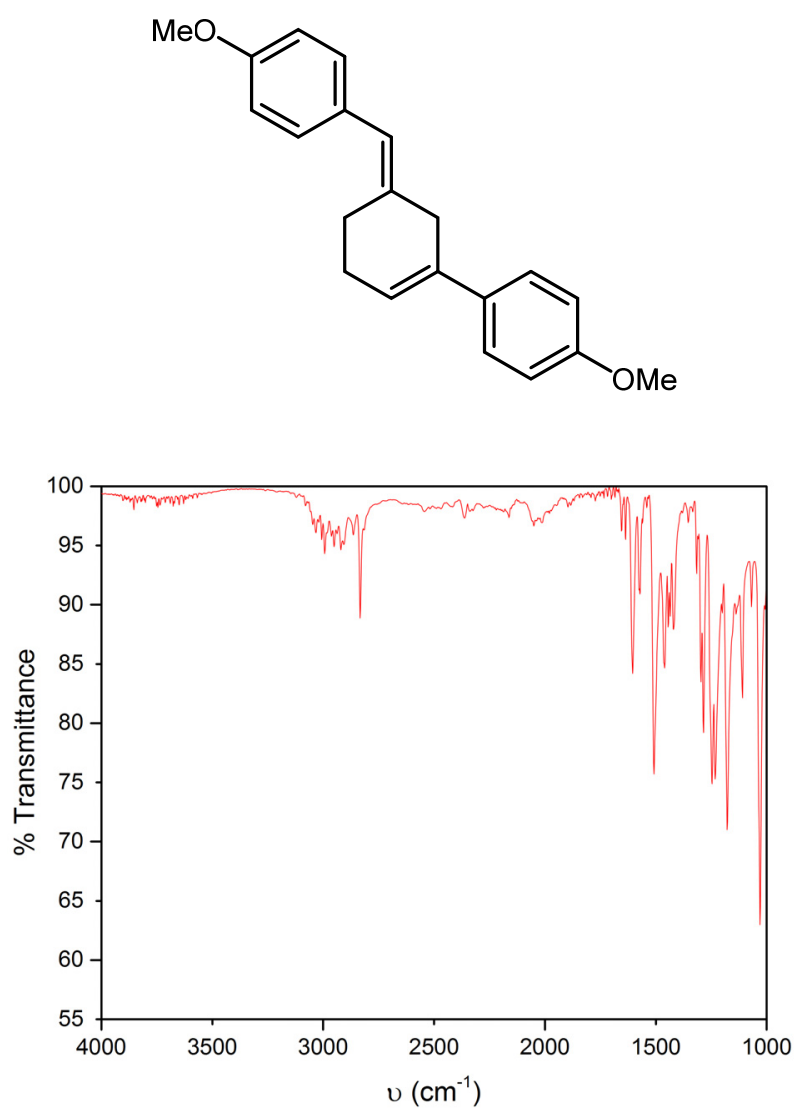

Figure S161. FT-IR of 25.

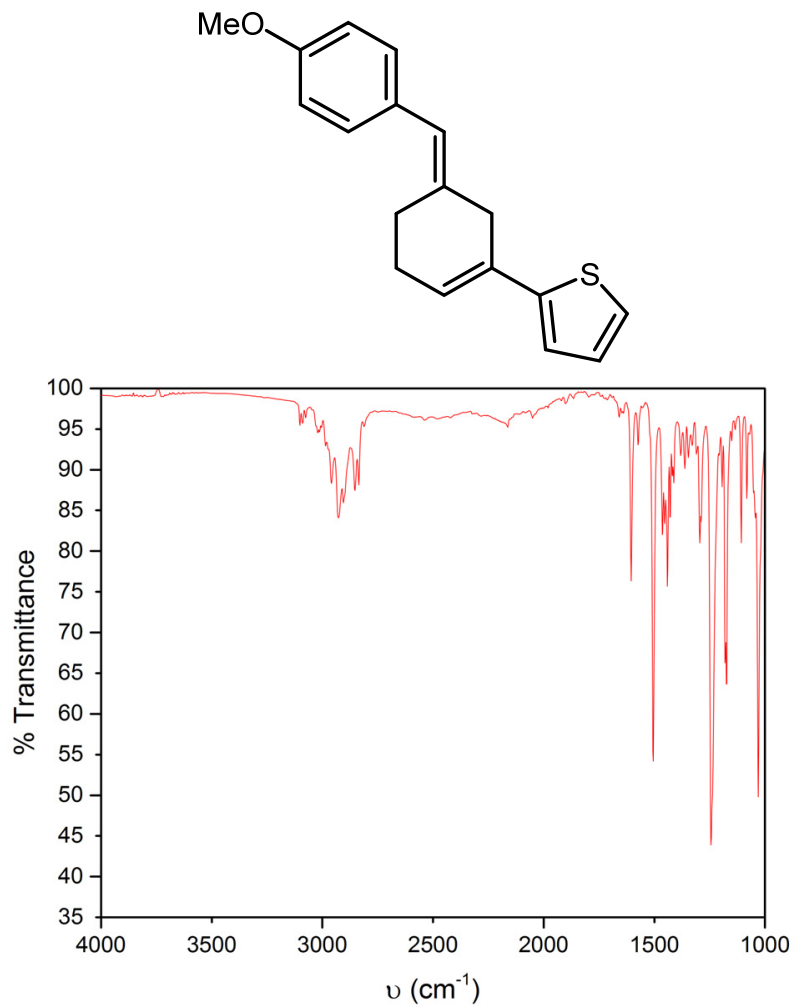

Figure S162. FT-IR of 26. 

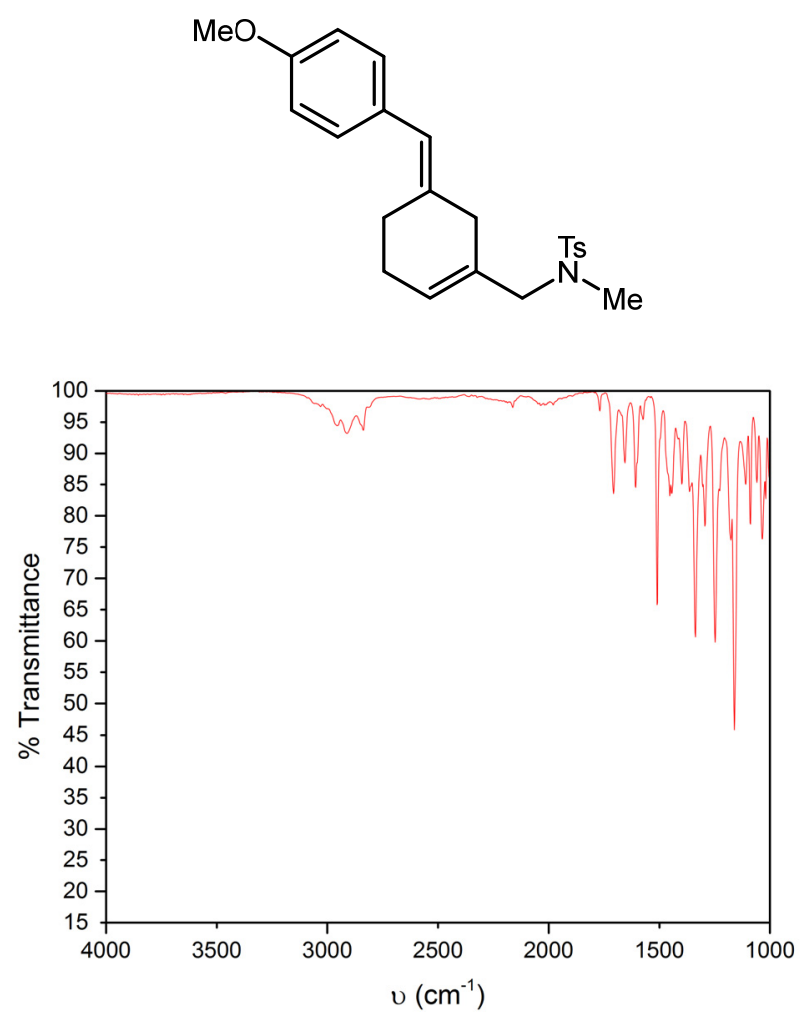

Figure S163. FT-IR of 27.
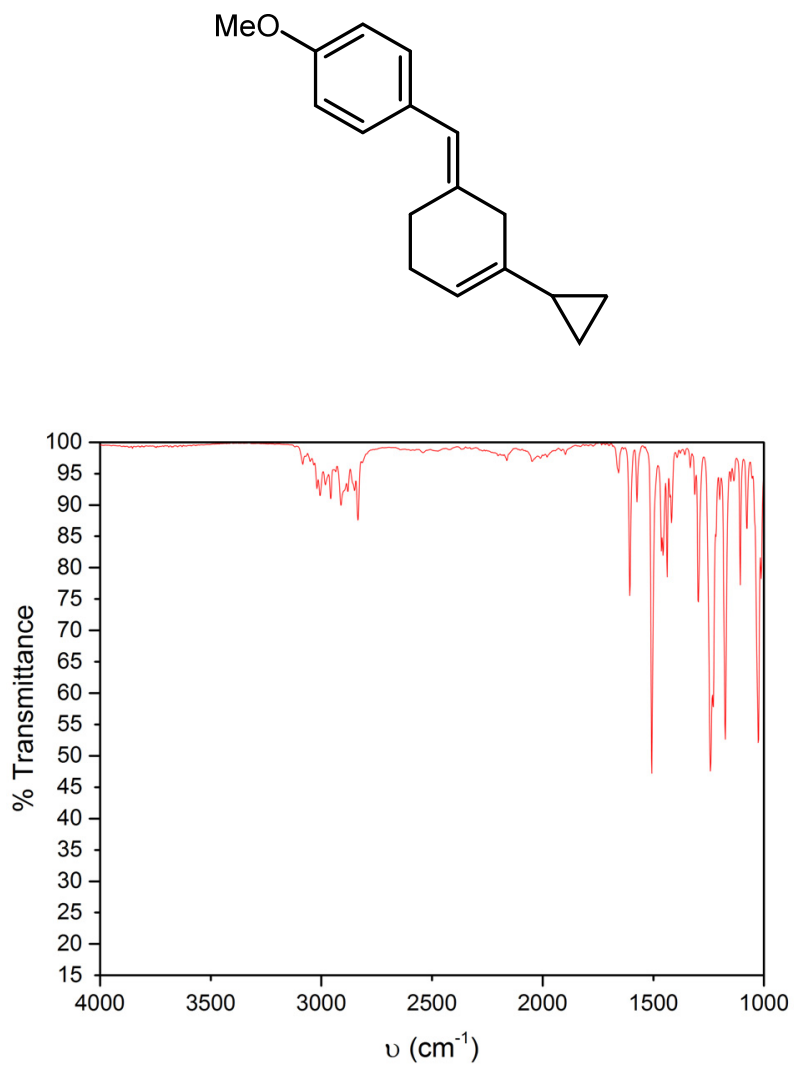

Figure S164. FT-IR of 28. 


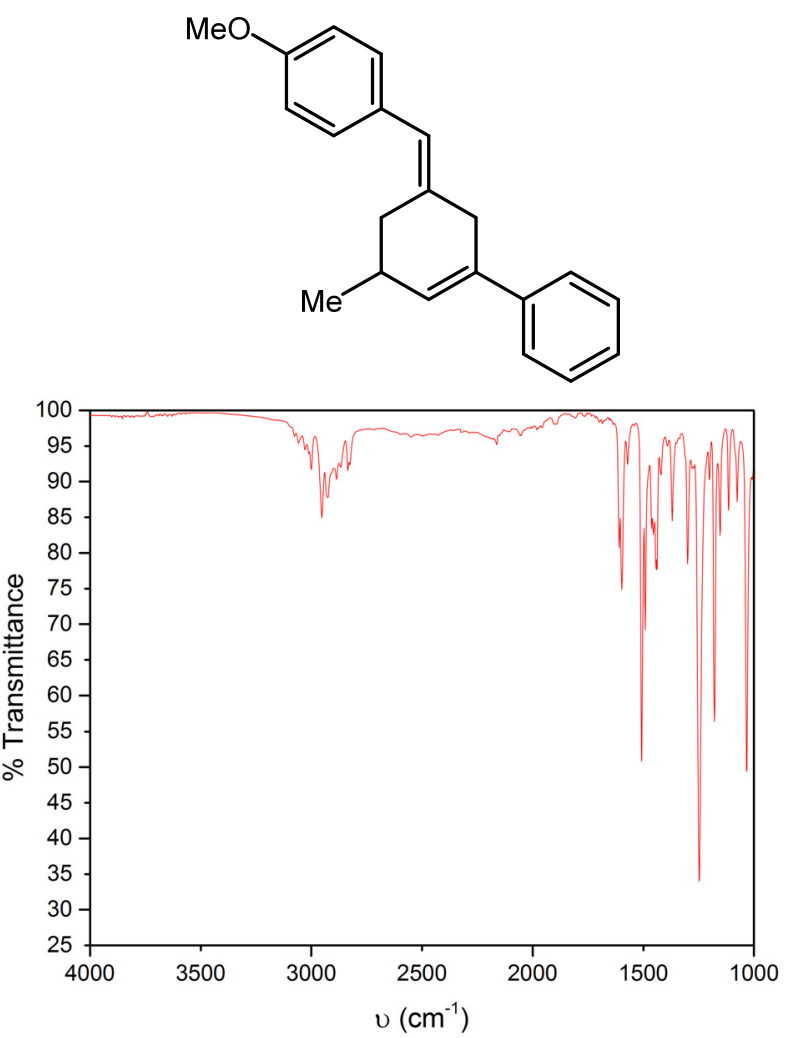

Figure S165. FT-IR of 30.
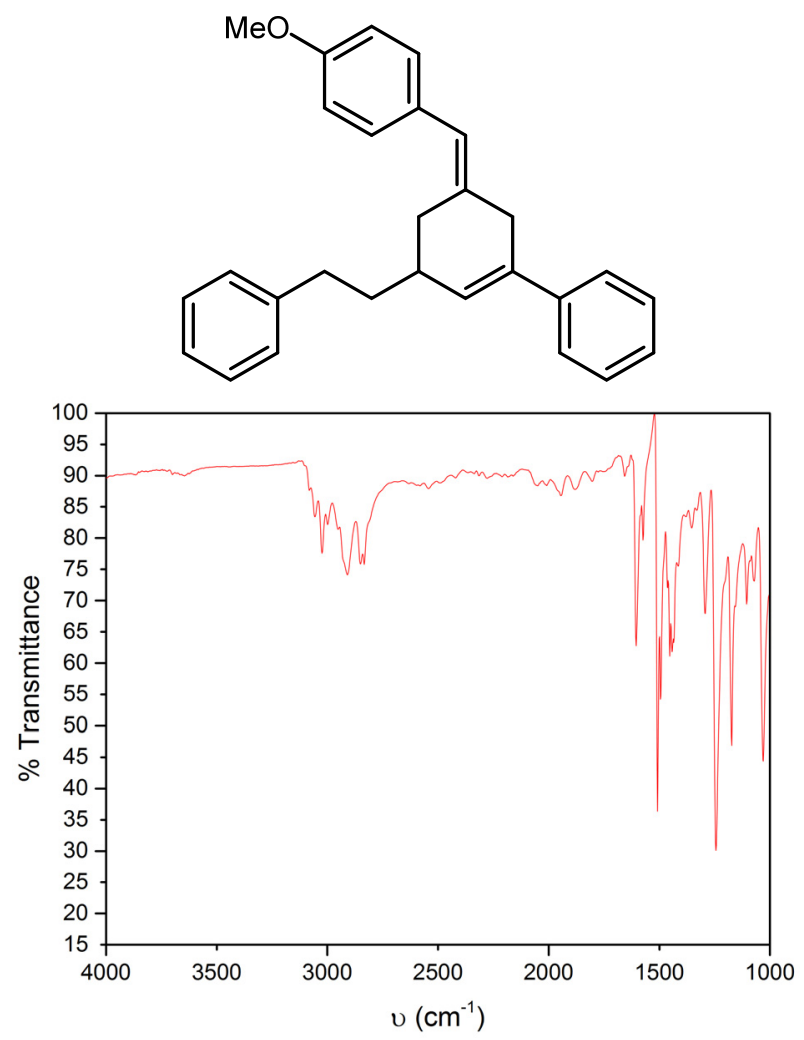

Figure S166. FT-IR of 31. 


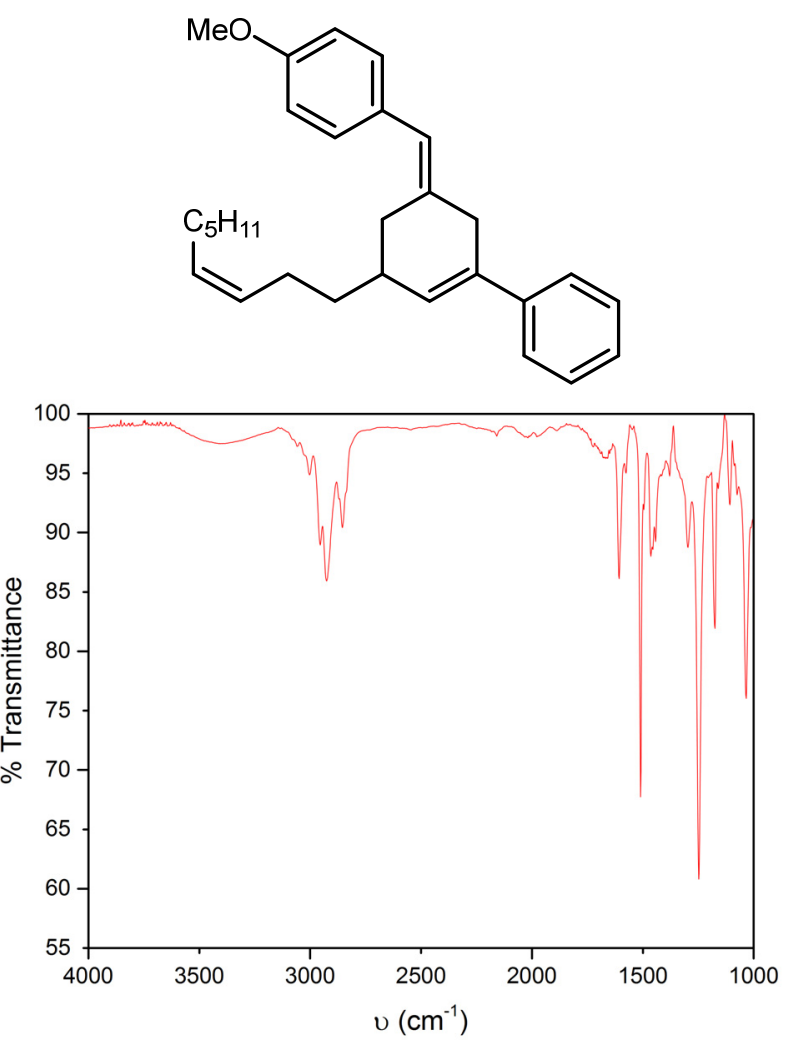

Figure S167. FT-IR of 32.
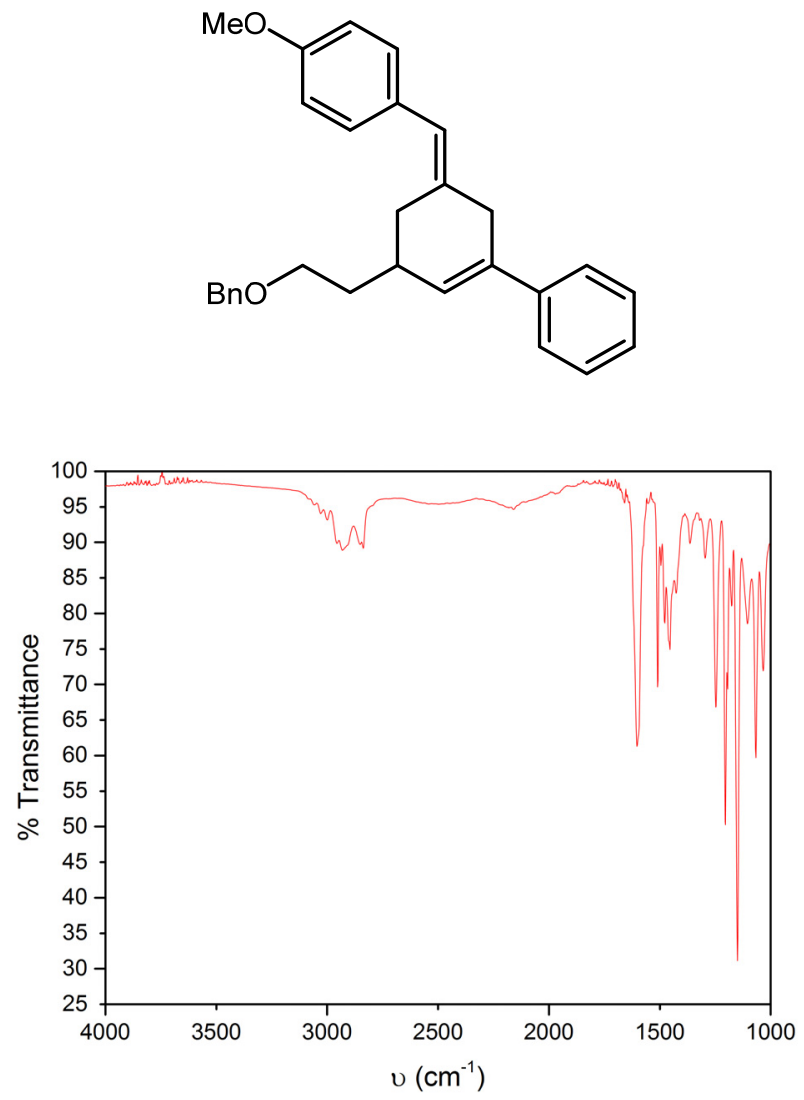

Figure S168. FT-IR of 33. 

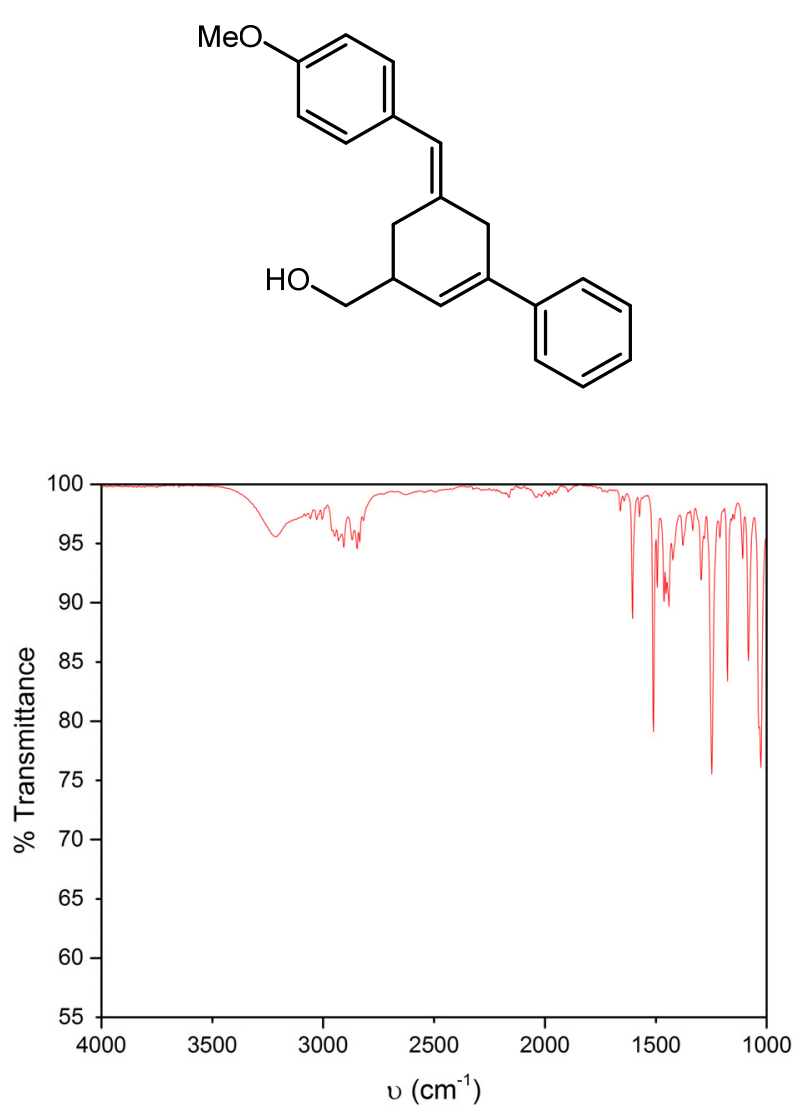

Figure S169. FT-IR of 34.

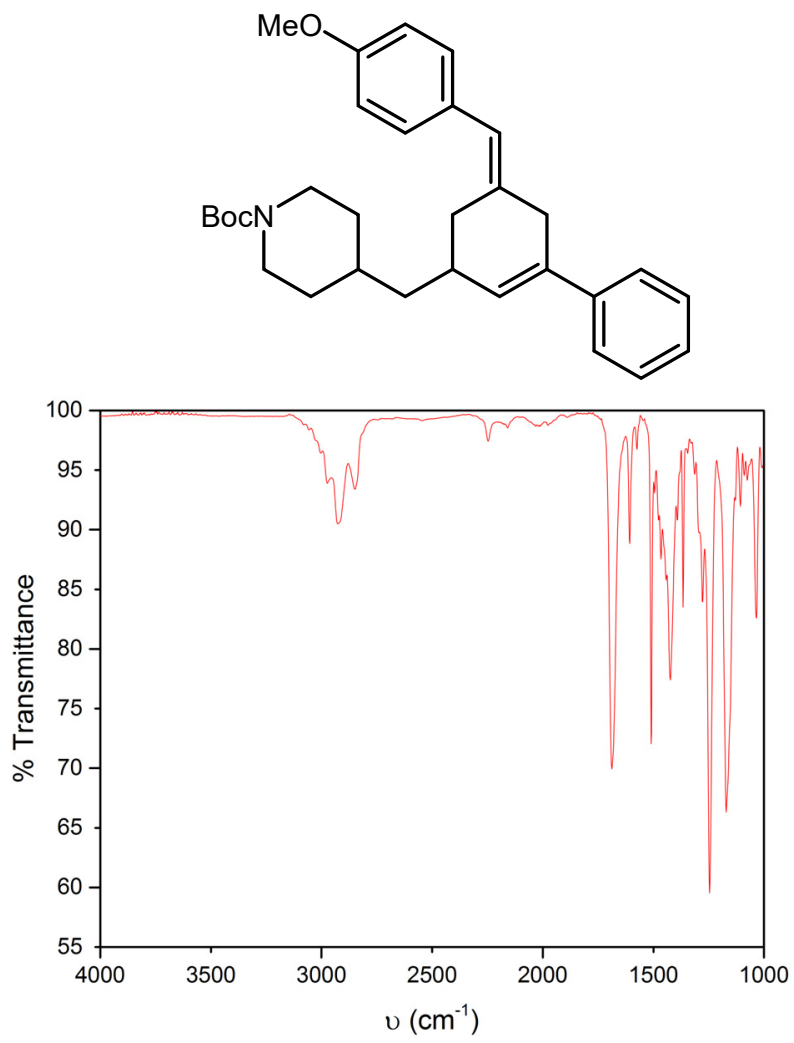

Figure S170. $\quad$ FT-IR of 35. 


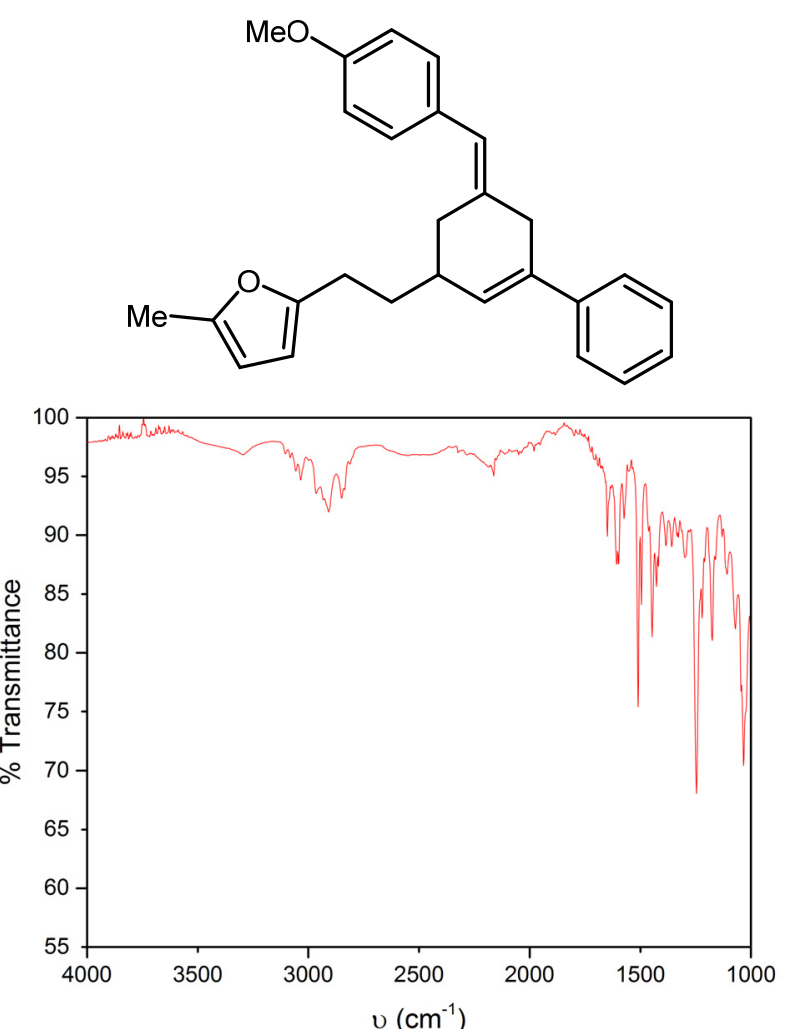

Figure S171. FT-IR of 36.

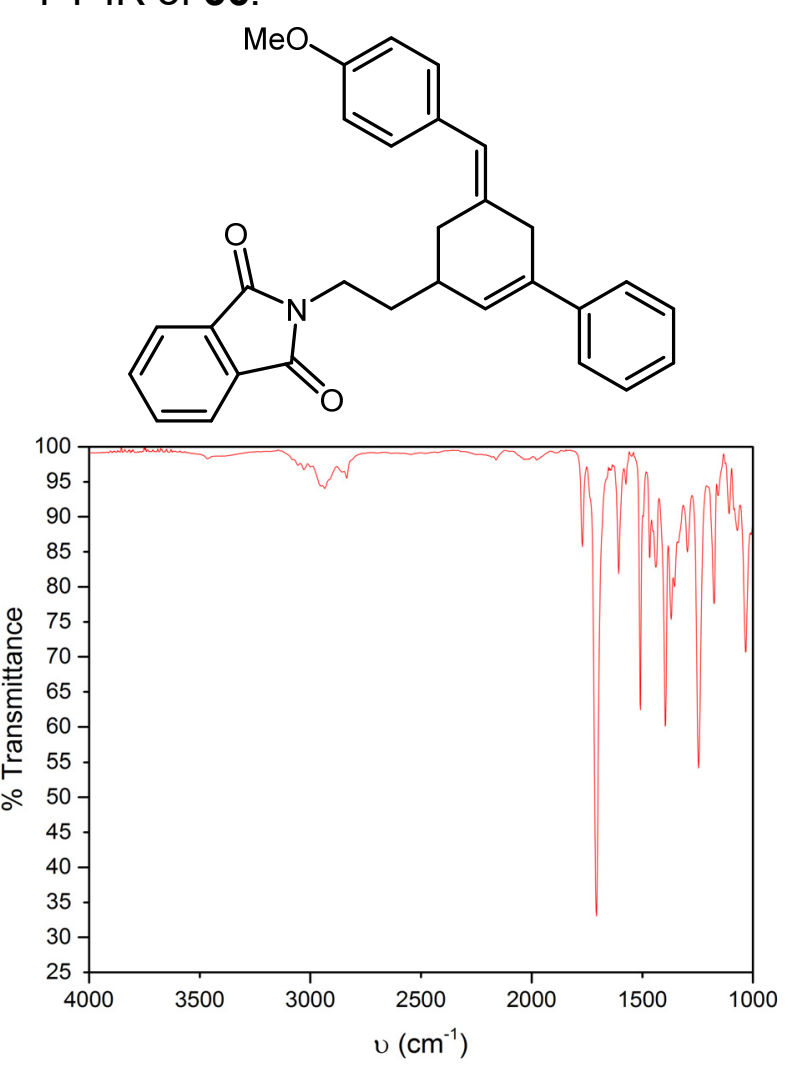

Figure S172. FT-IR of 37. 


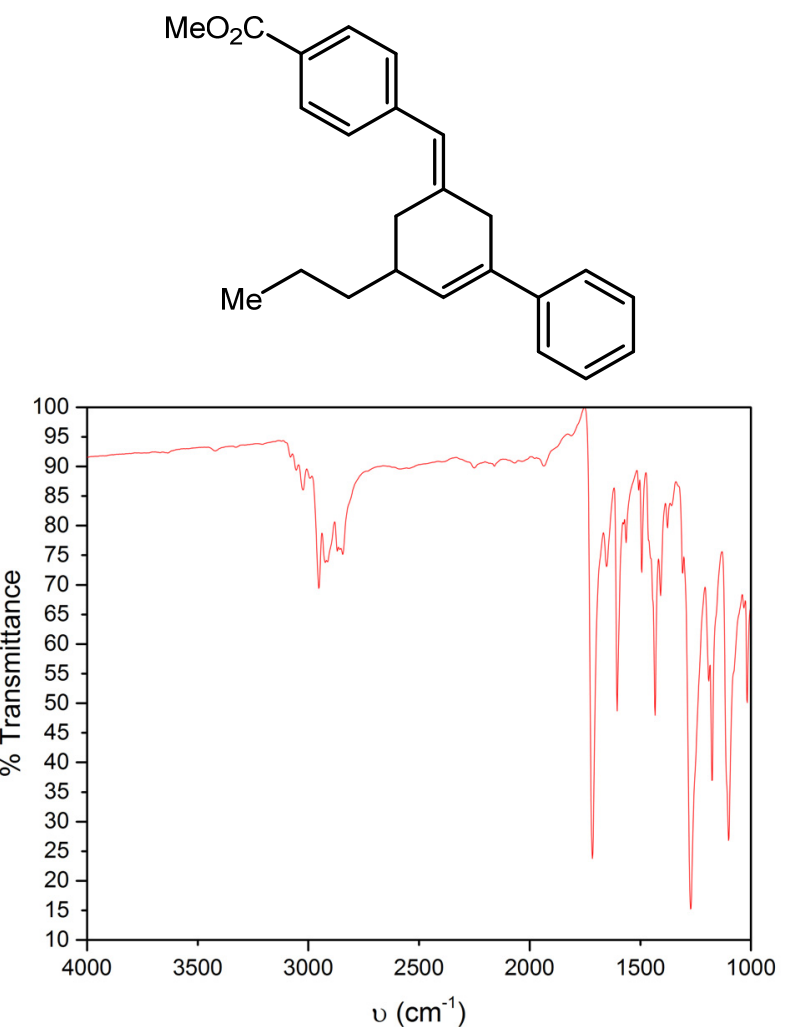

Figure S173. FT-IR of S18.

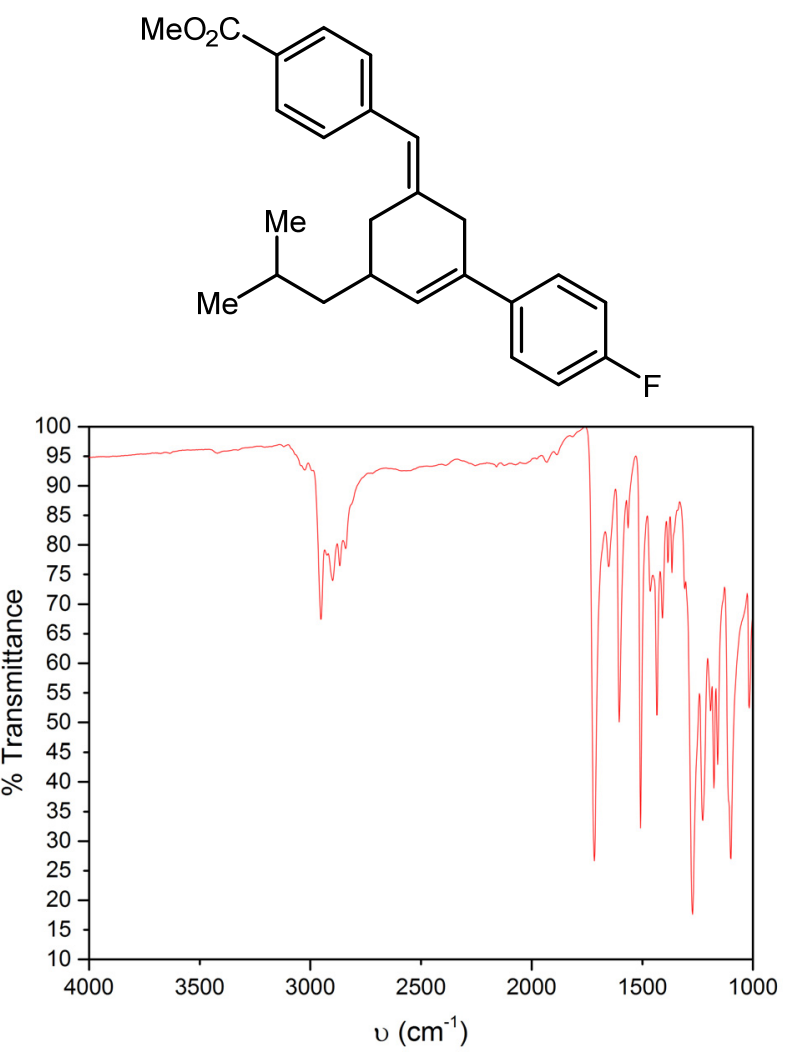

Figure S174. FT-IR of S19. 


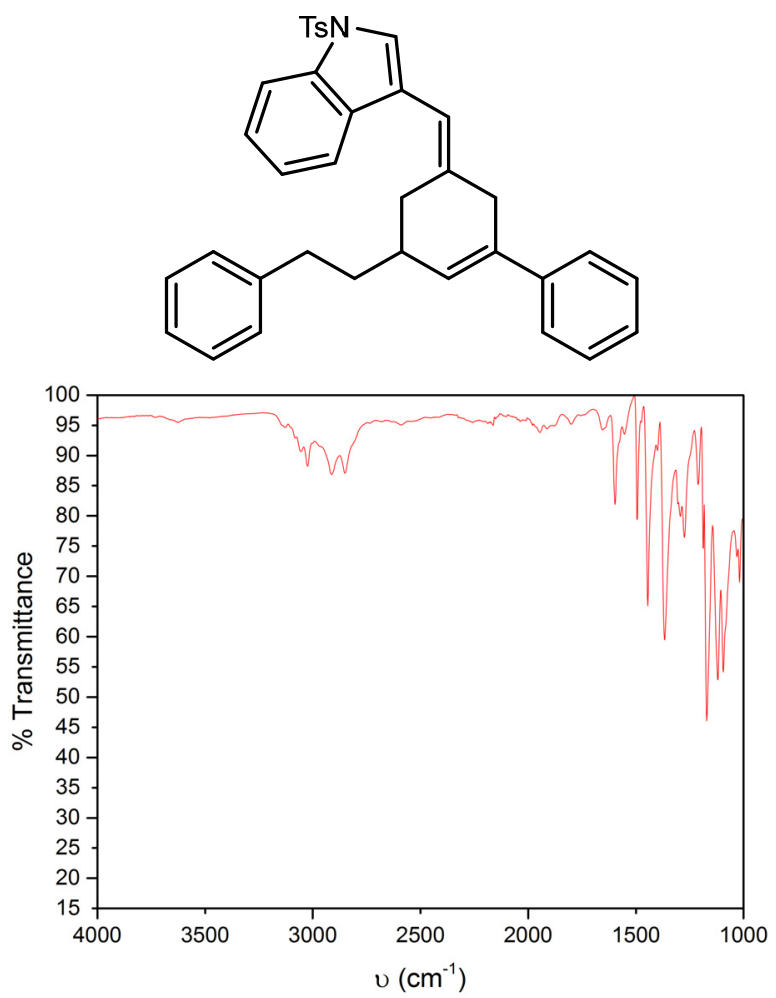

Figure S175. FT-IR of S20.

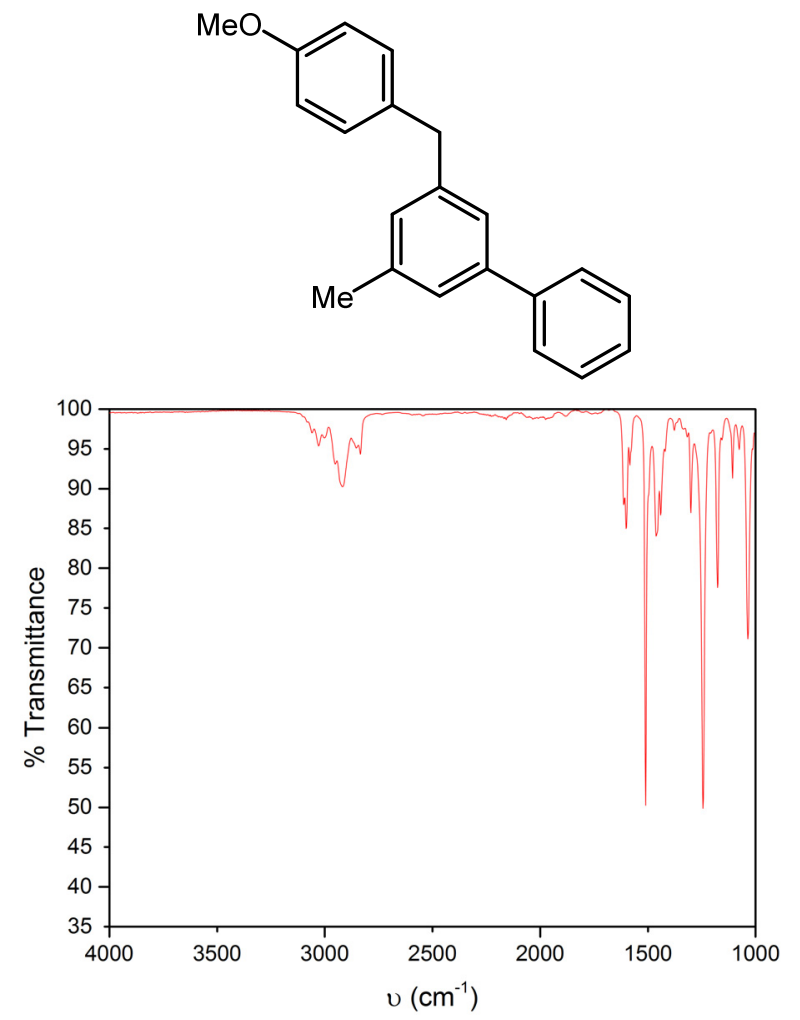

Figure S176. $\quad$ FT-IR of 38. 


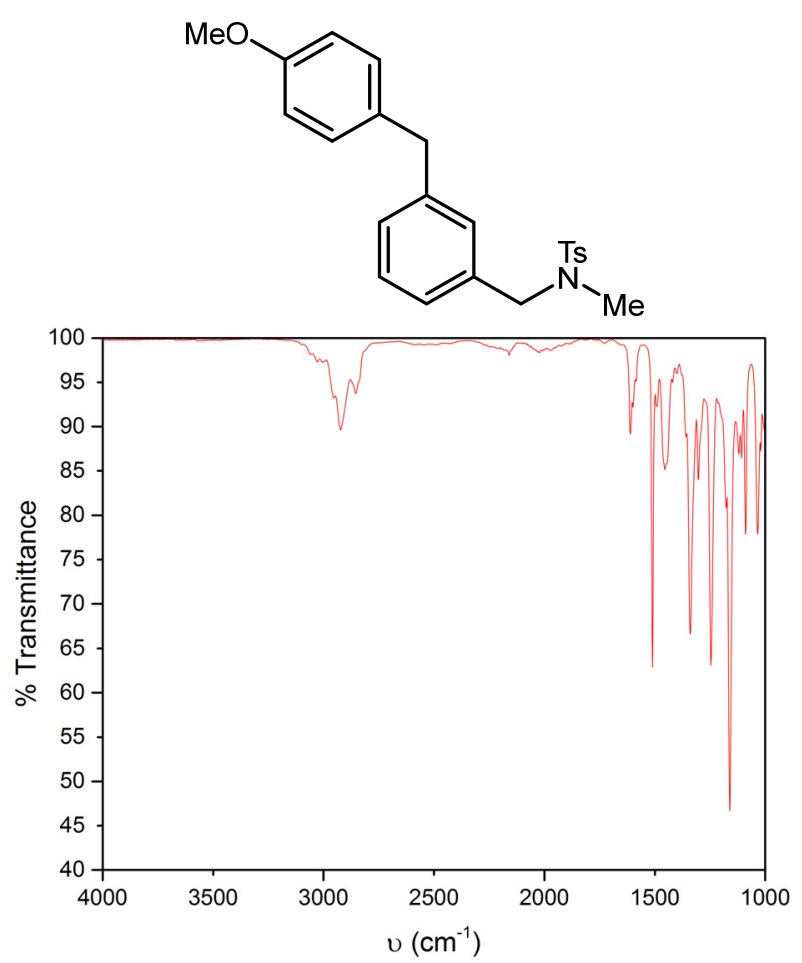

Figure S177. FT-IR of 39.
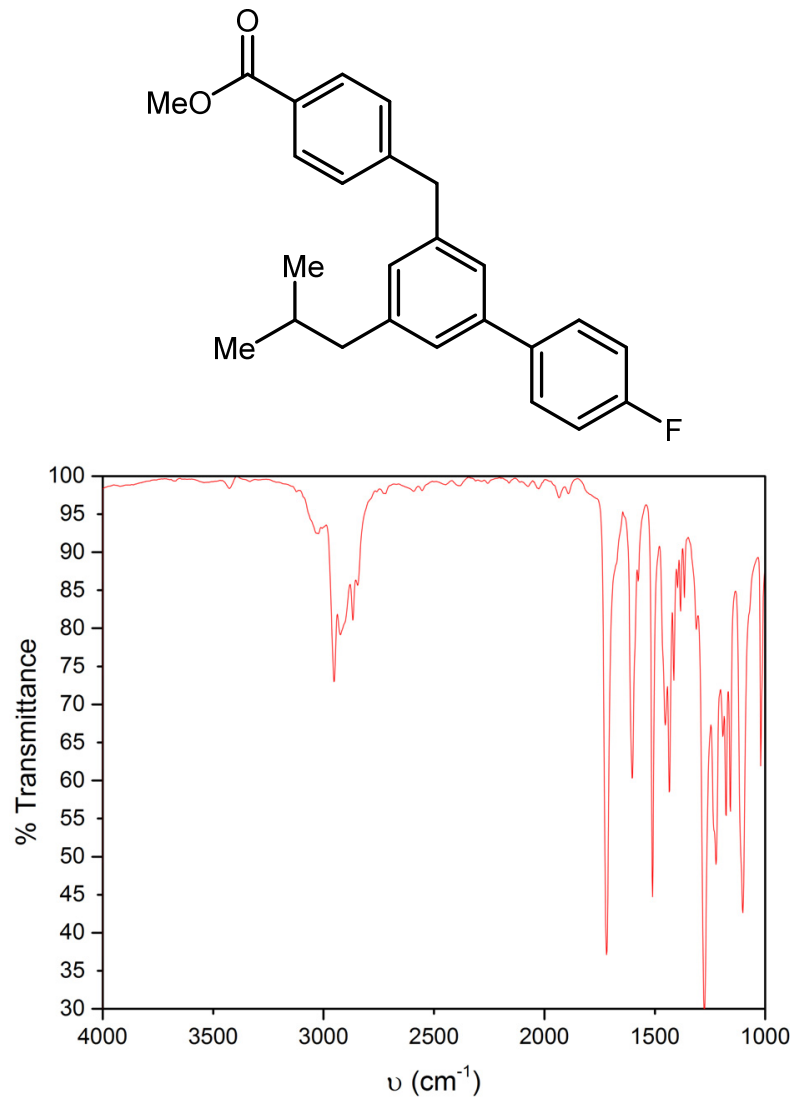

Figure S178. FT-IR of $\mathbf{4 0 .}$ 


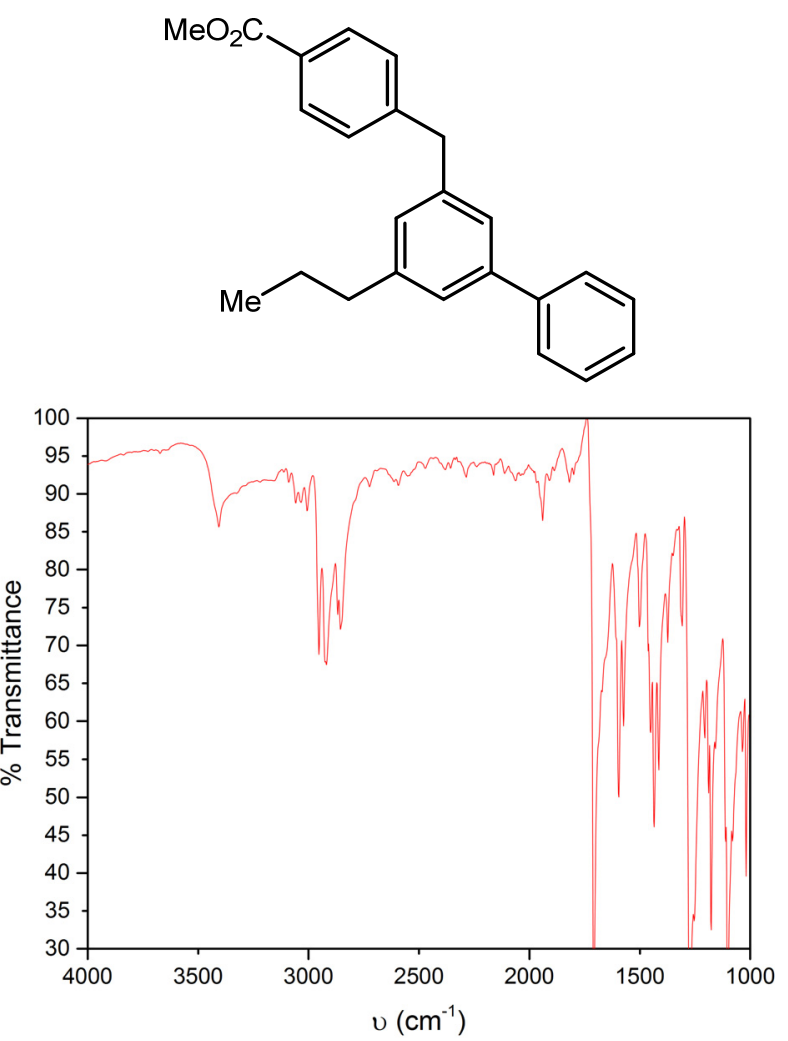

Figure S179. FT-IR of 41.
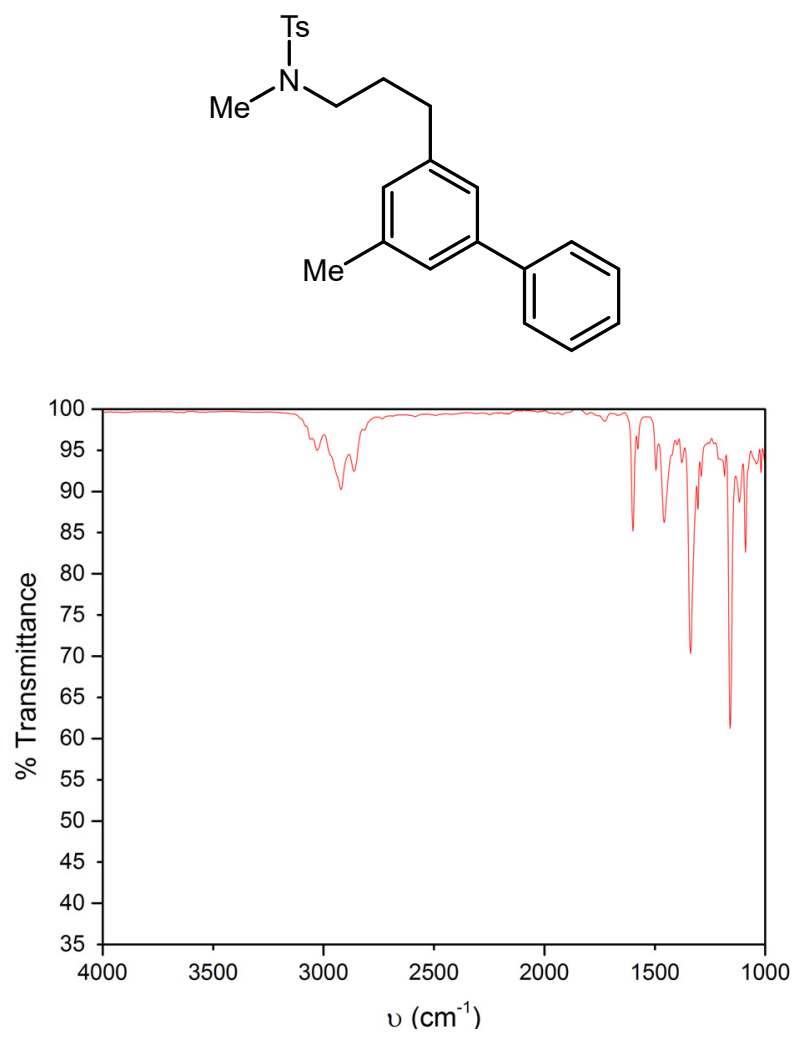

Figure S180. $\quad$ FT-IR of 42. 


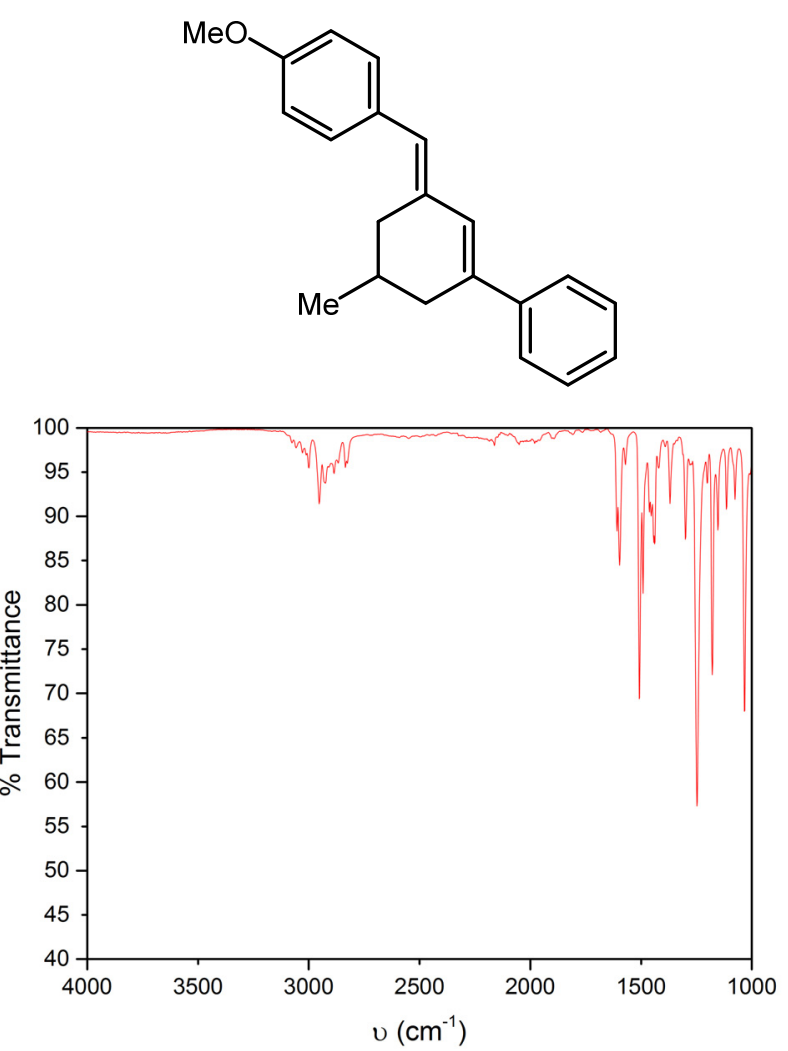

Figure S181. FT-IR of 43.<smiles>O=C(c1cc(CCc2ccccc2)cc(-c2ccccc2)c1)c1c[nH]c2ccccc12</smiles>

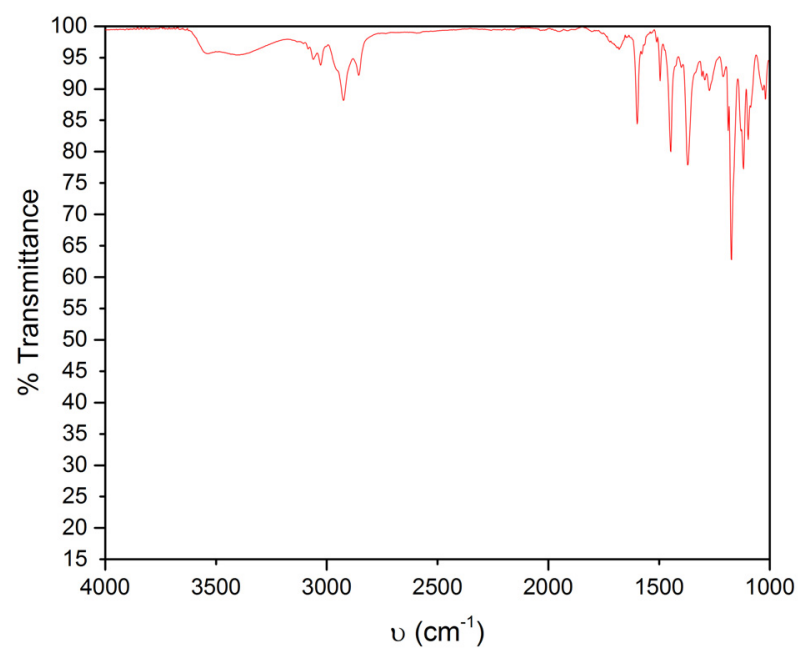

Figure S182. FT-IR of 44. 


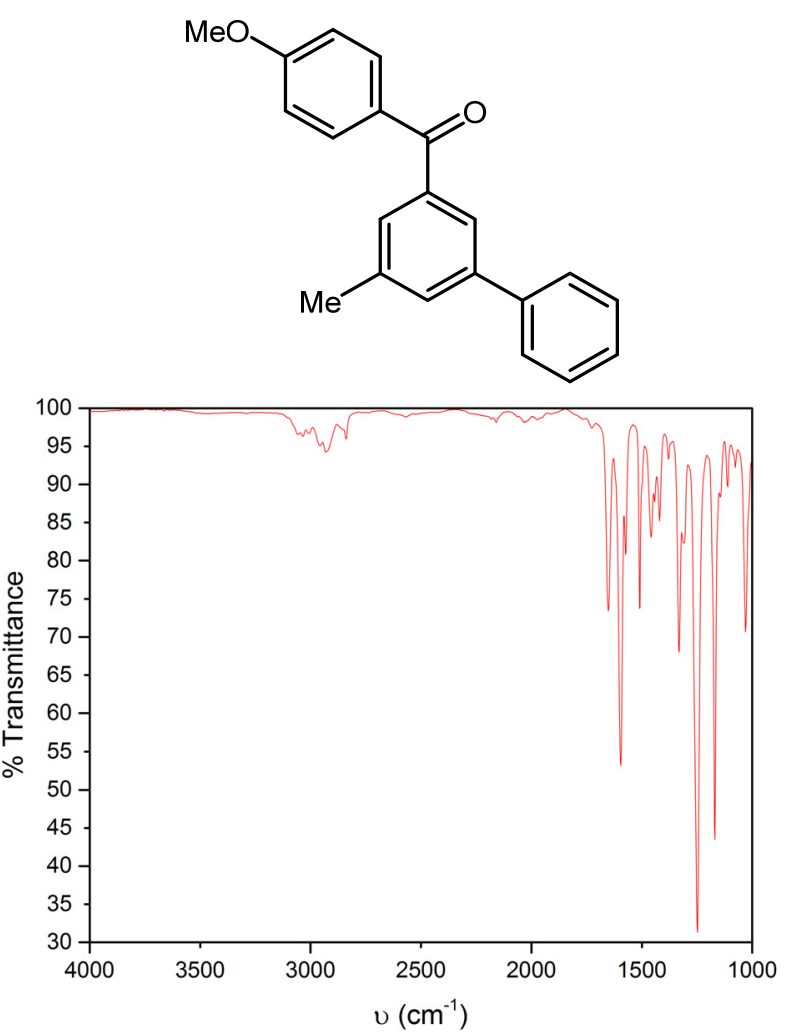

Figure S183. FT-IR of 45.

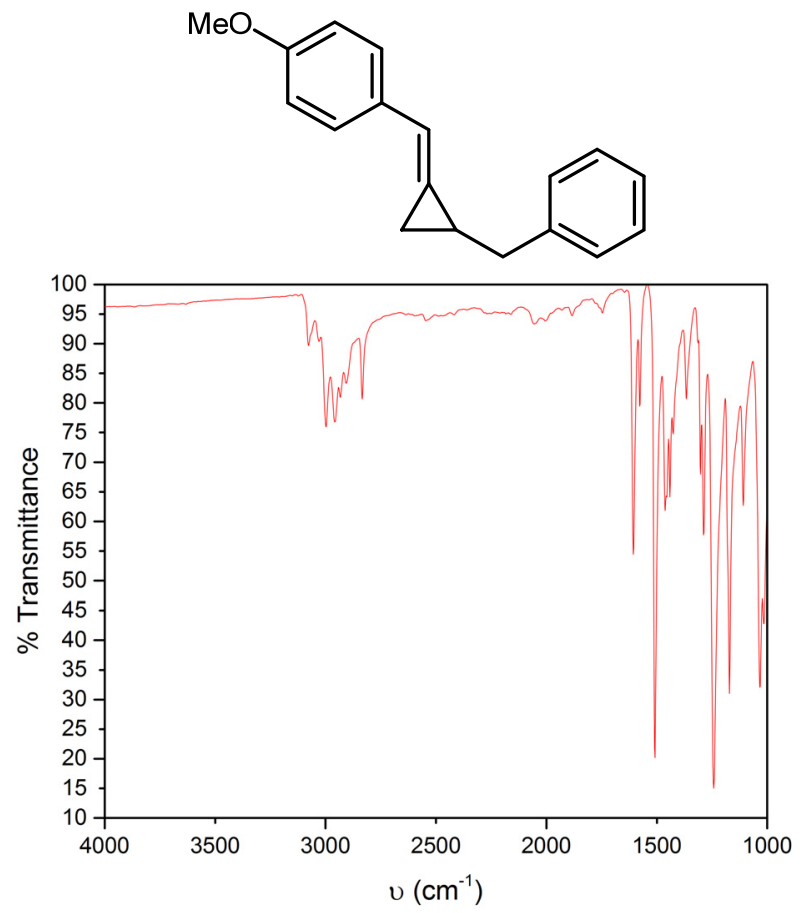

Figure S184. FT-IR of 47. 

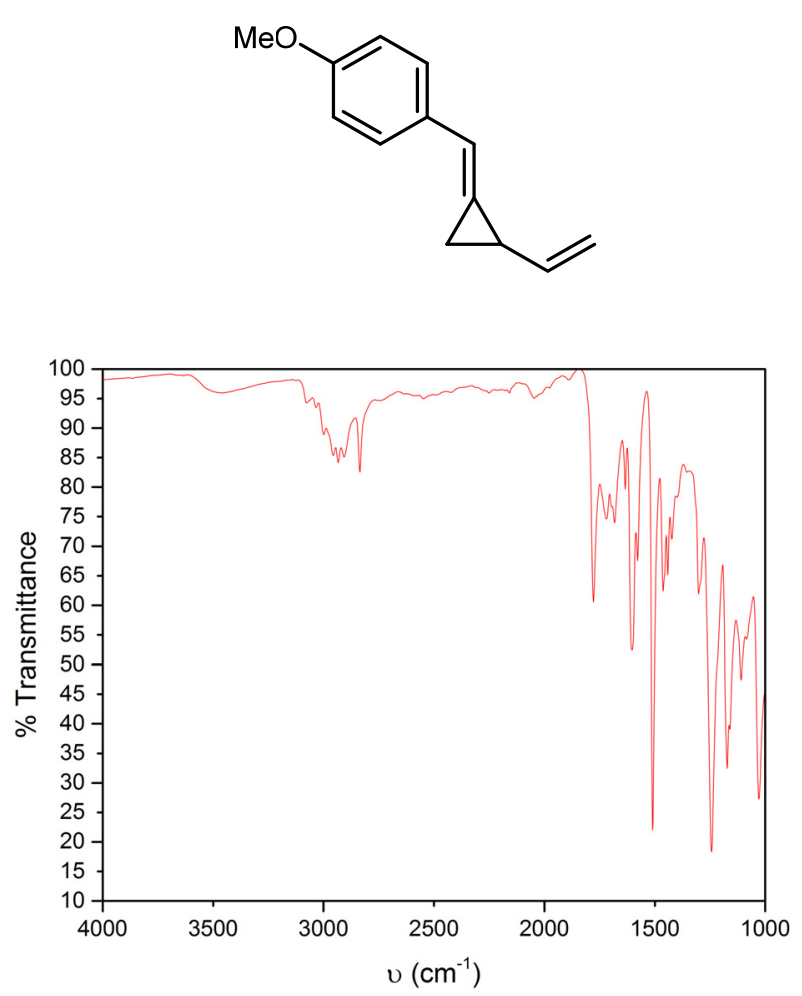

Figure S185. FT-IR of S21.
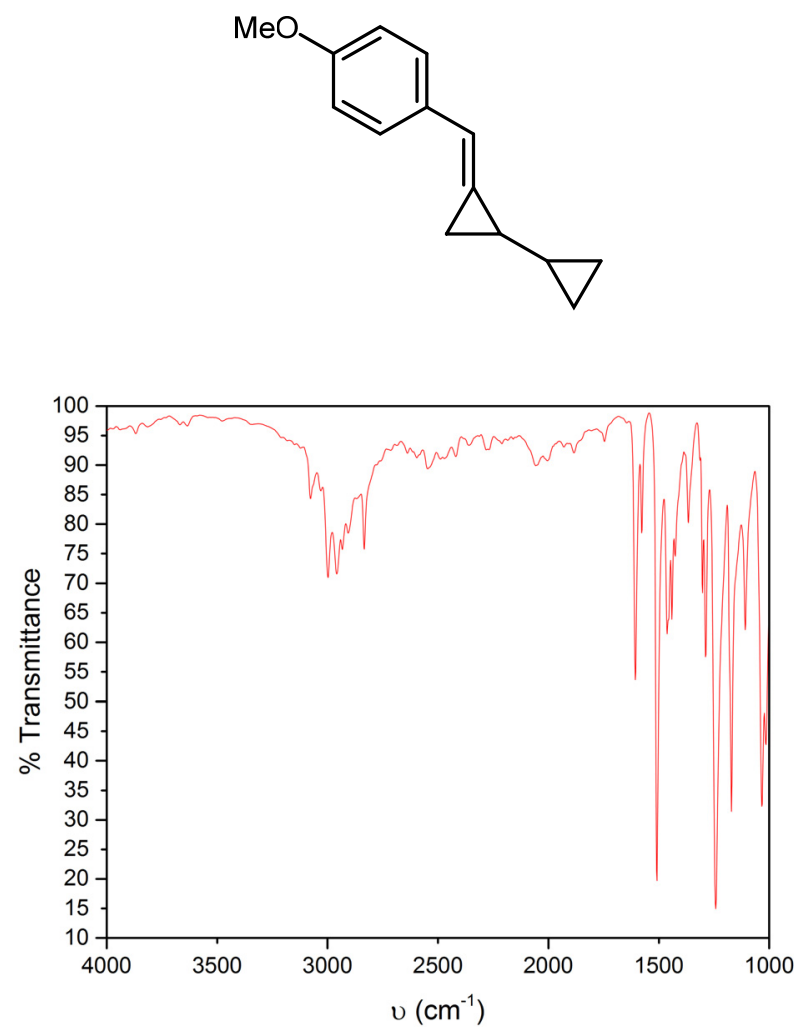

Figure S186. FT-IR of 4. 


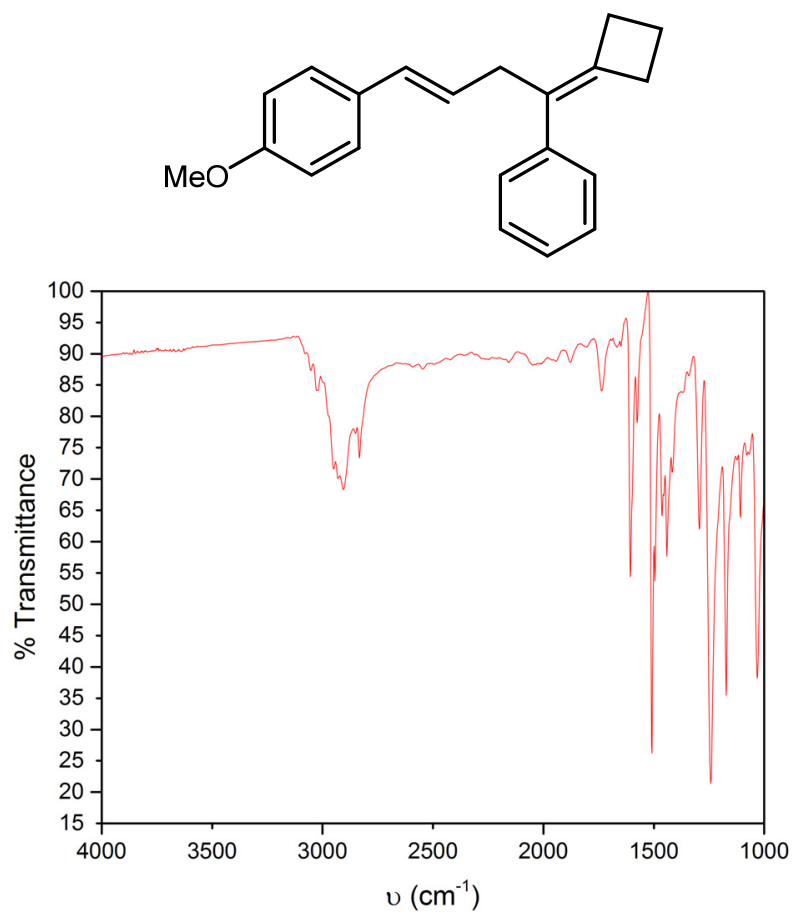

Figure S187. FT-IR of 49. 


\section{X-Ray Diffraction Data}

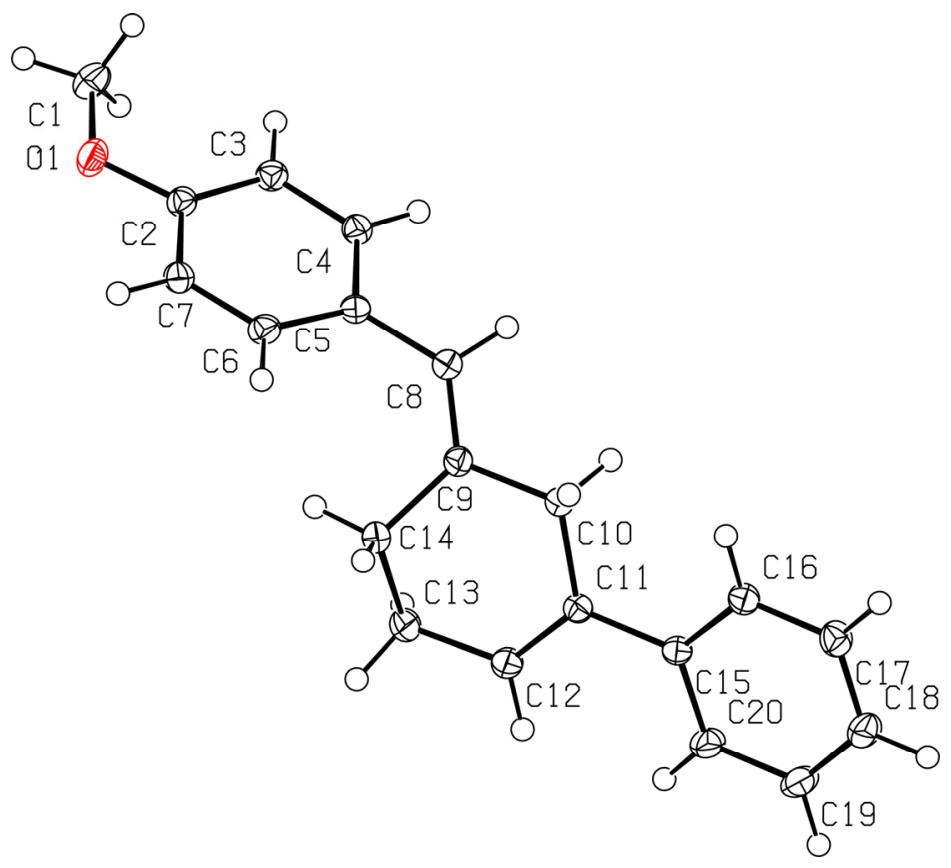

\section{Compound 6}

\section{Crystal data}

\begin{tabular}{|l|l|}
\hline Chemical formula & $\mathrm{C}_{20} \mathrm{H}_{20} \mathrm{O}$ \\
\hline
\end{tabular}

\begin{tabular}{|l|l|}
\hline Crystal system, space group & Triclinic, $P \overline{1}$
\end{tabular}

\begin{tabular}{|l|l|}
\hline Temperature $(\mathrm{K})$ & 150
\end{tabular}

\begin{tabular}{|l|l|}
\hline$a, b, c(\AA)$ & $9.6419(16), 9.7478(14), 9.9639(15)$
\end{tabular}

\begin{tabular}{|l|l|}
\hline$a, b, \gamma\left({ }^{\circ}\right)$ & $65.235(5), 62.617(4), 67.916(4)$
\end{tabular}

\begin{tabular}{|l|l|}
\hline$V\left(\AA^{3}\right)$ & $735.3(2)$ \\
\hline$Z$
\end{tabular}

\begin{tabular}{|l|l|}
\hline$Z$ & 2 \\
\hline
\end{tabular}

\begin{tabular}{|l|l|}
\hline$F(000)$ & 296 \\
\hline
\end{tabular}

\begin{tabular}{|l|l}
\hline$D_{x}\left(\mathrm{Mg} \mathrm{m}^{-3}\right)$ & 1.248 \\
\hline Radiation type
\end{tabular}

\begin{tabular}{|l|l|}
\hline Radiation type & Mo $K \alpha$
\end{tabular}

\begin{tabular}{|l|l} 
No. of reflections for cell & 9917
\end{tabular}

measurement

\begin{tabular}{|l|l|}
\hline$\theta$ range $\left(^{\circ}\right)$ for cell & $2.4-33.2$
\end{tabular}

measurement

\begin{tabular}{|l|l}
\hline$\mu\left(\mathrm{mm}^{-1}\right)$ & 0.08 \\
\hline
\end{tabular}

\begin{tabular}{|l|l|}
\hline Crystal shape & Block \\
\hline
\end{tabular}

\begin{tabular}{|l|l}
\hline Colour & Colorless \\
\hline
\end{tabular}

\begin{tabular}{|l|l}
\hline Crystal size $(\mathrm{mm})$ & $0.60 \times 0.40 \times 0.20$ \\
\hline
\end{tabular}

\section{Data collection}

\begin{tabular}{|l|l|}
\hline Diffractometer & Bruker AXS D8 Quest CMOS
\end{tabular}

diffractometer

Radiation source sealed tube X-ray source

Monochromator 


\begin{tabular}{|c|c|}
\hline Scan method & $\omega$ and phi scans \\
\hline Absorption correction & $\begin{array}{l}\text { Multi-scan } \\
\text { SADABS 2016/2: Krause, L., Herbst-Irmer, R., Sheldrick G.M. \& Stalke D., J. Appl. Cryst. } \\
48 \text { (2015) 3-10 }\end{array}$ \\
\hline$T_{\min }, T_{\max }$ & $0.721,0.747$ \\
\hline $\begin{array}{l}\text { No. of measured, } \\
\text { independent and } \\
\text { observed }[I>2 \sigma(I)] \\
\text { reflections }\end{array}$ & $20324,5621,4479$ \\
\hline$R_{\text {int }}$ & 0.027 \\
\hline$\theta$ values $\left({ }^{\circ}\right)$ & $\theta_{\max }=33.2, \theta_{\min }=2.4$ \\
\hline$(\sin \theta / \lambda)_{\max }\left(\AA^{-1}\right)$ & 0.771 \\
\hline Range of $h, k, l$ & $h=-14 \rightarrow 14, k=-15 \rightarrow 14, l=-15 \rightarrow 15$ \\
\hline \multicolumn{2}{|l|}{ Refinement } \\
\hline Refinement on & $F^{2}$ \\
\hline$R\left[F^{2}>2 \sigma\left(F^{2}\right)\right], w R\left(F^{2}\right), S$ & $0.046,0.138,1.03$ \\
\hline No. of reflections & 5621 \\
\hline No. of parameters & 191 \\
\hline No. of restraints & 0 \\
\hline H-atom treatment & H-atom parameters constrained \\
\hline Weighting scheme & $\begin{array}{l}w=1 /\left[\sigma^{2}\left(F_{0}^{2}\right)+(0.0786 P)^{2}+0.1416 P\right] \\
\text { where } P=\left(F_{0}^{2}+2 F_{\mathrm{c}}^{2}\right) / 3\end{array}$ \\
\hline$(\Delta / \sigma)_{\max }$ & 0.001 \\
\hline$\Delta \rho_{\max }, \Delta \rho_{\min }\left(\mathrm{e} \AA^{-3}\right)$ & $0.39,-0.23$ \\
\hline
\end{tabular}




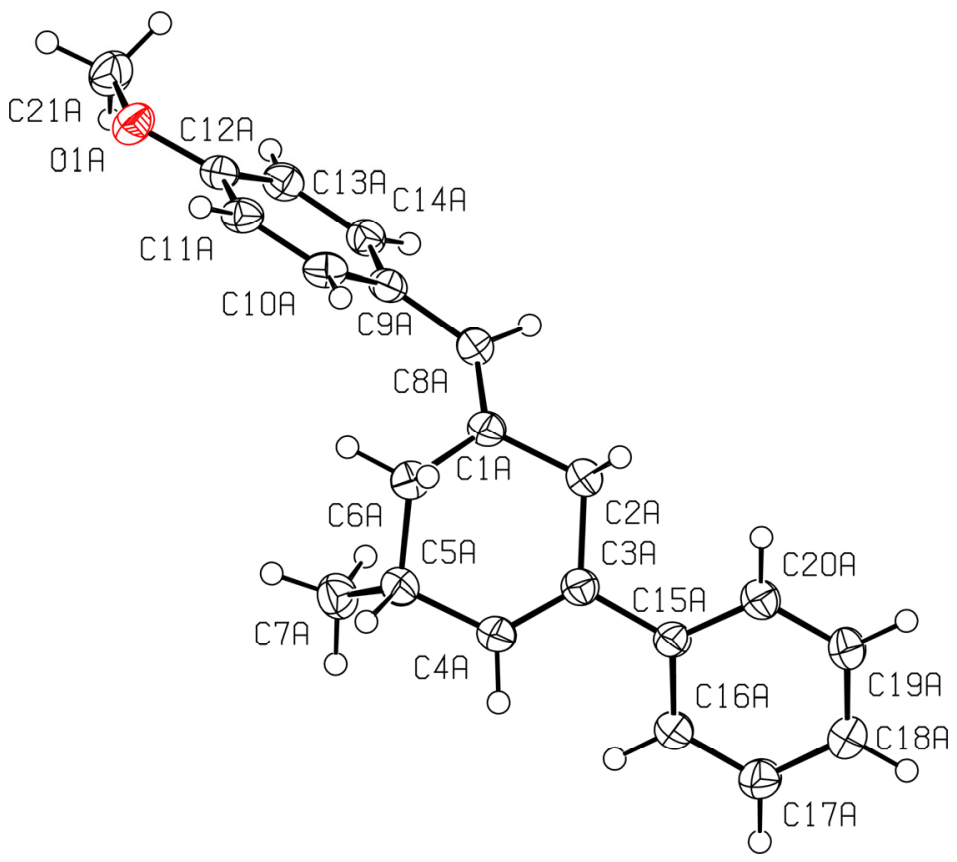

Compound 30

\begin{tabular}{|c|c|}
\hline \multicolumn{2}{|l|}{ Crystal data } \\
\hline Chemical formula & $\mathrm{C}_{21} \mathrm{H}_{22} \mathrm{O}$ \\
\hline$M_{\mathrm{r}}$ & 290.38 \\
\hline Crystal system, space group & Triclinic, $P \overline{1}$ \\
\hline \begin{tabular}{|l|l} 
Temperature $(\mathrm{K})$ \\
\end{tabular} & 150 \\
\hline$a, b, c(\AA)$ & $10.0693(2), 10.1210(2), 18.5597(4)$ \\
\hline$\alpha, \beta, \gamma\left(^{\circ}\right)$ & $85.6232(13), 85.2461(14), 60.4624(14)$ \\
\hline$V\left(\AA^{3}\right)$ & $1638.59(6)$ \\
\hline$Z$ & 4 \\
\hline$F(000)$ & 624 \\
\hline$D_{x}\left(\mathrm{Mg} \mathrm{m}^{-3}\right)$ & 1.177 \\
\hline Radiation type & $\mathrm{Cu} K \alpha$ \\
\hline $\begin{array}{l}\text { No. of reflections for cell } \\
\text { measurement }\end{array}$ & 9828 \\
\hline $\begin{array}{l}\theta \text { range }\left(^{\circ}\right) \text { for cell } \\
\text { measurement }\end{array}$ & $4.8-79.6$ \\
\hline$\mu\left(\mathrm{mm}^{-1}\right)$ & 0.54 \\
\hline Crystal shape & Block \\
\hline Colour & Colourless \\
\hline Crystal size $(\mathrm{mm})$ & $0.17 \times 0.15 \times 0.13$ \\
\hline \multicolumn{2}{|l|}{ Data collection } \\
\hline Diffractometer & $\begin{array}{l}\text { Bruker AXS D8 Quest CMOS } \\
\text { diffractometer }\end{array}$ \\
\hline Radiation source & I-mu-S microsource X-ray tube \\
\hline Monochromator & Laterally graded multilayer (Goebel) mirror \\
\hline Scan method & $\omega$ and phi scans \\
\hline Absorption correction & $\begin{array}{l}\text { Multi-scan } \\
\text { SADABS 2016/2: Krause, L., Herbst-Irmer, R., Sheldrick G.M. \& Stalke D., J. Appl. Cryst. }\end{array}$ \\
\hline
\end{tabular}




\begin{tabular}{|c|c|}
\hline & $48(2015) 3-10$ \\
\hline$T_{\min }, T_{\max }$ & $0.644,0.754$ \\
\hline $\begin{array}{l}\text { No. of measured, } \\
\text { independent and } \\
\text { observed }[I>2 \sigma(I)] \\
\text { reflections }\end{array}$ & $25527,12612,11829$ \\
\hline$R_{\text {int }}$ & 0.039 \\
\hline$\theta$ values $\left({ }^{\circ}\right)$ & $\theta_{\max }=80.6, \theta_{\min }=2.4$ \\
\hline$(\sin \theta / \lambda)_{\max }\left(\AA^{-1}\right)$ & 0.640 \\
\hline Range of $h, k, l$ & $h=-12 \rightarrow 12, k=-12 \rightarrow 12, l=-23 \rightarrow 23$ \\
\hline \multicolumn{2}{|l|}{ Refinement } \\
\hline Refinement on & $F^{2}$ \\
\hline$R\left[F^{2}>2 \sigma\left(F^{2}\right)\right], w R\left(F^{2}\right), S$ & $0.040,0.104,1.04$ \\
\hline No. of reflections & 12612 \\
\hline No. of parameters & 802 \\
\hline No. of restraints & 3 \\
\hline $\mathrm{H}$-atom treatment & H-atom parameters constrained \\
\hline Weighting scheme & $\begin{array}{l}w=1 /\left[\sigma^{2}\left(F_{0}^{2}\right)+(0.0449 P)^{2}+0.2845 P\right] \\
\text { where } P=\left(F_{0}^{2}+2 F_{c}^{2}\right) / 3\end{array}$ \\
\hline$(\Delta / \sigma)_{\max }$ & 0.001 \\
\hline$\left.\Delta\rangle_{\max }, \Delta\right\rangle_{\min }\left(\mathrm{e} \AA^{-3}\right)$ & $0.28,-0.20$ \\
\hline Extinction method & SHELXL2018/3 (Sheldrick 2018), $\mathrm{Fc}^{*}=\mathrm{kFc}\left[1+0.001 \mathrm{xFc}^{2} \lambda^{3} / \sin (2 \theta)\right]^{-1 / 4}$ \\
\hline Extinction coefficient & $0.0008(3)$ \\
\hline Absolute structure & $\begin{array}{l}\text { Flack x determined using } 4857 \text { quotients [(I+)-(I-)]/[(I+)+(I-)] (Parsons, Flack and } \\
\text { Wagner, Acta Cryst. B69 (2013) 249-259). }\end{array}$ \\
\hline $\begin{array}{l}\text { Absolute structure } \\
\text { parameter }\end{array}$ & $-0.04(11)$ \\
\hline
\end{tabular}




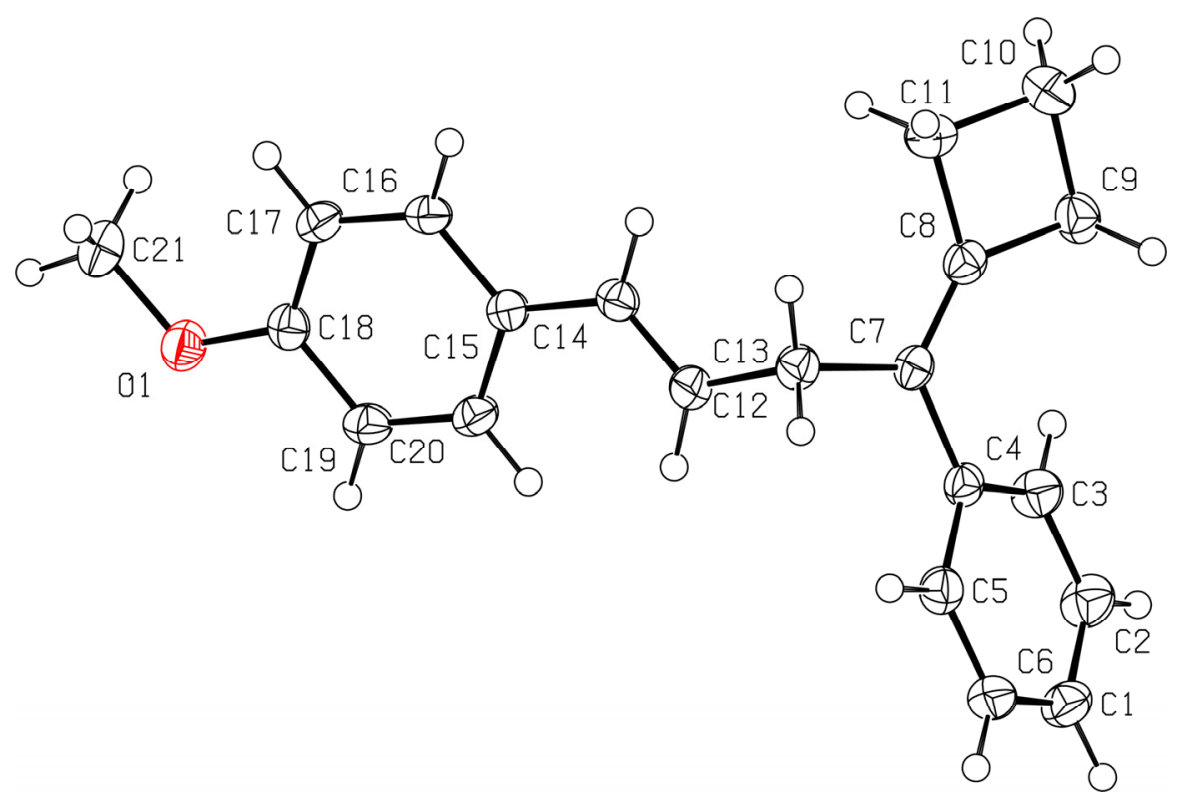

Compound 49

\begin{tabular}{|c|c|}
\hline \multicolumn{2}{|l|}{ Crystal data } \\
\hline Chemical formula & $\mathrm{C}_{21} \mathrm{H}_{22} \mathrm{O}$ \\
\hline$M_{\mathrm{r}}$ & 290.38 \\
\hline Crystal system, space group & Triclinic, $P \overline{1}$ \\
\hline Temperature $(\mathrm{K})$ & 150 \\
\hline$a, b, c(\AA)$ & 9.5996 (7), $9.8322(7), 18.0348(13)$ \\
\hline$\alpha, \beta, \gamma\left({ }^{\circ}\right)$ & 87.595 (3), $77.541(3), 75.984(3)$ \\
\hline$V\left(\AA^{3}\right)$ & $1612.6(2)$ \\
\hline$Z$ & 4 \\
\hline$F(000)$ & 624 \\
\hline$D_{x}\left(\mathrm{Mg} \mathrm{m}^{-3}\right)$ & 1.196 \\
\hline Radiation type & Mo $K \alpha$ \\
\hline $\begin{array}{l}\text { No. of reflections for cell } \\
\text { measurement }\end{array}$ & 4620 \\
\hline $\begin{array}{l}\theta \text { range }\left(^{\circ}\right) \text { for cell } \\
\text { measurement }\end{array}$ & $2.3-30.5$ \\
\hline$\mu\left(\mathrm{mm}^{-1}\right)$ & 0.07 \\
\hline Crystal shape & Plate \\
\hline Colour & Colorless \\
\hline Crystal size $(\mathrm{mm})$ & $0.5 \times 0.5 \times 0.5$ \\
\hline \multicolumn{2}{|l|}{ Data collection } \\
\hline Diffractometer & $\begin{array}{l}\text { Bruker AXS D8 Quest CMOS } \\
\text { diffractometer }\end{array}$ \\
\hline Radiation source & sealed tube X-ray source \\
\hline Scan method & $\omega$ and phi scans \\
\hline Absorption correction & $\begin{array}{l}\text { Multi-scan } \\
\text { SADABS 2016/2: Krause, L., Herbst-Irmer, R., Sheldrick G.M. \& Stalke D., J. Appl. Cryst. } \\
48 \text { (2015) 3-10 }\end{array}$ \\
\hline$T_{\min }, T_{\max }$ & $0.642,0.747$ \\
\hline
\end{tabular}




\begin{tabular}{|c|c|}
\hline $\begin{array}{l}\text { No. of measured, } \\
\text { independent and } \\
\text { observed }[I>2 \sigma(I)] \\
\text { reflections }\end{array}$ & $76183,9831,6241$ \\
\hline$R_{\text {int }}$ & 0.076 \\
\hline$\theta$ values $\left(^{\circ}\right)$ & $\theta_{\max }=30.5, \theta_{\min }=2.3$ \\
\hline$(\sin \theta / \lambda)_{\max }\left(\AA^{-1}\right)$ & 0.714 \\
\hline Range of $h, k, l$ & $h=-13 \rightarrow 13, k=-14 \rightarrow 14, l=-25 \rightarrow 25$ \\
\hline \multicolumn{2}{|l|}{ Refinement } \\
\hline Refinement on & $F^{2}$ \\
\hline$R\left[F^{2}>2 \sigma\left(F^{2}\right)\right], w R\left(F^{2}\right), S$ & $0.055,0.141,1.01$ \\
\hline No. of reflections & 9831 \\
\hline No. of parameters & 399 \\
\hline No. of restraints & 0 \\
\hline H-atom treatment & H-atom parameters constrained \\
\hline Weighting scheme & $\begin{array}{l}w=1 /\left[\sigma^{2}\left(F_{0}^{2}\right)+(0.0615 P)^{2}+0.412 P\right] \\
\text { where } P=\left(F_{0}^{2}+2 F_{\mathrm{c}}^{2}\right) / 3\end{array}$ \\
\hline$(\Delta / \sigma)_{\max }$ & 0.001 \\
\hline$\left.\Delta\rangle_{\max }, \Delta\right\rangle_{\min }\left(\mathrm{e} \AA^{-3}\right)$ & $0.35,-0.27$ \\
\hline
\end{tabular}




\section{References}

(1) Zhou, Y.-Y.; Hartline, D. R.; Steiman, T. J.; Fanwick, P. E.; Uyeda, C. Inorg. Chem. 2014, 53 (21), 11770.

(2) Farley, C. M.; Zhou, Y.-Y.; Banka, N.; Uyeda, C. J. Am. Chem. Soc. 2018, 140 (40), 12710.

(3) Zhou, Y.-Y.; Uyeda, C. Science. 2019, 363 (6429), 857.

(4) Pal, S.; Zhou, Y.-Y.; Uyeda, C. J. Am. Chem. Soc. 2017, 139 (34), 11686.

(5) Bruker (2016). Apex3 v2016.9-0, Saint V8.34A, SAINT V8.37A, Bruker AXS Inc.: Madison (WI), USA, 2013/2014.

(6) SHELXTL suite of programs, Version 6.14, 2000-2003, Bruker Advanced X-ray Solutions, Bruker AXS Inc., Madison, Wisconsin: USA.

(7) Sheldrick, G. Acta Crystallogr A 2008, 64 (1), 112.

(8) Sheldrick, G. University of Gottingen, Germany, 2018.

(9) Sheldrick, G. Acta. Crystallogr. Sect. C. Struct. Chem. 2015, 71 (1), 3.

(10) Clarke, C.; Foussat, S.; Fox, D. J.; Pedersen, D. S.; Warren, S. Org. Biomol. Chem. 2009, 7 (7), 1323.

(11) Amador, A. G.; Sherbrook, E. M.; Yoon, T. P. J. Am. Chem. Soc. 2016, 138 (14), 4722.

(12) Feldman, K. S.; Folda, T. S. J. Org. Chem. 2016, 81 (11), 4566.

(13) Chen, C.; Shen, X.; Chen, J.; Hong, X.; Lu, Z. Org. Lett. 2017, 19 (19), 5422.

(14) Majetich, George; Allen, S. Arkivoc 2010, 2010 (4), 104.

(15) Shao, L.-X.; Li, J.; Wang, B.-Y.; Shi, M. Eur. J. Org. Chem. 2010, 2010 (33), 6448.

(16) Werth, J.; Uyeda, C. Chem. Sci. 2018, 9 (6), 1604.

(17) Walker, J. C. L.; Oestreich, M. Org. Lett. 2018, 20 (20), 6411. 